\title{
A Precision Test of the Standard Model via Parity-Violating Electron Scattering in the $Q_{\text {weak }}$ Experiment
}

\author{
Emmanouil Kargiantoulakis \\ Charlottesville, VA \\ A Dissertation presented to the Graduate Faculty \\ of the Unversity of Virginia in Candidacy for the Degree of \\ Doctor of Philosophy \\ Department of Physics \\ University of Virginia \\ December, 2015
}




\section{Abstract}

The $Q_{\text {weak }}$ Collaboration has completed a challenging measurement of the parity-violating asymmetry in elastic $\overrightarrow{e p}$ scattering of longitudinally polarized electrons from protons at the Thomas Jefferson National Accelerator Facility (Jefferson Lab). The experiment released an early result for the parity-violating asymmetry $A_{e p}=-279 \pm 35$ (stat) \pm 31 (syst) parts-perbillion ( $\mathrm{ppb}$ ) from the commissioning period, constituting about $4 \%$ of the full data set. This result allowed the first determination of the weak charge of the proton $Q_{w}^{p}$ from a global fit of parity-violating elastic scattering (PVES) results from nuclear targets, where earlier data at higher $Q^{2}$ constrain uncertainties of hadronic structure. The value extracted from the global fit is $Q_{w}^{p}(\mathrm{PVES})=0.064 \pm 0.012$, in agreement with the standard model prediction $Q_{w}^{p}(\mathrm{SM})=$ $0.0710 \pm 0.0007$. The analysis of the full $Q_{\text {weak }}$ data is ongoing and expected to yield a value for the asymmetry within $10 \mathrm{ppb}$ of precision. Because of the suppression of $Q_{w}^{p}$, such a high precision measurement will allow the most precise extraction of the weak mixing angle below the $Z^{0}$ pole and place significant constraints to models of physics beyond the standard model.

The coupling of beam parameters to the electron spin direction can give rise to false asymmetries and bias the experimental measurement. These effects must be controlled at the 1-2 ppb level to achieve the challenging precision goals of $Q_{\text {weak }}$. We describe our efforts to suppress helicity-correlated beam parameters by optimizing the Jefferson Lab polarized source and arranging for cancellations. The correction methods to remove false asymmetries and their shortcomings are presented. Backgrounds generated at the beamline are considered to be connected to higher moments of helicity-correlated beam parameters and contributed the largest systematic uncertainty in the early result. An algorithm is developed to estimate the false asymmetry associated with this background source, improving the precision of this important systematic correction by an order of magnitude. We extract from a subset of the full $Q_{\text {weak }}$ dataset a preliminary and blinded result $A_{e p}=-226.95 \pm 9.44$ (stat) \pm 6.64 (syst) ppb, translating to a value for the weak charge $Q_{w}^{p}=0.0716 \pm 0.0053$ from a global fit of PVES asymmetries. In the absence of a blinding term this result would be in excellent agreement with the standard model prediction and constrain new physics at the multi-TeV scale. The status of ongoing analysis for important systematic corrections in the $Q_{\text {weak }}$ measurement will be presented. 
We approve the dissertation of Emmanouil Kargiantoulakis.

Prof. Kent D. Paschke, Dissertation Advisor

Provost Thomas Katsouleas, Committee Member

Prof. Nilanga Liyanage, Committee Member

Prof. Pham Q. Hung, Committee Member

Prof. James Fitz-Gerald, GSAS Representative 


\section{Acknowledgements}

It gives me great pleasure to acknowledge the contributions of the many people who have generously provided their help and support to me over the years. And I would like to begin by thanking my undergraduate advisors in the University of Athens in Greece, Professors Costas Papanicolas and Stathis Stiliaris. They introduced me to research in Nuclear Physics and inspired me to follow this path in life.

As a young graduate student coming to Jefferson Lab when the Qweak experiment was about to start taking data, I experienced what it takes to perform research at the frontiers of science during that frantic time. The totality of my doctoral studies has been a defining experience, and the hardships I encountered did not deter my love for fundamental research. I was very fortunate to have Kent as my advisor in this journey. His unwavering inquisitiveness has been incredibly valuable to the experiment and I have grown as a scientist through the challenge of keeping up with him. I thank him for being patient with me when I needed it. It has also been incredible working with Mark Dalton, whose contribution and direction in the beamline background analysis were sorely missed when he moved on. Many thanks to Mark Pitt for overseeing the beamline background analysis, along with many other analyses, and for being a generous and selfless unifying force in the experiment. I am very grateful to Greg for his relentless support and for believing in me more than I did at times. And to my senior collaborators, David Armstrong, Dave Gaskell, Dave Mack, Jay, Joe Grames, Matt Poelker, Paul, Roger: thank you for your input and openness and the many interesting conversations we shared. It has been an honour to address you as collaborators. And to my fellow Qweak students who contributed so much to the experiment: Katherine and John for leading the way, Buddhini for being so supportive during my first months in Jefferson Lab, my good friends Amrendra, Josh, Rakitha, Scott, Wade, and many more.

At the University of Virginia where I spent my later years in the program, I am thankful for the kindness and support of Nilanga Liyanage to me personally, and to our group of students who started the Graduate Physics Students Association in a spirit of togetherness and helping each other grow as physicists. To Cass, Donal, Gordon, Oscar, PQ: it has been a pleasure having 
conversations with you in the department hallways and taking your courses. Out of my many friends in Charlottesville I will mention John, who has been my very good friend for many years, and Alexandr and Sadie, for their unending inherent kindness. I am very grateful to Jennifer for being my best friend for many years, we have both learned so much from and through each other. And to Kate, my loving partner, for bringing me peace and happiness; I don't think it should be this easy and fun sharing my life with someone.

And lastly, I dedicate this dissertation to my loving family in Greece, and especially my mother and father and my brother. To you I owe the most of who I am today, and I miss you dearly. 


\section{Contents}

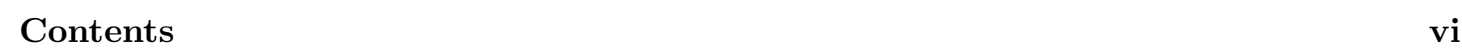

List of Figures $\quad x$

$\begin{array}{ll}\text { List of Tables } & \text { xxvii }\end{array}$

\begin{tabular}{|l} 
Acronyms \\
\hline
\end{tabular}

1 Introduction: The Electroweak Standard Model 1

\begin{tabular}{|lll}
\hline 2 & The Weak Charge of the Proton and Beyond & 7
\end{tabular}

$2.1 \quad$ Parity Violation in the Weak Interactions . . . . . . . . . . . . . . . . . . . 7

2.2 Parity-Violating Electron Scattering $\ldots \ldots \ldots \ldots \ldots \ldots$

2.3 The World PVES Program $\ldots \ldots \ldots \ldots \ldots \ldots$

$2.4 \quad$ First Determination of the Weak Charge of the Proton . . . . . . . . . . . 16

2.5 The Electroweak Mixing Angle $\ldots \ldots \ldots \ldots$

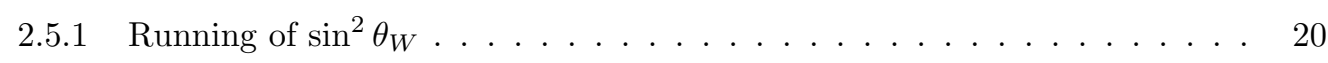

2.5 .2 Experimental determinations $\ldots \ldots \ldots \ldots \ldots \ldots$

$2.6 \quad$ Access to Physics Beyond the Standard Model $\ldots \ldots \ldots \ldots$. . . . . . . . . 25

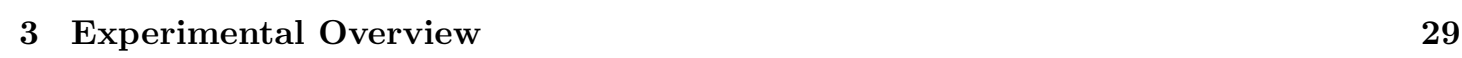

$3.1 \quad$ Parity-Violating Asymmetry . . . . . . . . . . . . . . . . . . . . . . . . . . . 31

3.2 The Continuous Electron Beam Accelerator Facility $\ldots \ldots$. . . . . . . . . . . . 33

3.3 Target System $\ldots \ldots \ldots \ldots$

$3.3 .1 \quad$ Target density fluctuations $\ldots \ldots \ldots \ldots \ldots \ldots$

3.3 .2 Aluminum windows and dummy targets . . . . . . . . . . . . . 36

$3.4 \quad$ Collimators and Shielding $\ldots \ldots \ldots \ldots$

3.5 Toroidal Spectrometer $\ldots \ldots \ldots \ldots \ldots$. . . . . . . . . . . . . . 40

3.6 Detector System $\ldots \ldots \ldots \ldots \ldots \ldots$. . . . . . . . . . . . . . . . . 40

3.6 .1 Main Detectors . . . . . . . . . . . . . . . . . . . . . . . 40 
$3.6 .2 \quad$ Luminosity monitors and background detectors $\ldots \ldots \ldots$. . . . . . . . . 42

$3.6 .3 \quad$ Kinematics and tracking detectors $\ldots \ldots \ldots \ldots$. . . . . . . 45

3.7 Beam Monitors . . . . . . . . . . . . . . . . . . . . . . . . . . 47

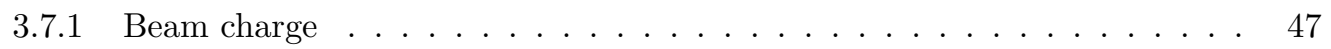

$3.7 .2 \quad$ Beam orbit and energy $\ldots \ldots \ldots \ldots \ldots$

$3.7 .3 \quad$ Beam halo and profile monitors . . . . . . . . . . . . . . . 51

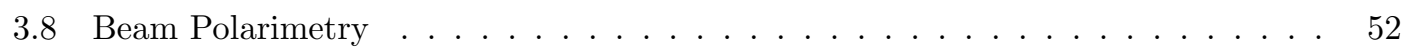

$3.8 .1 \quad$ Møller polarimeter . . . . . . . . . . . . . . . . . . . . . 52

3.8 .2 Compton polarimeter $\ldots \ldots \ldots \ldots \ldots \ldots$

$3.9 \quad$ Timeline and Datasets $\ldots \ldots \ldots \ldots$

\begin{tabular}{|lll}
4 & Optimization of the Jefferson Lab Polarized Source & 59
\end{tabular}

4.1 Polarized Source Specifications . . . . . . . . . . . . . . . . . . . . . . . . . 60

4.2 Helicity Signal $\ldots \ldots \ldots \ldots$

4.3 Laser Table Configuration $\ldots \ldots \ldots$. . . . . . . . . . . . . . . . . . . . . . . . . 64

$4.3 .1 \quad$ Laser system $\ldots \ldots \ldots$. . . . . . . . . . . . . . . . . . 64

4.3 .2 Polarization control $\ldots \ldots \ldots \ldots$. . . . . . . . . . . . . 65

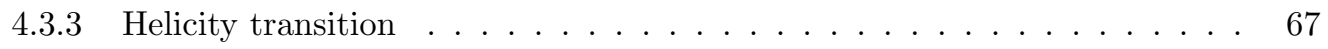

4.4 Photocathode . . . . . . . . . . . . . . . . . . . . . . . . . . . . . . . . . . 69

4.4 .1 Cathode lifetime $\ldots \ldots \ldots \ldots \ldots$

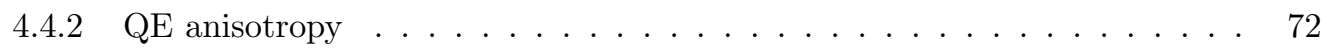

4.5 Injector Spin Manipulation System . . . . . . . . . . . . . . . . . . . . 72

4.6 Generation of False Beam Asymmetries . . . . . . . . . . . . . . . . . . . 74

$4.6 .1 \quad$ Generation of intensity (charge) asymmetry $\ldots \ldots \ldots . \ldots 74$

$4.6 .2 \quad$ PITA scans $\ldots \ldots \ldots \ldots \ldots \ldots \ldots$

$4.6 .3 \quad$ Helicity-correlated position differences $\ldots \ldots \ldots \ldots$

$4.6 .4 \quad$ Beam spot size asymmetry $\ldots \ldots \ldots \ldots \ldots$

$4.6 .5 \quad$ Higher-order effects $\ldots \ldots \ldots \ldots 1$

4.7 Polarized Source Optimization $\ldots \ldots \ldots \ldots$

$4.7 .1 \quad$ Optimization strategy $\ldots \ldots \ldots \ldots \ldots$

4.7 .2 Laser table studies . . . . . . . . . . . . . . . . . . . . . . 85

$4.7 .3 \quad$ Optimization on electron beam $\ldots \ldots \ldots \ldots$. . . . . . 86

4.7 .4 Results in the injector . . . . . . . . . . . . . . . . . . . 88

$4.8 \quad$ Adiabatic Damping $\ldots \ldots \ldots \ldots$

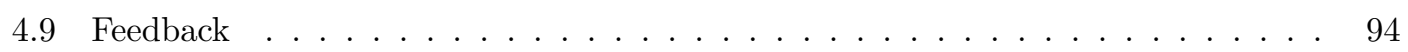

$4.9 .1 \quad$ Charge feedback $\ldots \ldots \ldots \ldots \ldots \ldots$ 
4.9 .2 Helicity magnets $\ldots \ldots \ldots \ldots \ldots \ldots$

4.10 Performance: HC Beam Properties in the Experiment . . . . . . . . . . . . . . 96

$\begin{array}{llr}5 \text { Beam Corrections } & 100\end{array}$

5.1 Regression . . . . . . . . . . . . . . . . . . . . . . . . . . . 101

$5.1 .1 \quad$ Potential issues with Regression $\ldots \ldots \ldots$. . . . . . . . . . 103

5.2 Modulation . . . . . . . . . . . . . . . . . . . . . . . . . . . . 109

5.2 .1 Interference with FFB $\ldots \ldots \ldots \ldots \ldots$

5.2 .2 Sine-cosine analysis and residuals $\ldots \ldots \ldots \ldots$. . . . . . . . 113

$5.2 .3 \quad$ Modulation residual responses $\ldots \ldots \ldots$. . . . . . . . . . . . . 114

5.3 Comparison Between Regression and Modulation . . . . . . . . . . . . . 115

5.4 Residual Beam Effects at Longer Time Scales . . . . . . . . . . . . . . . . . 117

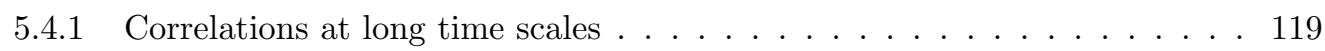

5.4 .2 Time scale and formulation of averaging . . . . . . . . . . . . 121

$5.4 .3 \quad$ Removing the sign-changing Physics asymmetry . . . . . . . . . . . 124

5.5 Residual Correlations to HC Differences over Run1 and Run2 . . . . . . . . . . . 127

5.6 Beam Corrections Results . . . . . . . . . . . . . . . . . . . . . . . . . . 130

5.6 .1 Beam corrections in Run2 $\ldots \ldots \ldots \ldots \ldots$

5.6 .2 Beam corrections in Run1 . . . . . . . . . . . . . . . . 135

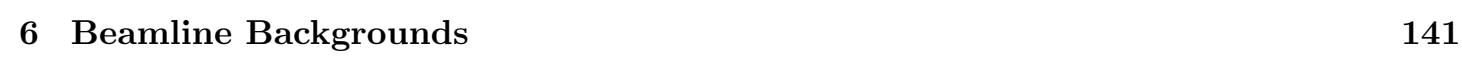

6.1 Background Detectors $\ldots \ldots \ldots \ldots \ldots$

$6.1 .1 \quad$ Background asymmetries . . . . . . . . . . . . . . . . . . . . . 142

6.1 .2 Correlations between background asymmetries in Run2 $\ldots . . . . . .143$

$6.1 .3 \quad$ Background yields and asymmetry widths . . . . . . . . . . . . . . . 144

6.1 .4 Residual correlations of background asymmetries to beam parameters . . 145

6.2 Connections with Beam Halo $\ldots \ldots \ldots \ldots$

6.3 Blocked-Octant Measurements . . . . . . . . . . . . . . . . . . . . . . . . . . . . 149

6.3 .1 Run1 blocked-octant studies . . . . . . . . . . . . . . . . . . . . . 150

6.3 .2 Run2 blocked-octant studies. . . . . . . . . . . . . . . . . . . 152

$6.4 \quad$ A Model for the Beamline Background Asymmetries . . . . . . . . . . . . . . . 156

6.5 Beamline Backgrounds Correction Algorithm $\ldots \ldots \ldots \ldots 1$

6.5 .1 Time scale of averaging $\ldots \ldots \ldots \ldots$

6.5 .2 Residual beam effects . . . . . . . . . . . . . . . . . 166

6.5 .3 Extraction of BB correction factor . . . . . . . . . . . . . . . . 168

6.5 .4 Comparison with other background detectors $\ldots \ldots \ldots \ldots$ 
$6.6 \quad$ Beamline Backgrounds Correction in Run2 $\ldots \ldots \ldots \ldots \ldots$

6.7 Model Uncertainty of BB Correction . . . . . . . . . . . . . . . . . . . 175

6.8 BB Correction in Run1 $\ldots \ldots \ldots \ldots \ldots$

\begin{tabular}{|lll}
\hline 7 & Statistical Properties of the Corrected Asymmetry & 181
\end{tabular}

7.1 Null Asymmetries $\ldots \ldots \ldots$. . . . . . . . . . . . . . . . . . . . . . 181

7.2 Beam and BB Corrections $\ldots \ldots \ldots \ldots \ldots \ldots$. . . . . . . . . . . 184

$7.3 \quad$ Azimuthal Dependence of Asymmetries $\ldots \ldots \ldots \ldots$

7.4 Auto-Correlation . . . . . . . . . . . . . . . . . . . . . . . . . 187

7.5 Dependence to Time Scale of Averaging $\ldots \ldots \ldots \ldots$

\begin{tabular}{lll}
\hline & Results and Conclusion & 190
\end{tabular}

$8.1 \quad$ Extracting the Parity-Violating Asymmetry $\ldots \ldots \ldots$. . . . . . . . . . . . 190

$8.1 .1 \quad$ Radiative corrections and experimental bias . . . . . . . . . . . . . . 191

8.1 .2 Polarization . . . . . . . . . . . . . . . . . . . . . . . 192

$8.1 .3 \quad$ Measured asymmetry . . . . . . . . . . . . . . . . . . . 193

$8.1 .4 \quad$ Background corrections . . . . . . . . . . . . . . . . . . . . . . . 199

8.1 .5 The parity-violating asymmetry . . . . . . . . . . . . . . 202

8.2 Extraction of the Weak Charge of the Proton . . . . . . . . . . . . . . . 203

8.3 Preliminary studies on model dependence . . . . . . . . . . . . . . . . 205

8.4 Extraction of the Weak Mixing Angle $\ldots \ldots \ldots$. . . . . . . . . . . 207

$8.5 \quad$ Concluding Discussion $\ldots \ldots \ldots \ldots$

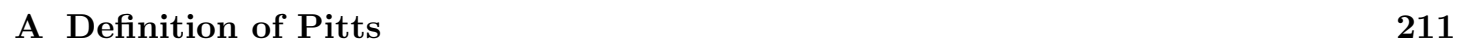

\begin{tabular}{|l|l|}
\hline B Development of a Kerr Cell for Future PVES Measurements & 213
\end{tabular}

\begin{tabular}{|ll}
\hline C Robust Algorithm to Extract Regression Parameters & 216
\end{tabular}

C.1 Ordinary least squares . . . . . . . . . . . . . . . . . . . . . . . . . . . 216

C.2 Symmetric algorithms … . . . . . . . . . . . . . . . . 217

C.3 York's algorithm . . . . . . . . . . . . . . . . . . . . . . . . . . . . . 219

C.4 Example with $Q_{\text {weak }}$ data $\ldots \ldots \ldots \ldots \ldots$

\begin{tabular}{ll}
\hline Bibliography & 222
\end{tabular} 


\section{List of Figures}

1.1 The particle content of the Standard Model. . . . . . . . . . . . . . . . . . 2

$1.2 \quad$ A neutral current $\bar{\nu}_{\mu}+e^{-} \rightarrow \bar{\nu}_{\mu}+e^{-}$event in the Gargamelle bubble chamber. Reproduced from 15.

2.1 The world data for the proton $G_{E}$ and $G_{M}$ form factors, normalized to the standard dipole. Reproduced from $[30 . . \ldots \ldots \ldots$. . . . . . . . . . 9

2.2 Tree-level diagrams of the neutral current weak and EM electron-proton interaction, characterized by the exchange of a $Z$ boson or a photon respectively. . . . . 10

2.3 Conceptual design of the "STAR" (Symmetric Toroidal ARray) spectrometer from the 1989 letter of intent [35]. The basic concepts of a large-acceptance magnetic spectrometer, azimuthaly symmetric detector array, and collimation to suppress backgrounds, would be employed by experiments such as $G 0$ and $Q_{\text {weak }}(c f$. Figure $3.1 \mid$ in the Jefferson Lab PVES program. . . . . . . . . . . . . . . . . . 14

2.4 Experimental constraints on $G_{E}^{s}$ and $G_{M}^{s}$ in the region of $Q^{2}=0.1 \mathrm{GeV}^{2}$ plotted as $1 \sigma$ bands. The result of a QCD calculation from Adelaide [48] is shown as a filled ellipse. The $68 \%$ CL contour of a global fit [49] incorporating data beyond what is included in this plot is shown as a solid elliptical contour. Reproduced

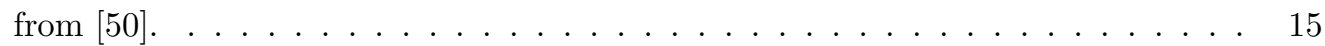

2.5 The reduced PV asymmetry from the world dataset of PVES results on nuclear targets, rotated to the forward angle limit $\theta \rightarrow 0$. The global fit result is the solid black line with the yellow band as its uncertainty. The $Q^{2}=0$ intercept corresponds to the weak charge of the proton $Q_{w}^{p}$ and is in good agreement with the SM prediction (black arrow). . . . . . . . . . . . . . . . . 17

2.6 The energy dependence of the $\mathcal{R} e \square_{\gamma Z}^{\mathrm{V}}$ correction from [66, where the total value is plotted as the solid black line along with the separate contributions from three kinematic regions in $W$ and $Q^{2}$ (Regions I,II, and III). The vertical dashed line corresponds to $Q_{\text {weak }}$ kinematics at $E=1.165 \mathrm{GeV}^{2}$. . . . . . . . . . . . . 19 
2.7 $\quad$ Global combined constraints (red ellipses) from the global PVES fit (blue ellipses)

and the APV measurement on ${ }^{133} \mathrm{Cs}$ (green ellipses) on the isovector and isoscalar

\begin{tabular}{l} 
combinations of the weak vector quark couplings $C_{1 u}$ and $C_{1 d}$. The constraints \\
\hline are in agreement with the SM prediction (black dot), quoted as a function of \\
\hline
\end{tabular}

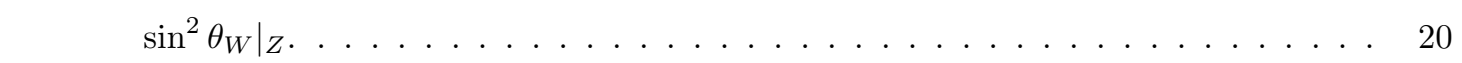

2.8 Left: running of the QED fine structure constant from the L3 Collaboration [71]. Right: running of the strong coupling constant $\alpha_{s}$ from [72. . . . . . . . . 21

$2.9 \quad \gamma-Z$ mixing (vacuum polarization) diagrams and $W$-loop contribution to the anapole moment for parity-violating elastic electron scattering. . . . . . . . . 21

2.10 The purely weak $\left(\square_{W W}\right.$ and $\left.\square_{Z Z}\right)$ and electroweak $\left(\square_{\gamma Z}\right)$ two-boson exchange

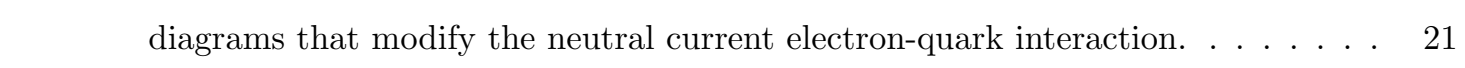

2.11 Theoretical prediction (blue line) for the scale dependence of the weak mixing angle $\sin ^{2} \theta_{W}$ in the $\overline{\mathrm{MS}}$ scheme [26], plotted versus the renormalization scale $\mu$.

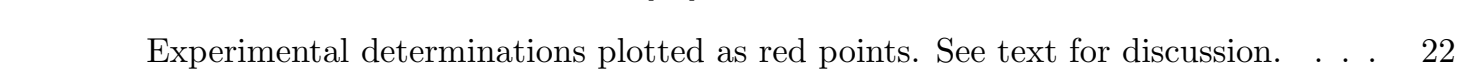

2.12 Feynman diagrams for models of new physics that could create a deviation from the SM prediction in the $Q_{\text {weak }}$ measurement: new $Z^{\prime}$ gauge bosons, Leptoquarks,

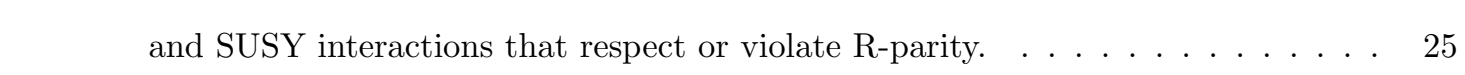

2.13 The $Q^{2}$-dependent modification of the running of $\sin ^{2} \hat{\theta}_{W}$ from a dark $Z$ boson with $m_{Z_{\mathrm{d}}}=15 \mathrm{GeV}$ is shown in blue [91]. Current experimental constraints are shown in black, while planned future measurements with their expected precision \begin{tabular}{|c|}
\hline are shown in red at arbitrary vertical location. A different theoretical calculation \\
\hline
\end{tabular} is employed here compared to Figure 2.11 . . . . . . . . . . . . . . 27

3.1 CAD design of the $Q_{\text {weak }}$ experimental apparatus. The beam is incident on the \begin{tabular}{l} 
target from the right. Elastically scattered events are selected by the triple col- \\
\hline limator system and focused by the field of the spectrometer magnet onto an az- \\
\hline imuthally symmetric array of quartz Cerenkov detectors, while inelastic electrons \\
\hline are swept away. The elements and design characteristics of the apparatus are \\
\hline described in this chapter. . . . . . . . . . . . . . . . . . . . . . . 30 30
\end{tabular}

3.2 Schematic of the Jefferson Lab main accelerator. Electrons are accelerated in the \begin{tabular}{|c|}
\hline two superconducting RF Linacs connected via recirculation arcs, to be delivered \\
\hline
\end{tabular} in the experimental end stations. . . . . . . . . . . . . . . . 33

$3.3 \quad$ CAD design of the $Q_{\text {weak }}$ target cryo-system. The $\mathrm{LH}_{2}$ flow in the conical cell that defines the beam interaction region is shown on the right. . . . . . . . . . 34 
3.4 The Fast-Fourier transform spectrum of detector bar MD1 current-normalized signal (in $\mathrm{V} / \mu \mathrm{A}$ ), showing that the frequency of target density fluctuations is $\lesssim 40 \mathrm{~Hz}$ at $150 \mu \mathrm{A}$. Noise spikes at $60 \mathrm{~Hz}$ harmonics are also observed. . . . . . . 35

3.5 The dependence of the target boiling contribution to the measured asymmetry width is examined versus the hydrogen recirculating pump frequency (left) and the beam spot raster size (right), in dedicated tests. . . . . . . . . . . . . 35

3.6 The simulated rate profile on the main detector bars from elastic aluminum events, shown separately for the upstream (top) and downstream (bottom) aluminum

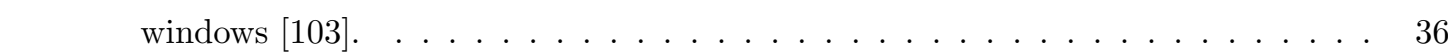

$3.7 \quad$ A simplified side elevation view illustrating possible sources of soft backgrounds in the main detectors and the shielding that was used to minimize the background rates. The vertical scale is amplified by a factor of three for clarity. The electron beam comes in from the left and interacts with the target. Subsequent scattering from different places in the beamline is shown with solid lines of different colors, while different line types show the particle trajectories after encountering shielding put in place to prevent them from reaching the main detectors. Three background detectors behind the main detectors (PMTLTG,PMTONL,MD9) and the upstream luminosity monitors on the upstream face of the defining collimator, were very sensitive to the beamline background component. . . . . . . . . . . . 38

3.8 Left: Engineering drawing of the tungsten plug collimator (dimensions in inches), where the electron beam is entering through the left side. Right: Picture of the plug collimator fit on the upstream collimator element. . . . . . . . . . . . . . 38

3.9 CAD drawing of the apparatus. Beam comes in from the right and scatters at the \begin{tabular}{|c|c|c|}
\hline target. Shown in blue is the simulated scattered elastic electron envelope through \\
\hline
\end{tabular} the collimator system and QTor spectrometer. Also shown are the horizontal and vertical drift chambers used for tracking reconstruction. . . . . . . . . . . . . . . 39

3.10 Main detector bars 7 and 8 before installation of the lead pre-radiators. The squares at the end of each bar is lead shielding covering the light-guides and PMTs. Also shown above the lower detector is the quartz sampling scanner. . .

3.11 A GEANT-IV simulation showing the elastic scattered electron profile on the quartz bars. See Figure 3.16 |for a detailed view of the rate profile on each bar. . 42

3.12 Distribution of the raw MDall measured asymmetry $A_{\text {raw }}$ from helicity quartets, collected over $\sim 5$ minutes. Data is blinded and no corrections are applied. The width of the measured asymmetry (here $\sigma_{A}=236 \mathrm{ppm}$ ) is an important figure- 
3.13 CAD drawing of the $Q_{\text {weak }}$ collimator system (red) upstream of the spectrometer magnet. Beam comes from the right and scatters from the target (light blue).

Also shown are the upstream luminosity monitors, on the upstream face of the defining collimator, and the horizontal drift chambers. . . . . . . . . . . . . . . 43

3.14 Left: Picture of the upstream luminosity monitors (USLumis) placed on the upstream face of the second (defining) collimator element. Lead shielding installed around the beamline is also visible. Right: The Run2 location of the background \begin{tabular}{|c|c|c|}
\hline detectors in the super-elastic region behind the MD focal plane, looking down- \\
\hline
\end{tabular} stream to the MD array. Background detector MD9 was placed behind MD5,

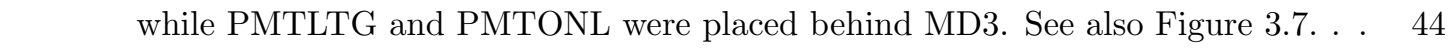

3.15 Vertical drift chambers (VDCs) can be placed upstream of MD bars for tracking

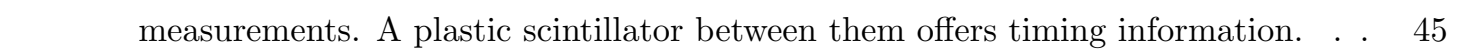

3.16 Scattered electron rate profile on on a main detector bar, reconstructed from the VDCs (top) and obtained from the focal plane scanner detector (bottom). The two measurements are in good agreement. The horizontal and vertical axes correspond to horizontal and vertical location on the detector bar. Color scale indicates relative rates. . . . . . . . . . . . . . . . . . . 46

3.17 Double difference (DD) distribution between BCMs 1-2 (blue histogram) and 7-8 (red histogram) in Run2. The much narrower distribution of $\mathrm{DD}_{78}$ suggests that BCMs 7 and 8 which use improved digital signal processing techniques have much better resolution than 1 and $2 . \ldots \ldots \ldots \ldots \ldots$. . . . . . . . . 48

3.18 Schematic of the antennae of SEE BPMs in the Hall C beamline, rotated by $45^{\circ}$ from the experimental Hall horizontal/vertical directions $\left(X_{H}, Y_{H}\right)$. Reproduced from $[113] . \ldots \ldots \ldots \ldots \ldots$

3.19 The position resolution of an SEE BPM estimated versus beam current, corresponding to quartets of $960 \mathrm{~Hz}$ helicity windows $[113 . \ldots \ldots \ldots$

3.20 Correlation between horizontal position and angle differences in Run2. Data is averaged over 8 -hour slugs (IHWP states). . . . . . . . . . . . . 52

3.21 Left: Design of the Hall C halo target. The beam was centered in the either the $13 \mathrm{~mm}$ diameter circular hole or the $8 \times 8 \mathrm{~mm}^{2}$ square hole, and the rates from \begin{tabular}{|c|}
\hline the aluminum frame were detected from halo monitors, their locations shown on \\
\hline
\end{tabular} the right downstream of the target. . . . . . . . . . . . . . . . . . . . 52

3.22 Schematic of the Hall C Møller polarimeter. Only quadrupole magnets Q1 and QQ2 were used during $Q_{\text {weak }} \ldots \ldots \ldots \ldots \ldots \ldots \ldots \ldots$ 
3.23 Dedicated measurement at intermediate current $(4.5 \mu \mathrm{A})$ where polarization is

\begin{tabular}{l} 
measured from both the Møller and Compton electron polarimeters. The Møller \\
\begin{tabular}{|l}
\hline measured polarization (black line with yellow band) is in good agreement with \\
\hline the polarization measured from Compton (red points, average is marked with the \\
\hline star symbol). . . . . . . . . . . . . . . . . . . . . . . . . . . . . 55
\end{tabular} \\
\hline
\end{tabular}

3.24 Layout of the Hall C Compton polarimeter developed for $Q_{w e a k}$. Electrons are

deflected $57 \mathrm{~cm}$ vertically to the Compton interaction region by dipole magnets and then returned to the nominal beamline during normal production. The total length of the dipole chicane is $11.1 \mathrm{~m}$. . . . . . . . . . . . . . . . . . 55

4.1 Schematic representation of the Jefferson Lab polarized source components used

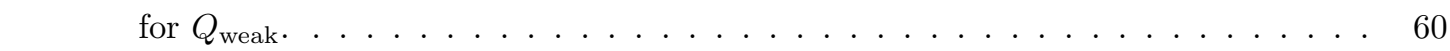

4.2 The helicity signals that drive the transition in the polarized source. . . . . . . . 62

4.3 The USLumi5 (upstream luminosity monitor in octant 5) asymmetry distribution from standard quartets (right) and from manually constructed pairs (left) of helicity windows, over the same dataset in a dedicated study with a relatively slow $480 \mathrm{~Hz}$ raster frequency. The quartet pattern suppresses noise which gives rise to

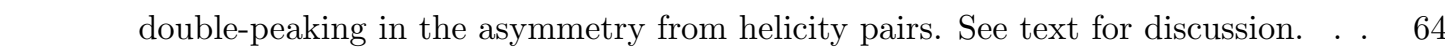

4.4 Left: Simulation of the FFT frequency spectrum for an "octo-quad" pattern of \begin{tabular}{|cl}
\hline helicity windows (eight quartets of helicity windows), using a tool that simulates \\
\hline
\end{tabular}

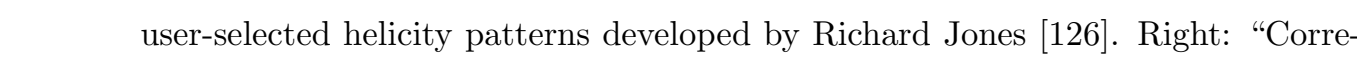
lated noise" on $\sigma_{A}$, defined as the remaining noise on the asymmetry measurement \begin{tabular}{|c|c|c|}
\hline after subtracting the BCM monitor noise and the MD statistical precision & 1 & The \\
\hline
\end{tabular} octo-quads pattern performs clearly better when more noise is present at lower raster frequencies, although results are statistically consistent at higher frequencies. 65

4.5 Picture of the Pockels cell on the Jefferon Lab source laser table, with the rotatable half-wave plate located immediately downstream. The HV switch driver case holding the optocouplers is placed on the cell and wrapped in tape for optical isolation. . . . . . . . . . . . . . . . . . . 66

4.6 Oscilloscope pictures of the signal upon helicity transition, before (left) and after \begin{tabular}{|l|}
\hline (right) optimization. Oscillation of the transmitted power through an analyzer is \\
\hline referred as "ringing", which can persist for the duration of the helicity window \\
\hline \\
before optimization. The optimized transition on the right has reduced ringing \\
\hline amplitude (approximately $4 \%$ ) and is completed within $60 \mu \mathrm{s}$, comfortably within \\
\hline
\end{tabular} $T_{\text {settle }}=70 \mu \mathrm{s}$. 
4.7 An initial version of the PC switch driver circuit, designed by John Hansknecht. The PD1,2 elements are the photodiodes cut out of the optocouplers, while the Pockels cell is seen as a purely capacitive device. The pulse-shaping RC circuits were later replaced by monostable multivibrators. .

4.8 Left: Commercial optocouplers OC100HG sawed off to remove the LEDs and isolate the optical diodes. Right: Diodes with polished housing [131]. . . . . . . 69

4.9 Band structure of GaAs. The numbers in circles represent relative transition probabilities for incident circularly polarized light. $E_{g}$ is the band gap energy and $E_{\mathrm{SO}}$ is the splitting of the valence $P_{1 / 2}$ and $P_{3 / 2}$ levels due to spin-orbit interaction. A strain applied by the lattice mismatch induces a splitting $\delta$ of the

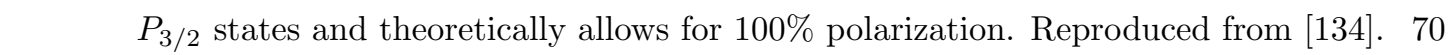

4.10 (a) The daily measured QE plotted against the charge extracted from the photocathode, during Run1 (red points, $0.5 \mathrm{~mm}$ beam spot size) and Run2 (blue points, $1 \mathrm{~mm}$ beam spot size). (b) QE profile scans before (left) and after (right) a four week period of high current running in Run2. In the figure on the right, the "dot" shows the electrostatic center and the "X" marks the spot from where beam was extracted for delivery to the experimental halls. The active area was $\sim 5 \mathrm{~mm}$ in diameter and the laser spot size was $1 \mathrm{~mm}$ FWHM. . . . . . . . . . . . . . . 71

4.11 Schematic of the injector design with the electron beam leaving the load-lock inverted photogun (top) with longitudinal polarization $\left(P_{z}\right)$, followed by focusing (FS) and spin solenoids (SS), vertical (VW) and horizontal (HW) Wien filters, quadrupoles (QW,QU), a buncher (PB) and beam position monitors (BP). The electron polarization may be rotated vertically by VW and then either "left" or "right" $\left(P_{x}\right)$ by SS for a slow spin reversal. The second Wien filter HW rotates the polarization in-plane to compensate for precession during transport. $\quad \ldots \quad$. 73

4.12 Left: An antisymmetric $\Delta$ phase will result in polarization ellipses with interchanged minor and major axes for the two helicity states. Right: The rotatable half-wave plate can be used to rotate the antisymmetric residual linear polarization to minimize the false asymmetry arising from the interaction with an asymmetric transport from the cathode analyzing power. . . . . . . . . . . 75

4.13 A non-zero horizontal angle of incidence $\xi_{x}$ with respect to the optic axis $z$ results in different indices of refraction $n_{1}, n_{2}$ experienced by the electric field in the two planes transverse to propagation. In the $x y$ plane (perpendicular to the page) the index of refraction is $n_{1}=n_{o}$, while in the $x z$ plane $n_{2}$ is a combination of the ordinary and extraordinary indices. Reproduced from [140]. . . . . . . . . . 76 
4.14 PITA scan where PC offset voltages are applied and the resulting charge asym-

metry $A_{Q}$ is measured on BCM8 in the experimental hall. The applied offsets of

4.15 Scan of charge asymmetry (top) and transverse position differences, as measured on an injector BPM (1I02), versus RHWP orientation angle. The fit corresponds

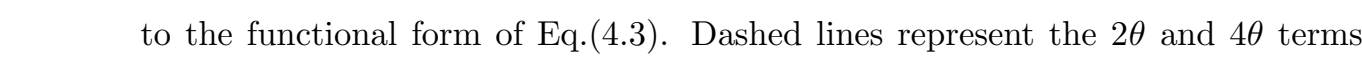

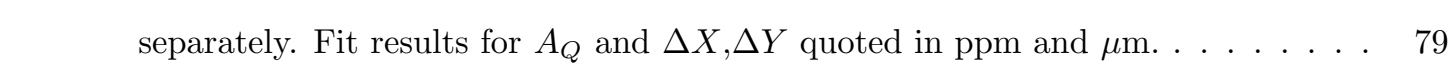

4.16 Measurement of the charge asymmetry (top), position difference (middle) and spot size difference (bottom) with a linear array of photodiodes, oriented vertically, during horizontal translation of the PC. The variation is a result of birefringence gradients. The position and spot size differences are seen to be proportional to \begin{tabular}{|c|c|c|}
\hline the gradient and curvature of the charge asymmetry, respectively. Reproduced \\
\hline
\end{tabular} from [140]. . . . . . . . . . . . . . . . . . . . . . . . 80

4.17 RHWP scan with the linear array as detector element. Responses are plotted

\begin{tabular}{l}
\hline for the charge asymmetry (top), horizontal position difference (middle) and beam \\
\begin{tabular}{|l}
\hline spot size asymmetry (bottom). The beam spot size asymmetry is constrained \\
\hline within 500 ppm with the analyzer. . . . . . . . . . . . . . . . . . . . . . . 82
\end{tabular}
\end{tabular}

4.18 Top row: $\Delta Y$ position differences on three different injector BPMs, after appropriate PITA voltage offset is applied (on each RHWP angle) to null $A_{Q}$. Bottom row: The average size of position differences on multiple injector BPMs, with appropriate PITA offset applied. This procedure was used to optimize the RHWP

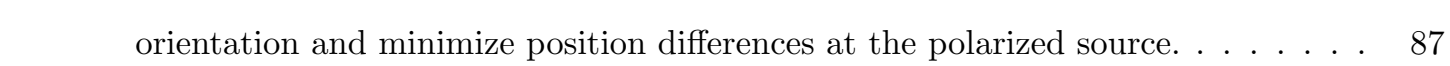

4.19 Horizontal position differences on successive injector BPMs. The purple band corresponds to $\pm 50 \mathrm{~nm}$ of position differences. . . . . . . . . . . . . . . . 88

4.20 Charge asymmetry on successive injector BPMs exhibits a troubling gradient in the location of the injector apertures. . . . . . . . . . . . . . . . . . 88

4.21 Interceptions on the A1, A2 and MS injector apertures plotted versus time and \begin{tabular}{l}
\hline color-coded, from the accelerator striptool. Interception on injector apertures \\
\hline slowly drifts with time and is highly correlated with observed parameters in the \\
\hline hall. Here the charge asymmetry width is offset vertically so that it superimposes \\
\hline almost exactly the A2 interception (they are both plotted with a similar green \\
\hline color which makes them hard to tell apart). . . . . . . . . . . . . . . . . . . . . 90 \\
\hline
\end{tabular} 
4.22 Top to bottom: Yield, horizontal position and charge asymmetry $A_{Q}$, as measured on BPM 0i05, versus injector chopper phase. By varying the chopper phase we access different longitudinal parts of the electron beam pulse, effectively getting a temporal profile of the beam. . . . . . . . . . . . . . . . . . . . 91

4.23 Top: Horizontal position differences in the experimental hall, plotted versus successive BPMs. This is the same run as for the injector position differences plotted \begin{tabular}{|r|l|l|}
\hline in Figure & 4.19 & The position differences at the $Q_{\text {weak }}$ target are higher than 100 \\
\hline
\end{tabular} nm. Bottom: Horizontal position differences in the injector and the experimental hall during the HAPPEX-II run. Kinematic damping through acceleration

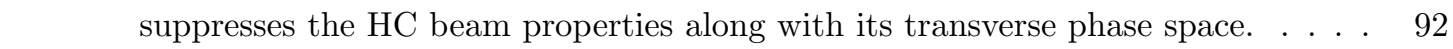

4.24 Active charge feedback minimizes the charge asymmetry faster than $1 / \sqrt{N}$ statistical convergence (dotted curves). Each data point is a running average of data up to that point. The horizontal axis is the number of 80s intervals [148]. . . . . 94

4.25 Responses of horizontal position differences in the experimental hall from large and opposite kicks from the injector helicity magnets. Large responses are observed, which were typically dependent on beam conditions. . . . . . . . . . . . 95

4.26 Horizontal and vertical (red and blue, respectively) position differences at the $Q_{\text {weak }}$ target versus time, over a period of six days. Each label in the horizontal axis corresponds to 12 hours. The black line marks the beginning of application of feedback from helicity magnets, achieving great improvement in the size of $\mathrm{HC}$ differences. Outlying measurements before the black line correspond to tests of the helicity magnet responses. The purple band that marks $\pm 50 \mathrm{~nm}$ of position differences is plotted again for comparison. . . . . . . . . . . . . . . . 97

4.27 Helicity-correlated differences in beam parameters, plotted versus slug through $Q_{\text {weak. }}$. Data is color-coded by IHWP state (blue for IHWP in, red for out). No sign-correction is applied. The red line marks the separation between Run1 and Run2. The dashed black lines mark separation between Wien periods. . . . . . . 98

$5.1 \quad$ MD sensitivities to horizontal (left) and vertical (right) beam position, plotted versus MD octant . . . . . . . . . . . . . . . . . . . . . . . . 102

5.2 Correlation between $\mathrm{HC}$ differences $\Delta X$ and $\Delta E$, over quartets in a $Q_{\text {weak }} 5$ minute runlet. The correlation coefficient approaches $0.999 . \quad \ldots \ldots \ldots$

$5.3 \quad$ Strange bi-modality in $\Delta Y$ to $\Delta X$ correlation (quartet scale) over Slug 40. Potential "double-peaking" in the $\Delta X$ distribution could give rise to this behaviour. 104 
5.4 Double-peaking in the $\Delta X$ measurement of the energy-sensitive arc BPM 3C12, resulting from Linac phase instabilities. The separation of the two peaks occurred sporadically, but was typically much smaller than in this case. . . . . . . . . . 104

5.5 Left:Quartet-level correlation of the regressed MDall asymmetry to $\Delta X$ over Slug 36. The "edges" of the $\Delta X$ distribution are fitted separately, in blue, from the "core" that is fitted in red. Right: Applying the fit for each MD bar, the extracted correlation slopes are plotted separately versus MD bar for the core (red) and edges (blue) of the distribution. The right-most correlation slope corresponds to the MDall combination, as extracted from the fit on the left. . . . . . . . . . . 105

5.6 Correlation between $\Delta X$ and $\Delta X^{\prime}$ at the target in Slug 110. Every red point corresponds to the average values in a 5-minute runlet within that slug, and the ellipse around that point is characteristic of the width and correlation of the quartet-scale $\Delta X$ and $\Delta X^{\prime}$ distributions. The red line is the linear fit for a forced zero offset. From $[152 . . \ldots \ldots \ldots$. . . . . . . . . . . . . 106

5.7 Regression sensitivity $S_{Q}$ to charge asymmetry in the $5+1$ scheme, plotted versus charge asymmetry width $\sigma_{Q}$ over Run1. Each point represents a 5-minute runlet where regression sensitivities are extracted. Color-coding corresponds to BCM monitor noise $\sigma_{\mathrm{BCM}}$, extracted from BCM double-difference. . . . . . . . . . . . 107

5.8 Schematic of the location of the dithering magnets with respect to the accelerator and Hall C beamline. . . . . . . . . . . . . . . . . . . . . . . . . 109

5.10 Yield responses of MD bars to modulation in horizontal position versus phase of the modulation. . . . . . . . . . . . . . . . . . . . . . . . . . . 110

5.9 Typical response of the target BPM to horizontal position modulation. . . . . . . 110

5.11 Phases of horizontal BPM responses to horizontal position modulation, plotted for BPMs along the beamline over many runs. Top: standard dithering modes for $X$ and $X^{\prime}$ modulation, where the $\mathrm{X} 1$ and $\mathrm{X} 2$ coils are pulsed together and FFB is active. Bottom: response phases during a short period where the two coils were pulsed separately and FFB was paused during modulation. . . . . . . . . . . . 112

5.12 The sine/in-phase (left) and the cosine/out-of-phase (right) responses of the un\begin{tabular}{|c|}
\hline corrected MD to driven modulation from coils X1 and X2 (top and bottom respec- \\
\hline tively) is plotted in red, versus MD octant. The sensitivities extracted from these \\
\hline responses are used to correct the MD yields in the same data. Residual responses \\
\hline to the modulation kicks after correction are plotted in blue and are inconsistent \\
\hline
\end{tabular} 
5.13 Residual response to $X^{\prime}$ modulation, plotted as fractional yield (normalized to the average yield) of background detectors USLsum (left) and PMTLTG (right) versus

the modulation phase. The residual response cannot be fitted by the standard sine/in-phase (blue and pink, for fixed or floating fit phase) and cosine/out-ofphase (red) functions. The residuals are highly correlated on all background detectors . . . . . . . . . . . . . . . . . . . . . 115

5.14 Correction sensitivities $S_{X}=\partial A / \partial \Delta X$ from regression (red) and dithering (blue), for the MD horizontal dipole. . . . . . . . . . . . . . . . . 116

5.15 Residual covariances of the MDall asymmetry, corrected through regression (blue) or modulation (red) sensitivities, to target $\Delta X$. Covariances have been extracted at the quartet-level in every runlet and averaged in each slug. . . . . . . . . . . 117

5.16 Noise removed from the MDall asymmetry width $\sigma_{A}$ through different correction

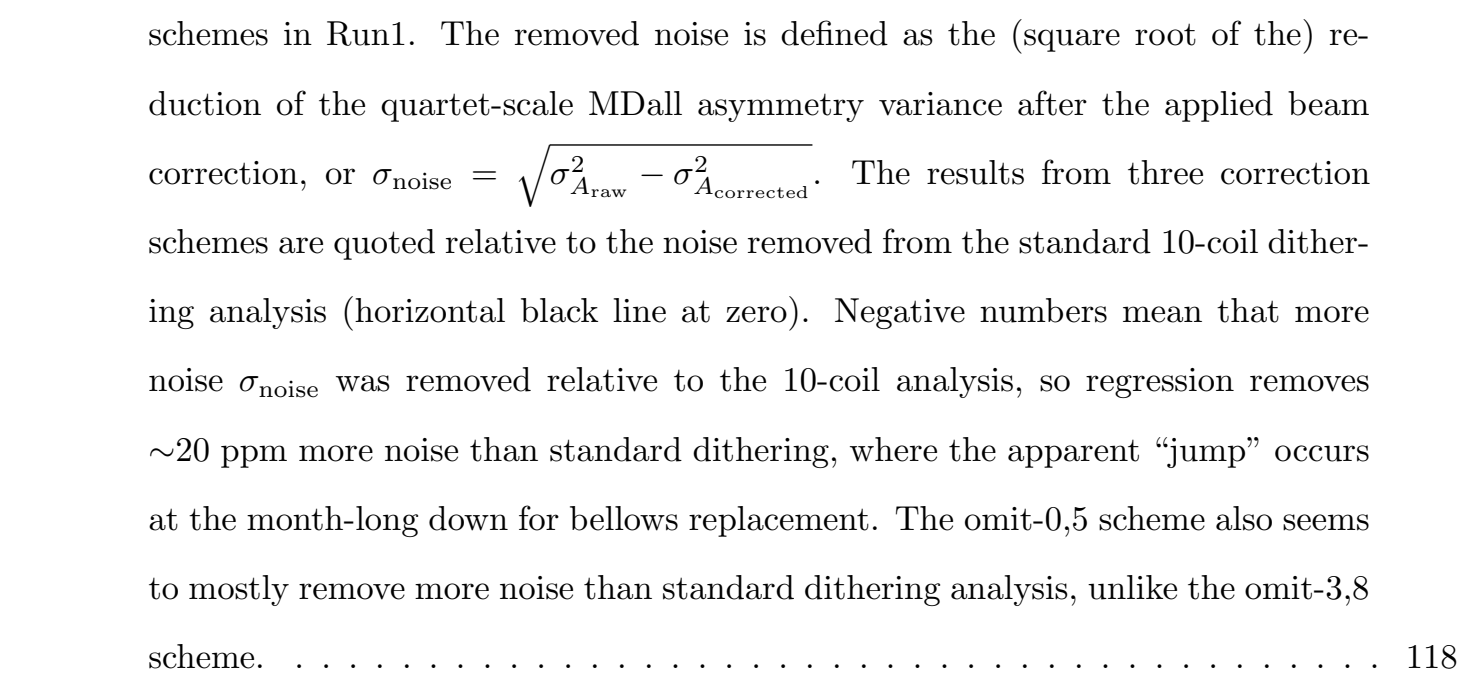

5.17 Left: Charge asymmetry $A_{Q}$ correlation to position difference $\Delta X$, in two opposite kicks from the helicity magnets. The large systematic position difference imparted by the kick of the helicity magnets does not affect the quartet-scale correlation between charge asymmetry and position difference, characteristic of beam noise/jitter. Right: The quartet-scale correlation slope between the PMTLTG asymmetry and $A_{Q}$ is not appropriate to correct for an offset $A_{Q}$, imparted by the source IA system. . . . . . . . . . . . . . . . . . . . . . . . 119

5.18 Correlation between the regressed asymmetry of bar MD5 and the HC difference $\Delta X^{\prime}$, one of the parameters it has been regressed against. Correlation extracted over all of Run1. Data binned at the slug scale. . . . . . . . . . . . . . . . 120 
5.19 Correlation between the regressed MDall asymmetry and $\Delta E$ versus time scale of averaging. A significant correlation develops at longer time scales, while the residual correlation of the regressed MDall asymmetry was zero by construction at the quartet scale.

5.20 Schematic representation of the HC differences in beam parameters, separated into a component that remains constant $(\overline{\Delta x})$ and one that changes sign $(\delta(\Delta x))$ under the IHWP reversal. $\sigma_{x}$ corresponds to random fluctuations. . . . . . . . . 122

$5.21 \mathrm{HC} \Delta X$ position difference in Run2, averaged over pairs of slugs. The differences are averaged in the Physics (blue) and Null (red) combinations separately in each slug pair. . . . . . . . . . . . . . . . . . . . 123

5.22 Correlation between regressed MDall $A_{\text {raw }}$ asymmetry and $\Delta X$ in Wien 8 , in the Sign-corrected formulation. A relative sign is applied to the IHWP OUT data (red) and they are fitted separately (solid lines) from the IHWP IN data (blue). The dashed black line is the combined fit. . . . . . . . . . . . . . . . . . . . . . 126

5.23 Correlation slopes of regressed MD asymmetries to $\Delta X^{\prime}$, extracted over Run1 at the slug scale, plotted versus MD octant. For example, the correlation slope of

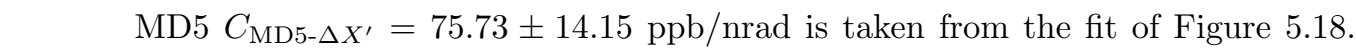

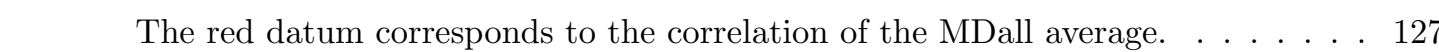

5.24 Physics (top) and Null (bottom) asymmetry combinations after beam corrections in Run2 for data binned at the pitt scale. Black points correspond to uncorrected asymmetries, blue and red points to asymmetries corrected through dithering and

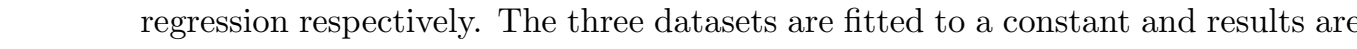
shown on the right. Vertical dashed lines mark different Wien periods. Polarization applied to all data. . . . . . . . . . . . . . . . . . . . . . . 132

5.25 Physics (top) and Null (bottom) asymmetry combinations after beam corrections in Run1 for data binned at the pitt scale. Black points correspond to uncorrected data, blue points for data corrected through dithering, and red for data corrected through regression. The three datasets are fitted to a constant and results are shown on the right. Vertical lines mark different Wien periods. Polarization applied to all data. . . . . . . . . . . . . . . . . . . . 135

5.26 Slug-scale correlations of MD asymmetries to charge asymmetry in Run1, plotted versus octant. The asymmetries are corrected in the standard 10-coil dithering

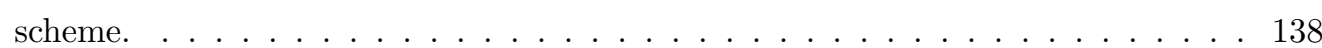

5.27 Scatterplot of the slug-averaged MDall asymmetry and charge asymmetry in Run1. Color scaling applied by slug number. . . . . . . . . . . . . . . . . . . 139 
5.28 Charge asymmetry in Run1 averaged in every Wien and separated into "Physics"

and "Null" combinations, corresponding to sign-correction for reversals applied and not applied respectively. . . . . . . . . . . . . . . . . . . . . 140

$6.1 \quad$ Background asymmetries in Run2 by the three background detectors and the US luminosity monitors, plotted versus slug and separated by IHWP state (blue for IHWP IN and red for OUT). Data for PMTLTG and PMTONL is missing for a period between Slugs 170-203 due to studies on their pre-amplifier channels. . . . 142

6.2 Correlation between background asymmetries over Run2, averaged at the slug \begin{tabular}{|c|c|c|}
\hline scale. The linear fit appears to be very good over a wide range of asymmetries. & 143
\end{tabular}

$6.3 \quad$ Yields of background detectors through Run2, averaged for every slug, in units of $\mathrm{V} / \mu \mathrm{A}$. Dashed vertical lines mark Wien transitions. . . . . . . . . . . . . . 145

6.4 Scatterplot of PMTLTG and USLsum yields through Run2, seen to be highly cor\begin{tabular}{|c|c|c|}
\hline related. Color gradient represents different periods in Run2, identified by segment \\
\hline
\end{tabular} number. . . . . . . . . . . . . . . . . . . . 146

6.5 Left: Data for the USLsum asymmetry width (black, vertical axis in units of $\mathrm{ppm}$ ) and yield (red, scaled and zero-suppressed), superimposed and plotted versus runlet number through Run2. Right: Profiled plot of the absolute USLsum asymmetry versus the USLsum asymmetry width. Larger $\sigma_{\text {USL }}$ generally comes with larger background asymmetries. . . . . . . . . . . . . . . . . 146

6.6 Residual slug-scale correlation slopes of the individual USLumi bars, corrected through regression, to HC beam parameters in Run1, plotted versus USLumi bar \begin{tabular}{|c|}
\hline number. The dipole shape of the residual correlations is clear, while the USLsum \\
\hline
\end{tabular} combination (red datum) is also significantly non-zero. . . . . . . . . . . . 147

6.7 Left: Dedicated test from 3/17/2012, where the field-integral $B d \ell$ of quadrupole magnet 0L06 was varied to modulate halo. The quadrupole $B d \ell$, halo rates, and USLsum asymmetry width $\sigma_{\text {USL }}$ are plotted versus time, using the accelerator EPICS strip tool. Right: The resulting halo rates in two halo monitors and the USLsum asymmetry width are highly correlated. . . . . . . . . . . . . . . . . . . 148

6.8 USLsum response during a dedicated study on 2/27/2012 where the Hall C laser phase was varied around its nominal value of $128^{\circ}$. Left: The USLsum regression \begin{tabular}{|c|}
\hline sensitivities to beam position (horizontal and vertical) clearly respond to the Hall \\
\hline
\end{tabular} C laser phase variation. Smaller regression sensitivities occur at smaller values of the USLsum asymmetry width $\sigma_{\text {USL }}$ and vice versa. Right: The USLsum

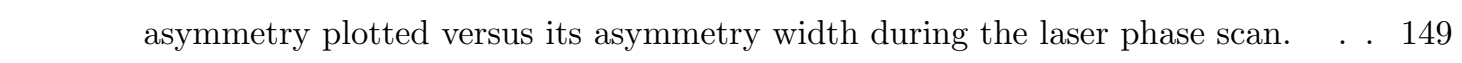


6.9 Normalized yield of blocked octant 7 versus raster size in the May 2011 studies, at the nominal elastic and inelastic magnetic field settings. Reproduced from [103].150

6.10 Picture of the tungsten shutters inserted on the downstream face of the first (most upstream) clean-up collimator, blocking the openings for elastic events in the horizontal octants 1 and 5. The downstream side of the tungsten plug collimator is also visible. . . . . . . . . . . . . . . . . . . . . . . . . . 153

6.11 Correlation between the average asymmetry in the blocked MD bars and PMTONL over all blocked-octant data. The data are binned over 5-minute runlets.

6.12 High correlation between horizontal and vertical USLumi bars. Data averaged at \begin{tabular}{|c|}
\hline the slug time scale over Run2, separated by IHWP states. The combination of \\
\hline
\end{tabular} opposite bars cancel out residual beam effects. . . . . . . . . . . . . . . . . . . . 159

6.13 Plot from the accelerator EPICS strip tool of halo rates (from detectors halo3 and halo4) versus time as the size of the raster is increased sequentially in $\mathrm{X}$ and $\mathrm{Y}$ (horizontal and vertical) from $1 \mathrm{~mm}$ to $6 \mathrm{~mm}$ per side. The raster size is increased in the vertical first without showing any response from the halo detectors. When the raster size is then increased in the horizontal, both halo detectors see a clear increase in halo rates. . . . . . . . . . . . . . . . . . . . . . . . 160

6.14 The extracted quartet-scale correlation slope $C_{\mathrm{MD}-U S \mathrm{U} L}^{\text {quatt }}$ between MD and USLsum asymmetries is plotted as the red solid line versus slug number in Run2. The predictions for the three separate contributions (Eq. 6.9) are plotted as dashed \begin{tabular}{|c|}
\hline lines, where the sum of the separate parts forms the total prediction (solid blue \\
\hline
\end{tabular} line). . . . . . . . . . . . . . . . . . . . . 164

6.15 Dependence of the MD-USLsum correlation slope to the USLsum width, extracted

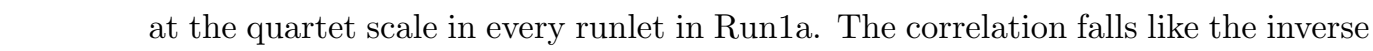
square of the USLsum width, asymptotically approaching the value of the correlation due to the BB component, exactly as we would expect from Eq.(6.9). Notice that the MD-USL correlation slope takes values about an order of magnitude higher than the $C_{\mathrm{MD}-\mathrm{BSL} u m}^{\mathrm{BB}}$ factor that we want to extract. $\ldots . . . . .165$ 
6.16 Correlation slope between MD bars and USLsum asymmetries plotted versus oc-

tant. Left: Correlation extracted at the quartet-scale over a subset of Wien8,

plotted for uncorrected (blue) and regressed (red) asymmetries. Sinusoidal fit

results correspond to the regressed asymmetries. Right: Correlations extracted at the Slug-scale over Run2, for asymmetries corrected through dithering. The characteristic dipole octant dependence can be seen at both the quartet and the slug time scale. . . . . . . . . . . . . . . . . . . . 166

6.17 The charge sensitivities between the PMTLTG and USLsum asymmetries are very highly correlated in Run2, with a correlation slope that is identical to that of their measured asymmetries. . . . . . . . . . . . . . . . . . 167

6.18 USLsum asymmetries in Run2, averaged over slug pairs (of opposite IHWP state, in the Sign-corrected formulation). The asymmetries are averaged in the Physics (blue) and Null (red) contributions in each slug pair. . . . . . . . . . . . . 168

6.19 The correlation between MDall and USLsum asymmetries in the Sign-corrected formulation, averaged at the slug scale over the Run2 Modulation dataset. The \begin{tabular}{|c|}
\hline extracted correlation slope is the correction factor $C_{\mathrm{MD}-\mathrm{USL}}^{\mathrm{BB}}$ for the $\mathrm{BB}$ correction \\
\hline
\end{tabular}

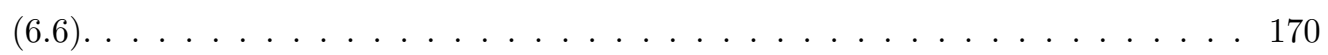

6.20 The correlation slope between regressed MDall and background asymmetries, ex-

\begin{tabular}{l}
\hline tracted over Run2 at the slug scale, plotted versus the scaling of the assigned \\
\hline background asymmetry resolution $\sigma_{\text {res }}^{i}$. Each color corresponds to a different \\
\hline background detector. The correlation slopes to all background detectors have \\
\hline been normalized to the value of the correlation to the USLsum, based on the very \\
\hline precise correlation slopes between background detectors. Results plotted as off- \\
\hline colored inverted triangles correspond to background uncertainties assigned from \\
\hline the distribution widths $\sigma_{A}^{i}$ rather than the detector resolution. See text for more \\
\hline
\end{tabular}

6.21 The MDall Physics asymmetry combination in Run2 averaged at the pitt scale.

Data correspond to uncorrected (orange), then corrected for beam effects through dithering (black), and finally corrected for beamline backgrounds (blue), where the latter includes beam corrections. . . . . . . . . . . . . . . . . . . 174

6.22 Correlation slopes between background asymmetries, extracted in every Wien period in Run2 for data binned at the slug scale. . . . . . . . . . . . . . . 177

6.23 Ensemble distributions for Physics and Null asymmetries in Run2 after 100,000

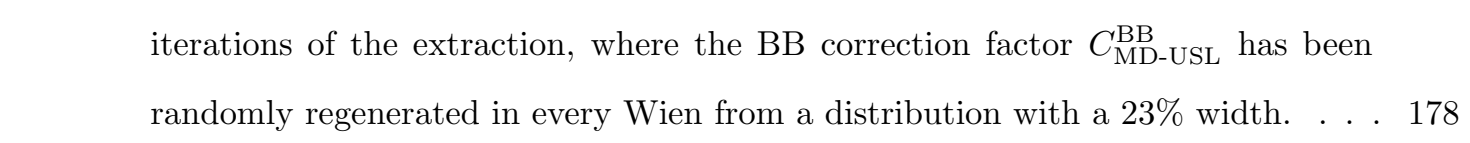


6.24 Slug-averaged USLsum asymmetry through $Q_{\text {weak }}$, uncorrected (blue) and regressed (red). Yellow dashed vertical lines mark Wien transitions, while the red dashed vertical line separates Run1 and Run2. . . . . . . . . . . . . . . . . 179

6.25 Fit between MD and USLsum asymmetries in Run1, averaged at the slug time scale. The correlation slope is the BB correction factor $C_{\mathrm{MD} \text {-USL }}^{\mathrm{BB}}$ for Run1. $\ldots 180$

$7.1 \quad$ MDall asymmetries averaged at the pitt scale in Run2 and separated by IHWP (top) or Spin state (bottom). Data correspond to uncorrected (orange), corrected for beam asymmetries $A_{\text {beam }}$ through dithering (black) and finally corrected for beamline backgrounds (blue/red, depending on IHWP/Spin separation). Fit results are quoted for the BB-corrected asymmetries. . . . . . . . . . . . . . . . 183

7.2 Three different definitions of the MDall Null asymmetry combination in Run2 averaged at the pitt scale. Fit probabilities and $\chi^{2} /$ NDF are quoted with respect to zero . . . . . . . . . . . . . . . . . . . . . 184

7.3 $\quad$ Applied corrections for false beam asymmetries $A_{\text {beam }}$ (black data) and beamline backgrounds $A_{\mathrm{BB}}$ (blue data) through Run2, averaged at the pitt scale and normalized for polarization. Corrections correspond to the Physics (top) and Null (bottom) MDall asymmetry combinations. . . . . . . . . . . . . . . . 185

7.4 Physics (top) and Null (bottom) asymmetry combinations, extracted over Run2 and plotted versus MD octant. The values have been extracted and plotted for every MD octant. The average asymmetries are quoted in every octant for uncorrected (orange), corrected for $A_{\text {beam }}$ through dithering (black) and then corrected for $A_{\mathrm{BB}}$ (blue) data in Run2. The MDall combination is the left-most point. . . 186

7.5 The fit probability for the Physics (top) and Null (bottom) asymmetry combi\begin{tabular}{|c|c|c|}
\hline nations, extracted over Run2 and plotted on a log scale versus MD octant. The \\
\hline
\end{tabular} probability is quoted in every octant for uncorrected (orange), corrected for $A_{\text {beam }}$ through dithering (black) and then corrected for $A_{\mathrm{BB}}$ (blue) asymmetries. The MDall combination is the left-most point. . . . . . . . . . . . . . . 187

7.6 The Auto-Correlation Function of the Physics (top) and Null (bottom) asymmetry combinations, for pitt-scale segmentation over Run2, is plotted versus the pitt

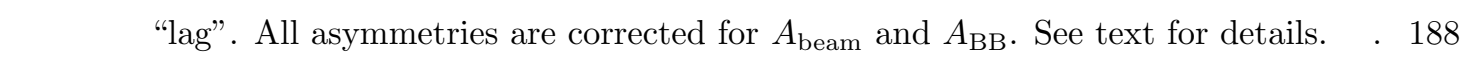

7.7 MDall Physics (top) and Null (bottom) asymmetry combinations in Run2, seg-

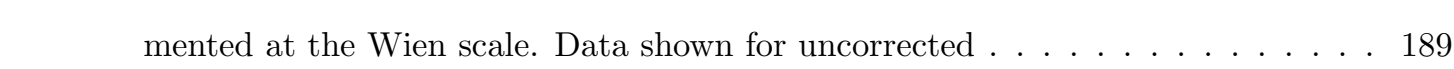

$8.1 \quad$ Initial (left) and final (middle) state QED radiative corrections in the $\overrightarrow{e p}$ interaction, and vertex loop correction (right). . . . . . . . . . . . . . . . 192 
8.2 Polarization measurements versus run number through Run2, from the Møller (red) and Compton (blue) polarimeters. Each data point for the Compton corresponds to an average over about 30 hours. The uncertainties shown represent statistical and estimated systematic errors added in quadrature. The dotted line with the yellow band is the central value and total uncertainty for the polarization in Run2, including a scaling uncertainty. . . . . . . . . . . . . . . . . 193

8.3 Comparison of the azimuthal dependence of the asymmetry measured with fully vertical (purple) and horizontal (green) transverse polarization, from a dedicated 2-hour long measurement in each case. The phase offset between the two orien-

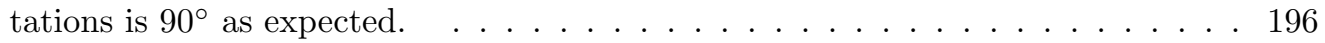

8.4 Placement of positive (red) and negative (blue) PMTs on every MD bar. . . . . 197

8.5 Left: The PV asymmetry $A_{\text {raw }}$ measured from the average of the positive (red) and negative (blue) PMTs on the main detector bars, plotted versus slug number in Run2. The difference between the two, referred as PMT double-difference $A_{\text {PMT-DD }}$ is also plotted in black. All three have been separately fitted to a constant over Run2. Right: The average asymmetries of positive (negative) PMTs over Run2, $A_{\text {raw }}^{+(-)}$, have been plotted in red (blue) versus MD octant and fitted to a sinusoid. . . . . . . . . . . . . . . . . . . . . . . . 198

8.6 Simulated rates on the MD versus current on the toroidal spectrometer. Rates from four different sources are plotted in different colours and blown-up by an order of magnitude relative to the elastic $\vec{e} p$ signal. Nominal value for the magnet current during production was $8921 \mathrm{~A}$, at the peak of the elastic contribution, plotted in red. The maximum inelastic rate occurs at a spectrometer current of $6700 \mathrm{~A}$, where dedicated studies for the measurement of $A_{4}$ were performed. . . 202

8.7 Fit over PVES data on nuclear targets, with our Run2 Modulation prelimi\begin{tabular}{|c|c|}
\hline nary/blinded result $(8.26)$ plotted at $Q^{2}=0.02455(\mathrm{GeV} / \mathrm{c})^{2}$. The fit is shown \\
\hline
\end{tabular} as the black line with the uncertainty given by the yellow band. The fit inter\begin{tabular}{|c|}
\hline cept is our result for the weak charge of the proton, in good agreement with the \\
\hline
\end{tabular} Standard Model prediction (black arrow). The dotted line is the 2013 result from \begin{tabular}{|l|l|l|l|}
\hline Figure & 2.5 & before our preliminary/blinded Run2 measurement. The insert on the \\
\hline
\end{tabular} right is zoomed in at the low- $Q^{2}$ region. . . . . . . . . . . . . . . . . 204 
8.8 Combined constraints on the isovector and isoscalar combinations of the $C_{1 u}, C_{1 d}$ couplings, from the PVES result shown in Figure 8.7 (blue ellipses) and from the APV measurement on cesium (green band). The global constraints are shown as red ellipses. The SM prediction is the black dot, shown as a function of the weak mixing value at the $Z$ resonance. . . . . . . . . . . . . . . . . . 205

8.9 The effect of different fit choices on the $Q_{w}^{p}$ result, for a trial value of the final $Q_{\text {weak }}$ parity-violating asymmetry $A_{\mathrm{PV}}^{\text {trial }}=-230 \pm 10 \mathrm{ppb}$, near its expected value from the SM. The black point and the dotted line correspond to the canonical fit choices used in this dissertation, which give $Q_{w}^{p}[$ trial $]=0.0702 \pm 0.0048$, and the solid horizontal lines are the $1 \sigma$ bound. The $Q_{w}^{p}[$ trial $]$ result is quoted under many different fit choices - see text for details. For reference the $Q_{w}^{p}$ without any $Q_{\text {weak }}$ data and from the 2013 result are shown on the far right, with much higher uncertainty. Reproduced from $[175]$. . . . . . . . . . . . . . . . 208

8.10 Our result for the weak mixing angle from Eq.(8.38) is the blue datum at $\mu=$ $0.157 \mathrm{GeV}$. This result is extracted from the global PVES fit, including our preliminary analysis on the blinded Run2 Modulation dataset of $Q_{\text {weak }}$. Red data points are existing experimental determinations, while the black line is the theoretical prediction for the running of the weak mixing angle in the $\overline{\mathrm{MS}}$ scheme. 209

B.1 Experimental data on a nitrobenzene Kerr cell from [181]. The vertical axis is proportional to the induced birefringence and the horizontal axis is distance along the entrance face of the Kerr cell. The different lines correspond to different applied voltages. The birefringence gradients arise from accumulation of space charge in the bulk of the dielectric near the electrodes, resulting in voltage-dependent optical non-uniformities. . . . . . . . . . . . . . . . . 215

C.1 Ordinary least squares fit to data. . . . . . . . . . . . . . . . . . . 217

C.2 Minimization of vertical versus perpendicular offsets. . . . . . . . . . . . 218

C.3 Correlation in the USLsum and PMTLTG asymmetries over a 5-minute runlet,

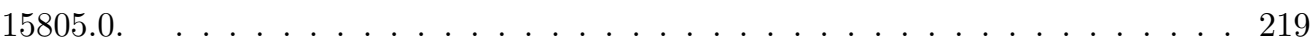

C.4 Fit between the USLsum and PMTLTG asymmetries, exchanging roles as independent and dependent variables (left and right plots) over the same data set. The black lines and fit statistics are extracted from the standard TH1::Fit ROOT command. The red lines correspond to the regression parameters extracted with the symmetric method. . . . . . . . . . . . . . . . . . . . . . . 221 


\section{List of Tables}

2.1 The SM prediction for the tree-level values of neutral weak vector and axial-vector charges of particles. . . . . . . . . . . . . . . . . . . . 10

2.2 The tree-level values of the EM and vector weak charges of quarks and nucleons, in terms of the weak mixing angle $\sin ^{2} \theta_{W}$ and the vector weak quark couplings $C_{1 u}, C_{1 d} \ldots \ldots \ldots \ldots \ldots \ldots \ldots$

$3.1 Q_{\text {weak }}$ design parameters. . . . . . . . . . . . . . . . . 31

$3.2 \quad$ Anticipated contributions to the experimental error budget, from the $2007 Q_{\text {weak }}$ proposal 33$].$

3.3 Systematic uncertainties of the Hall C Møller polarimeter during Run2. Based on analysis from $[119] . \ldots \ldots \ldots \ldots$

3.4 Systematic uncertainties of the Hall C Møller polarimeter during Run2. Based on analysis from $[119 . \ldots \ldots \ldots \ldots \ldots \ldots$

3.5 The duration and size of the three $Q_{\text {weak }}$ running periods. The size is quoted in number of helicity quartets. The size of the subset where Modulation sensitivities are available is quoted separately. Also listed are the Wien and slug sub-periods that define the slow reversals applied by the experiment. The result extracted in this dissertation is from the Run2 Modulation subset, listed in bold. . . . . . . . 57

$4.1 Q_{\text {weak }}$ specifications for control of helicity-correlated beam properties. . . . . . . 61

4.2 Average helicity-correlated beam properties in the Modulation datasets of Run1 and Run2, and comparison with specifications. . . . . . . . . . . . . . . . 99

5.1 List of regression schemes with the monitors included in them. "Target" corresponds to the constructed orbit and energy variables at the target, that enter

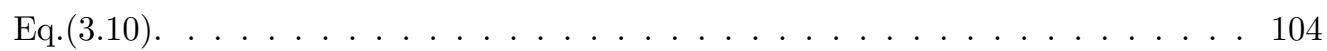

5.2 Dithering responses decomposed to 10 driving amplitudes, or "coils". . . . . . . 113 
5.3 Slug-scale correlation slopes of corrected MDall to beam parameters in Run1 for different correction schemes. Gray, orange and red font color corresponds to significance of $2 \sigma, 3 \sigma$ and $4 \sigma$ respectively. Schemes that are excluded based on these correlations are colored in red. . . . . . . . . . . . . . . . . . . . . 129

5.4 Slug-scale correlation slopes of corrected MDall to beam parameters in Run2 for different correction schemes. Gray, orange and red font color corresponds to significance of $2 \sigma, 3 \sigma$ and $4 \sigma$ respectively. Schemes that are excluded based on these correlations are colored in red. . . . . . . . . . . . . . . . . . . . 129

$5.5 \quad$ Regression and modulation correction schemes that are excluded, in Run1 and Run2 separately, based on the significance of slug-scale residual correlations to beam parameters. . . . . . . . . . . . . . . . . . . . 130

5.6 Polarization-weighted MDall Physics and Null asymmetry results in Run2, cor-

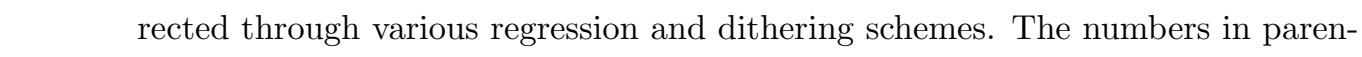
theses are the fit probabilities for each result. Schemes marked in red are excluded

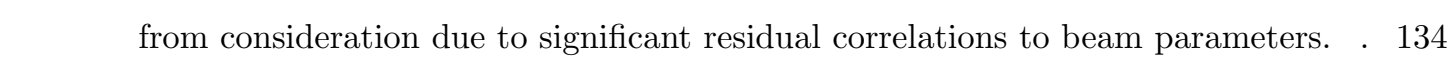

$5.7 \quad$ MDallbars Physics and Null asymmetry results in Run1 through different correc-

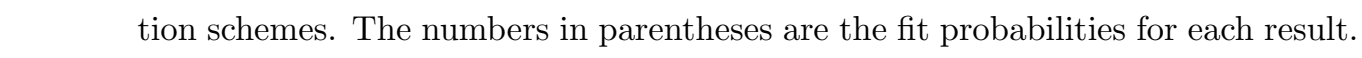
Rows marked in red have significant signs of residual false asymmetries and are excluded from consideration. . . . . . . . . . . . . . . . . 136

5.8 MDall Physics and Null asymmetry results in Run1, after "post-correction" is

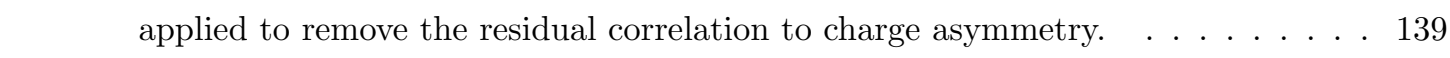

6.1 Correlation slopes between asymmetries from different background detectors in Run2, in units of $\mathrm{ppm} / \mathrm{ppm} . \quad \ldots \ldots \ldots \ldots \ldots \ldots$. . . . . . . . . . 144

$6.2 \quad$ BB signal fraction $f_{\mathrm{BB}}$ values measured directly in blocked-octant studies in Run1 versus raster size, from two separate periods of dedicated studies. . . . . . . . . . 151

$6.3 \quad$ Fractional signal size of detectors with the tungsten shutters installed in the upstream collimator openings in octants 1 and 5. The signal fraction is expressed as a ratio of the detector signal in the blocked-octant run to the signal of the same detector immediately before the shutters were inserted. The shutter tests were repeated in two different occasions, under "good" and "bad" halo conditions. From elogs $779,783,786,784 . \ldots \ldots \ldots \ldots \ldots$. . . . . . . . . . . . 154

6.4 Correlation slopes of the blocked MD bars to each of the background detectors, for the May 2012 blocked-octant dataset. All values quoted in units of ppm/ppm. 156

6.5 The BB signal fractions of background detectors are extracted from the correla-

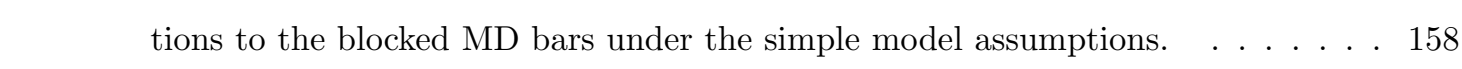


6.6 The BB signal fractions for each detector. For the background detectors the yield fractions may be only an effective description of their correlation, but it is quite a powerful and useful one in predicting the correlations between background asymmetries. The values correspond to a raster size of $4 \times 4 \mathrm{~mm}^{2}$. . . . . . . 160

6.7 Correlation slope between background detectors and the USLsum, in their measured asymmetries and charge sensitivities in Run2. . . . . . . . . . . . . 167

6.8 Correlation slope between MDall and USLsum over Run2, in various binning time scales and formulations. . . . . . . . . . . . . . . . . . . . . . 169

6.9 Correlation slopes of the MDall asymmetry to background asymmetries, averaged at the slug scale, in the subset of Run2 where all background detectors are available. Using the correlation slope to USLsum the expected result for other background detectors has been predicted (in parentheses) based on the very precise correlation slopes between background asymmetries in Run2. . . . . . . . . . 171

6.10 Assigned resolutions $\sigma_{\text {res }}^{i}$ and average distribution widths $\sigma_{A}^{i}$ (see text) for each background detector $i$, used in the study depicted in Figure $|6.20|$ All values in units of ppm. . . . . . . . . . . . . . . . . . . . 172

6.11 Physics and Null MDall asymmetry combinations, with BB correction applied through different background detectors. These results are quoted over the subset of Run2 where dithering data exists and all of the background detectors are available. 176

7.1 Fit probabilities of Physics and Null asymmetry combinations, segmented over different time scales in Run1 and Run2. . . . . . . . . . . . . . . . . . . . . 189

$8.1 \quad$ Assigned value and uncertainty for each term entering the extraction of $A_{\mathrm{PV}} \cdot \quad 203$

A.1 The range of slugs included in each pitt, separated by Run and Wien periods. The identifying number for each pitt is simply the first slug number in its range. 212 


\section{Acronyms}

ACF auto-correlation function

APV atomic parity violation

BB beamline background

BCM beam current monitor

BPM beam position monitor

CEBAF Continuous Electron Beam Accelerator Facility

CERN European Organization for Nuclear Research

CFD computational fluid dynamics

CL confidence level

CSV charge symmetry violation

CW continuous wave

DD double difference

DIS deep inelastic scattering

DOCP degree of circular polarization

DOF degrees of freedom

DSLumi downstream luminosity monitor

DV dependent variable

EM electromagnetic

EW electroweak 
FFB fast feedback

FOM figure of merit

HC helicity-correlated

HCBA helicity-correlated beam asymmetries

HDC horizontal drift chambers

HV high voltage

IA intensity asymmetry

IHWP insertable half-wave plate

IV independent variable

JLab Jefferson Lab

LAPD linear array of photodiodes

LEP Large Electron-Positron collider

$\mathbf{L H}_{2}$ liquid hydrogen

LHC Large Hadron Collider

Linac linear accelerator

MD main detector

OLS ordinary least squares

PC Pockels cell

PITA polarization-induced transport asymmetry

PMT photomultiplier tube

ppb parts per billion

ppm parts per million

PV parity violation

PVES parity-violating electron scattering 
QE quantum efficiency

QPD quad photodiode

$\mathbf{R F}$ radio frequency

RHWP rotatable half-wave plate

SEE Switch Electrode Electronics

SLAC Stanford Linear Accelerator Center

SLC Stanford Linear Collider

SM Standard Model

SRF superconducting radio-frequency

SUSY Supersymmetry

USLsum sum of upstream luminosity monitors

USLumi upstream luminosity monitor

VDC vertical drift chambers 


\section{Chapter 1}

\section{Introduction: The Electroweak}

\section{Standard Model}

The development of the Standard Model (SM), the quantum field theory of the known particles and fields, has been one of the great achievements of physics in the 20th century. The SM describes the strong, electromagnetic (EM), and weak interactions, with its internal symmetry defined by the gauge group $\mathrm{SU}(3)_{\mathrm{C}} \otimes \mathrm{SU}(2)_{\mathrm{L}} \otimes \mathrm{U}(1)_{Y}$. Here $\mathrm{SU}(3)_{\mathrm{C}}$ is the gauge (local) symmetry of the color charge, carried by the eight gluons that mediate the strong interactions, $\mathrm{SU}(2)_{\mathrm{L}}$ is the gauge symmetry of the weak isospin $I_{\mathrm{W}}$, and $\mathrm{U}(1)_{Y}$ is the gauge symmetry of the weak hypercharge $Y$, connected to the electric charge $Q$ and the third component of the weak isospin $I_{\mathrm{W}}^{3}$ through the relation

$$
I_{\mathrm{W}}^{3}=Q-Y / 2
$$

The symmetry structure $\mathrm{SU}(2)_{\mathrm{L}} \otimes \mathrm{U}(1)_{Y}$ characterizes the electromagnetic and weak interactions, mediated by the photon $\gamma$ and the $W^{ \pm}, Z^{0}$ bosons respectively.

One of the pillars of the SM is the concept of local gauge symmetries, pioneered for SU(2) by Yang and Mills in 1954 [1]. Yang and Mills explored the possibility of invariance under local isospin rotation: 11 , when EM interactions are neglected. They proposed the existence of an underlying field with the same relation to isospin that the electromagnetic field has to the electric charge. The quanta of the field should be bosons with spin 1 and three charge states, $\pm e$ and 0 . There was however no satisfactory answer to the issue of their mass: gauge symmetry forbids the gauge bosons from having any mass, and it was supposed that any massless gauge bosons would surely have been detected. On the other hand, inserting a mass by hand as many

\footnotetext{
${ }^{1}$ Heisenberg first proposed the idea that the proton and the neutron are the same particle in two different states of "isotopic spin" [2], with mathematical formalism similar to that of angular momentum. Isospin rotation refers to $p \rightarrow n$ or $u \rightarrow d$.
} 


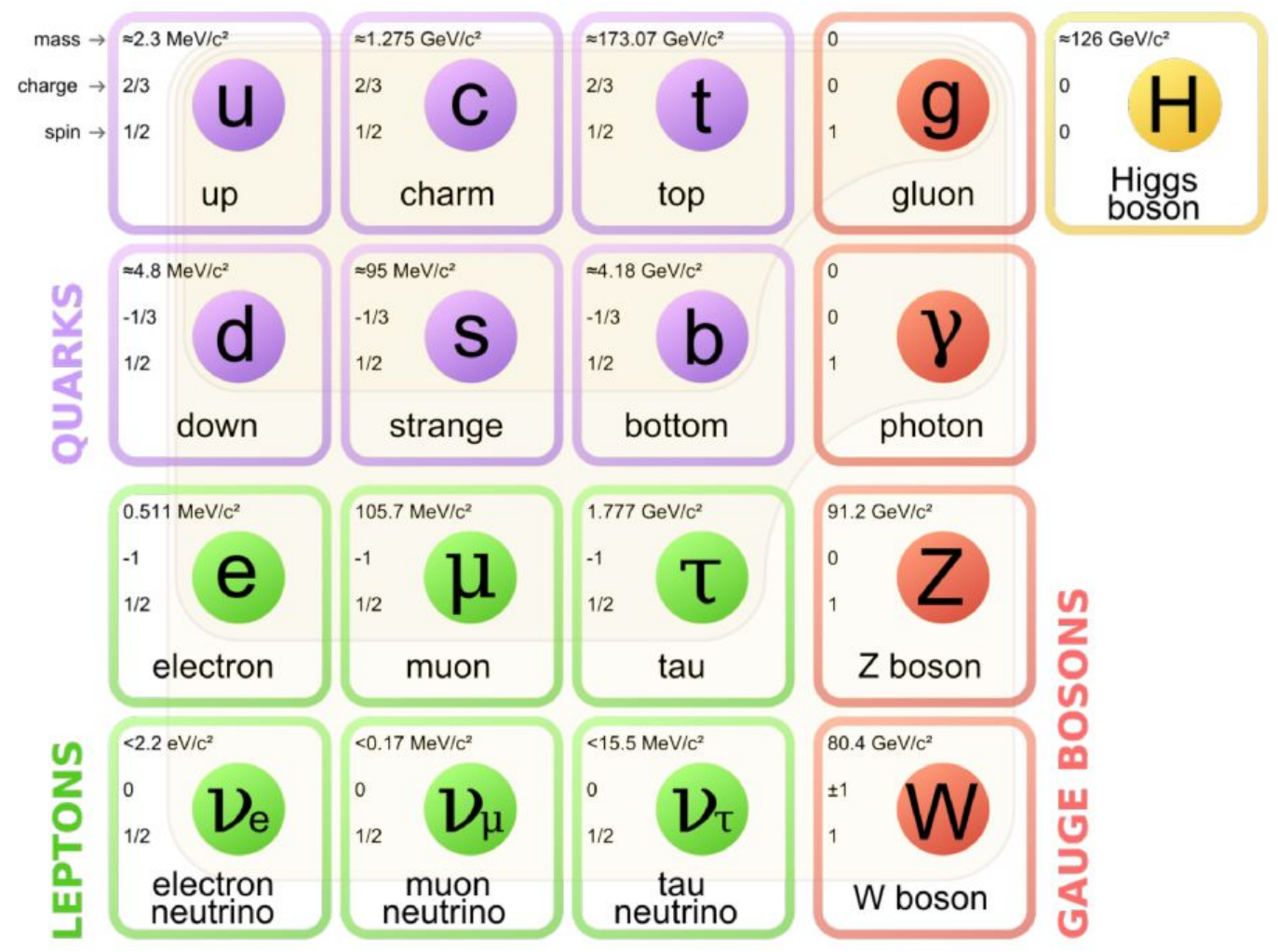

Figure 1.1: The particle content of the Standard Model.

authors did 3 would violate the local symmetry principle. This delayed the application of the Yang-Mills approach to theories of either the weak or the strong interactions. The concept of spontaneously broken symmetries was needed to make progress.

\section{Broken Symmetries}

Many symmetry principles were known and a large fraction of them were only approximate, including the isospin symmetry. Strangeness conservation was known from the beginning to be violated by the weak interactions [4]. Then in 1956 even the sacred symmetries of space and time, in the form of $P$ (parity) and $P T$ (parity-time) conservation, were found to be violated by the weak interactions in the $\mathrm{Wu}$ experiment (Section 2.1).

The concept of a spontaneously broken symmetry, inspired by the successful theory of superconductivity [5], refers to symmetries of the Lagrangian that are not symmetries of the vacuum, the ground state solution of the theory. Nambu suggested that the nucleon mass arises largely as a self-energy of some primary fermion field through the same mechanism as the appearance of energy gap in the theory of superconductivity [6]. But the Goldstone theorem [7, 8] proves that for every spontaneously broken symmetry there must be a massless spinless particle. Again it was considered that such Goldstone bosons would have been observed, which demotivated the 
ideas of spontaneously broken symmetries.

The necessary mechanism for a Nambu-type degenerate-vacuum theory without the issue of zero-mass Yang-Mills gauge bosons or Goldstone bosons was proposed by Anderson [9] in the context of superconductivity, where the two types of bosons "cancel each other out" leaving behind bosons of finite mass. Almost simultaneously in 1964, three independent groups (Higgs [10]; Englert-Brout [1]; and Guralnik-Hagen-Kibble [12]) adopted the mechanism to avoid the massless Goldstone bosons through introduction of vector gauge fields. Thus the spin-1 quanta of some of the gauge fields acquire mass, while the Goldstone bosons turn into their helicity-zero projection. Today this is usually referred as the Higgs mechanism.

\section{Electroweak Unification}

The electroweak (EW) theory was developed independently by Weinberg [13] and Salam [14], employing the ideas of spontaneous symmetry breaking. The symmetries relating the weak and EM interactions are exact symmetries of the Lagrangian but broken by the vacuum. Specifically, the weak isospin and weak hypercharge symmetries are broken by the non-zero vacuum expectation value of a hypothesized spin-zero Higgs doublet. Out of the "primordial" fields $W_{\mu}^{1,2,3}$ and $B_{\mu}$ that correspond to the generators of $\mathrm{SU}(2)_{\mathrm{L}}$ and $\mathrm{U}(1)_{\mathrm{Y}}$ respectively, the symmetry breaking mechanism leads to three massive and one massless vector gauge bosons:

$$
\begin{aligned}
W_{\mu}^{ \pm} & =\left(W_{\mu}^{1} \mp i W_{\mu}^{2}\right) / \sqrt{2} \\
Z_{\mu}^{0} & =W_{\mu}^{3} \cos \theta_{W}-B_{\mu} \sin \theta_{W} \\
A_{\mu} & =W_{\mu}^{3} \sin \theta_{W}+B_{\mu} \cos \theta_{W}
\end{aligned}
$$

The massive $W^{ \pm}$and $Z^{0}$ bosons mediate the weak interactions, whereas the massless $A$ field is associated with the photon. The mixing rotation between the primordial fields $B_{\mu}$ and $W_{\mu}^{3}$ to give the photon and the $Z$ is parametrized by the electroweak mixing angle $\sin \theta_{W}$, often referred to as the Weinberg angle. This is a fundamental parameter at the heart of the EW sector. At tree-level (before radiative corrections) it is connected to the masses of the $W$ and $Z$ vector bosons through the simple relation:

$$
\sin ^{2} \theta_{W}=1-\frac{M_{W}^{2}}{M_{Z}^{2}}
$$

It can also be expressed in terms of the coupling parameters of the electroweak and weak interactions:

$$
\sin ^{2} \theta_{W}=\frac{e^{2}}{g^{2}}=\frac{g^{\prime 2}}{g^{2}+g^{\prime 2}}
$$


where $\mathrm{g}$ and g' are the $\mathrm{SU}(2)_{\mathrm{L}}$ and $\mathrm{U}(1)_{\mathrm{Y}}$ gauge couplings, respectively. We will give a more in-depth discussion on the weak mixing angle in Section 2.5

The weak neutral current was first observed in 1973 in neutrino scattering at CERN in the Gargamelle bubble chamber [15] (Figure 1.2). After some confusing results from atomic experiments that suggested no parity violation at the $\mathrm{Bi}$ atom [16], parity violation in the neutral currents was discovered at the expected level in the scattering of longitudinally polarized electrons from deuterium and hydrogen at the pioneering SLAC E122 experiment in 1978 [17. This experiment gave birth to the field of parity-violating electron scattering (PVES) and provided the final confirmation that the proposed $\mathrm{SU}(2) \otimes \mathrm{U}(1)$ structure was correct.

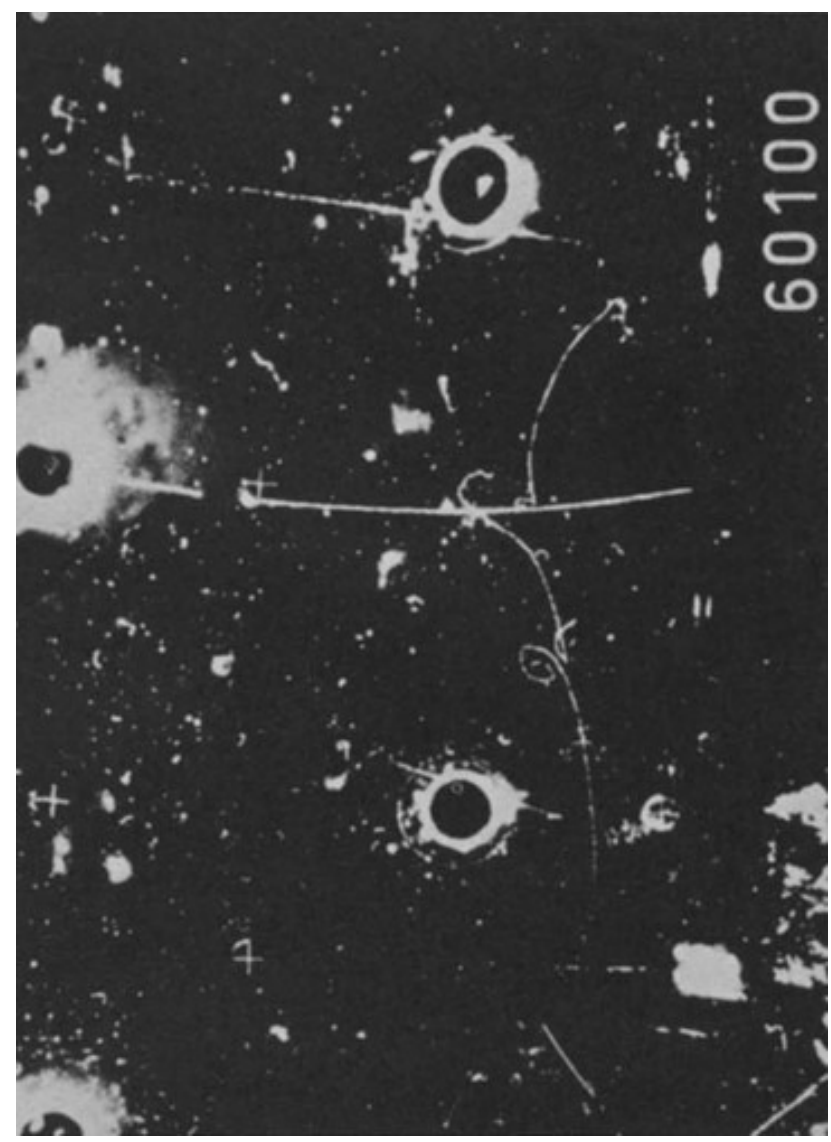

Figure 1.2: A neutral current $\bar{\nu}_{\mu}+e^{-} \rightarrow \bar{\nu}_{\mu}+e^{-}$event in the Gargamelle bubble chamber. Reproduced from [15].

The following year the Nobel Prize

in Physics was awarded to Sheldon Glashow, Steven Weinberg, and Abdus Salam for the development of the electroweak theory.

The intermediate weak vector bosons were detected in experiments at CERN [18, 19] and their measured masses $M_{W}=80.385 \pm 0.015(\mathrm{GeV} / \mathrm{c})^{2}$ and $M_{Z}=91.188 \pm 0.002(\mathrm{GeV} / \mathrm{c})^{2}$ were in agreement to the expectation from the SM. Carlo Rubia and Simon van der Meer were awarded the 1984 Nobel Prize in Physics for this discovery. Very recently, the last piece of the electroweak theory and the final particle predicted by the Standard Model, the Higgs boson, was discovered at the Large Hadron Collider (LHC) at CERN 20, 21. In a crowning triumph for the Standard Model, the detected particle so far appears to have properties consistent with theory. Many decades after their formulation of the spontaneous symmetry breaking mechanism, Higgs and Englert were awarded the 2013 Nobel Prize in Physics. 


\section{Physics Beyond the Standard Model}

The SM of Physics describes the observable world around us to an astounding precision. It has correctly predicted the existence of particles such as the $Z$, the top and the Higgs well before their discovery. Its predictions have stood up to experimental tests of increasing precision over many decades. And yet there are phenomena that the SM simply fails to account for, such as the observed matter-antimatter asymmetry in the universe, the existence of dark matter and dark energy, and the observation of neutrino oscillations and non-zero masses. We also still lack a complete description and integration of the fourth fundamental force, gravity. Furthermore there are theoretical issues within the SM itself, such as the hierarchy problem [22] which refers to the vast discrepancy between the electroweak and the Planck scale. There are 18 free parameters in the canonical Standard Model, not including neutrino masses and mixing angles, too many for a theory to be considered fundamental. Instead the SM is considered to be an effective low-energy approximation of a more fundamental structure.

These open issues are pursued with three basic approaches, often characterized as exploration along the Cosmic, Energy and Intensity Frontiers 23]. Each employs different tools and techniques, but they ultimately address the same fundamental questions. This allows a multipronged approach, where attacking basic questions from different angles furthers knowledge and provides deeper answers, so that the whole is more than a sum of the parts.

The Cosmic Frontier addresses issues at the cosmological scale through astrophysical observations. The Energy Frontier employs high energy machines such as the Tevatron and the Large Hadron Collider to directly excite matter into new forms. The Intensity Frontier explores fundamental physics with intense sources and ultra-sensitive detectors. It encompasses searches for extremely rare processes and for tiny deviations from Standard Model expectations, through a very diverse set of experimental probes that varies from table-top experiments to colliders. Intensity Frontier experiments use precision measurements to probe quantum effects. They typically investigate new laws of physics that manifest at higher energies or weaker interactions than those directly accessible at high-energy particle accelerators. An example is the very precise measurement of the muon anomalous magnetic moment $\alpha_{\mu}$ by the BNLE821 Collaboration [24], which revealed a $\sim 3.6 \sigma$ significant discrepancy from the SM prediction [25, 26]. This is one of the largest experimental deviations from a SM prediction and could potentially be a hint of undiscovered new interactions.

This dissertation describes the experimental effort by the $Q_{\text {weak }}$ Collaboration at the Thomas Jefferson National Accelerator Facility (Jefferson Lab) to measure the vector neutral weak charge of the proton, $Q_{w}^{p}$, using the lab's high-intensity polarized electron beam. $Q_{w}^{p}$ is the analog to the proton's electric charge for neutral-weak interactions, connected to the weak mixing 
angle $\sin ^{2} \theta_{W}$ of the electroweak structure. The weak charge of the proton is both precisely predicted and suppressed in the SM, and thus an excellent candidate for an indirect search for new parity-violating physics between electrons and light quarks. The $Q_{\text {weak }}$ experiment measured the parity-violating asymmetry in the scattering of longitudinally polarized electrons from protons, employing the same technique as the pioneering Prescott E122 experiment. The full $Q_{\text {weak }}$ result will determine $Q_{w}^{p}$ to high precision, such that the measurement becomes a test of the SM in the electroweak sector at the Intensity Frontier. Due to the suppression of the weak charge this measurement has enhanced sensitivity to many models of physics beyond the SM, with high complementarity to other experimental probes over a wide range of energy scales.

In Chapter 2 we introduce the formalism and world experimental program of parity-violating electron scattering, and provide the motivation and context for the $Q_{\text {weak }}$ measurement. An overview of the experimental design and apparatus is provided in Chapter 3 . In Chapter 4 we present our efforts to optimize the Jefferson Lab polarized source in order to suppress and cancel false asymmetries in our measurement arising from beam parameters that correlate to the beam helicity. Methods to correct such false asymmetries are described in Chapter 5 . In Chapter 6 we examine the large background asymmetries that we encountered during the experiment, believed to be connected with higher moments of helicity-correlated beam parameters, and develop a correction algorithm. The statistical properties of our corrected measurement are assessed in Chapter 7 offering a measure of the efficiency of our corrections. Finally in Chapter 8 we extract a blinded result for the $Q_{\text {weak }}$ parity-violating asymmetry from a limited dataset. This allows the determination of the weak charge of the proton from a global fit of PVES asymmetries, and an estimation of the mixing angle of the electroweak sector. 


\section{Chapter 2}

\section{The Weak Charge of the Proton}

\section{and Beyond}

This chapter introduces the formalism of parity-violating electron scattering, the technique utilized by the $Q_{\text {weak }}$ experiment. We will present the early result released by the experiment which allowed the first determination of the weak charge of the proton $Q_{w}^{p}$, relying on the world parity-violating electron scattering dataset to constrain uncertainties of hadronic structure. The physics motivation includes a precise extraction of the weak mixing angle $\sin ^{2} \theta_{W}$, a fundamental parameter of the electroweak sector. The full $Q_{\text {weak }}$ result is expected to be a stringent test of the theoretical prediction and place significant constraints on models of physics beyond the Standard Model.

\subsection{Parity Violation in the Weak Interactions}

Parity is the discrete transformation that refers to inversion of spatial coordinates, $(x, y, z) \rightarrow(-x,-y,-z)$. This corresponds to a reflection through the origin, equivalent to a rotation and a mirror (plane) reflection. Since physical laws are invariant under any rotation, the parity transformation can be thought of as a mirror reflection, $(x, y, z) \rightarrow(x, y,-z)$, which transforms a system from left-handed to right-handed and vice versa.

Lee and Yang first suggested [27] that weak interactions may violate the parity symmetry. That was indeed confirmed experimentally by C.S. Wu et al. 28, through a measurement suggested by Lee and Yang. The $\beta$-decay rate of ${ }^{60} \mathrm{Co}$ nuclei was measured with the nuclei placed in a strong magnetic field to align their spin. The experiment demonstrated in two opposite directions of the applied magnetic field that the electrons were emitted preferentially in the opposite direction of the magnetic field, and therefore opposite to the nuclear spin. There is an 
asymmetry in the rate between the two "mirror" processes, thus the parity symmetry is violated.

This led to a fundamental reformulation of the weak interaction theory in terms of a vector - axial-vector $(\mathcal{V}-\mathcal{A})$ structure for the charged weak current, making it maximally parityviolating: the charged weak current interacts only with left-handed particles or equivalently with right-handed antiparticles. For their revolutionary prediction of parity violation (PV) in the weak interactions, Lee and Yang received the 1964 Nobel Prize in Physics.

\subsection{Parity-Violating Electron Scattering}

Electron scattering has been used extensively as a tool to study the electromagnetic structure and properties of nuclei. The Sachs EM electric and magnetic form factors $G_{E}^{\gamma}$ and $G_{M}^{\gamma}[29$ ] have been measured with high precision down to very low momentum transfer $Q^{2}$. The latest results from the A1 Collaboration [30] and a fit over the world data of unpolarized and polarized electron scattering is shown in Figure 2.1 for the proton form factors. At the Breit (center-ofmass) frame, $G_{E}^{\gamma}$ and $G_{M}^{\gamma}$ are simply the Fourier transforms of the charge and magnetization distributions of the nucleon, such that their $Q^{2} \rightarrow 0$ limit gives the total charge and magnetic moment of the nucleon, respectively. The radii of the charge and magnetic distributions in the nucleon are related to the $Q^{2} \rightarrow 0$ derivatives of the Sachs form factors.

The electroweak symmetry suggests that the neutral weak interaction appears everywhere there is an electromagnetic interaction. However at low energies the weak interaction is weaker by orders of magnitude compared to the EM one, as the weak amplitude is suppressed by the heavy mass of the $Z$ propagator. Still the weak part of electron scattering can be employed to access parts of the interaction that are not available through the purely EM process.

The pioneering Prescott E122 experiment [17] which confirmed the structure of the weak neutral currents, introduced the powerful technique of measuring the helicity dependence of electron scattering rates. Helicity is defined as the expectation value of the intrinsic angular momentum (spin) projected onto the direction of motion:

$$
h=\frac{\mathbf{s} \cdot \mathbf{p}}{|s||p|}
$$

At the massless limit, helicity is equivalent to handedness (chirality). In a scattering process from an unpolarized target, reversing the helicity of the electrons is equivalent to a parity transformation. By measuring the asymmetry of scattering rates in states of opposite helicity, we gain access to the weak part of the interaction that is uniquely characterized by violation of the parity symmetry.

The leading-order Feynman diagrams for electron-proton scattering are shown in Figure 2.2 

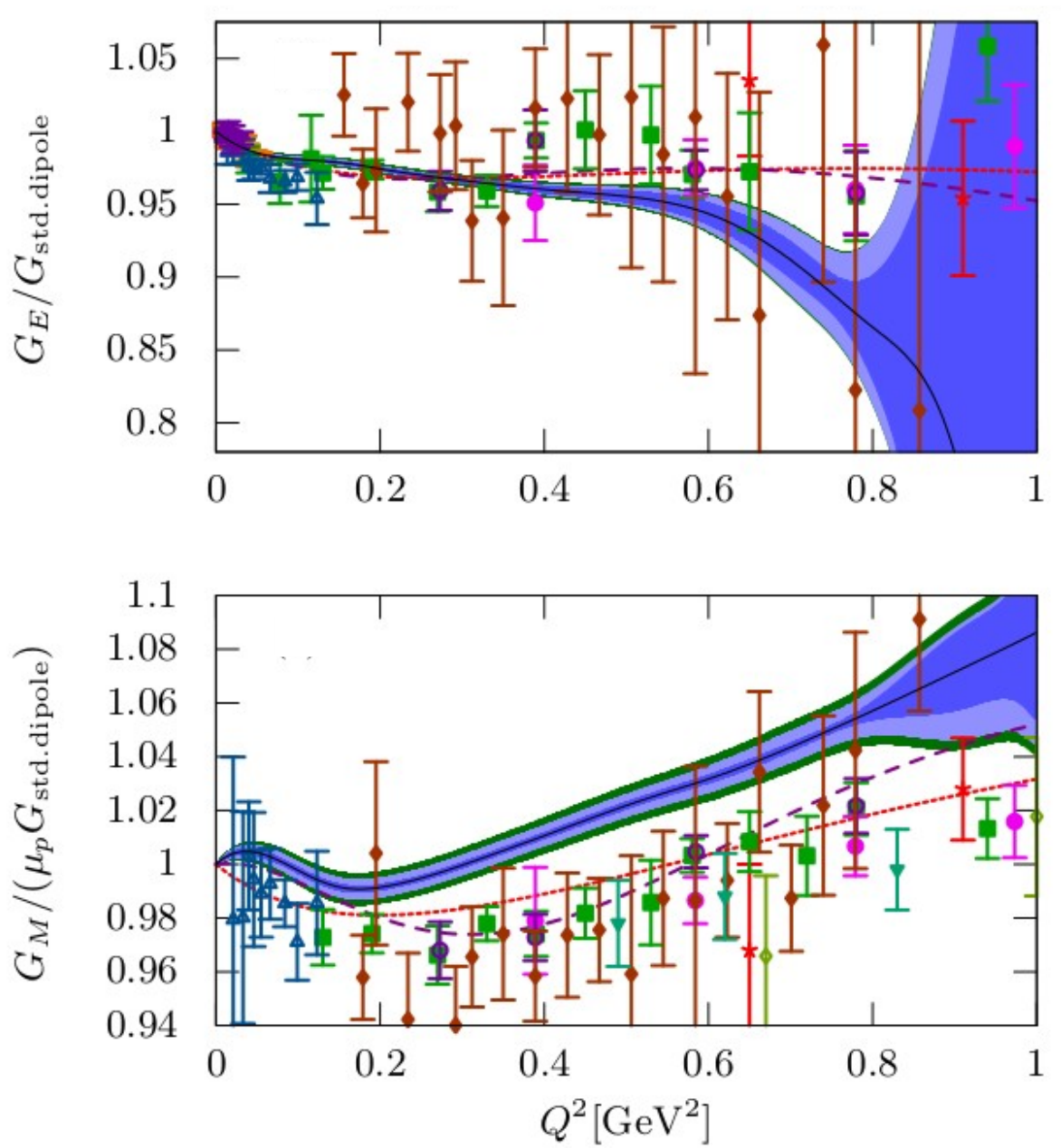

Figure 2.1: The world data for the proton $G_{E}$ and $G_{M}$ form factors, normalized to the standard dipole. Reproduced from [30.

where a photon $\gamma$ is exchanged in the EM interaction and a $Z$ boson in the neutral weak process. The corresponding amplitudes are:

$$
\begin{aligned}
\mathcal{M}_{\gamma} & =-\frac{e^{2}}{q^{2}} j_{\gamma}^{\mu} J_{\gamma \mu} \\
\mathcal{M}_{Z} & =-\frac{G_{F}}{2 \sqrt{2}} j_{Z}^{\mu} J_{Z \mu}
\end{aligned}
$$

where $j_{\gamma, Z}$ and $J_{\gamma, Z}$ are the neutral currents of the electron and nucleon respectively, that characterize the exchange of a photon or a $Z$ boson. The electron neutral currents are

$$
\begin{aligned}
j_{\gamma}^{\mu} & =\bar{u}_{e} \gamma^{\mu} u_{e} \\
j_{Z}^{\mu} & =\bar{u}_{e}\left(g_{V}^{e} \gamma^{\mu}+g_{A}^{e} \gamma^{\mu} \gamma_{5}\right) u_{e}
\end{aligned}
$$

The $g_{V}^{e}$ and $g_{A}^{e}$ parameters are the electron neutral weak vector and axial-vector coupling pa- 


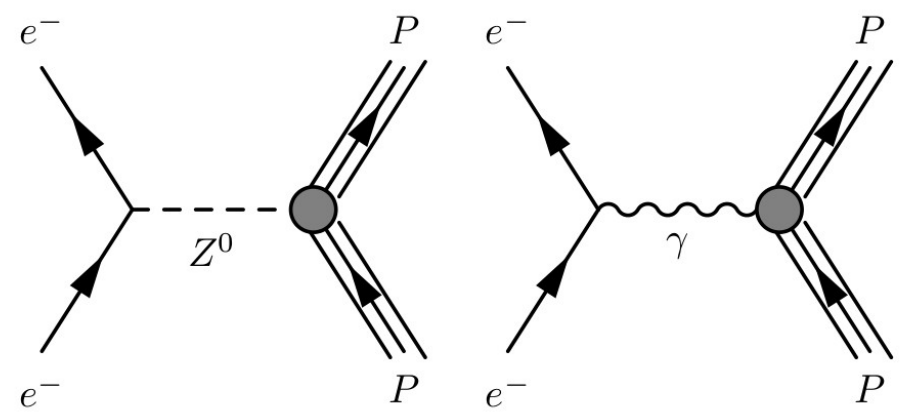

Figure 2.2: Tree-level diagrams of the neutral current weak and EM electron-proton interaction, characterized by the exchange of a $Z$ boson or a photon respectively.

Table 2.1: The SM prediction for the tree-level values of neutral weak vector and axial-vector charges of particles.

\begin{tabular}{c|cr} 
Particle & $g_{V}$ & $g_{A}$ \\
\hline$\nu_{e}$ & 1 & -1 \\
$e^{-}$ & $-1+4 \sin ^{2} \theta_{w}$ & 1 \\
$u, c, t$ & $1-\frac{8}{3} \sin ^{2} \theta_{w}$ & -1 \\
$d, s, b$ & $-1+\frac{4}{3} \sin ^{2} \theta_{w}$ & 1
\end{tabular}

rameters, respectively. For the charged weak current mediated by the $W^{ \pm}$bosons, both these parameters are equal to 1 , corresponding to maximal parity violation. The neutral weak interactions on the other hand acquire a parity-conserving component arising due to mixing with the EM interaction. In analogy to the electromagnetic coupling $e$ which defines the EM charge, the $g_{V}^{e}$ and $g_{A}^{e}$ parameters define the neutral weak vector and axial-vector charges of the electron. Their values for different particles are given in Table 2.1, defined in terms of the electric charge and weak isospin (Eq. 1.1) as:

$$
\begin{aligned}
& g_{V}^{f}=I_{W}^{3}(f)-2 Q^{f} \sin ^{2} \theta_{W} \\
& g_{A}^{f}=I_{W}^{3}(f)
\end{aligned}
$$

The nucleon vertices are more complicated due to hadronic structure, parametrized through the Dirac and Pauli form factors $F_{1}$ and $F_{2}$ :

$$
\begin{aligned}
& J_{\gamma}^{\mu}=\bar{u}_{N}\left[\gamma^{\mu} F_{1}^{N, \gamma}\left(Q^{2}\right)+\frac{i \sigma^{\mu \nu} q_{\nu}}{2 M} F_{2}^{N, \gamma}\left(Q^{2}\right)\right] u_{N} \\
& J_{Z}^{\mu}=\bar{u}_{N}\left[\gamma^{\mu} F_{1}^{N, Z}\left(Q^{2}\right)+\frac{i \sigma^{\mu \nu} q_{\nu}}{2 M} F_{2}^{N, Z}\left(Q^{2}\right)+\gamma^{\mu} \gamma_{5} G_{A}^{N, Z}\left(Q^{2}\right)\right] u_{N}
\end{aligned}
$$

$G_{A}$ is the axial form factor of the nucleon that only appears in the weak neutral-current exchange. The Dirac and Pauli form factors can be expressed through the previously introduced (Figure 2.1) 
Sachs electric and magnetic form factors:

$$
\begin{gathered}
G_{E}\left(Q^{2}\right)=F_{1}\left(Q^{2}\right)-\tau F_{2}\left(Q^{2}\right) \\
G_{M}\left(Q^{2}\right)=F_{1}\left(Q^{2}\right)-F_{2}\left(Q^{2}\right)
\end{gathered}
$$

where $\tau=Q^{2} / 4 M_{N}^{2}$ and $M_{N}$ is the mass of the nucleon. The neutral weak form factors of the nucleon that are accessed by the $Z$ exchange, $G_{E, M}^{Z}$, are not as well known as the EM form factors. For each nucleon the form factors can be decomposed in terms of their quark content, weighted by the appropriate charge. For the proton, neglecting heavier quarks [31, 32]:

$$
\begin{aligned}
G_{E, M}^{p, \gamma} & =\sum_{q=u, d, s} Q^{q} G_{E, M}^{q} \\
G_{E, M}^{p, Z} & =\sum_{q=u, d, s} g_{V}^{q} G_{E, M}^{q} \\
G_{A}^{p, Z} & =\sum_{q=u, d, s} g_{A}^{q} G_{A}^{q}
\end{aligned}
$$

where $Q^{q}, g_{V}^{q}$ and $g_{A}^{q}$ are the EM, weak vector and axial-vector charges of each light quark flavor $q=u, d, s$. Employing isospin symmetry we can write the neutral weak form factors for the proton and neutron in terms of the well studied proton and neutron EM form factors, and the strange form factors $G_{E, M}^{s}$ :

$$
\begin{aligned}
& G_{E, M}^{p, Z}=\left(1-4 \sin ^{2} \theta_{W}\right) G_{E, M}^{p, \gamma}-G_{E, M}^{n, \gamma}-G_{E, M}^{s} \\
& G_{E, M}^{n, Z}=\left(1-4 \sin ^{2} \theta_{W}\right) G_{E, M}^{n, \gamma}-G_{E, M}^{p, \gamma}-G_{E, M}^{s}
\end{aligned}
$$

The weak amplitude $\left|\mathcal{M}_{Z}\right|$ is weaker from the EM $\left|\mathcal{M}_{\gamma}\right|$ by $\sim 5$ orders of magnitude at a low $Q^{2} \ll M_{Z}^{2}$. Therefore the leading order PV contribution arises from the interference between the parity-conserving (PC) amplitude of the photon exchange, with the PV part of the weak amplitude mediated by $Z$ exchange. The purely weak term $\left|M_{Z}^{\mathrm{PV}}\right|^{2}$ is negligible by comparison:

$$
A_{\mathrm{PV}}=\frac{2 \mathcal{M}_{\gamma}^{*} \mathcal{M}_{Z}^{\mathrm{PV}}}{\left.\left|\mathcal{M}_{\gamma}^{2}+2 \mathcal{M}_{\gamma}^{*} \mathcal{M}_{Z}^{\mathrm{PV}}+\right| \mathcal{M}_{Z}^{\mathrm{PV}}\right|^{2}} \approx \frac{2 \mathcal{M}_{\gamma}^{*} \mathcal{M}_{Z}^{\mathrm{PV}}}{\left|\mathcal{M}_{\gamma}\right|^{2}}
$$

For the electron-proton interaction, in terms of the proton Sachs form factors [33]:

$$
A_{\mathrm{PV}}=-\frac{G_{F} Q^{2}}{4 \sqrt{2} \pi \alpha} \frac{g_{A}^{e}\left(\epsilon G_{E}^{p, \gamma} G_{E}^{p, Z}+\tau G_{M}^{p, \gamma} G_{M}^{p, Z}\right)+g_{V}^{e} \epsilon^{\prime} G_{M}^{p, \gamma} G_{A}^{p, Z}}{\epsilon\left(G_{E}^{p, \gamma}\right)^{2}+\tau\left(G_{M}^{p, \gamma}\right)^{2}}
$$

where $\epsilon \equiv\left[1+2(1+\tau) \tan ^{2}(\theta / 2)\right]^{-1}$ and $\epsilon^{\prime} \equiv\left[\tau(1+\tau)\left(1-\epsilon^{2}\right)\right]^{1 / 2}$ are kinematic quantities and $\theta$ is the electron scattering angle in the lab frame. Parity violation arises to leading order from the two terms in the numerator of Eq.2.10. The first term characterizes the coupling $\mathcal{V}(q) \times \mathcal{A}(e)$ 
between the vector quark current to the axial electron current; and the second term from the opposite coupling, $\mathcal{A}(q) \times \mathcal{V}(e)$. The corresponding tree-level axial-vector and vector couplings of the electron are (Table 2.1) $g_{A}^{e}=1$ and $g_{V}^{e}=-1+4 \sin ^{2} \theta_{w}$.

The lowest order parity-violating part of the effective contact interaction between an electron and a nucleon is characterized by the Lagrangian

$$
\mathcal{L}_{e q}^{\mathrm{PV}}=-\frac{G_{F}}{\sqrt{2}}\left[\bar{e} \gamma_{\mu} \gamma_{5} e \sum_{q=u, d, s} C_{1 q} \bar{q} \gamma^{\mu} q+\bar{e} \gamma_{\mu} e \sum_{q} C_{2 q} \bar{q} \gamma^{\mu} \gamma_{5} q\right]
$$

where $G_{F}$ is the Fermi constant, related to the weak coupling by $G_{F}=\sqrt{2}\left(g^{2} / 8 M_{W}^{2}\right)$ and measured to high precision from the muon lifetime by the MuLan Collaboration [34. We have neglected heavy quarks and summed over the light quarks, including strange quarks that only contribute through virtual quark-antiquark pairs in the sea. The two terms in the Langrangian correspond to parity violation arising from the $\mathcal{V}(q) \times \mathcal{A}(e)$ and $\mathcal{A}(q) \times \mathcal{V}(e)$ interactions. The quark-specific $C_{1 q}, C_{2 q}$ couplings parametrize the strength of the two types of electron-quark interaction:

$$
\begin{aligned}
& C_{1 q}=-\frac{1}{2} g_{A}^{e} g_{V}^{q}=-\frac{1}{2} g_{V}^{q} \\
& C_{2 q}=\frac{1}{2} g_{V}^{e} g_{A}^{q}=\frac{1}{2}\left(1-4 \sin ^{2} \theta_{W}\right) g_{A}^{q}
\end{aligned}
$$

They can also be used to parametrize the EW structure of the proton characterized by the $G_{E, M}^{Z}$ form factors. At $Q^{2} \rightarrow 0$ these coupling parameters are related to the quark vector and axial-vector weak charges:

$$
\begin{aligned}
& g_{V}^{u, d}=-2 C_{1 u, d} \\
& g_{A}^{u, d}=\frac{2 C_{2 u, d}}{1-4 \sin ^{2} \theta_{W}}
\end{aligned}
$$

The vector weak charge of the proton and neutron can be defined in terms of the $C_{1}$ couplings of their constituent quarks, at tree-level:

$$
\begin{aligned}
& Q_{w}^{p}=-2\left(2 C_{1 u}+C_{1 d}\right) \\
& Q_{w}^{n}=-2\left(C_{1 u}+2 C_{1 d}\right)
\end{aligned}
$$

More generally, the weak charge of a nucleus with $\mathrm{Z}$ protons and $\mathrm{N}$ neutrons is:

$$
Q_{w}(Z, N)=-2\left[(2 Z+N) C_{1 u}+(Z+2 N) C_{1 d}\right]
$$

Then the weak charge of the proton $Q_{w}^{p}$ can be expressed in terms of the weak mixing angle 
Table 2.2: The tree-level values of the EM and vector weak charges of quarks and nucleons, in terms of the weak mixing angle $\sin ^{2} \theta_{W}$ and the vector weak quark couplings $C_{1 u}, C_{1 d}$.

\begin{tabular}{c|ccc} 
& EM charge & \multicolumn{2}{c}{ Vector weak charge } \\
\hline$u$ & $2 / 3$ & $1-\frac{8}{3} \sin ^{2} \theta_{w}$ & $-2 C_{1 u}$ \\
$d$ & $-1 / 3$ & $-1+\frac{4}{3} \sin ^{2} \theta_{w}$ & $-2 C_{1 d}$ \\
$p(u u d)$ & 1 & $1-4 \sin ^{2} \theta_{w}$ & $-2\left(2 C_{1 u}+C_{1 d}\right)$ \\
$n(d d u)$ & 0 & -1 & $-2\left(C_{1 u}+2 C_{1 d}\right)$
\end{tabular}

$\sin ^{2} \theta_{W}$. Substituting the tree-level values of $g_{V}^{u}, g_{V}^{d}$ from Table 2.1

$$
Q_{w}^{p}=1-4 \sin ^{2} \theta_{W}
$$

Because the value of $\sin ^{2} \theta_{W}$ is numerically close to $1 / 4$, the weak charge of the proton is suppressed in the SM. This suppression is characteristic for protons but not for neutrons, and therefore it is absent in any other nucleus. This makes $Q_{w}^{p}$ very sensitive to the weak mixing angle and offers a unique place to extract it at low momentum transfer.

In complete analogy to the electric charge of the proton, which is the $Q^{2} \rightarrow 0$ limit of the EM Sachs form factor $G_{E}^{p, \gamma}$, the vector weak charge of the proton $Q_{w}^{p}$ is defined as the $Q^{2} \rightarrow 0$ limit of $G_{E}^{p, Z}$. It is convenient to define it at $Q^{2} \rightarrow 0$ so that it is a static property of the proton which doesn't depend on the energy of the interaction, unlike the $C_{1 q}$ couplings and the weak mixing angle. At forward angles and $Q^{2} \rightarrow 0$ the kinematic factors $\epsilon \rightarrow 1$ and $\tau, \epsilon^{\prime} \rightarrow 0$. At these kinematics the axial-vector quark contribution is heavily suppressed and only the vector weak quark charges are accessed. Then the expression 2.10 for the asymmetry can be reduced to:

$$
\overline{A_{\mathrm{PV}}}=Q_{w}^{p}+Q^{2} B\left(Q^{2}, \theta\right)
$$

where the leading $Q^{2}$ dependence has been absorbed in the reduced asymmetry $\overline{A_{\mathrm{PV}}}$ :

$$
\overline{A_{\mathrm{PV}}} \equiv A_{\mathrm{PV}}(-4 \sqrt{2} \pi \alpha) /\left(G_{F} Q^{2}\right)
$$

The leading term in Eq. 2.17) is the vector weak charge of the proton $Q_{w}^{p}$ that can be accessed through a measurement of $A_{\mathrm{PV}}$. Conceptually, at $Q^{2} \rightarrow 0$ the proton can be considered a point particle and the reduced asymmetry is equal to the weak charge. The second term parametrizes contributions of hadronic structure defined in terms of EM, strange and weak form factors. This contribution grows in significance with $Q^{2}$ and is suppressed at the low $Q^{2}$ of the $Q_{\text {weak }}$ measurement. As we will see, the global parity-violating electron scattering (PVES) data constrain this contribution and allow a relatively clean extraction of $Q_{w}^{p}$ from a measurement of $A_{\mathrm{PV}}$ at low $Q^{2}$. 


\subsection{The World PVES Program}

Through decades of progress after the seminal E122 experiment at SLAC, parity-violating electron scattering has become a precision tool. There is a continuous interplay between probing hadronic structure and electroweak physics.

At Jefferson Lab (JLab) in Newport News, VA, the vision for a strong parity-violating electron scattering program using the Continuous Electron Beam Accelerator Facility (CEBAF) was set very early in the life of the accelerator, in a 1989 letter of intent [35. It was realized that a high precision determination of $\sin ^{2} \theta_{W}$ could be made through the measurement of $A_{\mathrm{PV}}$ in elastic electron-proton scattering, while also accessing unique combinations of the $C_{1}$ coupling constants that test extensions to the SM theory, in complementarity to high-energy collider measurements. This was the scientific justification and experimental concept for the $Q_{\text {weak }}$ experiment, which was finally performed between 2010-2012 and culminated the Jefferson Lab PVES program in the $6 \mathrm{GeV}$ era.

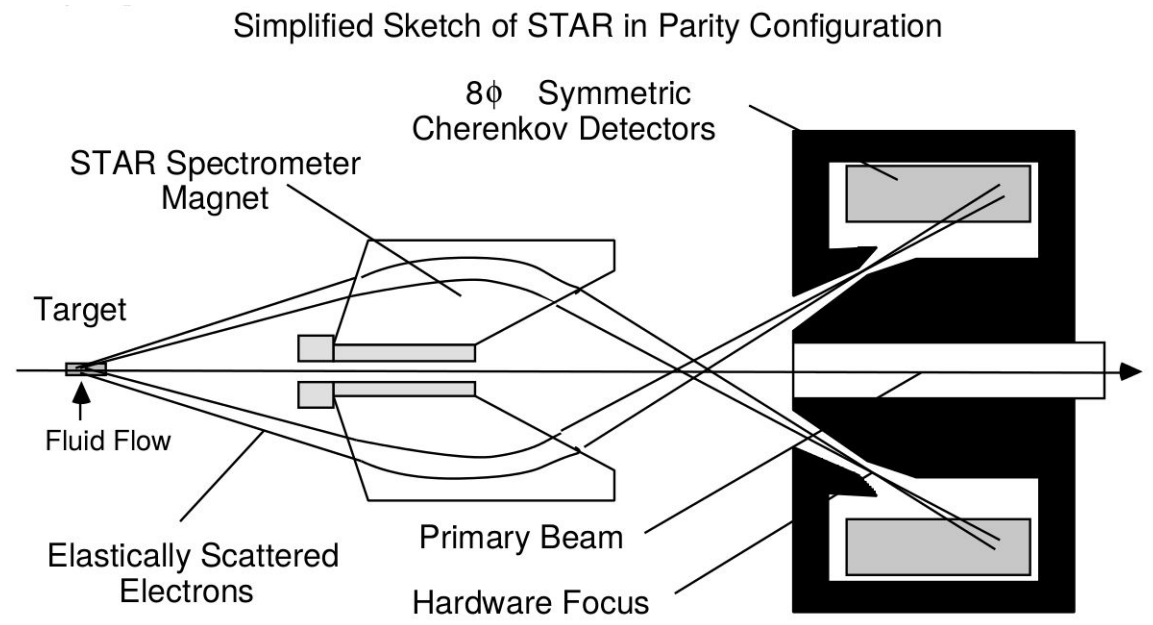

Figure 2.3: Conceptual design of the "STAR" (Symmetric Toroidal ARray) spectrometer from the 1989 letter of intent 35. The basic concepts of a large-acceptance magnetic spectrometer, azimuthaly symmetric detector array, and collimation to suppress backgrounds, would be employed by experiments such as $G 0$ and $Q_{\text {weak }}$ ( $c f$. Figure 3.1 in the Jefferson Lab PVES program.

However, before a precision test of the electroweak theory could be performed, it was first necessary to constrain theoretical uncertainties in the interpretation of a parity violation measurement in the electron-quark sector. These uncertainties mostly concern hadronic structure, and especially the role of strange quarks which is poorly known relative to the valence $u, d$ quarks. Semi-leptonic neutral weak current matrix elements offer unique access to strange quark-antiquark effects [32. The programs of HAPPEX [36, 37, 38, 39] and G0 [40, 41] at 


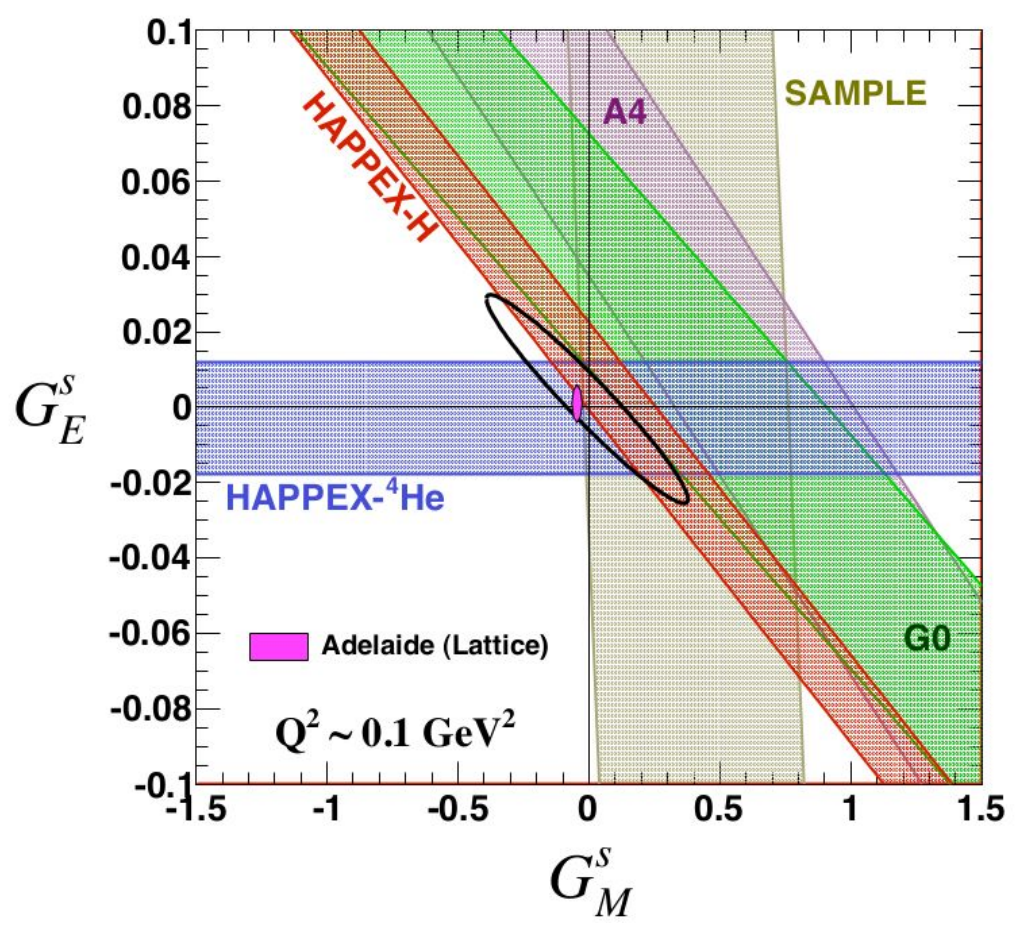

Figure 2.4: Experimental constraints on $G_{E}^{s}$ and $G_{M}^{s}$ in the region of $Q^{2}=0.1 \mathrm{GeV}^{2}$ plotted as $1 \sigma$ bands. The result of a QCD calculation from Adelaide [48] is shown as a filled ellipse. The $68 \%$ CL contour of a global fit 49 ] incorporating data beyond what is included in this plot is shown as a solid elliptical contour. Reproduced from [50].

Jefferson Lab, PVA4 [42, 43, 44] at Mainz, and SAMPLE [45, 46] at MIT-Bates, utilized the unique sensitivity of the PVES technique to study the strange quark content of the nucleon over a wide kinematic range. The motivation included theoretical suggestions of potentially large contributions of strange quarks to the nucleon vector form factors. However the results from this program mostly suggest that the strange quark contribution to the nucleon properties at low $Q^{2}$ is consistent with zero (Figure 2.4). A recent direct lattice QCD calculation achieves unprecedented statistical precision by implementing a variance reduction technique called hierarchical probing. The calculation shows for the first time that both $G_{E}^{s}$ and $G_{M}^{s}$ are non-zero with high significance [4], although future calculations at near-physical quark masses are needed to confirm these results.

The E158 experiment at the Stanford Linear Accelerator Center (SLAC) made the first observation of the parity-violating asymmetry in Møller scattering, via the scattering of longitudinally polarized electrons from unpolarized atomic electrons in a liquid hydrogen target [51]. Unaffected by nuclear structure effects this measurement provides the most precise determination of the weak mixing angle below the $Z$ pole to date, establishing its "running" with over $6 \sigma$ significance (Section 2.5. Important advances in the experimental technique were achieved by the E158 Collaboration to measure the very small PV asymmetry in the Møller process. More recently the PVDIS Collaboration at Jefferson Lab measured the parity-violating asymmetry 
in the deep inelastic scattering (DIS) of electrons from a deuterium target [52. This measurement yields a determination of the $C_{2 u}, C_{2 d}$ coupling parameters of the $\mathcal{A}(q) \times \mathcal{V}(e)$ interaction (Eq. 2.12 , constraining new physics over a complimentary set of parameters to the $C_{1 q}$ couplings, which are examined in this thesis.

The world PVES program achieved significant technological advances in polarized sources, cryo-target systems, polarimetry, and control of helicity-correlated beam parameters. This progress allows the feasibility of the challenging design parameters for the $Q_{\text {weak }}$ experiment. The constraints on the strange form factors from the global fits [49, [53, 54] and the suggested suppression of their contribution, allow the low- $Q^{2}$ forward-angle $Q_{\text {weak }}$ measurement to make the first determination of the weak charge of the proton with theoretical cleanliness and test the SM prediction.

\subsection{First Determination of the Weak Charge of the Proton}

The $Q_{\text {weak }}$ Collaboration proposed the measurement of the PV asymmetry $A_{\mathrm{PV}}$ at a very low momentum transfer $Q^{2}=0.025(\mathrm{GeV} / \mathrm{c})^{2}$, in order to extract the weak charge of the proton with suppressed hadronic structure uncertainties. Due to the very low $Q^{2}$ of the measurement and the suppressed value of $Q_{w}^{p}$, the weak charge contribution to $A_{\mathrm{PV}}$ (first term in Eq. 2.17) for the $Q_{\text {weak }}$ measurement is of order $-2 \times 10^{-7}$, or -200 parts per billion (ppb). The very small size of the asymmetry makes this a challenging measurement. Systematic uncertainties in the correction of false effects that arise experimentally become more important and have to be better controlled than in previous measurements. Even at this small $Q^{2}$ the hadronic term $B\left(Q^{2}, \theta\right)$ in Eq.2.17 contributes approximately $30 \%$ of $A_{\mathrm{PV}}$, demanding sufficient knowledge of the underlying form factors.

The $Q_{\text {weak }}$ experiment released an early result from a small subset of the data from the commissioning phase of the experiment, constituting only $4 \%$ of the full dataset [55]. That early result allowed for the first determination of the weak charge of the proton. For the extrapolation to $Q^{2} \rightarrow 0$ we rely on the existing world dataset of PVES data to constrain hadronic structure parameters.

The $Q_{\text {weak }}$ commissioning result for the parity-violating asymmetry in elastic $\vec{e} p$ scattering at $Q^{2}=0.025(\mathrm{GeV} / \mathrm{c})^{2}$ is $A_{\mathrm{PV}}^{e p}=-279 \pm 35$ (stat) \pm 31 (syst) ppb, shown as the red datum at the corresponding $Q^{2}$ in Figure 2.5. Following the procedure outlined in [49, 56, the weak charge of the proton is extracted from a global fit of Eq. 2.17) on PVES asymmetries measured on hydrogen, deuterium and ${ }^{4} \mathrm{He}$ targets. Data from the SAMPLE, HAPPEX, G0 and MAMI PVA4 Collaborations at higher $Q^{2}$ constrain hadronic strange and axial structure contributions 


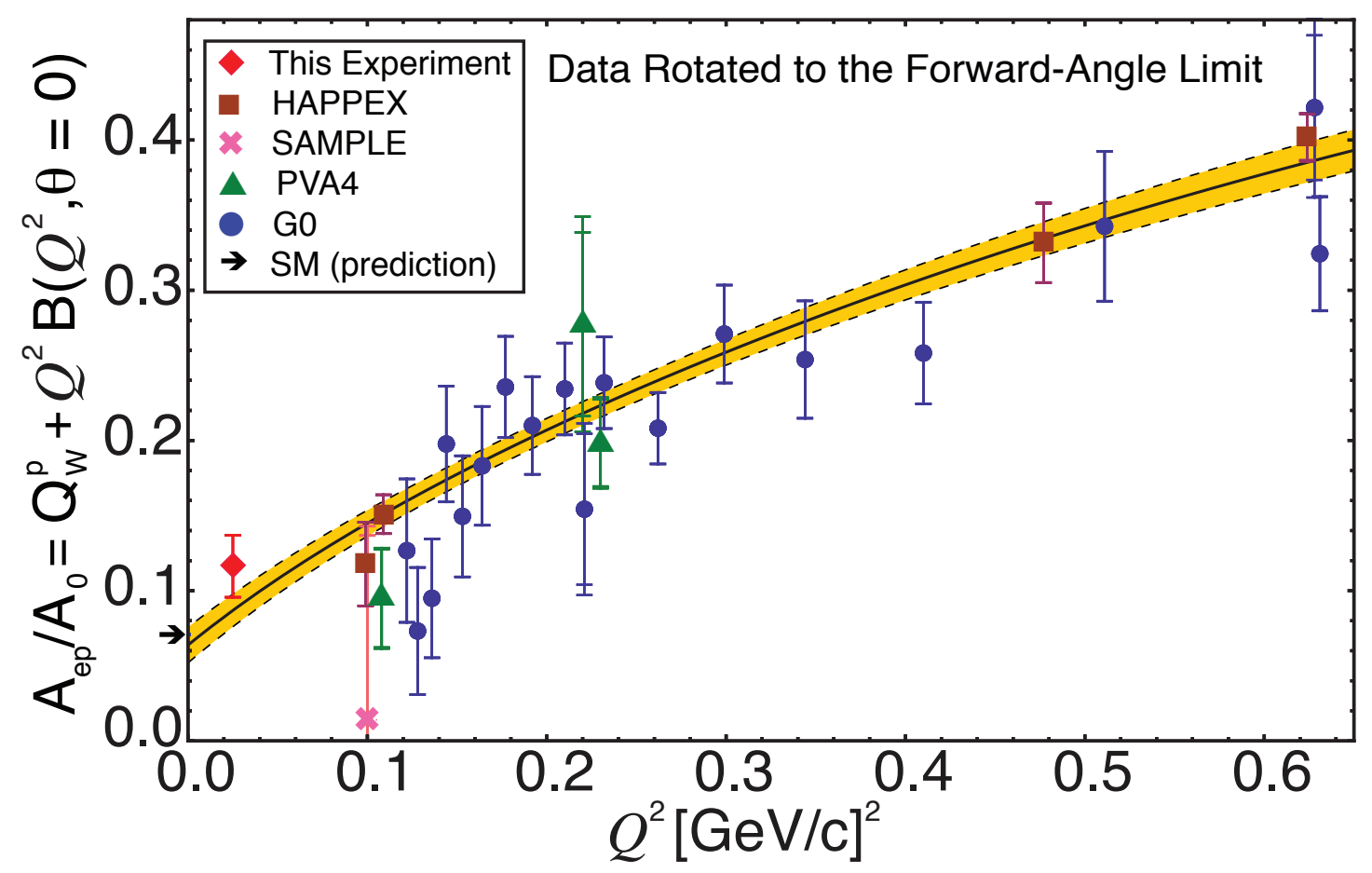

Figure 2.5: The reduced PV asymmetry from the world dataset of PVES results on nuclear targets, rotated to the forward angle limit $\theta \rightarrow 0$. The global fit result is the solid black line with the yellow band as its uncertainty. The $Q^{2}=0$ intercept corresponds to the weak charge of the proton $Q_{w}^{p}$ and is in good agreement with the SM prediction (black arrow).

to the asymmetry.

The Kelly parametrization [57] was used for the EM form factors $G_{E}^{p, \gamma}, G_{M}^{p, \gamma}$ and $G_{M}^{n, \gamma}$ :

$$
G\left(Q^{2}\right) \propto \frac{\sum_{k=0}^{n} \alpha_{k} \tau^{k}}{1+\sum_{k=1}^{n+2} b_{k} \tau^{k}},
$$

where $\tau=Q^{2} / 4 M_{p}^{2}$ and $n, \alpha_{k}, b_{k}$ are determined from fits to the data. The Galster parametrization is more successful for the $G_{E}^{n, \gamma}$ form factor:

$$
G_{E}^{n, \gamma}\left(Q^{2}\right)=\frac{A \tau}{1+B \tau} G_{D}\left(Q^{2}\right)
$$

where

$$
G_{D}=\left(1+Q^{2} / \lambda^{2}\right)^{-2}
$$

is the dipole form factor and $\lambda$ is the dipole "mass" parameter.

The fit has effectively five free parameters: the weak couplings $C_{1 u}$ and $C_{1 d}$, the strange charge radius $\rho_{s}$ and magnetic moment $\mu_{s}$ that parametrize the strange quark form factors $G_{E}^{s}$ and $G_{M}^{s}$, and the isovector axial form factor $G_{A}^{Z(T=1)} \sqrt[1]{1}$ The strange quark form factors as

\footnotetext{
${ }^{1}$ The value and uncertainty of the isoscalar axial form factor $G_{A}^{Z(T=0)}$ (which vanishes at tree level) is constrained by the calculation of 58
} 
well as $G_{A}^{Z(T=1)}$ employ the conventional dipole form 2.21 to make use of PVES data up to $Q^{2}=0.63(\mathrm{GeV} / \mathrm{c})^{2}$ :

$$
\begin{aligned}
G_{E}^{s} & =\rho_{s} Q^{2} G_{D} \\
G_{M}^{s} & =\mu_{s} G_{D}
\end{aligned}
$$

These four form factors $\left[G_{E, M}^{s}, G_{A}^{Z(T=0,1)}\right]$ have little influence on the result for the weak charge of the proton. The best value of the dipole mass $\lambda=1(\mathrm{GeV} / \mathrm{c})$ is taken from quasielastic neutrino scattering data [59, [58. Its variation within $30 \%$ of its size also had a small effect on the $Q_{w}^{p}$ result. The fit results for $\rho_{s}$ and $\mu_{s}$ are consistent with an earlier determination [49] but with uncertainties $\sim 4$ times smaller.

The proper treatment for the correction of the $\gamma-Z$ box diagram (Figure 2.10), and specifically the vector coupling at the proton vertex $\square_{\gamma Z}^{\mathrm{V}}$ which vanishes at threshold, has become contentious since a recent reanalysis through forward dispersion relations that uncovered a missing energy dependence [60. Three different theory groups agree on the size of this correction for the $Q_{\text {weak }}$ measurement but give different estimations for its uncertainty $61,62,63,64,65$. Here the energy-dependent $\gamma-Z$ box correction has been applied directly to each datum prior to the fitting procedure, based on the calculation of [66, with the associated uncertainties folded into the systematic error of each point. At $Q_{\text {weak }}$ kinematics the correction is (Figure 2.6):

$$
\mathcal{R} e \square_{\gamma Z}^{\mathrm{V}}\left(Q_{\text {weak }}\right)=(5.60 \pm 0.36) \times 10^{-3}
$$

The effect of either doubling or not including the nominally forward-angle $\square_{\gamma Z}$ radiative correction for the six larger angle data $>21^{\circ}$ used in the fit, has a negligible effect on the extracted $Q_{w}^{p}$ compared to its assigned uncertainty. It would be desirable to have a unified theory uncertainty on this correction before the full $Q_{\text {weak }}$ analysis is completed [67. Recent analysis however suggests that this contribution is now sufficiently under control with further constraints from the observation of quark-hadron duality in the PVDIS measurement from the deuteron [68, 69.

All data included in Figure 2.5 are from proton targets and rotated to the forward angle limit based on the fit result, in order to illustrate the two-dimensional global fit $\left(\theta, Q^{2}\right)$ in a single dimension $\left(Q^{2}\right)$, and plotted versus the reduced asymmetry (Eq. 2.17). Thus the angle dependence of the strange and axial form factors was removed through the subtraction

$$
A_{\mathrm{PV}}^{\theta=0^{\circ}}\left(Q^{2}\right)=A_{\mathrm{PV}}\left(\theta, Q^{2}\right)-\left[A_{\text {calc }}\left(\theta, Q^{2}\right)-A_{\text {calc }}\left(\theta=0^{\circ}, Q^{2}\right)\right]
$$

from the measured asymmetries $A_{\mathrm{PV}}\left(\theta, Q^{2}\right)$, where the calculated asymmetries $A_{\text {calc }}$ are deter- 


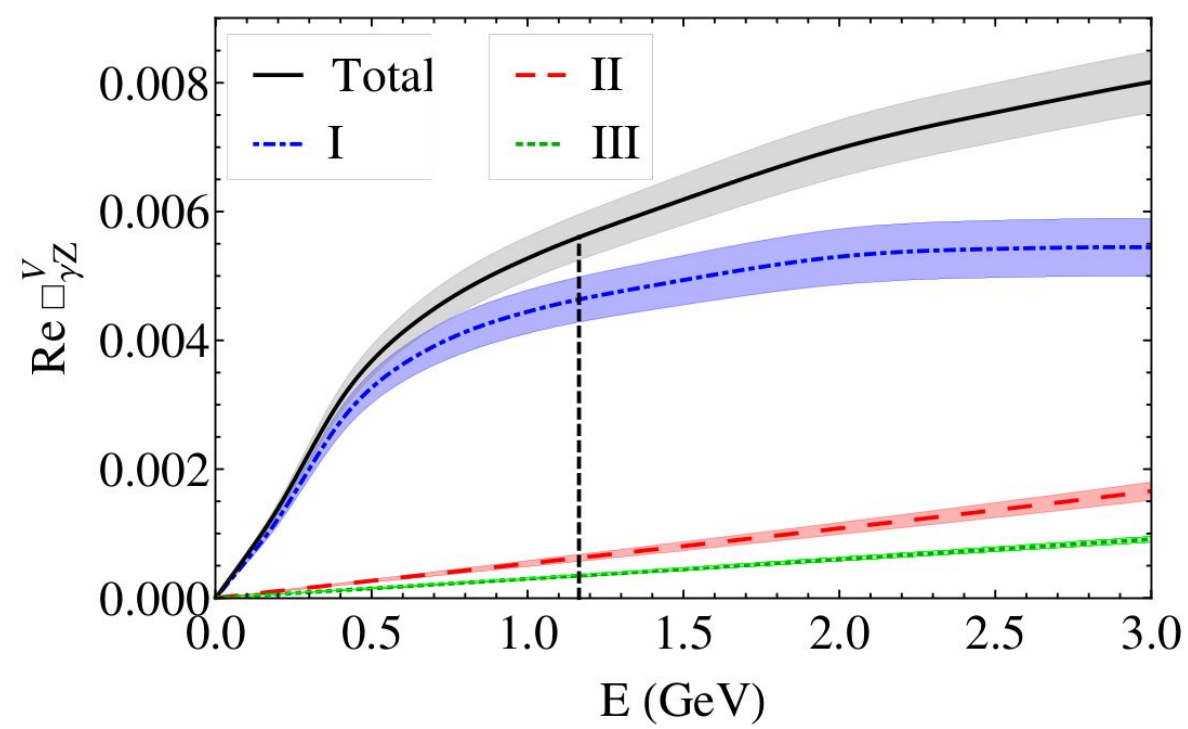

Figure 2.6: The energy dependence of the $\mathcal{R} e \square_{\gamma Z}^{\mathrm{V}}$ correction from 66, where the total value is plotted as the solid black line along with the separate contributions from three kinematic regions in $W$ and $Q^{2}$ (Regions I,II, and III). The vertical dashed line corresponds to $Q_{\text {weak }}$ kinematics at $E=1.165 \mathrm{GeV}^{2}$.

mined from Eq.2.10 using the results of the fit. The global fit result is the black solid line with its uncertainty plotted as the yellow band. The fit result for the intercept of the reduced asymmetry at $Q^{2}=0$ yields the first determination of the weak charge of the proton,

$$
Q_{w}^{p}(\mathrm{PVES})=0.064 \pm 0.012
$$

The result is in good agreement with the SM prediction

$$
Q_{w}^{p}(\mathrm{SM})=0.0710 \pm 0.0007
$$

When the global PVES fit result is further combined with the ${ }^{133} \mathrm{Cs}$ atomic parity violation (APV) measurement [70, significant constraints are obtained on the neutral-weak vector quark couplings $C_{1 u}$ and $C_{1 d}$. The APV experiment measured the atomic polarizability in the $6 \mathrm{~s} \rightarrow 7 \mathrm{~s}$ transition, a transition forbidden by parity selection rules which accesses the weak charge of the atomic cesium. The constraints from PVES and APV have sensitivity to nearly orthogonal combinations of $C_{1 u}$ and $C_{1 d}$ (Figure 2.7) and the global fit dramatically reduces the available parameter space. Thus both parameters can be extracted to high precision from the combined fit: $C_{1 u}=-0.1835 \pm 0.0054$ and $C_{1 d}=0.3344 \pm 0.0050$, with a correlation coefficient -0.980 . The results are in good agreement with the SM prediction, marked on the figure as a function of the value of the weak mixing angle on the $Z$ resonance, $\left.\sin ^{2} \theta_{W}\right|_{Z}(\mathrm{SM})=0.02312$ (see Eq. 2.28). 


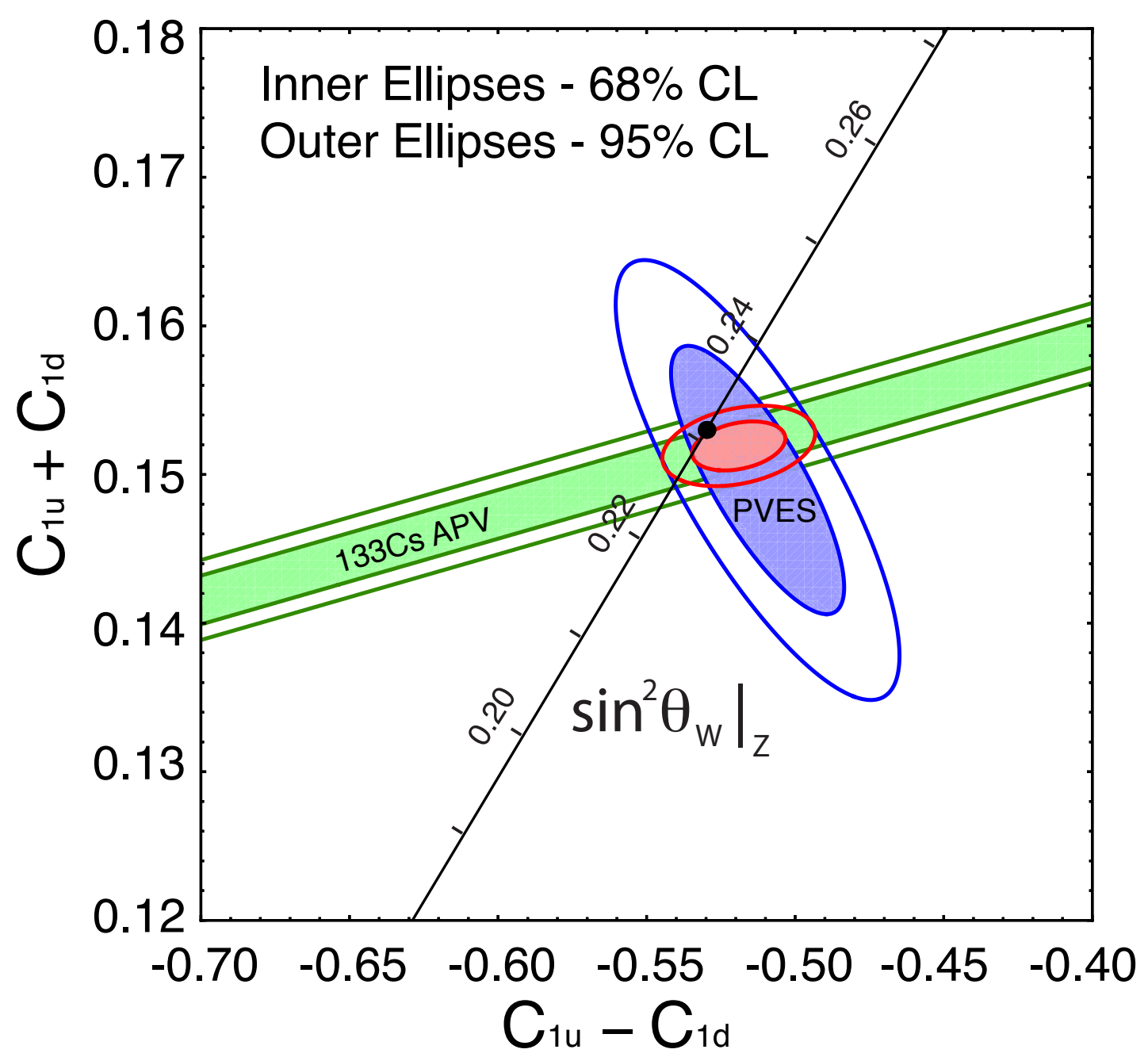

Figure 2.7: Global combined constraints (red ellipses) from the global PVES fit (blue ellipses) and the APV measurement on ${ }^{133} \mathrm{Cs}$ (green ellipses) on the isovector and isoscalar combinations of the weak vector quark couplings $C_{1 u}$ and $C_{1 d}$. The constraints are in agreement with the SM prediction (black dot), quoted as a function of $\sin ^{2} \theta_{W} \mid z$.

The weak charge of the neutron can then be extracted from the combination of $C_{1 u}$ and $C_{1 d}$, $Q_{w}^{n}(\mathrm{PVES}+\mathrm{APV})=-2\left(C_{1 u}+2 C_{1 d}\right)=-0.975 \pm 0.010$, also in agreement with the SM prediction $Q_{w}^{n}(\mathrm{SM})=-0.9890 \pm 0.0007$.

\subsection{The Electroweak Mixing Angle}

\subsubsection{Running of $\sin ^{2} \theta_{W}$}

Including higher order radiative corrections, the value of the weak mixing angle acquires a dependence on the energy scale of the interaction. This is the analog of the "running" of the QED fine structure constant $\alpha=e^{2} / 4 \pi$, seen on Figure 2.8. At low energies the fine structure has the familiar value $\alpha \approx 1 / 137$, but at the energy scale of the mass of the $Z$ boson its value becomes $\alpha\left(Q^{2}=M_{Z}^{2}\right) \approx 1 / 129[71$. The energy dependence of the fine structure constant arises 
due to EM radiative corrections that become more important at higher energies, conventionally described as a "screening" effect from the virtual creation and annihilation of electron-positron pairs. This "vacuum polarization" effect becomes more prominent at smaller distance scales that are probed by higher $Q^{2}$ values. Similarly the strong coupling constant $\alpha_{s}$ acquires energy dependence due to radiative interactions, running down to $\alpha_{s}\left(Q^{2}=M_{Z}^{2}\right) \approx 0.12[72$ as quarks and gluons inside hadrons tend towards asymptotic freedom.
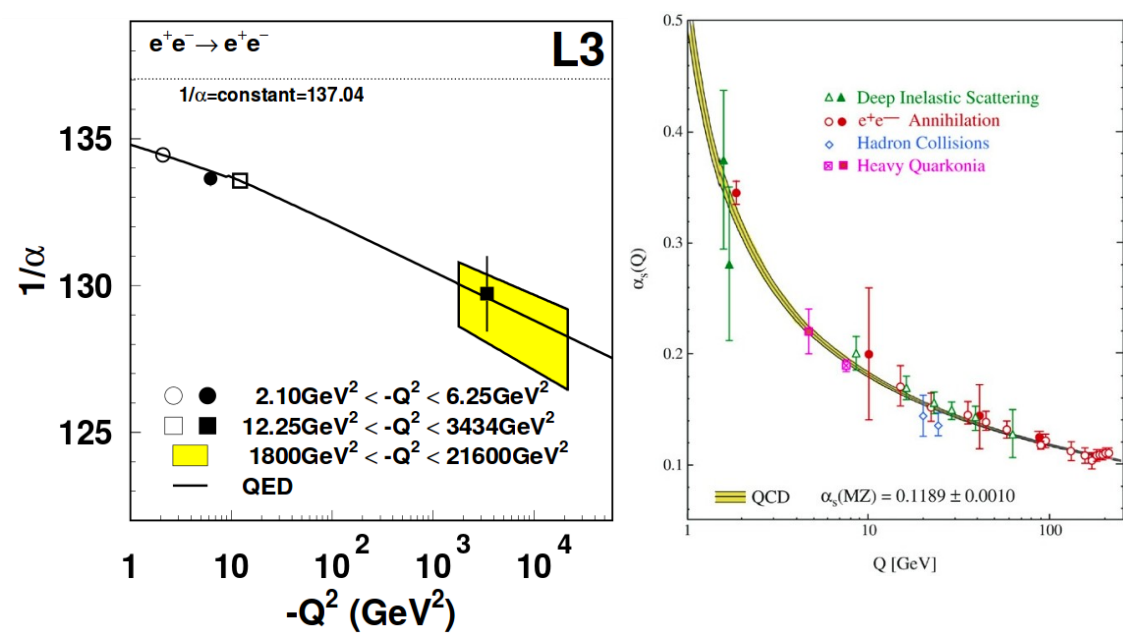

Figure 2.8: Left: running of the QED fine structure constant from the L3 Collaboration [71. Right: running of the strong coupling constant $\alpha_{s}$ from 72 .
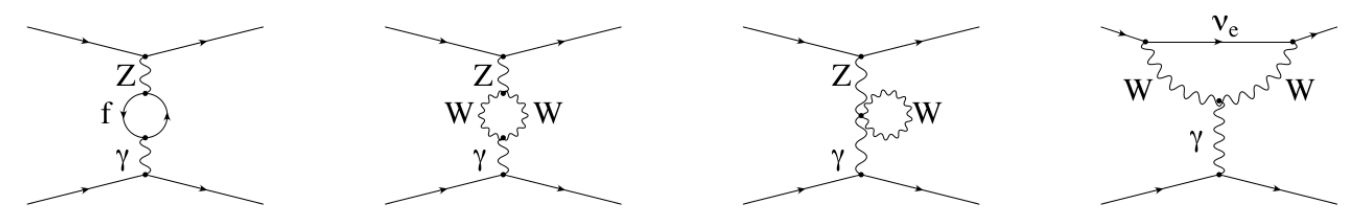

Figure 2.9: $\gamma-Z$ mixing (vacuum polarization) diagrams and $W$-loop contribution to the anapole moment for parity-violating elastic electron scattering.

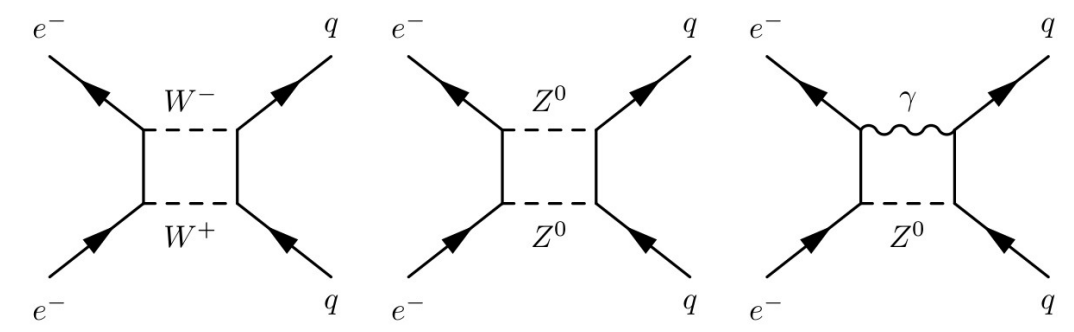

Figure 2.10: The purely weak $\left(\square_{W W}\right.$ and $\left.\square_{Z Z}\right)$ and electroweak $\left(\square_{\gamma Z}\right)$ two-boson exchange diagrams that modify the neutral current electron-quark interaction.

Vacuum polarization effects in the EW sector (Figure 2.9) give rise to the running of the weak mixing angle, which is a key prediction of the Standard Model. Including higher-order radiative corrections, the weak charge of the proton can be expressed in terms of the weak mixing angle 
as [73:

$$
Q_{w}^{p}=\left[\rho_{\mathrm{NC}}+\Delta_{e}\right]\left[1-4 \sin ^{2} \theta_{W}(0)+\Delta_{e}^{\prime}\right]+\square_{W W}+\square_{Z Z}+\square_{\gamma Z}(0)
$$

where the Veltman parameter $\rho_{\mathrm{NC}}=1+\Delta \rho[74]$ renormalizes the ratio of neutral to charged current interaction strengths at low energies, and $\Delta_{e}$ and $\Delta_{e}^{\prime}$ are non-universal corrections to the axial vector Zee and ree couplings respectively. The last three terms are higher order EW "box" diagrams, characterized by the exchange of two vector bosons (Figure 2.10. The purely weak $\square_{W W}$ and $\square_{Z Z}$ diagrams give corrections of $\sim 26 \%$ and $3 \%$ respectively, and can be treated perturbatively due to the large masses of the $W^{ \pm}$and $Z$ bosons. The $\square_{\gamma Z}$ diagram on the other hand cannot be treated perturbatively due to the massless photon leg and has been the subject of vigorous theoretical scrutiny, as was discussed in the previous section.

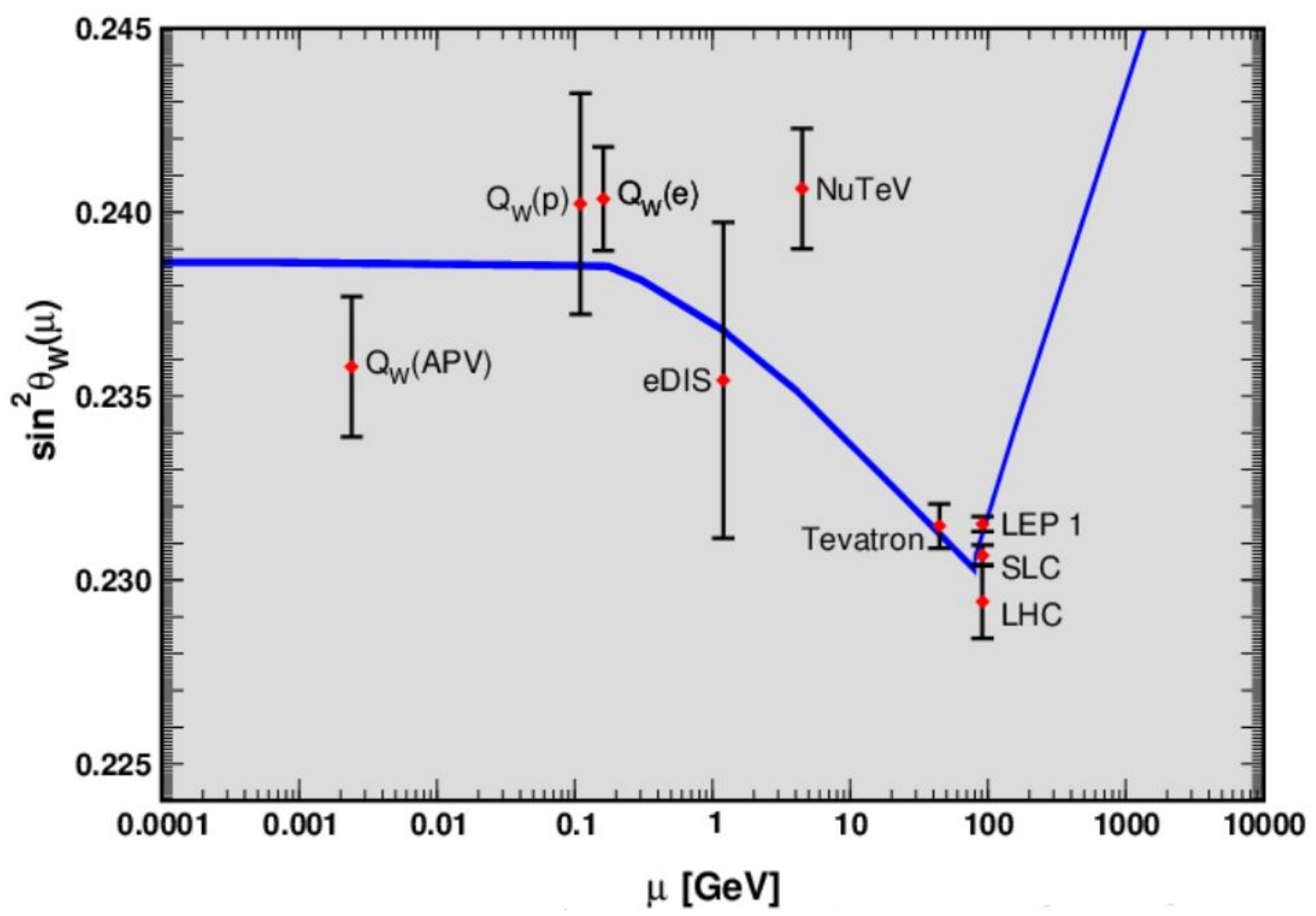

Figure 2.11: Theoretical prediction (blue line) for the scale dependence of the weak mixing angle $\sin ^{2} \theta_{W}$ in the $\overline{\mathrm{MS}}$ scheme [26], plotted versus the renormalization scale $\mu$. Experimental determinations plotted as red points. See text for discussion.

The curve of Figure 2.11 shows the variation of the mixing angle with the energy scale $\mu$ in the modified Minimal Subtraction $(\overline{\mathrm{MS}})$ renormalization scheme [75, 76, 77, which promotes the coupling-based definition 1.4 of $\sin ^{2} \hat{\theta}_{W}{ }^{2}$ to all orders in perturbation theory [78. The running is dominated by the vacuum polarization diagrams of Figure 2.9, which characterize the $\gamma-Z$ mixing due to fermionic and bosonic vacuum polarization loops. The horizontal axis $\mu$ can be considered as the renormalization scale of the theory, or the t'Hooft scale which appears in

\footnotetext{
${ }^{2}$ The caret denotes parameters defined in the $\overline{\mathrm{MS}}$ scheme.
} 
dimensional regularization. Choosing the renormalization scale equal to the momentum transfer of the process under consideration $\left(\mu^{2}=Q^{2}\right)$ will in general avoid spurious logarithms and reduce the truncation error associated with the perturbative expansion. The various discontinuities occur at the thresholds of particle masses, arising from scheme-dependent matching terms which are necessary to ensure that the various effective field theories within a given loop order describe the same physics [26]. Crossing a particle mass threshold from above, we switch to an effective theory with the particle integrated out. For example, the turning point between a screening and an anti-screening behaviour (the minimum of the curve) occurs at $\mu=M_{W}$, as the boson vacuum polarization diagrams have a contribution of opposite sign from the fermion diagrams. The running results in a $\sim 3 \%$ shift in the value of the weak mixing angle at the $Z$ resonance $\left(Q=M_{Z}\right)$ down to $Q^{2} \rightarrow 0$.

By comparison higher order diagrams that are specific to a given experimental probe should be applied to that specific result before converting to the value of the weak mixing angle. For the $Q_{\text {weak }}$ result obtained from the measurement of $A_{\mathrm{PV}}$ in $\overrightarrow{e p}$ scattering, the higher order EW "box" diagrams corresponding to the exchange of two vector bosons are shown in Figure 2.10 The corrections from these diagrams $\square_{W W}, \square_{Z Z}, \square_{\gamma Z}$ have been absorbed into the PVES result as discussed in the previous section, rather than as a global correction.

Thus the weak mixing angle cannot be equated to a physical observable to all orders, as its value depends on the choice of renormalization scheme and scale. Rather it is a theoretical construct, but a very useful one that provides the means to compare measurements at different energy scales and from various experimental probes to the predictions of the theory. Any observed deviation from the SM prediction could be a signature of a new interaction, entering through quantum corrections unaccounted for in the theory.

\subsubsection{Experimental determinations}

There is an impressive variety of experimental probes to test the SM through measurements of the weak mixing angle. The most precise determinations are from the Large Electron-Positron collider (LEP) at CERN and Stanford Linear Collider (SLC) measurements on the $Z$ resonance, where $Q^{2} \approx M_{Z}^{2}$ and $Z$ bosons are copiously produced. With the discovery of the Higgs boson the value of the weak mixing angle at the $Z$ resonance can be predicted theoretically, and there is an excellent consistency between the SM prediction and the experimental average [79]:

$$
\begin{array}{ll}
\sin ^{2} \hat{\theta}_{W}\left(M_{Z}\right)_{\mathrm{SM}}=0.23124(12) & \text { SM prediction } \\
\sin ^{2} \hat{\theta}_{W}\left(M_{Z}\right)_{\exp }=0.23125(16) & \text { Z-pole average }
\end{array}
$$


This level of agreement, combined with the very precisely measured $\alpha, G_{F}$ and $M_{Z}$, along with $m_{t}$ and $M_{H}=126 \mathrm{GeV}$, leads to significant constraints for very heavy new particle loop effects such as Technicolor or 4th generation fermions, parametrized through the electroweak precision ("oblique") $S$ and $T$ parameters [80, 81]. However there is a significant and longstanding $3.2 \sigma$ discrepancy between the two most precise determinations of $\sin ^{2} \theta_{W}$, from the forward-backward asymmetry of $Z$ bosons decaying into $b \bar{b}$ pairs, $A_{\mathrm{FB}}(b)$ at LEP1, and the SLC left-right polarization asymmetry for hadronic final states $A_{\mathrm{LR}}(\mathrm{had})$. It has been suggested that if new physics is responsible for the deviation, it is most likely of tree-level type affecting preferentially the third generation, although it is hard to accommodate other constraints [26]. The hadron collider $\sin ^{2} \theta_{W}$ determinations come from measurements of the Drell-Yan process, dominated by the invariant masses of the final state dilepton pair of $\mathcal{O}\left(M_{Z}\right)$ and should be considered as additional $Z$ pole data. The Tevatron determination has been moved to the left for clarity.

Precision measurements at lower energies test the theoretical renormalization group evolution for the weak mixing angle, but theoretical uncertainties cloud the interpretation of some results. The APV measurement on cesium 70 which was combined with the global PVES result to extract the $C_{1 u, d}$ parameters, also yields a determination for the weak mixing angle at very low $Q^{2}$. Evolving theoretical calculations [82] have shifted this result over time as additional atomic structure effects are incorporated into the calculations, for example due to the Breit (magnetic) interaction and radiative QED processes. The level of agreement with the SM prediction has changed with subsequent analyses $[83,84$. In a similar case, the NuTeV Collaboration performed a measurement of neutrino-nucleus deep inelastic scattering ( $\nu$ DIS) with a result for $\sin ^{2} \theta_{W}$ that was initially $3 \sigma$ away from the SM prediction [85. This was dubbed "the $\mathrm{NuTeV}$ anomaly" and created excitement and speculation on the nature of new physics that could give rise to such a discrepancy. However nuclear structure effects may also contribute to the discrepancy, for example after accounting for charge symmetry violation at the parton-level [86, 87] and the isovector nuclear EMC effect 88, that modifies light quark PDFs in the nuclear medium. The theoretical issues encountered by the APV and $\mathrm{NuTeV}$ measurements, relating to uncertainties of atomic and nuclear structure, cloud the interpretability of their results as clean measurements of the EW sector. These issues highlight the importance of a theoretically clean experimental probe, as well as the need for multiple experiments that may be affected by different theoretical uncertainties and radiative corrections.

The SLAC E158 experiment measured the weak charge of the electron $Q_{w}^{e}=-0.0369(52)$ through the parity-violating Møller (electron-electron) scattering at $Q^{2}=0.026(\mathrm{GeV} / \mathrm{c})^{2}$ [51, 89. It is currently the most precise measurement of the weak mixing angle at low $Q^{2}$, in fairly 
good agreement with the SM prediction. Very importantly, as a purely leptonic process it is quite clean of theoretical issues. This experimental result places stronger limits on electron compositeness scales than LEP, underscoring the power of measuring a small SM coupling to high precision.

The early result of the $Q_{\text {weak }}$ experiment for the weak charge of the proton through the global fit of PVES data on nuclear targets also yields a value for the weak mixing angle, albeit with limited precision. The full $Q_{\text {weak }}$ result is expected to replace E158 as the most precise measurement of the weak mixing angle at low $Q^{2}$. The suppression of the value of the weak charge of the proton implies significant enhancement in the relative precision of the weak mixing angle, by more than an order of magnitude. Neglecting radiative corrections:

$$
\frac{\Delta\left(\sin ^{2} \theta_{W}\right)}{\sin ^{2} \theta_{W}}=\frac{Q_{w}^{p}}{4 \sin ^{2} \theta_{W}} \frac{\Delta Q_{w}^{p}}{Q_{w}^{p}}=0.075 \frac{\Delta Q_{w}^{p}}{Q_{w}^{p}}
$$

The sensitivity to models of physics beyond the Standard Model is discussed in the following section.

\subsection{Access to Physics Beyond the Standard Model}

Low energy measurements are not yet mature enough to contribute significantly to the average value of the weak mixing angle at the $Z$ pole, or access new physics in terms of the wellconstrained oblique parameters. However they can access new kinds of interactions that may have gone unnoticed on the $Z$ resonance if the new particles don't couple strongly to the $Z$ boson. Particularly well motivated examples include new supersymmetric (SUSY) particles; Leptoquarks; or new neutral gauge bosons $Z^{\prime}$ of an extra $\mathrm{U}(1)^{\prime}$ interaction, predicted in most GUT and technicolor models as well as SUSY and string theories. $Q_{\text {weak }}$ is most sensitive to new SUSY interactions that violate R-parity ${ }^{3}$ as they would modify the electron-quark interaction at the tree level, compared to the R-parity conserving SUSY interactions which only enter through loops (Figure 2.12).
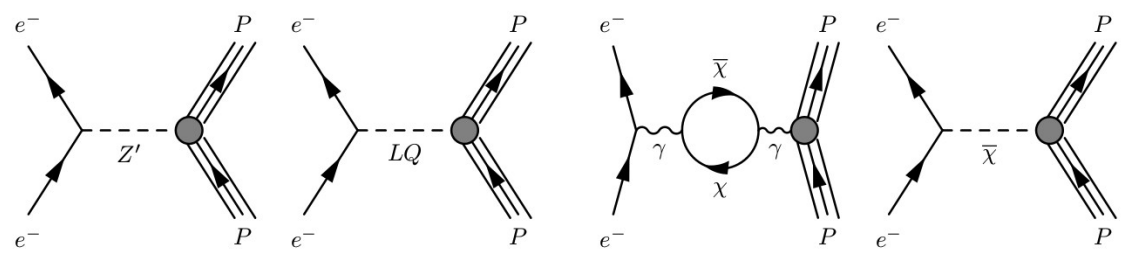

Figure 2.12: Feynman diagrams for models of new physics that could create a deviation from the SM prediction in the $Q_{\text {weak }}$ measurement: new $Z^{\prime}$ gauge bosons, Leptoquarks, and SUSY interactions that respect or violate R-parity.

\footnotetext{
${ }^{3} \mathrm{R}$-parity conservation refers to conservation of baryon number $B$ and lepton number $L$.
} 
Note that this kind of new physics that couples directly to fermions cannot be parametrized by the formalism of oblique parameters that describes heavy physics which affect only the gauge self-energies. Instead a new heavy interaction term can be parametrized by a 4 -fermion contact interaction that would interfere with the SM amplitude. The new physics term would modify the parity-violating part of the neutral current Langrangian 2.11) of the electron-quark interaction [79:

$$
\mathcal{L}_{\text {new }}^{\mathrm{PV}}=\frac{g_{\text {new }}^{2}}{2 \Lambda_{\text {new }}^{2}} \bar{e} \gamma_{\mu} \gamma_{5} e \sum_{\substack{q \\ i, j=L, R}} h_{i j}^{q} \bar{q} \gamma^{\mu} q
$$

where $g_{\text {new }}$ and $\Lambda_{\text {new }}$ are the coupling parameter and energy scale of the new interaction, respectively, and $h_{i j}^{q}$ are the quark-specific helicity projection operators. A typical convention sets $h_{i j}^{q}= \pm 1$ or 0 , commonly in the $\mathrm{LL}^{ \pm}$scenario which only considers models with positive- or negative-definite contributions. Then the precision of the $Q_{\text {weak }}$ measurement $\left|\Delta Q_{w}^{p}\right|$ can be used to set bounds on the scale of new physics, at the 95\% confidence level (CL) [90]:

$$
\begin{aligned}
& \frac{\Lambda_{\text {new }}^{ \pm}}{g_{\text {new }}}>v \sqrt{\frac{2 \sqrt{5}}{\left|2 g_{\mathrm{AV}}^{e u}+g_{\mathrm{AV}}^{e d}\right|^{ \pm}}} \quad, \quad \text { or } \\
& \frac{\Lambda_{\text {new }}}{g_{\text {new }}}>v \sqrt{\frac{4 \sqrt{5}}{1.96\left|\Delta Q_{w}^{p}\right|}}
\end{aligned}
$$

where $v=\left(\sqrt{2} G_{F}\right)^{-1 / 2}=246.22 \mathrm{GeV}$ is the Higgs vacuum expectation value. Note that indirect measurements at low $Q^{2}$ are only sensitive to the mass over the coupling of the new dynamics. Some discriminating power is restored by combining a broad set of measurements. For example the purely leptonic Møller interactions would be unaffected by Leptoquark interactions and act as control for a $Q_{w}^{p}$ deviation. On the other hand $Q_{w}(\mathrm{Cs})$ would be insensitive to SUSY loops while the effects on $Q_{w}^{e}$ and $Q_{w}^{p}$ would be similar over nearly all allowed parameter space, with the SUSY vertex corrections being heavily constrained by the oblique $T$ parameter. Measurements over a variety of chiral structures also offer complementary sensitivity, such as in the extraction of $C_{1 u}$ and $C_{1 d}$ from the combination of PVES and APV data.

But already very strong and competitive limits can be placed in the context of certain models. Contact interaction mass limits are usually quoted in terms of compositeness scales by setting $g_{\text {new }}^{2}=4 \pi$, where leptons or quarks are assumed to have a substructure and be composed of more fundamental objects, bound together by a new interaction of non-perturbative strength. Then precision measurements can be translated to bounds on $\Lambda_{\text {new }}$, with the strongest limits placed by the E158 measurement: $\Lambda_{\text {new }}^{+}>20.9 \mathrm{TeV}$ and $\Lambda_{\text {new }}^{+}>13.7 \mathrm{TeV}(95 \% \mathrm{CL})$, where the $(+,-)$ superscripts indicate constructive and destructive interference with the SM respectively [79]. For the $Q_{\text {weak }}$ measurement, a $4.5 \%$ precision on the full $Q_{w}^{p}$ result would probe compositeness scales of up to $33 \mathrm{TeV}$ [90]. 


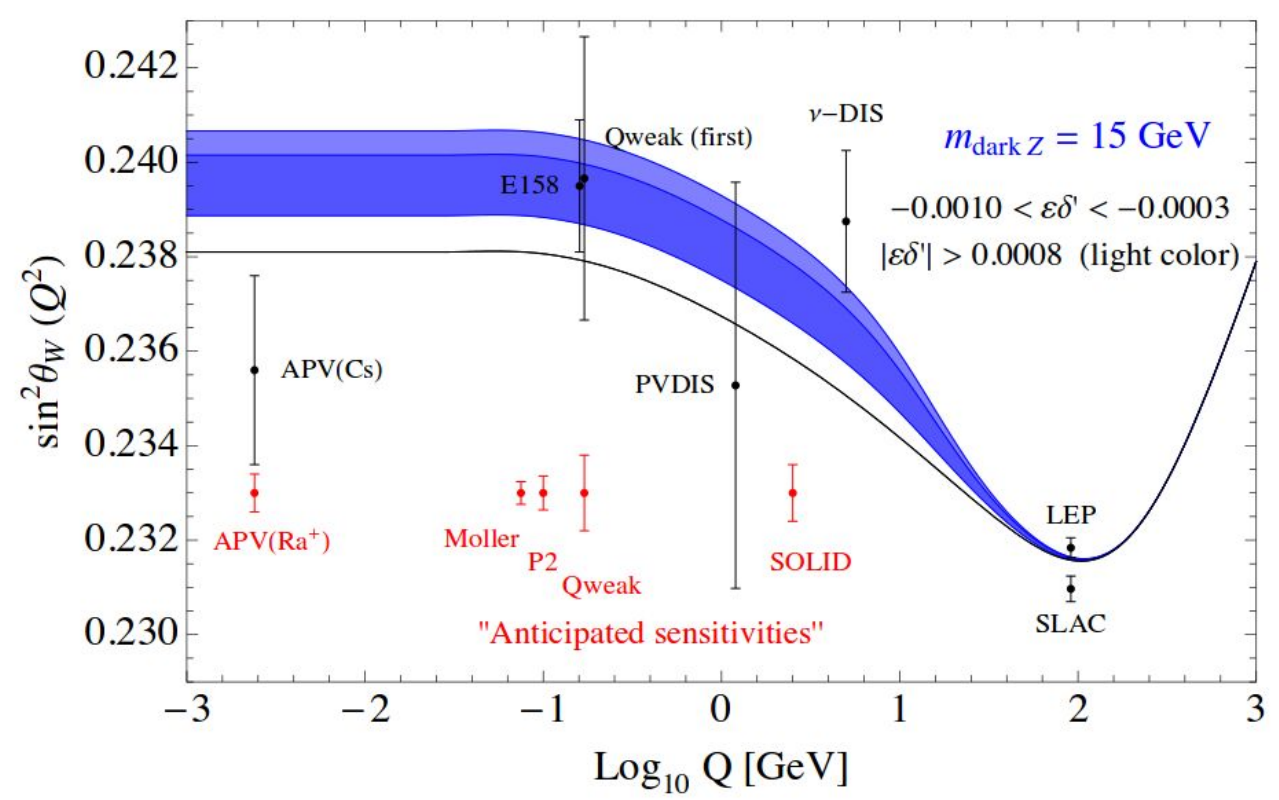

Figure 2.13: The $Q^{2}$-dependent modification of the running of $\sin ^{2} \hat{\theta}_{W}$ from a dark $Z$ boson with $m_{Z_{\mathrm{d}}}=15 \mathrm{GeV}$ is shown in blue 91]. Current experimental constraints are shown in black, while planned future measurements with their expected precision are shown in red at arbitrary vertical location. A different theoretical calculation is employed here compared to Figure 2.11

Another interesting scenario emphasizes the unique access of measurements at low $Q^{2}$ and their necessity as a complement to the collider measurements. In the model of "dark parity violation" 92, 93, a relatively light mediator (referred to as the "dark" $Z$ boson and denoted $Z_{\mathrm{d}}$ ) may offer access to a dark $\mathrm{U}(1)_{\mathrm{d}}$ gauge symmetry, but would have escaped detection if it couples very weakly to SM particles. It could however modify the running of the weak mixing angle in a $Q^{2}$-dependent manner, proportionally to $m_{Z_{\mathrm{d}}}^{2} /\left(Q^{2}+m_{Z_{\mathrm{d}}}^{2}\right)$ such that the low- $Q^{2}$ measurements would have unique access to it. The light mediator could not be parametrized as a contact interaction, but a new $Q^{2}$-dependent oblique parameter $X\left(Q^{2}\right) 94$ would be uniquely accessed by the low- $Q^{2}$ measurements. Depending on the model for the coupling and kinematic mixing of the new dark boson, a value of $m_{Z_{\mathrm{d}}} \approx 20-50 \mathrm{MeV}$ would satisfy the muon anomalous magnetic moment discrepancy. The access through parity violation could also evade current experimental constraints if $Z_{\mathrm{d}}$ preferentially decays to invisibles. Alternatively, a $m_{Z_{\mathrm{d}}} \approx 15-25$ $\mathrm{GeV}$ 91] would be consistent with the average of the low- $Q^{2}$ measurements extrapolated to $\sin ^{2} \hat{\theta}_{W}\left(M_{Z}\right)$, which is $1.8 \sigma$ higher than the SM prediction and collider average (Eq 2.28). Two interesting but tentative candidate events in this mass range $(\sim 20-25 \mathrm{GeV}$ at $1.7 \sigma)$ were seen recently by the $8 \mathrm{TeV}$ ATLAS search for $H \rightarrow Z_{\mathrm{d}} Z_{\mathrm{d}}$ [95. The $Q^{2}$-dependent modification of the $\sin ^{2} \hat{\theta}_{W}$ running in this scenario is shown in Figure 2.13

In this context, the full $Q_{\text {weak }}$ measurement is very important and highly anticipated. Its 
anticipated sensitivity is shown as a red point with arbitrary vertical location in Figure 2.13 along with future planned measurements that will test the theory with precision that even rivals that of the $Z$-factories. The MOLLER [96] Collaboration proposes to utilize the upgraded 11 $\mathrm{GeV}$ beam at Jefferson Lab for an ultra-precise measurement of $Q_{w}^{e}$ and $\sin ^{2} \theta_{W}$ at low $Q^{2}$, achieving a factor of 5 improvement over the E158 result. The P2 experiment at the MESA facility at Mainz will improve on the $Q_{\text {weak }}$ measurement at lower $Q^{2}$ for a more precise and theoretically cleaner extraction of $Q_{w}^{p}$. The progress achieved and the lessons learned from the $Q_{\text {weak }}$ measurement will be very important for these powerful future probes of the electroweak sector. 


\section{Chapter 3}

\section{Experimental Overview}

The $Q_{\text {weak }}$ experiment was performed in Hall C of the Thomas Jefferson National Accelerator Facility. The experiment determined for the first time the weak charge of the proton through a measurement of the parity-violating asymmetry $A_{e p}$ in the elastic scattering of $1.160 \mathrm{GeV}$ longitudinally polarized electrons from protons in a liquid hydrogen $\left(\mathrm{LH}_{2}\right)$ cryo-target system at a low 4-momentum transfer squared $Q^{2}$. The asymmetry is formed between two states of opposite electron helicity, defined by the direction of the longitudinal polarization of incoming electrons. The electron helicity was reversed at a rate of $960 \mathrm{~Hz}$ in a pseudorandom sequence of "helicity quartets" $(+--+)$ or $(-++-)$. Electrons scattered from the target at forward angles are selected by a series of lead collimators that define the kinematic acceptance. Elastically scattered electrons are then focused by an eight-segment toroidal magnet onto an azimuthally symmetric array of eight Čerenkov detectors, while inelastic electrons are swiped out of the acceptance. A schematic of the $Q_{\text {weak }}$ apparatus is shown in Figure 3.1 .

The experiment is performed at a nominal $Q^{2}=0.025(\mathrm{GeV} / \mathrm{c})^{2}$, selected as the optimal value to limit the contribution of hadronic uncertainties entering in the $B\left(Q^{2}, \theta\right)$ term, while avoiding very small asymmetries where corrections from helicity correlated beam parameters begin to dominate the systematic uncertainty. Higher beam energies would also increase the magnetic field requirements and the possibility for opening up secondary channels for production of backgrounds. The optimum $Q_{\text {weak }}$ kinematics correspond to a beam incident to the $\mathrm{LH}_{2}$ target at an energy of $E_{0}=1.165 \mathrm{GeV}$ and average scattering angle $\theta_{e}=7.9^{\circ}$, with a collimator acceptance of $5.8^{\circ}-11.6^{\circ}$.

At these kinematics $A_{e p}$ is expected to be very small, approximately -230 ppb. The $Q_{\text {weak }}$ proposal 33] for a $\sim 2.5 \%$ asymmetry measurement translates to a precision of $6 \mathrm{ppb}$, which will be the most precise measurement (relative and absolute) compared to the PVES dataset on nuclear targets. The ambitious precision goals of $Q_{\text {weak }}$ depend on maximizing the statisti- 


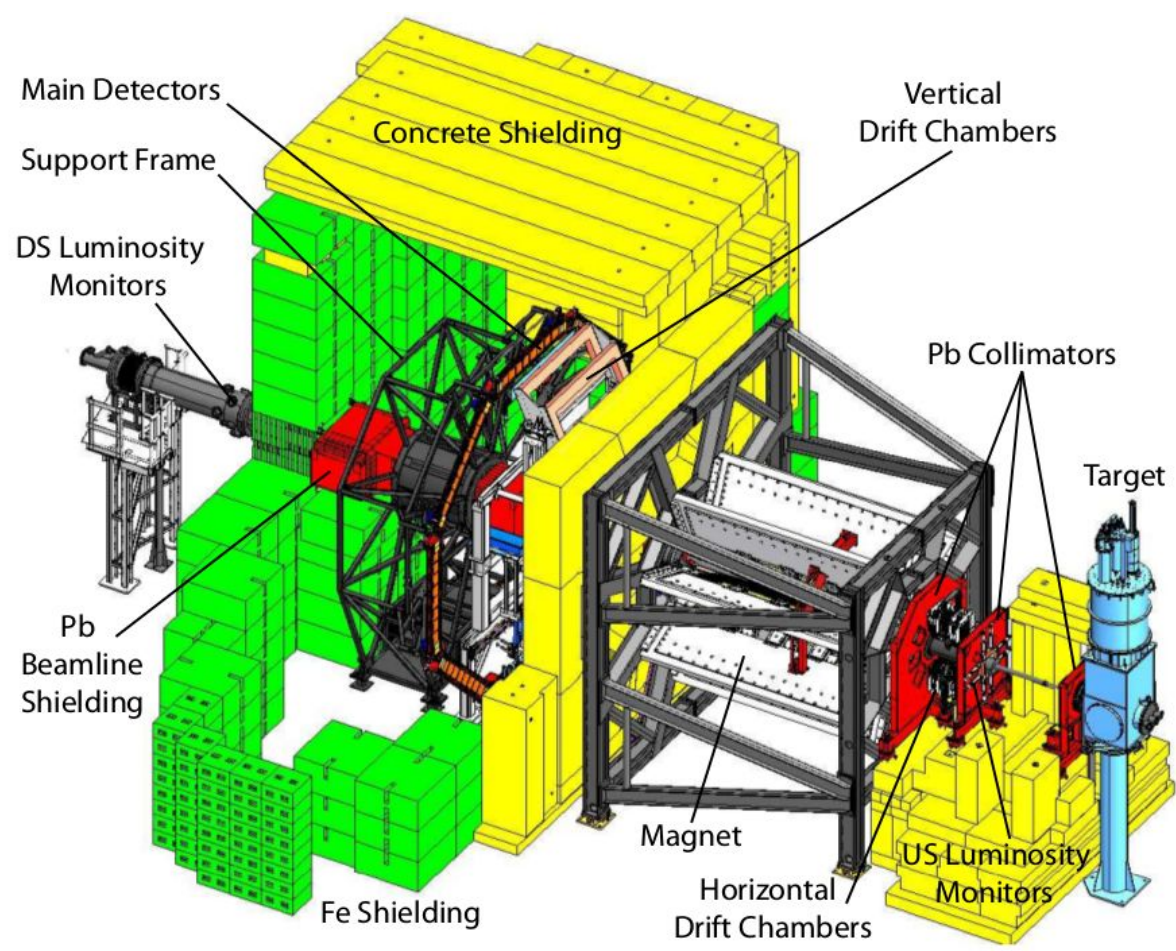

Figure 3.1: CAD design of the $Q_{\text {weak }}$ experimental apparatus. The beam is incident on the target from the right. Elastically scattered events are selected by the triple collimator system and focused by the field of the spectrometer magnet onto an azimuthally symmetric array of quartz Cerenkov detectors, while inelastic electrons are swept away. The elements and design characteristics of the apparatus are described in this chapter.

cal power of the measurement while controlling systematic contributions at the level of a few ppb. Building on technological advances achieved through the development of the world PVES program, the experiment requires:

- high beam current (up to $180 \mu \mathrm{A}$ ) with high longitudinal polarization (up to 90\%);

- a long $\mathrm{LH}_{2}$ target $(35 \mathrm{~cm})$ with minimal density fluctuations;

- a large-acceptance low-noise detector system operated in continuous-current mode in a high radiation environment; and

- suppression of false asymmetry contributions and backgrounds.

The $Q_{\text {weak }}$ design parameters are listed in Table 3.1. In this chapter the various elements of the $Q_{\text {weak }}$ apparatus [97 and design considerations will be presented, with special emphasis on elements that will be referenced later in this dissertation. 
Table 3.1: $Q_{\text {weak }}$ design parameters.

\begin{tabular}{l|c} 
Parameter & Value \\
\hline Incident beam energy & $1.160 \mathrm{GeV}$ \\
Beam polarization & $89 \%$ \\
Beam current & $180 \mu \mathrm{A}$ \\
Luminosity & $1.7 \times 10^{3} 9 \mathrm{~cm}^{-2} \mathrm{~s}^{-1}$ \\
Hydrogen target thickness & $34.4 \mathrm{~cm}$ \\
Beam power on target & $2.1 \mathrm{~kW}$ \\
Solid angle & $\Delta \Omega=43 \mathrm{msr}$ \\
Acceptance averaged $Q^{2}$ & $\left\langle Q^{2}\right\rangle=0.025(\mathrm{GeV} / \mathrm{c})^{2}$ \\
Scattering angle acceptance & $5.8^{\circ}-11.6^{\circ}$ \\
$\int|\vec{B}| d \ell$ & $0.9 \mathrm{~T} \cdot \mathrm{m}$ \\
Azimuthal acceptance & $49 \% \mathrm{of} 2 \pi$ \\
Integrated rate & $7 \mathrm{GHz}$
\end{tabular}

\subsection{Parity-Violating Asymmetry}

The PV asymmetry is formed between the scattering rates from the two states of opposite helicity:

$$
A_{\mathrm{PV}}=\frac{\sigma_{+}-\sigma_{-}}{\sigma_{+}+\sigma_{-}}
$$

where $\sigma_{+(-)}$is the scattering rate in the states of positive (negative) helicity, i.e. with the electron longitudinal spin aligned parallel (anti-parallel) to the direction of motion. The rate for the parity-conserving EM interaction is independent of electron helicity and its contribution vanishes from the numerator. Therefore $A_{\mathrm{PV}}$ is a direct signature of the weak interaction ${ }^{1}$ However the much larger EM amplitude dominates the total cross sections in the denominator, making the PV asymmetries very small and challenging to measure.

The raw asymmetry is measured from each detector element (Section 3.6.1) is formed in every quartet of helicity windows $(+--+$ or -++-$)$ as:

$$
A_{\text {raw }}=\frac{Y_{+}-Y_{-}}{Y_{+}+Y_{-}}
$$

where $Y_{ \pm}=S_{ \pm} / I_{\text {beam }}^{ \pm}$is the detector "yield", defined as the integrated detector signal $S_{ \pm}$ normalized to the beam current $I_{\text {beam }}^{ \pm}$in each helicity window. In Eq. 3.2 it is implied that $Y_{+(-)}$is averaged over the two positive (negative) helicity windows in a quartet. The beam current $I_{\text {beam }}$ is measured by one or more beam current monitors upstream of the target. This normalization is necessary to remove fluctuations of beam intensity and access the "physics" $\vec{e} p$ PV asymmetry.

While some systematic effects are suppressed by the azimuthal symmetry of the detector

\footnotetext{
${ }^{1}$ There is in fact a small parity-conserving asymmetry $A_{T}$ that arises from residual transverse electron polarization.
} 
Table 3.2: Anticipated contributions to the experimental error budget, from the $2007 Q_{\text {weak }}$ proposal 33 .

\begin{tabular}{c|cc} 
& $\Delta A_{P V} / A_{P V}$ & $\Delta Q_{w} / Q_{w}$ \\
\hline Counting statistics & $2.1 \%$ & $3.2 \%$ \\
Hadronic structure & - & $1.5 \%$ \\
Beam polarimetry & $1.0 \%$ & $1.5 \%$ \\
Absolute $Q^{2}$ & $0.5 \%$ & $1.0 \%$ \\
Backgrounds & $0.7 \%$ & $0.7 \%$ \\
HCBA $^{2}$ & $0.5 \%$ & $0.8 \%$ \\
\hline Total & $2.6 \%$ & $4.2 \%$
\end{tabular}

system, there are still residual false asymmetries that must be accounted for. $A_{\text {raw }}$ is corrected for false asymmetries arising from systematic effects and backgrounds, and also normalized for the beam polarization $P$, to form the elastic $\overrightarrow{e p}$ PV asymmetry $A_{e p}$ :

$$
\begin{aligned}
A_{\mathrm{PV}}^{e p} & =\frac{R_{\mathrm{tot}}}{1-\sum_{b=1}^{4} f_{b}}\left(\frac{1}{P} A_{\mathrm{msr}}-\sum_{b=1}^{4} f_{b} A_{b}\right), \\
A_{\mathrm{msr}} & =A_{\text {raw }}-A_{\text {beam }}-A_{T}-A_{L}
\end{aligned}
$$

Here $R_{\text {tot }} \approx 1$ includes small corrections that normalize the asymmetry to its tree-level value and account for sources of experimental bias. These corrections are described in Section 8.1.1. $P$ is the beam polarization which normalizes the "measured" asymmetry $A_{\mathrm{msr}}$, which includes "beam" corrections to the raw asymmetry. $A_{\text {beam }}$ refers to false asymmetries arising from correlations of beam properties with helicity, discussed in Chapter 5

The false asymmetry contributions from background sources must also be estimated and subtracted, where for each source we need to know the asymmetry $A_{b}$ and the fractional signal contribution ("dilution") in the main detector, $f_{b}=\left\langle Y_{b}\right\rangle /\left\langle Y_{\text {total }}\right\rangle$. The development of a correction for the soft background component generated at the beamline, which is the $b=2$ term in the sum of Eq. (3.3), will be presented in Chapter 6

$A_{T}$ accounts for the parity-conserving asymmetry arising from a residual transverse polarization component in the nominally longitudinally polarized electron beam. This effect should be suppressed by the azimuthal symmetry of the detector system. Finally $A_{L}$ accounts for potential non-linearity in the PMT response. The anticipated contributions to the proposed experimental error budget are listed in Table 3.2 . 


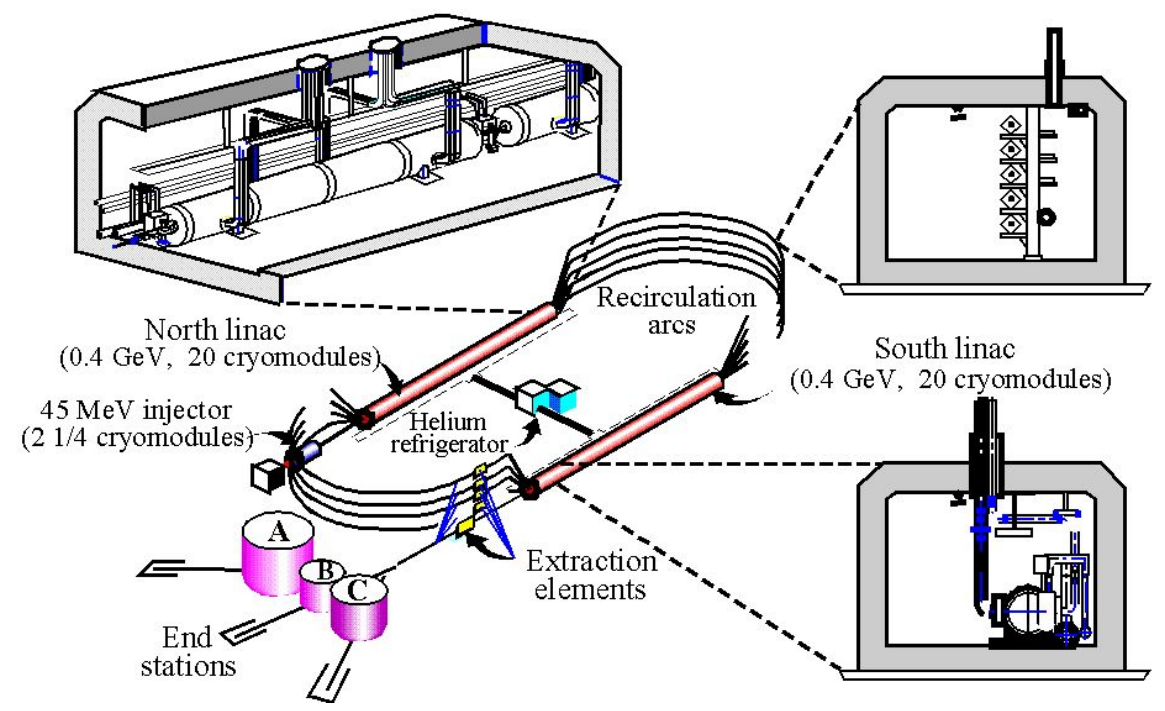

Figure 3.2: Schematic of the Jefferson Lab main accelerator. Electrons are accelerated in the two superconducting RF Linacs connected via recirculation arcs, to be delivered in the experimental end stations.

\subsection{The Continuous Electron Beam Accelerator Facility}

The Continuous Electron Beam Accelerator Facility (CEBAF) [98] at Jefferson Lab, located in Newport News VA, is one of the pioneering accelerating facilities in the world. The accelerator delivers continuous beam of high current and polarization with small emittance and energy spread [99, offering ideal conditions to support the lab's world-leading parity violation program. Until May of 2012 the electron beam was delivered to three experimental halls (A, B and C) with an energy of up to $6 \mathrm{GeV}$. $Q_{\text {weak }}$ in Hall C was one of the last experiments to take data under this configuration, which will be assumed in this dissertation. The lab is since being upgraded to double the beam energy up to $12 \mathrm{GeV}$ and also deliver beam to a fourth end station, experimental Hall D. Utilizing the capabilities of the upgraded facility to realize its forefront scientific program is of highest priority in the 2015 Long Range Plan for Nuclear Science [100].

The polarized beam for $Q_{\text {weak }}$ was created from a photocathode at the polarized source (Chapter 4). The fundamental accelerator radio frequency (RF) is $1497 \mathrm{MHz}$, with a third subharmonic RF chopping system in the injector providing a $499 \mathrm{MHz}$ independent continuous wave $(\mathrm{CW})$ bunch train to each experimental Hall. The beam receives an initial pre-acceleration to $5 \mathrm{MeV}$ and later up to $67 \mathrm{MeV}$ before being injected into the North linear accelerator (Linac). Each Linac provides $548 \mathrm{MeV}$ of acceleration through 5-cell superconducting radio-frequency (SRF) niobium cavities. The racetrack structure and the magnetic transport arcs allow up to five recirculation passes. When each beam is accelerated to the desired energy, it is extracted

\footnotetext{
${ }^{2}$ HCBA: Helicity-correlated beam asymmetries, referring to $A_{\text {beam }}$.
} 
at the end of the South Linac and transported to the corresponding experimental hall. $Q_{\text {weak }}$ in Hall $\mathrm{C}$ received single-pass beam of $E=1.165 \mathrm{GeV}$, except for a short period of two-pass operation.

\subsection{Target System}

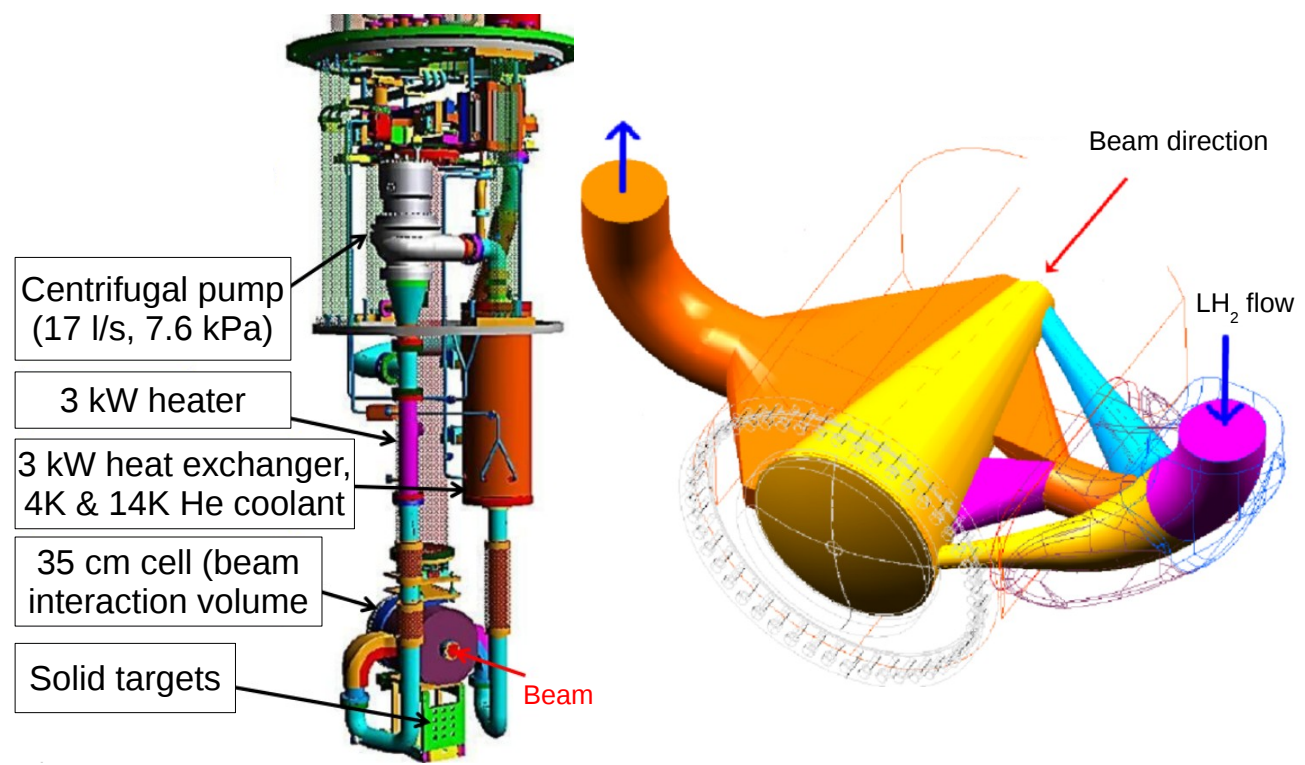

Figure 3.3: CAD design of the $Q_{\text {weak }}$ target cryo-system. The $\mathrm{LH}_{2}$ flow in the conical cell that defines the beam interaction region is shown on the right.

The 58 1, $20.00 \mathrm{~K}$ liquid hydrogen $\left(\mathrm{LH}_{2}\right)$ target cryo-system [101, 102] consisted of a recirculating loop driven by a centrifugal pump, a $3 \mathrm{~kW}$ resistive heater, and a $3 \mathrm{~kW}$ hybrid heat exchanger making use of both 14 and $4 \mathrm{~K}$ helium coolant (Figure 3.3). Due to the high statistical requirements the liquid hydrogen target is longer than in any previous experiment in the Jefferson Lab PV program. The conical aluminum cell which defined the beam interaction region was $34.4 \mathrm{~cm}$ long, designed using computational fluid dynamics (CFD) to maximize flow of $\mathrm{LH}_{2}$ transverse to the beam axis and suppress density variations. The combined requirements for high beam current and power $(180 \mu \mathrm{A}$ at $1.165 \mathrm{GeV})$ and a long target flask lead to a large deposition of beam power on the cryo-target. Sufficient cooling power must be provided to remove $\sim 2.5 \mathrm{~kW}$ of power, which makes this the highest power cryo-target in the world. A raster system was placed upstream of the target to reduce the current density on the target and prevent the beam from burning through the target windows. The intrinsic beam diameter of $\sim 250 \mu \mathrm{m}$ was rastered to a uniform square area of variable size at the target, typically 3.5 or $4 \mathrm{~mm}$ per side, using a $25 \mathrm{kHz}$ triangular waveform exciting iron-free magnets wound with aluminum wire. 


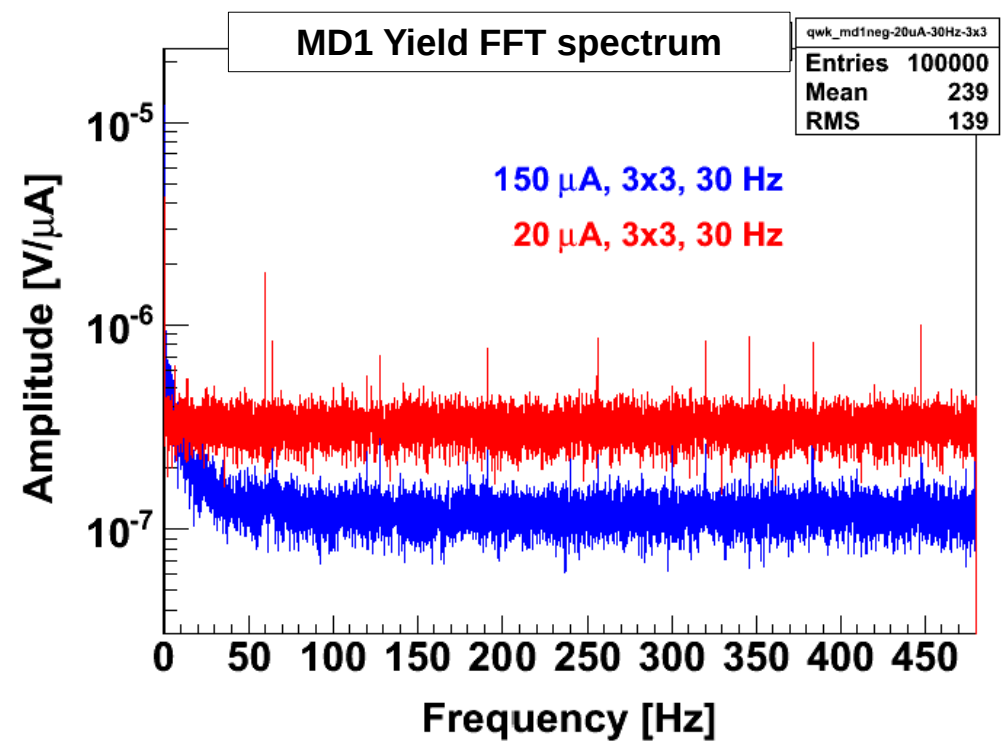

Figure 3.4: The Fast-Fourier transform spectrum of detector bar MD1 current-normalized signal (in $\mathrm{V} / \mu \mathrm{A}$ ), showing that the frequency of target density fluctuations is $\lesssim 40 \mathrm{~Hz}$ at $150 \mu \mathrm{A}$. Noise spikes at $60 \mathrm{~Hz}$ harmonics are also observed.
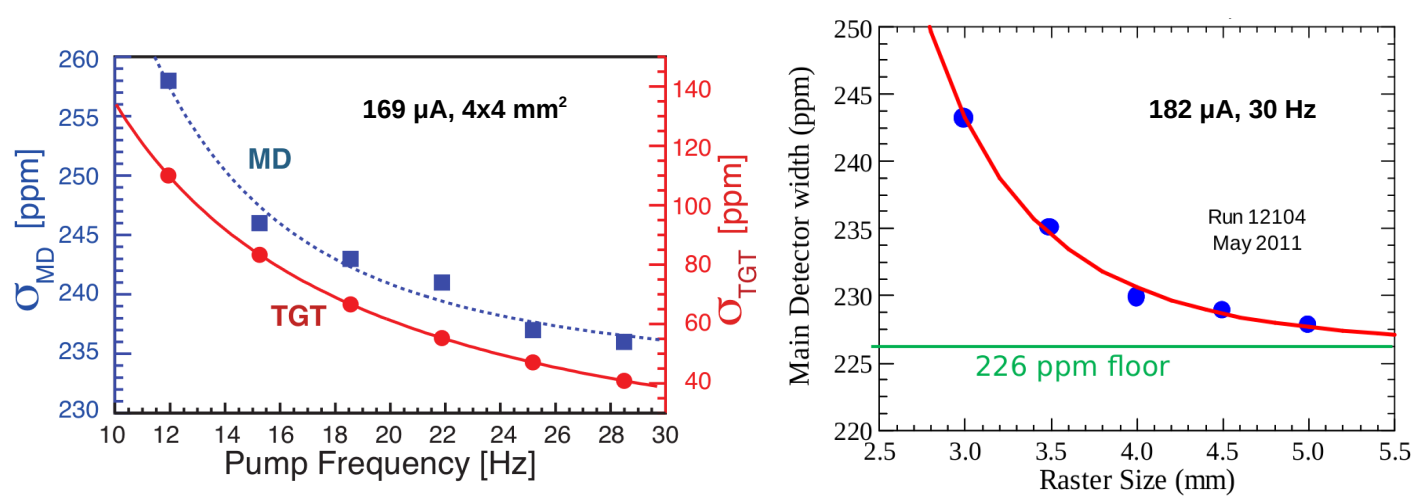

Figure 3.5: The dependence of the target boiling contribution to the measured asymmetry width is examined versus the hydrogen recirculating pump frequency (left) and the beam spot raster size (right), in dedicated tests.

\subsubsection{Target density fluctuations}

The width of the asymmetry that is measured in the main detectors is a fundamental figure of merit (FOM) for the experiment, as it defines the maximum statistical precision that can be achieved in a given time period. The width of the measured asymmetry was typically $\sim 225$ parts per million (ppm) at $180 \mu \mathrm{A}$ (Figure 3.12). Density fluctuations at the target due to high beam power deposition (often referred to as "target boiling") can contribute noise to the measured asymmetry and reduce the FOM of the experiment. Minimization of this noise contribution from density fluctuations was a main consideration in the design of the $Q_{\text {weak }}$ target.

The characteristic noise frequency of the target density fluctuations are shown to be $\lesssim 40 \mathrm{~Hz}$ at $150 \mu \mathrm{A}$ (Figure 3.4). To suppress the target noise contribution we pursued the development of the capability for a much faster helicity reversal at $960 \mathrm{~Hz}$ (Section 4.3.3), much faster than the 
reversal rate in previous PVES experiments (typically $30 \mathrm{~Hz}$ ). Thus the detector measurement is much shorter than the characteristic time scale for target boiling, suppressing its contribution to the asymmetry width. The dependence of the target noise contribution to beam current, raster size, and the rotational frequency of the hydrogen recirculation pump, was measured in dedicated tests (Figure 3.5) . Typical values where $29.4 \mathrm{~Hz}$ for the frequency of the $\mathrm{LH}_{2}$ pump and $4 \times 4 \mathrm{~mm}^{2}$ for the raster sizf 3 . At these conditions and with the fast $960 \mathrm{~Hz}$ helicity reversal the target boiling contribution is limited to $53 \pm 5 \mathrm{ppm}$ at $180 \mu \mathrm{A}$, negligible when added in quadrature to the main detector width from counting statistics and detector resolution.

\subsubsection{Aluminum windows and dummy targets}

Elastic scattering of electrons from the aluminum windows that encase the $\mathrm{LH}_{2}$ target is the largest background correction to the measured asymmetry, entering as the $b=1$ term in the sum of Eq.(3.3). The size of this background PV asymmetry is large relative to the $\overrightarrow{e p}$ asymmetry, as it is characterized by the weak charge of aluminum $Q_{w}\left({ }^{27} \mathrm{Al}\right)$ which from Eq. 2.15 is expected to be an order of magnitude larger than the suppressed $Q_{w}^{p}$, and of opposite sign. The rate contribution from this background source to the detector signal is $\sim 2.6 \%$ making this a significant correction.

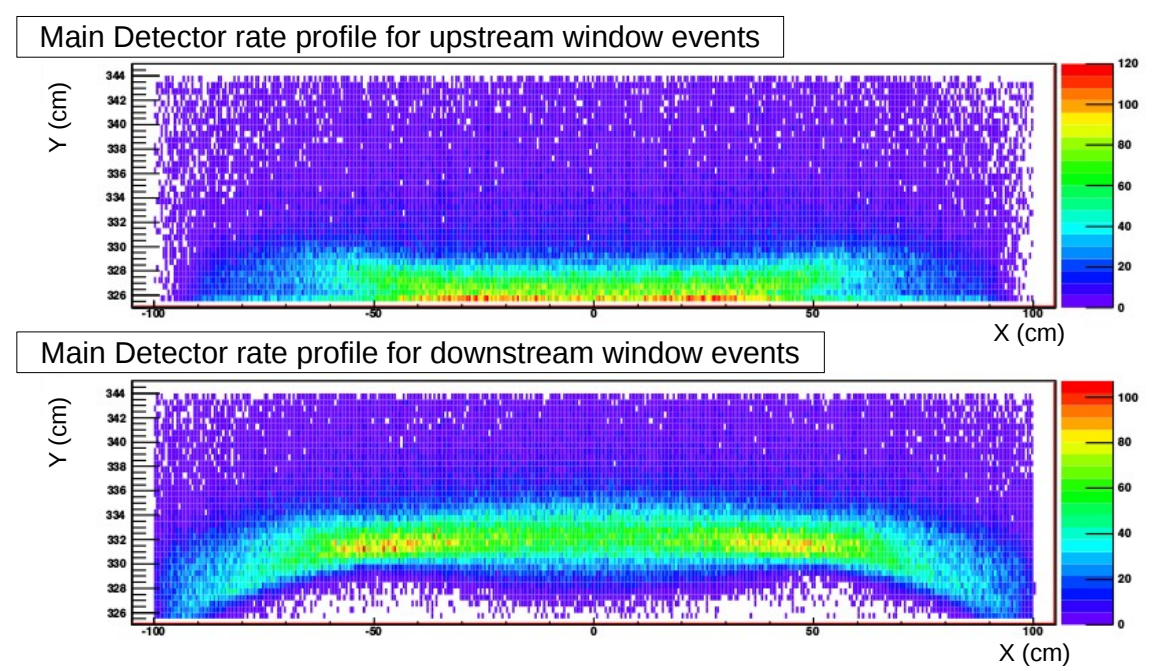

Figure 3.6: The simulated rate profile on the main detector bars from elastic aluminum events, shown separately for the upstream (top) and downstream (bottom) aluminum windows [103.

The aluminum background asymmetry is measured directly in dedicated data where the $\mathrm{LH}_{2}$ target is removed and scattering occurs from solid aluminum targets of different thickness that simulate the alloy of the upstream and downstream target windows. A remotely controlled 2-axis motion system with $600 \mathrm{~mm}$ of vertical travel and $86 \mathrm{~mm}$ of horizontal travel allows positioning the $\mathrm{LH}_{2}$ target or any of the 24 solid targets on the beam axis. The signal fraction $f_{1}$ in the

\footnotetext{
${ }^{3}$ The raster size was mostly $3.5 \times 3.5 \mathrm{~mm}^{2}$ in the first part of the $Q_{\text {weak }}$ run (Run1) where the average beam current and power deposition was lower.
} 
main detector from this background source is also measured from dedicated runs with the target cell evacuated and all detector signal coming from the aluminum windows.

An important effect that must also be considered is that the upstream and downstream windows have different rate profiles on the main detector bars (Figure 3.6). Much of the rate from the upstream window is cut off on the inner radius side of each detector bar. This was done purposefully to reduce the rate from the upstream window and lower the size of the factor $f_{1}$. However the sharp cut-off of the aluminum rate also increases energy sensitivity and potentially introduces higher-order effects due to fluctuations of the scattered rate.

\subsection{Collimators and Shielding}

The $Q_{\text {weak }}$ collimator system plays a crucial role in defining the $Q^{2}$ acceptance of the detector. The design was optimized through GEANT simulations and consists of three sequential elements, the middle of which is the acceptance-defining collimator, with the other two inserted for "cleanup" purposes and allowing ample clearance for elastic electrons. Each Pb collimator has eight sculpted openings for the elastic electrons that will be focused on each main detector octant. The defining collimator was $15 \mathrm{~cm}$ thick and placed $2.72 \mathrm{~m}$ downstream of the target center, as close to the spectrometer support structure as possible to maximize the acceptance of elastic events and make the target more "point-like". The acceptance in the scattering angle is defined by the opening on its downstream face, $5.8^{\circ} \leqslant \theta \leqslant 11.6^{\circ}$, where the smallest and largest values correspond to scattering from the upstream and downstream end of the target respectively.

Direct line-of-sight neutral events originating at the target are blocked by the collimators and shielding, and spatially separated by the field of the toroidal spectrometer. For indirect backgrounds we adopted a "2-bounce" design, demanding that events outside the defined acceptance must have scattered at least twice after leaving the target before reaching the main detector. Neutral background events arising from primary electrons scattering from the edges of the upstream and defining collimators (tracks 1 and 3 in Figure 3.7. respectively) should be outside the acceptance defined by the collimators. We still allow for this background source, referred as the "QTor transport channel background", which is the $b=4$ term in the sum of Eq. (3.3). Lead "lintel" collimators were installed between the coils of the magnet to shield the detectors from line-of- sight neutrals generated at the beamline between the upstream and the defining collimators (track 2 in Figure 3.7).

To eliminate possible direct line-of-sight backgrounds from the aluminum beampipe, a 21 cm long water-cooled tungsten-copper collimator was "plugged" snugly in the central aperture of the upstream collimator (Figure 3.8). The upstream face of this plug collimator was only 


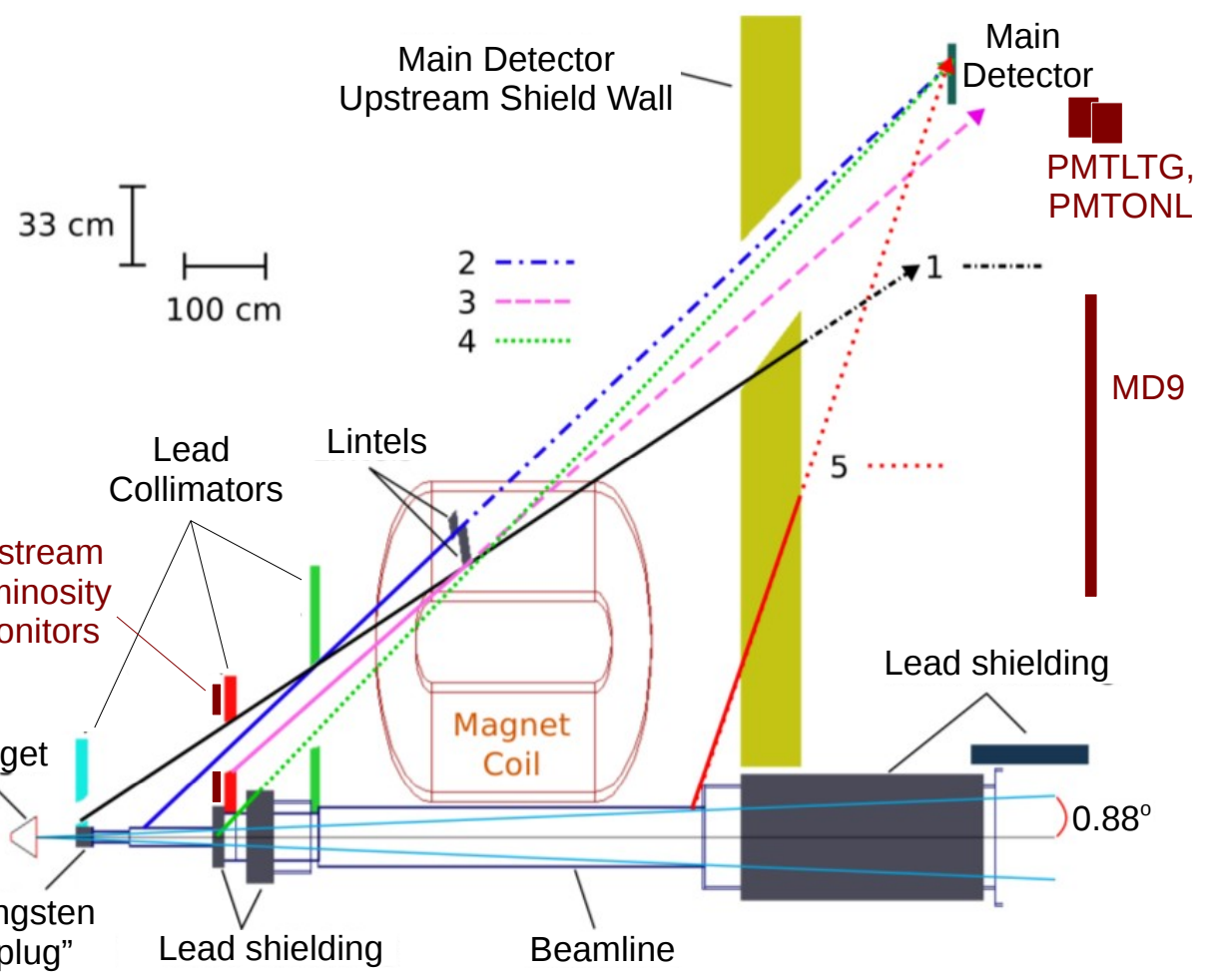

Figure 3.7: A simplified side elevation view illustrating possible sources of soft backgrounds in the main detectors and the shielding that was used to minimize the background rates. The vertical scale is amplified by a factor of three for clarity. The electron beam comes in from the left and interacts with the target. Subsequent scattering from different places in the beamline is shown with solid lines of different colors, while different line types show the particle trajectories after encountering shielding put in place to prevent them from reaching the main detectors. Three background detectors behind the main detectors (PMTLTG,PMTONL,MD9) and the upstream luminosity monitors on the upstream face of the defining collimator, were very sensitive to the beamline background component.
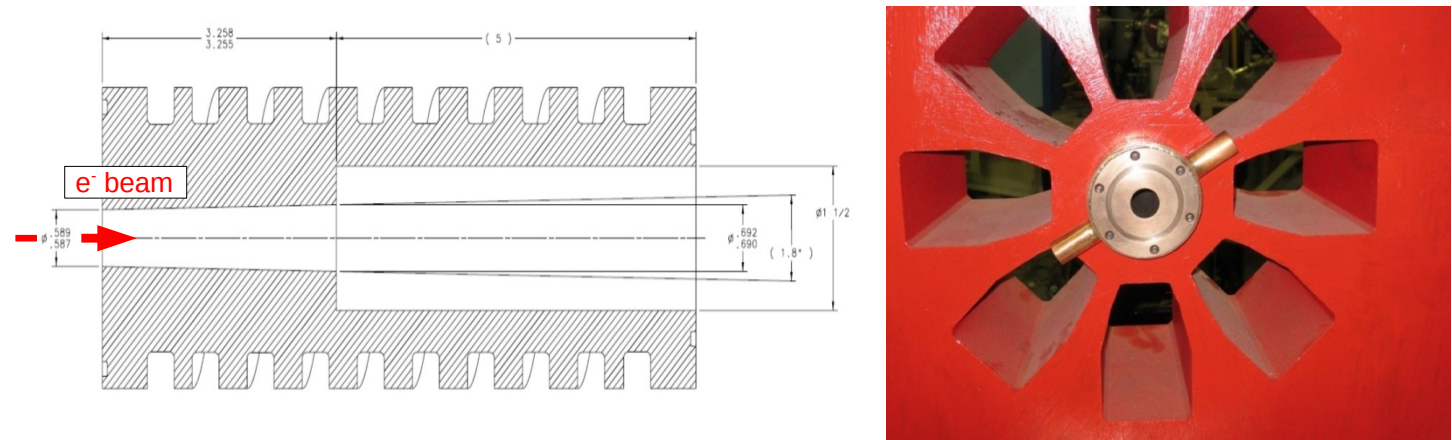

Figure 3.8: Left: Engineering drawing of the tungsten plug collimator (dimensions in inches), where the electron beam is entering through the left side. Right: Picture of the plug collimator fit on the upstream collimator element. 


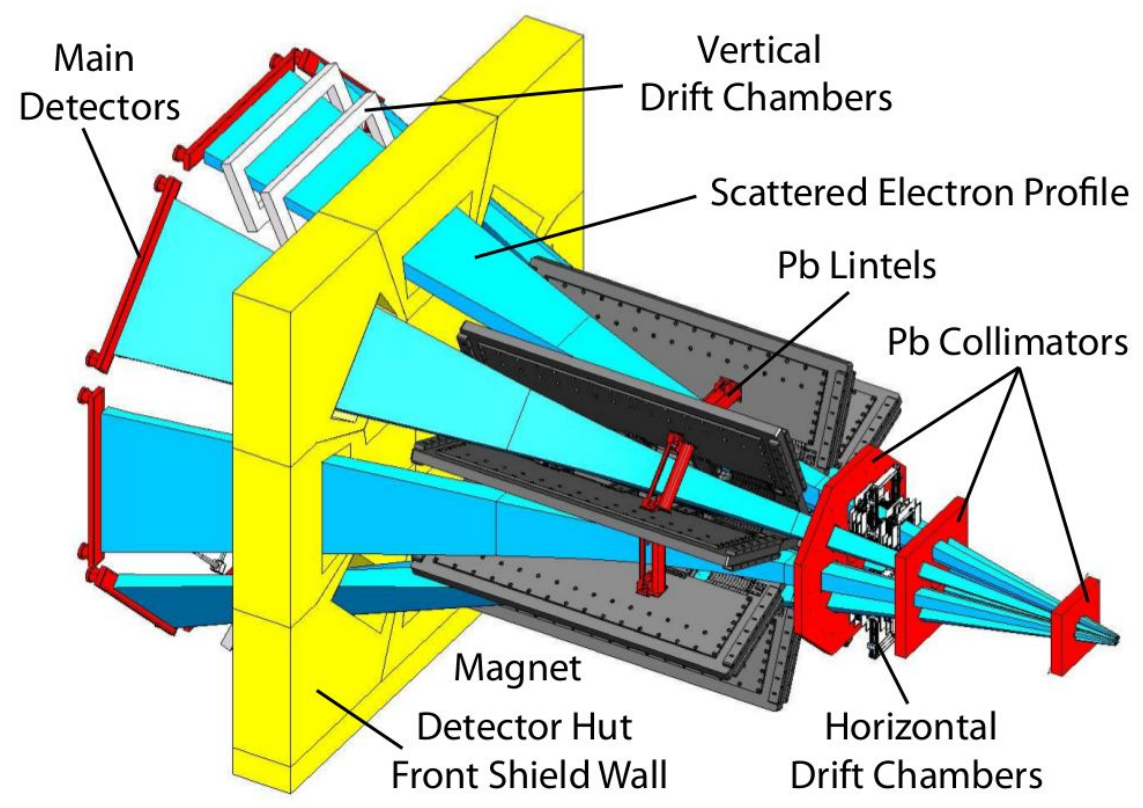

Figure 3.9: CAD drawing of the apparatus. Beam comes in from the right and scatters at the target. Shown in blue is the simulated scattered elastic electron envelope through the collimator system and QTor spectrometer. Also shown are the horizontal and vertical drift chambers used for tracking reconstruction.

$47 \mathrm{~cm}$ downstream of the target cell's exit window. The beam passed through an evacuated tapered conical section machined out of the center of the collimator which was $14.91 \mathrm{~mm}$ in diameter at the upstream end and $21.5 \mathrm{~mm}$ in diameter at the downstream end. This allowed a maximum angle $\theta_{\max }=0.88^{\circ}$ for electrons scattered from the downstream face of the target. This should in principle only allow scattered electrons to interact with the beamline downstream of the main detectors location, and simulations show that backgrounds are vastly reduced by this collimator. In practice however, some earlier interferences with the beamline may have been possible (tracks 4 and 5 from Figure 3.7) for electrons that scattered from the downstream end of the target. These are still separated from the main detectors by heavy lead shielding installed around the beamline and from the barite-loaded concrete $\left(\mathrm{Ba}_{2} \mathrm{SO}_{4}\right)$ wall of the detector hut, but a small fraction is still expected to reach the main detectors. This is the $b=2$ background component, referred as the "beamline background". It is considered to be associated with the outer part of the beam (beam "halo") which scatters preferentially from the collimators and beamline. Background detectors behind the main detector locus (PMTLTG,PMTONL,MD9) and the upstream luminosity monitors on the upstream face of the defining collimator, were very sensitive to this component. A correction for the effect of this large background asymmetry in the main detector will be developed in Chapter 6 


\subsection{Toroidal Spectrometer}

The $Q_{\text {weak }}$ resistive DC toroidal magnetic spectrometer "QTor" was centered $6.5 \mathrm{~m}$ downstream of the target center and consisted of eight coils arranged azimuthally about the beam axis. Each coil was composed [104] of a double pancake of 13 turns of copper conductor. Each racetrackshaped pancake had straight sections $2.20 \mathrm{~m}$ long, and semi-circular curved sections of inner (outer) radius $0.235 \mathrm{~m}(0.75 \mathrm{~m})$. A field integral $B d \ell$ of $\sim 0.9 \mathrm{~T} \cdot \mathrm{m} \mathrm{H}^{4}$ along the envelope of the elastic electron trajectories deflects them radially outwards by about $10^{\circ}$ and focuses them onto the main detector bars. The focusing properties must cleanly spatially separate elastic events from inelastics, which is the $b=3$ background source in Eq. (3.3), as well as neutrals (photons and neutrons) and low-energy Møller electrons. The spectrometer is also required to be highly symmetric around the azimuth to reduce sensitivity to systematic errors.

The coil geometry was optimized in a series of simulation studies using GEANT plus numerical integration over the conductor's current distribution to determine the magnetic field. Mapping indicated that the coil positions and angular displacement were mostly within specification, as were the coil-to-coil variations in $B d \ell$ 97. The total DC current during production was nominally $8921 \mathrm{~A}$ at $123 \mathrm{~V}$, corresponding to the maximum rate from elastic electrons. More details on the QTor spectrometer can be found in [104].

\subsection{Detector System}

\subsubsection{Main Detectors}

The $Q_{\text {weak }}$ main detector (MD) system consists of eight azimuthally symmetric fused quartz (Spectrosil $^{\circledR} 2000$ 105]) radiators, which produce Čerenkov light when high energy electrons pass through them. The radiator material consists of high-purity $\mathrm{SiO}_{2}$ which exhibits radiation hardness, insensitivity to backgrounds, uniformity of response, and low intrinsic noise. The eight MD bars were arrayed symmetrically about the beam axis $5.7 \mathrm{~m}$ downstream of the QTor magnet center at a radial distance of $3.3 \mathrm{~m}$. The eight-fold azimuthal symmetry of the MD array minimizes false asymmetries from helicity-correlated beam motion $A_{\text {beam }}$ and transverse asymmetry $A_{T}$, while also allowing characterization of these effects.

Each detector comprised two rectangular bars $100 \times 18 \times 1.25 \mathrm{~cm}$ thick, glued together into $2 \mathrm{~m}$ long bars. After many bounces, Cerenkov photons reach the ends of the rectangular bars by total internal reflection and are then collected by $12.7 \mathrm{~cm}$ diameter low-gain photomultiplier tubes (PMTs) with UV-transmitting windows, through $18 \mathrm{~cm}$ long quartz light-guides on each end of the bar assembly. To suppress the signal from soft neutral backgrounds, $2 \mathrm{~cm}$ thick lead

\footnotetext{
${ }^{4}$ Corresponds to the acceptance-averaged scattering angle $\theta=7.9^{\circ}$.
} 


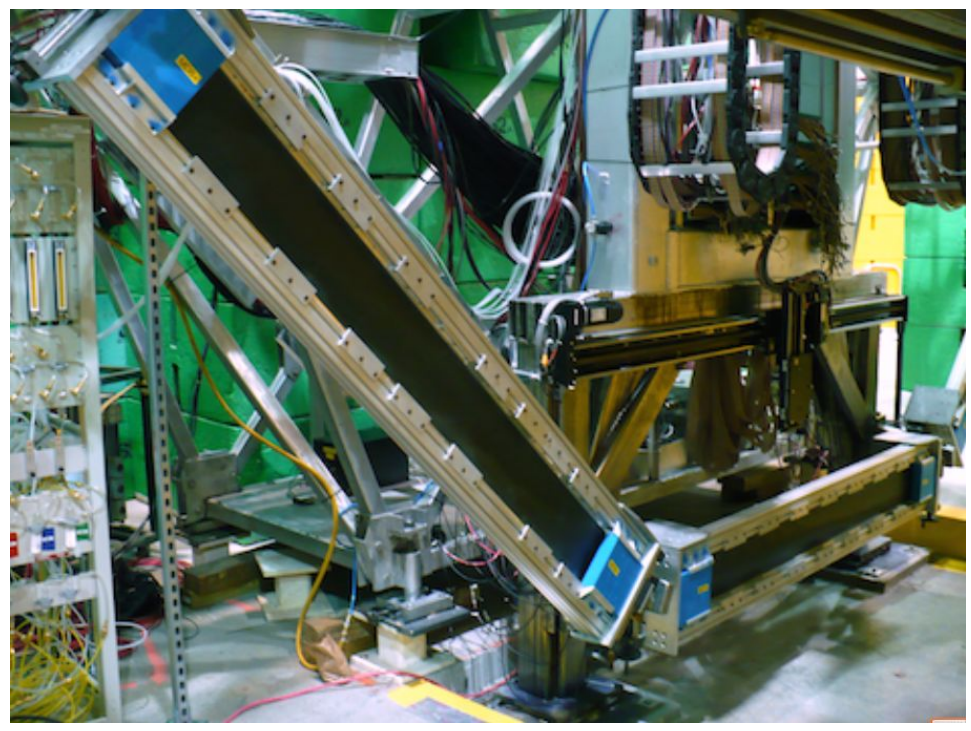

Figure 3.10: Main detector bars 7 and 8 before installation of the lead pre-radiators. The squares at the end of each bar is lead shielding covering the light-guides and PMTs. Also shown above the lower detector is the quartz sampling scanner.

pre-radiators were placed in front of each MD bar. These improve the signal-to-background ratio by a factor of $\sim 20$, achieving a suppression of systematic effects associated with the soft background.

During production at $180 \mu \mathrm{A}$ the rate on each detector is higher than $850 \mathrm{MHz}$ and integrating mode readout is required. The anode current from each PMT was converted to voltage using a custom low-noise preamplifier and digitized with a custom TRIUMF 18-bit ADC [106], its output phase-locked with the reversal of the beam helicity at $960 \mathrm{~Hz}$. Detector pedestals were measured every $\sim 8$ hours by taking data for a few minutes without beam in the experimental hall. This allowed estimation of the electronic noise contribution to the MD asymmetry width, and also provided an opportunity to search for false asymmetries due to electronic pickup.

The average signal from all PMTs is referred to as the "MDall" combination ${ }^{5}$. In every helicity quartet the raw asymmetry $A_{\text {raw }}$ is formed based on Eq. 3.2 . The raw quartet asymmetry distribution of the MDall average is plotted in Figure 3.12 for data collected over $\sim 7$ minutes at $165 \mu \mathrm{A}$. The width of the measured asymmetry $\sigma_{A}$ is a critical measure of the experiment's statistical power and figure-of-merit. The achievable relative statistical accuracy from a dataset with $N$ quartets is given by

$$
\frac{\Delta A}{A}=\frac{\sigma_{A}}{A P \sqrt{N}}
$$

where $A$ is the size of the asymmetry and $P$ is the beam polarization. It is therefore very

\footnotetext{
${ }^{5}$ The average may be formed unweighted or through weighting each PMT by its yield. Difference between the averaging methods is generally small, although relative weights may break the azimuthal symmetry of the detector.
} 


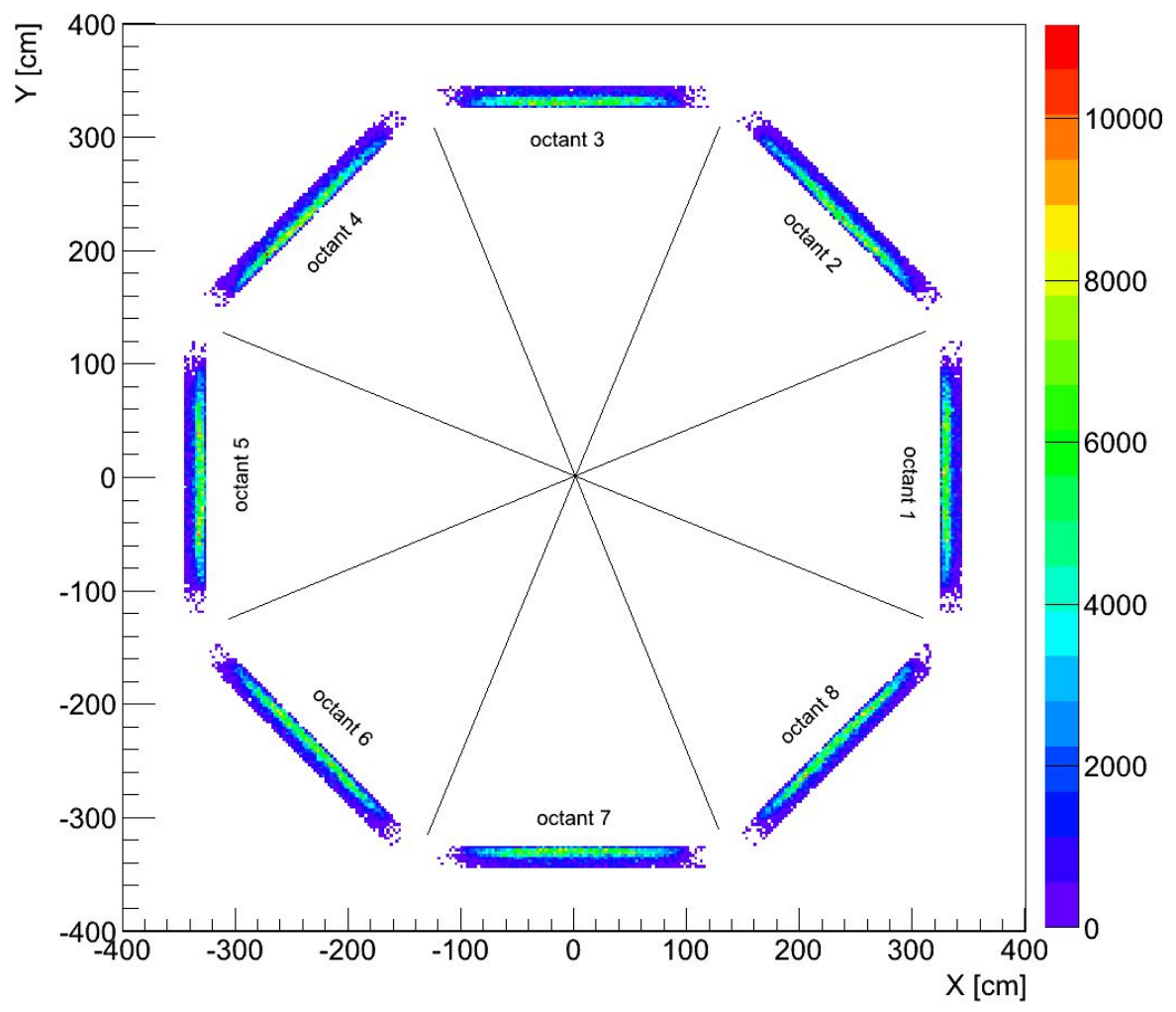

Figure 3.11: A GEANT-IV simulation showing the elastic scattered electron profile on the quartz bars. See Figure 3.16 for a detailed view of the rate profile on each bar.

important to suppress all sources of noise in the measurement. At $180 \mu \mathrm{A}$ the asymmetry width $\sigma_{A} \approx 225 \mathrm{ppm}$ is the quadrature sum of contributions from statistics per quartet ( $\sim 215 \mathrm{ppm}$, accounting for dead-time from a helicity transition time of $70 \mu$ s plus $42 \mu$ s gate delay at 960 $\mathrm{Hz}$ reversal, and for detector resolution), beam current monitor resolution ( $43 \mathrm{ppm})$, and noise from target density fluctuations ( $\sim 55 \mathrm{ppm})$. The health and efficiency of the experiment can be assessed by comparing the observed asymmetry width $\sigma_{A}$ with the expectation from the quadrature sum of these contributions.

\subsubsection{Luminosity monitors and background detectors}

The experimental apparatus included two azimuthally symmetric arrays of luminosity monitors [107], also based on fused silica Čerenkov radiators. The four upstream luminosity monitors (USLumis) consist of 4 Čerenkov detectors in the horizontal and vertical octants (octants $1,3,5,7$ ). They were placed in the upstream face of the defining collimator (Figure 3.14, $2.67 \mathrm{~m}$ from the target. The sum of the four luminosity monitors is referred as the "USLsum" combination. The eight downstream luminosity monitors (DSLumis) were located $17 \mathrm{~m}$ from the target (downstream of the target z-location) at a very small scattering angle. Both sets of luminosity monitors receive higher rates than the main detectors and offer high statistical precision. 


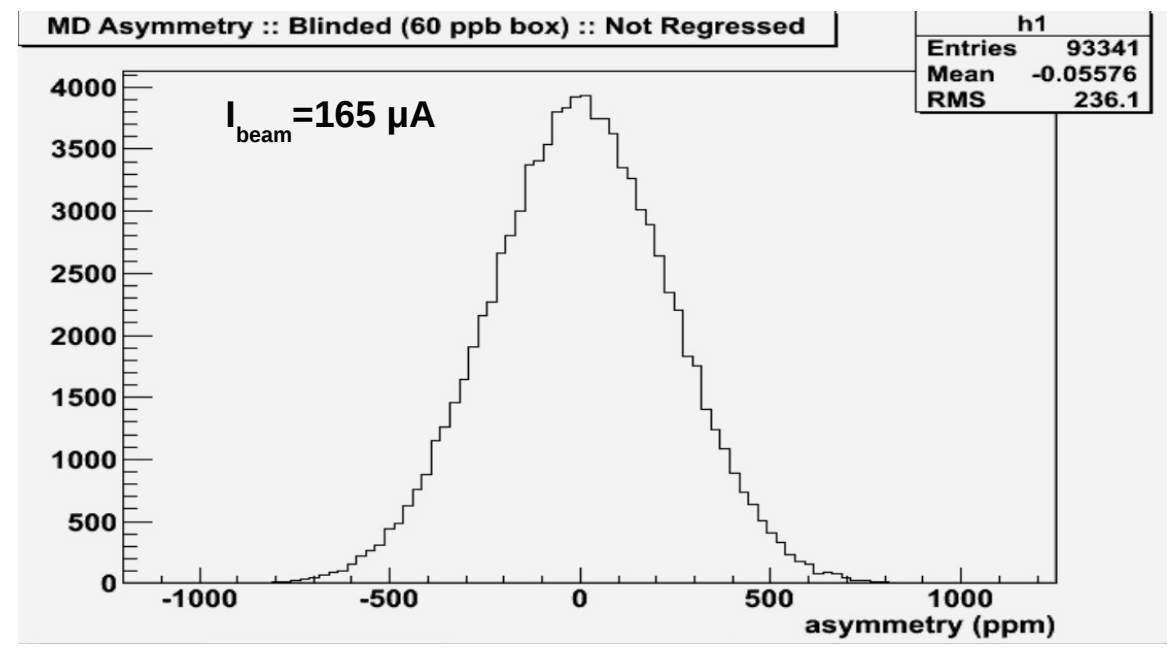

Figure 3.12: Distribution of the raw MDall measured asymmetry $A_{\text {raw }}$ from helicity quartets, collected over $\sim 5$ minutes. Data is blinded and no corrections are applied. The width of the measured asymmetry (here $\sigma_{A}=236 \mathrm{ppm}$ ) is an important figure-of-merit for the experiment.

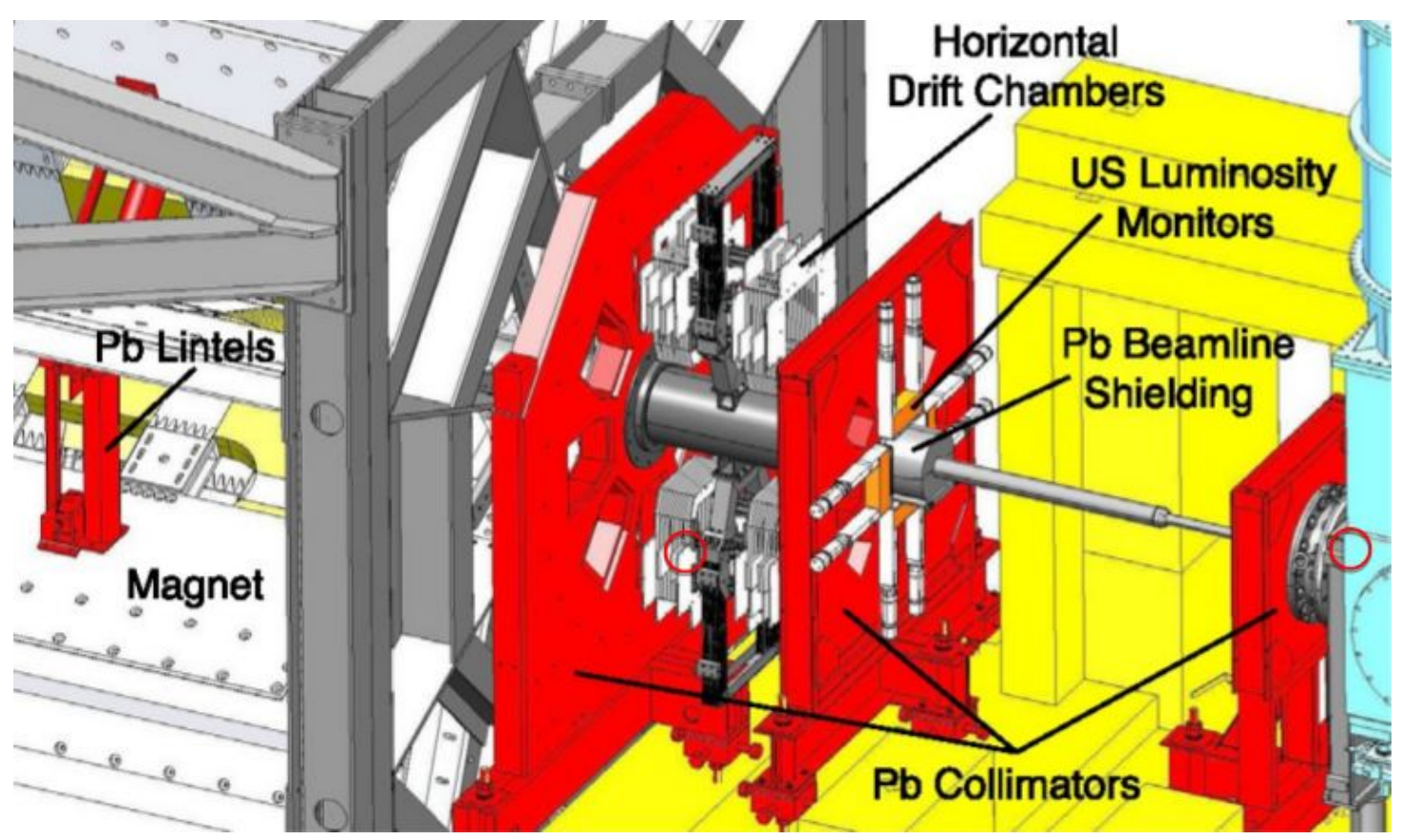

Figure 3.13: CAD drawing of the $Q_{\text {weak }}$ collimator system (red) upstream of the spectrometer magnet. Beam comes from the right and scatters from the target (light blue). Also shown are the upstream luminosity monitors, on the upstream face of the defining collimator, and the horizontal drift chambers. 
The USLumis were expected to detect Møller scattered electrons at small angles, $\sim 5^{\sqrt{6}}$ These events have much smaller asymmetry than the $\overrightarrow{e p}$ events at the main detectors, so the USLumis were expected to measure a "null" asymmetry, i.e. consistent with zero, and even be sensitive to the noise from target density fluctuations. Instead the luminosity monitors were sensitive to large background asymmetries, often more than an order magnitude higher than the physics $\vec{e} p$ asymmetry. These large asymmetries are considered to be associated with the soft neutral $b_{2}$ background component originating at the tungsten plug collimator and the beamline (Section 3.4).
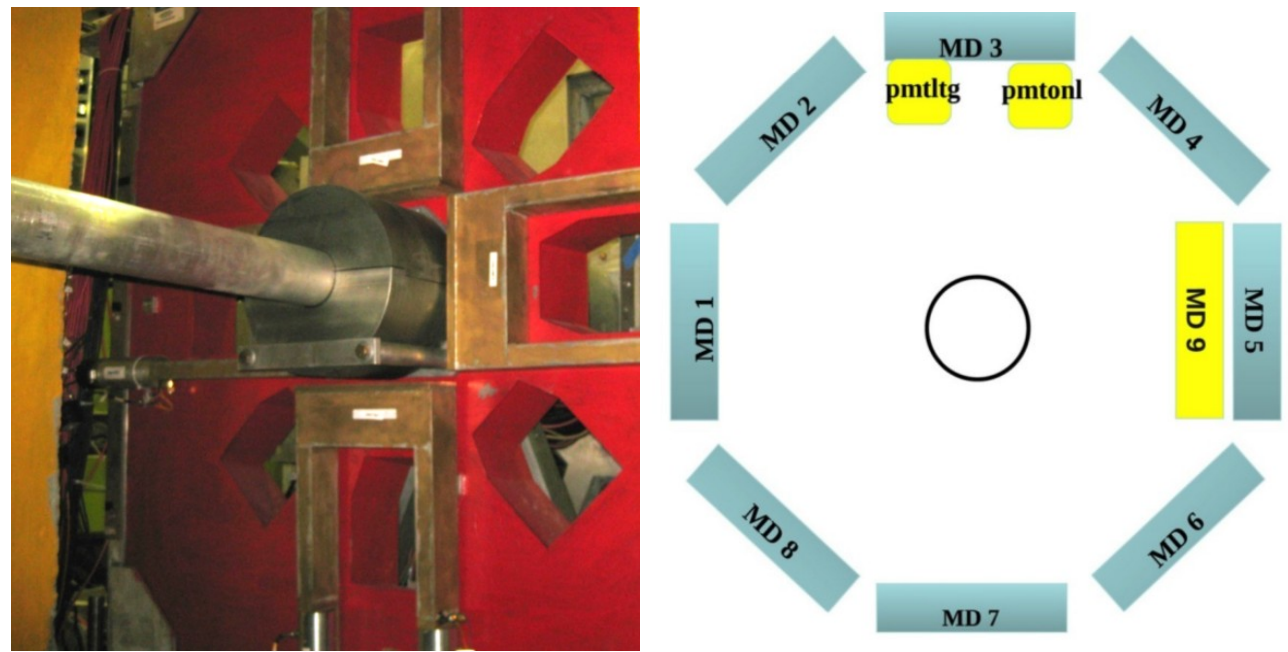

Figure 3.14: Left: Picture of the upstream luminosity monitors (USLumis) placed on the upstream face of the second (defining) collimator element. Lead shielding installed around the beamline is also visible. Right: The Run2 location of the background detectors in the super-elastic region behind the MD focal plane, looking downstream to the MD array. Background detector MD9 was placed behind MD5, while PMTLTG and PMTONL were placed behind MD3. See also Figure 3.7.

Any soft background in the experimental hall that reaches the main detector will produce a dilution of the elastic electron signal and potentially a false asymmetry. To help quantify such effects, and potentially search for leakage of the helicity reversal signal, three soft background detectors were placed inside the detector shield house. One was a complete detector assembly just like the eight main detectors, referred to as MD9; one was a PMT connected to a lightguide (PMTLTG); and the third was only a PMT in a small dark box (PMTONL). They were placed in different locations inside the detector hut to study the properties of the diffuse background component. Most pertinent for this dissertation is their location throughout the second part of the run (Run2), shown in Figure 3.14. They were placed at the "super-elastic" region immediately downstream of the main detector bars but at a smaller radius. These detectors were also sensitive to the soft background component and measured very large asymmetries.

Our effort to develop an algorithm for the correction of the the false asymmetries in the MD from this highly asymmetric background component will be discussed in Chapter 6. As we

\footnotetext{
${ }^{6}$ The openings of the upstream collimator allow such low-angle events to go through.
} 
will see, the upstream luminosity monitors and the background detectors will be crucial for this correction.

\subsubsection{Kinematics and tracking detectors}

Since the PV asymmetry is directly proportional to $Q^{2}$ (Eq. 2.10) it is essential that the interaction $Q^{2}$ is determined to very high precision. A dedicated tracking system was developed to extract the $Q^{2}$ and angle distributions of elastic events on the main detector bars, weighted by their light response. In conjunction with simulations this will allow the estimation of the $\left\langle Q^{2}\right\rangle$ averaged over the acceptance, in order to translate the measured $\left\langle A\left(Q^{2}\right)\right\rangle$ to $A\left(\left\langle Q^{2}\right\rangle\right)$ (Section 8.1.1)

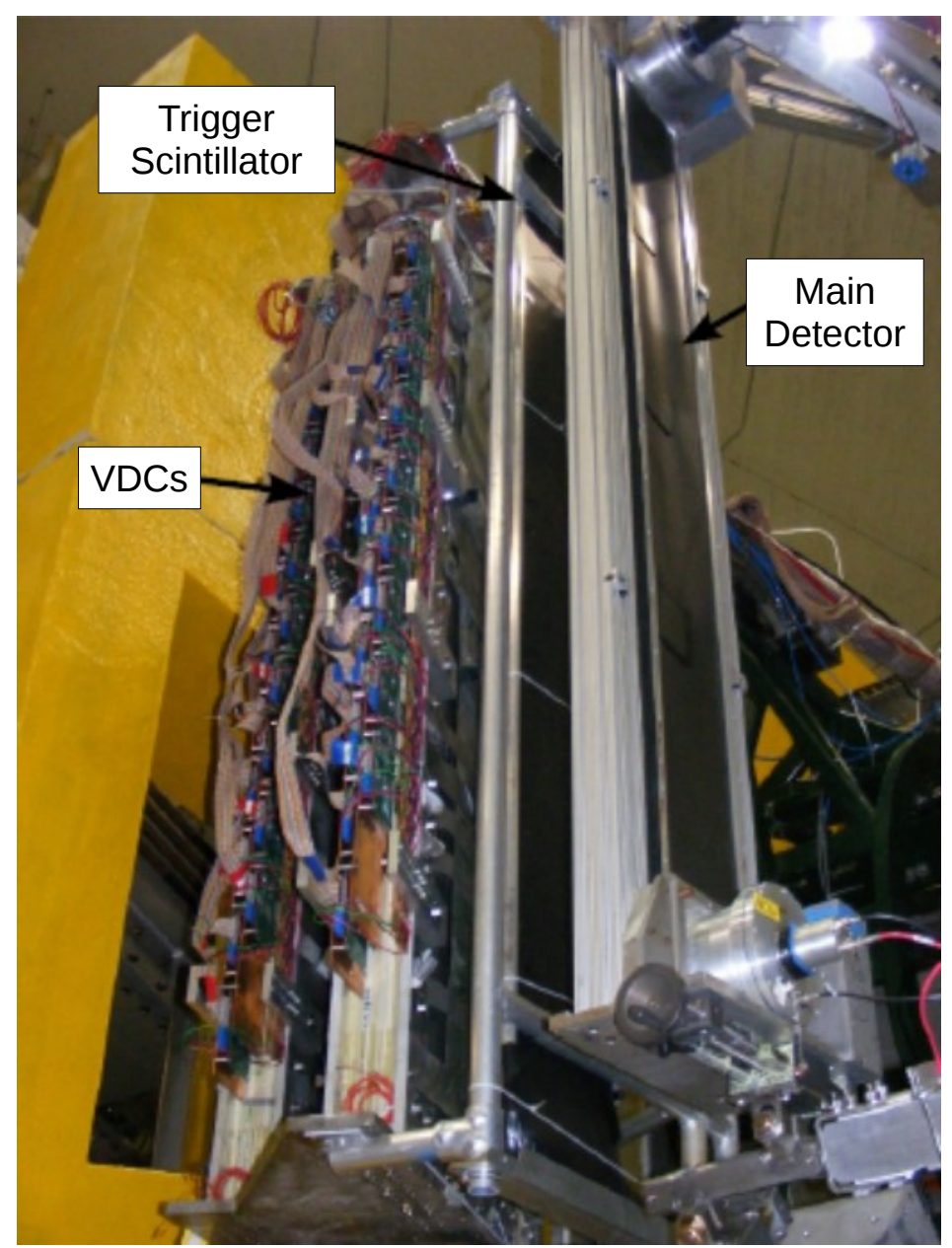

Figure 3.15: Vertical drift chambers (VDCs) can be placed upstream of MD bars for tracking measurements. A plastic scintillator between them offers timing information.

Tracking measurements were performed in dedicated runs with low beam currents $(50 \mathrm{pA}$ - $200 \mathrm{nA})^{7}$ where individual particle trajectories can be identified. Horizontal drift chamberss (HDCs) and vertical drift chamberss (VDCs) were placed on the upstream and downstream

\footnotetext{
${ }^{7}$ The limitation is set by the horizontal drift chambers which receive very high rates from Møller electrons.
} 
side of the QTor magnet, respectively. Plastic scintillation counters between the VDCs and the main detectors offer timing information (Figure 3.15). For the low beam current tracking runs a separate PMT base was used to read out the detectors with high gain in counting mode.

Each particle track is expected to provide a set of hits in 12 planes of HDCs and 4 planes of VDCs, each forming separate straight line partial tracks, since the magnetic field is close to zero at the location of the tracking chambers. A pattern recognition algorithm separately identifies valid line segments in the HDCs and in the VDCs by comparing the pattern of hit locations to a series of templates. The data from different orientations is combined in a least-squares fit to form a three-dimensional track segment. The electron scattering vertex and angle is reconstructed by the HDCs, while the VDCs confirm the momentum of the elastic events.

For elastic scattering, ignoring radiative and energy losses, the 4-momentum transfer squared $Q^{2}$ is given by

$$
Q^{2}=2 E^{2} \frac{(1-\cos \theta)}{1+\frac{E}{M}(1-\cos \theta)},
$$

where $M$ is the proton mass, $E$ is the known incident beam energy, and $\theta$ is the lab scattering angle. This allows the reconstruction of the interaction $Q^{2}$ from the tracking dedicated measurements with input from GEANT4 simulations to account for radiative effects. The detector's analog light response is correlated with each particle trajectory, and finally the "light-weighted" $Q^{2}$ distribution of events is formed on the main detector bar.
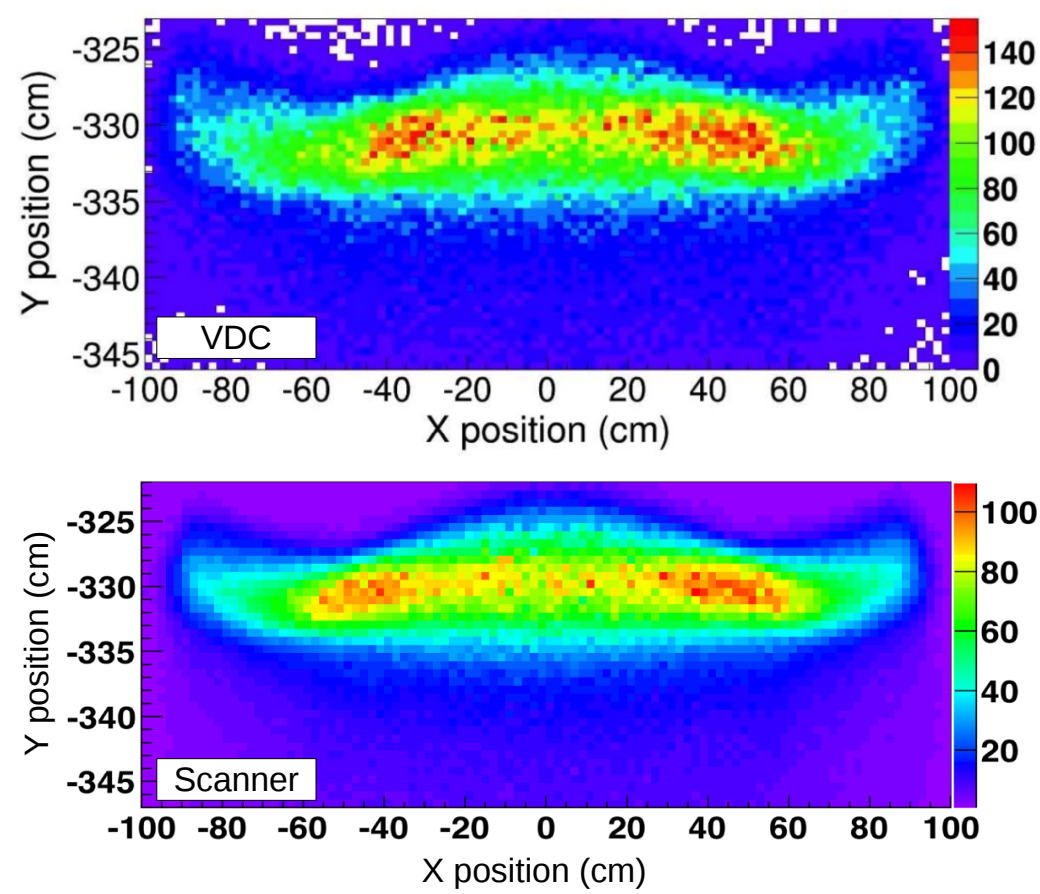

Figure 3.16: Scattered electron rate profile on on a main detector bar, reconstructed from the VDCs (top) and obtained from the focal plane scanner detector (bottom). The two measurements are in good agreement. The horizontal and vertical axes correspond to horizontal and vertical location on the detector bar. Color scale indicates relative rates. 
A small scanning Čerenkov detector was built and installed to measure the rate profile of events reaching the main detector [108]. Its small fiducial area of $1 \times 1 \mathrm{~cm}^{2}$ allows it to cope with the maximum electron flux of about $1 \mathrm{MHz} / \mathrm{cm}^{2}$ and operate at high currents. Employing a 2D linear motion system the detector is scanned across the face of a MD bar and measure the position-dependent event rate profile. Results from this measurement were in very good agreement with simulations and reconstruction from the VDCs (Figure 3.16).

\subsection{Beam Monitors}

Beam position monitors (BPMs) and beam current monitors (BCMs) were placed along the Hall $\mathrm{C}$ beamline in various locations. They offered continuous measurements of beam parameters, such as charge, position, angle, and energy.

\subsubsection{Beam charge}

The Hall C beam current monitors (BCMs) are "pillbox" RF cavities that offer a relative measurement of the beam current via resonance of the $\mathrm{TM}_{010}$ mode at $1497 \mathrm{MHz}$. The absolute calibration of the BCMs was performed using a Parametric Current Transformer [109] (Bergoz Unser monitor) in the Hall C beamline.

The charge measurement was important for the normalization of detector signals in Eq.(3.2). A BCM non-linearity would appear as

$$
I_{\mathrm{BCM}}=I_{\text {beam }}\left(1+\alpha_{L} I_{\text {beam }}\right),
$$

where $\alpha_{L}$ is the non-linearity parameter. The BCM linearity was observed to be better than $0.5 \%$ at the current range $20-180 \mu \mathrm{A}$.

In the first production period of $Q_{\text {weak }}$, defined as Run1 in Section 3.9, the beam current measurement used for charge normalization of the MD yields in Eq. 3.2 was provided by a pair of BCMs located $13.4 \mathrm{~m}$ upstream of the target, BCMs 1 and 2. The same BCMs were used for charge feedback (Section 4.9.1). They were housed in the same enclosure as the Unser monitor and had analog receivers, with their electronics placed outside the experimental hall to be protected from radiation damage. BCMs 5 and 6 , located $16 \mathrm{~m}$ and $2.7 \mathrm{~m}$ upstream of the target respectively, were also available in Run1 and were connected to prototype digital receivers. However they were seen to have significant noise associated with their local oscillator, so they were not used during Run1. In Run2, the second $Q_{\text {weak }}$ production period, two more BCMs (7 and 8) were located in the same enclosure as BCM5, $16 \mathrm{~m}$ upstream of the target, and offered 
very reliable charge normalization. BCMs 5-8 were outfitted with new digital receivers placed outside the experimental hall.

The intrinsic BCM resolution is an important measure of the BCM performance. It corresponds to the BCM monitor noise that is injected to the MD asymmetry width through charge normalization. The resolution was measured by taking the difference in two BCMs of the measured charge asymmetry between the two helicity states, a metric that was referred to as BCM "double-difference" (DD). The charge (or intensity) asymmetry $A_{Q}$ is defined as

$$
A_{Q}=\frac{I^{+}-I^{-}}{I^{+}+I^{-}}
$$

where $I^{+(-)}$is the beam intensity measured during the positive (negative) helicity window. Then the double-difference between two BCMs, e.g. BCMs 1 and 2, is formed as

$$
\mathrm{DD}_{12}=A_{Q}^{\mathrm{BCM} 1}-A_{Q}^{\mathrm{BCM} 2}
$$

If the two BCMs can be considered independent and of the same resolution, their individual resolution can be extracted from the width $\sigma_{\mathrm{DD}}$ of the DD distribution as $\sigma_{\mathrm{DD}} / \sqrt{2}$, where the noise from beam fluctuations will be cancelled. However if there is correlated noise in the measurement of two BCMs, relating to their common electronics or housing, then that noise will also be cancelled in the width of the DD distribution.

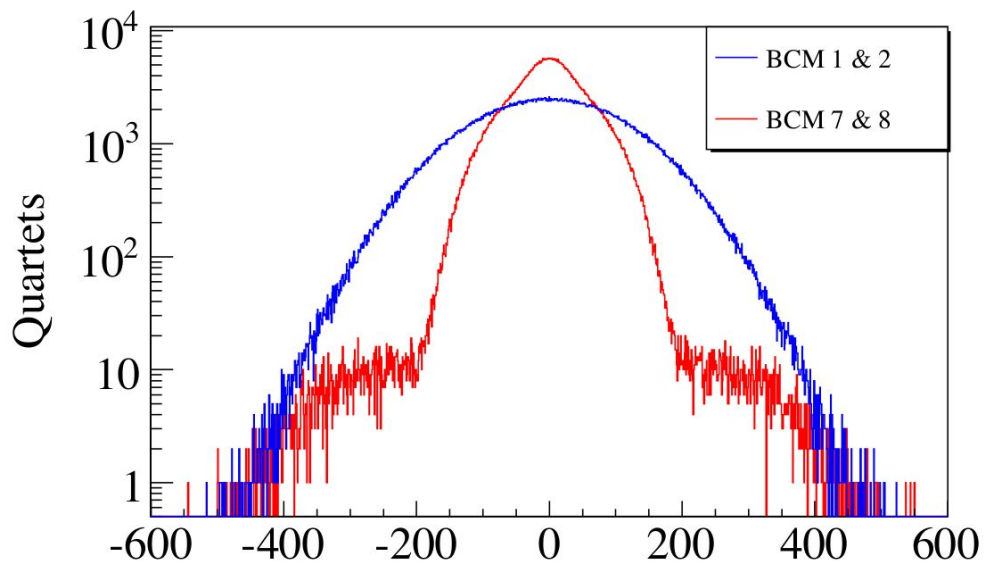

Figure 3.17: DD distribution between BCMs 1-2 (blue histogram) and 7-8 (red histogram) in Run2. The much narrower distribution of $\mathrm{DD}_{78}$ suggests that BCMs 7 and 8 which use improved digital signal processing techniques have much better resolution than 1 and 2 .

Typically the average of BCMs 1 and 2 was used in Run1 for charge normalization, thus achieving cancellation of the monitor noise relative to the individual BCMs. The monitor noise 
injected to the measured asymmetry through charge normalization was $\sigma_{\text {avg BCM1+2 }}^{\text {Run1 }} \approx 60 \mathrm{ppm}$. Charge normalization in Run2 was performed through BCM8. Benefiting from the improved digital signal processing and the higher beam current the monitor noise was significantly reduced, $\sigma_{\mathrm{BCM} 28}^{\mathrm{Run} 2} \approx 40 \mathrm{ppm} 8$

\subsubsection{Beam orbit and energy}

Beam properties such as position, angle and energy, must be measured with high linearity and low noise to correct for false asymmetries arising from correlations of these parameters with the beam helicity. These measurements were provided by stripline Switch Electrode Electronics (SEE) beam position monitors (BPMs) [11] in the injector and in the Hall $\mathrm{C}$ beamline. The BPM consists of two pairs of perpendicular antennae positioned transverse to the beam axis and rotated by $45^{\circ}$ from vertical to minimize effects from synchrotron radiation along the vertical and horizontal planes (Figure 3.18). The antennae are tuned to the RF structure of the beam and the signal of each antenna is proportional to its proximity to the beam orbit. Therefore the central beam position on the rotated axes can be determined from the antennae output signals as 112:

$$
X^{\prime}=\kappa\left[\frac{\left(X P-X P_{o f f}\right)-\alpha_{X}\left(X M-X M_{o f f}\right.}{\left(X P-X P_{o f f}\right)+\alpha_{X}\left(X M-X M_{o f f}\right)}\right]
$$

where $\kappa$ is a mutual inductance-like constant of the BPM characteristic of its geometry, which converts the voltage signals into $\mathrm{mm}$ units, and $X_{\text {off }}$ are the antennae pedestals. $\alpha_{X}$ parametrizes the relative gain of the two wires. For these SEE BPMs the opposite wires are alternately connected to the same RF front-end electronics, which leads to the measured positions $\mathrm{X}$ and $\mathrm{Y}$ being insensitive to changes in the electronics gain $\left(\alpha_{X, Y}=1\right)$.

The typical resolution of an SEE BPM is $\lesssim 0.1 \mathrm{~mm}$ at $1 \mathrm{~Hz}$ in a current range of 1-1000 $\mu \mathrm{A}$ [111. The resolution corresponding to $Q_{\text {weak }}$ quartets of $960 \mathrm{~Hz}$ helicity windows can be calculated from a similar methodology to the DD for the BCMs. Using three BPMs in the drift region upstream of the target, the position from the first two was projected onto the third and a distribution is formed for the difference between the projected and the measured position on the third. The width of that distribution is a measure of the resolution of the BPMs. In Figure 3.19 the BPM resolution is estimated for different values of beam current. For the high currents of $Q_{\text {weak }}$ the BPM position resolution is $0.9-1 \mu \mathrm{m}$.

In a drift region without fields that alter the beam orbit, the position measurement from two successive BPMs can be used to extract the beam angle. The beam position and angle at the

\footnotetext{
${ }^{8}$ While this resolution was perfectly adequate for the $Q_{\text {weak }}$ measurement, it was limited by an observed "noise floor" [110]. The next-generation ultra-precise MOLLER PVES measurement will require improved resolution and understanding of the apparent BCM noise floor. On the other hand the MOLLER specifications for BPM resolution are already met by the $Q_{\text {weak }}$ performance.
} 


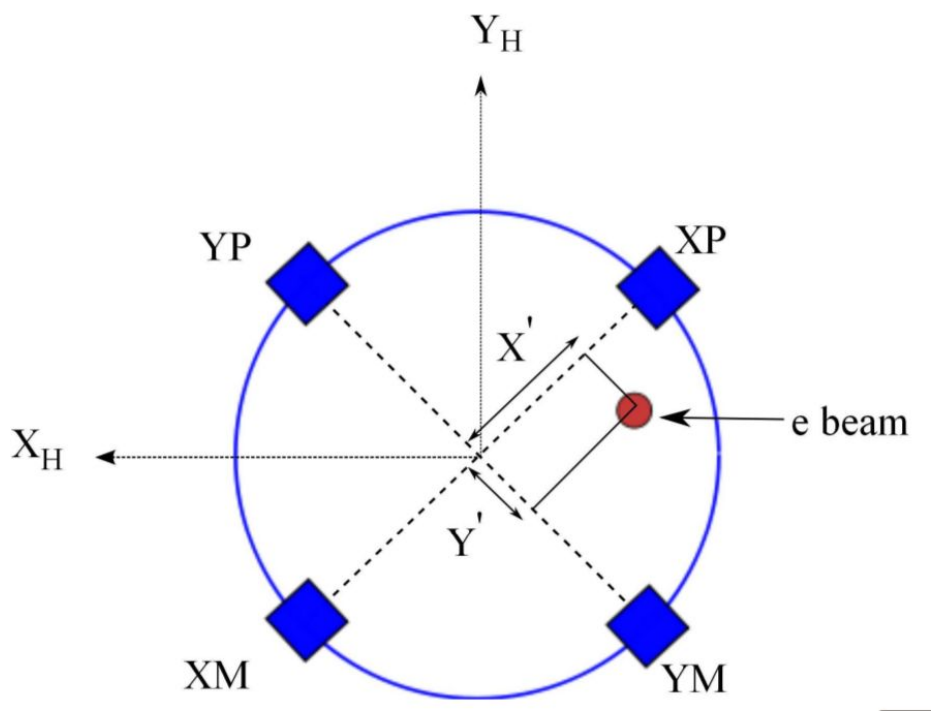

Figure 3.18: Schematic of the antennae of SEE BPMs in the Hall C beamline, rotated by $45^{\circ}$ from the experimental Hall horizontal/vertical directions $\left(X_{H}, Y_{H}\right)$. Reproduced from 113 .

$\mathrm{LH}_{2}$ target is determined from a linear least squares fit of 4 or 5 BPMs in the drift region between $1.5 \mathrm{~m}$ and $10.5 \mathrm{~m}$ upstream of the target. A slow (1 s update) position lock was implemented to maintain the desired beam trajectory at the target, using corrector magnets upstream of the BPMs feeding back on the calculated position at the target.

The BPM located in the region of highest dispersion (typically $4 \mathrm{~cm} / \%$ ) in the Hall $\mathrm{C}$ arc (BPM 3C12) was used for continuous energy monitoring. By effectively using the arc as a spectrometer, the horizontal position measurement of BPM 3C12 is sensitive to its momentum and energy [114. The BPM 3C12 measurement in the arc is combined with the BPMs in the drift region to derive the beam energy at the target, using first order beam transport matrices determined through the OptiM model [115:

$$
\frac{\Delta E_{\text {target }}}{E}=\frac{\Delta X_{3 \mathrm{C} 12}}{411}-\frac{\Delta X_{\text {target }}}{596}+\frac{\Delta X_{\text {target }}^{\prime}}{0.443},
$$

where $\Delta E_{\text {target }}, \Delta X_{\text {target }}$ and $\Delta X_{\text {target }}^{\prime}$ are the helicity-correlated differences in beam energy, position and angle at the target. Any residual dispersion at the target or coupling between beam parameters are higher order effects that are not considered in this first order calculation. In fact residual dispersion and coupling between beam parameters were often significant (Figure 3.20). This was most likely a result of imperfect matching between the beam envelope and accelerating cavities in the injector, as will be discussed in Chapter 4 


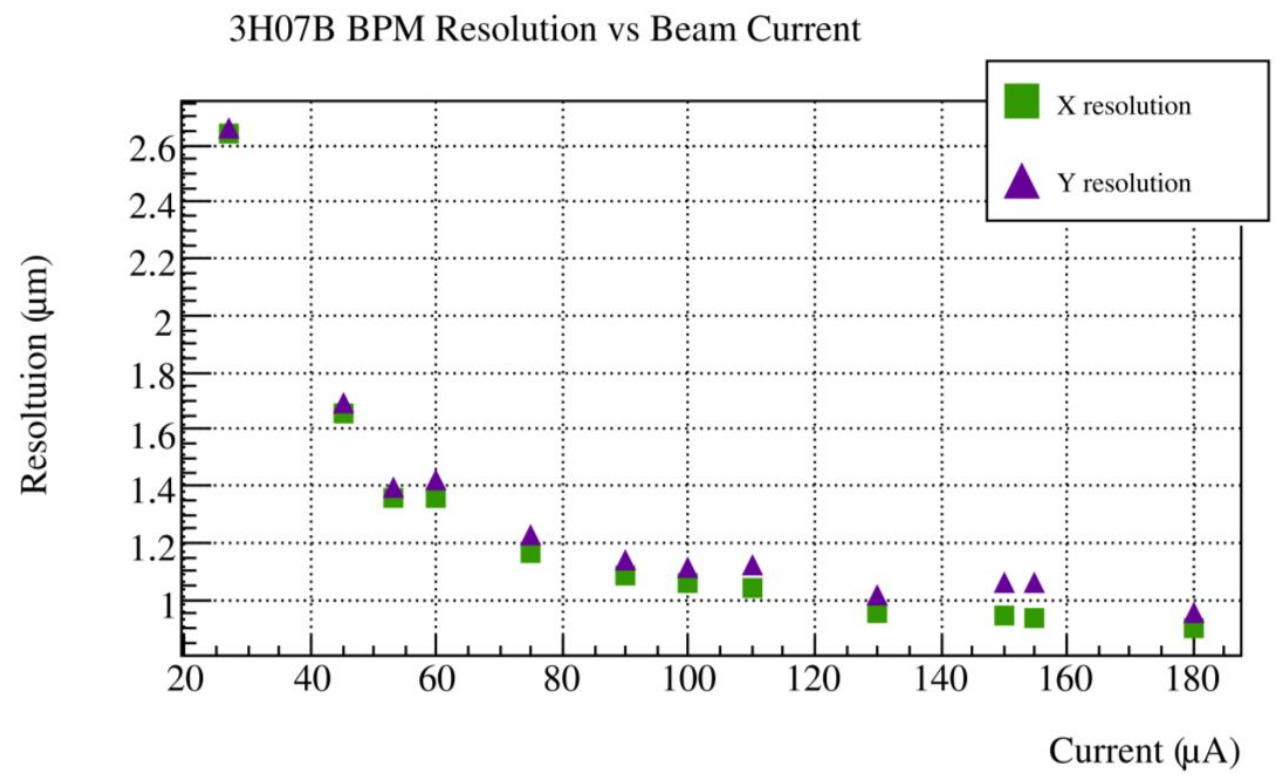

Figure 3.19: The position resolution of an SEE BPM estimated versus beam current, corresponding to quartets of $960 \mathrm{~Hz}$ helicity windows [113.

\subsubsection{Beam halo and profile monitors}

Beam "halo" refers to electrons at large transverse displacements from the beam propagation axis, typically larger than $5 \sigma_{\text {beam }}$ although different definitions can be found in the literature. Some beam halo generation mechanisms include space-charge effects of electrons during bunching, scraping in the beamline or injector apertures, and poor vacuum. Halo was monitored near the beamline upstream of the $\mathrm{LH}_{2}$ target by halo PMT detectors, made of lucite or plastic scintillation material. These monitors were shielded with lead and facing upstream $(75 \mathrm{~cm})$ to a halo "target", a $1 \mathrm{~mm}$ thick aluminum plate with two openings: an $8 \times 8 \mathrm{~mm}^{2}$ square opening and a $13 \mathrm{~mm}$ diameter circular hole (Figure 3.21). Halo rates were calibrated by steering the low current primary beam onto the halo target.

The target could be retracted or positioned so that the beam passes through one of the openings. The beam went through the circular halo target for most of Run2 and provided a continuous measurement of beam halo, which was typically of order 0.1-1 ppm. The circular hole nearly matches the opening of the tungsten plug collimator so the scattered halo rates should be similar, ignoring the effect of the $\mathrm{LH}_{2}$ target. Halo is the part of the beam that scatters preferentially from the beamline and collimators, and is considered to be connected to the very large diffuse background asymmetries that were observed in the USLumi monitors and background detectors. We will refer explicitly to these connections in the study of that background component in Chapter 6 . 


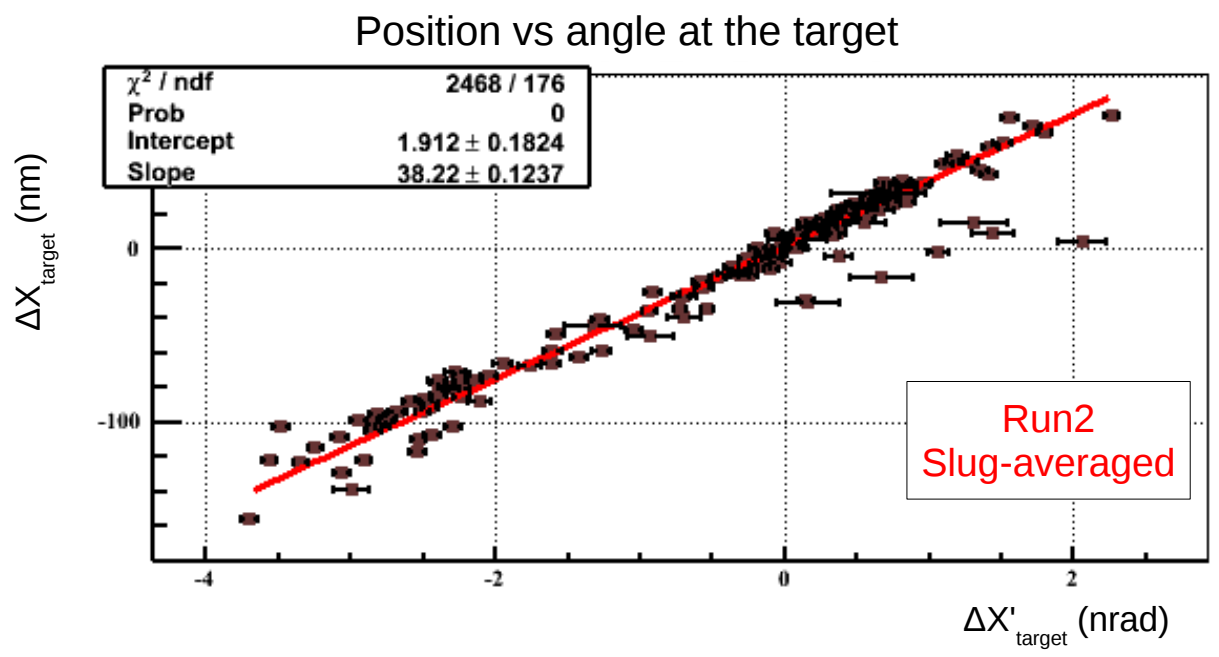

Figure 3.20: Correlation between horizontal position and angle differences in Run2. Data is averaged over 8-hour slugs (IHWP states).

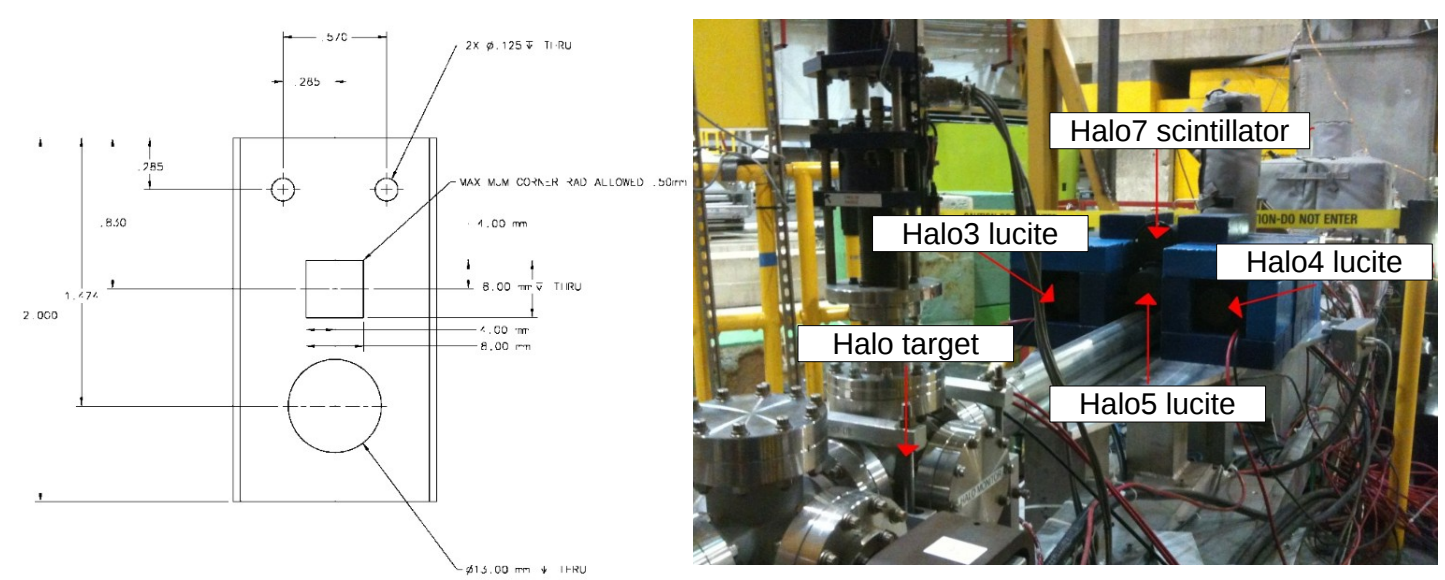

Figure 3.21: Left: Design of the Hall $\mathrm{C}$ halo target. The beam was centered in the either the 13 $\mathrm{mm}$ diameter circular hole or the $8 \times 8 \mathrm{~mm}^{2}$ square hole, and the rates from the aluminum frame were detected from halo monitors, their locations shown on the right downstream of the target.

\subsection{Beam Polarimetry}

Beam polarization is one of the largest systematic corrections for the experiment and must be estimated with a precision at the $1 \%$ level. Two redundant and independent polarimetry systems were employed for the $Q_{\text {weak }}$ measurement. We augmented the existing Hall C Møller polarimeter, which only operates invasively at low currents (typically $\lesssim 2 \mu \mathrm{A}$ ), with a new non-invasive Compton polarimeter which provided continuous polarization measurements during production at $180 \mu \mathrm{A}$. The two polarimeters are described in the following sections.

\subsubsection{Møller polarimeter}

The Hall C Møller polarimeter [116] has provided polarization measurements with $\leqslant 1.5 \%$ precision for many years. These measurements however require dedicated operation at low currents 
$(\lesssim 2 \mu \mathrm{A})$ for approximately $4-6$ hours, usually $2-3$ times per week.

The polarimeter measures the parity-conserving $\vec{e} \vec{e}$ cross section asymmetry, for which the analyzing power is precisely known. The cross section for this purely QED process can be calculated to high precision. A superconducting solenoid polarizes a $1 \mu \mathrm{m}$ thick pure iron target foil along the beam direction. For the ferromagnetic foil magnetized to saturation the polarization of the atomic electrons is known with great accuracy. Scattered and recoil electrons were detected in coincidence using a near-symmetric apparatus (Figure 3.22), with one electron detector aperture slightly smaller than the other to cleanly define the acceptance. Coincidence in a narrow time window allows us to eliminate the dominant background from Mott scattering from the iron nucleus [117. The relatively large acceptance enhances statistical precision and reduces the influence of the atomic motion of the electrons in the target.

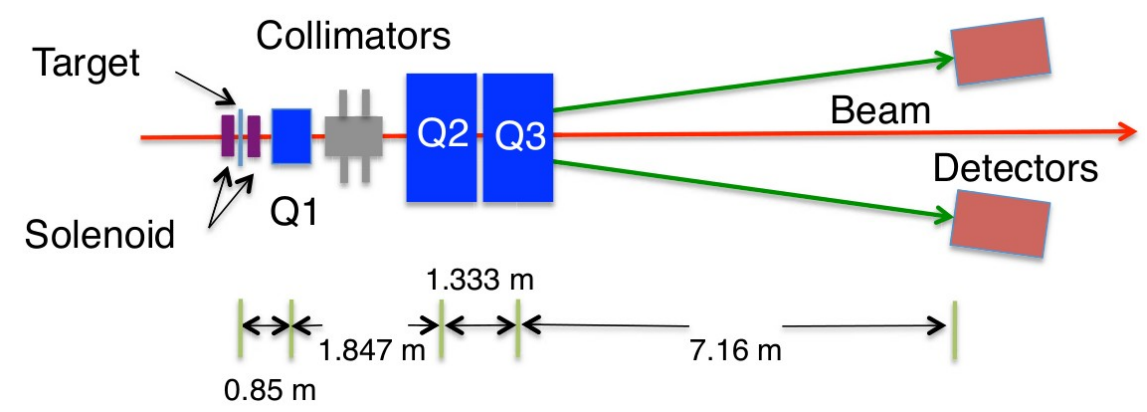

Figure 3.22: Schematic of the Hall C Møller polarimeter. Only quadrupole magnets Q1 and Q2 were used during $Q_{\text {weak }}$.

During Run1, an intermittent short in one of the coils of quadrupole Q3 (Figure 3.22) affected the acceptance and therefore the analyzing power of the polarimeter at the few-percent level. To account for this, the Møller simulation used to provide the polarimeter acceptance was modified to include a correspondingly altered quadrupole field map using a POISSON magnetostatic field generator [118. Hall probes in the quad were used to compare to simulations of the polarimeter response with and without the short. An uncertainty of $0.89 \%$ was added to the Run0 commissioning Møller polarization measurements to account for this effect, and will also be added in the Run1 analysis.

The effect on quadrupole Q3 was absent in the second production run of the experiment, Run2. All systematic uncertainties of the Møller polarimeter during Run2 are listed in Table 3.3 . where the polarimeter achieved a precision of $0.83 \%$. The largest systematic contribution to the uncertainty is from the extrapolation of the Møller measurement at low currents $(\lesssim 2 \mu \mathrm{A})$ to the high currents that are relevant for $Q_{\text {weak }}$ production. Any potential effect was constrained directly with a dedicated measurement at intermediate current $(4.5 \mu \mathrm{A})$ where the Møller polarimeter was found to be in good agreement with the Compton electron detector (Figure 3.23), 
Table 3.3: Systematic uncertainties of the Hall C Møller polarimeter during Run2. Based on analysis from [119.

\begin{tabular}{l|cc} 
Source & Uncertainty & $d A / A(\%)$ \\
\hline Beam position X & $0.5 \mathrm{~mm}$ & 0.17 \\
Beam position Y & $0.5 \mathrm{~mm}$ & 0.28 \\
Beam direction X' & $0.5 \mathrm{mrad}$ & 0.1 \\
Beam direction Y' & $0.5 \mathrm{mrad}$ & 0.1 \\
Q1 current & $2 \%$ & 0.07 \\
Q3 current & $3 \%$ & 0.05 \\
Q3 position & $1 \mathrm{~mm}$ & 0.01 \\
Multiple scattering & $10 \%$ & 0.01 \\
Levchuk effect & $10 \%$ & 0.33 \\
Collimator position & $0.5 \mathrm{~mm}$ & 0.03 \\
Target temerature & $100 \% 0.14$ & \\
B-field direction & $2^{\circ}$ & 0.14 \\
B-field strength & $5 \%$ & 0.03 \\
Spin depolarization & - & 0.25 \\
Electronic dead time & $100 \%$ & 0.05 \\
Solenoid focusing & $100 \%$ & 0.21 \\
Solenoid position (X,Y) & $0.5 \mathrm{~mm}$ & 0.23 \\
High current extrapolation & - & 0.5 \\
Monte Carlo statistics & - & 0.14 \\
\hline & Total: & 0.83 \\
& &
\end{tabular}

and also with the polarization measured from the Compton polarimeter at high current immediately before and after this dedicated measurement. While no evidence exists of an effect from the extrapolation to high currents, a conservative $0.5 \%$ uncertainty is assigned.

\subsubsection{Compton polarimeter}

The $Q_{\text {weak }}$ Colaboration undertook a major effort to develop a Compton polarimeter in Hall C to complement the Møller, based on the successful Hall A Compton polarimeter. The Compton polarimeter provided continuous on-line measurement of the beam polarization at full current $(180 \mu \mathrm{A})$ which increases precision of polarimetry and reduces the necessary time for the invasive Møller measurements.

During production the electron beam is deflected vertically by two dipole magnets to the Compton $(\vec{e} \vec{\gamma} \rightarrow e \gamma)$ interaction region with photons in a moderate-gain laser cavity (Figure 3.24). The $99.9 \%$ circular polarization of the photons in the laser cavity was continuously monitored using reflected light at the entrance mirror of the optical cavity. The unscattered electrons are deflected back to the production beamline by a second pair of dipoles, while the Compton recoil electrons are spatially separated according to their momentum and detected in a multi-plane diamond strip detector. The background spectrum is determined from laser-off data and subtracted. The momentum dependence of the asymmetry is a function of the electron beam polarization, which is extracted from a fit to a QED calculation [120]. The Compton 


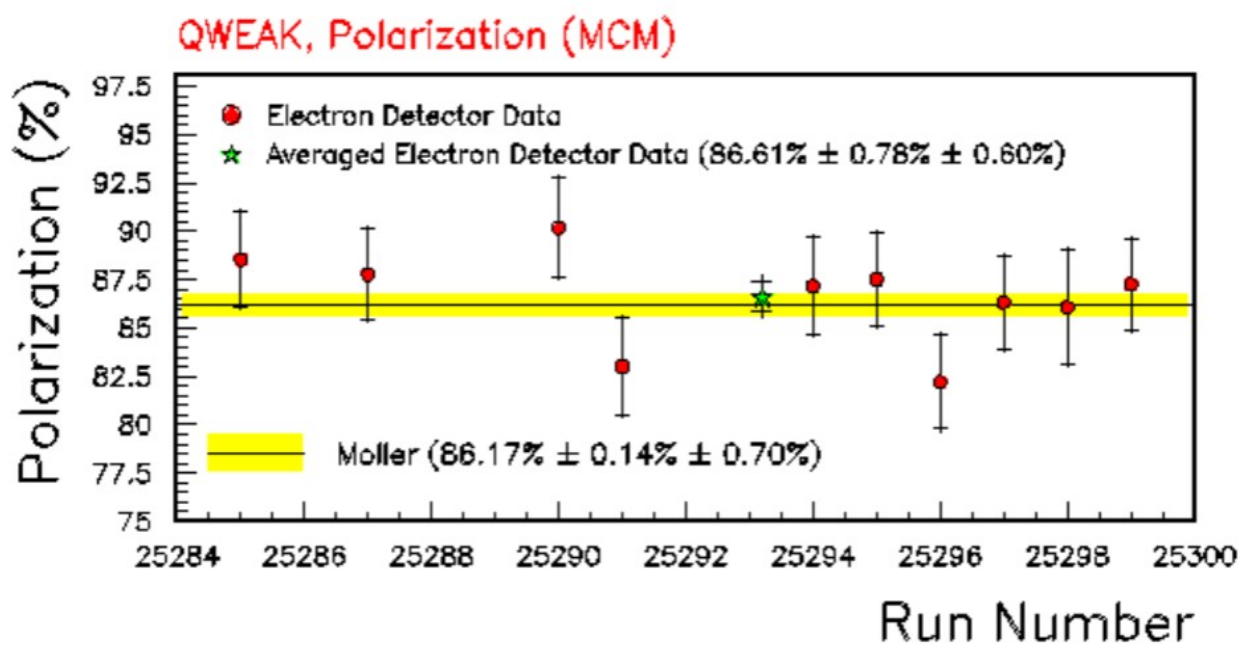

Figure 3.23: Dedicated measurement at intermediate current $(4.5 \mu \mathrm{A})$ where polarization is measured from both the Møller and Compton electron polarimeters. The Møller measured polarization (black line with yellow band) is in good agreement with the polarization measured from Compton (red points, average is marked with the star symbol).

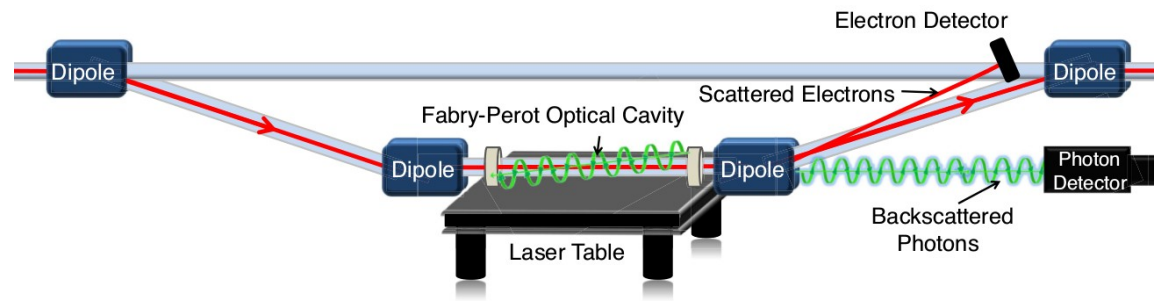

Figure 3.24: Layout of the Hall C Compton polarimeter developed for $Q_{w e a k}$. Electrons are deflected $57 \mathrm{~cm}$ vertically to the Compton interaction region by dipole magnets and then returned to the nominal beamline during normal production. The total length of the dipole chicane is $11.1 \mathrm{~m}$.

back-scattered photons passed through the magnet and were also detected in an array of $\mathrm{PbWO}_{4}$ crystals.

The Compton polarimeter was only expected to provide a relative polarization measurement which would be calibrated by the Møller measurements. However the Compton electron detector was able to provide continuous absolute polarization measurements to an accuracy of better than $0.5 \%$ per hour in Run2. The obtained beam polarization was consistent with the Møller polarimeter results at low currents, and with stability that justifies the intermittent Møller measurements. Beam polarization was expected to change in response to changes in the polarized source, notably laser spot moves and photocathode reactivation (Section 4.4), and Møller measurements were performed immediately before and after such changes.

The systematic uncertainties in the Compton analysis during Run2 are listed in Table 3.4 where the dominant uncertainties are associated with DAQ inefficiencies. A detailed description of these corrections can be found in [121. While commissioning of the Compton polarimeter was only completed half way through Run1, both Compton and Møller polarimeters were operational 
Table 3.4: Systematic uncertainties of the Hall C Møller polarimeter during Run2. Based on analysis from 119 .

\begin{tabular}{l|cc} 
Source & Uncertainty & $\Delta P / P(\%)$ \\
\hline Laser polarization & $0.14 \%$ & 0.14 \\
$3^{\text {rd dipole field }}$ & $0.0011 \mathrm{~T}$ & 0.13 \\
Beam energy & $1 \mathrm{MeV}$ & 0.08 \\
Detector Z position & $1 \mathrm{~mm}$ & 0.03 \\
Trigger multiplicity & $1-3$ plane & 0.19 \\
Trigger clustering & $1-8$ strips & 0.01 \\
Detector tilt (X) & $1^{\circ}$ & 0.03 \\
Detector tilt (Y) & $1^{\circ}$ & 0.02 \\
Detector tilt (Z) & $1^{\circ}$ & 0.04 \\
Strip eff. variation & $0.0-100 \%$ & 0.1 \\
Detector noise & $20 \%$ of rate & 0.1 \\
Fringe field & $100 \%$ & 0.05 \\
Radiative corrections & $20 \%$ & 0.05 \\
DA ineff.correction & $40 \%$ & 0.3 \\
DAQ ineff. pt-to-pt & & 0.3 \\
Beam vert. angle variation & $0.5 \mathrm{mrad}$ & 0.2 \\
Helicity-correl. beam pos. & $5 \mathrm{~nm}$ & $<0.05$ \\
Helicity-correl. beam angle & $3 \mathrm{nrad}$ & $<0.05$ \\
Spin precession through chicane & $20 \mathrm{mrad}$ & $<0.05$ \\
\hline & Total: & 0.58
\end{tabular}

for most of Run2, where the polarization analysis is complete.

\subsection{Timeline and Datasets}

The $Q_{\text {weak }}$ dataset can be separated into three distinct running periods, which are listed in Table 3.5. A different blinding factor has been applied to each of the three periods, allowing them to be treated (and potentially published) separately. The size is quoted in the number of helicity quartets, the basic time unit of forming the asymmetry. Each quartet of helicity windows at $960 \mathrm{~Hz}$ corresponds to $4 / 960 \approx 4.17 \mathrm{~ms}$.

The Run periods are subdivided in ten $\sim$ month-long "Wien" periods, each characterized by a stable setting of the Wien spin manipulation system (Section 4.5) which reverses the electron beam helicity in the low-energy injector. Two of these Wien periods $(\# 8,9)$ were much longer and were broken into two parts. Each Wien is further subdivided into "slug" periods of approximately 8 hours (321 slug periods in total), characterized by the setting of an insertable half-wave plate that reverses the helicity on the polarized source laser table (Section 4.3). Combinations of nominally four neighbouring slugs define a "pitt", which allow formation of asymmetry combinations over opposite states of the insertable half-wave plate. These combinations will often be used to segment the data in the statistical analyses that will be presented in following chapters. The pitt definitions used in this dissertation are defined in Appendix A. 
Table 3.5: The duration and size of the three $Q_{\text {weak }}$ running periods. The size is quoted in number of helicity quartets. The size of the subset where Modulation sensitivities are available is quoted separately. Also listed are the Wien and slug sub-periods that define the slow reversals applied by the experiment. The result extracted in this dissertation is from the Run2 Modulation subset, listed in bold.

\begin{tabular}{|c|c|c|c|c|}
\hline Period & $\begin{array}{c}\text { Dates } \\
\text { (from/to) }\end{array}$ & $\begin{array}{c}\text { Total size } \\
\text { (\#quartets) }\end{array}$ & $\begin{array}{l}\text { Modulation size } \\
\text { (\#quartets) }\end{array}$ & $\begin{array}{l}\text { Wiens included } \\
\text { (Slug range) }\end{array}$ \\
\hline Run0 & $\begin{array}{l}\text { Jan. } 312011 \\
\text { Feb. } 082011\end{array}$ & $\sim 53 \cdot 10^{6}$ & 0 & $\# 0$ (34-40) \\
\hline Run1 & $\begin{array}{l}\text { Feb. } 112011 \\
\text { May } 132011\end{array}$ & $\sim 440 \cdot 10^{6}$ & $\sim 394 \cdot 10^{6}$ & $\begin{array}{l}\# 1(42-58) \\
\# 2(59-80) \\
\# 3(81-98) \\
\# 4(99-116) \\
\# 5(117-136)\end{array}$ \\
\hline Run2 & $\begin{array}{l}\text { Nov. } 242011 \\
\text { May } 172012\end{array}$ & $\sim 870 \cdot 10^{6}$ & $\sim 760 \cdot 10^{6}$ & $\begin{array}{l}\# 6(137-155) \\
\# 7(156-171) \\
\# 8 \mathrm{a}(172-198) \\
\# 8 \mathrm{~b}(199-225) \\
\# 9 \mathrm{a}(226-255) \\
\# 9 \mathrm{~b}(256-306) \\
\# 10(307-321)\end{array}$ \\
\hline
\end{tabular}

Of specific interest to this dissertation is the availability of data where beam parameters were deliberately modulated to measure the sensitivity of detector rates to helicity-correlated beam parameters (Section 5.2). These sensitivities are crucial for the correction of false asymmetries $A_{\text {beam }}$ in our measurement arising from correlation of beam parameters to the beam helicity, which is the subject of Chapter 5 . Also quoted in Table 3.5 is the size of the subset in each Run where Modulation sensitivities are available.

The Run0 dataset was taken over the last few days of commissioning and constitutes only $\sim 4 \%$ of the full dataset. It was from this small data sample that $Q_{\text {weak }}$ released its early result that allowed the first determination of the weak charge of the proton, described in Section 2.4 Several subsystems of the experiment were still unavailable at that time, including the Modulation subsystem. The bulk of the $Q_{\text {weak }}$ dataset is contained in Run1 and Run2, two periods spanning many months and separated by the 6-month scheduled CEBAF "summer-down" of 2011. During the down period many aspects of the experiment were improved based on lessons learned from the Run1 experience. A month-long halt to production occurred in the middle of Run1 due to a vacuum leak on a flange near the end of the beamline. As a result Run2 is by far the largest dataset, containing $\sim 70 \%$ of the total number of quartets. Additionally the average beam current and polarization were higher in Run2, while several systematic uncertainties were better controlled. Therefore Run2 is even more significant compared to Run1 than their relative size in number of quartets would suggest.

In this dissertation we will extract a result for the parity-violating asymmetry from the Run2 dataset only, for two reasons. Firstly, because it is much larger and the different blinding factors 
between Run1 and Run2 do not allow a combined analysis; and secondly, the systematic analyses that will be examined in this dissertation are much more mature for Run2. We will still present an overview of the analysis in Run1.

Further, we will also constrain the analysis in the subset where Modulation sensitivities are available, as these are considered more reliable in the correction of false asymmetries $A_{\text {beam }}$ arising from helicity-correlated beam properties. Therefore the results from this dissertation will be extracted from the Run2 Modulation dataset, constituting approximately $56 \%$ of the full dataset (including Run0)

\footnotetext{
${ }^{9}$ There is a very small amount of Modulation data in Wien10 that has been removed due to different systematic properties. That is reflected in the Run2 Modulation dataset size quoted in Table 3.5 The size of the removed data is so small that it doesn't change the $56 \%$ fraction.
} 


\section{Chapter 4}

\section{Optimization of the Jefferson Lab}

\section{Polarized Source}

The polarized electrons delivered to the experimental halls originate at the Jefferson Lab polarized source, where circularly polarized photons from a laser source impinge on a strained superlattice GaAs/GaAsP doped photocathode. Photoelectrons of the same helicity are then released and accelerated in an electrostatic field. The polarization of the light is controlled by optical components on the source laser table, at the heart of which is an electro-optic device, the Pockels cell (PC). The Pockels crystal varies its birefringence in linear response to an applied electric field. Operating at a quarter-wave voltage of $\sim 2.5 \mathrm{kV}$ and with its fast axis aligned at $45^{\circ}$ from the linear polarization of incoming light, the $\mathrm{PC}$ acts as quarter-wave plate retarder turning the linear light into circular of either right or left helicity, depending on the polarity of the applied voltage.

In this chapter the various components that make up the Jefferson Lab polarized source are described, with emphasis on relevant parameters and technological advances achieved in the polarized source, driven by the ambitious precision goals of the $Q_{\text {weak }}$ experiment. These advances include a much higher rate of helicity reversal $(960 \mathrm{~Hz})$ which required the development of a new high-voltage switch driver circuit. After successive upgrades over many years the source was able to robustly deliver the high current at high polarization required by $Q_{\text {weak }}$. In the low-energy injector a two-Wien spin manipulation system was developed to offer important cancellations of false beam asymmetries $A_{\text {beam }}$. Corrector magnets at the $500 \mathrm{MeV}$ line were recommissioned for $Q_{\text {weak }}$ and were integral in achieving the tight specifications for helicitycorrelated beam properties.

The importance of the polarized source is so great for $Q_{\text {weak }}$ that it is considered a subsystem 


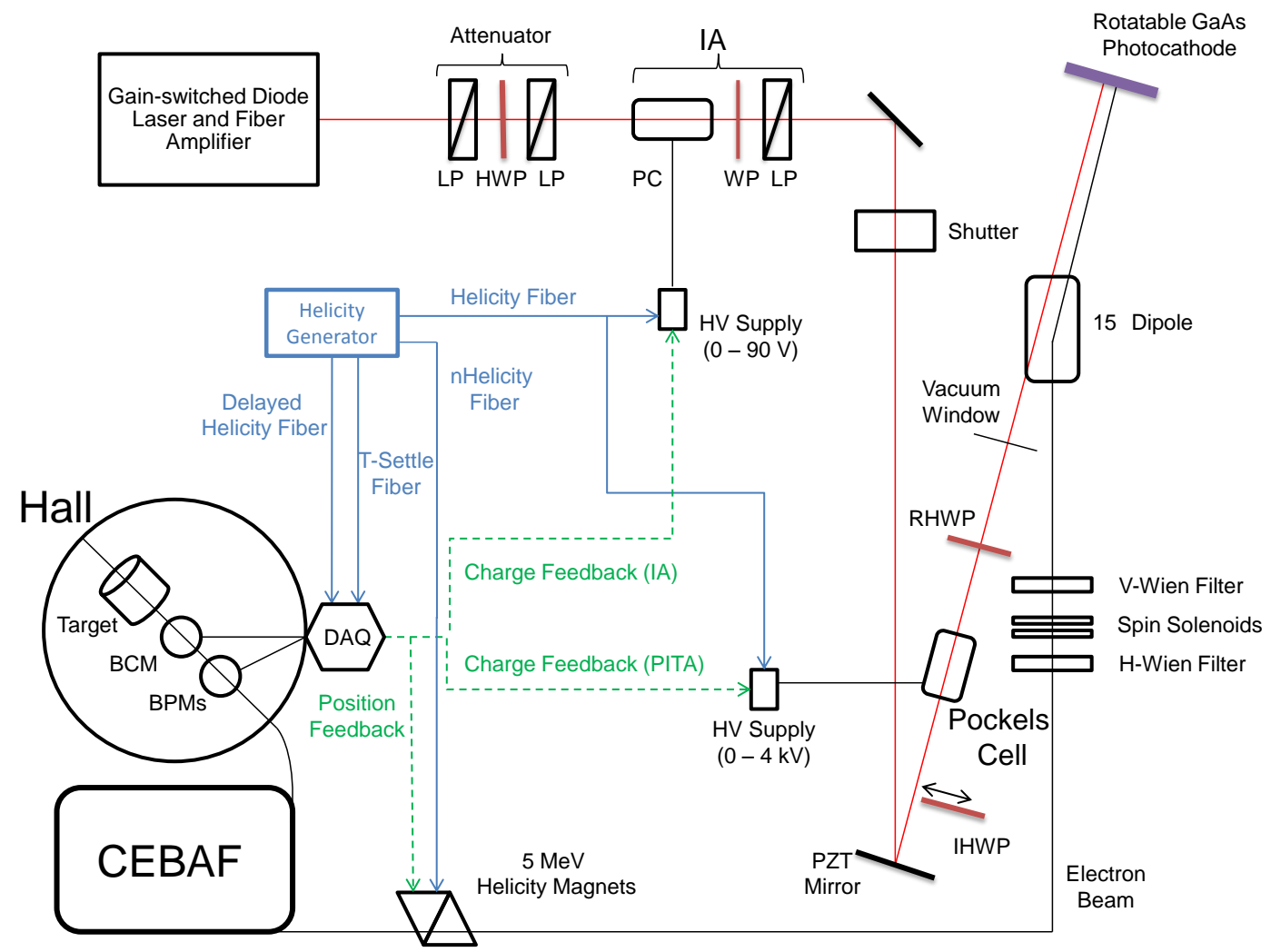

Figure 4.1: Schematic representation of the Jefferson Lab polarized source components used for $Q_{\text {weak }}$.

of the experiment. This author worked with the UVA group towards optimization of the source parameters in preparation and throughout the data-taking period, in close collaboration with scientists from the Jefferson Lab Center for Injectors and Sources. We relied heavily on the knowledge and experience gained from many years of running parity violation experiments, in understanding the sources of beam asymmetries and developing methods to suppress or cancel their effect.

\subsection{Polarized Source Specifications}

Parity violation experiments have more stringent requirements from the Jefferson Lab polarized source compared to typical experiments performed at the lab. Some of these requirements include high beam polarization, high photocathode quantum efficiency with low anisotropy, small phase noise in the laser system, and fast helicity control. Very importantly, parity violation experiments require tight control of differences in beam parameters between the opposite helicity states. These helicity-correlated (HC) differences can result in false asymmetries $A_{\text {beam }}$ in the experimental measurement, often referred to as helicity-correlated beam asymmetries (HCBA).

As the PVES experiment with the most aggressive precision goals to date, $Q_{\text {weak }}$ is also the most demanding in its requirements from the polarized source. With the precision goal of 
Table 4.1: $Q_{\text {weak }}$ specifications for control of helicity-correlated beam properties.

\begin{tabular}{l|ccc} 
Parameter & Max DC value & $\begin{array}{c}\text { Max run-averaged } \\
\text { HC value } \\
(2544 \text { hours })\end{array}$ & $\begin{array}{c}\text { Max noise during } \\
\text { quartet pattern } \\
(4.2 \mathrm{~ms})\end{array}$ \\
\hline Beam intensity & $\Delta E / E \leq 10^{-3}$ & $\langle\Delta E / E\rangle \leq 10^{-9}$ & $\Delta E / E \leq 3 \times 10^{-6}$ \\
Beam energy & $2.5 \mathrm{~mm}$ & $\langle\delta x\rangle<2 \mathrm{~nm}$ & $7 \mu \mathrm{m}$ \\
Beam position & $\theta_{0}=60 \mu \mathrm{rad}$ & $\langle\delta \theta\rangle<30 \mathrm{nrad}$ & $100 \mu \mathrm{rad}$ \\
Beam angle & $4 \mathrm{~mm}$ rastered & $\langle\delta \sigma\rangle<0.7 \mu \mathrm{m}$ & $<2 \mathrm{~mm}$ \\
Beam diameter & $(\sim 100 \mu \mathrm{m}$ unrastered $)$ & $($ unrastered $)$ &
\end{tabular}

measuring $A_{\mathrm{PV}}$ within a few ppb, the systematic uncertainties of HCBA can easily dominate the uncertainty of the measurement if not treated carefully. The error budget of $0.5 \%$ contribution to $\Delta A_{\mathrm{PV}} / A_{\mathrm{PV}}$ from the $A_{\text {beam }}$ correction (Table 3.2 sets the specifications for the level of control required on the run-averaged HC beam parameter differences, listed in Table 4.1 For example a specification for $Q_{\text {weak }}$ is that the run-averaged asymmetry in beam intensity (charge) $\left\langle A_{Q}\right\rangle$ between the opposite helicity states must be kept smaller than $0.1 \mathrm{ppm}$. Similarly stringent specifications at the nanometer scale were set for the beam position, angle, and energy. To achieve that suppression over the entire run, we typically require that $\mathrm{HC}$ beam parameter differences are never larger than order of magnitude from their run-averaged specification.

It is highly non-trivial to achieve this level of control for helicity-correlated differences in beam parameters, but the necessary techniques were already developed by previous PVES measurements, especially by SLAC E158 [122]. The HAPPEX-II Collaboration was very successful at controlling these position differences at the CEBAF source through a combination of carefully selected laser optics components and novel alignment techniques [123, working closely with the electron gun group. In addition, significant work was performed by CASA 1 scientists to maintain the electron beam optics throughout the machine close to design specification, thereby avoiding phase space correlations which might exaggerate intrinsically small helicity-correlated effects (Section 4.8). As a result, helicity-correlated position differences averaged over the HAPPEX-II run were held to $\langle\delta x\rangle<2 \mathrm{~nm}$ and angle differences to $\langle\delta \theta\rangle<1 \mathrm{nrad}$, without active feedback on the beam trajectory. Therefore the $Q_{\text {weak }}$ specifications had already been achieved by previous experiments.

\subsection{Helicity Signal}

The helicity transition was driven by fiber signals in an electrically isolated VME2 2 crate. Helicity Gates are generated with a frequency of $960.015 \mathrm{~Hz}$, or every $1.042 \mathrm{~ms}$, in a quartet pattern

\footnotetext{
${ }^{1}$ The Jefferson Lab Center for Advanced Studies of Accelerators.

${ }^{2}$ VERSA Module Eurocard
} 


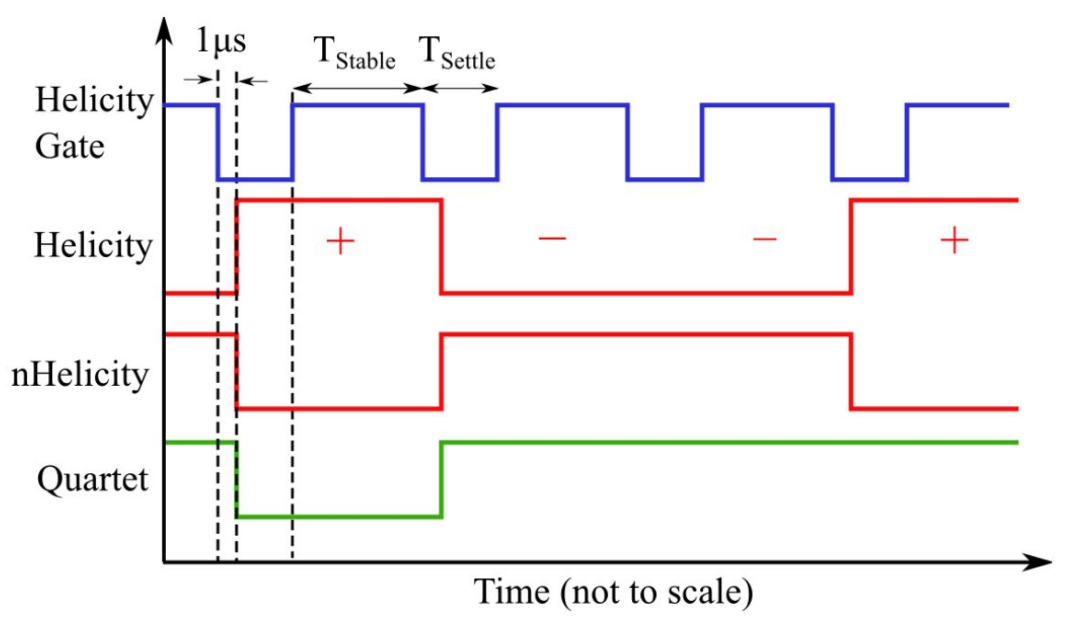

Figure 4.2: The helicity signals that drive the transition in the polarized source.

where the first and fourth gates correspond to the same helicity (+- -+ as shown in Figure 4.2 or -++-$)$. The helicity state of the first gate in each quartet is determined via a 30-bit pseudo-random algorithm. The Helicity signal is used to switch the Pockels cell high voltage $(\mathrm{HV})$ and drive the helicity transition between the two states. For a period of $T_{\text {settle }}=70 \mu \mathrm{s}$ the Pockels cell is allowed to complete the helicity transition by changing its birefringence. For the remaining time $T_{\text {stable }} \approx 972 \mu$ s the helicity is considered stable in every window. However a further gate delay of $\sim 42 \mu$ s was applied as the integrating modules showed a drawdown of the measured values in the period too close to the gate input. The integration period during which data is collected and integrated by detectors and monitors in the experiment lasted for $928 \mu \mathrm{s}$.

Great care was taken to suppress sources of electrical pickup in the experiment by isolating the helicity-correlated reversal signals from cables and ground paths, as these can result in a significant and varying false asymmetry. Feedback through the corrector magnets (Section 4.9.2) was applied though the nHelicity signal, the complement to the Helicity signal. This way the helicity board always drew the same current regardless of helicity state and further protected against any electrical pickup. The helicity state reported to the DAQ was delayed by eight helicity gates, suppressing potential electrical pickup.

Isolation is also important for the helicity-correlated high voltage applied on the Pockels cell in the source laser table (Section 4.3.2 which can act as an antenna. A small mV-level leakage would give rise to a significant false asymmetry, which would show up even in the absence of any electron beam. Searches for helicity-correlation in MD pedestals constrain such effects. These measurements were performed daily, usually as short 5-minute dedicated runs with beam-off, and occasionally 1-hour long runs with higher statistics. HC pedestals over the course of the 
experiment constrain pickup false asymmetries at the $1 \mathrm{ppb}$ level. The applied slow reversals that will be described in this chapter should cancel any residual pickup effects on the measured asymmetry.

\section{Noise suppression}

As discussed in Section 3.6.1, the width $\sigma_{A}$ of the measured asymmetry in the main detector is a fundamental figure-of-merit for the experiment. The quartet pattern and $960 \mathrm{~Hz}$ reversal rate of helicity were chosen with the intent of minimizing noise contributions to $\sigma_{A}$ from target boiling, power line harmonics and other sources. The fast reversal rate makes the measurement much faster than the characteristic time scales of these noise sources and thereby suppressing them. Several helicity gating options were tested and it was found that higher reversal rates were performing clearly better in minimizing $\sigma_{A}$. That was especially true at higher currents and slower frequencies of the hydrogen recirculating pump, where the target boiling contribution is greater [124. On the other hand the improvement at higher reversal frequencies was not so pronounced on a solid carbon target, highlighting the effect on $\mathrm{LH}_{2}$ target boiling.

Note that higher reversal rates offer a trade-off between noise suppression and increase of fractional dead-time, defined as the fraction of each helicity window where we are not collecting data due to the helicity transition and gate delay. The integration period of $928 \mu$ s out of the 1.042 ms window translates to a significant dead-time of $\sim 11 \%$. This is an inherent limitation from going to higher reversal rates, and motivated the developments for faster Pockels cell helicity transition (Section 4.3.3).

The quartet pattern was also aimed at suppression of noise from $60 \mathrm{~Hz}$ harmonics and slow linear drifts. An example is given in Figure 4.3 from a dedicated study where the raster frequency was varied below the helicity reversal frequency of $960 \mathrm{~Hz}$, performed on April 282011 [125]. The asymmetry distribution of the upstream luminosity monitor in octant 5 (USLumi5) is plotted for a $480 \mathrm{~Hz}$ raster frequency, in two different helicity patterns. For standard quartets of 960 $\mathrm{Hz}$ helicity windows, meaning that the asymmetry is formed at a rate of $240 \mathrm{~Hz}$; and for pairs of $960 \mathrm{~Hz}$ windows (manually constructed by this author from neighbouring windows), so the asymmetry is formed at a rate of $480 \mathrm{~Hz}$. For the standard quartets, any slow drifts will affect the positive and negative helicity states equally and therefore cancel in the asymmetry. For the pairs however, the effect of a slow drift (especially one of $480 \mathrm{~Hz}$ ) will depend on the polarity of the pair $(+-$ or -+$)$, resulting in double-peaking and even a large systematic false asymmetry.

This author also examined properties of more complicated patterns. One example is the "octo-quads" pattern, a combination of eight quartets of helicity windows, organized so that the polarity of the first four quartets is opposite to that of the last four. Other than reducing 


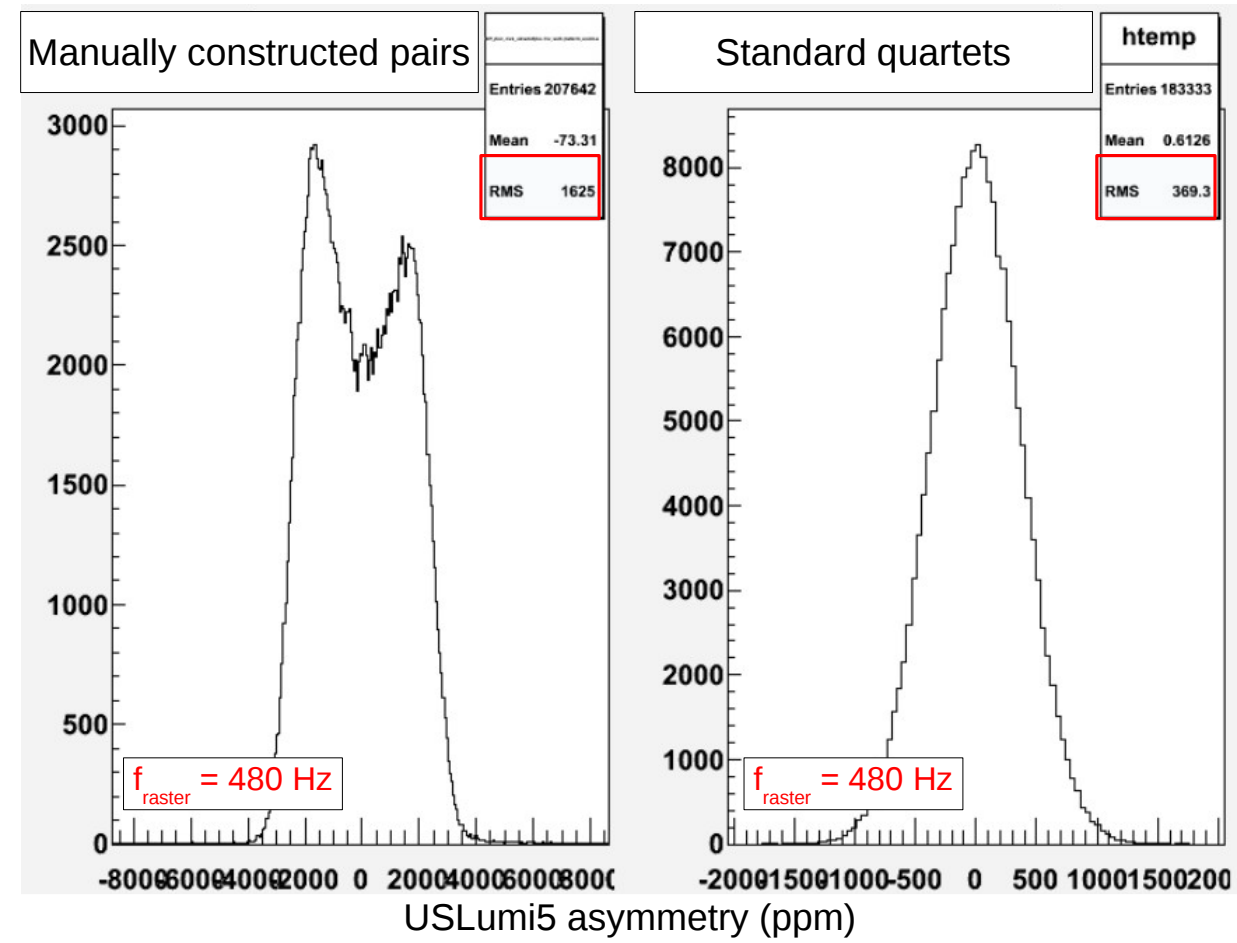

Figure 4.3: The USLumi5 (upstream luminosity monitor in octant 5) asymmetry distribution from standard quartets (right) and from manually constructed pairs (left) of helicity windows, over the same dataset in a dedicated study with a relatively slow $480 \mathrm{~Hz}$ raster frequency. The quartet pattern suppresses noise which gives rise to double-peaking in the asymmetry from helicity pairs. See text for discussion.

the data size by a factor of $q^{3}$, this pattern was also shown through simulations and manually constructed data to have better noise cancellation properties (Figure 4.4). This pattern was however never adopted as we had already been collecting data with the quartet pattern.

\subsection{Laser Table Configuration}

\subsubsection{Laser system}

On the source laser table, the laser light was provided by a gain-switched RF pulsed diode operating at $1560 \mathrm{~nm}$, the seed laser, focused into and amplified by a tapered-stripe travelingwave diode laser amplifier, and then frequency-doubled to $780 \mathrm{~nm}$ in a periodically poled lithium niobate crystal (PPLN) [127].

Three lasers operating at a repetition rate of $499 \mathrm{MHz}$ were used to separately supply beam to each of the three experimental halls. Attenuators comprised of fixed linear polarizers and stepper motor controlled half-wave plates are in each laser beam line. Mirror mounts with

\footnotetext{
${ }^{3}$ Note that we would have $8 \times$ fewer asymmetry formulations, each more precise by a factor of $\sqrt{8}$ relative to quartet asymmetries, therefore statistical considerations are equal. Dead-time is also equal in both cases. The asymmetry pattern only has an effect on noise cancellation.

${ }^{4}$ The MD statistical precision is estimated from the width of the difference between even and odd-numbered bars, in the same fashion as the BPM and BCM resolution.
} 


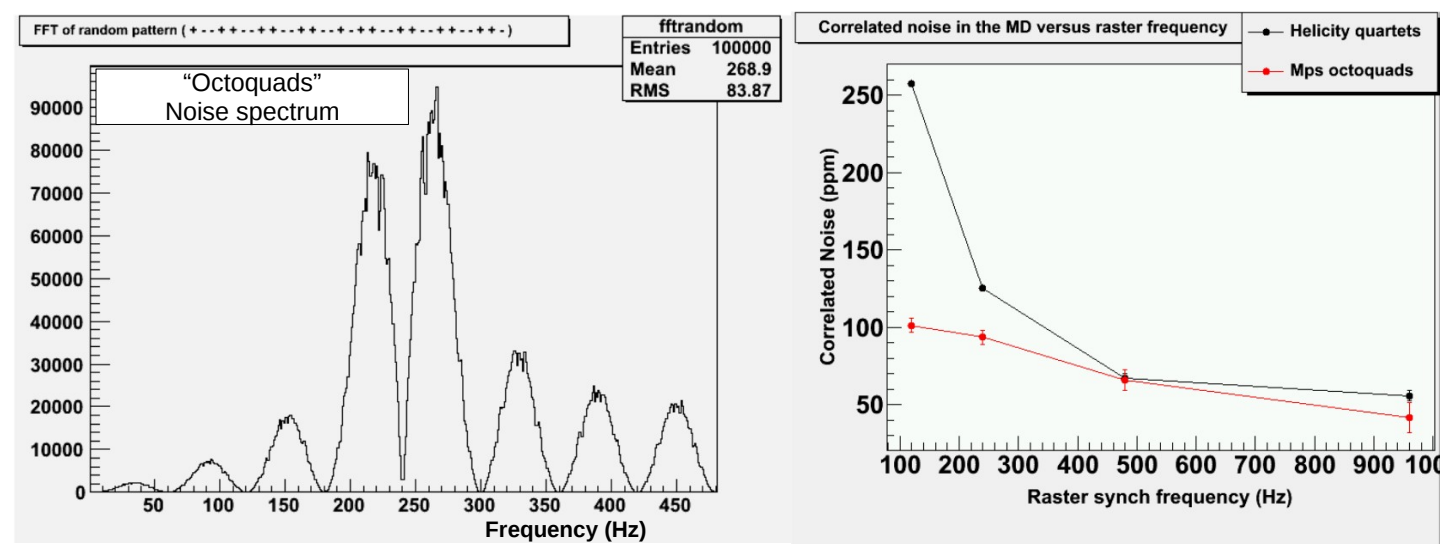

Figure 4.4: Left: Simulation of the FFT frequency spectrum for an "octo-quad" pattern of helicity windows (eight quartets of helicity windows), using a tool that simulates user-selected helicity patterns developed by Richard Jones [126]. Right: "Correlated noise" on $\sigma_{A}$, defined as the remaining noise on the asymmetry measurement after subtracting the BCM monitor noise and the MD statistical precision 4 . The octo-quads pattern performs clearly better when more noise is present at lower raster frequencies, although results are statistically consistent at higher frequencies.

picomotor adjustment screws allow precise alignment of the illuminated spot from each laser on the photocathode. The $499 \mathrm{MHz}$ frequency is synchronized to the frequency of the injector chopping system. As the seed laser is driven by RF power derived directly from the accelerator RF, synchronization and phase stability are automatically obtained without feedback loops. The produced electron beam is also endowed with the RF time structure. The width of the laser pulses was typically 60 to 80 ps FWHM, well below the pulse duration passed by the chopper system.

\subsubsection{Polarization control}

A large number of optical components, many remotely controlled, are located between the lasers and the entrance window to the electron gun vacuum system. Beams from the three lasers are combined to be on a single axis by a combination of beam splitters, polarizers, and dichroic mirrors. The beams were combined using a polarizing beam-splitter for the high-current halls (Halls A and C) and a partially transmissive mirror for the low-intensity hall (Hall B). A consequence of this arrangement was that the $Q_{\text {weak }}$ Hall C beam had opposite polarization to the others. Guided by a series of dielectric mirrors that were minimally sensitive to the laser light polarization, all beams are combined in a beam splitting polarizing cube and directed through a single Pockels cell (PC), which controlled the polarization for all halls.

A PC is a uniaxial crystal which becomes triaxial and behaves like a retardation plate, with its birefringence linearly proportional to the applied voltage along its optical axis. It was mounted with its longitudinal axis along the direction of beam propagation and its fast and slow axes, orthogonal between them, aligned at $45^{\circ}$ to the incoming linear polarization orientation. The 
high voltage applied to the $\mathrm{PC}$ was appropriate so that it functions like a quarter-wave retarder, therefore the light emerged from the PC circularly polarized. The appropriate quarter-wave voltage for a $\mathrm{KD}^{*} \mathrm{I}^{5} \mathrm{PC}$ for $780 \mathrm{~nm}$ light is typically $\sim 2.6 \mathrm{kV}$. Reversing the polarity of the applied high voltage at $960 \mathrm{~Hz}$ would reverse the birefringence of the crystal and therefore the helicity of the light.

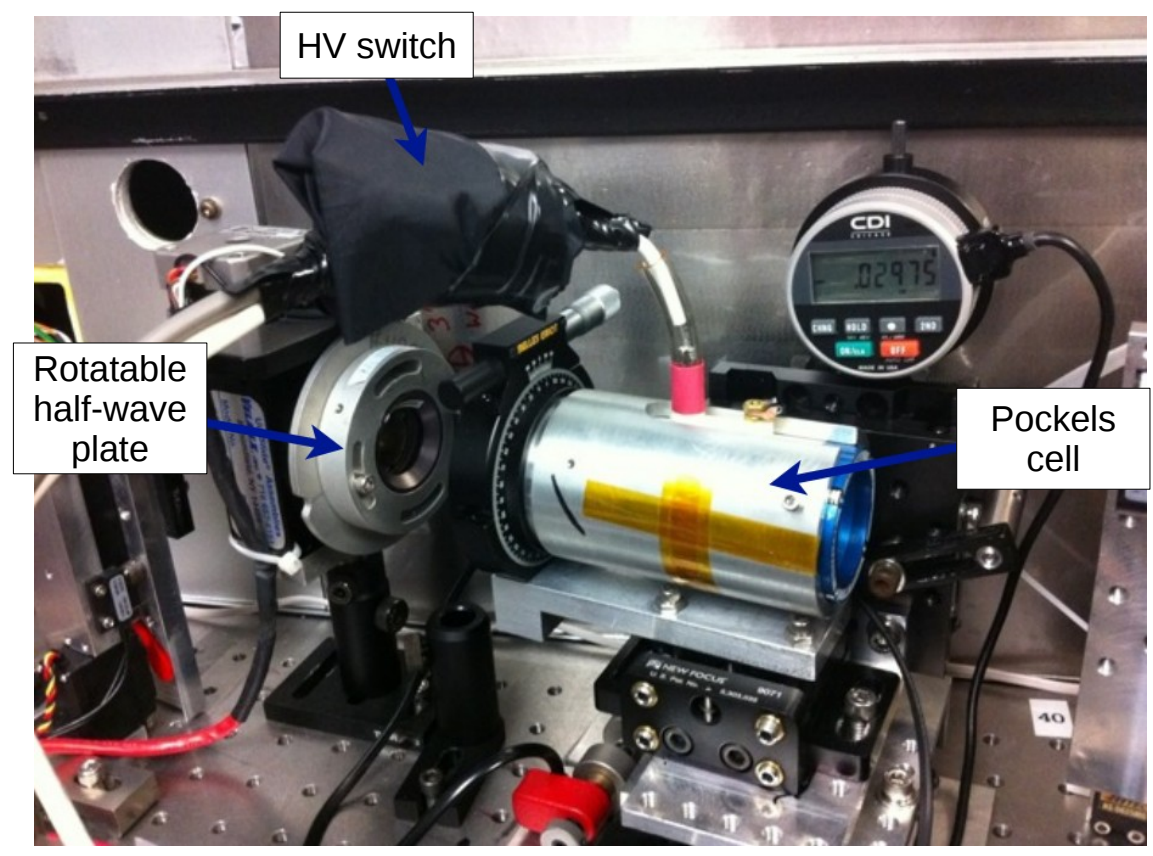

Figure 4.5: Picture of the Pockels cell on the Jefferon Lab source laser table, with the rotatable halfwave plate located immediately downstream. The HV switch driver case holding the optocouplers is placed on the cell and wrapped in tape for optical isolation.

In general the polarization of light emerging from the $\mathrm{PC}$ will not be perfectly circular but rather elliptical in character, with a residual component of linear polarization. Residual linear light from the PC arising from misalignment, crystal imperfections, or deviation in the applied voltage from perfect quarter-wave, can couple to the analyzing power of the cathode and generate false asymmetries (Section 4.6). A rotatable half-wave plate (RHWP) was placed downstream of the $\mathrm{PC}$ in order to rotate the residual linear polarization relative to the analyzing power of the cathode and provide cancellations of our sensitivity to it. In practice a small residual sensitivity to linear polarization was allowed, so that asymmetric shifts in the Pockels cell voltages could be used to counteract effects from downstream elements.

An insertable half-wave plate (IHWP) was placed immediately upstream of the Pockels cell, and its function was to reverse the polarization of the beam without changing the trajectory through the cell. It was alternately inserted and removed approximately every 8 hours, defining a slug period. This is a "slow" helicity reversal (compared to the fast reversal of the PC voltages at $960 \mathrm{~Hz}$ ) that serves to cancel sources of HCBAs that would remain constant under this reversal.

\footnotetext{
${ }^{5}$ The Pockels crystal was potassium dideuterium phosphate, or KD*P.
} 
The two states of the IHWP are referred as "IN" and "OUT".

\subsubsection{Helicity transition}

By construction the Pockels cell is a crystalline dielectric with an electrode on each end, and therefore appears essentially as a pure capacitance of approximately 6-10 $\mathrm{pF}$ to its driver circuit. The helicity transition that is initiated by the Helicity signal will not be complete until this capacitance is "charged", which constitutes dead-time for the measurement. Therefore minimization of the transition time is critical for the FOM and statistical power of the experiment, especially for the much faster $960 \mathrm{~Hz}$ reversal rate. This motivated studies from the source group to vastly reduce the time required to complete the helicity transition from $500 \mu \mathrm{s}$ down to $T_{\text {settle }}=70 \mu \mathrm{s}$.
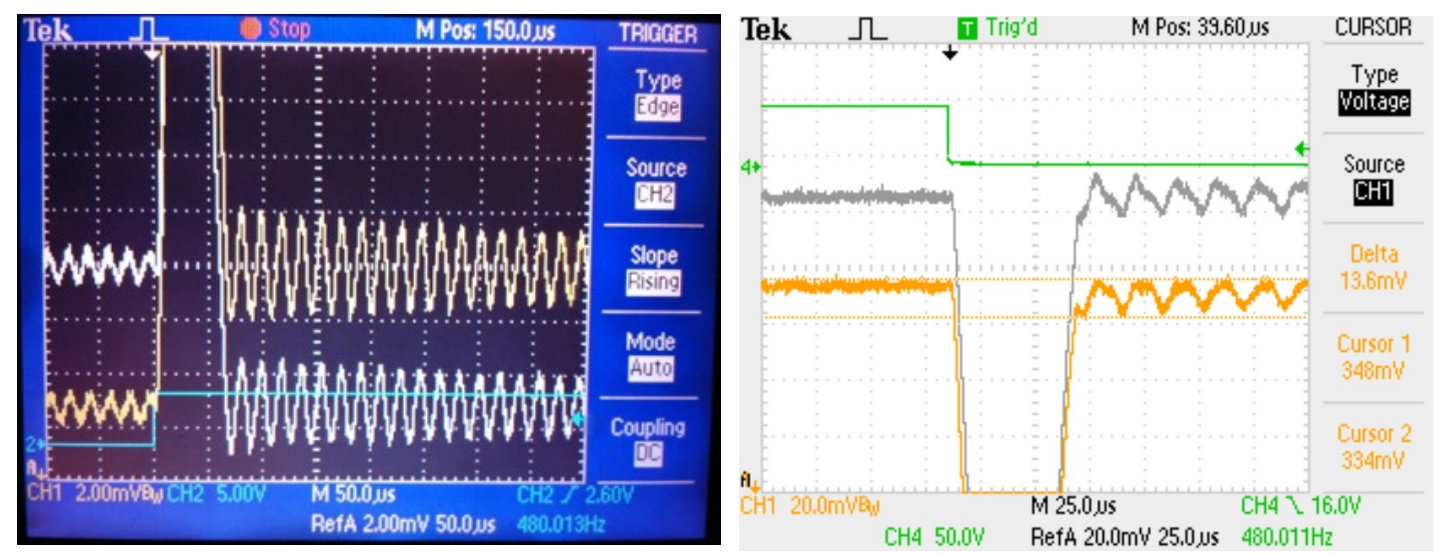

Figure 4.6: Oscilloscope pictures of the signal upon helicity transition, before (left) and after (right) optimization. Oscillation of the transmitted power through an analyzer is referred as "ringing", which can persist for the duration of the helicity window before optimization. The optimized transition on the right has reduced ringing amplitude (approximately 4\%) and is completed within $60 \mu$ s, comfortably within $T_{\text {settle }}=70 \mu \mathrm{s}$.

Another important consideration is the piezoelectric "ringing" effect that appears immediately after the helicity transition. This phenomenon appears as an oscillation in the transmitted laser power through an analyzer (Figure 4.6), corresponding to oscillations of residual linear light in the emerging laser beam; and these in turn arise from oscillations in the crystal birefringence upon the transition. Due to the piezoelectric response of the crystal, the high voltage pulses that drive the transition cause the crystal to physically change shape [128. This mechanical stress acts as the "clapper" of a bell and the cell tends to "ring" for some period after it has been "struck" with a high voltage pulse. At low repetition frequencies this effect can be ignored because there is plenty of time for these acoustic oscillations to damp out, but it becomes an important consideration at our high reversal frequency.

The first HV switch circuit used at JLab was home-made and utilized two MOSFET switches that would ring for approximately $500 \mu$ s before stabilizing. In addition, MOSFET drive circuitry 
requires relatively high current and this caused a voltage droop of the regulated HV power supplies powering the circuitry. This voltage droop introduced a long settling time-constant. This was not necessarily a problem for experiments that ran in "toggle" mode, where helicity states were deterministically alternating, therefore every window looked just like the previous one even with the drooping. But for a pseudo-random helicity pattern, distinct asymmetry values were observed depending on the state of the previous helicity pattern.

After a commercial drive system [129] that also used MOSFET technology produced the same problems, it became clear that ringing was not a problem of the drive circuitry but rather was associated with the piezoelectric properties of the Pockels crystal. Since high-speed MOSFET switches were not desirable, a new PC switch was constructed using "optocouplers", high-voltage optical diodes that reverse-conduct when light is applied via LEDs shining on the diode [130]. A custom TTL drive circuit was designed by John Hansknecht to sequentially apply light to two optical diodes in series, and thereby flip the polarity of the voltage applied to the pockels cell. The original design is shown in Figure 4.7 where the PC can be considered as a purely capacitive device.

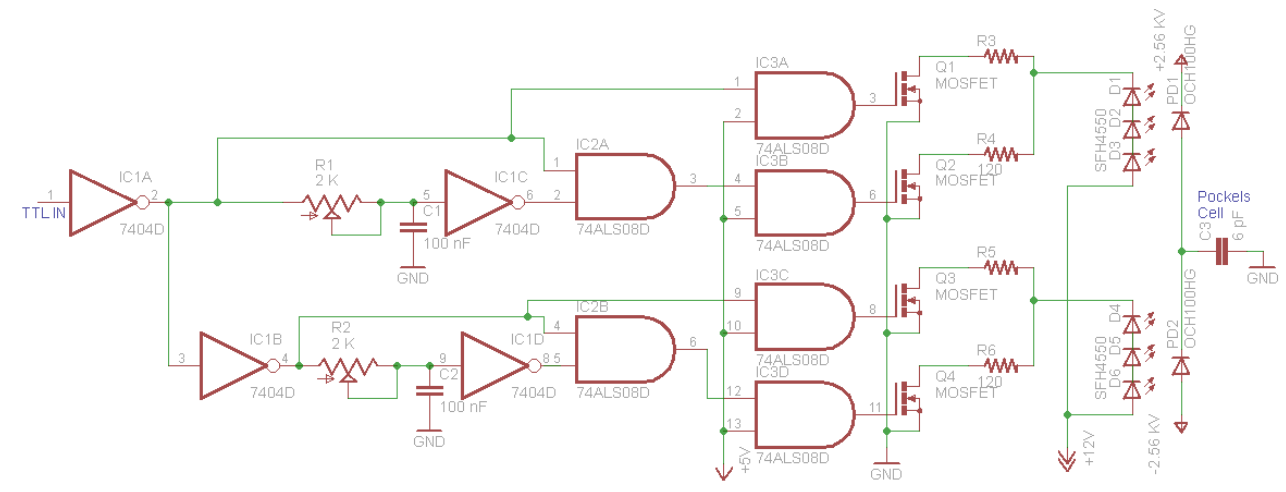

Figure 4.7: An initial version of the PC switch driver circuit, designed by John Hansknecht. The PD1,2 elements are the photodiodes cut out of the optocouplers, while the Pockels cell is seen as a purely capacitive device. The pulse-shaping RC circuits were later replaced by monostable multivibrators.

The optical diodes can provide fast rise/fall times and the capacitance of the switch circuit is very small. Consequently, voltage stability throughout each helicity state is markedly better compared to previous switches. This also improves the electrical isolation to parasitic currents to prevent leakage or radiation of the helicity signal into the electronics.

Note that the commercial OC100HG optocoupler includes both the LED and the optical diode. It was discovered 131 that we could saw off the case of the OC100HG and cut away the LEDs (Figure 4.8), then polish the housing of the diodes and mount our own LEDs. This provides the advantage of being able to mount multiple LEDs in series and therefore achieve faster transition as the PC capacitance will be charged faster by higher currents. It also allows 
to attach the case holding the LED immediately on the PC, shortening the high voltage leads as much as possible to remove their capacitance and stray inductance. Finally the commercial packaging may have had some flaws that destroyed the focusing properties of the original LEDs, and we can get higher current transfer ratios with a custom design 6 . At UVA we improved the design by using six LEDs is series to drive each transition, with a custom LED holding case that maximizes the current transfer ratio.
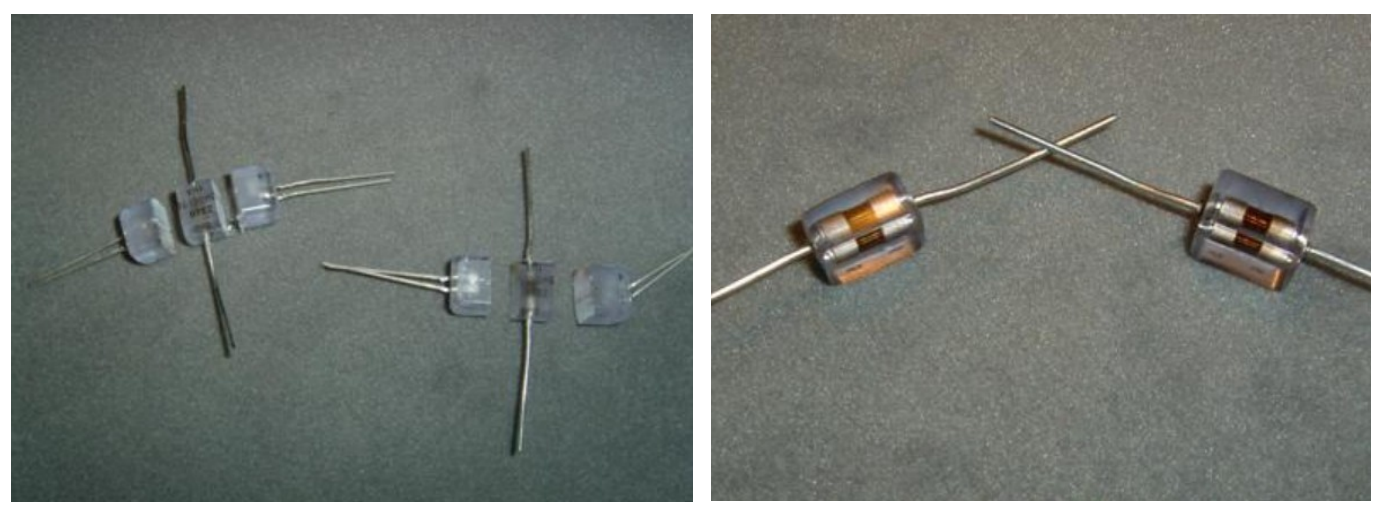

Figure 4.8: Left: Commercial optocouplers OC100HG sawed off to remove the LEDs and isolate the optical diodes. Right: Diodes with polished housing [131.

While driving the transition with higher currents decreases the transition time, a trade-off occurs as the amplitude and duration of ringing increase. The switch design explores higher dimensions of that trade-off by allowing a high-current pulse of short duration that is only applied during the helicity transition. The duration of the extra pulse was of order 10-50 $\mu \mathrm{s}$, adjustable through one-shot monostable multivibrators that eventually replaced the $\mathrm{RC}$ circuit of the design in Figure 4.7. Through a variable resistance we could easily control the duration of the pulse and experiment with different values to find an optimal balance of fast transition and minimal ringing. We observed that by increasing the duration of the driving high-current pulse the transition would become monotonically faster, as expected, whereas the ringing amplitude and duration would go through successive minima and maxima. We hypothesize that these correspond to destructive and constructive interference of the transverse acoustic waves in the cell created by the piezoelectric response to the HV pulse. Eventually we achieved transition times smaller than $60 \mu \mathrm{s}$ (Figure 4.6), well within the $T_{\text {settle }}=70 \mu$ s period and with good ringing properties.

\subsection{Photocathode}

The three drive lasers, one for each hall, illuminate the same location on the strained-superlattice GaAs-GaAsP photocathode [132, 133] with a $5 \mathrm{~mm}$ diameter active area. A focusing lens near

\footnotetext{
${ }^{6}$ The diodes have since become commercially available without the LEDs.
} 
the entrance window to the gun sets the beam size. This lens is mounted on two orthogonal stepper motor controlled translation stages to move the laser spot on the cathode. For $Q_{\text {weak }}$ this lens was changed between Run1 and Run2 to increase the beam spot size on the photocathode from 0.5 to $1 \mathrm{~mm}$ FWHM 7

The strained GaAs-GaAsP superlattice material provides both higher polarization and higher quantum efficiency $(\mathrm{QE})$, defined as the ratio of emitted electrons per incident photon. The superlattice material is created by alternating layers of GaAs and GaAsP only a few nm thick. The dopant induces a strain on the photocathode crystal which breaks the four-fold degeneracy of the valence band found in "bulk" GaAs through spin-orbit interaction, where the splitting is proportional to the strain. This allows spin-selective promotion of electrons to the conduction band using circularly polarized laser light. It is theoretically possible to achieve $100 \%$ polarization from a strained GaAs-GaAsP superlattice cathode, although in practice the energy bands in a GaAs crystal are broadened due to crystalline defects and thermal agitation. The achieved polarization then is proportional to the induced strain and resulting splitting of the valence states. The photocathodes are prepared by activation of the clean semiconductor surface to negative electron affinity using cesium and oxidation, allowing the polarized electrons to exit from the conduction band and be accelerated in an electrostatic field.

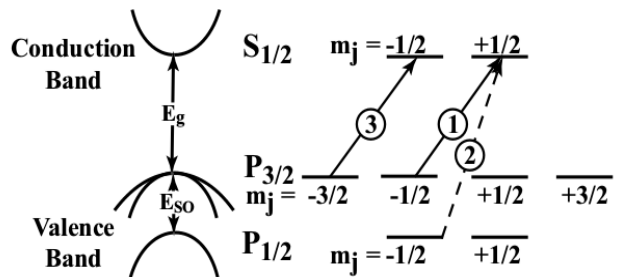

(a) Bulk or unstrained GaAs.

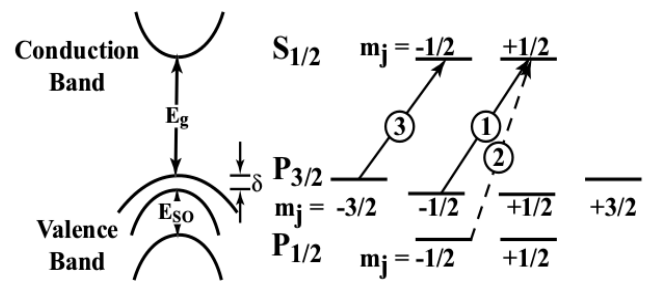

(b) Strained GaAs.

Figure 4.9: Band structure of GaAs. The numbers in circles represent relative transition probabilities for incident circularly polarized light. $E_{g}$ is the band gap energy and $E_{\mathrm{SO}}$ is the splitting of the valence $P_{1 / 2}$ and $P_{3 / 2}$ levels due to spin-orbit interaction. A strain applied by the lattice mismatch induces a splitting $\delta$ of the $P_{3 / 2}$ states and theoretically allows for $100 \%$ polarization. Reproduced from [134].

\subsubsection{Cathode lifetime}

The cathode lifetime is a metric describing the amount of charge that can be extracted from the photogun before the $\mathrm{QE}$ falls to 1/e of its initial value. There is no inherent mechanism of photoemission that degrades the quantum efficiency. QE is degraded by physical processes such as chemical poisoning and affected by the vacuum level within the high voltage chamber, but the dominant mechanism is radiation damage by ion back-bombardment [135]. The electrons emitted from the cathode follow a trajectory toward the electro- static axis, creating ions along

\footnotetext{
${ }^{7}$ Full width at half maximum.
} 
their path. The ions are accelerated back to the cathode with very little transverse deflection. Thus, the signature of ion back-bombardment is a pattern of $\mathrm{QE}$ degradation along a line from the illuminated spot toward the electrostatic center of the cathode, while the $\mathrm{QE}$ of the remaining unilluminated area is unchanged.

Initial QE from a strained-superlattice GaAs/GaAsP photocathode is high $(\sim 1 \%)$ and degrades until an unacceptably low value is reached, typically $\sim 0.2 \%$. At that point the beam spot must be moved on the cathode via the focusing lens to extract electrons from a fresh high-QE location. Spot moves should only require a few minutes, however "resteering" may be needed in the injector to re-establish beam delivery to the halls. Eventually the photocathode will have to be heated and reactivated when QE becomes too low across the active area and there are no good spots left. This involves a full vacuum bakeout followed by cathode cleaning and reactivation, a task that requires $\sim 8$ hours during which no beam delivery is possible. The downtime of a cathode reactivation is disruptive for our statistical goals and for stable beam delivery. Cathode reactivation occurred once in Run1 and once in Run2. Due to the higher beam spot size during Run2 there were fewer available spots on the cathode, but lifetime from each spot was significantly improved (Figure $4.10 \mathrm{z}$ ) as the ion damage was distributed over a larger region.
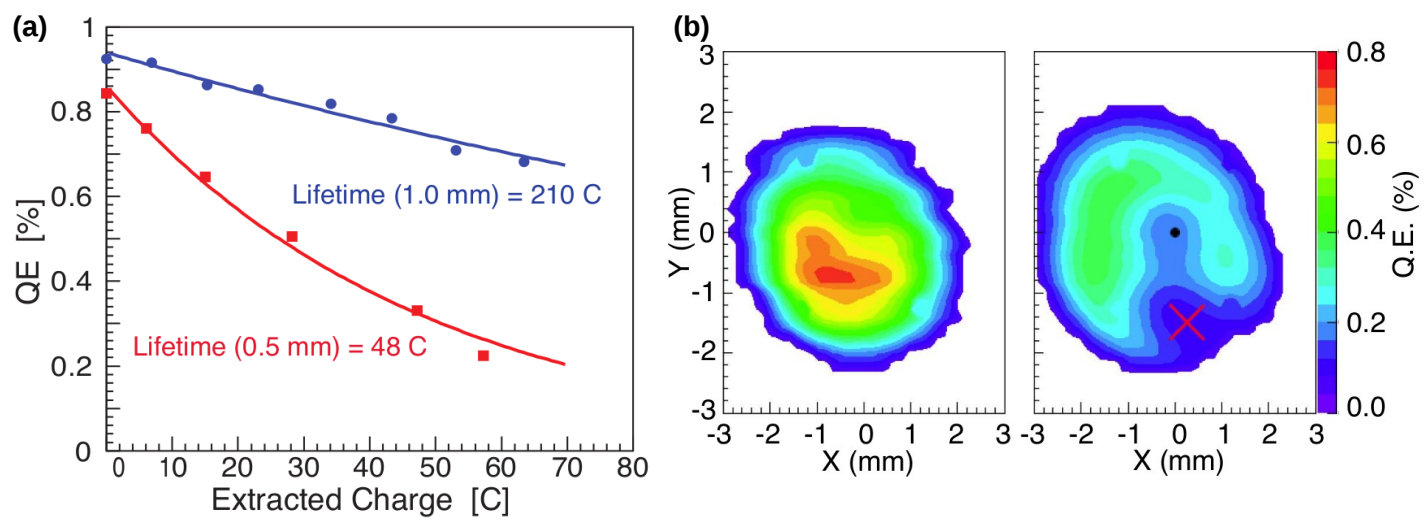

Figure 4.10: (a) The daily measured QE plotted against the charge extracted from the photocathode, during Run1 (red points, $0.5 \mathrm{~mm}$ beam spot size) and Run2 (blue points, $1 \mathrm{~mm}$ beam spot size). (b) QE profile scans before (left) and after (right) a four week period of high current running in Run2. In the figure on the right, the "dot" shows the electrostatic center and the "X" marks the spot from where beam was extracted for delivery to the experimental halls. The active area was $\sim 5 \mathrm{~mm}$ in diameter and the laser spot size was $1 \mathrm{~mm}$ FWHM.

When both high-current halls $\mathrm{A}$ and $\mathrm{C}$ were receiving electrons the average current from the gun exceeds $200 \mu \mathrm{A}$. During Run2 with $Q_{\text {weak }}$ running consistently at $180 \mu \mathrm{A}$, the highest ever instantaneous amounts of current were extracted from the CEBAF cathode, up to $\sim 280 \mu \mathrm{A}$. At these conditions $\mathrm{QE}$ degrades much faster than before and we can operate for approximately a week before the QE becomes too low and the laser beams must be moved to a fresh location on the photocathode.

A new gun design was developed in preparation for the high current requirements of $Q_{\text {weak }}$, 
employing a compact, tapered ceramic insulator that extends into the vacuum chamber [136]. This gun geometry is commonly referred to as an "inverted" gun design, a reference to the first such implementation at SLAC [137]. The inverted insulator design helped to eliminate field emission by reducing the amount of metal biased at high voltage and increasing the distance between biased and grounded parts of the photogun. The gun has improved vacuum at the photocathode to minimize QE decay associated with ion back-bombardment. The elimination of electrons originating from the large-radius region of the photocathode was a very significant improvement.

Quantum efficiency profiles are obtained by scanning a drive laser beam across the active area of the photocathode using the scanning lens and measuring the emitted current. The damage pattern observed during the experiment is shown in Figure 4.10b, before and after a four week period of high current running. QE degradation is most severe at the illuminated area and along a trench towards the electrostatic center, a characteristic pattern of ion back-bombardment.

\subsubsection{QE anisotropy}

The amount of strain in the active layer of the cathode varies in direction with respect to the crystalline lattice. When linearly polarized light illuminates the strained GaAs photocathode, the $\mathrm{QE}$ shows a dependence on the azimuth angle of the polarization plane [138, i.e. the $\mathrm{QE}$ is anisotropic. This anisotropy provides an analyzing power for residual linear polarization when the circular polarization is imperfect. The typical analyzing power for the superlattice cathode is $3-4 \%$, which is an improvement over a typical $12 \%$ for a strained-layer cathode. Through the rotation of the RHWP element we attempt to provide equal QE for the orthogonal residual linear polarization in each helicity state and suppress resulting HC differences (Section 4.7). Higher order effects arise from polarization gradients in the beam and spatial variations of the cathode QE and its anisotropy.

\subsection{Injector Spin Manipulation System}

The $4 \pi$ spin manipulation system was installed during Summer 2010 in the Jefferson Lab injector to meet $Q_{\text {weak }}$ requirements. It consists of two orthogonal Wien "filters" (spin manipulators) separated by two solenoid magnets. A Wien filter is a device with static electric and magnetic fields perpendicular to each other and to the velocity of charged particles passing through it. Charged particles traversing the Wien filters should have their spin rotated in the plane of the electric field without any orbit deflection. The optical focusing depends on the square of the magnetic field, to first order, and should also in principle remain unaffected during spin 
manipulation.

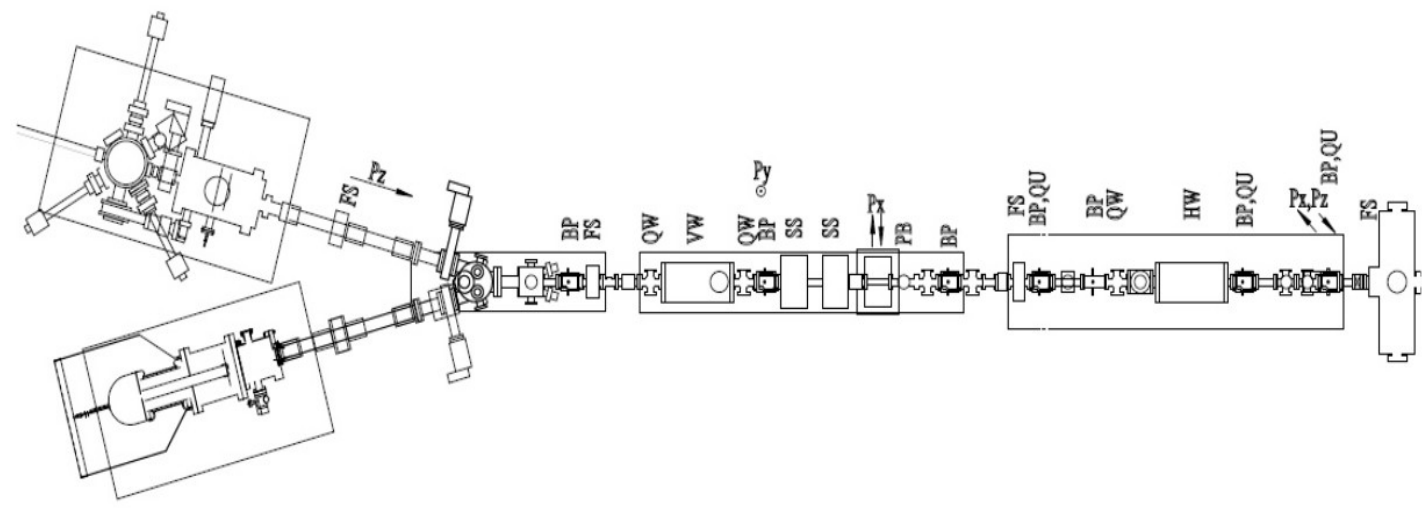

Figure 4.11: Schematic of the injector design with the electron beam leaving the load-lock inverted photogun (top) with longitudinal polarization $\left(P_{z}\right)$, followed by focusing (FS) and spin solenoids (SS), vertical (VW) and horizontal (HW) Wien filters, quadrupoles (QW,QU), a buncher (PB) and beam position monitors (BP). The electron polarization may be rotated vertically by VW and then either "left" or "right" $\left(P_{x}\right)$ by SS for a slow spin reversal. The second Wien filter HW rotates the polarization in-plane to compensate for precession during transport.

The system is used to set the optimal launch polarization angle of the electrons in order to compensate for the in-plane spin precession resulting from beam transport through the CEBAF arcs and hall transport lines, thus ensuring fully longitudinal polarization at the experimental target. Imperfections in the launch angle can result in residual components of transverse polarization, which give rise to a parity-conserving false asymmetry $A_{T}$ (Eq. 3.3 through the two-photon exchange term in the elastic $\vec{e} p$ scattering process [139], referred as beam normal single spin asymmetry. The asymmetry of this component is of order 5 ppm, more than an order of magnitude higher than $A_{e p}$ at $Q_{\text {weak }}$ kinematics. Therefore it is very important to minimize the presence of transverse polarization through the injector spin manipulation system. A Mott polarimeter in the $5 \mathrm{MeV}$ region was used to calibrate the Wien system and maximize longitudinal polarization, and confirming with the Hall $\mathrm{C}$ polarimeters. Conversely the spin manipulation system can also be used to deliver fully transversely polarized beam (vertical or horizontal) in the experimental hall for dedicated studies of the $A_{T}$ asymmetry.

The injector spin manipulation system was also employed as a slow helicity reversal at the time scale of a month, by flipping the electron beam helicity with respect to the Pockels cell voltages via the second Wien filter (Figure 4.11). This defines a "Wien" periods listed in Table 3.5 In theory the reversal on the spin of the electron beam can be applied without affecting any $\mathrm{HC}$ beam properties, thereby cancelling the effect of all $\mathrm{HC}$ beam differences out of the polarized source, including higher-order effects that are impossible to measure. In practice however the spin reversal affects the beam envelope and requires resteering the beam through the injector which alters HC beam properties, therefore a perfect reversal was not achieved. Still, having another slow reversal to complement the IHWP is preferable, as the two can be sensitive to 
different classes of false effects and will allow for complementary cancellations and tests, as we will see in Section 7.1 ,

\subsection{Generation of False Beam Asymmetries}

In an ideal parity-violating electron scattering experiment, the electron beam would be identical for the two helicity states. In practice however, imperfections in the laser optics system that controls the polarization at the source will produce some level of coupling of the helicity state to beam properties. Such correlations to helicity can be dangerous to the experiment as they can give rise to false asymmetries $A_{\text {beam. }}$. In this section we describe the various methods of generation of $\mathrm{HC}$ differences in the polarized source.

\subsubsection{Generation of intensity (charge) asymmetry}

Ideally the retardation induced by the PC on the laser light is exactly $\pi / 2$ (quarter-wave) and results in perfect circular polarization. In practice however the retardation induced by the PC is not perfect. Following the prescription of [134] and the treatment of [140], the phase shift introduced by the $\mathrm{PC}$ can be expressed as

$$
\delta^{\mathrm{R}}=-\left(\frac{\pi}{2}+\alpha\right)-\Delta ; \quad \delta^{\mathrm{L}}=+\left(\frac{\pi}{2}+\alpha\right)-\Delta,
$$

where $\delta^{\mathrm{R}(\mathrm{L})}$ refers to the phase shift induced to the right (left) helicity light. The $\alpha$ and $\Delta$ phases parametrize deviations from perfect $\pi / 2$ retardation and they are referred to as symmetric and antisymmetric phase shifts, respectively. Both residual phase shifts will turn the circular polarization into elliptical by creating a component of residual linear polarization.

A residual $\alpha$ phase shift is symmetric in that it will induce residual linear polarization that will be along the same axis for both helicity states, i.e. the two states will have symmetric polarization ellipses with the same minor and major axes. A residual $\Delta$ antisymmetric phase shift on the other hand will induce residual linear polarization along complementary axes for the two helicity states, with interchanged minor and major axes (Figure 4.12).

When the polarization ellipses are propagated through an asymmetric transport, such as through a system with an analyzing power, a non-zero $\Delta$ phase will induce an intensity asymmetry while a symmetric $\alpha$ phase will not. The asymmetric transport can be provided by an analyzer with transmission coefficients $T_{x^{\prime}}$ and $T_{y^{\prime}}$ along some axes $x^{\prime}$ and $y^{\prime}$. The resulting charge asymmetry will depend only on the $\Delta$ phase and can be expressed as

$$
A_{Q}=-\frac{\epsilon}{T}\left[\Delta \cos \left(2 \psi-2 \psi^{\prime}\right)\right]
$$




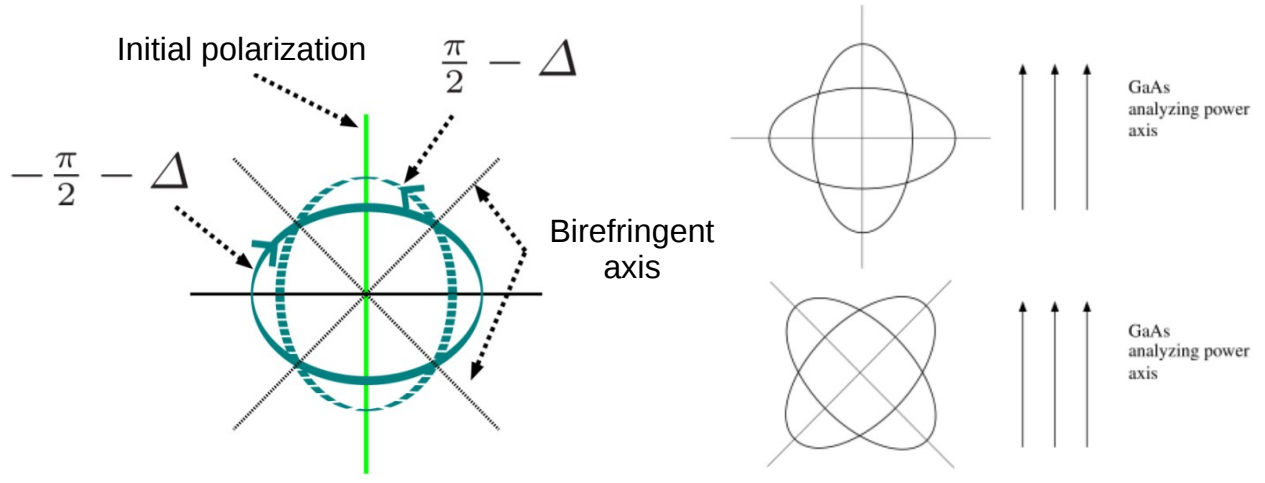

Figure 4.12: Left: An antisymmetric $\Delta$ phase will result in polarization ellipses with interchanged minor and major axes for the two helicity states. Right: The rotatable half-wave plate can be used to rotate the antisymmetric residual linear polarization to minimize the false asymmetry arising from the interaction with an asymmetric transport from the cathode analyzing power.

where $\epsilon=T_{x^{\prime}}-T_{y^{\prime}}$ and $T=\left(T_{x^{\prime}}+T_{y^{\prime}}\right) / 2$. The angle difference $\left(\psi-\psi^{\prime}\right)$ is the angle subtended between $x^{\prime}$ and an axis of the polarization ellipses, so that $\psi$ is the angle between $x^{\prime}$ and an arbitrary reference axis which can be taken as the horizontal.

Here it is assumed that $\Delta \ll 1$ and $\alpha \ll 1$ which allows the small-angle approximation, retaining only first-order terms in phase shifts [134. The quantity $\epsilon / T$ is what we have referred to as the analyzing power, and $x^{\prime}, y^{\prime}$ are the corresponding analyzing axes. Notice that if the polarization ellipses are rotated so that $\psi=45^{\circ}$ then from Eq.4.2. we get zero charge asymmetry. In fact this is a method of cancellation of false asymmetries that the RHWP is designed to provide, as illustrated in Figure 4.12 .

Other than the PC antisymmetric phase, relative rotation of the polarization ellipses can also be created on the laser table by imperfect half-wave retardation of the RHWP, as well as any residual birefringence introduced by the vacuum window or any other element downstream of the RHWP. All these effects will couple with the analyzing power, typically provided by the photocathode, to give rise to a charge asymmetry. As expressed in [134]:

$$
A_{Q}=-\frac{\epsilon}{T}[\underbrace{\beta \sin (2 \rho-2 \psi)}_{\text {Offset }}+\underbrace{\gamma \sin (2 \theta-2 \psi)}_{2 \theta}+\underbrace{\left(\Delta-\Delta^{0}\right) \cos (4 \theta-2 \psi)}_{4 \theta}]
$$

All angles in the above equation are defined with respect to a common reference axis, which can be taken arbitrarily as the horizontal. The parameter $\beta$ is a small phase shift induced by the vacuum window or any other element between the RHWP and the analyzer, and $\rho$ is the corresponding orientation angle of that element. $\gamma$ is the retardation due to the RHWP's deviation from perfect half-wave retardation, and $\theta$ is the angle subtended by the RHWP fast axis. The last term describes the charge asymmetry arising from the PC asymmetric phase shift as in Eq. 4.2 but expressed in terms of $\theta$, and with the added effect of an asymmetric phase 


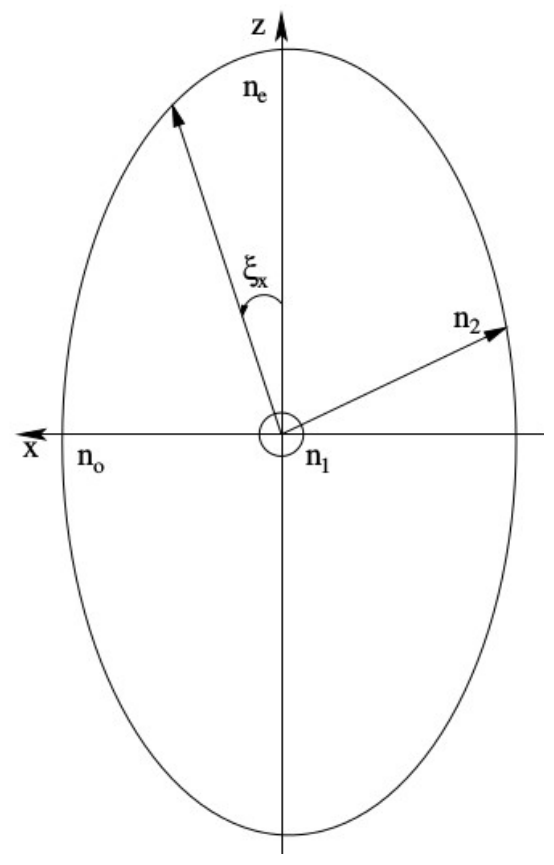

Figure 4.13: A non-zero horizontal angle of incidence $\xi_{x}$ with respect to the optic axis $z$ results in different indices of refraction $n_{1}, n_{2}$ experienced by the electric field in the two planes transverse to propagation. In the $x y$ plane (perpendicular to the page) the index of refraction is $n_{1}=n_{o}$, while in the $x z$ plane $n_{2}$ is a combination of the ordinary and extraordinary indices. Reproduced from [140].

shift $\Delta^{0}$ arising from residual birefringence due to latent stress in the crystal.

The three terms in Eq.4.3 are referred to as offset, $2 \theta$ and $4 \theta$, depending on the factor multiplying the RHWP angle $\theta$ in the trigonometric function. All effects included in Eq.4.3. are identified as polarization effects, as they depend on the polarization of the laser light. Note that under the insertion of the IHWP upstream of the PC, which provides a slow reversal of the sign of the $A_{e p}$ "physics" asymmetry with respect to the $\mathrm{PC}$ voltages, all polarization effects will similarly change sign. This means that polarization effects mimic the physics asymmetry under IHWP insertion, and therefore will not be cancelled by this reversal.

Charge asymmetry may also arise from misalignment of the Pockels cell. When the light direction of propagation makes a small angle with the PC optic axis due to angular misalignment of the $\mathrm{PC}$, its electric vector will experience different indices of refraction in the two transverse directions, as shown in Figure 4.13. This results in a retardation offset from perfect quarter-wave that will give rise to a charge asymmetry. The charge asymmetry arising from such misalignment is referred as a "Skew/Paschke" effect and is treated in detail in [140].

\subsubsection{PITA scans}

Via the electro-optic effect the Pockels cell induces a phase shift that is proportional to the applied voltage. We can induce an antisymmetric phase shift $\Delta$ by adjusting the PC voltages 
anti-symmetrically for the two helicity states, i.e. increasing the applied voltage for one state and reducing it for the other. In this manner we can give rise to a charge asymmetry $A_{Q}$ that will be proportional to the antisymmetric voltage offset $\Delta V$ :

$$
A_{Q}=-k(\psi)(\Delta V)
$$

The antisymmetric voltage offset accesses effects that are historically referred to as polarizationinduced transport asymmetry (PITA) 141. The PITA slope $k(\psi)$ is a very important parameter that characterizes the response of the optical system to a residual component of linear polarization.

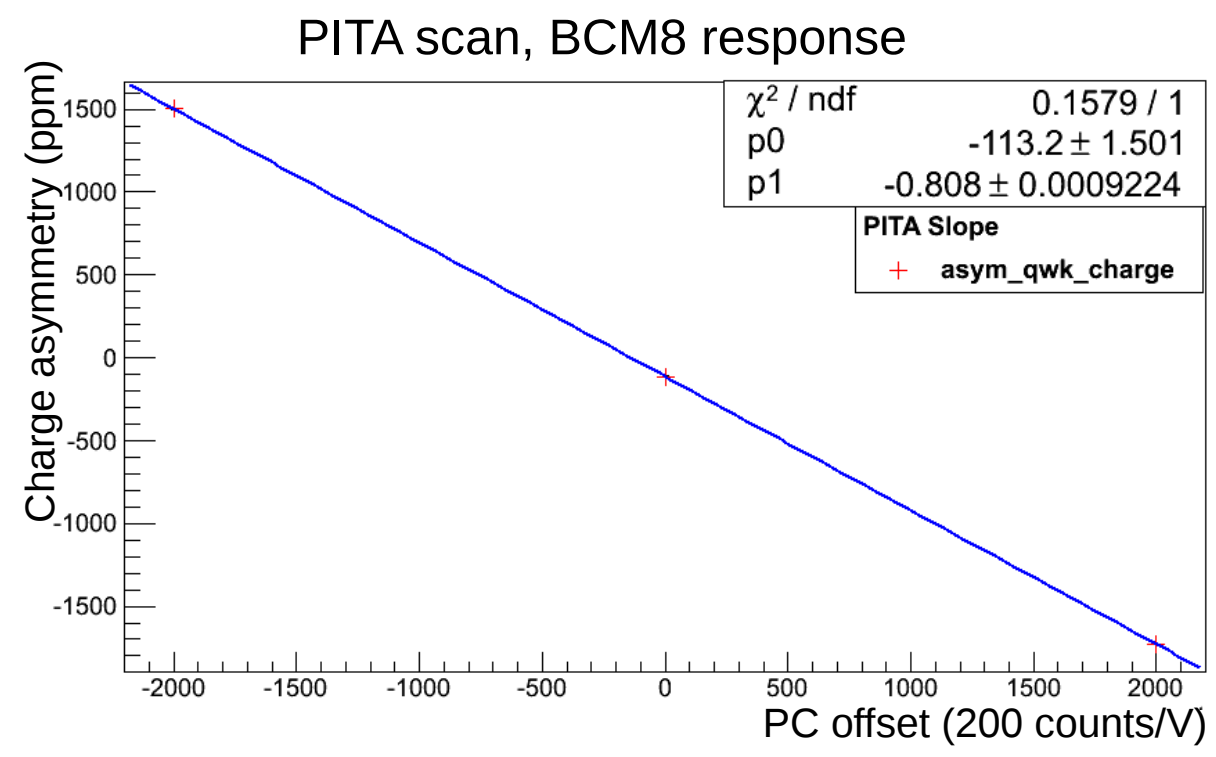

Figure 4.14: PITA scan where PC offset voltages are applied and the resulting charge asymmetry $A_{Q}$ is measured on BCM8 in the experimental hall. The applied offsets of \pm 2000 "hardware units" correspond to $\pm 100 \mathrm{~V}$ offset voltage.

The system under examination can extend all the way to the experimental hall. In the PITA scan of Figure 4.14 large offset voltages $(\Delta V= \pm 100 \mathrm{~V})$ are applied to the $\mathrm{PC}$ and the response of BCM8 is measured in Hall C. The response is very clear as the induced PITA effect is large and completely dominates. The extracted PITA slope from this scan will be used for charge feedback (Section 4.9.1). For example, the slope extracted from this scan suggests that the voltages should be modified along the antisymmetric line to null the intercept of $113.2 \mathrm{ppm}$. This suggests an antisymmetric shift of $(-113.2[\mathrm{ppm}]) /(-0.808[\mathrm{ppm} /$ count $])=-140$ counts, which corresponds to a small shift of $7 \mathrm{~V}$ in applied voltage. PITA scans are similarly used regularly during alignment on the laser table to optimize the $\mathrm{PC}$ offset voltages $\Delta V$ such that charge asymmetry $A_{Q}$ is minimized. 


\subsubsection{Helicity-correlated position differences}

The charge asymmetry is generated by phase offsets coupling with an asymmetric transport. A higher-moment effect arises from spatial variation of the phase offset across the beam spot, or equivalently a spatial gradient of the residual linear light component. Coupled with an asymmetric transport this gradient will result in a relative shift of the beam centroid of the two states. This helicity-correlated position difference arises from a linear gradient of the $\Delta$ phase across the beam spot, which also results in a gradient of the generated charge asymmetry. This polarization effect is the dominant mechanism for creation of position differences.

The functional form of Eq. 4.3 for the charge asymmetry is examined in scans where the RHWP orientation angle $\theta$ is rotated (Figure 4.15). This corresponds to rotation of the linear light component with respect to the cathode analyzing power. The horizontal and vertical position differences $\Delta X$ and $\Delta Y$ are also responding with the same functional form. The fit results allow important decomposition of the various sources that contribute to helicity-correlated effects. Note in Figure 4.15 that it is very challenging to constrain position differences in the injector to better than few 100's nm. We generally require that the running average for position differences remains within an order of magnitude of the maximum run-averaged specification $|\Delta x|<2 \mathrm{~nm}$ (Table 4.1). To maintain position differences $|\Delta X| \lesssim 50 \mathrm{~nm}$ we must also rely on kinematic suppression through acceleration (Section 4.8.

Separate from the polarization effects described in Eq. 4.3 is another class of effects, referred as non-polarization effects. An example is helicity-correlated "steering" of the laser beam, an effect that occurs due to the piezoelectric nature of the PC which causes it to deform in response to the applied voltage and act as a voltage-activated (and helicity-correlated) lens. This is an example of a static effect that depends only on the applied voltage and not on the polarization state of the incoming laser beam, therefore it would remain unchanged in both states of the IHWP. We expect such static effects to cancel well under the IHWP reversal, when data from opposite IHWP states are averaged together.

\subsubsection{Beam spot size asymmetry}

Using nomenclature similar to moments of a distribution, helicity-correlated effects can be thought to be connected to different moments of asymmetric phase shift induced from the Pockels cell. The zero moment is the average phase shift induced over the beam spot and gives rise to a charge asymmetry. The spatial gradient of the phase shift across the beam spot is the first moment and is connected to the generation of position differences. The phase shift curvature (second spatial derivative) will give rise to helicity-correlated asymmetries in beam spot size and shape, a second-moment effect. 
RHWP scan, Run 2207, IHWP OUT, qwk_1i02, PITA=0
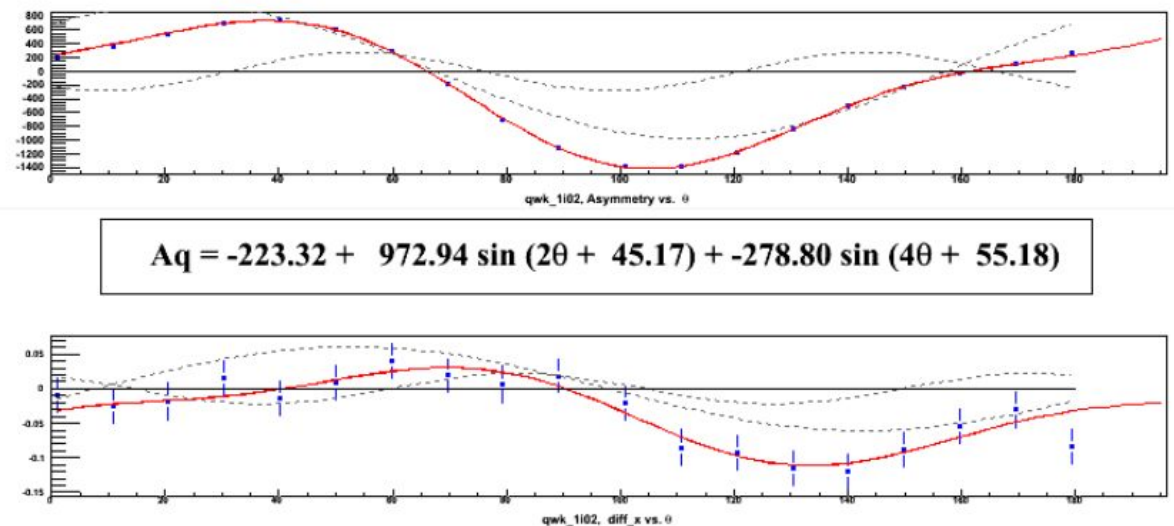

$\mathrm{Dx}=-0.03+-0.06 \sin (2 \theta+164.25)+0.02 \sin (4 \theta+122.05)$

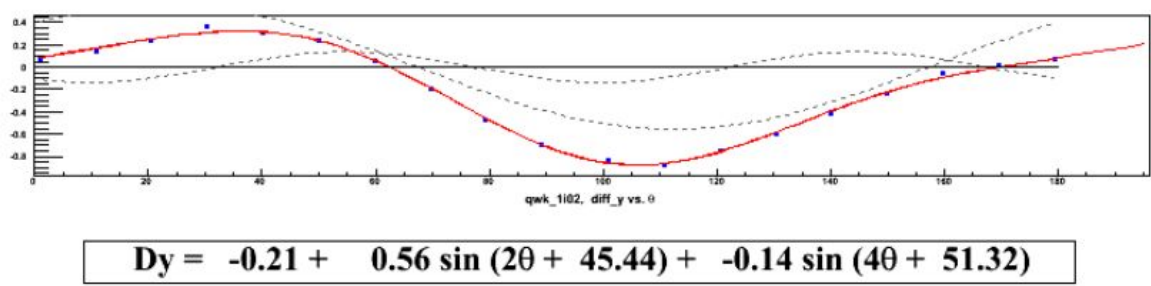

Figure 4.15: Scan of charge asymmetry (top) and transverse position differences, as measured on an injector BPM (1I02), versus RHWP orientation angle. The fit corresponds to the functional form of Eq. (4.3). Dashed lines represent the $2 \theta$ and $4 \theta$ terms separately. Fit results for $A_{Q}$ and $\Delta X, \Delta Y$ quoted in $\mathrm{ppm}$ and $\mu \mathrm{m}$.

The birefringence gradients on the PC can be accessed directly through scans where the $\mathrm{PC}$ is translated on its mount. The beam profile remains stationary and samples the birefringence and its spatial gradients along the translation axis. A scan where the $\mathrm{PC}$ was translated horizontally can be seen in Figure 4.16. The charge asymmetry $A_{Q}$ is varying over the translation due to birefringence gradients which give rise to the higher moments of position and spot size differences.

While the second-moment effect of beam spot size and shape difference was previously assumed to be negligible, calculations suggest that it may become important. Especially since the sensitivity of $Q_{\text {weak }}$ detectors to the beam spot size asymmetry is probably not suppressed by the azimuthal symmetry, unlike for other HC differences. We may attempt to estimate the effect by expanding the $Q_{\text {weak }}$ detector rate about the average scattering angle $\theta_{0} \approx 8^{\circ}$ :

$$
\frac{d R}{d \theta}=\left(\frac{d R}{d \theta}\right)_{\theta_{0}}+\frac{d}{d \theta}\left(\frac{d R}{d \theta}\right)_{\theta_{0}}(\Delta \theta)+\frac{1}{2} \frac{d^{2}}{d \theta^{2}}\left(\frac{d R}{d \theta}\right)_{\theta_{0}}(\Delta \theta)^{2}
$$

For a first-order approximation we assume that the acceptance-defining collimator is a pinhole at a distance $D \approx 3 \mathrm{~m}$ from the target. Then if $\sigma_{x}$ is the beam spot size, the scattering angle from the edge of the beam spot will be different from $\theta_{0}$ by $\Delta \theta=\sigma_{x} / D$. A helicity-correlated beam spot size asymmetry $\sigma_{ \pm}=\sigma_{x} \pm \Delta \sigma_{x} / 2$ will then give rise to a rate asymmetry $\delta R$ from 

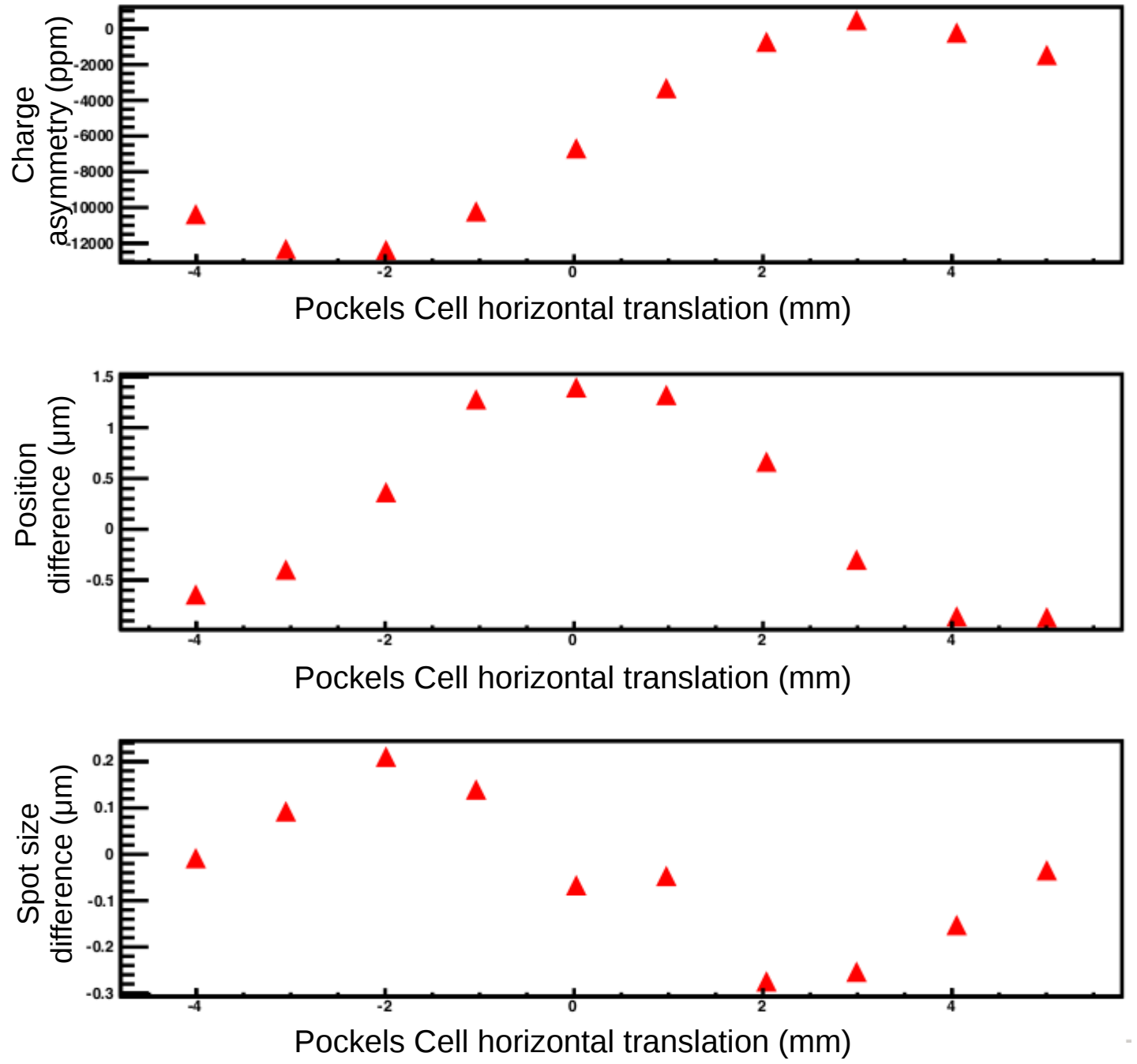

Figure 4.16: Measurement of the charge asymmetry (top), position difference (middle) and spot size difference (bottom) with a linear array of photodiodes, oriented vertically, during horizontal translation of the PC. The variation is a result of birefringence gradients. The position and spot size differences are seen to be proportional to the gradient and curvature of the charge asymmetry, respectively. Reproduced from [140].

the second-order term in Eq.4.5):

$$
\delta R=\frac{d^{2}}{d \theta^{2}}\left(\frac{d R}{d \theta}\right)_{\theta_{0}} \frac{\sigma_{x} \Delta \sigma_{x}}{D^{2}}
$$

At $Q_{\text {weak }}$ kinematics the differential cross-section depends on the scattering angle as $d R / d \theta \sim$ $\theta^{-3}$. Therefore we can write the fractional helicity-correlated change in rate as

$$
\frac{\delta R}{R}=\frac{24 \sigma_{x}^{2}}{D^{2} \theta_{0}^{2}}\left(\frac{\Delta \sigma_{x}}{\sigma_{x}}\right)
$$

Through this calculation we can limit the false asymmetry induced in the detector for a given spot size asymmetry. For $Q_{\text {weak }}$ the distance from the target to the defining collimator openings 
is $D \approx 3 \mathrm{~m}$, and the average scattering angle is $\theta_{0} \approx 8^{\circ}=0.396$ radians. The intrinsic beam spot size is $\sigma_{x}=100 \mu \mathrm{m}$. If we can bound the spot size asymmetry to

$$
\frac{\Delta \sigma_{x}}{\sigma_{x}} \lesssim 10^{-3}
$$

then we can limit the effect on the measured asymmetry to a few ppb:

$$
\frac{\delta R}{R}=\frac{24(100 \mu \mathrm{m})^{2}}{(3 \mathrm{~m})^{2}(0.396)^{2}} 10^{-3} \approx 2 \mathrm{ppb}
$$

We can then rely on cancellations from slow reversals (the IHWP and the Wien spin reversals) to render this effect negligible.

While it is very hard to constrain $\Delta \sigma_{x}$ on the electron beam, better bounds can be achieved at the laser table. The spot size asymmetry of the laser beam was measured with a linear array of photodiodes and bound within $\Delta \sigma_{x} / \sigma_{x} \lesssim 5 \cdot 10^{-4}$ with an analyzer (100\% analyzing power), as seen in Figure 4.17. We expect a suppression by a factor of $\sim 25$ on the electron beam due to the much smaller analyzing power of the cathode. $\Delta \sigma_{x} / \sigma_{x}$ was also measured within $3 \cdot 10^{-5}$ without the analyzer, thereby constraining non-polarization effects. However there may have been an enhancement of up to an order of magnitude by higher-order effects, such as the QEhole effect (Section 4.6.5), thus the spot size asymmetry should be considered constrained at the $10^{-4}$ level for $Q_{\text {weak }}$. Future ultra-precise PVES experiments should aim to better constrain this potential false asymmetry contribution by monitoring the spot size on the electron beam.

\subsubsection{Higher-order effects}

\section{Higher-order Skew/Paschke effects}

For our discussion on the generation of a charge asymmetry between the helicity states, we have mostly assumed that the laser light traverses the PC along its optic axis. The charge asymmetry that arises due to misalignment of the Pockels cell was briefly mentioned in Section 4.6.1 as the "Skew/Paschke" effect, which makes it critical that we achieve good alignment.

While the treatment of [140] considers misalignment effects on a single light ray, in practice the laser beam has a finite size and transverse properties. Therefore different components of the beam will form different angles of incidence to the PC optic axis and experience different misalignment effects. Thus residual linear components acquire spatial variation across the beam spot, where we expect the largest misalignment effects to be experienced by the outer part of the beam. This spatial variation can potentially couple to other higher-order effects considered next. 

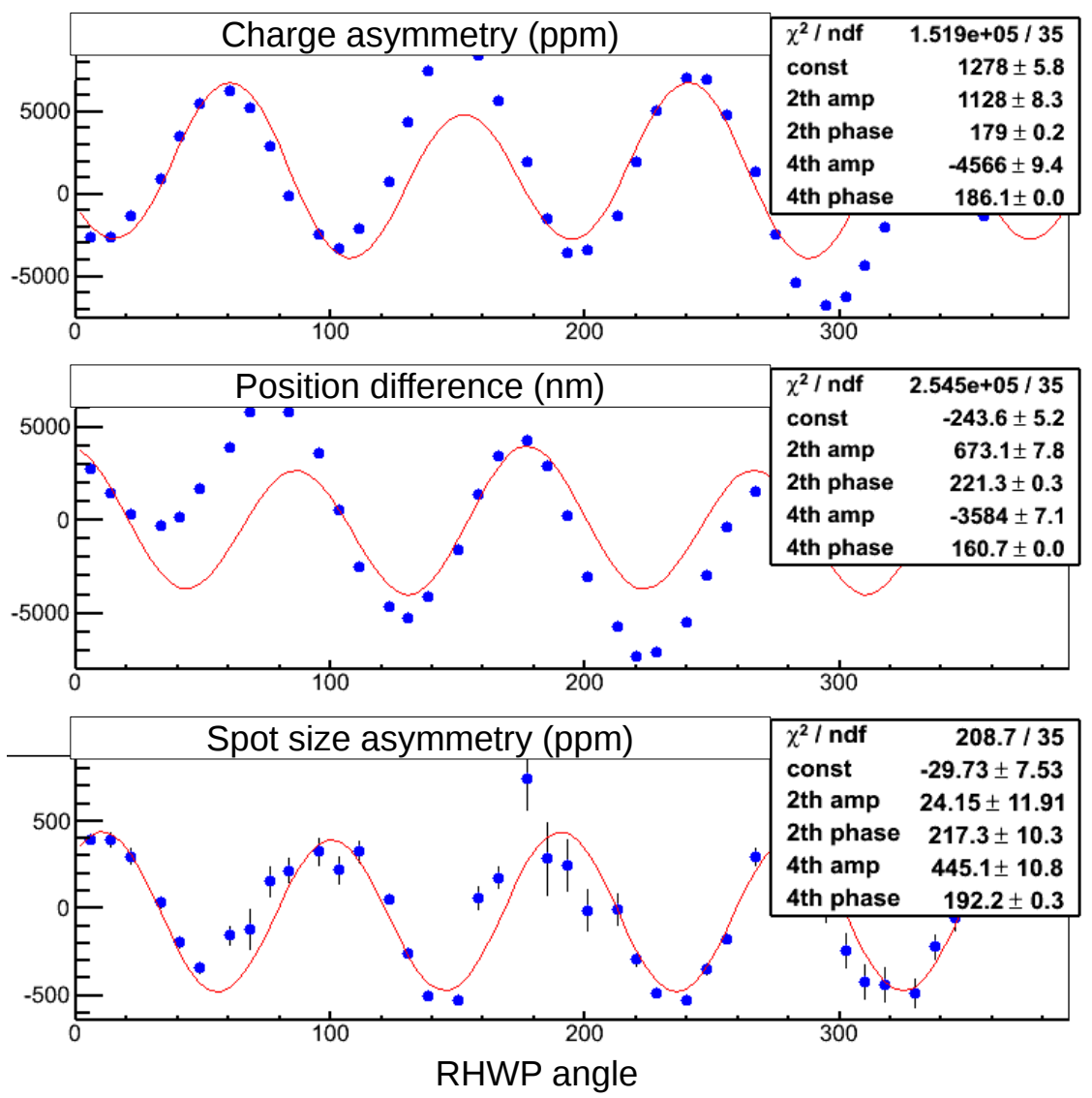

Figure 4.17: RHWP scan with the linear array as detector element. Responses are plotted for the charge asymmetry (top), horizontal position difference (middle) and beam spot size asymmetry (bottom). The beam spot size asymmetry is constrained within 500 ppm with the analyzer.

In Section 4.7 .4 we will see a dedicated study with the injector chopper where we accessed a variation of the beam $\mathrm{HC}$ properties across the temporal profile of the electron beam. The potential for a small outer part of the beam (halo) to carry a large asymmetry may have also been associated with the large background asymmetries measured in the experimental hall.

\section{Quantum efficiency hole}

Higher moments can appear not only in the phase shift induced by the PC but also on the cathode QE, or the QE anisotropy, giving rise to higher-order effects. For example, a spatial gradient in the QE of the cathode can couple to position differences in the beam. In this case each helicity state experiences a different average QE, thereby a "secondary" charge asymmetry is created.

Spatial variations of the QE can occur quite naturally through continuous operation. In a gaussian beam profile there is less power distributed in the "tails" (larger radii) of the beam spot, resulting in slower degradation of the cathode QE. We refer to this effect as a "QE hole" that is dug deeper in the center of the gaussian beam profile. The resulting spatial QE variation 
around the beam spot can be seen by eye in the right picture of Figure $4.10 \mathrm{~b}$. This effect can be especially problematic if the outer part of the beam profile is associated with larger helicitycorrelated parameter differences, which is perhaps expected. Note also that the QE discrepancy between the edges and the central part of the beam would get progressively larger over extraction from the same spot on the cathode, thus creating increasingly larger QE spatial gradients.

The non-trivial effect of a QE spatial variation on the spot size asymmetry was estimated by a calculation from this author [142]. The spot size asymmetry after the photocathode can be expressed as

$$
\frac{\Delta \sigma}{\sigma_{0}}=\frac{1}{4 \sigma_{0}^{2} Y_{0}} \int_{x} Y(x) \operatorname{QE}(x) A_{Q}(x) r(x)^{2} d x \quad,
$$

where the integration runs from the center to the edge of the beam spot. $\sigma_{0}$ is the average beam spot size, $Y_{0}$ is the total beam intensity, $r(x)$ is distance from center, $Y(x)$ is the position-dependent beam intensity from a beam slice of thickness $r(x) d x$, and $\mathrm{QE}(x)$ is the $\mathrm{QE}$ experienced by that slice. Therefore the spot size asymmetry is a convolution of many different properties. $\Delta \sigma$ may arise even for a zero net charge asymmetry $A_{Q}$ if there are non-uniform helicity-correlated properties across the beam spot. Since in general we expect HC properties to be larger on the edges of the beam spot, the QE hole effect will likely amplify $\Delta \sigma$.

\section{Aperture interception}

Another mechanism involves beam interception at injector apertures or other places in the beamline. Any differences in beam position or size and shape could give a helicity-correlated component to this interception, thus again giving rise to secondary charge asymmetry and higherorder differences. This effect was observed directly in the experiment and will be presented in Figure 4.20 This effect is also amplified if $\mathrm{HC}$ properties are larger at the outer part of the beam, and becomes more important at the high currents of $Q_{\text {weak }}$ where increased emittance due to space-charge effects lead to increased beam losses on apertures. Further complications from aperture interception are discussed in Figure 4.21

\subsection{Polarized Source Optimization}

In this section we give an overview of the optimization strategies we adopted and the studies that were performed to suppress HC beam differences within experimental specifications. These techniques had been developed and implemented successfully by previous experiments in the PVES program. In Section 4.7 .4 we characterize the control of HC properties we achieved in the injector. 


\subsubsection{Optimization strategy}

Eq. (4.3) parametrizes various mechanisms that give rise to charge asymmetry and higher order effects. The terms in that equation can be conceptually thought of as [140]

$$
A_{Q}=\text { Photocathode } \times[\text { Vacuum window }+ \text { RHWP }+\mathrm{PC}]
$$

The most direct approach to achieve optimization of $\mathrm{HC}$ parameters would be to use a photocathode with no analyzing power, thereby outright suppressing all $\mathrm{HC}$ differences created from imperfections of the optical elements that control polarization. Unfortunately some analyzing power on the cathode is unavoidably arising from the strain on the crystal lattice 8 Instead we must arrange for the sum of the three terms in brackets to be minimized.

The most direct option would be to set each of the three terms separately to zero. This approach is more desirable as it should also suppress higher-order effects and their unpredictable coupling mechanisms (Section 4.6.5). It is easy to null the charge asymmetry from the PC, corresponding to the $4 \theta$ term in Eq. 4.3 , by adjusting the offset voltages after a PITA scan. The other two terms however are harder to access as they are independent of the $\Delta$-phases. Some imperfections on the RHWP plate and phase shifts due to the vacuum window stress-induced birefringence are unavoidable.

Therefore we have to adopt the approach of arranging the sum of the three terms to be zero, accepting that the charge asymmetry from each individual term does not vanish. Effectively this means that we allow residual linear light from the PC to cancel effects generated downstream, such as the retardation induced by the vacuum window. This is not ideal as it allows generation of higher-order effects, which can be hard to access and measure. In practice we attempt to suppress the charge asymmetry and higher-order effects from each term until practical limits are reached in laser table studies, which ensures good alignment of the PC and the IHWP. The vacuum window and the cathode are necessarily downstream of the PC and all elements on the laser table, so we can only arrange for cancellations of the effects they induce with studies on the electron beam. After achieving the best possible setup for the polarized source we rely on further damping of $\mathrm{HC}$ differences through kinematic suppression (Section 4.8) and feedback (Section 4.9). Finally, slow helicity reversals from the IHWP and the Wien provide important cancellations of residual false asymmetries $A_{\text {beam }}$.

\footnotetext{
${ }^{8}$ Superlattice cathodes have already achieved smaller analyzing power and higher polarization compared to a strained-layer cathode [143]. Future experiments may receive benefits from further advancements in photocathode technology.
} 


\subsubsection{Laser table studies}

Studies on the laser table serve to optimally align the optical elements that control the light polarization and set initial values for the Pockels cell offset voltages and RHWP orientation, before effects from the vacuum window and photocathode are taken into account. Laser table studies were initially performed in Lab 26 at UVA, where optical elements were characterized and the PC driver switch circuit and optocouplers case were developed. We then moved our setup for optimization at the laser table in the Jefferson Lab polarized source before the beginning of $Q_{\text {weak }}$ production. Studies on the laser table were destructive to production so they were only performed opportunistically after the experiment started, whenever access was possible (if beam could not be delivered beyond the injector for other reasons) and necessary to recalibrate source parameters.

\section{Laser table elements}

During laser table studies a remotely operable dielectric mirror is inserted upstream of the vacuum window to measure and optimize the $\mathrm{HC}$ properties of the laser beam. A separate beamline was setup to test different Pockels cells and half-wave plates. The detector element typically used was a quad photodiode (QPD), comprised of four photodiodes that allow measurement of both beam intensity and position. We used a linear array of photodiodes (LAPD) along with a cylindrical lens that expanded the beam spot elliptically along the LAPD, to measure the HC beam spot size difference $\Delta \sigma_{x}$ (Figure 4.17 .

A linear polarizer was installed immediately upstream of the detector element to provide the analyzing power in the system in lieu of the photocathode, and is commonly referred as the analyzer. By limiting $\mathrm{HC}$ effects on the laser table with the analyzer (100\% analyzing power) we expect a further suppression by a factor of 20-25 on the electron beam due to the much smaller analyzing power of the cathode $(\sim 4 \%)$. The analyzer's orientation can be modified to characterize complementary components of the light polarization. By aligning the analyzing axis along the horizontal and then rotating it by $45^{\circ}$ we access the polarization components corresponding to the S1 and S2 Stokes parameters [ref], respectively. The analyzer should be installed immediately upstream of the detector element to minimize the lever arm of steering/lensing effects. Data was also taken without the analyzer, with a much smaller analyzing power provided by the optical elements downstream of the PC, potentially including the insertable mirror, the insertable lens, and the detector element. Finally, another Pockels cell is part of the intensity asymmetry (IA) system, located immediately downstream the Hall $\mathrm{C}$ laser and before it is combined with the lasers of the other halls (Figure 4.1). Driven by the Helicity signal, small offsets in its applied voltage couple with the wave plate and linear polarizer to give an intensity asymmetry, aimed 
at cancelling residual intensity asymmetries when no voltage is applied on the main Pockels cell.

\section{Optimization techniques}

As a passive technique that contributes to suppression of all sources of $A_{\text {beam }}$, it is absolutely to our benefit to achieve optimal alignment of the optical elements and suppress misalignment effects. Optimal alignment of the PC is achieved through established techniques including observation of the isogyre pattern. Translation scans as in Figure 4.16 are implemented, along with scans of the PC pitch and yaw angles by rotating the mounting stage. These scans directly access birefringence gradients and model false asymmetries arising from misalignment. In establishing the optimal PC position and orientation we especially aim to suppress the first moment position differences, especially those arising from skew/Paschke effects. The charge asymmetry can more easily be adjusted via the $\Delta$ phases, controlled through the PC offset voltages.

We can test for residual PC misalignment by allowing the light to propagate through the crystal without any applied voltage, when in theory we expect no phase retardation if the beam propagates along the optic axis. In practice there will be some irreducible effects arising from finite beam spot size and imperfections in the crystal. Phase shifts are detected by placing the PC between crossed polarizers. We are satisfied with PC alignment when we achieve extinction ratios of order $5 \%$. If there is an irreducible intensity asymmetry arising even with the PC voltages off, then we cancel it by inducing a small and opposite intensity asymmetry via the IA Pockels cell.

Our first estimation for the optimal PC voltages that correspond to quarter-wave retardation are established quickly and efficiently using a spinning linear polarizer downstream of the PC. Rotation of the analyzing direction allows a quick reading of the variation of transmitted light, which establishes the degree of circular polarization (DOCP). The PC voltages are adjusted to minimize this variation (i.e. dependence on orientation of analyzing power, indicative of residual linear light) and maximize DOCP, where typically values of $99.9 \%$ are achieved.

A variety of scans are then performed to characterize the response of the system and achieve optimal suppression of HC properties, including PC translation and orientation scans, PITA scans and RHWP scans as described previously. The optimization procedures on the laser table are described in more detail in [140].

\subsubsection{Optimization on electron beam}

Upon achieving satisfying control of $\mathrm{HC}$ differences on the laser table, we retract the reflecting mirror and perform the optimization procedure on the electron beam, using injector BPMs as our detector elements. The final stage of optimization is to achieve suppression of helicity-correlated 
beam properties taking into account the vacuum window birefringence and the cathode analyzing power, parametrized by the $\beta, \rho, \epsilon / T$ and $\psi$ parameters in Eq. 4.3 .

These studies could be performed by remotely controlling and scanning PITA voltages and RHWP orientation. While remote operation did not allow access to translation and alignment of optical elements, it was less invasive and could be performed parasitically with other accelerator beam studies.

$\triangle \mathrm{Y}, \mathrm{BPM} 0 \mathrm{OO} 2$

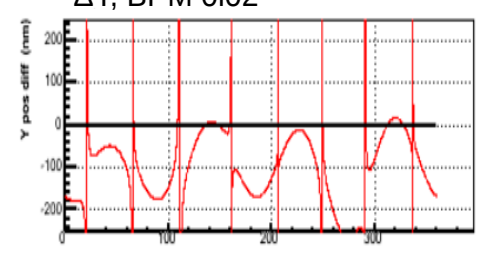

$\triangle \mathrm{X}$, all injector BPMs

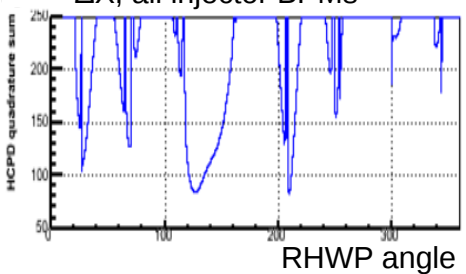

$\triangle \mathrm{Y}, \mathrm{BPM} 0 \mathrm{0i02 \textrm {a }}$

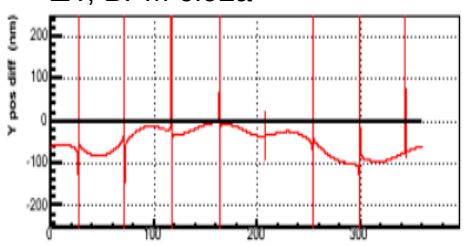

$\triangle \mathrm{Y}$, all injector BPMs

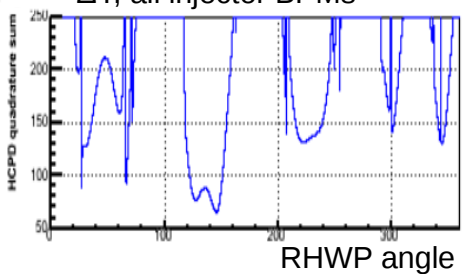

$\triangle \mathrm{Y}, \mathrm{BPM} 0 \mathrm{O05}$

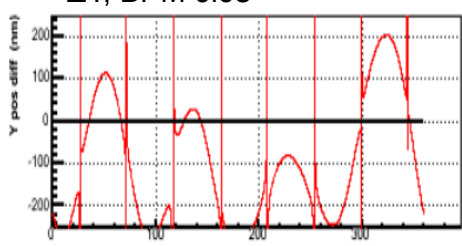

Combined, all injector BPMs

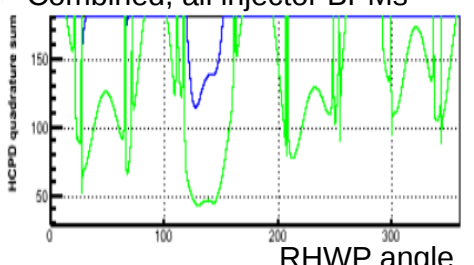

Figure 4.18: Top row: $\Delta Y$ position differences on three different injector BPMs, after appropriate PITA voltage offset is applied (on each RHWP angle) to null $A_{Q}$. Bottom row: The average size of position differences on multiple injector BPMs, with appropriate PITA offset applied. This procedure was used to optimize the RHWP orientation and minimize position differences at the polarized source.

For the final optimization of PC voltages and RHWP orientation we implement two RHWP scans at $\mathrm{PC}$ voltages that are $\sim 100 \mathrm{~V}$ apart, collecting data at multiple BPMs along the injector. By comparing the data from the two scans we can define a PITA slope for the charge asymmetry and position differences measured on each BPM, for each RHWP orientation. This allows us to create the plots of Figure 4.18, where in each pane on the top row the red line corresponds to the $\Delta Y$ difference on each BPM versus RHWP angle, taking into account the effect of the PITA voltage adjustment that we would have to make to null the charge asymmetry at the given RHWP orientation. The discontinuities correspond to RHWP angles that completely cancel the sensitivity of the PC offset voltages (the $\Delta$ phases) with respect to the cathode orientation. Through this complicated plot we can quickly read the RHWP angles that minimize position differences on each BPM. Considering a single BPM may be inadequate as that gives us no information on the angle degrees of freedom which access a complementary part of the beam transverse phase space. Therefore we determine the optimal RHWP orientation by constructing the average horizontal and vertical magnitude of position differences (after taking into account the necessary PITA adjustment) over the injector BPMs, shown in the bottom row of the figure. We select an optimal orientation for the RHWP such that the combined (horizontal and vertical) average is suppressed on multiple BPMs along the injector. 


\subsubsection{Results in the injector}

\section{Position differences}

Figure 4.19 shows the position differences (in $\mathrm{nm}$ ) as measured on individual injector BPMs, plotted versus each BPM labeled by its name. The BPMs are plotted in the order they are located in the injector. The position differences of a few 10's of ppb are the smallest ever achieved in the Jefferson Lab injector. By comparison position differences during HAPPEX-II were typically larger by an order of magnitude in the same locations, as we will see in Figure 4.23. The excellent control of $\mathrm{HC}$ beam properties in the injector suggests that our setup of the polarized source was very well optimized, although source parameters would drift and optimization is required again after some time.

The purple band corresponds to position differences within $\pm 50 \mathrm{~nm}$, which is an acceptable magnitude for the running average size of $\mathrm{HC}$ differences at the $Q_{\text {weak }}$ target (about an order of magnitude larger than the $\langle\Delta X\rangle$ specification from Table 4.1. We would theoretically expect a suppression of $\mathrm{HC}$ parameters at the $Q_{\text {weak }}$ target by a factor of $\sim 60$ relative to what we achieved in the injector, due to kinematic damping, placing us very comfortably within specifications. However as we will see in Section 4.8, in practice that theoretical suppression was not realized.

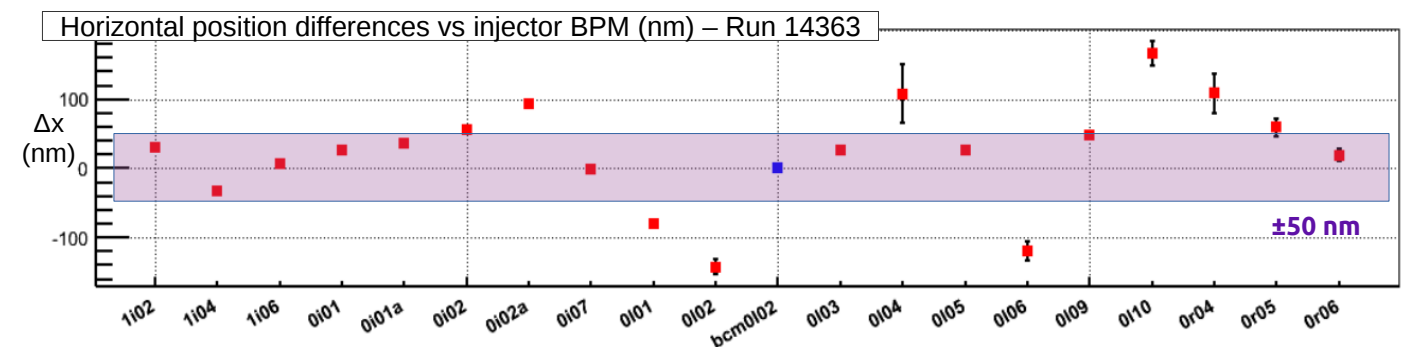

Figure 4.19: Horizontal position differences on successive injector BPMs. The purple band corresponds to $\pm 50 \mathrm{~nm}$ of position differences.

\section{Charge asymmetry}

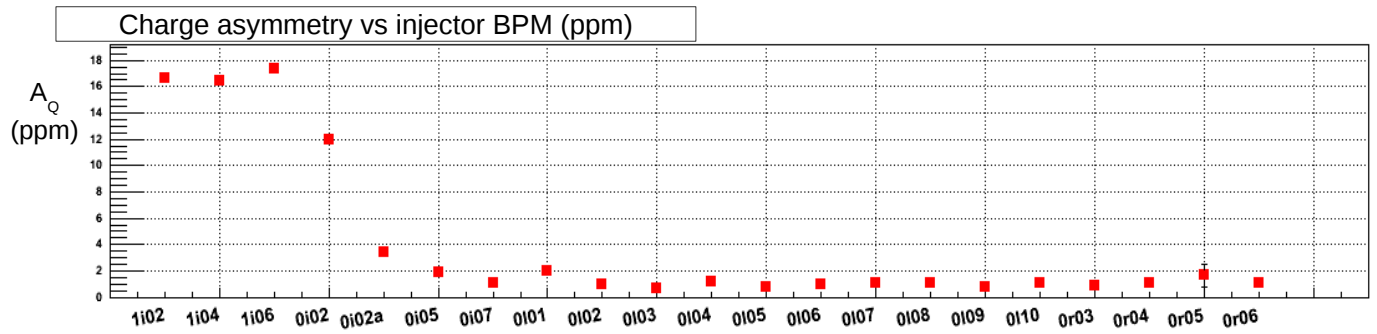

Figure 4.20: Charge asymmetry on successive injector BPMs exhibits a troubling gradient in the location of the injector apertures.

In Figure 4.20 the measured charge asymmetry $A_{Q}$ is similarly plotted on successive injector 
BPMs. The gradient of charge asymmetry as measured between BPMs $1 \mathrm{i} 06$ and 0i05 is quite troubling. Between these BPMs are located the injector apertures A1, A2 and MS. The $A_{Q}$ gradient suggests that the interception in the apertures is helicity-correlated, a higher-order mechanism described in Section 4.6.5. The character of this helicity-correlated interception generally varies with time, as injector parameters are adjusted to establish beam delivery.

That the charge asymmetry converges to zero after interception in the apertures is ensured by the charge feedback (Section 4.9.1) that is applied through the PC voltages. It is highly questionable however whether $\Delta$-phase offsets are an appropriate form of feedback for helicitycorrelated interception at the apertures. Complicated phase-space correlations may arise through feedback between charge asymmetry and higher helicity-correlated moments.

Beam delivery parameters were often unstable and had to be tuned to establish acceptable beam to the experimental halls. For $Q_{\text {weak }}$ indications of bad beam conditions include high halo and background rates in the experimental hall, as well as large asymmetry widths at the luminosity monitors (a very good indicator of backgrounds, as we shall see in Chapter 6) and width of $\mathrm{HC}$ monitor differences. In Figure 4.21 , interceptions at the three injector apertures are plotted versus time and shown to correlate highly (specifically interception at the A2 aperture in this case) with the width of the measured charge asymmetry from the normalizing BCM, which comes from the average of BCMs 1 and 2. Significant drift is observed here on the time scale of just an hour. When parameters in the experimental hall would become unacceptable (for example the specification from Table 4.1 is that charge asymmetry width, which is plotted here, must remain below $300 \mathrm{ppm}$ ), beam delivery parameters are tuned which creates the abrupt changes in the figure. It is unclear what is the drift mechanism on such short time scale. In general beam delivery parameters do not remain stable for more than a few days, and the same is true for higher-order effects and responses in the experimental hall.

\section{Longitudinal beam profile}

A very interesting dedicated study was performed in the injector on June 28-29 2012. This data was taken in a systematic study after $Q_{\text {weak }}$ was completed, and was in fact the very last data taken with the electron beam by users before the CEBAF shutdown for the $12 \mathrm{GeV}$ upgrade.

The test involved varying the phase of the chopper cavity as a means to access different temporal (longitudinal) "slices" of the electron pulses 9 The dependence of beam parameters (as measured downstream of the chopper cavity) to the chopper phase allows access to the longitudinal structure of the beam. Indeed we measured a significant variation of beam properties along the temporal beam profile (Figure 4.22). The total charge has a near-gaussian temporal

\footnotetext{
${ }^{9}$ Unbeknown to us at the time, a similar test with the chopper is reported in 99 .
} 


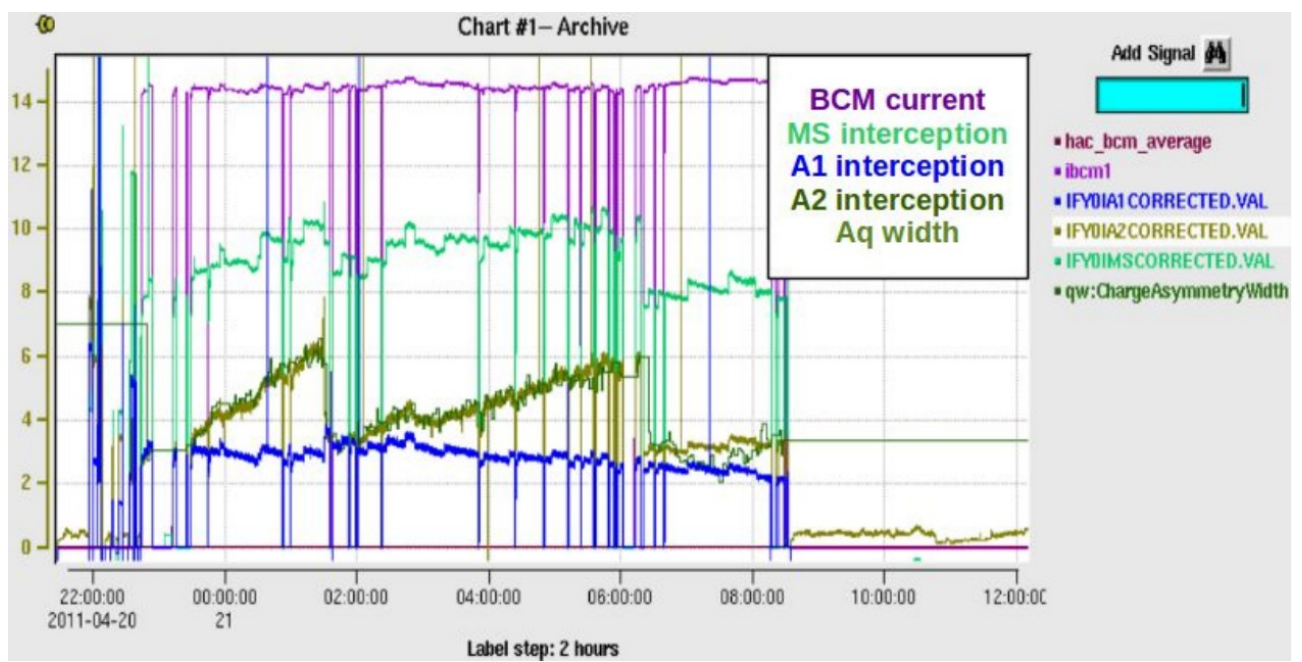

Figure 4.21: Interceptions on the $\mathrm{A} 1, \mathrm{~A} 2$ and $\mathrm{MS}$ injector apertures plotted versus time and colorcoded, from the accelerator striptool. Interception on injector apertures slowly drifts with time and is highly correlated with observed parameters in the hall. Here the charge asymmetry width is offset vertically so that it superimposes almost exactly the A2 interception (they are both plotted with a similar green color which makes them hard to tell apart).

profile with most charge being carried in the core of the beam, and less on the the leading and trailing beam tails. The transverse position of the beam varies significantly along the longitudinal profile. The temporal tails also seem to carry significantly different charge asymmetry $A_{Q}$ relative to the beam core.

That is at least a proof of principle that a small part of the beam may carry very large $\mathrm{HC}$ beam properties. These longitudinal tails may well evolve into the transverse tails in the experimental hall that we referred to as beam halo and was likely associated with the large background asymmetries that will be presented in Chapter 6. A dedicated test at the Jefferson Lab FEL revealed that the injector buncher cavity was most sensitive to a transverse tail appearing in the experimental hall, suggesting that the beam dynamics in the injector couple via space-charge the longitudinal and transverse planes [144.

\subsection{Adiabatic Damping}

Under the assumptions of linear beam optics (small inclination angles and only constant or linearly increasing magnetic restoring forces) and in the absence of acceleration, the equations of transverse motion of particles in the electron beam can be solved to give the phase-space ellipse in the $X-X^{\prime}$ plane, which parametrizes the beam's transverse phase-space in terms of the Twiss parameters [145]. The area of this phase-space ellipse is simply $\pi \epsilon$, where $\epsilon$ is the emittance. For the case of no acceleration, Liouville's theorem states that the area of the phase-space ellipse (and therefore the emittance) remains constant, even though its shape may 


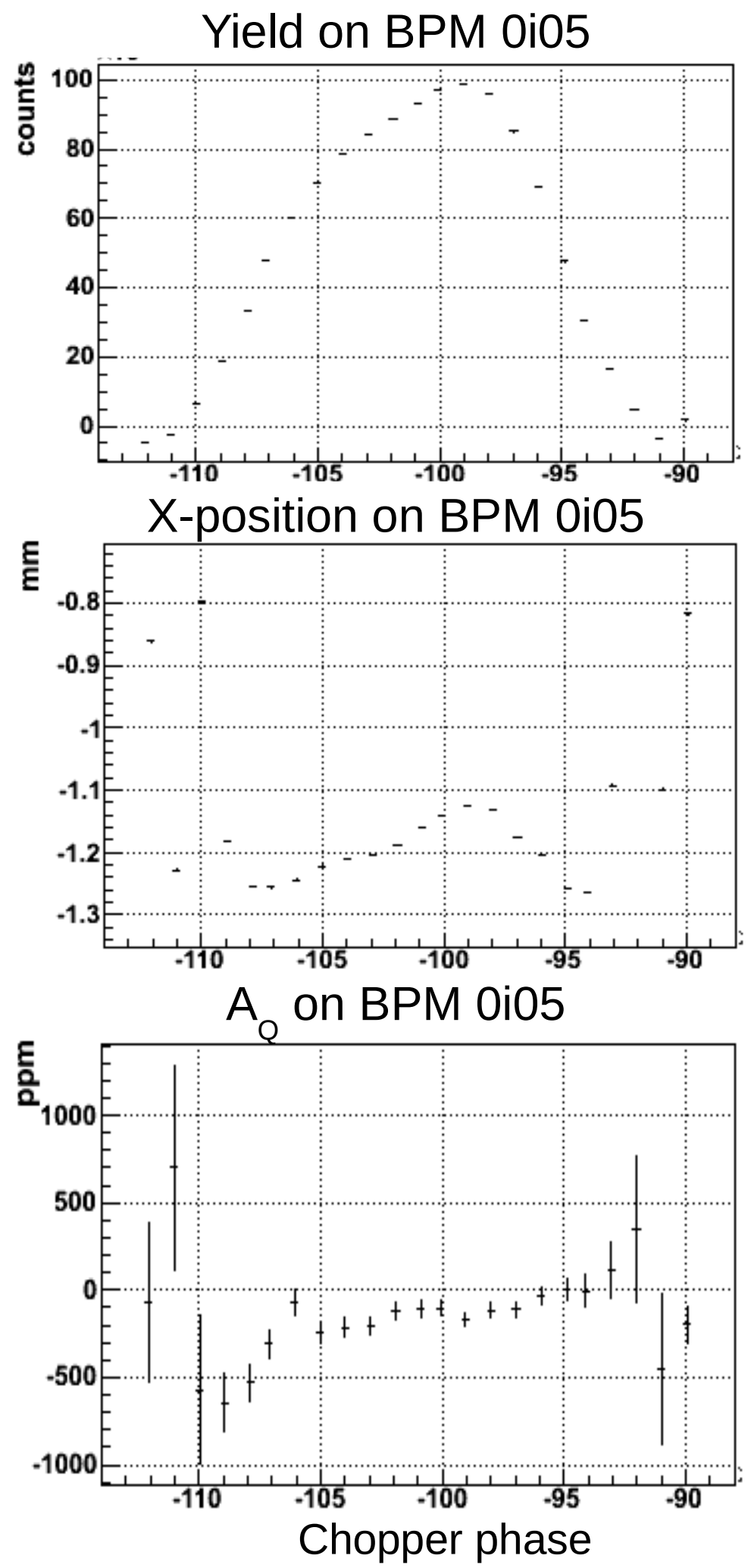

Figure 4.22: Top to bottom: Yield, horizontal position and charge asymmetry $A_{Q}$, as measured on BPM 0i05, versus injector chopper phase. By varying the chopper phase we access different longitudinal parts of the electron beam pulse, effectively getting a temporal profile of the beam. 
change. Under acceleration the size of the transverse phase-space is theoretically modified in a simple way:

$$
X, X^{\prime} \propto \sqrt{\frac{p_{0}}{p}},
$$

where $p_{0}$ and $p$ are the initial and final beam particle longitudinal momenta, respectively. This corresponds to a reduction in the amplitude of betatron motion (transverse oscillations around beam axis) and helicity-correlated differences as the beam momentum is adiabatically increased, and is referred to as adiabatic damping. Therefore acceleration through CEBAF is theoretically expected to reduce the amplitude of the $\mathrm{HC}$ differences.

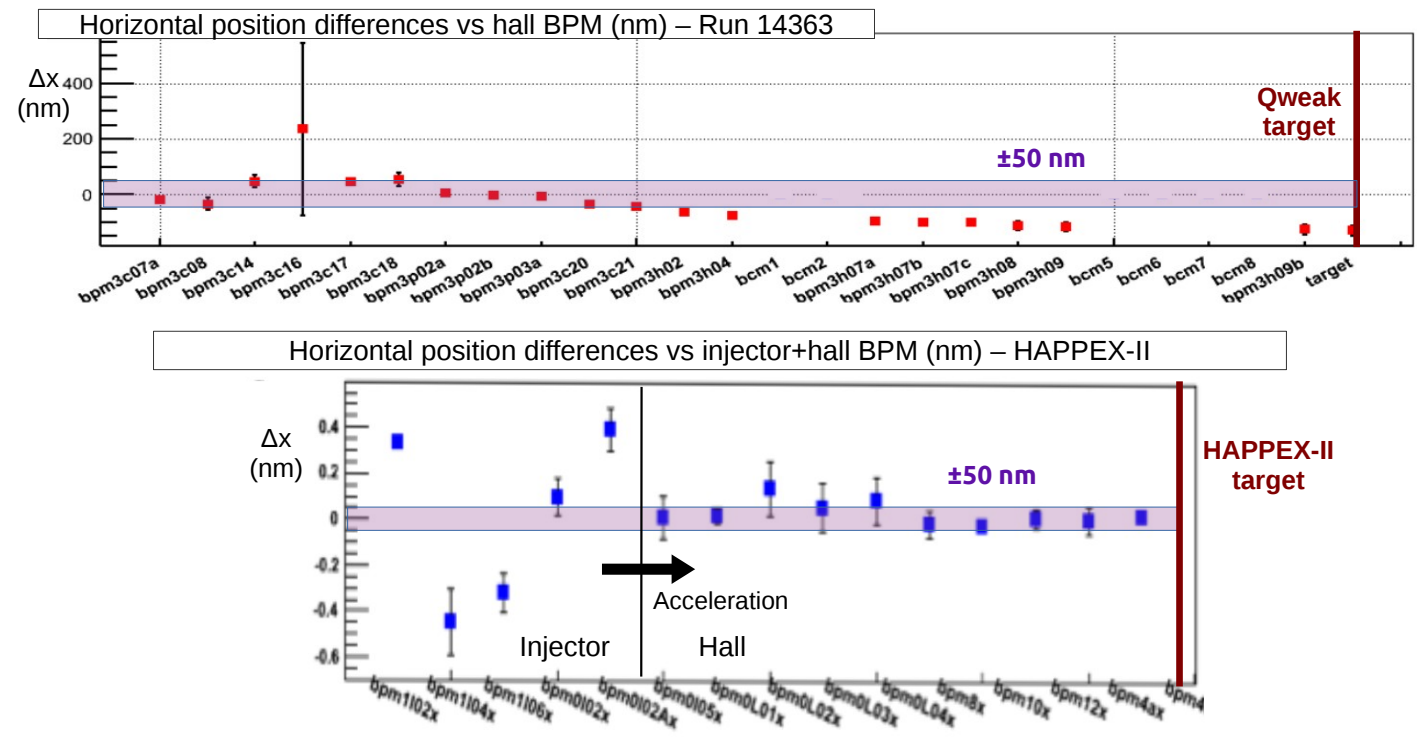

Figure 4.23: Top: Horizontal position differences in the experimental hall, plotted versus successive BPMs. This is the same run as for the injector position differences plotted in Figure 4.19 The position differences at the $Q_{\text {weak }}$ target are higher than $100 \mathrm{~nm}$. Bottom: Horizontal position differences in the injector and the experimental hall during the HAPPEX-II run. Kinematic damping through acceleration suppresses the HC beam properties along with its transverse phase space.

For $Q_{\text {weak }}$ the beam energy at the target is $1.155 \mathrm{GeV}$, so for a reference injector energy of 300 $\mathrm{keV}$ we expect a theoretical suppression factor of $\sim 60$. In Figure 4.23 the position differences are plotted in the experimental hall for the same run as for the injector position differences plotted in Figure 4.19. We see that, not only we didn’t receive the theoretical suppression, but the differences in fact blow-up and are increased in the experimental hall compared to their size in the injector. Due to insufficient suppression of the transverse phase-space the position differences evolve over the drift region to $\sim 100 \mathrm{~nm}$ at the target.

The bottom plot in Figure 4.23 is a comparison to data from HAPPEX-II. While the position differences off the cathode are much higher $(\sim 400 \mathrm{~nm})$ than what was achieved in $Q_{\text {weak }}$, that experiment got the benefit of adiabatic damping and achieved suppression of HC properties as the beam accelerated. The purple band that marks $\pm 50 \mathrm{~nm}$ serves to remind that the scale is 
different comparing to Figure 4.19 .

The theoretical suppression factor of Eq.4.11) assumes that the optical transport system is perfectly matched to the beam envelope. However various types of imperfections can lead to deviations from the theoretical expectation. A mismatched transport setup can lead to a distorted (dilated) phase-space ellipse and large betatron amplitude ("orbit blow-up") which can manifest as high correlation between transverse beam parameters. This is exactly what was observed in $Q_{\text {weak }}$ running, as presented previously in Figure 3.20 . The HC horizontal position and angle parameters ideally should be independent, but are instead shown to be fully coupled over the entirety of Run2.

This strong coupling suggests that $X$ and $X^{\prime}$ are hardly separate degrees of freedom, while the phase-space distortion crushes beam noise in the anti-correlated direction in $X-X^{\prime}$ phasespace (the direction perpendicular to the fitted line of Figure 3.20). This strong coupling can also be problematic for correcting the false asymmetries $A_{\text {beam }}$ that arise from HC differences in these parameters, as we will discuss in Chapter 5

Matching the sections of the accelerator is an empirical procedure in which the CourantSnyder parameters [146] are measured by making kicks in the beam orbit and the quadrupole magnets are adjusted to fine-tune the optics [147. The HAPPEX Collaboration and CASA scientists made the necessary effort and time investments to optimize matching and get the benefit of suppressed transverse beam phase-space. For various reasons that investment was not made for the $Q_{\text {weak }}$ experiment. As a result, even though we achieved the smallest-ever position differences right after the photocathode, we often had to take data with large $>100 \mathrm{~nm}$ position differences.

A significant investment to match focusing properties in the injector ${ }^{10}$ was undertaken towards the end of Wien8. Immediately after that injector tuning, HC beam parameter differences and background asymmetries were suppressed well within specification. The period until the end of Wien8 (Slugs 217-225) was thus dubbed "golden period", and we will refer to it as we discuss systematic corrections in the experiment. It is considered that systematic uncertainties in the $Q_{\text {weak }}$ measurement would be far smaller if all the data was taken under such conditions. Unfortunately the excellent beam quality lasted only for a few days until the Wien spin manipulation system was reversed. This highlights that the benefits from matching are only temporary and a new matching investment is required, but also that the Wien spin reversal was imperfect and effected the beam envelope and HC properties.

\footnotetext{
${ }^{10}$ Matching is most important in the injector where the relative acceleration is large and focusing elements have a stronger effect due to the lower beam energy. The large field and focusing gradients of the chopper solenoids are especially important.
} 


\section{$4.9 \quad$ Feedback}

The best approach is always to eliminate as best as possible all sources of false asymmetries in the polarized source, such as misalignment of optical elements and residual linear light in the circularly polarized beam. This would also achieve better suppression of higher-order effects that may be immeasurable. Optimal setup of the polarized source offers a passive suppression of false beam asymmetries.

As an active method of suppressing $\mathrm{HC}$ differences in beam parameters due to drifts, feedback is also an important part of our strategy to suppress $A_{\text {beam }}$. Through feedback we cannot cancel higher order effects, so ideally it should only be used for small corrections while most of the suppression is achieved through optimally setting up the source. Feedback is also limited by the precision of the monitors used to measure HC differences. Ideally feeding back on a specific beam parameter should leave other beam parameters unaffected, otherwise a complicated coupling between $\mathrm{HC}$ parameters may arise. During $Q_{\text {weak }}$ feedback was implemented on charge asymmetry via the $\mathrm{PC}$ voltages, and on position/angle differences through corrector magnets.

\subsubsection{Charge feedback}

The charge asymmetry $A_{Q}$ in the experimental hall is monitored continuously from BCMs. Since the response of the BCM charge asymmetry to a PITA offset is known from dedicated PITA scans (as in Figure 4.14), we can feed back on the measured charge asymmetry by adjusting the PC voltages.

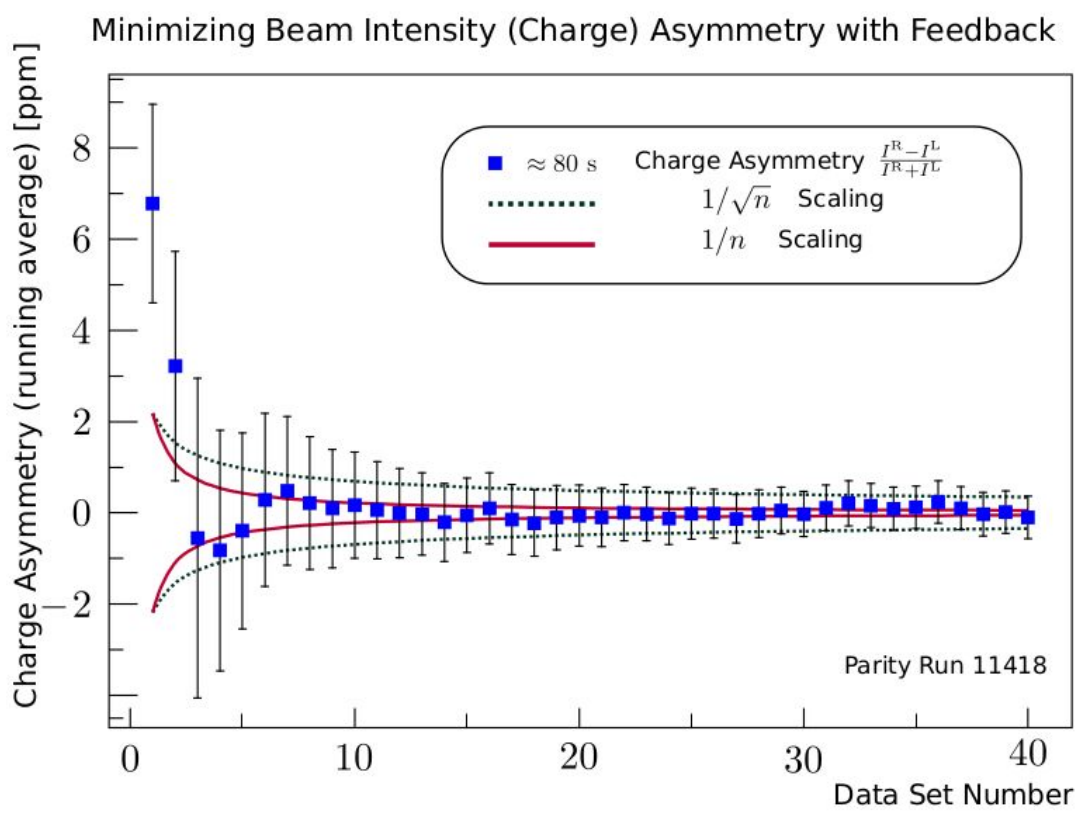

Figure 4.24: Active charge feedback minimizes the charge asymmetry faster than $1 / \sqrt{N}$ statistical convergence (dotted curves). Each data point is a running average of data up to that point. The horizontal axis is the number of 80 s intervals [148. 
The BCM measurement was integrated for a time period before the PITA slope was used to correct the average. The integration period was $80 \mathrm{~s}$ in Run1 and $40 \mathrm{~s}$ in Run2 [148]. At this author's suggestion the optimal PITA offset was saved at the end of every slug (IHWP state), to be used as the optimal initial value for the next slug with the same IHWP state. This greatly improved convergence time of bringing $A_{Q}$ within the instantaneous specification from Table 4.1 . thus saving a few minutes of data per slug.

The charge feedback is generally limited to $1 \%$ by the accuracy of the $A_{Q}$ measurement due to BCM resolution or a non-linearity in the experimental apparatus. Any other issues with the BCM monitor would also create offsets in the correction, as may have been the case in Run1 (Section 5.6.2). Ideally by adjusting the PC $\Delta$-phases we improve cancellation of residual linear light from the source, thereby improving-higher order parameters as well. However mechanisms such as helicity-correlated interception at the apertures (Figure 4.20 ) are not optimally treated by feeding back through the PC voltages, and complicated correlations between charge asymmetry and $\mathrm{HC}$ position and angle differences may arise.

\subsubsection{Helicity magnets}

A set of fast, pulsed magnets [149] are installed at the $5 \mathrm{MeV}$ injector (at locations 0L01-0L03). These fast dipole magnets can alter the beam trajectory in a helicity-correlated manner and therefore produce, or correct for, helicity-correlated position or angle differences. The controls of the magnets are electrically isolated to avoid cross-talk false asymmetries. Since the magnets are downstream of injector apertures they avoid creating correlations between intensity and trajectory due to scraping, which was a drawback of the previous position feedback system employed by the G0 experiment, by moving the beam spot on the photocathode via the PZT mirror.

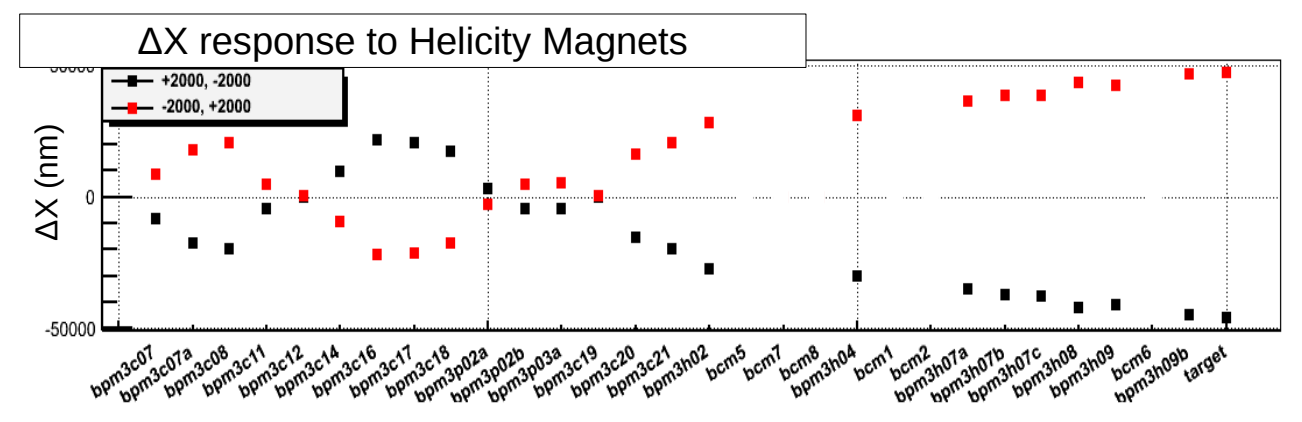

Figure 4.25: Responses of horizontal position differences in the experimental hall from large and opposite kicks from the injector helicity magnets. Large responses are observed, which were typically dependent on beam conditions.

We decided to use these corrector magnets due to the large $\mathrm{HC}$ differences outside $Q_{\text {weak }}$ 
specifications that we were often burdened with, even with a very good setup of the polarized source. This author was charged with recommissioning and recalibrating the helicity corrector magnets. We experimented with measuring the responses immediately downstream of the magnets in order to achieve suppression of transverse phase-space that would hopefully cancel higher-order differences, but we couldn't get reliably stable results on the $Q_{\text {weak }}$ target. Instead the responses of the $\mathrm{HC}$ orbit to the magnets were measured directly at the target. The responses to large and opposite kicks from the magnets are shown in Figure 4.25 . These responses are the equivalent to the PITA slope that is used for the charge asymmetry feedback. While statistical significance on the measurement of position differences is achieved much more slowly compared to the charge asymmetry, in general the $\Delta X, \Delta X^{\prime}$ were fairly stable until a change in beam delivery tune occurred.

After some trials the helicity magnets were continuously implemented beginning in February 7th 2012. The application of the magnets immediately marked great improvement in the HC differences $\Delta X, \Delta Y$ at the target, bringing them within specifications (Figure 4.26). HC angle differences $\Delta X^{\prime}, \Delta Y^{\prime}$ were similarly suppressed as they are highly coupled to position. The corrector magnets were operated at the same setting for both IHWP states, achieving good suppression of HC differences in both states. This is a testament to the good setup of the polarized source: we were able to use the same setting because the position differences don't change much under IHWP reversal, suggesting good control of polarization effects from residual linear light components. Applying the same kick in both IHWP states is also a safety measure in case of electrical pickup from the magnet signal. While we found no indication of this, any residual small effect would be cancelled by the IHWP reversal.

The responses of position and angle differences at the target to the helicity magnets were confirmed and updated daily by this author. We confirmed that the response of the system didn't change unless there was a change in the optics of beam delivery. Unfortunately such changes occurred quite often, usually on the time scale of a few days, as CEBAF operation with all three experimental halls was very demanding. We were however able to measure the new responses quite quickly and apply the helicity magnet feedback as necessary, and the HC beam properties were mostly well suppressed for the remainder of the experiment.

\subsection{Performance: HC Beam Properties in the Experi- ment}

The helicity-correlated differences in beam parameters are plotted in Figure 4.27 through the full Run1 and Run2 $Q_{\text {weak }}$ dataset. The data in the figure are averaged by slug (IHWP state) and 


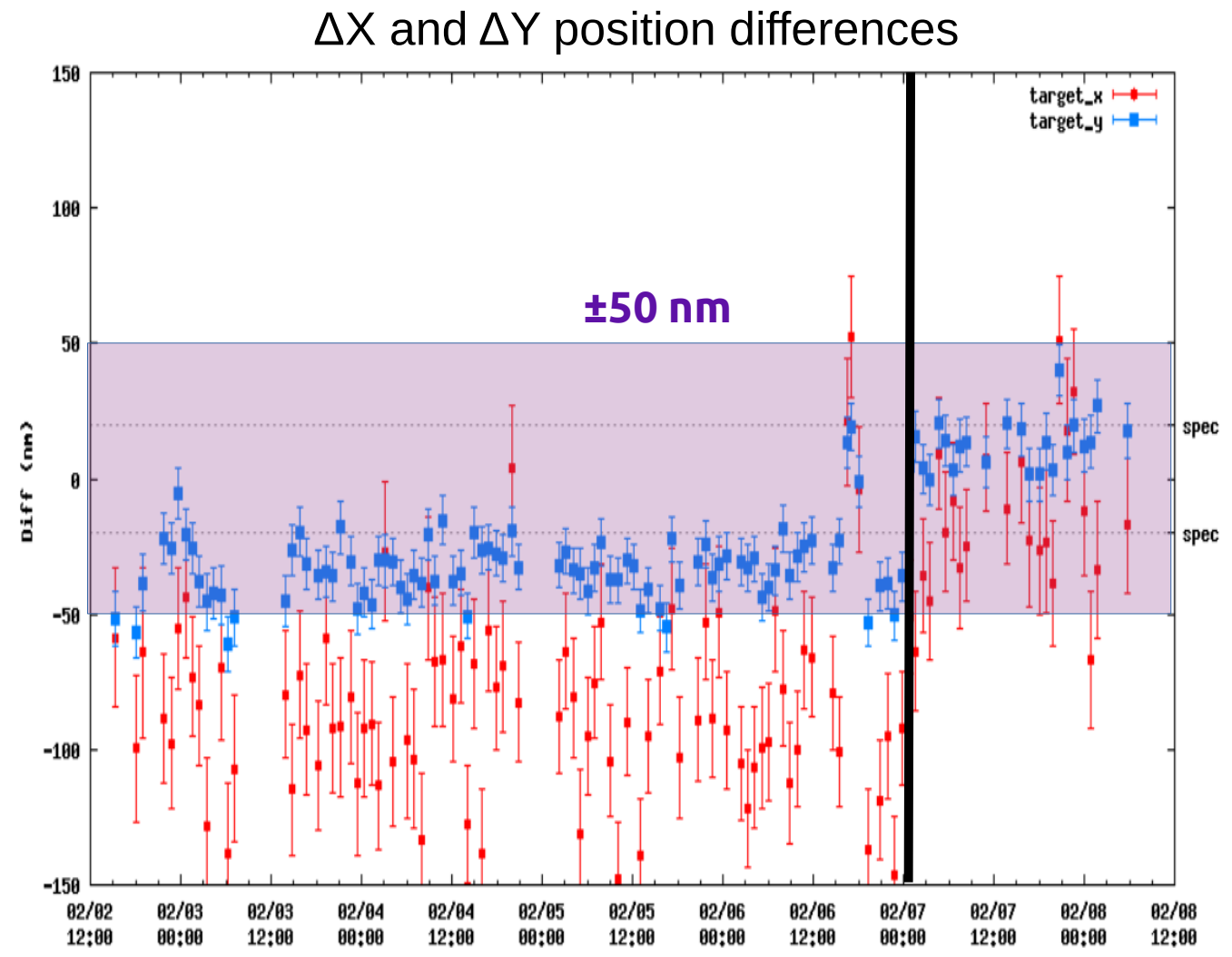

Figure 4.26: Horizontal and vertical (red and blue, respectively) position differences at the $Q_{\text {weak }}$ target versus time, over a period of six days. Each label in the horizontal axis corresponds to 12 hours. The black line marks the beginning of application of feedback from helicity magnets, achieving great improvement in the size of HC differences. Outlying measurements before the black line correspond to tests of the helicity magnet responses. The purple band that marks $\pm 50 \mathrm{~nm}$ of position differences is plotted again for comparison.

color-coded for the different IHWP states. The average values of the helicity-correlated beam properties for the Modulation datasets of Run1 and Run2 (Section 3.9) are listed in Table 4.2. with their run-averaged specification given for comparison.

Some comments can be made that characterize the behaviour of $\mathrm{HC}$ differences in $Q_{\text {weak }}$ :

- The HC differences averaged in individual slugs are very often rather large compared to experimental specifications. For example in many slugs the average position differences are of order $100 \mathrm{~nm}$, much higher than the running average specification of 20-50 nm. This was far less than ideal.

- The HC differences are vastly reduced beginning around Slug 215, when we started implementing position feedback through the helicity magnets, and remain smaller and generally within specification until the end of the run. This feedback was greatly beneficial in keeping the experiment within specification for a significant portion of the dataset.

- The HC differences in general seem to be rather stable within each Wien period, although 
diff_qwk_targetX vs Slug, Qweak

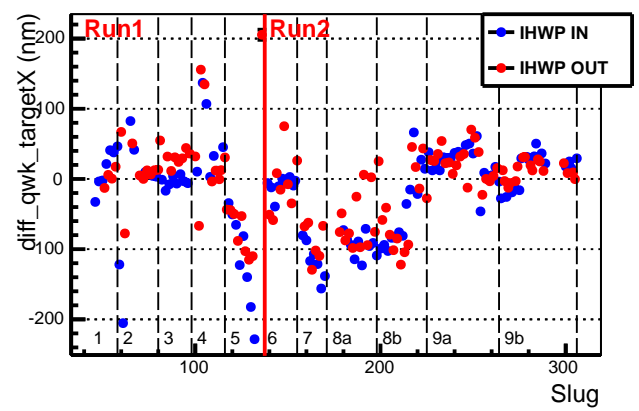

diff_qwk_targetXSlope vs Slug, Qweak

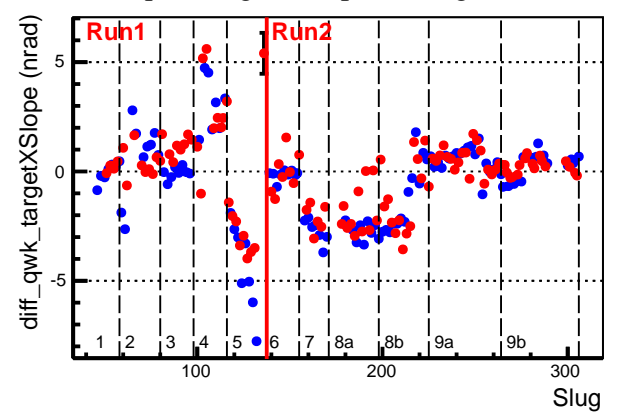

diff_qwk_energy vs Slug, Qweak

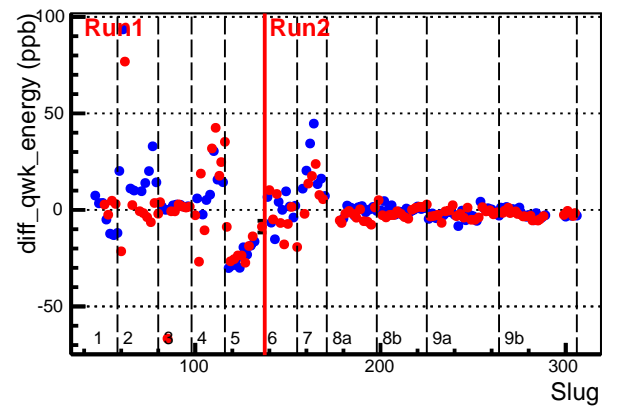

diff_qwk_targetY vs Slug, Qweak

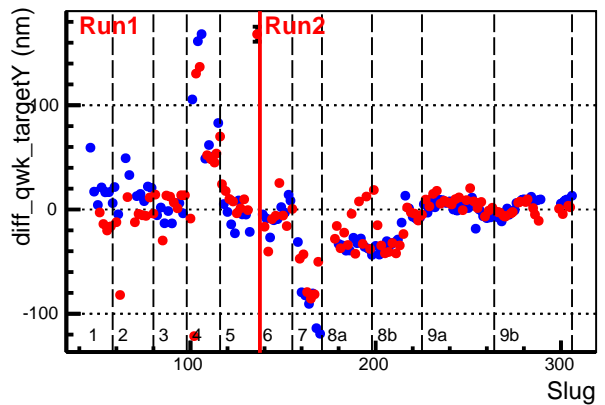

diff_qwk_targetYSlope vs Slug, Qweak

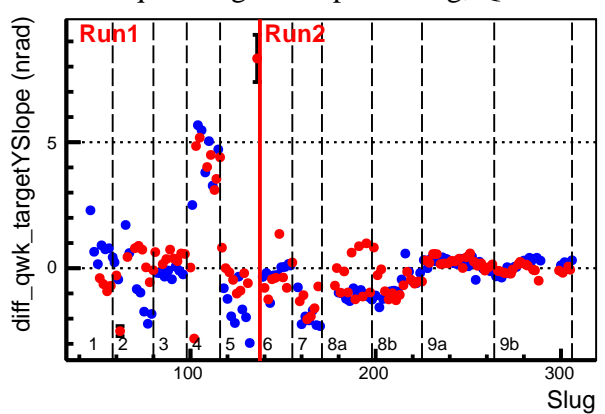

offoff.asym_qwk_charge vs Slug, Qweak

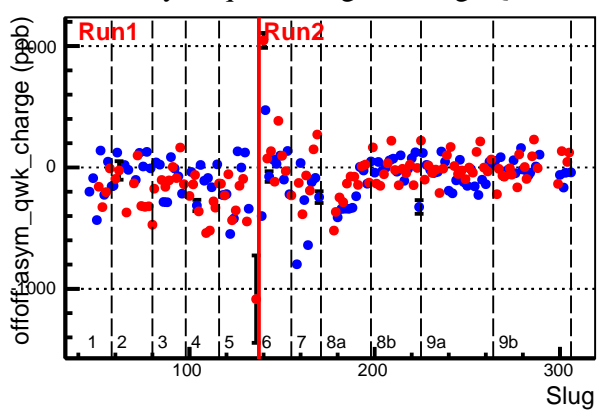

Figure 4.27: Helicity-correlated differences in beam parameters, plotted versus slug through $Q_{\text {weak }}$. Data is color-coded by IHWP state (blue for IHWP in, red for out). No sign-correction is applied. The red line marks the separation between Run1 and Run2. The dashed black lines mark separation between Wien periods.

occasionally a slow drift is observed on a time scale that may be consistent with the development of a "QE hole" effect. On the other hand we usually get abrupt changes in the size and behaviour of the HC properties upon a Wien reversal (marked by dashed vertical black lines). Other large changes in beam conditions that don't involve a Wien flip, include the long down-time for bellows replacement (middle of Wien 2), a significant injector resteering (middle of Wien 4), commencement of position feedback (Slug 215) followed by the "golden period" (Slugs 217-225) ${ }^{11}$ and spot moves on the photocathode.

- Note that the HC differences plotted in Figure 4.27 are not sign-corrected for reversals applied to the Physics asymmetry from the IHWP and Wien reversals. Therefore when HC

\footnotetext{
${ }^{11}$ The injector retune was motivated by the same large HC properties that motivated feedback from the corrector magnets. Feedback from the corrector magnets was not applied during the "golden period", however position differences became large again after the Wien reversal in Slug 226 and feedback had to be applied again.
} 
Table 4.2: Average helicity-correlated beam properties in the Modulation datasets of Run1 and Run2, and comparison with specifications.

\begin{tabular}{l|ccc} 
Parameter & $\begin{array}{c}\text { Max run-averaged } \\
\text { HC value }\end{array}$ & $\begin{array}{c}\text { Run1 } \\
\text { (Modulation set) }\end{array}$ & $\begin{array}{c}\text { Run2 } \\
\text { (Modulation set) }\end{array}$ \\
\hline Beam intensity & $\left\langle A_{Q}\right\rangle<10^{-7}$ & $-5.0 \pm 2.9\left(10^{-} 8\right)$ & $2.8 \pm 1.4\left(10^{-} 8\right)$ \\
Beam energy & $\langle\Delta E / E\rangle \leq 10^{-9}$ & $-2.0 \pm 0.3\left(10^{-} 9\right)$ & $0.36 \pm 0.18\left(10^{-} 9\right)$ \\
Beam position & $\langle\Delta X\rangle<2 \mathrm{~nm}$ & $1.6 \pm 1.2 \mathrm{~nm}$ & $2.2 \pm 0.9 \mathrm{~nm}$ \\
& $\langle\Delta Y\rangle<2 \mathrm{~nm}$ & $-6.3 \pm 0.9 \mathrm{~nm}$ & $0.2 \pm 0.4 \mathrm{~nm}$ \\
Beam angle & $\left\langle\Delta \theta_{X}\right\rangle<30 \mathrm{nrad}$ & $-0.15 \pm 0.04 \mathrm{nrad}$ & $-0.05 \pm 0.02 \mathrm{nrad}$ \\
& $\left\langle\Delta \theta_{Y}\right\rangle<30 \mathrm{nrad}$ & $0.04 \pm 0.04 \mathrm{nrad}$ & $-0.05 \pm 0.01 \mathrm{nrad}$
\end{tabular}

differences remain constant in consecutive slugs of opposite IHWP state, the false asymmetries $A_{\text {beam }}$ will be opposite in sign and cancel. The good stability of the HC differences under IHWP reversal is excellent news for successfully removing false asymmetries; it suggests that the polarized source was set-up very well to remove the "polarization" class of false asymmetries, which would not cancel under IHWP reversal.

- With the exception of $\langle\Delta E / E\rangle$ and $\langle\Delta Y\rangle$ in Run1, average helicity-correlated values for beam parameters are within specification. All beam parameters are within specification in the Run2 Modulation dataset, where we will extract the result of this dissertation.

While achieving excellent control of HC properties at the polarized source, we were often forced to collect data with beam conditions outside our specifications. Higher-order effects and correlations between beam parameters may have created issues in correcting for the effect of false beam asymmetries, as we will see in the following chapter. Higher-order effects of HC differences may have also been related to the presence of halo and large background asymmetries in the experimental hall, which will be studied in Chapter 6). It is considered that the systematic issues that will be discussed in the following chapters could have been avoided if we had received the benefits of kinematic suppression. We were however able to achieve significant suppression of first-order HC beam parameters through position feedback. Furthermore, HC beam parameters are relatively insensitive to the IHWP reversal due to good suppression of polarization-related effects in the source setup, which should result in cancellation of the false asymmetries $A_{\text {beam }}$. 


\section{Chapter 5}

\section{Beam Corrections}

Since the $\overrightarrow{e p}$ scattering rate at the $Q_{\text {weak }}$ target depends on beam parameters such as orbit and energy, the helicity-correlated differences in these parameters that were described in the previous chapter will give a helicity-dependent response in the main detector, which translates to a false asymmetry $A_{\text {beam }}$ in our measurement:

$$
A_{\text {beam }}=\sum_{i=1}^{N} \frac{\partial A_{\text {raw }}}{\partial \Delta x_{i}} \Delta x_{i}
$$

where $\partial A_{\text {raw }} / \partial \Delta x_{i}$ is the sensitivity of the measured asymmetry $A_{\text {raw }}$ to changes in beam parameter $x_{i}$, and $\Delta x_{i}$ is the HC difference in that beam parameter .

To first order we consider a set of 5 beam parameters $(N=5)$ : horizontal and vertical beam position $(X$ and $Y)$ and angle $\left(X^{\prime}\right.$ and $\left.Y^{\prime}\right)$, and beam energy $(E)$ at the $Q_{\text {weak }}$ target. It was considered favourable to work in terms of the constructed target beam parameters as these were the coordinates used in the simulations before the experiment to determine the detector sensitivities. The numerous BPMs in the drift region upstream of the target facilitate the calculation and monitoring of the beam parameters at the target. Alternative combinations of monitors were also tested, sometimes with $N>5$ and even including the BCM $A_{Q}$ measurement in the sum of Eq.(5.1).

Ideally the constructed variables should isolate and decouple the position/angle/energy information in an orthogonal way, however the beam orbit parameters were often highly correlated due to imperfect matching. The energy calculation at the target (Eq. 3.10 was complicated by residual dispersion after the arc and into the Hall $\mathrm{C}$ beamline, that modifies the transport matrix elements of the OptiM model.

To estimate and correct the false asymmetry $A_{\text {beam }}$ generated by $\mathrm{HC}$ differences $\Delta x_{i}$, we still need to know the sensitivities $\partial A_{\text {raw }} / \partial \Delta x_{i}$ of the measured asymmetry to each of these beam 
parameters. It is from estimations of these sensitivities, suppressed by the azimuthal symmetry of the $Q_{\text {weak }}$ apparatus, that the experimental specifications for control of $\mathrm{HC}$ beam parameters were set (Table 4.1).

Detector sensitivities to beam parameters were measured from two independent techniques:

- "Regression" refers to the extraction of detector sensitivities from natural beam motion during production, and

- "Modulation" offers a completely independent extraction from deliberate modulation (or "dithering") of individual beam parameters.

Ideally the correction for $A_{\text {beam }}$ should be consistent from the two independent techniques. However separate systematic issues associated with each technique may give rise to a discrepancy. The redundancy of the two independent techniques allows for an important cross-check that can in principle be used to estimate the systematic uncertainty of the correction.

In this chapter we present the two methods and their variations to extract detector sensitivities $\partial A_{\text {raw }} / \partial \Delta x_{i}$. Several issues are discussed that complicate the $A_{\text {beam }}$ correction. The size and uncertainty of the $A_{\text {beam }}$ correction will be extracted for Run2, and the analysis status for Run1 will be presented.

\subsection{Regression}

Regression is the procedure to extract the detector sensitivities $S_{x_{i}}=\partial A_{\text {raw }} / \partial \Delta x_{i}$ from multivariate linear regression on normal (random) beam jitter, from regular production data. This procedure is designed to completely remove the correlations of the dependent variable (DV), the detector asymmetry $A_{\text {raw }}$, to the set of $N$ independent variables (IVs), the HC beam parameters $\Delta x_{i}$. From Eq 5.1, the covariance between the measured asymmetry and a regression IV is:

$$
\operatorname{Cov}\left(A_{\text {raw }}, \Delta x_{k}\right)=\frac{\partial A_{\text {raw }}}{\partial \Delta x_{k}} \operatorname{Var}\left(\Delta x_{k}\right)+\sum_{\substack{i=1 \\ i \neq k}}^{N} \frac{\partial A_{\text {raw }}}{\partial \Delta x_{i}} \operatorname{Cov}\left(\Delta x_{k}, \Delta x_{i}\right) \quad, \quad k=1 \rightarrow N
$$

for each regression variable $\Delta x_{k}$. The covariances $\operatorname{Cov}\left(A_{\text {raw }}, \Delta x_{k}\right)$ and $\operatorname{Cov}\left(\Delta x_{k}, \Delta x_{i}\right)$, as well as the variances $\operatorname{Var}\left(\Delta x_{k}\right)$ for $k=1 \rightarrow N$, are all calculated analytically from production quartets. From the $N$ equations then we can extract the detector sensitivities $S_{x_{k}}=\left(\partial A_{\text {raw }} / \partial \Delta x_{k}\right)$. The analytical calculation and extraction of detector sensitivities is repeated in every 5-minute run segment ("runlet").

The detector sensitivities to horizontal and vertical beam position, $S_{X}$ and $S_{Y}$ respectively, have been extracted for every individual MD bar plotted versus octant in Figure 5.1 The max- 

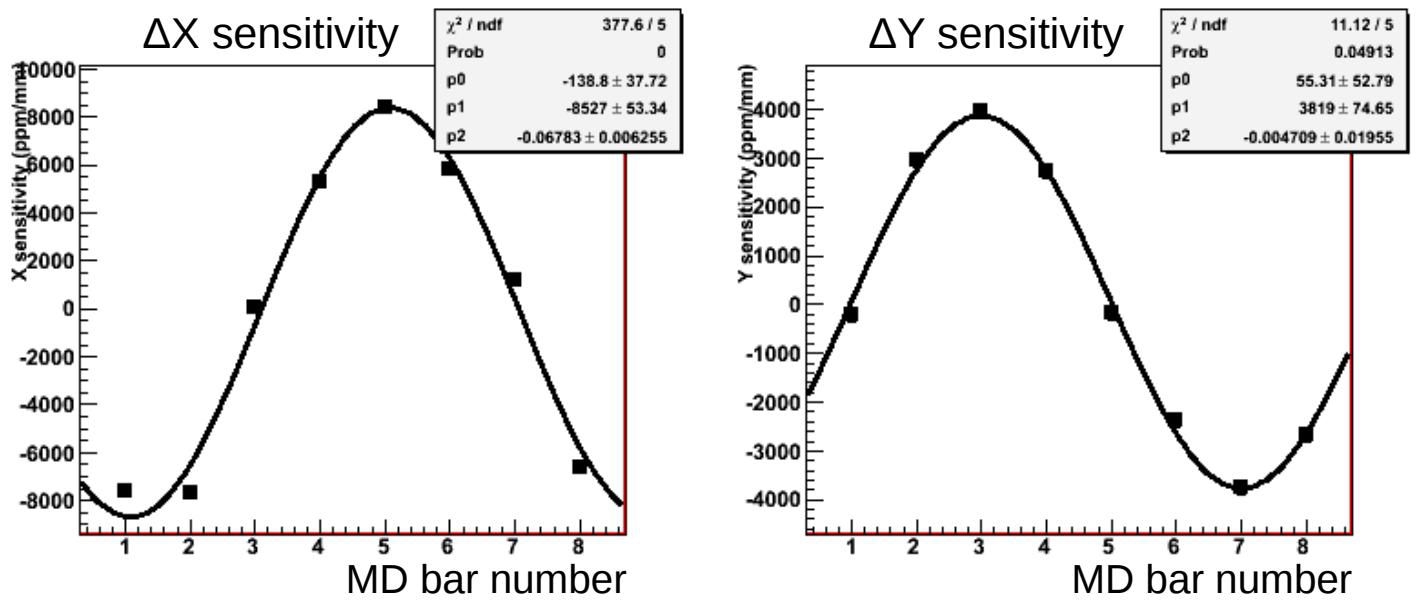

Figure 5.1: MD sensitivities to horizontal (left) and vertical (right) beam position, plotted versus MD octant.

imum sensitivities to horizontal beam motion are observed on the horizontal bars 1 and 5 , as expected, and similarly the vertical bars 3 and 7 have maximum sensitivity to vertical beam motion. The sensitivities exhibit a characteristic sinusoidal ("dipole") pattern across the azimuthal coverage, as the sensitivity on a single detector bar is opposite to its diametrically symmetric detector. Thus cancellation of false asymmetries is achieved when we take the combination MDall of all detector bars, owing to the azimuthal symmetry of the detector system.

In the absence of systematic problems, the regression procedure should accurately produce sensitivities to correct for false beam asymmetries $A_{\text {beam }}$, as long as the set of $N$ beam monitors holds the complete information on the effect of beam differences. We use $N \geq 5$ monitors to access the 5 degrees of freedom (DOF) of beam energy and horizontal/vertical position/angle. However, measurements of BPMs can be highly correlated such that one contributes no extra information. As a result the constructed target variables are often highly coupled, at the quartet time scale (Figure 5.2) as well as averaged over longer time scales (averaged over slugs in Figure 3.20 . The highly correlated BPMs can hardly be considered as separate degrees of freedom and we are motivated to try including different BPMs and BCMs that may provide better resolution for orthogonal information.

Various combinations of regression monitors were considered, with some characteristic schemes listed in Table 5.1. Regression schemes are defined by including different BPM combinations to extract position and angle information, and some also include the BCM $A_{Q}$ measurement to the algorithm as a charge IV. The BPM 3C12X was always used to access beam energy, either as a stand-alone IV or within the constructed $E_{\text {target }}$ variable for energy at the target. For example, that is the only difference between the standard scheme and set11. In retrospect this made no significant difference, as we gain no additional information by forming different combinations 


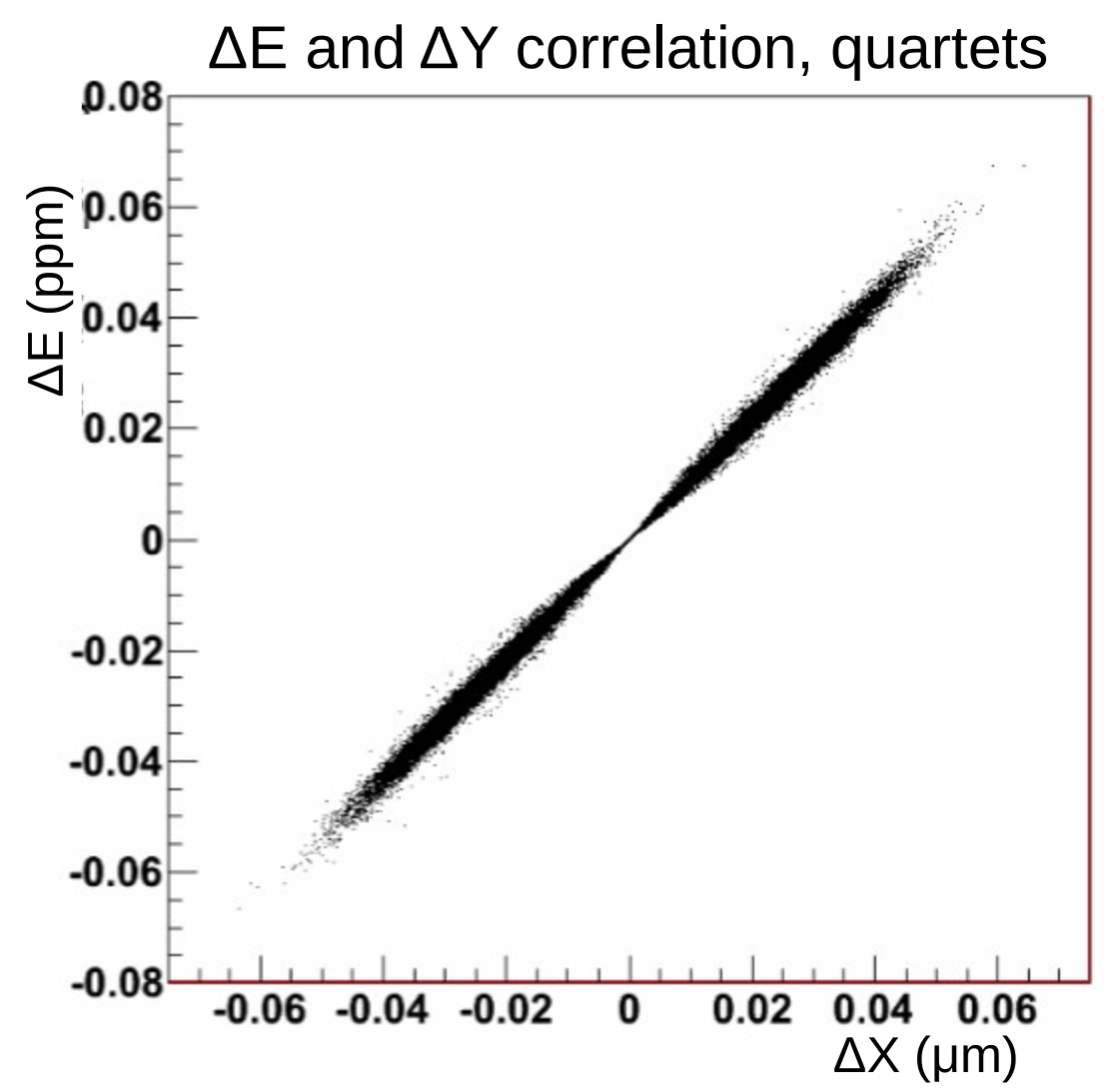

Figure 5.2: Correlation between $\mathrm{HC}$ differences $\Delta X$ and $\Delta E$, over quartets in a $Q_{\text {weak }} 5$-minute runlet. The correlation coefficient approaches 0.999 .

from the same monitors. However since our regression basis consists of highly correlated variables, we can glean insight on the underlying issues by recombining the variables into orthogonal constructs.

\subsubsection{Potential issues with Regression}

There are several issues that may affect the Regression procedure. In this section we list some strong indications of these issues. Most of the potential issues appear to have been more important in Run1 compared to Run2.

\section{Bi-modality of parameter distributions}

An interesting effect first discovered by this author is an intermittent bi-modality in the profiled correlation between beam parameters, possibly associated with non-gaussian tails in the distribution of horizontal position differences (Figure 5.3). The same bi-modality is injected through regression to the MD asymmetries and in their correlation to $\Delta X$, although it was absent before regression. 
Table 5.1: List of regression schemes with the monitors included in them. "Target" corresponds to the constructed orbit and energy variables at the target, that enter Eq. 3.10 .

\begin{tabular}{c|c|c|c|}
$\begin{array}{c}\text { Regression } \\
\text { schemes }\end{array}$ & Orbit & Energy & Charge \\
IVs & IV & IV \\
\hline std & target & energy & - \\
$5+1$ & target & energy & charge \\
set4 & target & BPM 3C12X & BCM5 \\
set6 & BPMs 9b+4 & BPM 3C12X & charge \\
set10 & BPMs 9b+4y & BPM3C12X & BCM6 \\
set11 & target & BPM3C12X & -
\end{tabular}
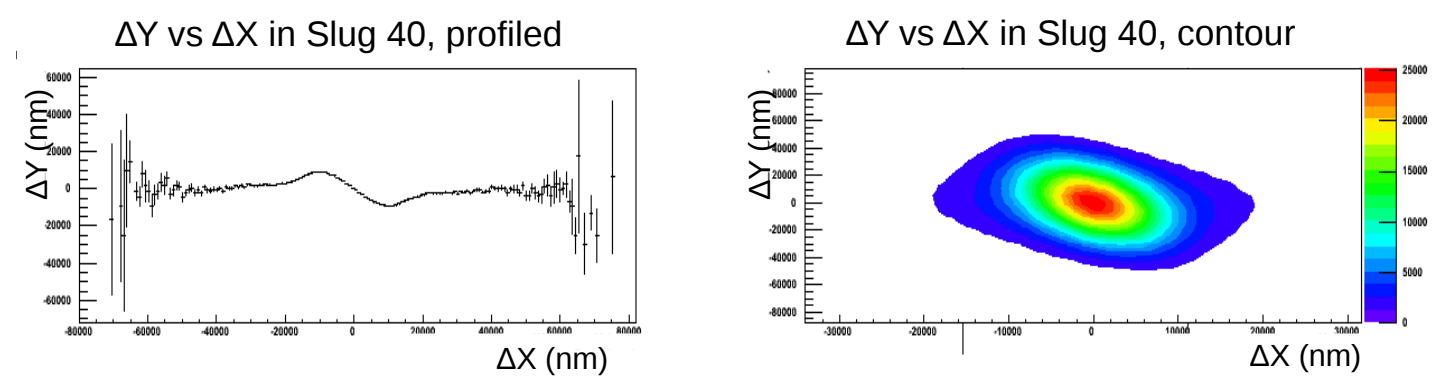

Figure 5.3: Strange bi-modality in $\Delta Y$ to $\Delta X$ correlation (quartet scale) over Slug 40. Potential "double-peaking" in the $\Delta X$ distribution could give rise to this behaviour.

This phenomenon was modeled under different assumptions [150] that involve two separate components in the $\Delta X$ distribution having identical mean but different RMS, or conversely identical RMS but different mean. If the separate components were characterized by different correlations

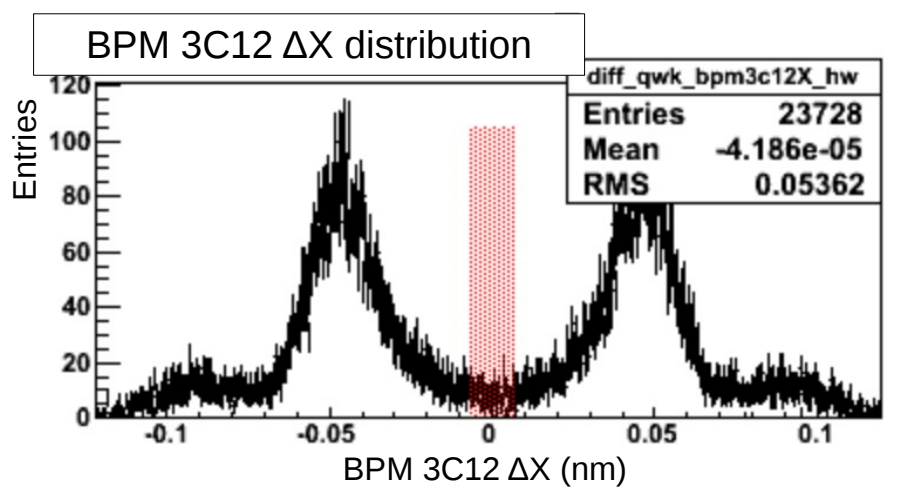

Figure 5.4: Double-peaking in the $\Delta X$ measurement of the energysensitive arc BPM 3C12, resulting from Linac phase instabilities. The separation of the two peaks occurred sporadically, but was typically much smaller than in this case.

to other regression parameters

or the MD asymmetry, this could create issues with the regression procedure. A possible source for this effect is an oscillation in the beam energy induced by a diagnostic/lock mechanism, the Master Oscillator Modulator (MOMod) and the associated "phase-lock" PID lock, at times operating "off-crest" relative to the RF timing of the Linacs [151]. Off-crest operation was an attempt to improve criteria of beam quality in the experimental hall, such as background and halo rates. The oscillation resulted in an energy modulation which also manifested as doublepeaking in the $\Delta X$ distribution of the arc BPM 3C12X. The typical peak separation, when 

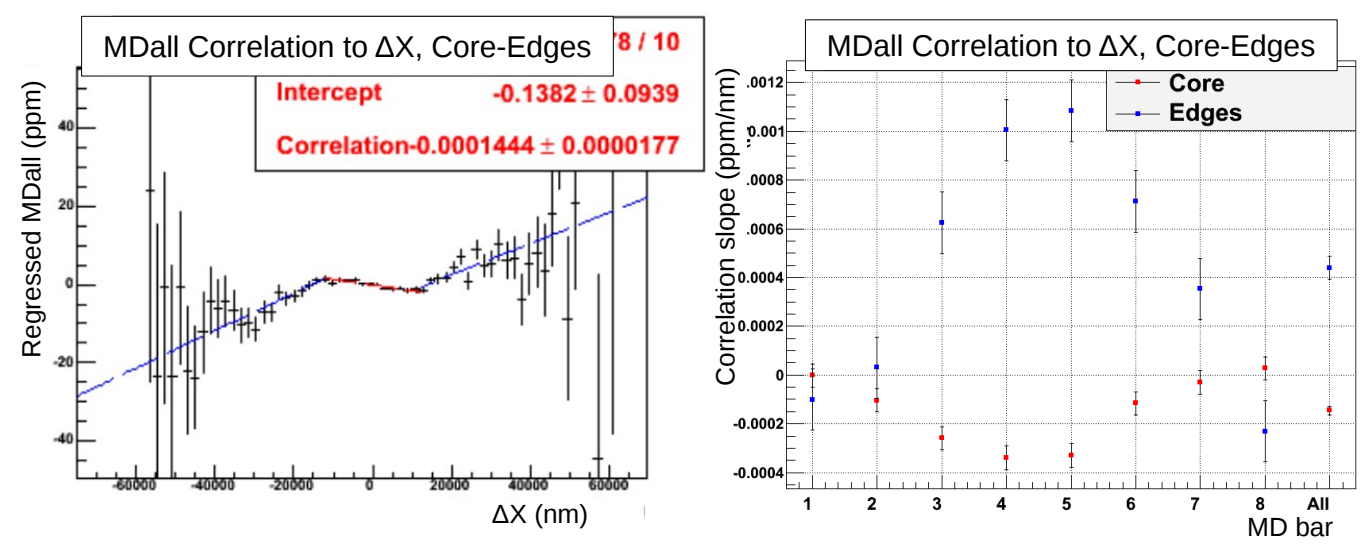

Figure 5.5: Left:Quartet-level correlation of the regressed MDall asymmetry to $\Delta X$ over Slug 36 . The "edges" of the $\Delta X$ distribution are fitted separately, in blue, from the "core" that is fitted in red. Right: Applying the fit for each MD bar, the extracted correlation slopes are plotted separately versus MD bar for the core (red) and edges (blue) of the distribution. The right-most correlation slope corresponds to the MDall combination, as extracted from the fit on the left.

observed, was usually significantly smaller than in the unique case of Figure 5.4 .

While regression is guaranteed by construction to remove the average correlation to the IVs at the quartet scale, it is possible that higher-order effects are folded into this procedure with unpredictable effects. A study by this author demonstrates that while the average correlation of regressed asymmetries vanishes by construction, it is still possible to have residual correlations in different regions of the beam phase-space as long as the average remains consistent with zero. In this study the correlation of the regressed asymmetry of individual MD bars was extracted separately for the smaller and larger values of $\Delta X$, referred to as "core" and "tails" of the $\Delta X$ distribution. In Figure 5.5 the correlations of individual MD bars over Slug 36 are fitted separately for $|\Delta X|<12 \mu \mathrm{m}$ (the "core" of the distribution, which contains most quartets) and for $|\Delta X|>12 \mu \mathrm{m}$ (the possibly non-gaussian "tails"). The latter is fitted separately for the positive and negative large values of $\Delta X$ and a single number is obtained from the average of the two. Then the extracted correlation slopes for the core and tails of the distribution are plotted separately versus MD bar. Because the MD asymmetries are regressed (separately on every 5minute runlet of the slug), the average of the two values is consistent with zero by construction on every bar, and yet each separate contribution is significantly non-zero. Furthermore these residual correlations exhibit the characteristic "dipole" pattern of false asymmetries $A_{\text {beam }}$ across the MD octants, suggesting residual higher-order effects left over from the regression correction.

Again, this study exhibits the possibility of separate regions of the beam phase space having different HC properties, which is consistent with the longitudinal variation of HC properties observed in the chopper scan described in Section 4.7.4 


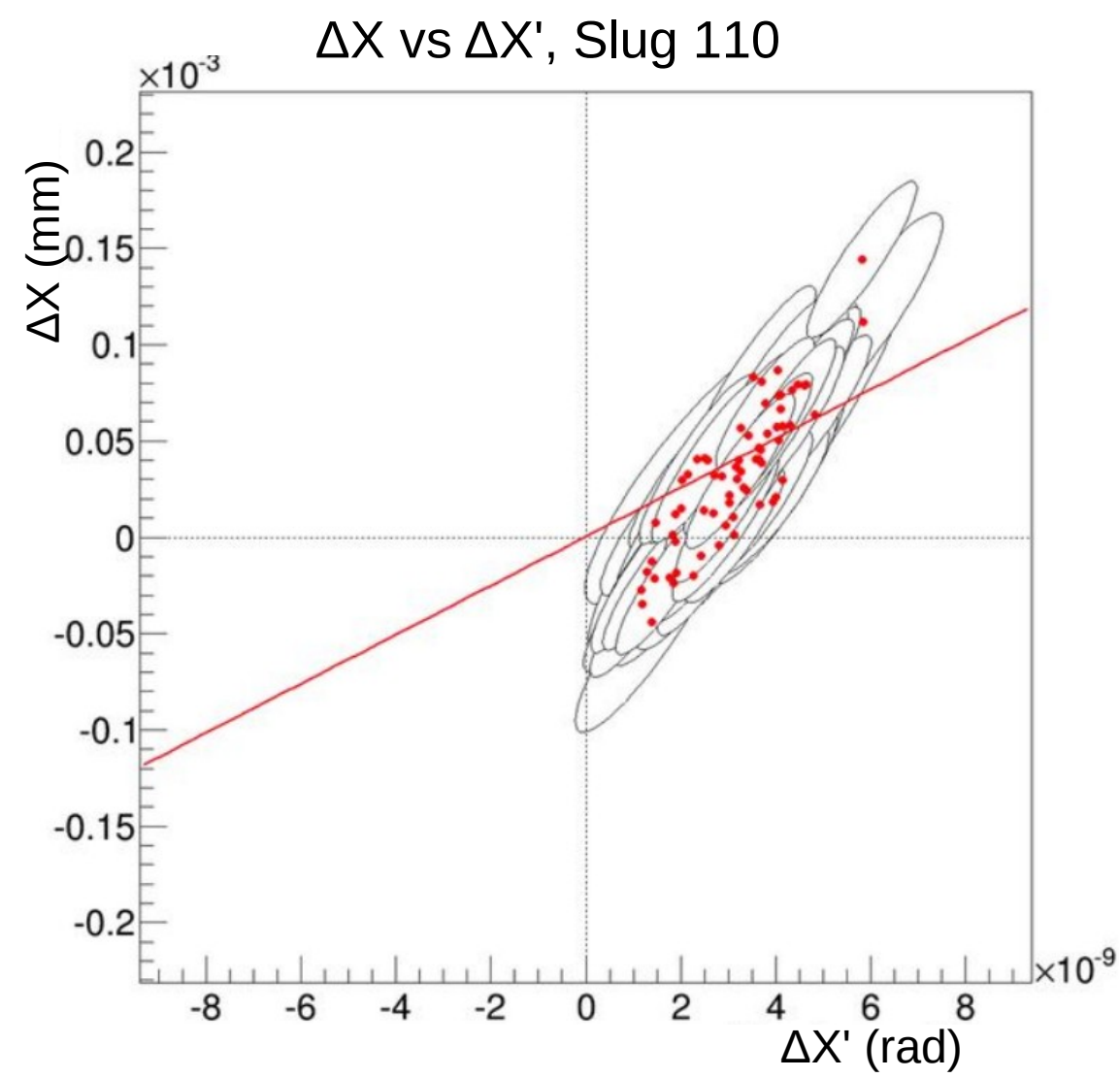

Figure 5.6: Correlation between $\Delta X$ and $\Delta X^{\prime}$ at the target in Slug 110. Every red point corresponds to the average values in a 5 -minute runlet within that slug, and the ellipse around that point is characteristic of the width and correlation of the quartet-scale $\Delta X$ and $\Delta X^{\prime}$ distributions. The red line is the linear fit for a forced zero offset. From [152].

\section{Hidden variables}

Higher-order beam parameters, such as beam halo and spot size/shape, are not adequately accessed by the BPMs that form the regression basis. Their contribution then is hidden and may be responsible for residual false asymmetries that are inaccessible by regression.

Figure 5.6 is a powerful depiction of what is possibly an important issue with regression. The ellipses in the figure are characteristic of the quartet-scale correlation between $\Delta X$ and $\Delta X^{\prime}$ in every runlet in Slug 110. The quartet-scale correlation slope (the tilt of the ellipses) appears to be consistent with the slope of the runlet-binned data, and the correlation coefficient between the two parameters is high.

However, while the two highly correlated parameters are effectively reduced to a single degree of freedom, the two don't simultaneously go to zero. Rather a significant offset exists which describes a separate degree of freedom. The offset however is not included in the regression formalism; it is removed in the calculation of the centered covariances in Eq. 5.2 , where only the correlation slopes effectively matter. Then the resulting sensitivities $S_{X}=\partial A_{\text {raw }} / \partial \Delta X$ 
and $S_{X^{\prime}}=\partial A_{\text {raw }} / \partial \Delta X^{\prime}$ will be highly coupled but won't have access to the "hidden" degree of freedom represented by the offset. In other words the regression correction will shift the corrected asymmetry along the true correlation $\Delta X-\Delta X^{\prime}$ correlation slope (not the red line shown in the figure) but it will be unable to access the $\left(\Delta X, \Delta X^{\prime}\right)=(0,0)$ point where the false asymmetries are presumably fully removed. The significant offset, considered to be characteristic of effects that are inaccessible to Regression, was shown to be largely absent during Run2 [152].

\section{Real and apparent "non-linearity": Residual correlation to charge asymmetry}

An important suggestion for residual effects after the detector has been corrected through regression is provided by the residual correlation of regressed detectors to charge asymmetry $A_{Q}$, at the quartet time scale. This was often referred as detector "non-linearity", since it mimics a non-linear detector response after the detector has been normalized to the BCM charge. The true detector non-linearity was constrained within $\lesssim 0.5 \%$ through dedicated LED studies on the test bench, and yet the observed values from the residual correlation to $A_{Q}$ were of order $5-10 \%$.

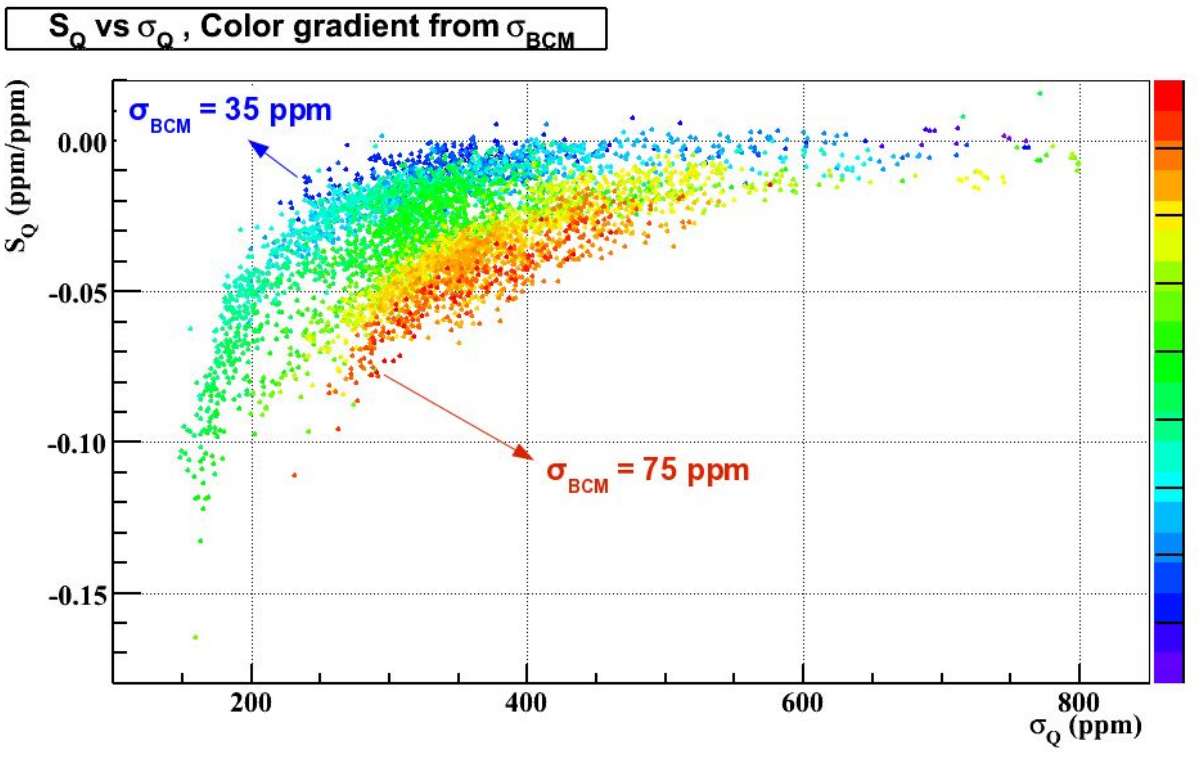

Figure 5.7: Regression sensitivity $S_{Q}$ to charge asymmetry in the $5+1$ scheme, plotted versus charge asymmetry width $\sigma_{Q}$ over Run1. Each point represents a 5-minute runlet where regression sensitivities are extracted. Color-coding corresponds to BCM monitor noise $\sigma_{\mathrm{BCM}}$, extracted from BCM doubledifference.

At first it was considered that residual correlation to $A_{Q}$ was an indication of imperfect beam corrections: a residual false asymmetry, perhaps associated with a hidden variable (such as beam halo) that is inaccessible by the BPM basis but potentially couples to intensity through 
jitter in beam parameters. This motivated the extra regression schemes that incorporate charge asymmetry (like the $5+1$ scheme in Table 5.1) in an attempt to address residual beam effects that are potentially accessed via the charge asymmetry. By construction in these schemes all correlations to charge asymmetry (the apparent non-linearity) will be removed.

However, nearly all of the apparent residual correlation to $A_{Q}$ can be accounted by BCM monitor noise (the BCM resolution $\sigma_{\mathrm{BCM}}$ ) leaking into the detector asymmetry through normalization [153]. Indeed in Figure 5.7 the sensitivity $S_{Q}$ to charge asymmetry (from the $5+1$ regression scheme which includes a charge IV) is trivially always negative and inversely proportional to the charge asymmetry width $\sigma_{Q}$. Increased $\sigma_{Q}$ due to larger uncorrelated beam noise dilutes the monitor noise $\sigma_{\mathrm{BCM}}$ component that is the source of the correlation 11 For higher values $\sigma_{Q} \gtrsim 400 \mathrm{ppm}$ the BCM monitor noise $\sigma_{\mathrm{BCM}}$ is almost completely diluted and $S_{Q}$ near-vanishes. On the other hand, the charge regression sensitivity $S_{Q}$ increases for higher BCM monitor noise $\sigma_{\mathrm{BCM}}$, as measured via the BCM double-difference (Section 3.7.1), which is also expected in this model.

Conversely to the effect described above, the real detector non-linearity $d A / d Q$ (of order $0.5 \%$ ) is expected to inject some noise in the detector asymmetry proportionally to the charge asymmetry width $\sigma_{Q}$. This can be problematic if the regression parameters are correlated through charge jitter, as they are expected to some extent, thus forcing the extracted regression slopes to account for that correlated noise in a regression scheme that doesn't include charge. The regression correction for $\Delta x$, in the presence of a correlation $C_{Q x}$ between $\Delta x$ and charge asymmetry $A_{Q}$ binned at the quartet scale, would be :

$$
\Delta A_{\mathrm{reg}}=S_{x} * \Delta x=\left[d A / d x+(d A / d Q) \frac{\sigma_{Q}}{\sigma_{x}} C_{Q x}\right] \Delta x
$$

In this simplified picture (where other regression parameters are neglected) the true sensitivity is given by the first term, whereas the second term is bias from charge noise. This effect is amplified in the symmetric $Q_{\text {weak }}$ detector as the bias from charge non-linearity is not suppressed by the azimuthal symmetry, unlike the geometric sensitivities to HC position an angle differences.

At longer time scales the noise contributions will average to zero, potentially revealing residual false asymmetries due to systematic bias. We will study this effect in Section 5.4 .

\footnotetext{
${ }^{1}$ Or in other words, increased charge asymmetry noise $\sigma_{Q}$ reduces the reliability of the BCM to represent the source of the correlation, the monitor noise.
} 


\subsection{Modulation}

The beam modulation system uses an independent approach to measure detector sensitivities, by periodically inducing controlled changes in beam parameters and measuring the response of monitors and detectors. The beam position and angle parameters were modulated using a system of 4 air-core dipole magnets located in the Hall $\mathrm{C}$ beamline, that could nominally create decoupled excursions on the $Q_{\text {weak }}$ target for each of the 4 orbit parameters $\left(X, Y, X^{\prime}, Y^{\prime}\right)$ separately. In the standard dithering modes, two coils were pulsed for modulation in a specific beam parameter. The coils referred to as X1 and X2 were used in different combinations of driving amplitudes to give modulation in $X$ and $X^{\prime}$, while the other two coils (Y1 and Y2) give modulation in $Y$ and $Y^{\prime}$. The beam energy was modulated via a superconducting RF cavity in the South Linac of the accelerator.

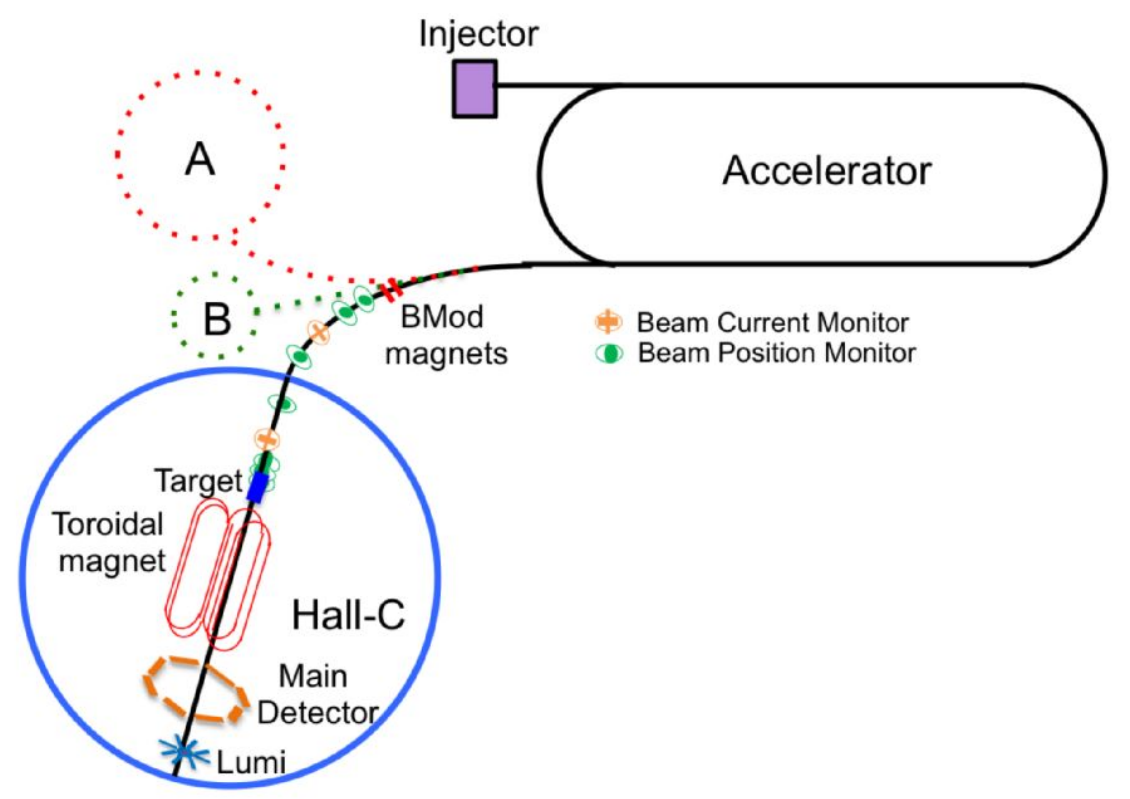

Figure 5.8: Schematic of the location of the dithering magnets with respect to the accelerator and Hall C beamline.

Typical responses of MD bar yields to horizontal position modulation versus the modulation phase are plotted in Figure 5.10. Horizontal octants 1 and 5 have maximum response to modulation of horizontal position, while vertical octants 3 and 7 have minimum response, consistently with the relative size of regression sensitivities from Figure 5.1. The response of the constructed target BPM for horizontal position to the same modulation is a sinusoidal of amplitude $\sim 200 \mu \mathrm{m}$ (Figure 5.9. The driven modulation suppresses correlations between beam parameters, therefore it is generally considered a preferable method to extract the detector sensitivities compared to regression. 

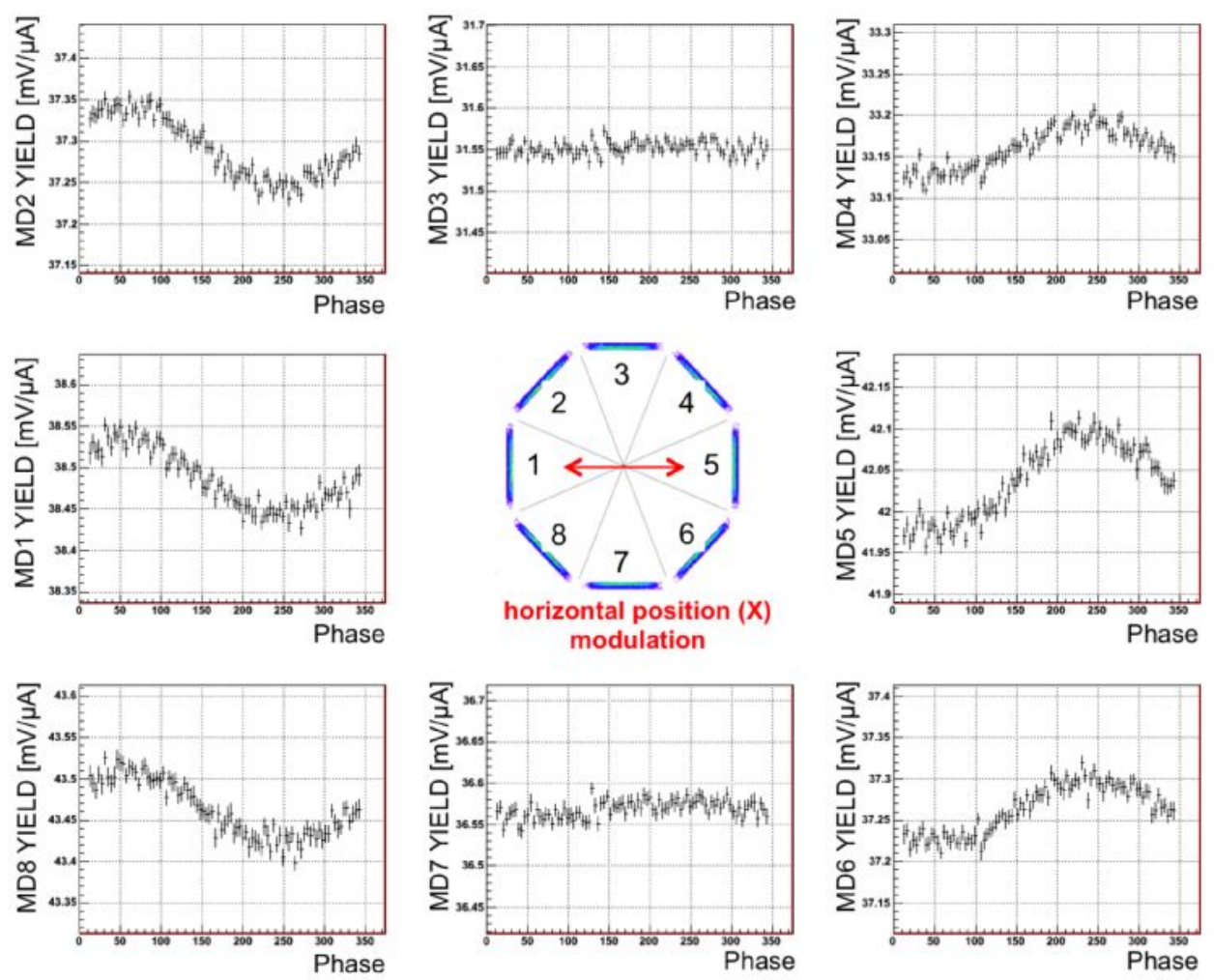

Figure 5.10: Yield responses of MD bars to modulation in horizontal position versus phase of the modulation.

We refer to the modulation of beam parameter $\alpha$ from the driving coils as $C_{\alpha}$. Then the responses of a detector and each BPM to the modulation BPM $X$ response to Modulation

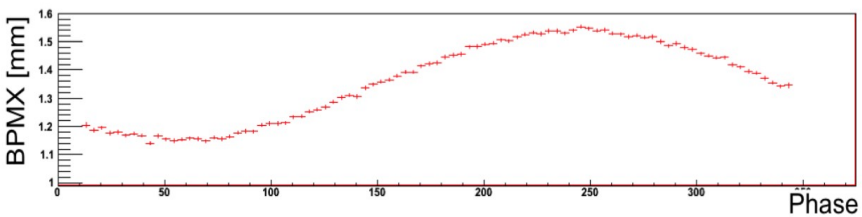

of parameter $\alpha, \partial D / \partial C_{\alpha}$ and $\partial M_{i} / \partial C_{\alpha}$, are measured di-

Figure 5.9: Typical response of the target BPM to horizontal position modulation.

rectly from the modulation periods. The response in the detector is decomposed in terms of the responses in the monitor basis:

$$
\frac{\partial D}{\partial C_{\alpha}}=\sum_{i=1}^{N} \frac{\partial D}{\partial M_{i}} \frac{\partial M_{i}}{\partial C_{\alpha}}
$$

The sum is over the $\mathrm{N}=5$ monitors whose response is measured during modulation. The typical 5-monitor set consisted of the constructed variables for orbit at the target and BPM 3C12X for energy, although more combinations were tested.

The duration and magnitude of the driven modulation were defined such that natural beam jitter and BPM monitor noise would be negligible in the measurement of the responses. A "microcycle" of modulation $C_{\alpha}$ in each parameter consists of 510 iterations of induced dithering at a 
nominal frequency of $125 \mathrm{~Hz}$, with a total duration of 510 iterations $\times(1 / 125 \mathrm{~Hz})=4.08 \mathrm{~s}$. A micro-cycle of modulation in another beam parameter follows after $\sim 75 \mathrm{~s}$ of production, thus modulation has a duty factor of $\sim 6 \%$. A "macro-cycle" consists of completed micro-cycles in each of the 5 modulation parameters, $X, X^{\prime}, Y, Y^{\prime}$ and $E$. When modulation micro-cycles to all five parameters exist then the macro-cycle is complete and a matrix inversion is applied to extract the detector sensitivities from the dithering algorithm. From the 5 equations (5.4) we are able to extract the correction sensitivities $\partial D / \partial M_{i}$ for detector $D$ with respect to each BPM $M_{i}$ included in the monitor basis. The detector sensitivities were extracted from this algorithm with a precision of a few $\%$ in every slug. In principle this level of precision should be more than adequate to correct for false beam asymmetries over the whole experiment, in the absence of systematic errors.

\subsubsection{Interference with FFB}

The beam orbit and energy parameters of the electron beam generally fluctuate, mostly at the power line frequency of $60 \mathrm{~Hz}$ and higher harmonics, due to electromagnetic fields generated by the accelerator electronic equipment. These deviations are largely nulled via the fast feedback (FFB) system by applying real-time corrections targeted at the power line harmonics through four air-core corrector dipoles (located just upstream of the dithering dipoles) and the last RF zone in the South Linac, using the same SRF cavity as we used during modulation. The corrections are calculated using the measurement of BPMs with a sampling rate of $2-5 \mathrm{kHz}$, limited by the BPM resolution.

The FFB system was seen to completely counter the energy modulation from the dithering algorithm such that all response to it was suppressed, since FFB uses the same cavity for feedback, therefore FFB was paused during the energy modulation micro-cycles. To minimize the loss of production time due to the overhead associated with pausing and restarting the FFB system, the energy modulation was applied less frequently than position and angle in Run2. It was also decided that FFB would not be paused during modulation in the beam orbit variables, as its effect on the size of BPM responses to the coil kicks was deemed negligible.

Unfortunately the FFB had subtler effects during position and orbit modulation that were only realized late in the experimental run through studies by this author. Evidence of the interference of modulation with FFB is the variation of the response phase on various BPMs along the beamline to the modulation kick. This phase variation is thought to be the result of interference with the FFB which is attempting to "correct" the deviations induced by the dithering coils. The responses can be considered as the sum of opposing modulations from different locations, with a possible time-lag in the FFB application. The amplitude and phase to 


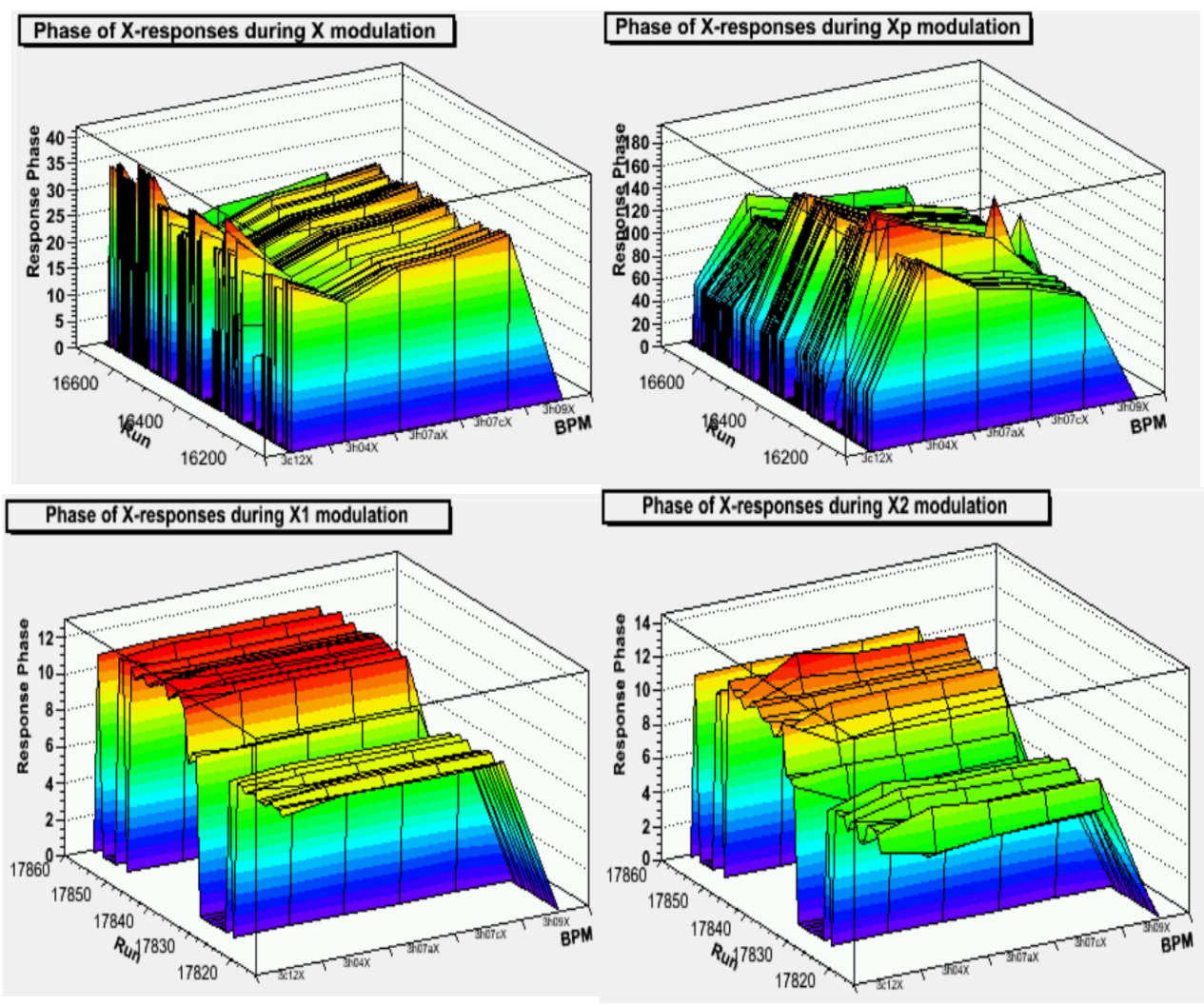

Figure 5.11: Phases of horizontal BPM responses to horizontal position modulation, plotted for BPMs along the beamline over many runs. Top: standard dithering modes for $X$ and $X^{\prime}$ modulation, where the $\mathrm{X} 1$ and $\mathrm{X} 2$ coils are pulsed together and FFB is active. Bottom: response phases during a short period where the two coils were pulsed separately and FFB was paused during modulation.

the sum of the modulation and FFB kicks will vary with position on the beamline as we consider the two independent transfer functions from each of the driving locations to the measurement location.

The phase variation is seen during standard dithering mode on the top plots of Figure 5.11 where the phase of the sinusoidal pattern of horizontal position measurements of BPMs along the beamline is plotted over many runs for $\mathrm{X}$ and $\mathrm{X}$ ' modulation, both driven by the same two coils X1 and X2. The variation of the response phase to modulation kick can be as high as $80^{\circ}$ on various BPMs along the beamline in the same modulation cycle. The bottom two plots show the response phases during modulation with the FFB paused, as part of a test study after the findings on the phase variation ${ }^{2}$ With the FFB paused the phase variation on consecutive BPMs along the beamline is limited within the fit precision of a few degrees.

\footnotetext{
${ }^{2}$ The two coils X1 and X2 were also pulsed separately as part of the study, although the pausing of the FFB is the main reason for the much increased stability of responses.
} 
Table 5.2: Dithering responses decomposed to 10 driving amplitudes, or "coils".

\begin{tabular}{c|c|c} 
Coil number & Modulation type & Response \\
\hline 0 & $X$ & sine \\
1 & $Y$ & sine \\
2 & $E$ & sine \\
3 & $X^{\prime}$ & sine \\
4 & $Y^{\prime}$ & sine \\
5 & $X$ & cosine \\
6 & $Y$ & cosine \\
7 & $E$ & cosine \\
8 & $X^{\prime}$ & cosine \\
9 & $Y$ & cosine
\end{tabular}

\subsubsection{Sine-cosine analysis and residuals}

In the dithering analysis the maximum response amplitude is considered to occur synchronously on every BPM and the detector, therefore the phase variation due to FFB interference can be problematic. The correlation of the modulation responses in the detector and monitors is problematic if the maxima occur up to $80^{\circ}$ apart.

A simple sine fit fails to fully incorporate the action of the FFB. The response on each location on the beamline can be decomposed in terms of a sine and a cosine function, each with different amplitude but with the same phase as the modulation drive signals. Thus effectively the sine part of the FFB action becomes part of the driving signal, while the time-orthogonal cosine part averages out over the micro-cycle. The sine and cosine amplitudes are thus referred to as "in-phase" and "out-of-phase" responses respectively.

This is a more complete approach to understanding the responses during modulation, however the $\partial D / \partial C_{\alpha}$ responses must be extracted separately for the sine and cosine signals, considered as separate driving signals. Thus the dithering analysis of Eq. (5.4) is performed with 10 driving coils $C_{\alpha}, \alpha=1 \rightarrow 10$, a sine and a cosine for each of the 5 modulation parameters. This became the standard scheme for the dithering algorithm and was referred to as 10-coil analysis. The full list and numbering scheme of the 10 coils and responses is given in Table 5.2 .

Note that the sum in Eq. (5.4) is still over $N=5$ monitors and we still extract the $5\left(\partial D / \partial M_{i}\right)$ sensitivities, but we now have 10 equations. Thus the problem of extracting the detector sensitivities from dithering becomes over-determined. Different dithering schemes are created by omitting combinations of coils from the full 10-coil analysis, i.e. reducing the number of responses considered in the analysis and thereby the over-determinacy of the system of equations. Results from various dithering schemes will be presented in Section 5.6 . As we will see, the dithering schemes created by omitting coil combinations often give inconsistent results. We will eventually assign an uncertainty to the dithering correction by comparing results from schemes 
that should be equivalent.

\subsubsection{Modulation residual responses}

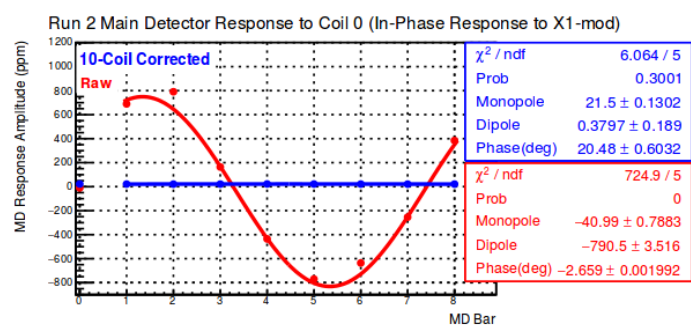

Run 2 Main Detector Response to Coil 3 (In-Phase Response to X2-mod)

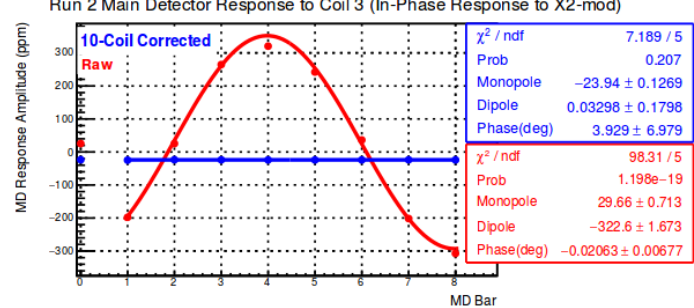

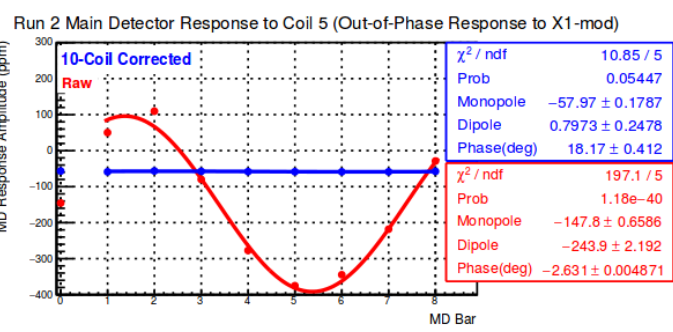

Run 2 Main Detector Response to Coil 8 (Out-of-Phase Response to X2-mod)

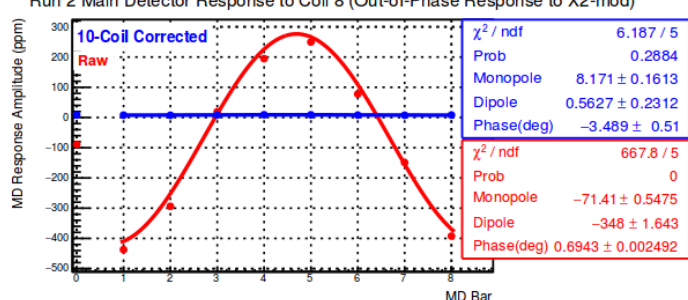

Figure 5.12: The sine/in-phase (left) and the cosine/out-of-phase (right) responses of the uncorrected MD to driven modulation from coils X1 and X2 (top and bottom respectively) is plotted in red, versus MD octant. The sensitivities extracted from these responses are used to correct the MD yields in the same data. Residual responses to the modulation kicks after correction are plotted in blue and are inconsistent with zero.

In Figure 5.12 the responses of MD bars to the driven modulation are plotted versus MD octant. In red are the responses of the uncorrected detectors exhibiting the characteristic dipole shape. After responses have been measured from modulation on all beam parameters the detector sensitivities $\partial D / \partial M_{i}$ are extracted in the 10-coil dithering scheme. Using these sensitivities to correct the MD, we fit again the corrected MD octants to the modulation signal and the responses are plotted in blue.

While heavily suppressed, the corrected (blue) detectors have non-zero responses to the modulation signal. The residual responses have a small dependence on octant ("dipole" fit parameter). The geometrically averaged residual response (the "monopole" fit parameter) is unambiguously significant, suggesting that the residual is mostly consistent among octants. The residuals can be defined as:

$$
R_{a}=\frac{\partial D}{\partial C_{\alpha}}-\sum_{i=1}^{N} \frac{\partial D}{\partial M_{i}} \frac{\partial M_{i}}{\partial C_{\alpha}}
$$

Therefore the extracted dithering sensitivities fail to correct for the detector responses in the same dataset whence they were extracted. This is a worrying internal inconsistency for the dithering analysis. These residuals persist for all dithering schemes and for various combinations of BPM in the monitor basis, including when adding the BCM measurement as a sixth monitor. We remind that regression always removes the dependence of the corrected MD asymmetries to 
the regression parameters by construction, in the same dataset (and time scale of binning) where the regression sensitivities were extracted, and is therefore not subject to such a self-consistency check.

\section{Residual responses of background detectors}

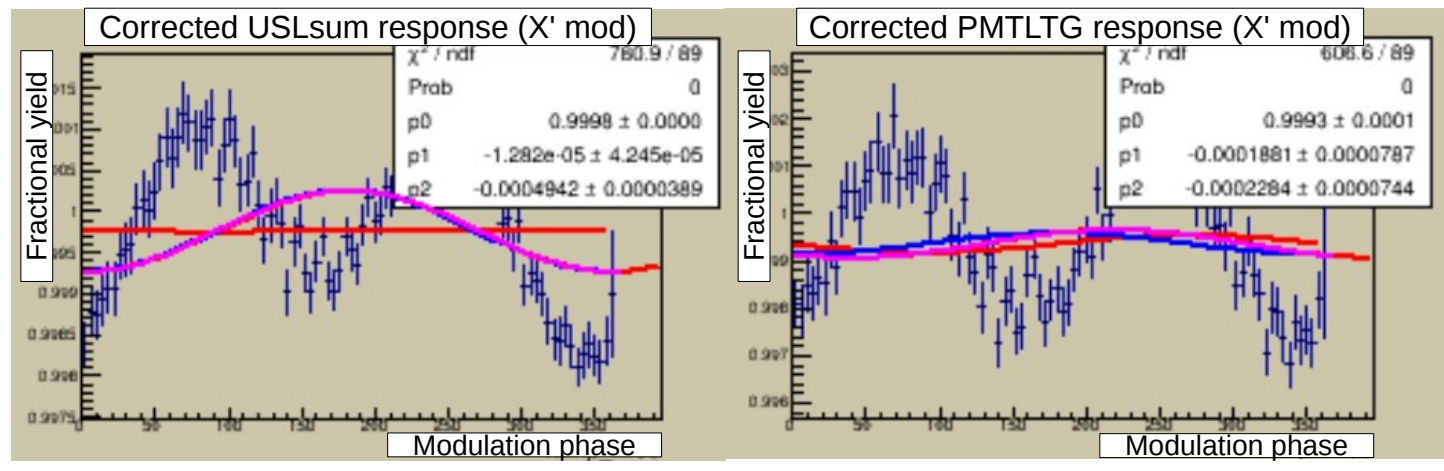

Figure 5.13: Residual response to $X^{\prime}$ modulation, plotted as fractional yield (normalized to the average yield) of background detectors USLsum (left) and PMTLTG (right) versus the modulation phase. The residual response cannot be fitted by the standard sine/in-phase (blue and pink, for fixed or floating fit phase) and cosine/out-of-phase (red) functions. The residuals are highly correlated on all background detectors.

The dithering residuals are generally significantly larger for the background detectors. In fact the decomposition into a sine and a cosine component often fails to describe the residual response of background detectors, as we see in Figure 5.13 It is likely that these responses of background detectors are related to scattering from the plug collimator at the large beam excursions of the induced modulation. This hypothesis is strengthened by the correlation of residual responses on different background detectors, each sensitive to the same beamline background component.

If the beamline background component is correlated to the beam modulation, then it is possible that the same effect is responsible for the residual responses in the main detector. In this scenario it is not surprising that the residuals are much smaller in the MD, as the signal from beamline scattering is significantly suppressed from collimation and shielding compared to the background detectors.

\subsection{Comparison Between Regression and Modulation}

The correction sensitivities $\partial A / \partial \Delta X$ that correspond to horizontal position differences $\Delta X$ are plotted in Figure 5.14 through Run1 and Run2, averaged in every slug. Sensitivities from regression and dithering are compared in the figure. These correction factors correspond to the horizontal MD dipole, defined as the difference between "beam-left" and "beam-right" detector: 3

${ }^{3}$ Specifically the horizontal dipole is defined as the weighted combination
$\left(\mathrm{MD} 1+\frac{1}{\sqrt{2}} \mathrm{MD} 2-\frac{1}{\sqrt{2}} \mathrm{MD} 4-\mathrm{MD} 5-\frac{1}{\sqrt{2}} \mathrm{MD} 6+\frac{1}{\sqrt{2}} \mathrm{MD} 8\right)$.


This specific combination enhances sensitivity to geometric effects of horizontal beam motion, while cancelling contributions that are common to all octants.

Modulation sensitivities are much more stable as they are less dependent to changes in beam conditions and correlations between parameters in beam jitter than regression. Strength-sharing between beam parameters is also suppressed in modulation compared to regression resulting in discrepant correction slopes for each parameter: it is not necessary that the same sensitivity $S_{X}=\partial A / \partial \Delta X$ is extracted from regression and dithering, although ideally the total correction $A_{\text {beam }}$ (summing corrections from all $N$ monitors) will be consistent.

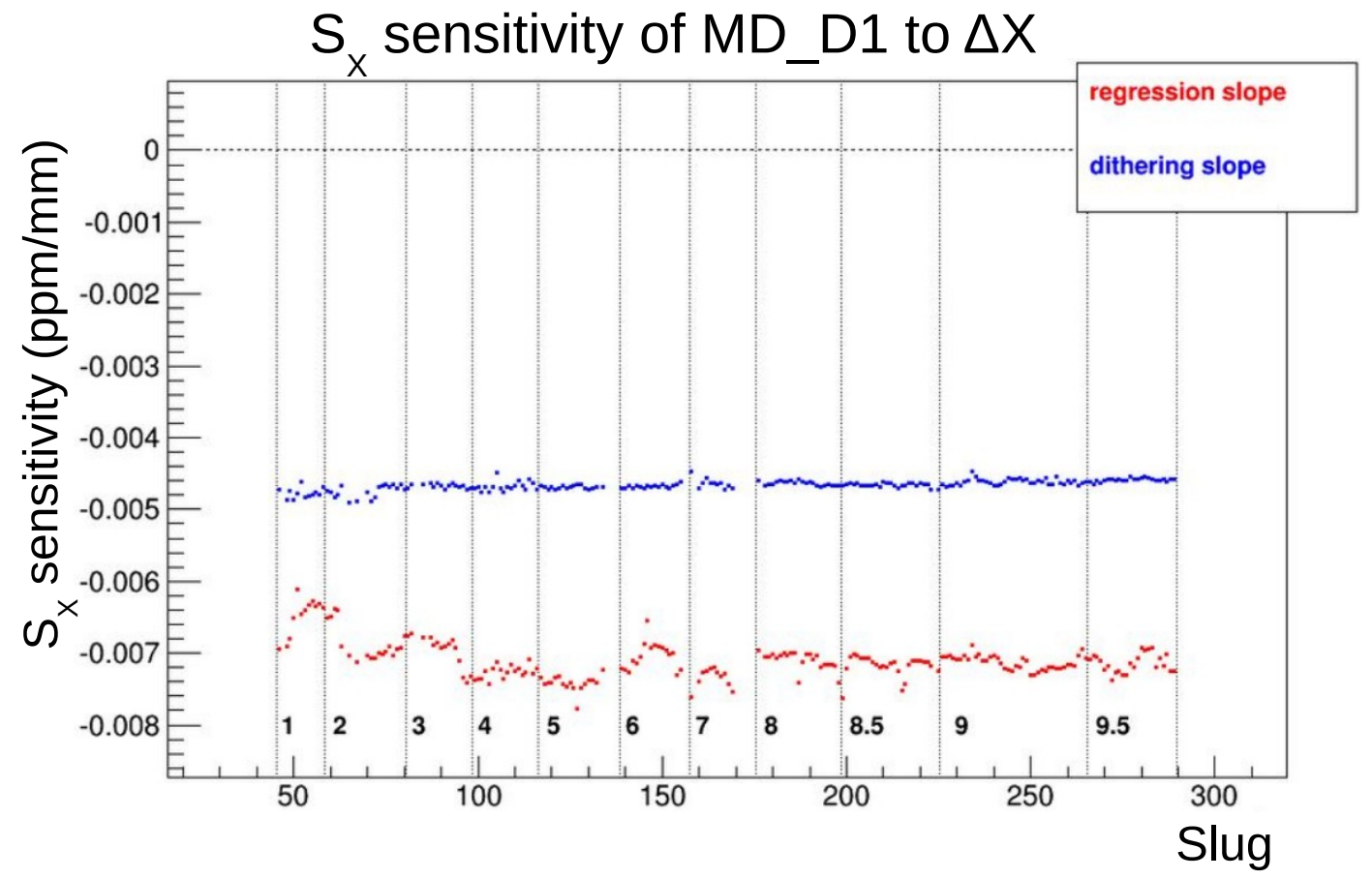

Figure 5.14: Correction sensitivities $S_{X}=\partial A / \partial \Delta X$ from regression (red) and dithering (blue), for the MD horizontal dipole.

Regression provides correction sensitivities from the same dataset that is being corrected, and is guaranteed to remove the effect of beam noise/jitter in the detectors. By construction of the procedure, regressed detectors will have no residual covariance to the monitors that were used in the correction, in that same dataset. Dithering on the other hand provides correction slopes from a separate dedicated period and is not necessarily expected to eliminate residual covariances that are characteristic of beam jitter. This can be seen in Figure 5.15 where the residual covariance of the regressed MD asymmetry to $\Delta X$ is consistent with zero within the precision of the measurement, orders of magnitude smaller than the residual covariance after correcting through dithering sensitivities. This independence of modulation responses to ephemeral characteristics of beam jitter, makes dithering the preferred method to extract the detector sensitivities.

A corollary to the statement that regression completely removes covariances of the corrected 


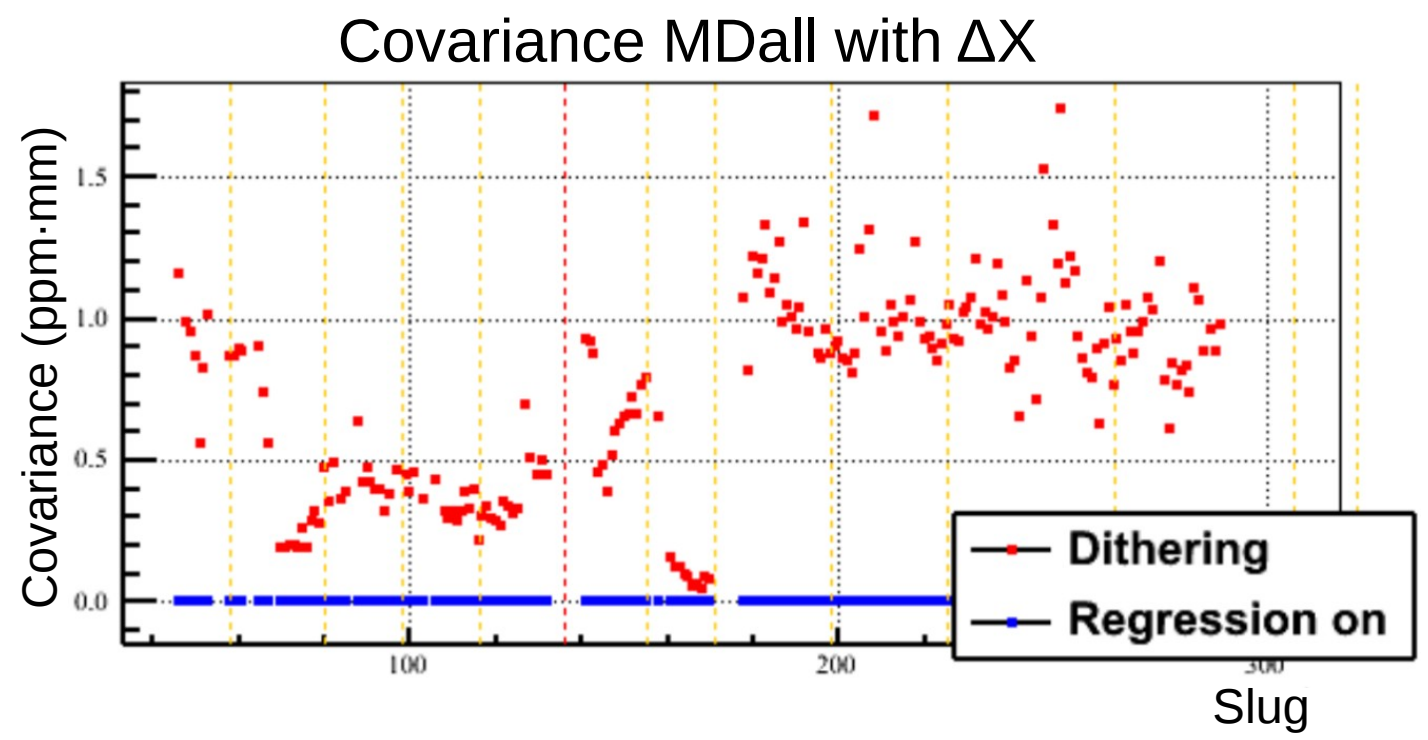

Figure 5.15: Residual covariances of the MDall asymmetry, corrected through regression (blue) or modulation (red) sensitivities, to target $\Delta X$. Covariances have been extracted at the quartet-level in every runlet and averaged in each slug.

detector to the monitor basis, is that the regressed detector has its asymmetry width $\sigma_{A}$ minimized compared to any other possible correction using that specific monitor set. The reduction of $\sigma_{A}$ through the applied correction is characteristic of the size of the beam noise that was removed. In Figure 5.16 the noise removed from different correction schemes is quoted relative to the noise removed from the standard modulation analysis. Negative green points mean that regression removed more beam noise than the standard 10-coil modulation or any of its variants, which is expected since regression is designed to minimize $\sigma_{A}$. Two alternative dithering schemes are also presented in the figure, where the alternative modulation scheme "omit-0,5" (defined by omitting coils 0 and 5 from the analysis) often removes more noise than standard modulation, whereas the alternative scheme "omit-3,8" is always more noisy. In Section 5.5 we will identify that scheme as failing to correct false beam asymmetries.

\subsection{Residual Beam Effects at Longer Time Scales}

It is important to keep in mind the time scale associated with the data presented so far. The covariances plotted in Figure 5.15 correspond to the time scale of a quartet $(4.164 \mathrm{~ms})$ : the covariances were calculated from quartets in each 5-minute runlet, and then averaged over each slug. The regression procedure is also applied at the quartet time scale, therefore we expect these quartet-scale covariances to be consistent with zero for the regressed detectors. However it is possible that these covariances will not remain consistent with zero when data is averaged (binned) at longer time scales. 


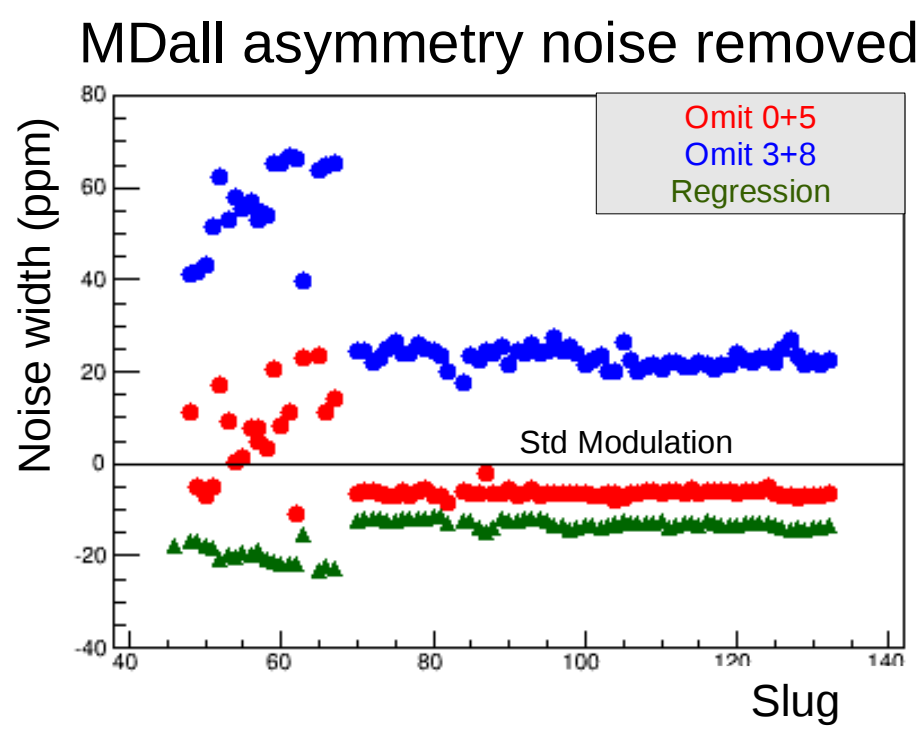

Figure 5.16: Noise removed from the MDall asymmetry width $\sigma_{A}$ through different correction schemes in Run1. The removed noise is defined as the (square root of the) reduction of the quartet-scale MDall asymmetry variance after the applied beam correction, or $\sigma_{\text {noise }}=\sqrt{\sigma_{A_{\text {raw }}}^{2}-\sigma_{A_{\text {corrected }}^{2}}^{2}}$. The results from three correction schemes are quoted relative to the noise removed from the standard 10-coil dithering analysis (horizontal black line at zero). Negative numbers mean that more noise $\sigma_{\text {noise }}$ was removed relative to the 10-coil analysis, so regression removes $\sim 20 \mathrm{ppm}$ more noise than standard dithering, where the apparent "jump" occurs at the month-long down for bellows replacement. The omit- 0,5 scheme also seems to mostly remove more noise than standard dithering analysis, unlike the omit- 3,8 scheme.

Studies by this author revealed for the first time that residual non-zero covariances (or equivalently, correlation slopes) of regressed detectors may appear at longer time scales, even though they are suppressed by construction at the quartet scale. Correlations between parameters at the quartet time scale are characteristic of beam jitter, and the same is true for the regression sensitivities. Jitter in each parameter will average to zero over longer scales, but the effect and correlations of a systematic offset value can be different than the beam jitter correlations.

Two illustrative examples are given in Figure 5.17. On the left, the quartet-scale correlations between $A_{Q}$ and $\Delta X$ are plotted in two different runs where the helicity magnets induce large and opposite position differences, without any effect on $A_{Q}$. Their jitter correlation is consistent in both settings of the magnets. Therefore we should not attempt to "correct" (if $A_{Q}$ was a detector to be corrected) the large systematic offsets in $\Delta X$ via the quartet-scale correlation slope, which is very much like the correction sensitivities extracted from regression. Regression can remove quartet-scale beam noise and minimize the asymmetry width, however it may fail to correct for a systematic offset that doesn't average to zero, such as the position differences of order $\sim 100 \mathrm{~nm}$ that we often experienced. The failure of regression to correct for an offset that is inaccessible through the quartet-scale correlations was discussed in the context of Figure 5.6 On the other hand, the offset applied through the helicity magnets is very much like the induced 

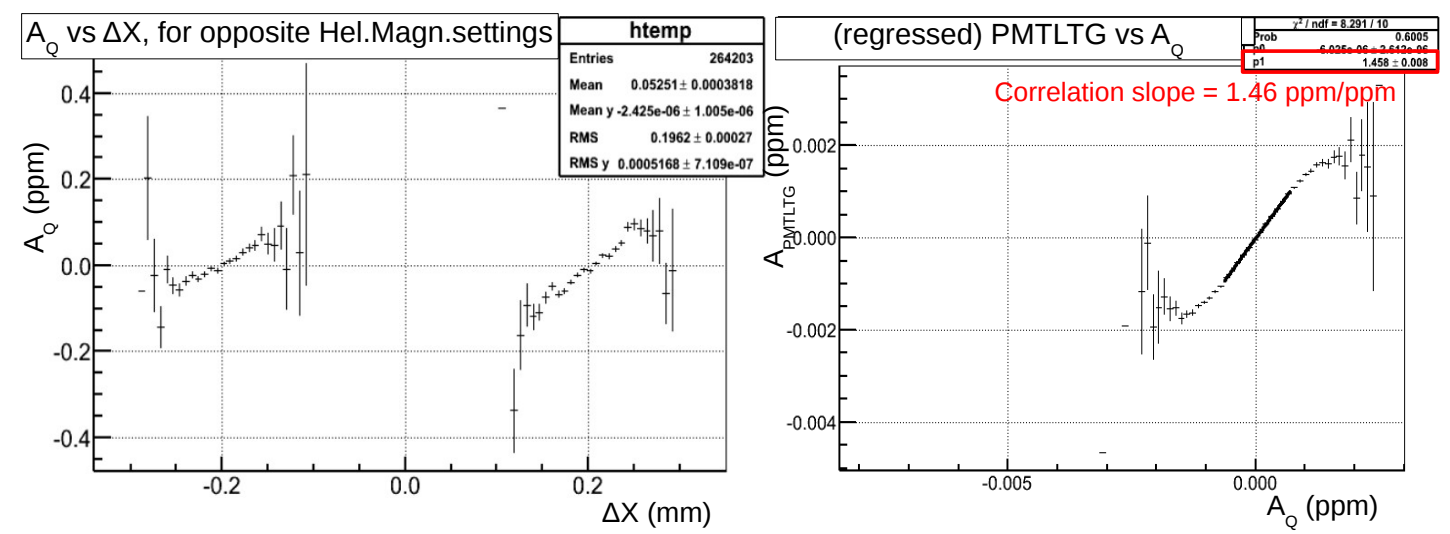

Figure 5.17: Left: Charge asymmetry $A_{Q}$ correlation to position difference $\Delta X$, in two opposite kicks from the helicity magnets. The large systematic position difference imparted by the kick of the helicity magnets does not affect the quartet-scale correlation between charge asymmetry and position difference, characteristic of beam noise/jitter. Right: The quartet-scale correlation slope between the PMTLTG asymmetry and $A_{Q}$ is not appropriate to correct for an offset $A_{Q}$, imparted by the source IA system.

modulation which measures the false asymmetries induced by a systematic offset.

The example on the right of Figure 5.17 directly involves regression applied incorrectly during dedicated runs with the PC voltages turned off. With the PC off feedback could not be applied and a significant $A_{Q} \approx 6 \mathrm{ppm}$ was present on the beam, probably due to drift of parameters in the polarized source setup and especially the appropriate IA Pockels cell voltage to counteract intensity asymmetries with the main PC off. While the asymmetry of uncorrected detectors was consistent with zero as expected, a large asymmetry was induced on background detectors as an artifact of $5+1$ regression. The sensitivity to charge asymmetry $S_{Q}=1.6 \mathrm{ppm} / \mathrm{ppm}$ was not appropriate to correct the $A_{Q} \approx 6 \mathrm{ppm}$ offset. Therefore a large correction was applied through regression, falsely implying a large background asymmetry $A_{\mathrm{PMTLTG}} \approx-10 \mathrm{ppm}$. In the same regression scheme a false correction would be similarly applied on the MD, but it would be smaller and not as evident due to the limited MD statistical power. Again this shows that the quartet-scale sensitivities extracted by regression can be inappropriate to correct for systematic offsets in beam parameters, even if they are small relative to the range of jitter.

\subsubsection{Correlations at long time scales}

This author first developed the technique and tools to examine correlations at longer time scales, demonstrating that corrected detector asymmetries can develop significant correlations to the monitors that were used in the correction. An example is shown in Figure 5.18 where the correlation between the regressed MD5 asymmetry to $\Delta X^{\prime}$ is extracted over Run1, with data

\footnotetext{
${ }^{4}$ This huge sensitivity was not characteristic of normal production. The dedicated studies discussed here were attempts to generate and study beam halo (see Section 6.2, during which the $5+1$ charge regression sensitivities increased by more than an order of magnitude. This is quite interesting in itself and suggests significant coupling between the induced halo and charge jitter.
} 


\section{Regressed MD5 asymmetry to $\Delta \mathrm{X}^{\prime}$, Slug-scale, Run1}

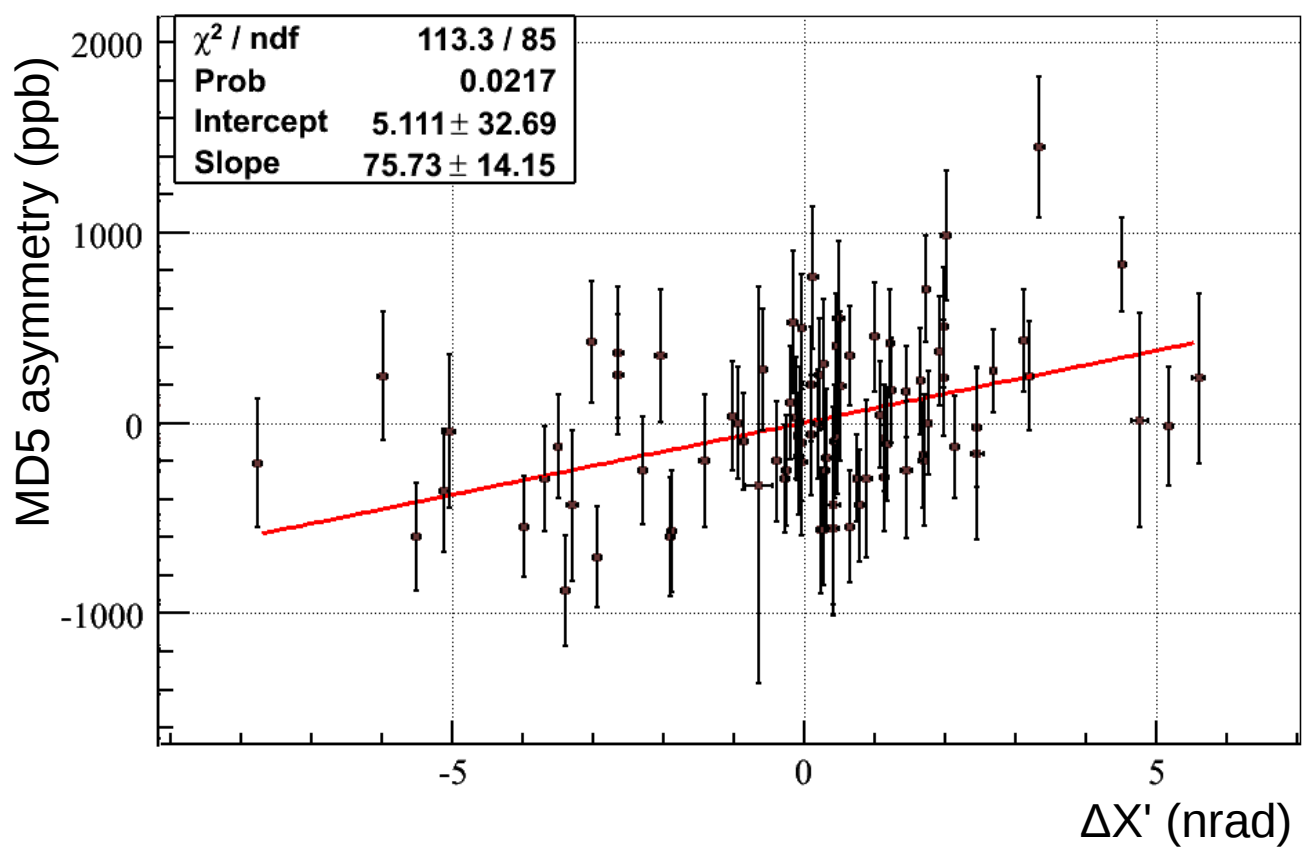

Figure 5.18: Correlation between the regressed asymmetry of bar MD5 and the HC difference $\Delta X^{\prime}$, one of the parameters it has been regressed against. Correlation extracted over all of Run1. Data binned at the slug scale.

averaged over individual slugs. At the much longer time scale of a slug $(\sim 8 \text { hours })^{5}$ the beam noise is averaged to zero and potential systematic effects are revealed. Since the beam correction schemes (both regression and dithering) are designed explicitly to remove the effect of HC differences, any significant "residual" correlation slope at longer time scales could be a sign that the correction has failed and there are residual false asymmetries. The correlation slope in the figure is more than $5 \sigma$ significant, suggesting that regression has failed to adequately remove false beam asymmetries in Run1. Similarly large and significant correlations of the regressed asymmetry to other beam parameters are also observed in Run1 (Section 5.5.

This author also developed a method to exhibit the development of these correlations as data is averaged at longer time scales. The data for the regressed MDall asymmetry $A_{\mathrm{MD}}$ and HC energy difference $\Delta E$ from a single slug (Slug 35) were averaged in successively longer time scales, by combining more and more quartets into a single average. We refer to these averages as "bursts" of quartets. The $A_{\mathrm{MD}}-\Delta E$ correlation slope was extracted in each time scale of averaging over the exact same dataset. The results for the correlation slope are plotted in Figure 5.19 versus the number $N_{\text {quartets }}$ of quartets that are included in each burst, so the

\footnotetext{
${ }^{5}$ Note the vast change in time scales. Regression is applied at the quartet scale of $\sim 4.164 \mu \mathrm{s}$, meaning that every term in the covariance sums of Eq. 5.2 has values averaged over a quartet and calculated over 5-minute segments. On the other hand data in Figure 5.18 is binned at the slug scale (8-10 hours) and the correlation is extracted over all of Run1 (3 months).
} 


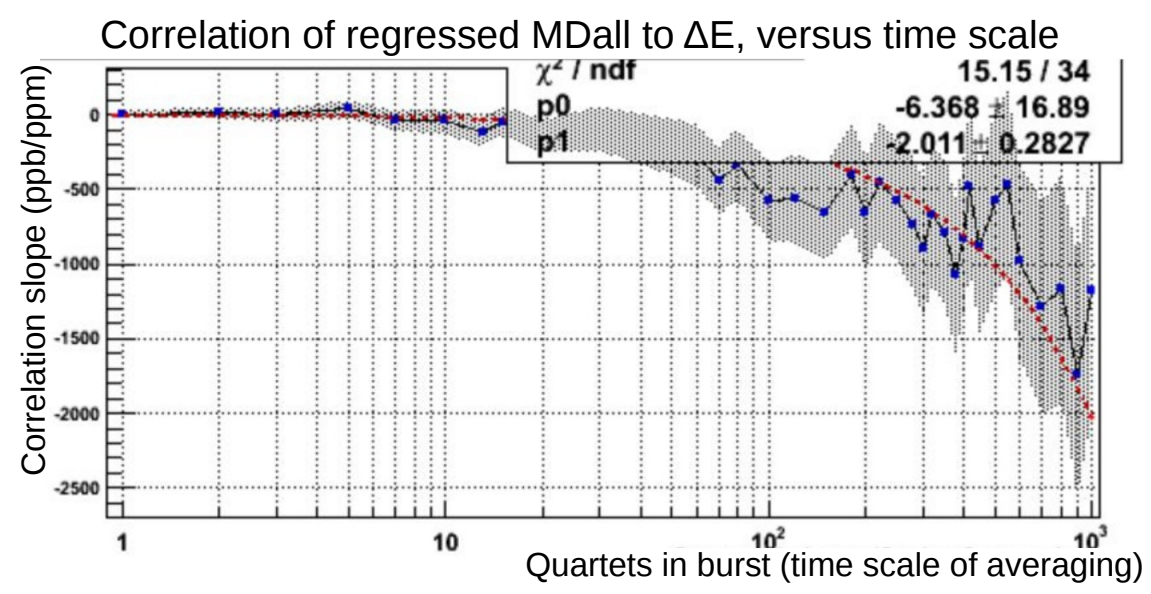

Figure 5.19: Correlation between the regressed MDall asymmetry and $\Delta E$ versus time scale of averaging. A significant correlation develops at longer time scales, while the residual correlation of the regressed MDall asymmetry was zero by construction at the quartet scale.

horizontal axis corresponds to the time scale of averaging. For $N_{\text {quartets }}=1$ the correlation is extracted at the quartet scale where it is zero by construction of the regression procedure, and yet at longer time scales (up to $N_{\text {quartets }}=1000$ ) a significant correlation develops. A linear fit is attempted (although there is no reason that this should be the correct functional form) and a $7 \sigma$ significant dependence to the averaging scale is revealed. That time scale corresponds to a few seconds, still well below the time scale of a slug. The same study was repeated with simulated uncorrelated pseudodata where the extracted correlation slope remained consistent with zero and independent of the scale of averaging. The significant correlation developing at longer time scales in the real data suggests a true systematic effect that is missed by the regression correction.

Correlations at longer time scales proved to be a very important tool for the qualification of $A_{\text {beam }}$ correction schemes, as well as the development of a correction for beamline background asymmetries. In the rest of this section we examine special considerations in the extraction of these correlations at longer time scales, before we present our results over Run1 and Run2 from various correction schemes.

\subsubsection{Time scale and formulation of averaging}

The appropriate time scale of averaging to optimally isolate true systematic effects is an important consideration. The effect of random jitter is not suppressed sufficiently and still influences results at the time scale of the 5-minute runlets. Notice however that the uncertainty of the extracted correlation slope in Figure 5.19 increases with the time scale of averaging $N_{\text {quartets }}$. By increasing the number of quartets in a burst we create fewer bursts of higher precision. But the averaging induces a reduction of the available range over which the correlation is extracted (the "lever-arm" of the fit) thereby reducing the fit precision. This is an issue that must be 


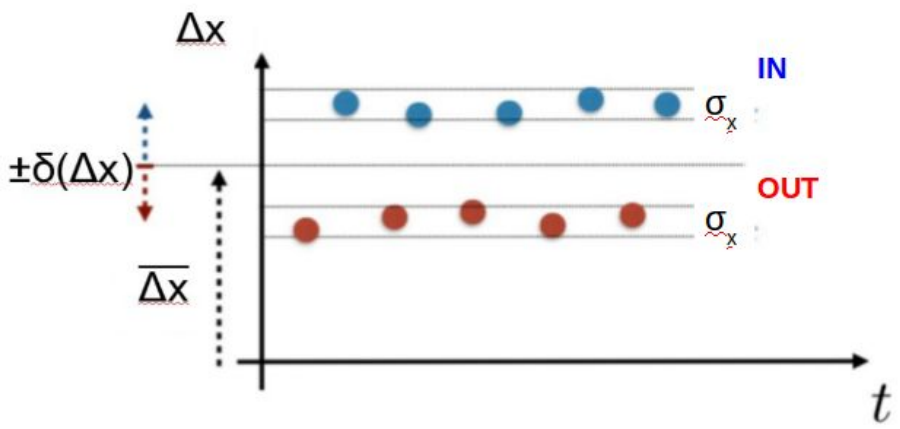

Figure 5.20: Schematic representation of the HC differences in beam parameters, separated into a component that remains constant $(\overline{\Delta x})$ and one that changes sign $(\delta(\Delta x))$ under the IHWP reversal. $\sigma_{x}$ corresponds to random fluctuations.

considered in longer averaging scales. At quartet scale the correlation slope can be extracted with significance over a 5-minute segment, but averaging at the slug scale we usually have to fit over the entire Run1 or Run2 for a significant result.

By considering the correlations over the entirety of Run1/2, we should be mindful that HC differences are rather consistent in consecutive slugs, usually over a Wien period. This makes the fit assumption of independent data points not very good, i.e. the size of a $\mathrm{HC}$ difference in a slug is a good predictor for its size in the following slug. It would perhaps be more appropriate to average the data at yet longer time scales to ensure that they are as independent as possible. We have experimented with different time scales of averaging, combining data from consecutive slugs. We soon however ran into problems when we suppress a lot of the range of variation (the fit lever-arm) by averaging at longer time scales.

We should also consider that in time scales longer than a slug we combine data from different IHWP and perhaps Wien states. To do this we can either apply a sign-correction for the slow reversals or not. The two combinations are referred as:

- the Physics combination, where a sign-correction is applied to account for the sign reversal of the "physics" $\overrightarrow{e p}$ asymmetry; and

- the Null combination, where no sign-correction is applied and therefore the physics is "nulled", or cancelled.

More simply, the expected average Physics combination is the size of the physics $\overrightarrow{e p}$ asymmetry, while the expected size of the Null asymmetry is zero, in the absence of false asymmetries. That makes the Null asymmetry an excellent tool to search for residual systematic effects. 
In combining data over two or more slugs we unavoidably cancel some systematic effects. We can deconstruct the HC differences into a part that changes sign under a IHWP reversal, like the Physics asymmetry does, and a part that doesn't. These components are referred as $\overline{\Delta x}$ and $\delta(\Delta x)$ respectively (Figure 5.20 . Then the HC differences in the two IHWP states can be expressed as:

$$
\begin{aligned}
& \Delta x_{\mathrm{IN}}=\overline{\Delta x}+\delta(\Delta x) \\
& \Delta x_{\mathrm{OUT}}=\overline{\Delta x}-\delta(\Delta x)
\end{aligned}
$$

Therefore in combining data from two successive slugs of opposite IHWP states, The Physics combination (sign-correction applied) will cancel the $\overline{\Delta x}$ component, and the Null combination (no sign-correction) will cancel the $\delta(\Delta x)$ component. The two combinations are plotted in Figure 5.21 over pairs of slugs in Run2. The sign-changing $\delta(\Delta X)$ component that survives in the Physics combination is seen to have suppressed range compared to the sign-constant $\overline{\Delta X}$ component, which is consistent with our observations from Figure 4.27. Again this is a sign that the polarized source was very well setup to suppress the polarization effects that change sign with IHWP. Only the sign-changing $\delta(\Delta X)$ component will contribute potential false asymmetries towards our measurement of the Physics asymmetry.

$\Delta \mathrm{X}$ combinations vs Slug-pair, Run2

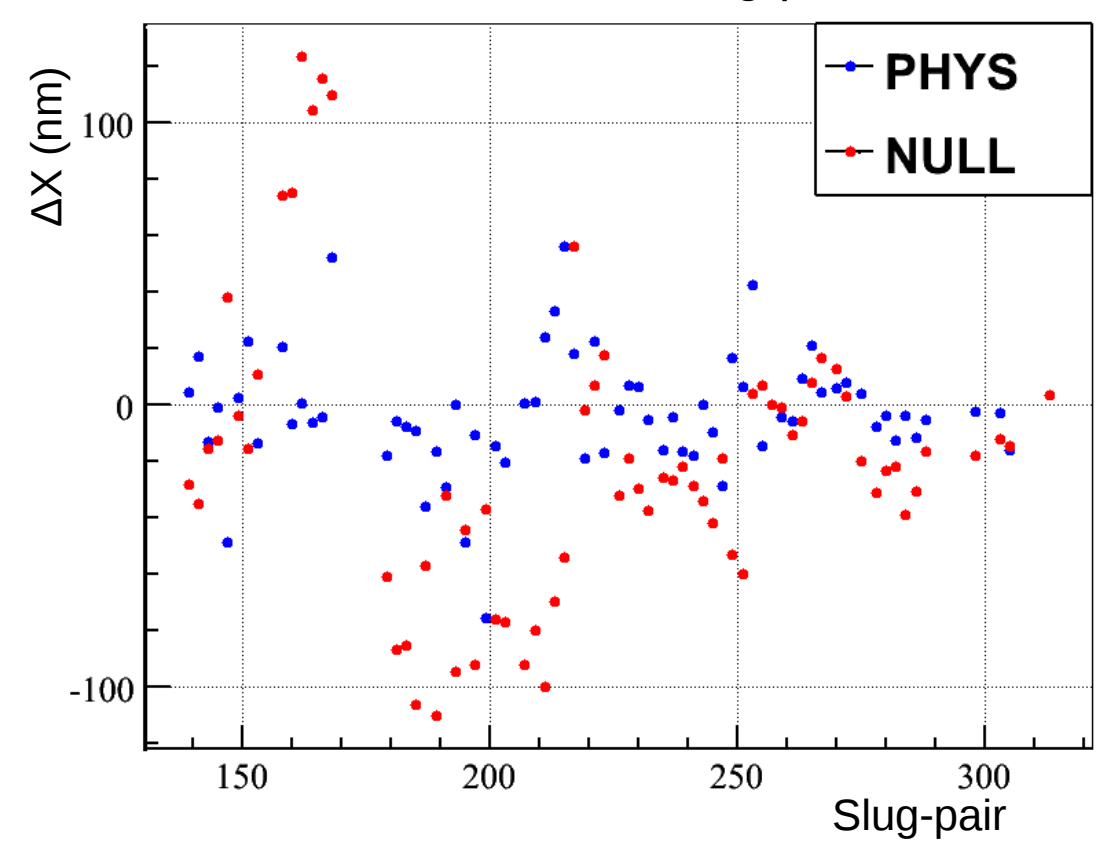

Figure 5.21: HC $\Delta X$ position difference in Run2, averaged over pairs of slugs. The differences are averaged in the Physics (blue) and Null (red) combinations separately in each slug pair.

Of course cancelling parts of HC beam parameter differences is very good news, and suggests that our applied reversals are successful at cancelling residual systematic effects. But it does mean that the lever-arm of the fit is reduced at time scales longer than a slug due to this 
cancellation, making it harder to access the systematic effects of the HC differences. Correlations to $\mathrm{HC}$ differences were studied over longer time scales (averages of two or four slugs) and found to be consistent within their statistical precision. In this dissertation we will limit our study of residual correlations to the slug time scale, which offers a good balance between precision and cancellation of random noise.

\subsubsection{Removing the sign-changing Physics asymmetry}

When the correlation is extracted over a period that is longer than a single slug, then we must consider that the Physics $\overrightarrow{e p}$ asymmetry $A_{e p}$ will have different sign in consecutive slugs due to the IHWP reversal, and similarly for Wien reversals. The sign-reversal of $A_{e p}$ is incoherent to the false asymmetry induced by a $\mathrm{HC}$ difference, the effect of which we want to access. Therefore the sign-changing $A_{e p}$ can be considered as uncorrelated "noise" in our attempt to isolate the false asymmetry $A_{\text {beam }}$ and correlate it to the monitor difference $\Delta x$. If unaccounted, the sign-changing Physics asymmetry would constitute a large noise superimposed on a small correlation. Removing the effect of the Physics asymmetry can be challenging, given that it is unknown (of course, the goal of $Q_{\text {weak }}$ is to measure this quantity) and hard to separate from the sign-changing false asymmetries. Two approaches were developed by this author and are described in the following paragraphs.

\section{Residual formulation}

In the first approach we attempt to estimate and remove the Physics asymmetry from each average with the appropriate sign. The remaining ("residual") asymmetries would therefore be centered to zero, and the correlation of any potentially systematic deviation from zero to the HC differences would be examined. This was termed the Residual formulation of asymmetries.

Of course the true Physics asymmetry is unknown, so we estimate it as the average (signcorrected) MDall asymmetry $\overline{A_{\mathrm{MD}}^{\text {Phys }}}$ in Run2. Then the residual MD asymmetry $A_{\text {Residual }}$ in each slug is:

$$
A_{\text {Residual }}=A_{\text {raw }}-[\operatorname{sgn}] \overline{A_{\mathrm{MD}}^{\text {Phys }}},
$$

where the average MDall asymmetry $\overline{A_{\mathrm{MD}}^{\text {Phy }}}$ is subtracted with the appropriate sign [sgn]. The correlation is then formed between $A_{\text {Residual }}$ and $\Delta x$. Note that even the blinding factor in the measured asymmetry will be also properly subtracted. However for data in Run1 the average MDall asymmetry $\overline{A_{\mathrm{MD}}^{\text {Phy }}}$ should be extracted over Run1, so that the correct blinding term is included.

The estimation of $\overline{A_{\mathrm{MD}}^{\text {Phy }}}$ is unavoidably injecting some noise through the subtraction, due 
to its limited statistical precision precision and potentially unaccounted systematic effects. The statistical precision in the Run2 average should be of order $\sim 20 \mathrm{ppb}$, quite small compared to the $\sim 110 \mathrm{ppb}$ precision of individual $A_{\text {raw }}$ slug averages. However the sign-corrected subtraction

of $\overline{A_{\mathrm{MD}}^{\text {Phy }}}$, if not precisely estimated, will inject a small "false asymmetry" in each slug-average that is coherent with the sign-changing component of a HC difference $\Delta x, \delta(\Delta x)$ (Figure 5.20). This will introduce unavoidable bias in the extracted correlation, amplified in periods where $\delta(\Delta x)$ is large.

\section{Sign-corrected formulation}

The second approach calls for applying an appropriate sign-correction for every reversal to both the MD asymmetry and the HC difference. In the Sign-corrected formulation, the asymmetry in every slug is

$$
A_{\text {Sign-corrected }}=[\operatorname{sgn}] A_{\text {raw }}=A^{\text {Phys+blind }}+[\operatorname{sgn}] C_{\mathrm{MD}-\Delta x} \Delta x
$$

where $C_{\mathrm{MD}-\Delta x}$ is the correlation slope that reveals a systematic false effect arising from $\Delta x$. The correlation to $[\operatorname{sgn}] \Delta x$ should then allow us to access $C_{\mathrm{MD}-\Delta x}$, while $\overline{A_{\mathrm{MD}}^{\text {Phy }}}$ will be the intercept of the fit. This approach in fact unambiguously achieves cancellation of the Physics $A_{e p}$ asymmetry and the blinding factor in the correlation.

However we uncovered potential issues with this formulation as well. There is an implicit assumption in the formalism of Eq. 55.8 that the monitor $\Delta x$ can account for all false asymmetries in the measurement, i.e. there are no residual false asymmetries at $\Delta x=0$. But this need not be the case, in fact we know that false asymmetries can also arise from the other HC beam parameters; while some are highly correlated, we certainly expect some effects that cannot be fully explained by a single parameter $\Delta x$. False asymmetries from backgrounds and other sources are also expected.

The presence of a false asymmetry that does not correlate to the independent variable could create problems in the extraction of $C_{\mathrm{MD}-\Delta x}$ from the Sign-corrected formulation. An illustrative example is given in Figure 5.22 where the Sign-corrected correlation of the regressed MDall asymmetry to $\Delta X$ is extracted over Wien 8 for data averaged at the slug scale. A relative sign is applied to IHWP OUT data (red), to account for the reversal. The correlation is extracted for the two IHWP states separately, marked in blue and red, and then also for both states combined in black. The sign-correction ensures that both IHWP states have the same sign of intercept, although the significant discrepancy in the intercepts for the two states suggests a residual false asymmetry that is decoupled from $\Delta X$. While $\Delta X$ is mostly negative in Wien 8 and has small 


\section{Sign-corrected Regressed Mdall- $\Delta \mathrm{X}$, Wien8}

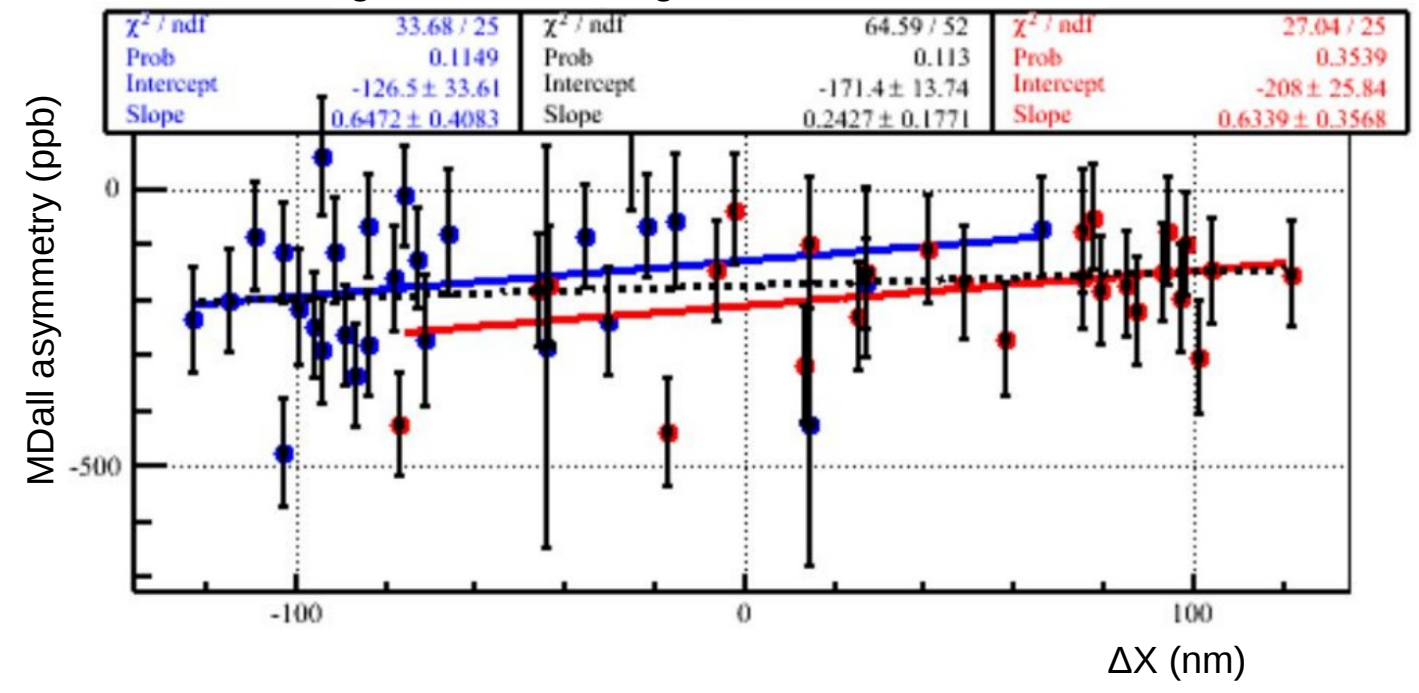

Figure 5.22: Correlation between regressed MDall $A_{\text {raw }}$ asymmetry and $\Delta X$ in Wien 8 , in the Signcorrected formulation. A relative sign is applied to the IHWP OUT data (red) and they are fitted separately (solid lines) from the IHWP IN data (blue). The dashed black line is the combined fit.

dependence to IHWP state (small $\delta(\Delta X)$ component, IHWP cancellation expected to be good), as shown in Figure 4.27, the relative sign applied on IHWP OUT data makes $\Delta X_{\text {OUT }}$ mostly positive.

It is generally very difficult to identify a significant residual correlation slope to beam parameters in a single Wien period, but Wien8 is a period where beam corrections appear to have failed significantly and we can glean a residual dependence in Figure 5.22 Both IHWP states fitted separately see a consistent residual correlation slope to $\Delta X$ developing at the slug scale, $C_{\mathrm{MD}-\Delta X} \approx 0.64 \mathrm{ppb} / \mathrm{nm}$ although only slightly above $1 \sigma$ significance. The combined statistical power of both IHWP states is needed to achieve better precision. The combined fit in the Residual formulation (not shown) gives a correlation slope $C_{\mathrm{MD}-\Delta X}=0.66 \pm 0.26 \mathrm{ppb} / \mathrm{nm}$, very close to the average of the separate IHWP states and revealing a significant effect. However when combining the two IHWP states in the Sign-corrected formulation (dashed black line in figure) the correlation slope appears to be falsely consistent with zero. A residual false asymmetry that is decoupled from $\Delta X$ biases the result in the Sign-corrected formulation. A general formalism for this effect has been developed in [154, where it is shown that the resulting biased correlation slope can be suppressed or magnified due to an unaccounted systematic offset of either sign.

Both the Residual and Sign-corrected formulations have been extensively studied in the extraction of correlations over long time scales. When the correlations are extracted over the entire Run2, we generally don't expect large residual systematic offsets that would bias the result in the Sign-corrected formulation. The results that will be presented in the following section are mostly well consistent from both formulations. 


\subsection{Residual Correlations to HC Differences over Run1 and Run2}

The residual correlations of the corrected asymmetry to beam parameters developing at longer time scales are extracted over the entirety of Run1 and Run2, separately. With data averaged at the slug scale it is necessary to extract correlations over very long periods to get statistically significant results. A single Wien is in some cases a natural time scale for the effect of $\mathrm{HC}$ differences to be considered relatively constant, but beam conditions are expected to change upon Wien reversal. By extracting the correlations over the full Run1 or Run2 we are able to get a statistically significant result, which will be the average systematic effect over different periods included in the fit.

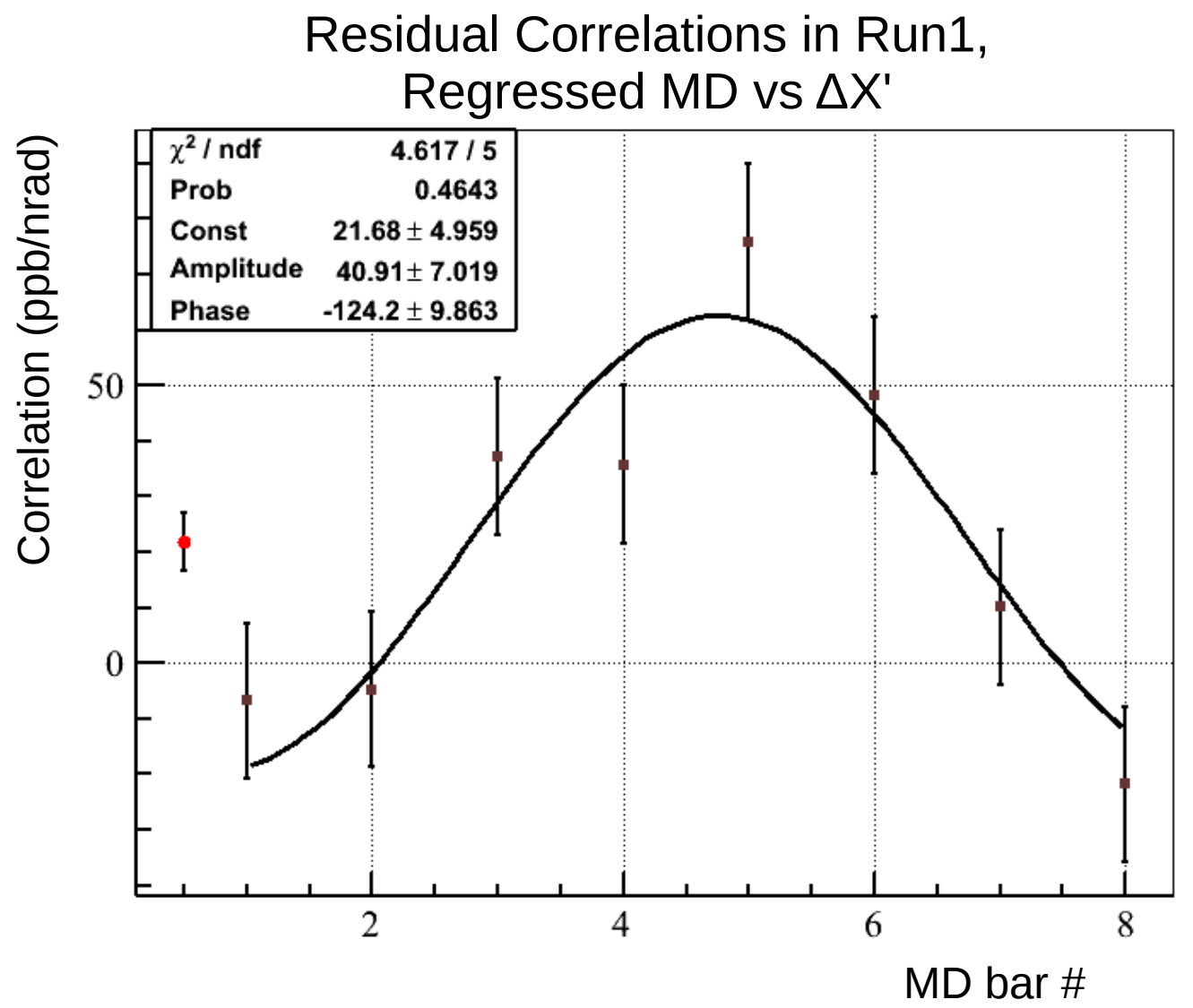

Figure 5.23: Correlation slopes of regressed MD asymmetries to $\Delta X^{\prime}$, extracted over Run1 at the slug scale, plotted versus MD octant. For example, the correlation slope of MD5 $C_{\mathrm{MD} 5-\Delta X^{\prime}}=75.73 \pm$ $14.15 \mathrm{ppb} / \mathrm{nrad}$ is taken from the fit of Figure 5.18. The red datum corresponds to the correlation of the MDall average.

The correlations of the regressed asymmetry of every MD bar to HC differences have been extracted in Run1 and Run2. In Figure 5.23 the extracted correlation slopes to $\Delta X^{\prime}$ are plotted versus detector octant. These values have been extracted from fits such as shown in Figure 5.18 
for MD5. A strong sinusoidal octant dependence is observed with significant amplitude and high fit probability, a sign of residual false asymmetries from beam corrections becoming apparent at longer time scales. Even so we still expect some geometric cancellation of false asymmetries when the azimuthally symmetric detectors are combined, but the regressed MDall combination (red datum) also exhibits a significant residual dependence. This is a strong suggestion that regression has failed to remove the systematic effect of HC beam differences in Run1.

By definition the residual MDall correlation suggests that there is a remaining false asymmetry in Run1, even after correcting for $A_{\text {beam }}$ through regression and getting the benefit of azimuthal cancellation. The Run1 average of $\left\langle\Delta X^{\prime}\right\rangle=-0.15 \pm 0.04$ nrad suggests a large and significant false asymmetry of $A_{\text {beam }}^{\Delta X^{\prime}}=C_{\mathrm{MD} 5-\Delta X^{\prime}} \Delta X^{\prime} \approx-11.4 \pm 3.7 \mathrm{ppb}$, although correlations with other beam parameters should also be considered in the estimation of the full residual false asymmetry.

Fortunately, not all beam correction schemes fail to suppress correlations to HC beam parameters at long time scales. Thus the residual correlations to $\mathrm{HC}$ beam parameters become an important qualifying criterion between the various correction schemes that we tested. A significant residual dependence after beam corrections and azimuthal cancellation suggests failure of the examined scheme.

All results for residual correlation slopes of the corrected MDall asymmetry to beam parameters, extracted over Run1 and Run2 at the slug scale, are listed in Tables 5.3 and 5.4 for a variety of regression and dithering schemes. Results are extracted over the Modulation datasets of Run1 and Run2, i.e. only data for which modulation correction sensitivities are available (Section 3.9). Regression results are also limited to the Modulation datasets for direct comparison.

Some correction schemes give clearly very large residual correlations to beam parameters at long time scales. This method allows us to identify the correction schemes that fail to correct the systematic effect of $\mathrm{HC}$ differences in our measurement and exclude them. Correction schemes with large $>4 \sigma$ residual correlations to beam parameters (table entries in red) are identified as failing and are excluded. There are some correction schemes with $3 \sigma$-level residual correlations (orange entries) which may be considered suspect, but we conservatively accept them.

It appears to be a lot more difficult to suppress residual systematic dependencies in Run1 compared to Run2, as evidenced by the higher amount of significant residual correlations and excluded schemes. In particular all regression schemes fail to sufficiently remove false beam asymmetries in Run1 and are excluded. In Section 5.1.1 we pointed to several mechanisms that would create problems for the regression correction and that appeared mostly in Run1.

In the modulation algorithm, we can clearly see that omitting certain "coils" should be avoided. Especially omitting coil 3 (the sine response during X' modulation, Table 5.2 always 
Table 5.3: Slug-scale correlation slopes of corrected MDall to beam parameters in Run1 for different correction schemes. Gray, orange and red font color corresponds to significance of $2 \sigma, 3 \sigma$ and $4 \sigma$ respectively. Schemes that are excluded based on these correlations are colored in red.

\begin{tabular}{|c|c|c|c|c|c|c|}
\hline & $\begin{array}{c}\Delta X \\
(\mathrm{ppb} / \mathrm{nm})\end{array}$ & $\begin{array}{c}\Delta Y \\
(\mathrm{ppb} / \mathrm{nm})\end{array}$ & $\begin{array}{c}\Delta X^{\prime} \\
(\mathrm{ppb} / \mathrm{nrad})\end{array}$ & $\begin{array}{c}\Delta Y^{\prime} \\
(\mathrm{ppb} / \mathrm{nrad})\end{array}$ & $\begin{array}{c}\Delta E \\
(\mathrm{ppb} / \mathrm{ppb})\end{array}$ & $\begin{array}{c}A_{Q} \\
(\mathrm{ppb} / \mathrm{ppb})\end{array}$ \\
\hline \multicolumn{7}{|l|}{ Regression } \\
\hline on & $0.61 \pm 0.20$ & $0.44 \pm 0.30$ & $22.52 \pm 5.49$ & $15.46 \pm 6.52$ & $1.13 \pm 0.63$ & $0.23 \pm 0.08$ \\
\hline $5+1$ & $0.63 \pm 0.20$ & $0.51 \pm 0.30$ & $24.28 \pm 5.49$ & $15.78 \pm 6.52$ & $1.71 \pm 0.63$ & $0.26 \pm 0.08$ \\
\hline set10 & $0.59 \pm 0.20$ & $0.43 \pm 0.30$ & $22.03 \pm 5.49$ & $15.25 \pm 6.52$ & $1.06 \pm 0.63$ & $0.23 \pm 0.08$ \\
\hline set11 & $0.60 \pm 0.20$ & $0.43 \pm 0.30$ & $22.47 \pm 5.49$ & $15.38 \pm 6.52$ & - & $0.23 \pm 0.08$ \\
\hline \multicolumn{7}{|l|}{ Dithering } \\
\hline 10-coil & $0.46 \pm 0.20$ & $-0.10 \pm 0.30$ & $8.43 \pm 5.50$ & $-3.95 \pm 6.53$ & $-0.78 \pm 0.63$ & $0.31 \pm 0.08$ \\
\hline omit 0,3 & $-1.22 \pm 0.20$ & $-2.01 \pm 0.30$ & $-49.49 \pm 5.50$ & $-54.83 \pm 6.54$ & $-5.33 \pm 0.63$ & $0.29 \pm 0.08$ \\
\hline omit 0,5 & $0.21 \pm 0.20$ & $-0.10 \pm 0.30$ & $6.05 \pm 5.50$ & $-3.87 \pm 6.53$ & $0.07 \pm 0.63$ & $0.29 \pm 0.08$ \\
\hline omit 0,8 & $-0.67 \pm 0.20$ & $-0.90 \pm 0.30$ & $-19.42 \pm 5.50$ & $-23.13 \pm 6.53$ & $-1.52 \pm 0.63$ & $0.21 \pm 0.08$ \\
\hline omit 3,8 & $-0.61 \pm 0.20$ & $-1.67 \pm 0.30$ & $-38.51 \pm 5.50$ & $-45.91 \pm 6.54$ & $-6.70 \pm 0.63$ & $0.29 \pm 0.08$ \\
\hline omit 1,6 & $0.65 \pm 0.20$ & $-0.08 \pm 0.30$ & $11.35 \pm 5.50$ & $-4.04 \pm 6.53$ & $-0.95 \pm 0.63$ & $0.28 \pm 0.08$ \\
\hline omit 1,9 & $0.44 \pm 0.20$ & $-0.11 \pm 0.30$ & $8.12 \pm 5.50$ & $-4.52 \pm 6.53$ & $-0.66 \pm 0.63$ & $0.30 \pm 0.08$ \\
\hline omit 4,6 & $0.43 \pm 0.20$ & $-0.05 \pm 0.30$ & $7.74 \pm 5.50$ & $-3.67 \pm 6.53$ & $-0.69 \pm 0.63$ & $0.30 \pm 0.08$ \\
\hline omit 4,9 & $0.43 \pm 0.20$ & $-0.15 \pm 0.30$ & $7.62 \pm 5.50$ & $-5.58 \pm 6.53$ & $-0.74 \pm 0.63$ & $0.30 \pm$ \\
\hline omit 0 & $-0.23 \pm 0.20$ & $-0.51 \pm 0.30$ & $-7.32 \pm 5.50$ & $-14.01 \pm 6.53$ & $-0.84 \pm 0.63$ & $0.25 \pm 0.08$ \\
\hline omit 3 & $-0.56 \pm 0.20$ & $-1.57 \pm 0.30$ & $-35.71 \pm 5.50$ & $-43.14 \pm 6.53$ & $-6.24 \pm 0.63$ & $0.29 \pm 0.08$ \\
\hline omit 4 & $0.43 \pm 0.20$ & $-0.13 \pm 0.30$ & $7.57 \pm 5.50$ & $-5.26 \pm 6.53$ & $-0.72 \pm 0.63$ & $0.30 \pm 0.08$ \\
\hline omit 5 & & $0.14 \pm 0.30$ & $16.73 \pm 5.50$ & $1.89 \pm 6.53$ & $0.31 \pm 0.63$ & $0.22 \pm 0.08$ \\
\hline omit 8 & $0.46 \pm 0.20$ & $-0.09 \pm 0.30$ & $8.64 \pm 5.50$ & $-4.09 \pm 6.53$ & $-0.62 \pm 0.63$ & $0.30 \pm 0.08$ \\
\hline
\end{tabular}

Table 5.4: Slug-scale correlation slopes of corrected MDall to beam parameters in Run2 for different correction schemes. Gray, orange and red font color corresponds to significance of $2 \sigma, 3 \sigma$ and $4 \sigma$ respectively. Schemes that are excluded based on these correlations are colored in red.

\begin{tabular}{|c|c|c|c|c|c|c|}
\hline & $\begin{array}{c}\Delta X \\
(\mathrm{ppb} / \mathrm{nm})\end{array}$ & $\begin{array}{c}\Delta Y \\
(\mathrm{ppb} / \mathrm{nm})\end{array}$ & $\begin{array}{c}\Delta X^{\prime} \\
(\mathrm{ppb} / \mathrm{nrad})\end{array}$ & $\begin{array}{c}\Delta Y^{\prime} \\
(\mathrm{ppb} / \mathrm{nrad})\end{array}$ & $\begin{array}{c}\Delta E \\
(\mathrm{ppb} / \mathrm{ppb})\end{array}$ & $\begin{array}{c}A_{Q} \\
(\mathrm{ppb} / \mathrm{ppb})\end{array}$ \\
\hline $\begin{array}{l}\text { Regression } \\
\text { on } \\
5+1 \\
\text { set10 } \\
\text { set11 } \\
\end{array}$ & $\begin{array}{l}-0.06 \pm 0.15 \\
-0.06 \pm 0.15 \\
-0.09 \pm 0.15 \\
-0.06 \pm 0.15\end{array}$ & $\begin{array}{l}-0.05 \pm 0.29 \\
-0.08 \pm 0.29 \\
-0.13 \pm 0.29 \\
-0.06 \pm 0.29 \\
\end{array}$ & $\begin{array}{l}-0.48 \pm 5.70 \\
-0.47 \pm 5.71 \\
-1.52 \pm 5.71 \\
-0.52 \pm 5.71\end{array}$ & $\begin{array}{l}-10.64 \pm 9.96 \\
-11.29 \pm 9.96 \\
-12.40 \pm 9.95 \\
-10.61 \pm 9.96\end{array}$ & $\begin{array}{c}1.54 \pm 1.14 \\
1.73 \pm 1.14 \\
1.79 \pm 1.14 \\
-\end{array}$ & $\begin{array}{l}0.07 \pm 0.05 \\
0.07 \pm 0.05 \\
0.05 \pm 0.05 \\
0.07 \pm 0.05\end{array}$ \\
\hline Dithering & & & & & & \\
\hline $\begin{array}{l}\text { 10-coil } \\
\text { omit } 0,3\end{array}$ & $\begin{array}{l}-0.10 \pm 0.15 \\
-0.91 \pm 0.17\end{array}$ & $\begin{array}{l}-0.11 \pm 0.29 \\
-1.28 \pm 0.38\end{array}$ & $\begin{array}{c}-2.79 \pm 5.72 \\
-34.24 \pm 6.22\end{array}$ & $\begin{array}{c}-10.42 \pm 9.97 \\
-43.47 \pm 11.41\end{array}$ & $\begin{array}{l}1.37 \pm 1.14 \\
3.47 \pm 2.18\end{array}$ & $\begin{array}{c}0.05 \pm 0.05 \\
-0.02 \pm 0.05\end{array}$ \\
\hline omit 0,5 & $-0.16 \pm 0.15$ & $-0.17 \pm 0.29$ & $-5.35 \pm 5.75$ & $-12.46 \pm 10.02$ & $1.59 \pm 1.16$ & $0.05 \pm 0.05$ \\
\hline omit 0,8 & $-1.27 \pm 0.15$ & $-1.39 \pm 0.29$ & $-49.17 \pm 5.77$ & $-56.98 \pm 10.10$ & $2.41 \pm 1.16$ & $-0.05 \pm 0.05$ \\
\hline omit 3,8 & $-1.01 \pm 0.15$ & $-1.22 \pm 0.29$ & $-38.92 \pm 5.76$ & $-47.70 \pm 10.05$ & $2.05 \pm 1.16$ & $-0.04 \pm 0.05$ \\
\hline omit 1,6 & $-0.08 \pm 0.15$ & $-0.63 \pm 0.29$ & $-0.61 \pm 5.75$ & $-31.20 \pm 10.03$ & $1.55 \pm 1.16$ & $0.05 \pm 0.05$ \\
\hline omit 1,9 & $-0.09 \pm 0.15$ & $-0.45 \pm 0.29$ & $-1.82 \pm 5.75$ & $-25.64 \pm 10.03$ & $1.44 \pm 1.16$ & $0.05 \pm 0.05$ \\
\hline omit 4,6 & $-0.09 \pm 0.15$ & $-0.57 \pm 0.29$ & $-1.20 \pm 5.75$ & $-29.17 \pm 10.02$ & $1.56 \pm 1.16$ & $0.06 \pm 0.05$ \\
\hline omit 4,9 & $-0.09 \pm 0.15$ & $-0.63 \pm 0.29$ & $-1.00 \pm 5.75$ & $-31.12 \pm 10.03$ & $1.54 \pm 1.16$ & $0.06 \pm 0.05$ \\
\hline omit 0 & $-0.69 \pm 0.15$ & $-0.72 \pm 0.29$ & $-26.28 \pm 5.75$ & $-32.68 \pm 10.03$ & $1.88 \pm 1.16$ & $0.00 \pm 0.05$ \\
\hline omit 3 & $-0.87 \pm 0.15$ & $-1.10 \pm 0.29$ & $-33.45 \pm 5.76$ & $-45.14 \pm 10.04$ & $1.78 \pm 1.16$ & $-0.03 \pm 0.05$ \\
\hline omit 5 & $0.09 \pm 0.15$ & $0.12 \pm 0.29$ & $4.89 \pm 5.75$ & $-2.44 \pm 10.02$ & $1.29 \pm 1.16$ & $0.07 \pm 0.05$ \\
\hline omit 8 & $-0.09 \pm 0.15$ & $-0.10 \pm 0.29$ & $-2.24 \pm 5.75$ & $-9.87 \pm 10.02$ & $1.43 \pm 1.16$ & $0.06 \pm 0.05$ \\
\hline
\end{tabular}


Table 5.5: Regression and modulation correction schemes that are excluded, in Run1 and Run2 separately, based on the significance of slug-scale residual correlations to beam parameters.

\begin{tabular}{c|cc} 
& Regression & Modulation \\
\hline \multirow{4}{*}{ Run1 } & on & omit-0,3 \\
& $5+1$ & omit-0,8 \\
& set10 & omit-3,8 \\
& set11 & omit-3 \\
\hline \multirow{3}{*}{ Run2 } & & omit-0,3 \\
& & omit-0,8 \\
& & omit-3,8 \\
& & omit-0 \\
& & omit-3
\end{tabular}

leads to large failures, suggesting perhaps that this coil alone grants an important access to a region of beam phase space that we cannot afford to omit.

It is also very interesting that all correction schemes in Run1 have consistent and significant residual correlations to the charge asymmetry $A_{Q}$ of order $30 \%$ (Table 5.3p. While charge asymmetry is not included in the monitor basis of most of these correction schemes, these residual correlations are quite surprising given that the detectors should be normalized for charge. We will return to this issue in Section 5.6.2.

As we will see in the following section, the result for the final corrected asymmetry varies significantly depending on the choice of correction scheme. Perhaps unsurprisingly, the most discrepant results come from schemes that are failing to suppress residual correlations to beam parameters over long time scales.

\subsection{Beam Corrections Results}

In this section we present the results from various beam correction schemes. More than just the size of the correction, it is important to inspect the statistical consistency of the corrected asymmetries. Here we will have a first look on the statistical properties after beam corrections of the Physics and Null asymmetry combinations, defined in Section 5.4.2. The expectation value of these combinations is the "physics" $A_{e p}$ asymmetry and 0 , respectively, in the absence of residual systematic effects. Therefore statistical properties of these combinations are sensitive to indications of residual false asymmetries.

A more complete treatment of statistical properties will be presented in Chapter 7] after the beamline backgrounds correction has been applied as well. As we will see there are actually multiple ways to define a Null combination $A_{\text {Null }}$, each with access to different classes of systematic effects. Here we will only examine the simplest Null combination, which results if we apply no sign correction for either the IHWP or the reversal of the spin of the electron beam. We will 
refer to this Null combination as "IHWP+Spin", where the naming convention will be clarified in Chapter 7

Here the Physics and Null asymmetries are segmented in combinations of $\sim 4$ slugs, referred as "itts" and defined in Appendix $\mathrm{A}$ Notice that we need at least one slug of each IHWP state to define a Null combination. The pitt time scale is more characteristic of the inherent time scale of variation of the beam asymmetries than the slug scale. Longer yet time scales such as Wiens could arguably be better. A study on the dependence of results to the time scale of averaging will be presented in Section 7.5 .

In the following paragraphs the statistical consistency of $A_{\text {Phys }}$ and $A_{\text {Null }}$ combinations after beam corrections will be presented separately for Run1 and Run2. All data is weighted by polarization on a run-by-run basis, based on the prescription for the beam polarization that will be presented in Section 8.1 .2

\subsubsection{Beam corrections in Run2}

We will first examine the statistical properties in the Modulation dataset of Run2, the main dataset that will be examined in this dissertation. It constitutes a much larger data fraction and, as we will see, is much better behaved in terms of systematic corrections.

The Run2 Physics asymmetry combination is plotted in Figure 5.24, for uncorrected data (black) and then for data corrected through the standard dithering or regression analyses (blue and red respectively). The three datasets have been fitted to a constant, with the fit results in each scheme shown to the right of the figure. Note that for the Null combination the fit probability is quoted with respect to zero, i.e. the probability that the deviation of the data sample from $A_{\text {Null }}=0$ is statistical and not systematic. The raw uncorrected asymmetry in Run2, appropriately weighted for polarization, is:

$$
A_{\text {raw }}^{P}(\text { Run2 })=-179.39 \pm 9.30 \mathrm{ppb}
$$

We can immediately draw some important conclusions from the fit results. For the Physics asymmetry combination:

- The fit probability is significantly improved through beam corrections, and is consistent with the absence of residual systematic effects. The fit probability is slightly better for dithering compared to regression.

- The total polarization-weighted correction is small compared to the size of the asymmetry and its statistical uncertainty, of order $2 \mathrm{ppb}$ for the dithering correction. The size of the applied beam corrections on individual pitts (the deviation between the black and the 


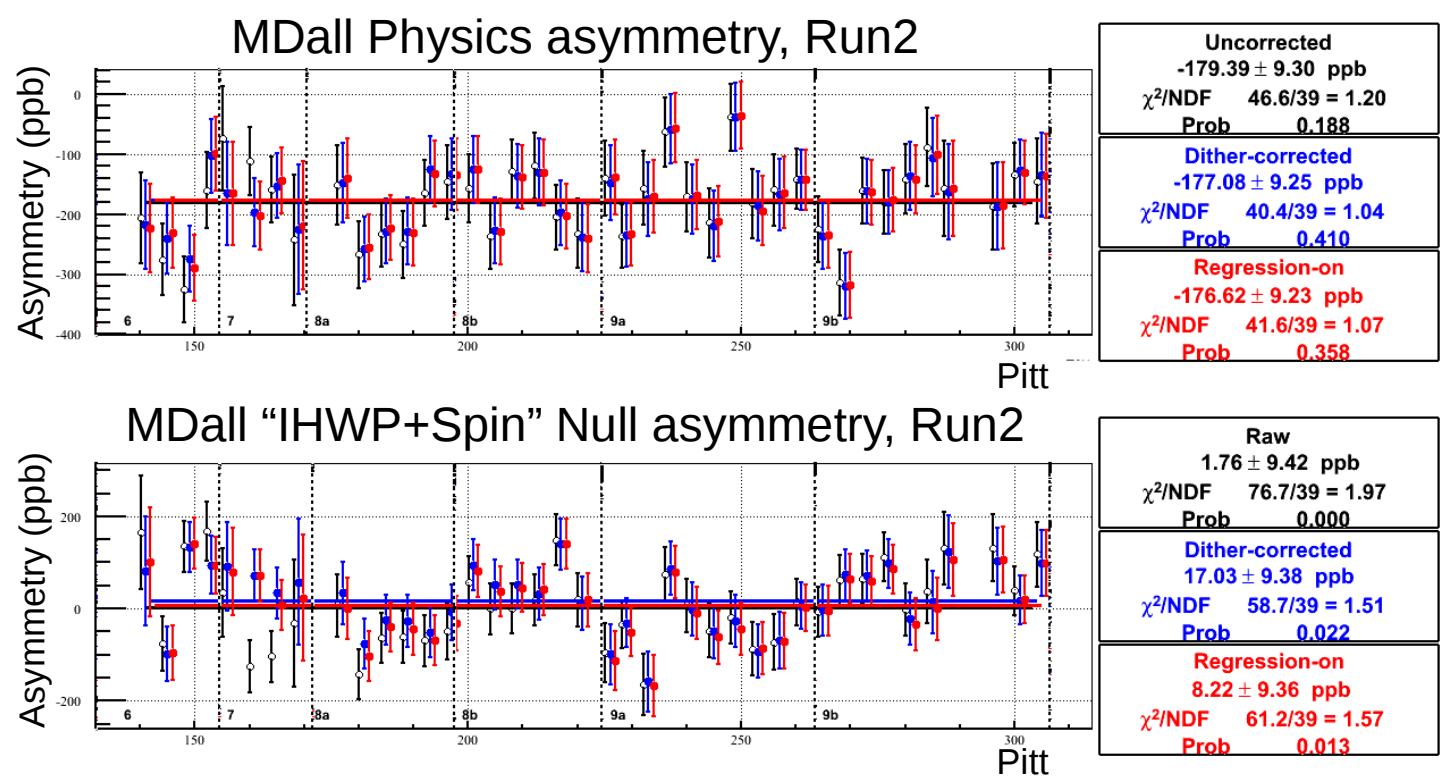

Figure 5.24: Physics (top) and Null (bottom) asymmetry combinations after beam corrections in Run2 for data binned at the pitt scale. Black points correspond to uncorrected asymmetries, blue and red points to asymmetries corrected through dithering and regression respectively. The three datasets are fitted to a constant and results are shown on the right. Vertical dashed lines mark different Wien periods. Polarization applied to all data.

blue/red points) appears to be larger during Wiens 6 and 7, then suppressed especially after position feedback is applied around Slug 215.

- The discrepancy between regression and dithering is also small, $\sim 0.6 \mathrm{ppb}$. This level of agreement is excellent from the two completely independent correction algorithms, with sensitivities extracted from fully separate periods.

However, for the Null asymmetry combination:

- The Null probability is very low suggesting imperfect removal of residual systematic effects.

Dithering still gives a slightly better probability.

- The size of the average Null asymmetry over Run2 is mostly consistent with zero, up to $\sim 1.7 \sigma$ deviation for the dither-corrected asymmetries. This indicates that even if there are residual systematic effects over short periods, suggested by the low fit probability, the average residual systematic effect over Run2 should be consistent with zerd

- The difference in fit probability between the Physics and Null asymmetries is solely due to the effect of the applied reversals, suggesting that the IHWP and spin reversals achieved

\footnotetext{
${ }^{6}$ Notice that this statement translates to the residual systematic effect on the Physics combination up to the statistical parity between the two IHWP states, i.e. assuming that they have roughly equal amounts of data. That is because the Physics combination is formed by appropriately weighting the two IHWP states according to their statistical precision, whereas the Null combination is formed as their unweighted sum to ensure that the elastic $\overrightarrow{e p}$ asymmetry cancels in this combination. Since we do in fact have approximately equal amounts of data from both IHWP states, we expect the cancellation of systematic effects over Run2 to translate to the Physics asymmetry as well.
} 
significant cancellation of residual false asymmetries that appear on the Null. Of course the important criterion are the statistical properties of the Physics asymmetries, which appear to be consistent with no residual false asymmetries after we get the benefit of cancellations. This highlights the indispensable contribution of the reversals we applied to cancel residual systematic effects that are imperfectly corrected.

The asymmetry combinations and fit probabilities are extracted through all correction schemes that are not excluded due to large residual correlations to beam parameters (Section 5.5). The results are listed in Table 5.6 , along with the corresponding fit probabilities.

Results are also quoted for some excluded correction schemes, marked in red on the table. These schemes are seen to give the most discrepant results for both the Physics and Null asymmetry combinations, as well as the lowest probabilities. The result for the Null combination also exhibits the most significant deviation from zero. All these are strong signs of residual false asymmetries in these schemes, giving us confidence in excluding them.

Following the prescription suggested in [155, we accept all examined schemes and consider the range of the results as characteristic of the systematic uncertainty in the $A_{\text {beam }}$ correction. The full range of $1.06 \mathrm{ppb}$ in Physics asymmetry results is inscribed by the dithering schemes omit-0,5 (-176.28 ppb) and omit-5 (-177.34 ppb).

For the mean value of the corrected asymmetry we will accept the result from the standard 10-coil dithering analysis,

$$
A_{10-\text { coil }}^{P}=-177.08 \pm 9.25 \mathrm{ppb},
$$

where the $P$ superscript reminds us that the asymmetry is weighted for polarization. This is the most unambiguous choice from the dithering analysis and is one of the schemes that showed no signs of residual false asymmetries. We will conservatively treat the largest discrepancy from the 10-coil Physics asymmetry as a gaussian error, corresponding to the "model" uncertainty of the correction:

$$
\delta A_{\text {beam }}^{P}(\text { model })=0.80 \mathrm{ppb}
$$

We will further assign an error on the correction due to the noise of the extracted dithering sensitivities in each slug and the limited resolution of the BPMs [156]. As shown in Figure 5.14 the dithering sensitivities are generally very precise and stable. The "noise" error in the correction is [155]:

$$
\delta A_{\text {beam }}^{P}(\text { noise })=0.11 \mathrm{ppb}
$$

Thus the Run2 correction for false beam asymmetries, appropriately weighted for polarization, is calculated as the difference between the 10-coil dithering scheme and the raw uncorrected 
Table 5.6: Polarization-weighted MDall Physics and Null asymmetry results in Run2, corrected through various regression and dithering schemes. The numbers in parentheses are the fit probabilities for each result. Schemes marked in red are excluded from consideration due to significant residual correlations to beam parameters.

\begin{tabular}{|c|c|c|}
\hline & $\begin{array}{c}\text { Physics } \\
\text { (fit probability) }\end{array}$ & $\begin{array}{c}\text { Null (IHWP+Spin) } \\
\text { (fit probability) }\end{array}$ \\
\hline \multicolumn{3}{|l|}{ Regression } \\
\hline on & $\begin{array}{l}-176.62 \pm 9.23 \\
\quad(0.431)\end{array}$ & $\begin{array}{l}8.22 \pm 9.36 \\
(0.021)\end{array}$ \\
\hline $5+1$ & $\begin{array}{l}-176.81 \pm 9.23 \\
(0.361)\end{array}$ & $\begin{array}{c}8.41 \pm 9.36 \\
(0.009)\end{array}$ \\
\hline set10 & $\begin{array}{l}-177.09 \pm 9.23 \\
\quad(0.351)\end{array}$ & $\begin{array}{l}9.16 \pm 9.36 \\
(0.014)\end{array}$ \\
\hline set11 & $\begin{array}{c}-177.05 \pm 9.23 \\
(0.343)\end{array}$ & $\begin{array}{c}7.88 \pm 9.36 \\
(0.011)\end{array}$ \\
\hline \multicolumn{3}{|l|}{ Dithering } \\
\hline 10-coil & $\begin{array}{l}-177.08 \pm 9.25 \\
\quad(0.410)\end{array}$ & $\begin{array}{l}17.03 \pm 9.38 \\
\quad(0.022)\end{array}$ \\
\hline omit 0,3 & $\begin{array}{c}-175.15 \pm 9.62 \\
(0.168)\end{array}$ & $\begin{array}{l}42.79 \pm 9.74 \\
(0.000)\end{array}$ \\
\hline omit 3,8 & $\begin{array}{l}-179.05 \pm 9.30 \\
(0.340)\end{array}$ & $\begin{array}{c}26.96 \pm 9.43 \\
(0.000)\end{array}$ \\
\hline omit 0,5 & $\begin{array}{l}-176.28 \pm 9.30 \\
\quad(0.400)\end{array}$ & $\begin{array}{l}14.93 \pm 9.43 \\
(0.032)\end{array}$ \\
\hline omit 1,6 & $\begin{array}{l}-177.09 \pm 9.30 \\
\quad(0.406)\end{array}$ & $\begin{array}{l}17.14 \pm 9.43 \\
(0.024)\end{array}$ \\
\hline omit 1,9 & $\begin{array}{l}-177.00 \pm 9.30 \\
\quad(0.412)\end{array}$ & $\begin{array}{l}17.25 \pm 9.43 \\
(0.023)\end{array}$ \\
\hline omit 4,6 & $\begin{array}{l}-177.22 \pm 9.30 \\
\quad(0.402)\end{array}$ & $\begin{array}{l}17.56 \pm 9.43 \\
(0.021)\end{array}$ \\
\hline omit 4,9 & $\begin{array}{l}-176.94 \pm 9.30 \\
\quad(0.410)\end{array}$ & $\begin{array}{l}17.51 \pm 9.43 \\
(0.020)\end{array}$ \\
\hline omit 3 & $\begin{array}{c}-177.44 \pm 9.30 \\
\quad(0.382)\end{array}$ & $\begin{array}{c}30.62 \pm 9.43 \\
(0.000)\end{array}$ \\
\hline omit 5 & $\begin{array}{l}-177.34 \pm 9.30 \\
\quad(0.403)\end{array}$ & $\begin{array}{l}13.53 \pm 9.43 \\
(0.016)\end{array}$ \\
\hline omit 8 & $\begin{array}{l}-177.26 \pm 9.30 \\
\quad(0.409)\end{array}$ & $\begin{array}{l}17.74 \pm 9.43 \\
(0.019)\end{array}$ \\
\hline
\end{tabular}


result, $A_{\text {beam }}^{P}=A_{\text {raw }}^{P}-A_{10 \text {-coil }}^{P}$

$$
A_{\text {beam }}^{P}(\text { Run2) }=-2.31 \pm 0.18(\text { noise }) \pm 0.80(\text { model }) \mathrm{ppb}
$$

Note that we have avoided referring to the error contributions as "statistical" and "systematic" to avoid confusion, as $A_{\text {beam }}$ is a systematic correction in the asymmetry measurement.

The achieved suppressions and cancellations of HC differences make for a very small $A_{\text {beam }}$ correction and uncertainty relative to the statistical precision of the raw asymmetry (5.9), 9.30 ppb. Identifying failing schemes through long-scale correlations was critical in limiting the model uncertainty of the correction. The total correction uncertainty is $0.36 \%$ of the Standard Model prediction for $A_{\mathrm{PV}} \sqrt{8}$ well within the proposed $0.5 \%$ uncertainty for the $A_{\text {beam }}$ correction (Table 3.2).

\subsubsection{Beam corrections in Run1}

We will not quote a result from Run1 in this dissertation, however we will give a brief discussion of the analysis status. As we will see the beam corrections analysis is more difficult in Run1, as indicated also by the generally larger residual correlations from Table 5.3 .
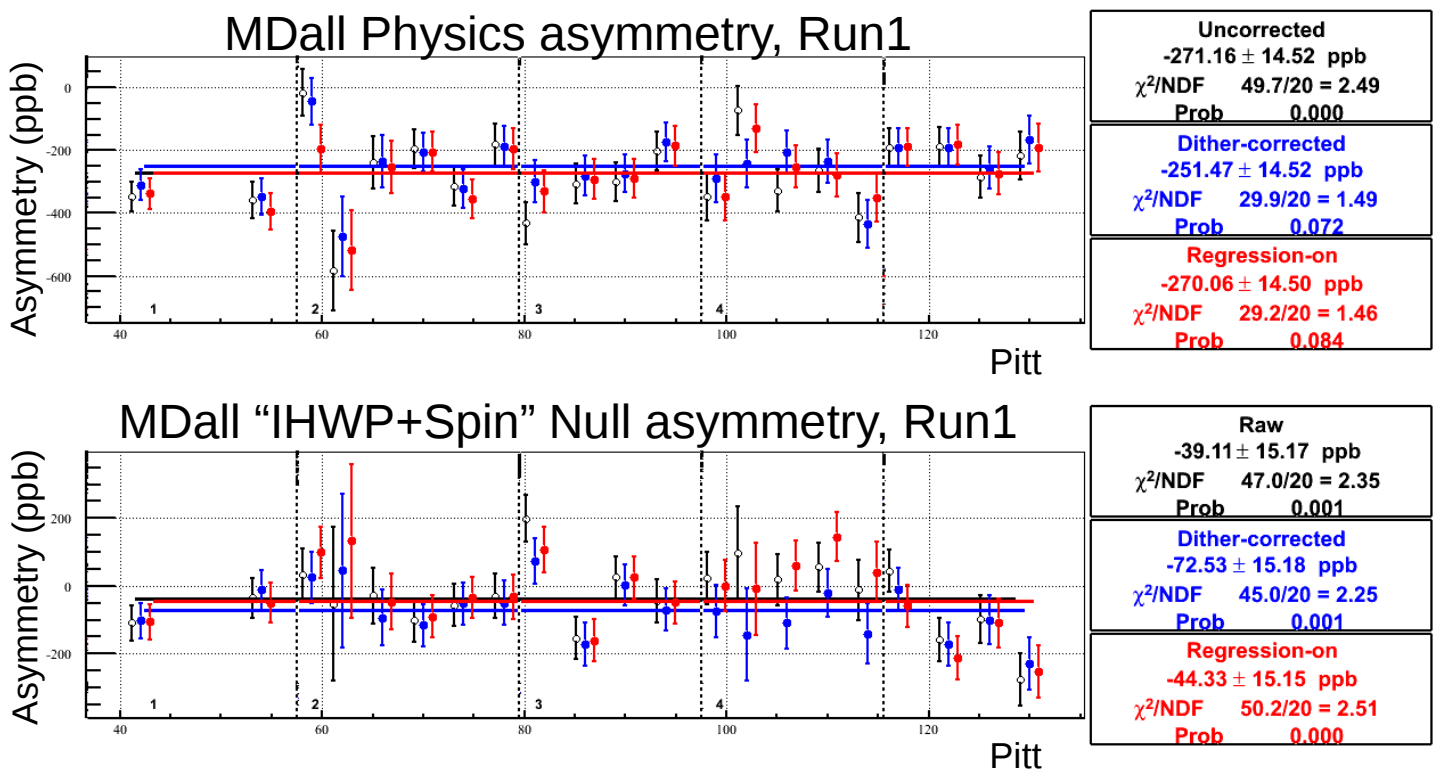

Figure 5.25: Physics (top) and Null (bottom) asymmetry combinations after beam corrections in Run1 for data binned at the pitt scale. Black points correspond to uncorrected data, blue points for data corrected through dithering, and red for data corrected through regression. The three datasets are fitted to a constant and results are shown on the right. Vertical lines mark different Wien periods. Polarization applied to all data.

The Physics and Null asymmetry combinations in Run1 are plotted in Figure 5.25 after beam

\footnotetext{
${ }^{7}$ The sign is in agreement with the convention of Eq. 3.3 .

${ }^{8}$ The comparison is valid because the value $\sqrt{5.11}$ ) is weighted for polarization, although there is a small missing normalizing factor $R / \sum f_{b}(\mathrm{Eq} 3.3$.
} 
Table 5.7: MDallbars Physics and Null asymmetry results in Run1 through different correction schemes. The numbers in parentheses are the fit probabilities for each result. Rows marked in red have significant signs of residual false asymmetries and are excluded from consideration.

\begin{tabular}{c|cc} 
& $\begin{array}{c}\text { Physics } \\
\text { (fit probability) }\end{array}$ & $\begin{array}{c}\text { Null } \\
\text { (fit probability) }\end{array}$ \\
\hline Regression & & \\
on & $-270.06 \pm 14.50$ & $-44.33 \pm 15.15$ \\
& $(0.084)$ & $(0.000)$ \\
set10 & $-269.52 \pm 14.50$ & $-43.72 \pm 15.16$ \\
& $(0.081)$ & $(0.000)$ \\
\hline \multirow{2}{*}{ Dithering } & & \\
10-coil & $-251.47 \pm 14.52$ & $-72.53 \pm 15.18$ \\
& $(0.072)$ & $(0.001)$ \\
omit 0,3 & $-220.74 \pm 14.52$ & $-88.31 \pm 15.17$ \\
& $(0.000)$ & $(0.000)$ \\
omit 0,5 & $-255.15 \pm 14.52$ & $-68.50 \pm 15.17$ \\
& $(0.382)$ & $(0.002)$ \\
omit 1,6 & $-251.15 \pm 14.52$ & $-63.19 \pm 15.17$ \\
& $(0.002)$ & $(0.004)$ \\
omit 1,9 & $-248.94 \pm 14.52$ & $-71.13 \pm 15.17$ \\
& $(0.070)$ & $(0.002)$ \\
omit 4,6 & $-245.66 \pm 14.52$ & $-71.39 \pm 15.17$ \\
& $(0.111)$ & $(0.002)$ \\
omit 4,9 & $-249.26 \pm 14.52$ & $-71.66 \pm 15.17$ \\
& $(0.048)$ & $(0.002)$ \\
omit 5 & $-257.32 \pm 14.52$ & $-69.35 \pm 15.17$ \\
& $(0.074)$ & $(0.000)$ \\
omit 8 & $-249.18 \pm 14.52$ & $-71.29 \pm 15.17$ \\
& $(0.071)$ & $(0.002)$ \\
& &
\end{tabular}

corrections from the standard dithering (blue) and regression (red) analyses. Unlike Run2 the fit probability is unfortunately very small, basically zero for the Null asymmetry and still very small after we get the benefit of cancellations for the Physics combination. There is a large discrepancy of $20 \mathrm{ppb}$ between regression and dithering, and even larger discrepancies can be seen on individual pitts. Note however that in Run1 all regression schemes fail to suppress residual correlations to beam parameters at longer time scales, therefore they can be excluded from consideration.

The average Physics and Null asymmetries in Run1 from various correction schemes are given in Table 5.7 along with their fit probabilities. Even just considering the dithering results the range is much larger than in Run2, at $11.66 \mathrm{ppb}$. The total correction is also much larger than we would be comfortable with, at almost $20 \mathrm{ppb}$ and larger than the statistical precision of $A_{\text {raw }}$. Worryingly, the average Null asymmetry combination in Run1 deviates very significantly from zero, $A_{\text {Null }}^{P}($ Run 1$) \approx-70 \pm 15$ ppb, with high consistency among all accepted schemes. These are all strong indications of residual false asymmetries in Run1. 
The omit- 0,5 scheme gives a result with significantly higher probability than any other scheme, but we will again quote the correction from the standard 10-coil scheme for consistency, and since it lays near the middle of the accepted range. We give a preliminary estimation for the size of the correction in Run1 following the Run2 procedure, although there is clearly more analysis required to fully understand these results:

$$
A_{\text {beam }}^{P}(\text { Run } 1)=-19.69 \pm 0.17(\text { stat }) \pm 5.85(\text { model }) \mathrm{ppb}
$$

The correction is much larger and with much larger uncertainty compared to the Run2 correction (5.11), indicative of more complicated issues with the beam corrections analysis in Run1. The total uncertainty is $\sim 2.5 \%$ of the Standard Model $A_{\mathrm{PV}}$, a factor of several larger than specification. The analysis is still ongoing to improve the precision of the $A_{\text {beam }}$ correction in Run1. A specific issue relating to the residual correlations to the charge asymmetry $A_{Q}$ is discussed in the following paragraph.

\section{Residual beam corrections in Run1}

Up to now we have been able to quote results from the schemes that are seemingly successful at suppressing correlations to beam parameters at long scales, and simply exclude the schemes that fail. It is not obvious how to treat a correction scheme with significant residual correlations. The author of [157] faced this situation in quoting a result for Run1 in the standard regression scheme. That author applied a "post-regression" correction to the regressed asymmetries, in order to remove the false asymmetry implied by the significant residual correlation slope $C_{\mathrm{MD}-\Delta x}$ to beam parameters $\Delta x$ :

$$
A \underset{\text { post-regression }}{\text { beam }}=C_{\mathrm{MD}-\Delta X^{\prime}} \Delta X^{\prime}
$$

The post-regression correction was applied through a single variable, $\Delta X^{\prime}$, which seemed to remove residual correlations to all five considered beam parameters. That attempt however was partial and incomplete: later analysis showed that the result varies significantly based on the choice of post-regression variable, and the same full removal of residual correlations could not be achieved for the background post-regressed asymmetries.

In a similar attempt to fix the issues developing with regression, this author attempted to apply the regression procedure at longer time scales, with data binned for every 5-minute segment and the full regression algorithm applied in every Wien. While this approach succeeded (by construction) in removing long-scale correlations in Run1, the correction slopes extracted through this procedure were very noisy (due to the reduced range of $\mathrm{HC}$ differences) so that quartet-scale asymmetry width of the corrected detectors was unacceptably large. 


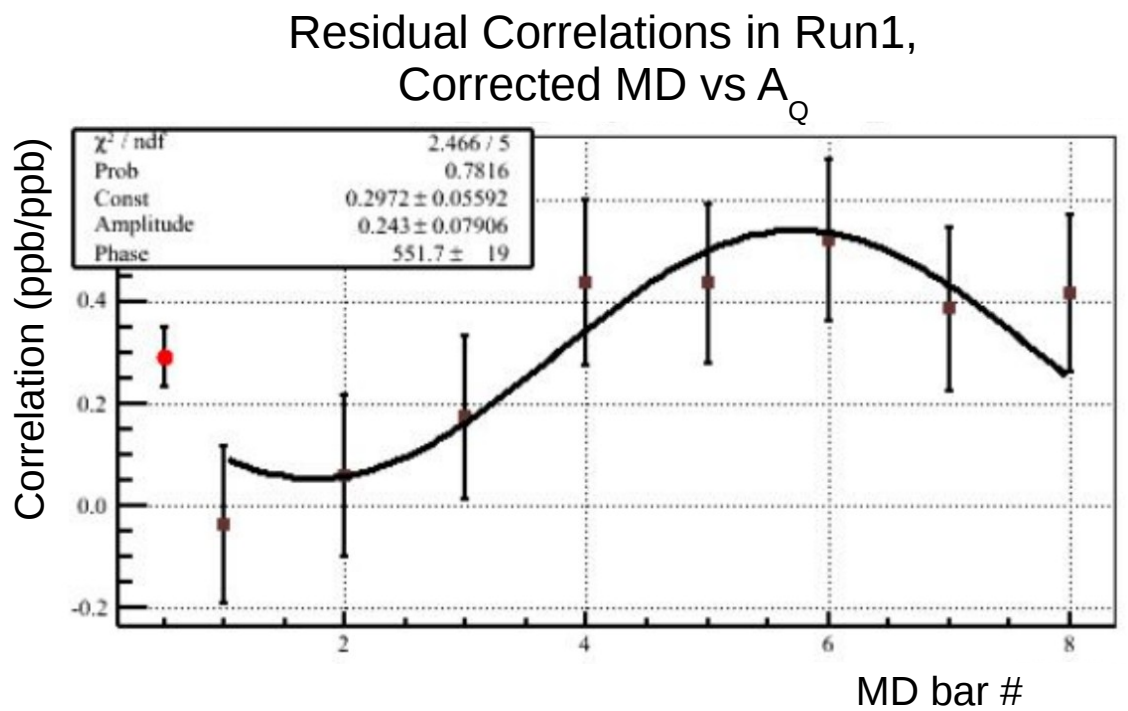

Figure 5.26: Slug-scale correlations of MD asymmetries to charge asymmetry in Run1, plotted versus octant. The asymmetries are corrected in the standard 10-coil dithering scheme.

It would seem that with dithering data available we don't have to worry about a "postcorrection" in Run1. This author however showed that the significant residual correlations to charge asymmetry in Run1 (Table 5.3) may still pose an issue. The dipole shape in the octant dependence of that correlation (Figure 5.26 ) is characteristic of residual beam effects, and after azimuthal cancellation the MDall combination still has significant residual dependence to charge asymmetry. This $30 \%$ residual correlation to charge (up to $50 \%$ on individual bars) is much larger than the expected $d A_{\text {raw }} / d A_{Q} \sim 0.5 \%$ detector non-linearity. At the slug time scale any noise effects (as described in Section 99) should cancel, therefore this correlation is indicating systematic effects. In Figure 5.27 a scatterplot of the slug-averaged $A_{\text {raw }}$ and $A_{Q}$ reveals that the correlation to charge asymmetry is rather consistent throughout Run1 and not dominated by individual periods, more clearly than correlations to other beam parameters.

We inquired what would be the effect on the asymmetry if we attempted a "post-correction" on charge asymmetry to remove the residual correlation. The correction is defined as

$$
A_{\text {beam }} \rightarrow A_{\text {beam }}+A_{\text {post-correction }}=A_{\text {beam }}+C_{\mathrm{MD}-A_{Q}} A_{Q}
$$

where the correction factor $C_{\mathrm{MD}-A_{Q}}$ is the scheme-dependent slug-scale residual correlation slope to $A_{Q}$ from Table 5.3 . The charge post-correction was applied on every run segment to ensure proper statistical and polarization weighting. The results for the Physics and Null asymmetry in Run1 after the charge post-correction are given in Table 5.8. The post-correction on charge is seen to significantly improve the fit probability of the Null asymmetry in Run1, and making its magnitude consistent with zero. 


\section{Sign-corrected MDall vs Aq, Run1. Color-coded by Slug}

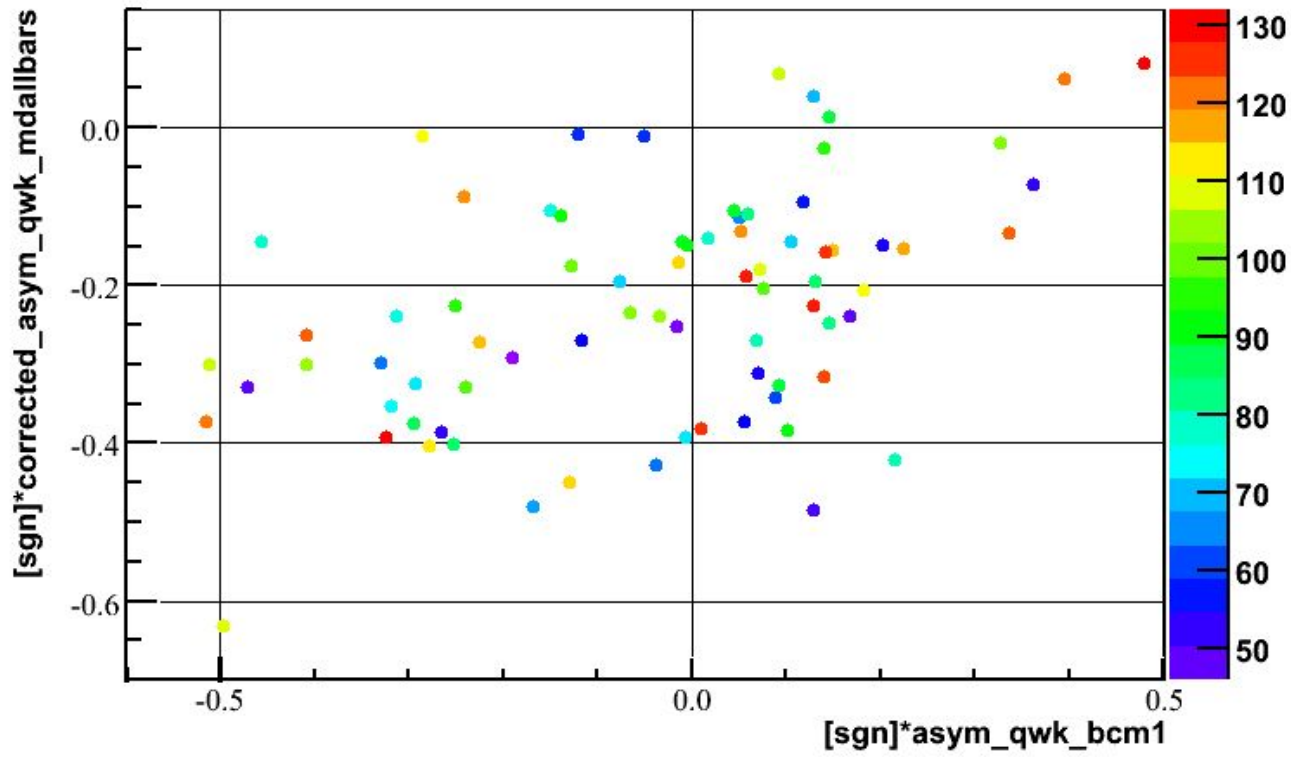

Figure 5.27: Scatterplot of the slug-averaged MDall asymmetry and charge asymmetry in Run1. Color scaling applied by slug number.

Table 5.8: MDall Physics and Null asymmetry results in Run1, after "post-correction" is applied to remove the residual correlation to charge asymmetry.

\begin{tabular}{c|cc} 
& Physics & Null \\
\hline 10-coil & $\begin{array}{c}234.8 \pm 14.5 \\
(0.048)\end{array}$ & $-19.9 \pm 15.2$ \\
& $(0.624)$
\end{tabular}

The residual correlation to $A_{Q}$ seems to be associated with a consistently non-zero charge asymmetry in Run1, before any sign-correction for reversals is applied. This corresponds to the Null combination of $A_{Q}$ plotted in Figure 5.28. The consistently non-zero "Null" combination of $A_{Q}$ combines with the residual correlation to charge, to produce the large Null asymmetries in the MD (Table 5.7). It is not clear what gives rise to the significantly non-zero value of the charge asymmetry; after all the charge feedback is designed to make the charge asymmetry converge to zero.

The reasons behind the unexpected residual correlation and significantly non-zero charge asymmetry are currently being investigated. It has been suggested that there may be problems associated with the normalizing BCMs 1 and 2 in Run1, which are also used for feedback. Preliminary analysis suggests that normalizing through BCM6 achieves very good suppression of the Null asymmetry as well as residual correlations to all beam parameters. However these improvements, either through a post-correction or normalizing from a different BCM, are associated with a significant shift in the MDall Physics asymmetry (of order 15 ppb, comparing 


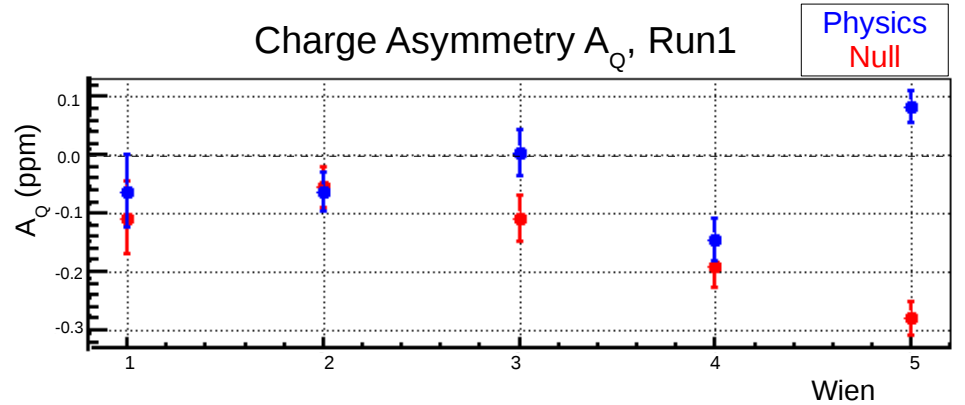

Figure 5.28: Charge asymmetry in Run1 averaged in every Wien and separated into "Physics" and "Null" combinations, corresponding to sign-correction for reversals applied and not applied respectively.

Tables 5.7 and 5.8. . More analysis is required in Run1 before such a large shift is justified. None of these issues however appears to be present in Run2, which is the main dataset examined in this dissertation. 


\section{Chapter 6}

\section{Beamline Backgrounds}

The "beamline" background (BB) is considered to be a soft background component in the main detector that is generated from electrons scattering in the beamline and in the tungsten "plug" collimator, located in the central aperture of the upstream collimator (Section 3.4). The tungsten plug was placed there specifically to intercept electrons at small angles (up to $\theta_{\max }=0.88^{\circ}$ ) that would otherwise scatter from the beamline, therefore we expect most of the $\mathrm{BB}$ component to originate at the plug. This soft background component in the MD is expected to be predominantly composed of neutral particles (photons), as simulations suggest that low-energy charged particles are highly suppressed by the lead preradiators [103.

The BB component is the $b=2$ background contribution to the $A_{e p}$ asymmetry from Eq. (3.3). The background correction for the false asymmetry $A_{\mathrm{BB}}=f_{2} A_{2}$ generated in the MD from this component, contributed the largest systematic uncertainty in the $2013 Q_{\text {weak }}$ result [55, $\delta A_{\mathrm{BB}}=23.2 \mathrm{ppb}$. This author led the development of an algorithm for the beamline background correction $A_{\mathrm{BB}}$ with the goal of reducing the uncertainty by an order of magnitude. In this chapter we will present the properties of the BB asymmetries, accessed through dedicated studies, and we will develop a correction for their effect in the $Q_{\text {weak }}$ measurement.

\subsection{Background Detectors}

The three background detectors presented in Section 3.6.2 referred to as PMTLTG, PMTONL, and MD9, were placed inside the detector shield house and were sensitive to the diffuse background component. They were located in the super-elastic region immediately downstream of the MD plane but at a smaller radius, their placement in Run2 shown in Figure 3.14. Their placement in Run1 was different which complicates the comparison between the two datasets. For the most part we will focus on the Run2 analysis, before we give an overview of the status 
of the Run1 $A_{\mathrm{BB}}$ analysis in Section 6.8

\subsubsection{Background asymmetries}

The background asymmetries in Run2 are plotted in Figure6.1, averaged in every slug and colorcoded by IHWP state. The size of these background asymmetries average $>20 \mathrm{ppm}$ in some slugs. That is 2 orders of magnitude higher than the $A_{e p}$ asymmetry and 4 orders of magnitude higher than the precision $\delta A_{\mathrm{BB}}$ we need to achieve for this correction. The presence of these large background asymmetries was not predicted in the original $Q_{\text {weak }}$ proposal, such that their contribution is absent from the original error budget (Table 3.2. where the "Backgrounds" budget accounts mostly for backgrounds from the aluminum target windows). And yet the uncertainty from this correction dominated the error of the $2013 Q_{\text {weak }}$ result with $\delta A_{B B}=23.2 \mathrm{ppb}$, from the combined uncertainties of the $f_{2}$ and $A_{2}$ parameters in Eq.3.3.

USLsum vs Slug, Run2

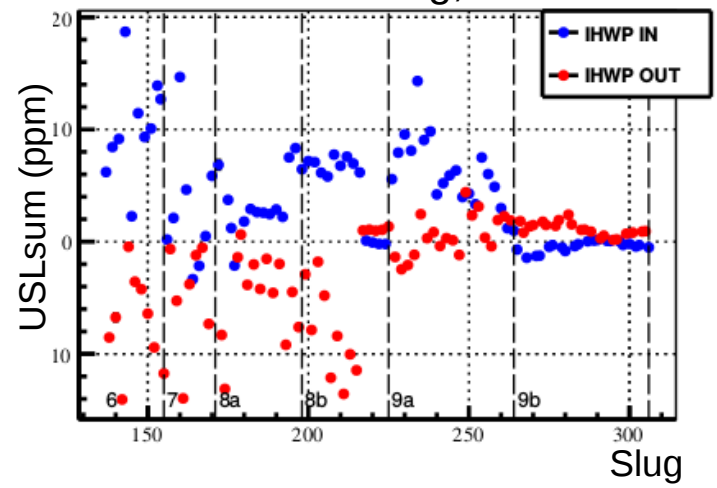

PMTLTG vs Slug, Run2

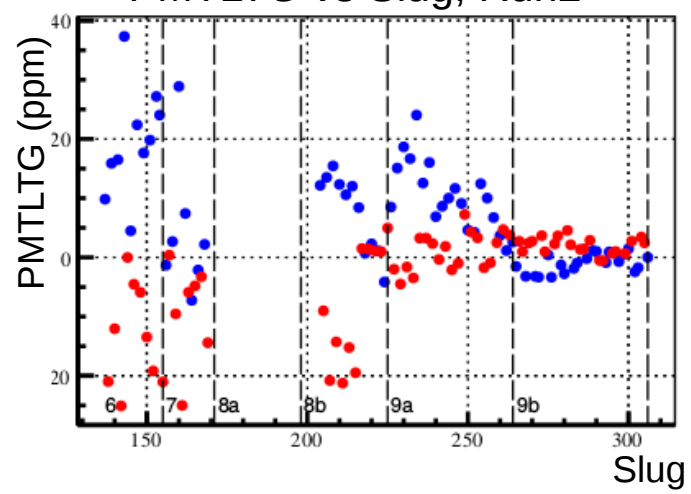

MD9 vs Slug, Run2

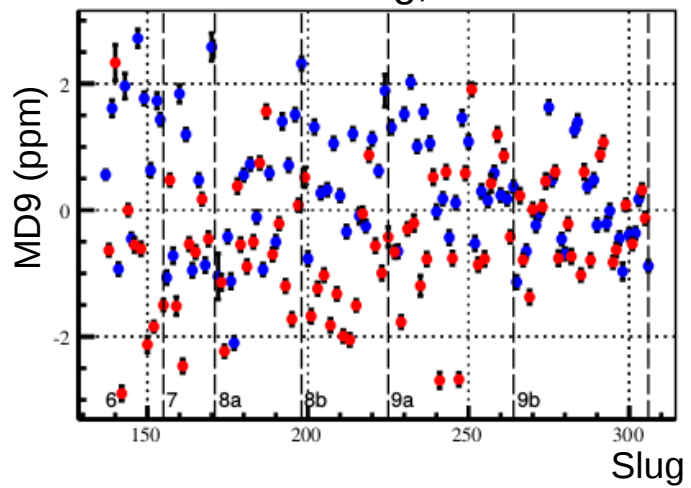

PMTONL vs Slug, Run2

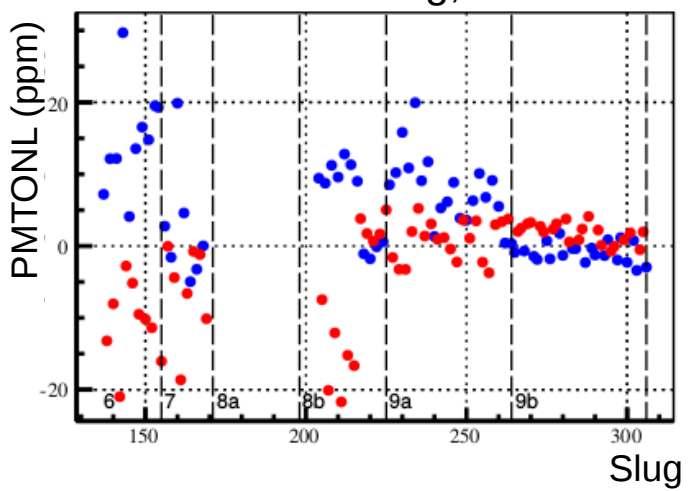

Figure 6.1: Background asymmetries in Run2 by the three background detectors and the US luminosity monitors, plotted versus slug and separated by IHWP state (blue for IHWP IN and red for OUT). Data for PMTLTG and PMTONL is missing for a period between Slugs 170-203 due to studies on their pre-amplifier channels.

The asymmetry sum of the upstream luminosity monitors (USLsum) is also included in the figure. The USLumis are treated as background detectors in this chapter, as their asymmetry is considered to be dominated by the same beamline background component. The asymmetries of 
all background detectors are visibly quite consistent and dominated by the same highly asymmetric background component that originates at the tungsten plug. This consistency will be a fundamental consideration in developing a correction for the $\mathrm{BB}$ asymmetry $A_{\mathrm{BB}}$.

\subsubsection{Correlations between background asymmetries in Run2}

Even by eye one can tell from Figure 6.1 that the asymmetries measured by different background detectors are very highly correlated and track the same changes in the beamline background component. This can be shown more rigorously with correlations between the asymmetries measured on different detectors, as in Figure 6.2. The linear fit is impressively consistent throughout Run2 with a good $\chi^{2} /$ DOF. Background asymmetries here are corrected for $A_{\text {beam }}$ through regression, although the beam correction scheme doesn't matter much for correlations between background asymmetries.
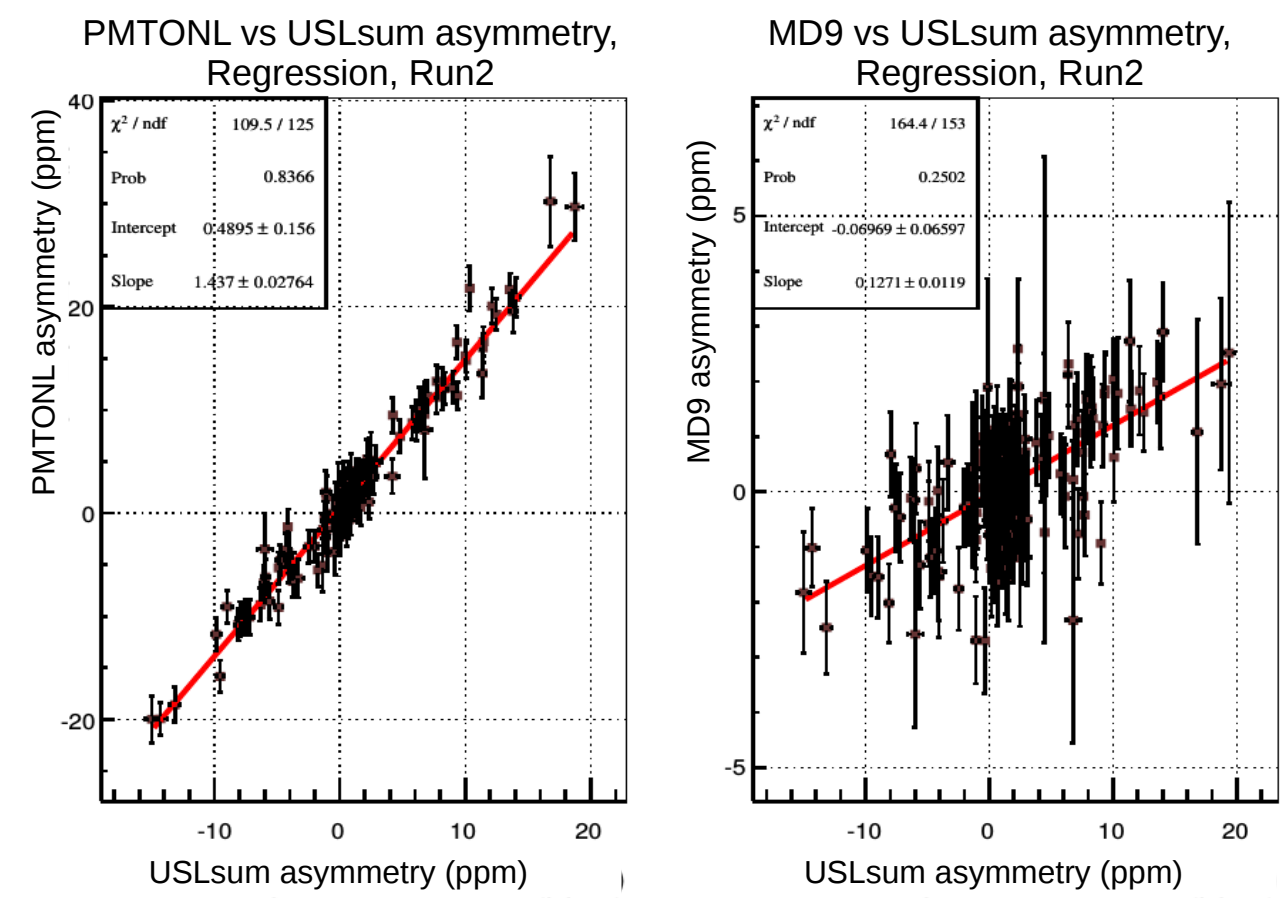

Figure 6.2: Correlation between background asymmetries over Run2, averaged at the slug scale. The linear fit appears to be very good over a wide range of asymmetries.

The USLsum asymmetry appears to be a very good predictor of the asymmetries measured by the background detectors in the MD hut. This is quite impressive considering that they are separated by two collimators, the spectrometer magnet, and the detector hut shielding. Especially since we were expecting the USLumi signal to be dominated by Møller electrons that carry negligible asymmetry and cannot be related to the BB component. It appears that the background component is isotropic, i.e. the same component is seen everywhere in the experimental hall. 
Table 6.1: Correlation slopes between asymmetries from different background detectors in Run2, in units of $\mathrm{ppm} / \mathrm{ppm}$.

\begin{tabular}{c|cccc} 
& USLsum & PMTLTG & PMTONL & MD9 \\
\hline USLsum & 1 & $0.55 \pm 0.01$ & $0.70 \pm 0.01$ & $7.87 \pm 0.74$ \\
PMTLTG & $1.82 \pm 0.02$ & 1 & $1.27 \pm 0.03$ & $14.22 \pm 1.41$ \\
PMTONL & $1.44 \pm 0.03$ & $0.79 \pm 0.02$ & 1 & $11.31 \pm 1.15$ \\
MD9 & $0.13 \pm 0.01$ & $0.07 \pm 0.01$ & $0.09 \pm 0.01$ & 1
\end{tabular}

The full list of correlation slopes between asymmetries from different background detectors in Run2 is given in Table6.1. The correlations are extracted in the same manner as in Figure 6.2 for example the correlation slope of PMTONL to USLsum $C_{\text {ONL-USL }}=1.44 \pm 0.03$ is taken from the fit in the figure. The fit applied between the background asymmetries is the symmetric fit discussed in Appendix C. Opposite correlations in the table are inverse of each other (e.g. $\left.C_{\mathrm{USL}-\mathrm{ONL}} \approx 1 / C_{\mathrm{ONL}-\mathrm{USL}}=0.70\right)$, as expected from the symmetric fit algorithm. All correlations are unambiguously significant and appear to be consistent through Run2.

We should observe the scaling of the asymmetries measured in different detectors, suggesting that they don't all measure the same size for the background asymmetry, or in other words the correlation slopes in Table 6.1 are not equal to 1. In Section 6.4 we will develop a model through which we will explain and predict these scaling factors in terms of the yield fraction each detector receives from the $\mathrm{BB}$ component.

\subsubsection{Background yields and asymmetry widths}

Like the background asymmetries, the yields of background detectors similarly appear to be sensitive to fluctuations of the same component (Figure 6.3) and are clearly correlated on different background detectors (Figure 6.4. This is not surprising if we assume that the BB component originates at the tungsten plug. If there is a bigger fraction of the electron beam scattering at the plug and beamline then we expect the yields of background detectors to increase in a correlated manner. Superimposed on the fluctuations of the BB component is a gradual yield reduction due to natural PMT yield degradation, most clearly on the MD9 detector where the $\mathrm{BB}$ component is smaller.

The asymmetry widths of the background detectors were also responding to the same variations of the BB component. On Figure 6.5 the asymmetry width of USLsum is plotted over Run2 with the USLsum yield superimposed. The agreement on the fluctuations tracked from these quantities is clear. When the yield of USLsum (and all background detectors) increases, signaling an increase in the presence of the BB component, the USLsum asymmetry width $\sigma_{\mathrm{USL}}$ increases also. 

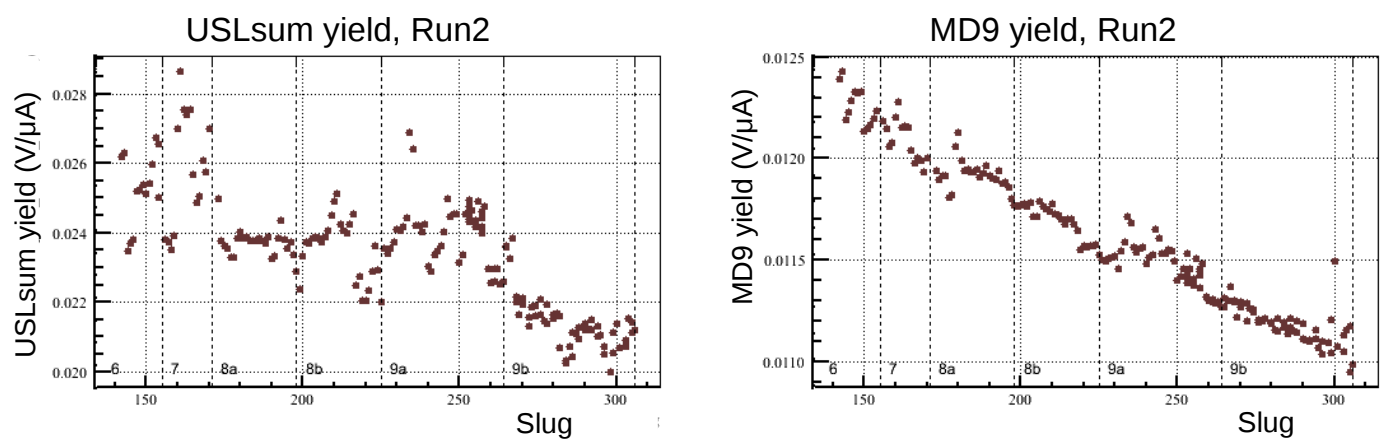

PMTLTG yield, Run2
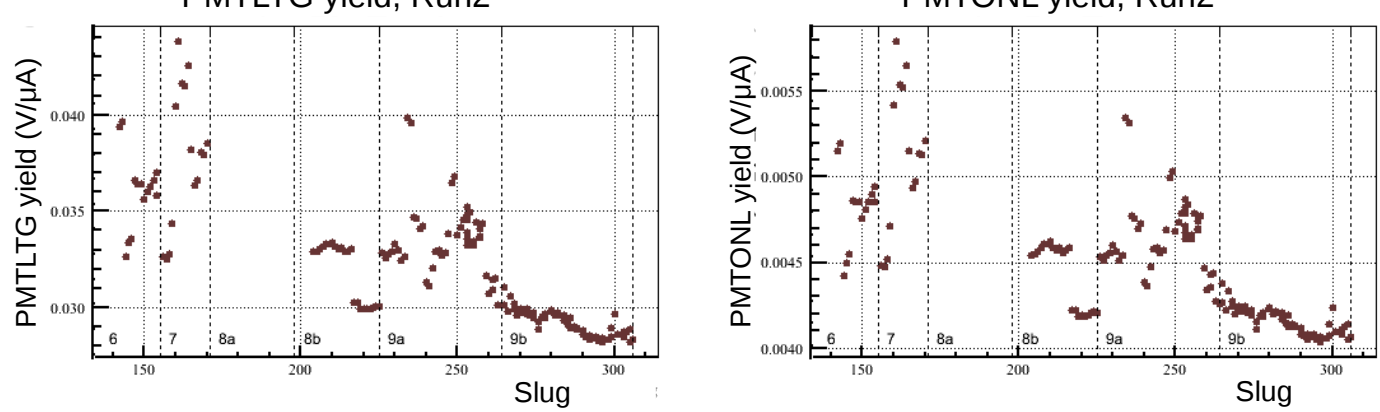

Figure 6.3: Yields of background detectors through Run2, averaged for every slug, in units of $\mathrm{V} / \mu \mathrm{A}$. Dashed vertical lines mark Wien transitions.

The size of the background asymmetries, while generally random and changing sign, is on absolute value clearly larger when the USLsum asymmetry width $\sigma_{\text {USL }}$ grows larger also. In fact $\sigma_{\mathrm{USL}}$ became our fastest metric to inform us of the presence of beamline backgrounds, which are considered to dominate above $\sigma_{\mathrm{USL}} \gtrsim 400 \mathrm{ppm}$. The background asymmetries are not only highly correlated among different background detectors, but also the background yields and asymmetry widths clearly respond to fluctuations of the same diffuse background component as well.

\subsubsection{Residual correlations of background asymmetries to beam pa- rameters}

The background detectors also need to be corrected for false beam asymmetries. And just as for the MD, significant residual correlations to beam parameters develop at longer time scales. In Figure 6.6, the residual correlation slopes of the regressed individual USLumi bars to HC beam parameters at the slug scale are plotted versus USLumi bar octant. This is the equivalent of Figure 5.23 for the main detector. The dipole shape of the residual correlations is characteristic of false beam asymmetries, while the USLsum average still exhibits significant non-zero residual correlation. The size of the residual correlation slope, up to $5 \mathrm{ppb} / \mathrm{nm}$ for residual dependence to position differences $\Delta X$ and $\Delta Y$, suggests a rather significant false asymmetry in periods of large $\mathrm{HC}$ position differences (a false asymmetry of $0.5 \mathrm{ppm}$ is implied for $\mathrm{HC}$ position differences of $100 \mathrm{~nm})$. 
PMTLTG vs USLsum yields, Run2

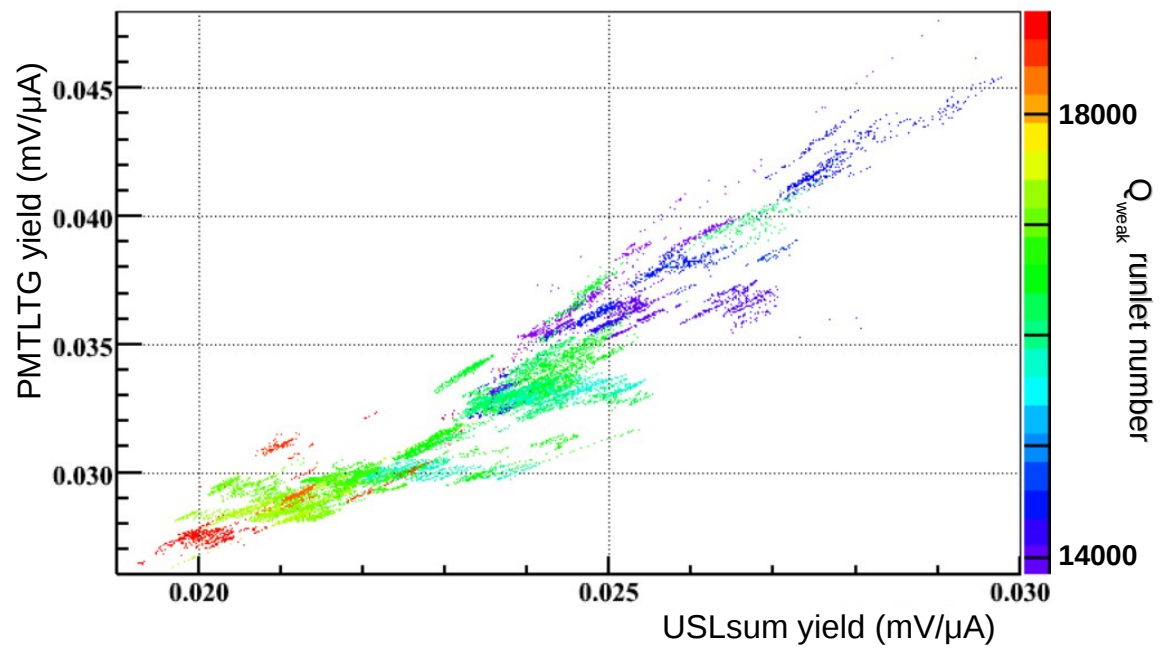

Figure 6.4: Scatterplot of PMTLTG and USLsum yields through Run2, seen to be highly correlated. Color gradient represents different periods in Run2, identified by segment number.
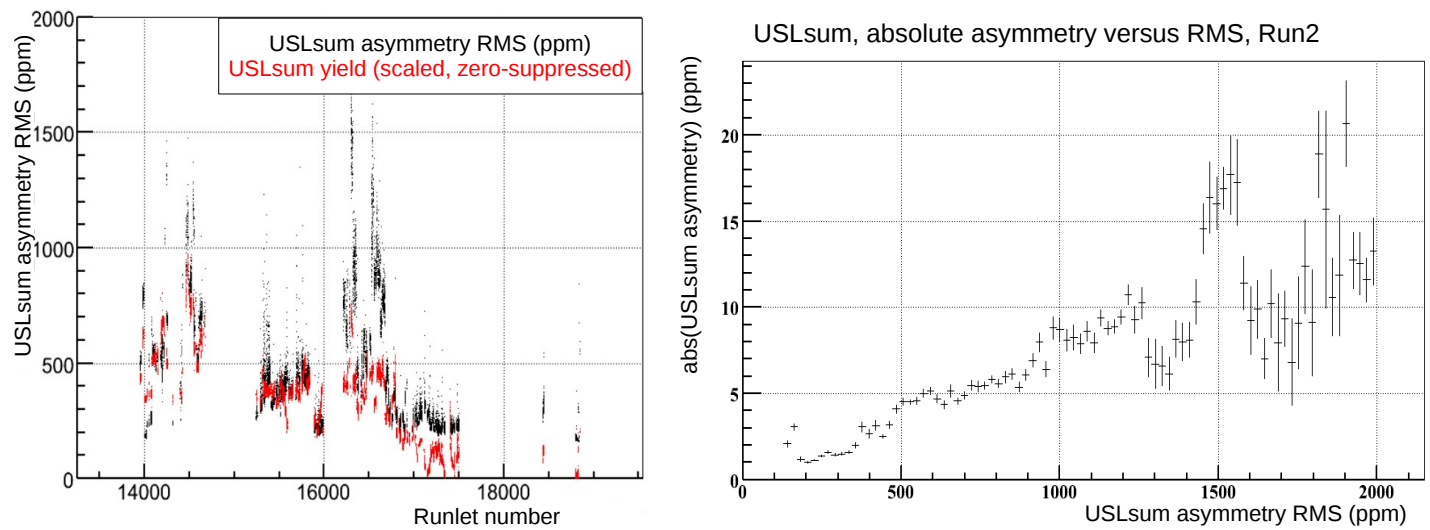

Figure 6.5: Left: Data for the USLsum asymmetry width (black, vertical axis in units of ppm) and yield (red, scaled and zero-suppressed), superimposed and plotted versus runlet number through Run2. Right: Profiled plot of the absolute USLsum asymmetry versus the USLsum asymmetry width. Larger $\sigma_{\text {USL }}$ generally comes with larger background asymmetries.

The residual correlation slopes are suggestive of coupling between the asymmetries measured in the background detectors and $\mathrm{HC}$ beam differences. Remember that background detectors had larger residual responses to the modulated beam parameters, as was discussed in Section 5.2.3. We hypothesized that the dithering residuals, correlated among different background detectors just as the background asymmetries and yields are, were connected to scattering from the plug collimator. Then the residual beam effects and the large background asymmetries may in fact have a common source. 

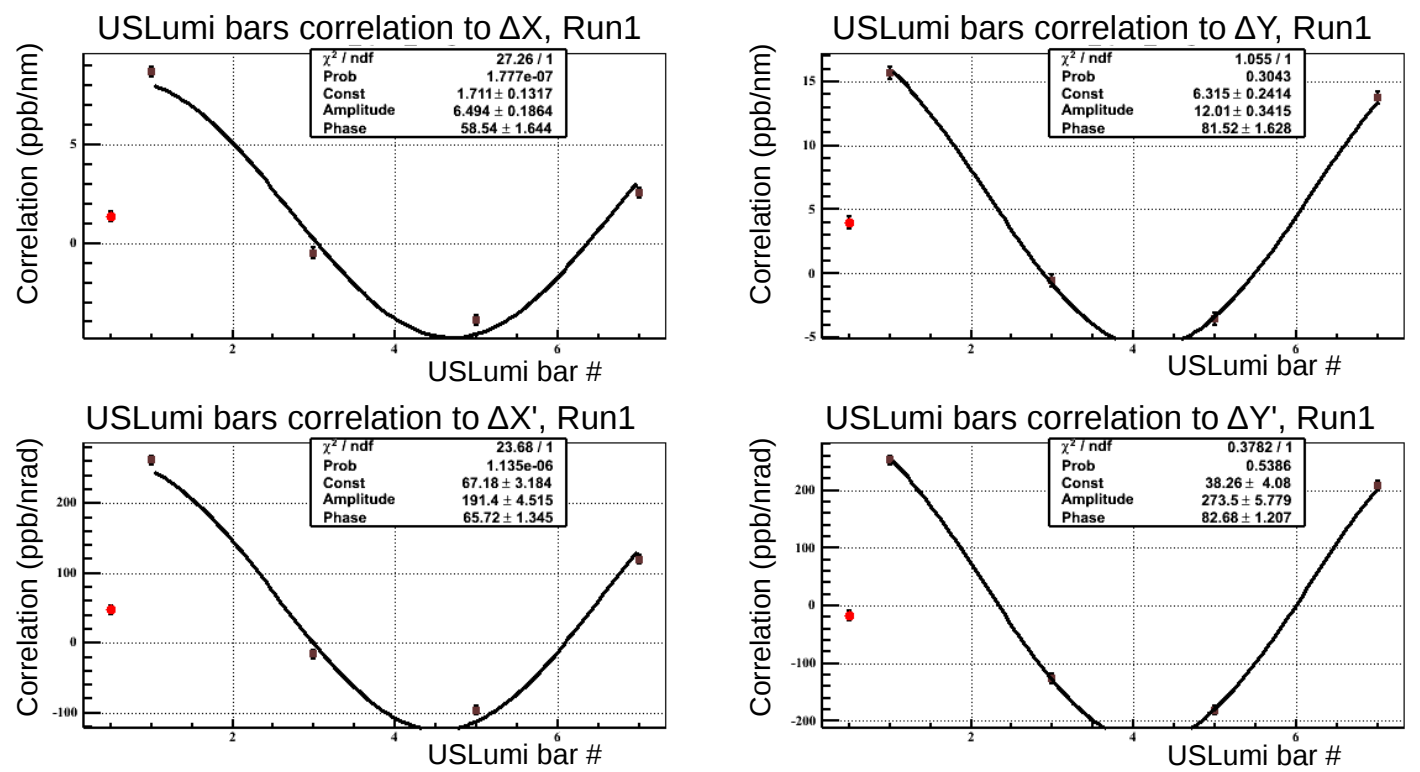

Figure 6.6: Residual slug-scale correlation slopes of the individual USLumi bars, corrected through regression, to HC beam parameters in Run1, plotted versus USLumi bar number. The dipole shape of the residual correlations is clear, while the USLsum combination (red datum) is also significantly non-zero.

\subsection{Connections with Beam Halo}

The large asymmetries seen by the background detectors are thought to be connected to a highly asymmetric beam "halo" component. It makes sense that beam halo, the outer part of the electron beam, will scatter preferentially from the beamline and plug collimator, and will be therefore connected to the beamline background component. This hypothesis was tested directly through dedicated studies.

To understand the response to beam halo and potential connections with the beamline background component, we worked with the accelerator operators to find ways to detune the beam optics in a controlled way and induce large halo rates. We experimented with changes in the field-integral $B d \ell$ of quadrupole magnets in the 3C12 (Hall C arc) or 0L06 locations, and also the phase of the Hall C source laser. These parameters were typically adjusted by accelerator operators during production to optimize beam parameters, such as halo and background rates. We were able to detune the beam and induce large halo rates and measure responses and properties of background detectors, although we found that the halo response was not fully reproducible after a few days. It should be noted that the induced halo rates and background asymmetries were not outside the range of beam conditions we encountered over the experiment.

The induced increase in halo rates was clearly correlated with a simultaneous increase in USLsum asymmetry width, as can be seen in Figure 6.7 in response to variation of the 0L06 quad $B d \ell$. Since $\sigma_{\mathrm{USL}}$ is a reliable indicator for the asymmetric beamline background component, this is direct indication of the connection with beam halo. Besides, the halo target (Section 3.7.3) 

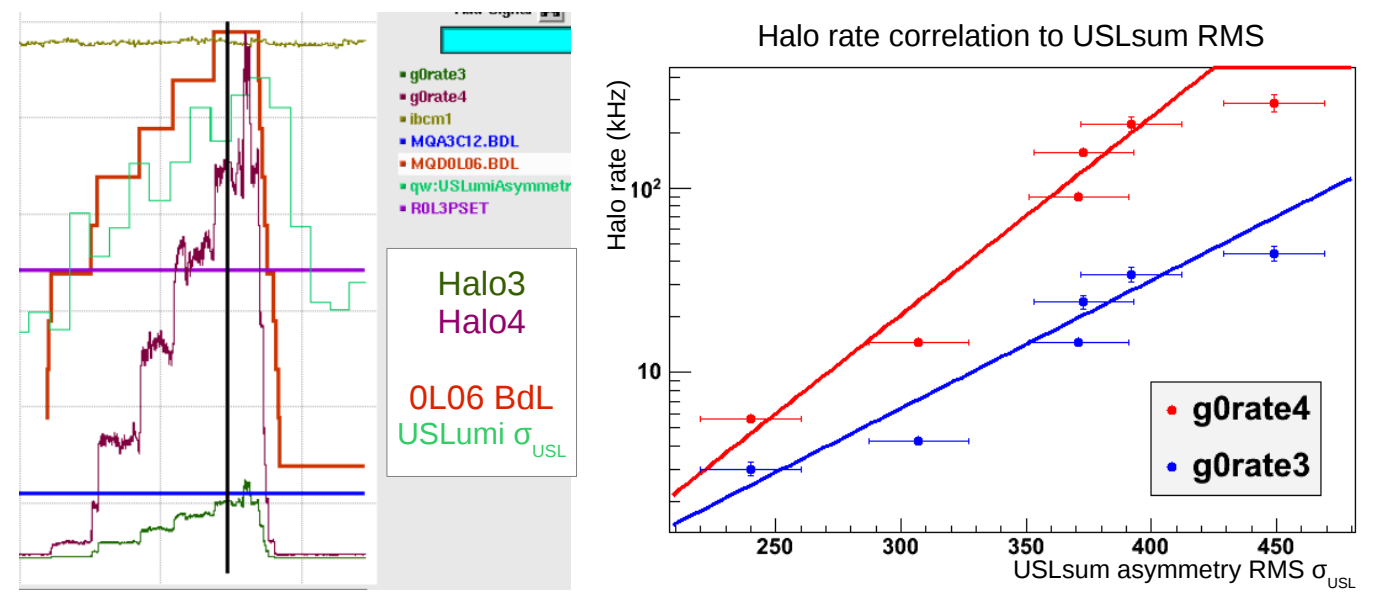

Figure 6.7: Left: Dedicated test from 3/17/2012, where the field-integral $B d \ell$ of quadrupole magnet 0L06 was varied to modulate halo. The quadrupole $B d \ell$, halo rates, and USLsum asymmetry width $\sigma_{\mathrm{USL}}$ are plotted versus time, using the accelerator EPICS strip tool. Right: The resulting halo rates in two halo monitors and the USLsum asymmetry width are highly correlated.

was specifically designed to be sensitive to electrons that would interact with the tungsten plug collimator, which is the source of beamline backgrounds in the experimental hall.

The response of the USLsum asymmetry width was also clear during a dedicated study where the Hall C laser phase was varied (Figure 6.8). Consistent with Figure 6.5 the USLsum asymmetry is larger in magnitude when its asymmetry width $\sigma_{\text {USL }}$ is also large. Very interestingly there is a connection with the USLsum regression sensitivities to position, which are also larger in magnitude (more negative) for larger $\sigma_{\mathrm{USL}}$. It is unclear whether this connection is characteristic of correlations through beam jitter (where regression sensitivities are derived from) between beam halo and first-order beam parameters like beam position. This would be expected to some degree as halo is a higher-order parameter generated by the same mechanisms (Section 4.6). We will see another interesting and related connection between background asymmetries and sensitivity to charge asymmetry in Section 6.5 .2

It is worth noting that the nominal operating phase for the Hall $\mathrm{C}$ laser at the time of the study was at $128^{\circ}$, which is not the point of minimum background asymmetries, asymmetry widths, regression sensitivities, and even false background asymmetries in the MD by some indications [158]. The significant improvement on all these qualities (at values of the $\mathrm{C}$ laser phase above $128^{\circ}$ ) coincides with a significant increase in aperture interception (up to $50 \mu \mathrm{A}$ on the A2 aperture, versus virtually zero on the nominal laser phase setting). It is conceivable that backgrounds in the experimental hall are removed due to an asymmetric halo component that is intercepted in the apertures ${ }^{1}$

The parameters that were modulated to induce halo during these studies, like the quadrupole

\footnotetext{
${ }^{1}$ We often encountered a similar situation during production, when background rates at the Compton region were anti-correlated with backgrounds rates and asymmetry widths in the experimental hall. It is possible that a halo component was being intercepted upstream before it had the chance to interact with the collimator.
} 

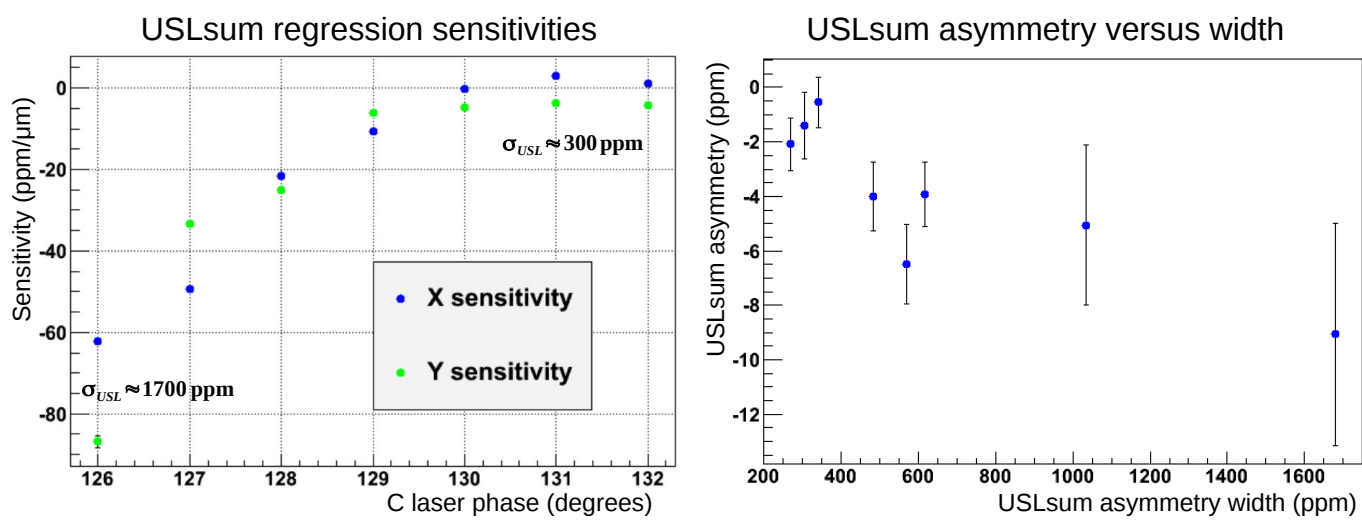

Figure 6.8: USLsum response during a dedicated study on $2 / 27 / 2012$ where the Hall C laser phase was varied around its nominal value of $128^{\circ}$. Left: The USLsum regression sensitivities to beam position (horizontal and vertical) clearly respond to the Hall $\mathrm{C}$ laser phase variation. Smaller regression sensitivities occur at smaller values of the USLsum asymmetry width $\sigma_{\mathrm{USL}}$ and vice versa. Right: The USLsum asymmetry plotted versus its asymmetry width during the laser phase scan.

field-integral and Hall C laser phase, are the same that were often adjusted during production by accelerator operators to optimize beam delivery criteria. These criteria include interception at injector apertures, as well as halo and background rates and asymmetry widths in the experimental hall. The functional form of halo is not known and depends on the combination of mechanisms that generate it, and is generally expected to vary through all the changes in beam delivery parameters. These variations were likely manifested in the experimental hall through the fluctuations of the beamline background asymmetries, as seen in Figure 6.1 .

\subsection{Blocked-Octant Measurements}

The beamline background component in the MD can be accessed in dedicated studies where an opening of a collimator is blocked and the yield of the corresponding MD octant is measured. This should be a direct measurement of the signal in the "blocked octant" from the BB component scattering from the tungsten collimator and downstream beamline, as simulations show that the primary signal leakage in the blocked octant is less than $0.0002 \%$. This is the BB signal fraction $f_{\mathrm{BB}}$, defined for any detector $i$ as the ratio of the signal from the beamline background component to the total signal+background:

$$
f_{\mathrm{BB}}^{i}=\frac{Y_{\mathrm{BB}}^{i}}{Y_{\text {total }}^{i}}
$$

Note that for the main detector $f_{\mathrm{BB}}^{\mathrm{MD}} \equiv f_{2}$, the factor which enters Eq. 3.3. For the MD the $\mathrm{BB}$ yield $Y_{\mathrm{BB}}^{\mathrm{MD}}$ is measured directly in the blocked-octant studies. The $Y_{\text {total }}^{\mathrm{MD}}$ is known from data immediately before the octant was blocked, or estimated by the yield of unblocked octants during the dedicated studies. 


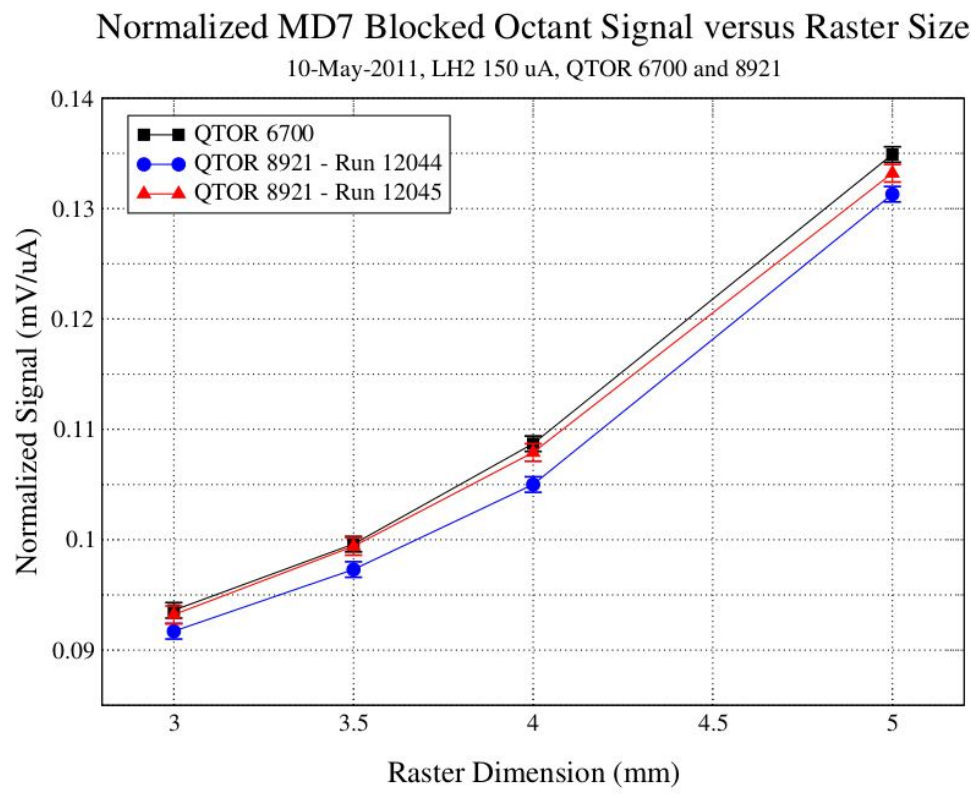

Figure 6.9: Normalized yield of blocked octant 7 versus raster size in the May 2011 studies, at the nominal elastic and inelastic magnetic field settings. Reproduced from [103.

Data was collected in these dedicated studies under different raster sizes and spectrometer settings, to examine the dependence of the beamline background component to these parameters.

\subsubsection{Run1 blocked-octant studies}

Several blocked-octant studies were performed during Commissioning and Run1, with different shielding configurations. Here we will give an overview of the Run1 studies, which were presented in more detail in 103 .

In the blocked-octant studies of May 2011, towards the end of Run1, the opening of octant 7 of the defining collimator was blocked with 3 " of lead. The beamline background component was measured at the nominal magnetic field setting of $8921 \mathrm{~A}$ and the inelastic field setting of $6700 \mathrm{~A}$, with a beam current of $150 \mu \mathrm{A}$ on the $\mathrm{LH}_{2}$ target. The beam raster size was also varied to study the dependence of the dilution factor on raster size.

The normalized signal of blocked octant MD7 is plotted versus raster size in Figure 6.9 for both magnetic field settings. The beamline background signal clearly increases with raster size with a dependence that seems quadratic. This makes sense as the raster increases the beam size and therefore the fraction of beam that is scattering on the plug collimator and the beamline, forming the BB component $\mathrm{D}^{2}$ The signal is also independent the magnetic field setting, confirming that it is dominated by neutrals.

Very interesting is the $\sim 2 \%$ discrepancy between sequential runs 12044 and 12045, taken

\footnotetext{
${ }^{2}$ Note however that the dependence on raster size for the aluminum target appeared to be quite linear, i.e. consistent with a zero quadratic component [103].
} 
Table 6.2: $\mathrm{BB}$ signal fraction $f_{\mathrm{BB}}$ values measured directly in blocked-octant studies in Run1 versus raster size, from two separate periods of dedicated studies.

\begin{tabular}{l|cc}
$\begin{array}{l}\text { Raster size } \\
(\mathrm{mm})\end{array}$ & $\begin{array}{c}\text { BB fraction } f_{\mathrm{BB}} \\
\text { (blocked defining collimator) }\end{array}$ & $\begin{array}{c}\text { BB fraction } f_{\mathrm{BB}} \\
\text { (blocked upstream collimator) }\end{array}$ \\
\hline 3 & $0.00129 \pm 0.00005$ & $0.00169 \pm 0.00040$ \\
3.5 & $0.00138 \pm 0.00006$ & $0.00178 \pm 0.00040$ \\
4 & $0.00150 \pm 0.00006$ & $0.00190 \pm 0.00040$ \\
5 & $0.00185 \pm 0.00008$ & $0.00225 \pm 0.00041$
\end{tabular}

at the same magnetic field setting. This discrepancy is larger than characteristic run-to-run variations of the unblocked detector signals over several hours, observed to be $\sim 0.75 \%$. The two runs were separated by about an hour during which several parameters in the injector were changed to re-establish beam delivery, and afterwards the halo rates were much higher and the beamline background component in the MD increased by $2 \%$. This is consistent with the assumed connection of the BB component with beam halo, which depends on beam delivery parameters in an unpredictable manner.

Another period of dedicated blocked-octant studies was performed in January 2011 during the Commissioning phase of the experiment. The final shielding configuration was not in place at that point, but a tray of three lead bricks installed around the beampipe between the second and third collimators closely resembled the final shielding configuration in that region. In this study it was the opening of the upstream collimator that was blocked, again on octant 7 . Due to high radiation and the need for multiple accesses, beam current was limited to $10 \mu \mathrm{A}$.

The signal in this configuration was measured for two raster sizes $\left(3 \times 3 \mathrm{~mm}^{2}\right.$ and $\left.4 \times 4 \mathrm{~mm}^{2}\right)$ on the $\mathrm{LH}_{2}$ target at the nominal elastic magnet setting. The fractional yield $f_{\mathrm{BB}}$ in this configuration was found to be higher from the May 2011 studies by an absolute $\sim 0.04 \%$. Extrapolating for the other raster sizes using that difference, the results from the two periods are listed in Table 6.2

Note that both configurations should be accessing the same background component that is mostly generated at the tungsten plug and perhaps rescatters from the beamline downstream. Only the part of the BB component that goes through the opening of the defining collimator may be affected differently in the two configurations. That is precisely the component that the "lintel" collimators were blocking, the blue line in Figure 3.7. It is possible that the discrepancy of $0.04 \%$ in the two configurations is due to this pathway. Systematic effects that may also contribute to the discrepancy include potential differences in halo and background conditions during the two periods, differences of the installed tray of lead bricks from the final shielding configuration, and possibly pedestal imperfections that become important due to the very small signals. To account for the discrepancy we will follow the prescription of [103] and assign a $100 \%$ 
uncertainty on the component that was missing from the May 2011 studies, such that its signal fraction is estimated to be $0.04 \% \pm 0.04 \%$.

The raster size most commonly used in Run2 was $4 \times 4 \mathrm{~mm}^{2}$. The corresponding BB signal fraction from Table 6.2 is:

$$
\left.f_{\mathrm{BB}}\right|_{4 \times 4 \mathrm{~mm}^{2}} ^{\mathrm{Run} 1}=0.19 \% \pm 0.04 \%
$$

We may still need to account for variations of the BB signal fraction due to changing beam conditions and halo rates, although it is possible that the discrepancy in the two periods was solely due to different beam conditions and therefore the assigned uncertainty is an estimation of these variations. During the studies in Run2, presented in the following subsection, we attempted to access this uncertainty more directly by inducing a broad range of halo conditions.

\subsubsection{Run2 blocked-octant studies}

A more comprehensive and reproducible method of blocking collimator openings by remotely inserting tungsten shutters was in place for Run2. The shutters could be inserted to block the openings in octants 1 and 5 of the upstream collimator. In this configuration not only the MD octants 1 and 5, but also the corresponding USLumi octants can be considered blocked. For the upstream luminosity monitors this means that the Møller electrons coming through the opening of the upstream collimator will be blocked, therefore this measurement should be directly accessing the $\mathrm{BB}$ component and signal fraction $f_{\mathrm{BB}}^{\mathrm{USL}}$. We will also attempt to extract the $\mathrm{BB}$ signal fraction for the other background detectors, although the non-BB signal is not directly blocked on them.

In the blocked-octant studies of Run2 we experimented with intentionally varying the beam halo through 0L06 or 3C12 quadropole field integral, or through the Hall $\mathrm{C}$ laser phase, as described in Section 6.2. These studies allowed us to measure the BB signal fraction under different beam conditions and get an estimation for its range of variation. Specifically the blocked-octant studies in Run2 were performed in two separate periods, a period with "good" halo conditions, where halo rates were as low as ever observed, and one with "bad" halo conditions where very large halo rates were artificially induced. All data was taken with a $4 \times 4 \mathrm{~mm}^{2}$ raster at high beam currents of $180 \mu \mathrm{A}$.

\section{Fractional rates}

The current-normalized signals of background detectors and MD bars were measured in the two different periods of Run2 blocked-octant studies, under "good" and "bad" beam halo conditions. The fractional signal of various detectors, defined as the signal in the blocked-octant studies over 


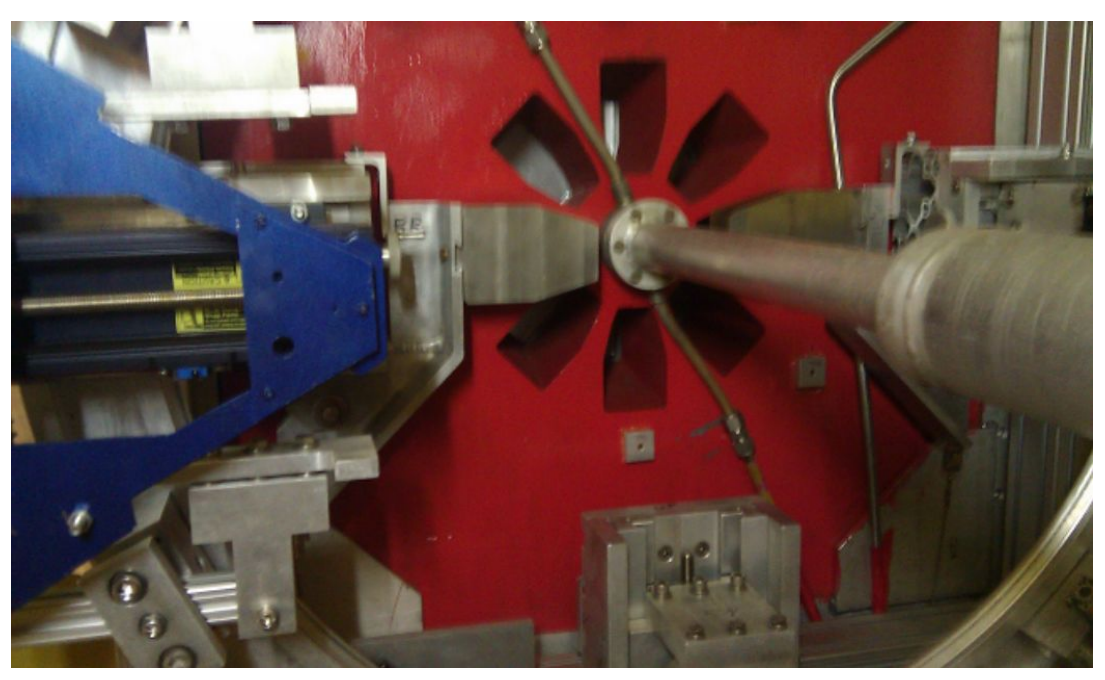

Figure 6.10: Picture of the tungsten shutters inserted on the downstream face of the first (most upstream) clean-up collimator, blocking the openings for elastic events in the horizontal octants 1 and 5. The downstream side of the tungsten plug collimator is also visible.

the unblocked signal, is listed in Table 6.3 under good and bad halo conditions. Note that this fraction is equivalent to the $\mathrm{BB}$ signal fraction $f_{\mathrm{BB}}$ from Eq. 6.1 if all signal during the blockedoctant studies is coming from the BB component. This is probably only true for the blocked octants in the MD and the USLumi monitors, but not the other background detectors that may be only partially blocked.

There is a clear dependence of the signal fraction to halo rates. The blocked signal increases with halo in both octants 1 and 5, as well as on all background detectors. This is consistent with the assumption that the beamline backgrounds component is connected to halo scattering at the collimators and beamline. The variation of the yield fraction on the MD is $\sim 4 \%$, which happens to be the same uncertainty assigned to the Run1 estimation for the BB signal fraction in Eq. 6.2. There is also a $\sim 3 \%$ variation between octants 1 and 5 that is consistent for both periods. The average value for the blocked $\mathrm{MD}$ octants is $f_{\mathrm{BB}}^{\mathrm{MD}}=0.196 \%$, in very good agreement with the Run1 estimation of $0.19 \%$. For the MD beamline background signal fraction we will accept the average of the two estimations with a very conservative $33 \%$ fractional uncertainty:

$$
f_{\mathrm{BB}}^{\mathrm{MD}} \equiv f_{2}=0.193 \% \pm 0.064 \%
$$

The uncertainty accounts for the variation over different halo conditions and the apparent discrepancy between blocked octants 1 and 5 in Table 6.3. As we will see this dilution factor will be used for the entire Run2 $A_{\mathrm{BB}}$ correction, so the uncertainty partially absorbs variations of the signal fraction due to short periods of production under different raster sizes. 
Table 6.3: Fractional signal size of detectors with the tungsten shutters installed in the upstream collimator openings in octants 1 and 5. The signal fraction is expressed as a ratio of the detector signal in the blocked-octant run to the signal of the same detector immediately before the shutters were inserted. The shutter tests were repeated in two different occasions, under "good" and "bad" halo conditions. From elogs 779,783,786,784.

\begin{tabular}{c|cc} 
& \multicolumn{2}{|c}{ Fractional Signal } \\
& "Good" halo conditions & "Bad" halo conditions \\
\hline MD1 & $0.191 \%$ & $0.232 \%$ \\
MD5 & $0.159 \%$ & $0.203 \%$ \\
USLumi1 & $50.5 \%$ & $59.1 \%$ \\
USLumi3 & $93.1 \%$ & $106.8 \%$ \\
USLumi5 & $49.1 \%$ & $58.9 \%$ \\
USLumi7 & $94.2 \%$ & $102.5 \%$ \\
PMTLTG & $94.3 \%$ & $117.6 \%$ \\
PMTONL & $94.2 \%$ & $115.3 \%$ \\
MD9- & $4.68 \%$ & $5.89 \%$ \\
MD9+ & $11.2 \%$ & $12.9 \%$ \\
MD9sum & $8.0 \%$ & $9.1 \%$
\end{tabular}

We generally believe detectors USLumi3, USLumi7, PMTLTG and PMTONL to be unaffected from the shutters inserted in octants 1 and $5^{3}$ This is confirmed by the fact that correlations between background asymmetries in the blocked-octant dataset are consistent with the unblocked correlations over Run2 [159]. Therefore their signal fraction deviation from 100\% with respect to their signal prior to the shutters insertion is solely due to the change in halo conditions. Again it makes sense that the background rates are proportional to halo rates based on the connections discussed in Section 6.2

For the blocked USLumi bars 1 and 5 , the yield fraction is likely a good estimation of the BB signal fraction: $f_{\mathrm{BB}}^{\mathrm{USL} 15} \approx 55 \%$. Expecting that the USLumi signal would be dominated by Møller electrons, as described in the $Q_{\text {weak }}$ proposal, it was an important revelation that more than half of their signal is not coming through the openings of the upstream collimator. Instead the USLumis will be indispensable as a background monitor and essential in our algorithm for the $A_{\mathrm{BB}}$ correction.

The average signal fraction for MD9 is $\sim 8.5 \%$. This is also an important point: MD9, as well as the other background detectors in the superelastic region, receive part of their signal through the collimator openings, very likely due to "leakage" signal from MD bars. This is confirmed through correlations of these detectors to individual MD bars, and also through simulations. For MD9 we see that more than $90 \%$ of its signal comes through octant 5 . This is the reason why MD9 measures the smallest background asymmetries and is the most noisy in its ability to determine $A_{\mathrm{BB}}$ : the $\mathrm{BB}$ component in its signal is diluted more than in any other background detector. The

\footnotetext{
${ }^{3}$ Except for possible cross-talk between USLumi octants, i.e. scattering from the edges of collimator openings in octants 1 and 5 that make it into USLumis 3 and 7 ; this signal would be removed when the shutters are inserted, but it should be a negligible component. Conversely however, cross-talk from the unblocked collimator openings could contribute a significant signal fraction in the "blocked" USLumi octants.
} 


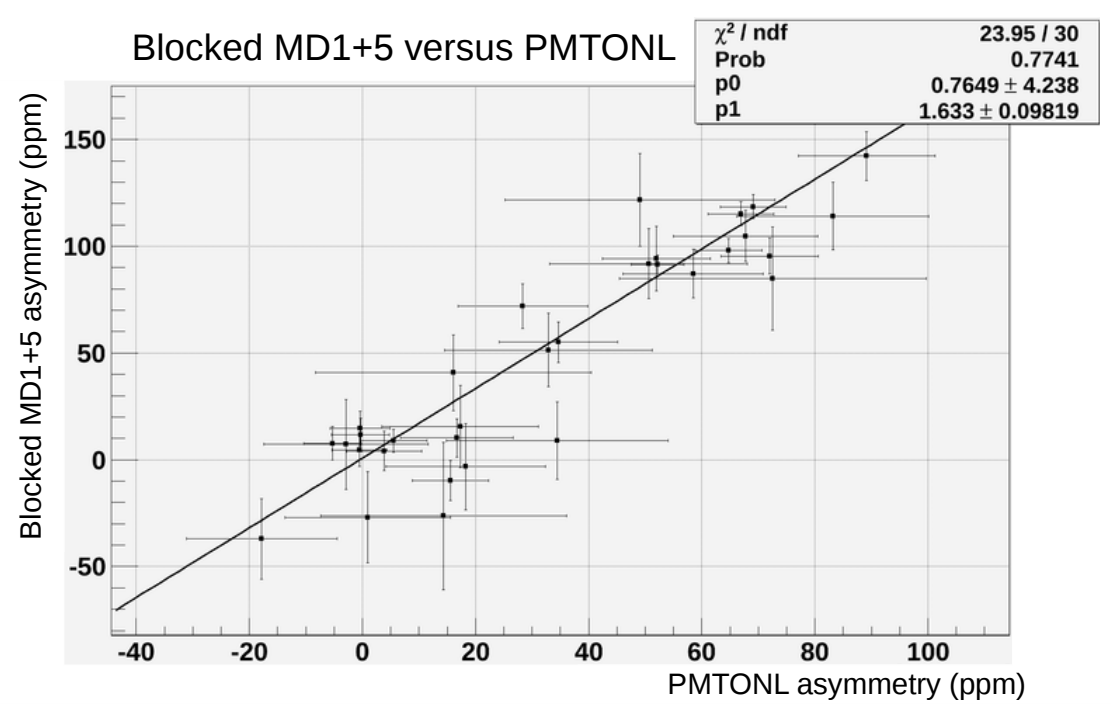

Figure 6.11: Correlation between the average asymmetry in the blocked MD bars and PMTONL over all blocked-octant data. The data are binned over 5-minute runlets.

fractional signal on the positive and negative MD9 PMTs are not equal as they don't receive equal signals from MD5 leakage due to their relative positioning. Through correlations this author has shown that MD9 also receives some leakage from MD6, which was unblocked in the dedicated studies and further complicates interpretation of the numbers on the table. Similarly the PMTLTG and PMTONL detectors receive leakage signal from MD3, which however is not blocked in these studies.

\section{Correlations of asymmetries}

Combining the data over the different periods of blocked-octant studies in Run2 we get a wide range of halo rates and background asymmetries. Forming correlations between the asymmetries measured in background detectors over these different conditions is conceptually the same as the correlations extracted over all of Run2. In Section 6.1.2 we showed that the correlation slopes between background asymmetries are remarkably constant over the entire Run2.

We repeat the extraction of correlations over the range of induced halo conditions in the blocked-octant data, where now some of the detectors may be partially blocked. We also include the average of the fully blocked MD bars 1 and 5 , where their signal is considered to be $100 \%$ from beamline backgrounds. As an example the correlation of the blocked MD bars to PMTONL is shown in Figure6.11. The asymmetries measured by the blocked MD bars were very large during the bad halo conditions, up to $150 \mathrm{ppm}$. In fact the blocked MD bars consistently measured higher asymmetries than any background detector. The large asymmetries on the blocked MD bars, highly correlated to the background detector asymmetries, constitutes conclusive evidence that the $\mathrm{BB}$ component generates a false asymmetry in the MD. 
Table 6.4: Correlation slopes of the blocked MD bars to each of the background detectors, for the May 2012 blocked-octant dataset. All values quoted in units of $\mathrm{ppm} / \mathrm{ppm}$.

\begin{tabular}{ccccc}
$\begin{array}{c}\text { USLumi15 } \\
\text { (partially blocked) }\end{array}$ & $\begin{array}{c}\text { USLumi37 } \\
\text { (unblocked) }\end{array}$ & $\begin{array}{c}\text { MD9 } \\
\text { (partially blocked) }\end{array}$ & $\begin{array}{c}\text { PMTLTG } \\
\text { (unblocked) }\end{array}$ & $\begin{array}{c}\text { PMTONL } \\
\text { (unblocked) }\end{array}$ \\
\hline $1.88 \pm 0.07$ & $2.67 \pm 0.10$ & $1.99 \pm 0.16$ & $1.29 \pm 0.06$ & $1.63 \pm 0.10$
\end{tabular}

The correlation slopes of the asymmetry measured on the blocked MD bars to all background detectors are listed in Table 6.4 extracted as in Figure 6.11. Since the measured asymmetry was higher in the blocked MD bars than in any other detector, these slopes are always larger than $1 \mathrm{ppm} / \mathrm{ppm}$.

Our observations on the fractional rates and background asymmetry correlations from the blocked-octant dedicated studies, will be incorporated in the model for the beamline backgrounds that we develop in the next section.

\subsection{A Model for the Beamline Background Asymmetries}

The beamline background component is created through electrons scattering at the collimators and beamline, and assumed to be isotropic. In a simple model, we assume that the asymmetry measured on each background detector is proportional to the yield fraction $f_{\mathrm{BB}}^{i}$ that detector $i$ receives from the $\mathrm{BB}$ component:

$$
A_{\mathrm{BB}}^{i}=f_{\mathrm{BB}}^{i} A_{2},
$$

where $A_{2}$ is a "source" term for the beamline background asymmetry, assumed to be fluctuating and even changing sign under different beam conditions. This is the asymmetry associated with the beamline background component that enters $\mathrm{Eq} 3.3$.

This model is only a first-order attempt at a framework that accommodates our observations, but already has some satisfying characteristics:

- It is reasonable to assume that the asymmetry of all detectors is dominated by the same component, given their high correlation coefficients.

- All other asymmetric sources, such as electrons carrying the $\overrightarrow{e p}$ or Møller asymmetry, can usually be assumed negligible compared to the large BB asymmetries. Therefore from the asymmetry formula $(3.2)$, and since the non-BB signal on every detector does not contribute in the numerator, we expect the asymmetry measured by each detector $i$ to be proportional to its $\mathrm{BB}$ signal fraction $f_{\mathrm{BB}}^{i}$. In other words, all signal on a background detector that is not coming from beamline scattering is only diluting the BB asymmetry 
$A_{2}$.

- This model also naturally explains why the blocked MD bars in the dedicated studies see higher asymmetries compared to the background detectors: their BB signal fraction is $f_{\mathrm{BB}}^{\text {blocked } \mathrm{MD}} \rightarrow 100 \%$ and therefore $A_{\mathrm{BB}}$ is undiluted compared to the background detectors. The asymmetries measured by the blocked MD suggest that the source BB asymmetry $A_{2}$ can be higher than 100 ppm under "bad" halo conditions.

- Similarly the MD9 detector always measures smaller asymmetries during production because its $\mathrm{BB}$ signal fraction $f_{\mathrm{BB}}^{\mathrm{MD} 9}$ approx $8.5 \%$ is expected to be much lower than other background detectors.

- Finally this model offers a nice way to explain the "scaling" of asymmetries measured on different background detectors, described by the scaling factors of Table 6.1 The correlation slope between two background detectors is simply equal to the ratio of the $\mathrm{BB}$ fractional signals in each detector:

$$
\operatorname{Corr}\left(A^{\text {Det1 }}, A^{\text {Det2 }}\right) \approx \frac{A_{\mathrm{BB}}^{\text {Det1 }}}{A_{\mathrm{BB}}^{\text {Det2 }}} \approx \frac{f_{\mathrm{BB}}^{\text {Det1 }}}{f_{\mathrm{BB}}^{\text {Det2 }}}
$$

The last bullet point suggests the most satisfying feature of this model - that it makes falsifiable predictions. If we can estimate the BB signal fraction of a background detector then we can test our scaling hypothesis. Unfortunately only USLumi bars 1 and 5 are considered blocked and therefore should directly access their BB signal fraction. As we can see on Table 6.3 this fraction varies between 50-60\%. The BB signal fraction $f_{\mathrm{BB}}^{i}$ of every detector $i$ varies with beam conditions and is expected to be roughly proportional to the presence of beam halo.

While we don't have a direct signal fraction measurement for any other background detector, we can use our simple model to estimate their signal fractions from the blocked-octant asymmetry correlations. Under the assumption of Eq. (6.5) that the correlation slope between two detectors is equal to the ratio of their signal fractions, then we can extract the suggested fraction $f_{\mathrm{BB}}^{i}$ for every background detector $i$ from the correlation with the blocked MD bars, assuming $f_{\mathrm{BB}}^{\text {blocked MD }} \rightarrow 100 \%$. The suggested BB signal fraction for every detector is given in Table 6.5 These numbers are practically the inverse of the correlations given in Table 6.4

We can compare these numbers suggested by the model with the correlations extracted over Run2. For PMTLTG and PMTONL, which are the simplest since they are unobstructed in the blocked-octant study and can be directly translated to normal production data, the ratio of signal fractions gives

$$
f_{\mathrm{BB}}^{\mathrm{PMLTG}} / f_{\mathrm{BB}}^{\mathrm{PMTONL}}=78 \% / 61 \% \approx 1.28
$$


Table 6.5: The BB signal fractions of background detectors are extracted from the correlations to the blocked MD bars under the simple model assumptions.

\begin{tabular}{ccccc}
$\begin{array}{c}f_{\mathrm{BB}}^{\mathrm{USLumi} 15} \\
(\text { blocked) }\end{array}$ & $\begin{array}{c}f_{\mathrm{BB}}^{\mathrm{USLumi} 37} \\
(\text { unblocked) }\end{array}$ & $\begin{array}{c}f_{\mathrm{BB}}^{\mathrm{MD} 9} \\
\text { (partially blocked) }\end{array}$ & $\begin{array}{c}f_{\mathrm{BB}}^{\mathrm{PMTLTG}} \\
\text { (unblocked) }\end{array}$ & $\begin{array}{c}f_{\mathrm{BB}}^{\mathrm{PMTONL}} \\
\text { (unblocked) }\end{array}$ \\
\hline $53.1 \% \pm 4 \%$ & $37.5 \% \pm 4 \%$ & $50.2 \% \pm 8 \%$ & $77.3 \% \pm 5 \%$ & $61.2 \% \pm 6 \%$
\end{tabular}

which is in excellent agreement with the correlation slope of $1.27 \mathrm{ppm} / \mathrm{ppm}$ over Run2, from Table 6.1

For the blocked and partially blocked detectors, translation to numbers for their unblocked values is complicated. We can see that the blocked USLumi bars do not measure the same large background asymmetries as the blocked MD bars. This could mean that there is a significant part of the beam scattering at the collimator which carries much smaller asymmetries, such as the cross-talk component from the unblocked octants. If this is the case then the USLumi octants 1 and 5 should be considered only partially blocked in the dedicated studies.

\section{Deviation from isotropy}

Looking at the USLumi horizontal and vertical bars separately in Run2, we will see that the proposed simplistic model cannot be exactly correct. Averaged at the slug time scale the horizontal and vertical USLumi asymmetries are highly correlated in Run2, but the asymmetries measured in the horizontal are consistently higher from the vertical by $33 \%$, i.e. the correlation slope $C_{\mathrm{USL15}-\mathrm{USL} 37} \approx 1.33$ (Figure 6.12 . This is certainly a deviation from the isotropy assumption which would require that horizontal and vertical USLumi bars measure the same asymmetry. Instead it seems as if the combinations of horizontal and vertical USLumi bars might as well be considered separate background detectors with a scaling factor of 1.33 .

Perhaps related to the anisotropy between the horizontal and vertical USLumi asymmetries, is a raster scan performed on 03/12/2012. In this scan (Figure 6.13) the raster size was increased from $1 \mathrm{~mm}$ to $6 \mathrm{~mm}$ in steps of $1 \mathrm{~mm}$, with the increase in the horizontal size (raster X) always delayed compared to the increase in the vertical size (raster $\mathrm{Y}$ ). The halo rates consistently increased with the $\mathrm{X}$ raster size, as expected from the dedicated raster scan of Figure 6.9. However the halo rates showed no response to increases of the $\mathrm{Y}$ raster size. This is more evidence against the isotropy hypothesis, possibly associated with residual energy dispersion in the horizontal plane.

In any case, assigning a single number to each background detector that nominally corre-

\footnotetext{
${ }^{4}$ Note that we couldn't have seen such a clear picture from correlations of individual USLumi bars because of relatively large residual effects from beam corrections that cloud this correlation; we need the combination of opposite bars to cancel residual beam effects and isolate the correlation due to the BB component.
} 


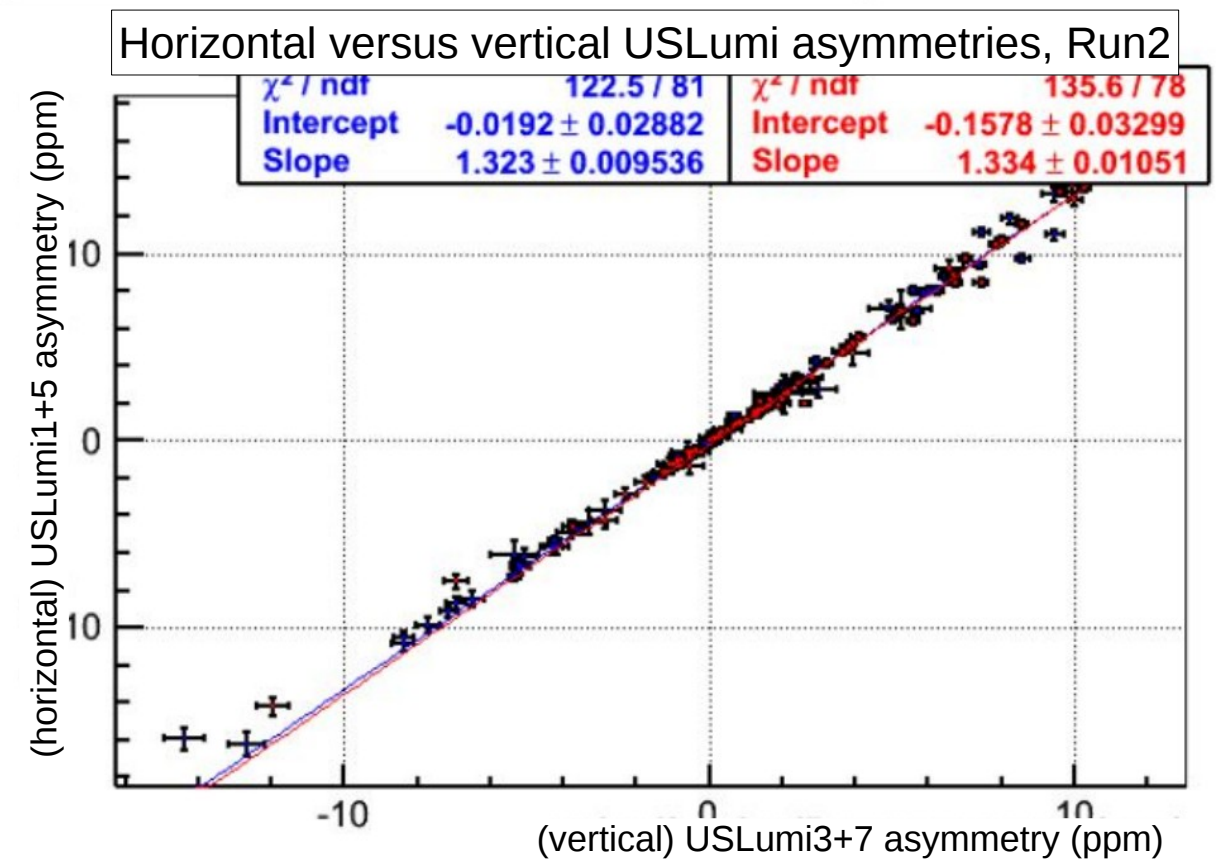

Figure 6.12: High correlation between horizontal and vertical USLumi bars. Data averaged at the slug time scale over Run2, separated by IHWP states. The combination of opposite bars cancel out residual beam effects.

sponds to its background signal fraction appears to work very well for describing the background correlations, even if the yield ratios are only an effective description. The correlation of the blocked MD bars to the vertical USLumis suggests an "effective" signal fraction of $f_{\mathrm{BB}}^{\mathrm{USLumi} 37} \approx 37 \%$. From the correlation between horizontal and vertical USLumi bars we expect

$$
f_{\mathrm{BB}}^{\mathrm{USL} 15}=C_{\mathrm{USL} 15-\mathrm{USL} 37} \times f_{\mathrm{BB}}^{\mathrm{USL} 37} \approx 49.9 \%
$$

and therefore for the USLsum combination of all bars:

$$
f_{\mathrm{BB}}^{\mathrm{USLsum}}=\left(f_{\mathrm{BB}}^{\mathrm{USL} 15}+f_{\mathrm{BB}}^{\mathrm{USL} 37}\right) / 2 \approx 43.7 \%
$$

According to the simple model of Eq. (6.4) we should be able to predict the correlations between background detectors from their signal fractions:

$$
\begin{aligned}
& C_{\text {PMTLTG-USLsum }}=f_{\mathrm{BB}}^{\text {PMTLTG }} / f_{\mathrm{BB}}^{\text {USLum }} \approx 1.77 \\
& C_{\text {PMTONL-USLsum }}=f_{\mathrm{BB}}^{\text {PMTONL }} / f_{\mathrm{BB}}^{\text {USLum }} \approx 1.40
\end{aligned}
$$

These values are nicely consistent with the results we get from the Run2 correlations in Table 6.1 Our final estimations for the effective BB signal fractions for the unblocked detectors are given in Table 6.6. They have impressive power and consistency to predict the correlations between 


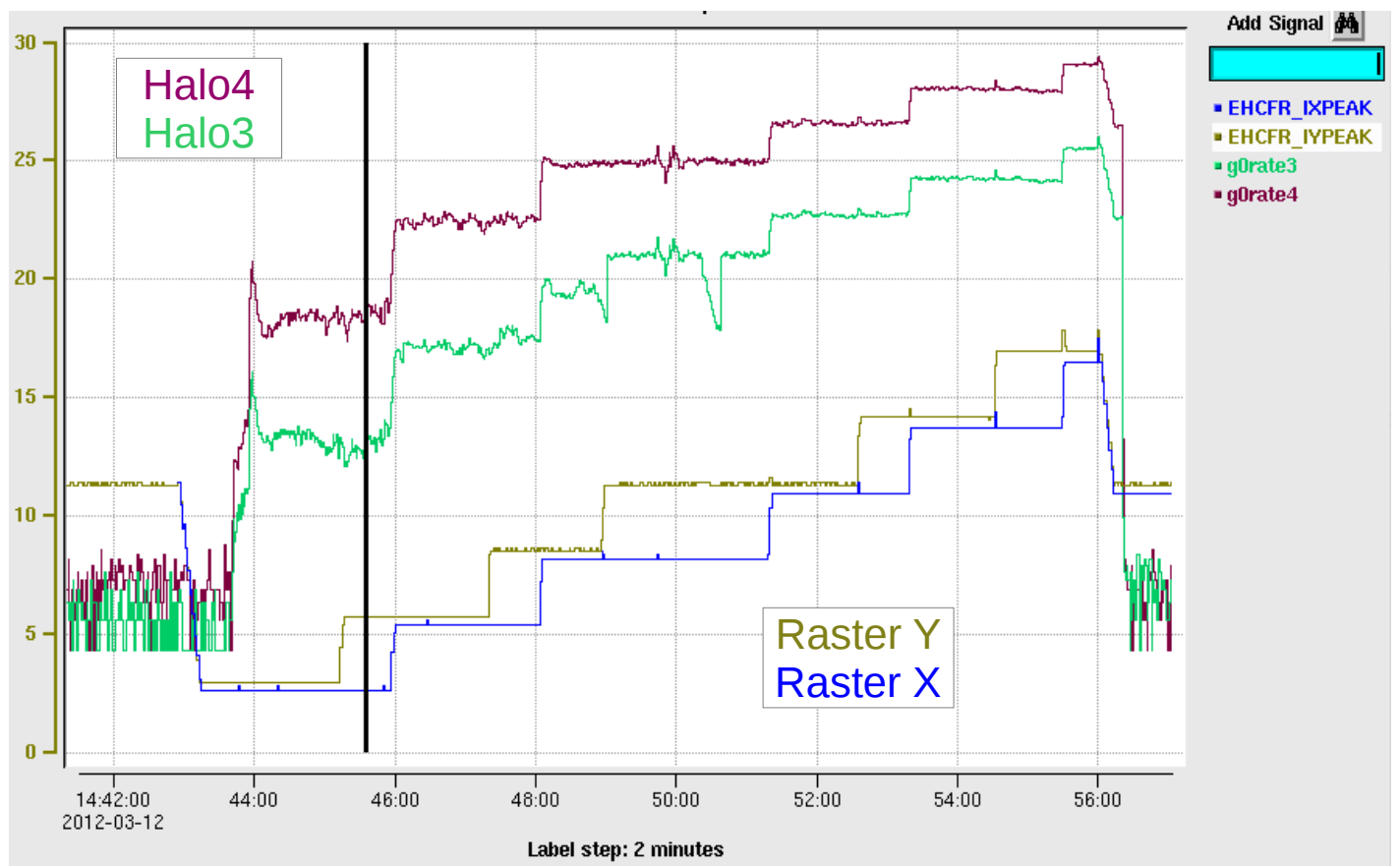

Figure 6.13: Plot from the accelerator EPICS strip tool of halo rates (from detectors halo3 and halo4) versus time as the size of the raster is increased sequentially in $\mathrm{X}$ and $\mathrm{Y}$ (horizontal and vertical) from $1 \mathrm{~mm}$ to $6 \mathrm{~mm}$ per side. The raster size is increased in the vertical first without showing any response from the halo detectors. When the raster size is then increased in the horizontal, both halo detectors see a clear increase in halo rates.

Table 6.6: The BB signal fractions for each detector. For the background detectors the yield fractions may be only an effective description of their correlation, but it is quite a powerful and useful one in predicting the correlations between background asymmetries. The values correspond to a raster size of $4 \times 4 \mathrm{~mm}^{2}$.

\begin{tabular}{cccc}
$f_{\mathrm{BB}}^{\mathrm{MD}}$ & $f_{\mathrm{BB}}^{\mathrm{USLsum}}$ & $f_{\mathrm{BB}}^{\mathrm{PMTLT}}$ & $f_{\mathrm{BB}}^{\mathrm{PMTONL}}$ \\
\hline $0.19 \% \pm 0.06 \%$ & $43.7 \% \pm 4 \%$ & $77.3 \% \pm 5 \%$ & $61.2 \% \pm 6 \%$
\end{tabular}

background asymmetries in Run2.

Notice that we saw a large variation of the yield fractions between "good" and "bad" halo conditions during the blocked-octant studies; and more generally the yields of background detectors vary significantly with beam conditions through the run, presumably due to variations of the BB component. So the signal fractions in Table 6.6 are also expected to vary within that range. And yet the correlation slope appears to be remarkably stable through very different beam conditions. The implication is that the fractional yields of different background detectors scale by the same factor under a change in beam conditions.

An example of the correlation of background yields was given in Figure 6.4, where the yields are indeed correlated although significant noise remains at the runlet (5-minute) time scale. A calculation by this author has also constrained any possible non-linearity effect in the relative 
scaling due to the unitarity bound (the yield fraction cannot exceed 100\%), which can be a consideration on the PMTLTG and PMTONL detectors that are assumed to have a high BB signal fraction. However as we will see in Section 6.7 we do not rely on the hypothesis that the correlation slope between detectors is constant over a wide range of beam conditions, and instead allow for its variation by assigning a systematic uncertainty.

\subsection{Beamline Backgrounds Correction Algorithm}

Through the high correlation between background asymmetries over Run2, the USLsum asymmetry is an excellent predictor of the asymmetry measured on all other background detectors. This is true regardless of shortcomings in the description of Eq. 6.4 based on the BB signal fractions $f_{\mathrm{BB}}$.

We can then similarly use the correlation to the USLsum asymmetry to predict the false asymmetry $A_{\mathrm{BB}}$ from the $\mathrm{BB}$ component on the MD. The correlation slope between the MD and the USLsum asymmetries $C_{\mathrm{MD} \text {-USLsum }}^{\mathrm{BB}}$ is the beamline background correction factor, as long as it isolates the correlation due to the BB component from other contributions. Then the BB false asymmetry on the MD can be estimated as

$$
A_{\mathrm{BB}}^{\mathrm{MD}}=C_{\mathrm{MD}-\mathrm{USL} s u m}^{\mathrm{BB}} \cdot A_{\mathrm{USLsum}}
$$

In this section we develop an algorithm to extract the correlation between the MD and USLsum asymmetries in a manner that isolates the effect from the BB component in both detectors, and can then be used to correct for the BB false asymmetry $A_{\mathrm{BB}}$.

The USLsum is preferred over other background detectors for the BB correction as the luminosity monitors offer higher precision. They are also the only background detectors that remained in the same location throughout Run1 and Run2. Further it was available for the entirety of Run2, unlike PMTLTG and PMTONL5, and they measure the BB component with much better reliability (less noise) than MD9. Regardless, as we will see in Run2 there is very good agreement in the $A_{\mathrm{BB}}$ correction no matter what background detector we use as reference, owing to their high correlation.

Issues that should be considered in the extraction of the correction factor $C_{\text {MD-USL }}$ are discussed in the following paragraphs.

\footnotetext{
${ }^{5}$ PMTLTG and PMTONL underwent pre-amplifier studies during Slugs 170-203 and were unavailable in that period.
} 


\subsubsection{Time scale of averaging}

As we discussed in Section 5.4 the time scale of averaging the asymmetries is an important consideration which can influence the result. In the case of regressed asymmetries we saw that residual correlations to beam parameters develop at longer time scales as random beam jitter averages away. For the correlation between $A_{\mathrm{MD}}$ and $A_{\mathrm{USL}}$ there are two clearly identifiable sources of common noise that influence the result:

- noise from target density fluctuations, and

- BCM monitor noise, that enters through charge normalization.

The target "boiling" contribution was presented in Section 3.3.1 limitations of BCM resolution and its induced bias through charge normalization were discussed in Sections 3.7.1 and 99 These two sources inject common noise to the MD and USLsum asymmetry measurements, and therefore contribute positively to their covariance. This contribution will be suppressed at longer time scales.

A study by this author shows that the common noise from these sources dominates the MDUSLsum correlation at the quartet time scale. The quartet-scale correlation slope $C_{\mathrm{MD}-\mathrm{qSL}}^{\text {uartet }}$ is extracted analytically in every 5-minute runlet in Run2, then averaged for every slug and plotted versus slug number as the red line in Figure 6.14. The blue line in the figure corresponds to the modeling of this correlation using only 3 components that may affect the covariance: target boiling, BCM monitor noise, and the BB component.

Based on the studies described in Section 3.3.1 an empirical estimation of the MD asymmetry width contribution from target boiling noise has been extracted in terms of beam current and raster size, for a given target pump frequency [160]:

$$
\sigma_{\text {Tgt noise }}^{\mathrm{MD}}\left(A_{\text {raster }}, I_{\text {beam }}\right) \approx\left(98-3.17 * A_{\text {raster }}\left[\mathrm{mm}^{2}\right]\right)\left(I_{\text {beam }}[\mu \mathrm{A}] / 169\right)^{3}
$$

where $A_{\text {raster }}$ and $I_{\text {beam }}$ is the raster area and beam current respectively. For reference this width contribution is $\sim 55 \mathrm{ppm}$ with a $4 \times 4 \mathrm{~mm}^{2}$ raster at $180 \mu \mathrm{A}$, a rather small contribution to the total MDall asymmetry width $\sigma_{\mathrm{MD}} \approx 225 \mathrm{ppm}$ (see also Figure 3.5). The BCM monitor noise is estimated from the width of the difference between charge asymmetries from different BCMs (the "double-difference" described Section 3.7.1), and should contribute equally to both $\mathrm{MD}$ and USLsum asymmetries, approximately $\sigma_{\mathrm{BCM}} \approx 40 \mathrm{ppm}$ in Run2.

Finally a simple model was developed for the contribution of the $\mathrm{BB}$ component to the $\mathrm{MD}$ and USLsum asymmetry widths. Estimating a base value for the USLsum statistics+monitor resolution (of order 100-150 ppm, a little lower than the smallest USLsum asymmetry width 
that we observed) we assume that all variance in the USLsum asymmetry above the Resolution $\bigoplus$ Boiling $\bigoplus \mathrm{BCM}$ noise contributions, can be attributed to the presence of beamline backgrounds. This model was motivated by the correlation between the USLsum width and size of the asymmetry (Figure 6.5, both considered to be dominated by the BB component. Then the $\mathrm{BB}$ contribution to the MD-USLsum covariance is simply the variance of the BB component on the USLsum asymmetry $\operatorname{Var}(\mathrm{USL})_{\mathrm{BB}}$ multiplied by the correlation slope $C_{\mathrm{MD} \text {-USLsum }}^{\mathrm{BB}}$ between the $\mathrm{BB}$ components of the MD and USLsum asymmetries:

$$
\operatorname{Cov}(\mathrm{MD}, \mathrm{USL})_{\mathrm{BB}} \equiv \operatorname{Cov}_{\mathrm{BB}}=C_{\mathrm{MD}-\mathrm{USL}}^{\mathrm{BB}} * \operatorname{Var}(\mathrm{USL})_{\mathrm{BB}}
$$

Note that $C_{\mathrm{MD}-\text { USLsum }}^{\mathrm{BB}}$ is the correction factor entering Eq.6.6. which we seek to extract in the correction algorithm.

The quartet-scale correlation between the MD and the USLsum asymmetries is modeled as the sum of the three covariance contributions:

$$
C_{\mathrm{MD}-\mathrm{USL}}^{\mathrm{quartet}}=\frac{\operatorname{Cov}_{\mathrm{Tgt}}+\operatorname{Cov}_{\mathrm{BCM}}+\operatorname{Cov}_{\mathrm{BB}}}{\operatorname{Var}(\mathrm{USL})}
$$

where $\operatorname{Var}(\mathrm{USL})=\sigma_{\mathrm{USLsum}}^{2}$ is the USLsum variance. The value of this correlation can be predicted in every runlet based on this model, where the values for beam current, raster size, BCM monitor noise (through the width of the double-difference of BCM asymmetries) and USLsum asymmetry width are inputs, and some further model assumptions are made on the effect of target boiling and BB on the USLsum asymmetry width. For the $C_{\mathrm{MD} \text {-USL }}^{\mathrm{BB}}$ factor we assigned the value that was used in the $2013 Q_{\text {weak }}$ publication, $4.5 \mathrm{ppb} / \mathrm{ppm}{ }^{6}$. With these inputs and assumptions we were able to predict the correlation between MD and USLsum asymmetries at the quartet time scale within the model.

The model prediction for the correlation is plotted as a solid blue line in Figure 6.14, and the three separate contributions to the correlation are plotted as dashed lines. Notice that the $\mathrm{BB}$ contribution is usually swamped by the boiling and BCM contributions at the quartet scale, suppressed by the very small factor $C_{\mathrm{MD} \text {-USLsum }}^{B B}=4.5 \mathrm{e}-3$. It is least effected by variations of the USLsum variance, since very often $\operatorname{Var}(\mathrm{USL})_{\mathrm{BB}} \rightarrow \operatorname{Var}(\mathrm{USL})$. In fact sometimes the USLsum variance gets so large that the $\mathrm{BB}$ component dominates the correlation (grows larger than the other two contributions), even at the quartet scale. As discussed in previous sections, very large values of USLsum asymmetry width is a sign that large amounts of halo are scattering from the plug and the $\mathrm{BB}$ component increases the yields and asymmetries of background detectors.

\footnotetext{
${ }^{6}$ We arrived at this value for the 2013 publication through considerations similar to the simple model of Section 6.4
} 


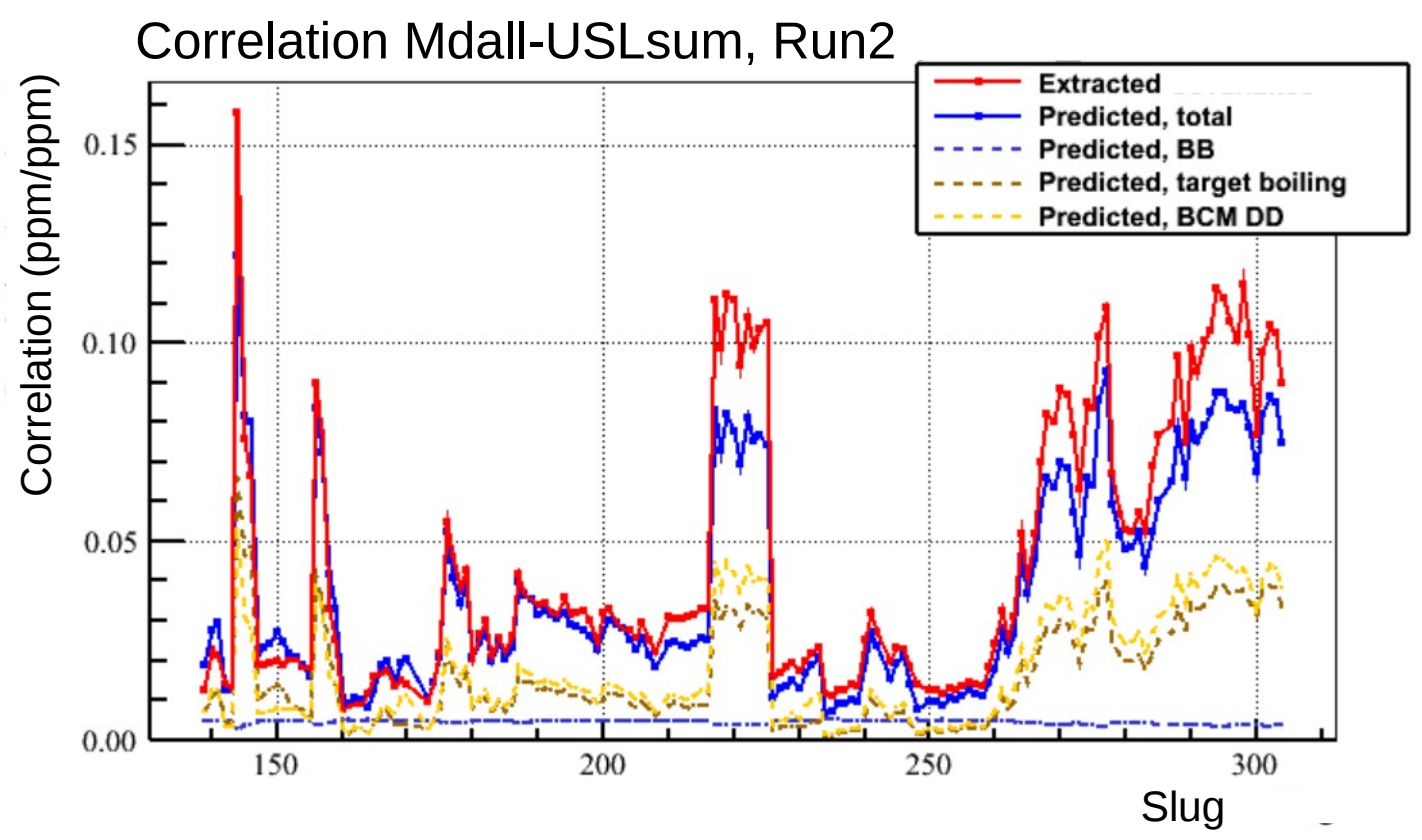

Figure 6.14: The extracted quartet-scale correlation slope $C_{\mathrm{MD}-\mathrm{qu} L \mathrm{arte}}$ between MD and USLsum asymmetries is plotted as the red solid line versus slug number in Run2. The predictions for the three separate contributions (Eq. 6.9) are plotted as dashed lines, where the sum of the separate parts forms the total prediction (solid blue line).

The very high values of the quartet-scale correlation correspond to periods where the USLsum variance is very small, an example being the "golden period" between Slugs 217-225 (described in Section 4.8). For a study of the BB contribution these are the least interesting periods, as the relative $\mathrm{BB}$ contribution is suppressed and the background asymmetries are expected to be very small. This is also where we are most sensitive to uninteresting details of the model parametrization for the BB contribution in terms of the USLsum width. Therefore the larger deviations of the model prediction from the extracted correlations at these regions are not worrisome.

It is interesting however that the data-model agreement appear to have a time dependence. This study also revealed inconsistencies in the correlation of different individual MD bars to the USLsum asymmetry. There is no feature in the simple model of Eq. 6.9 to account for octant dependence in the extracted correlation. These inconsistencies suggest an effect from residual beam asymmetries that can shift the value of the correlation. Referring to the residual beam asymmetries in both the MD and the USLsum as $\delta_{\text {beam }}$, we can now write the MD-USLsum asymmetry correlation slope as:

$$
C_{\mathrm{MD}-\mathrm{USL}}=\frac{C_{\mathrm{MD}-\mathrm{USL}}^{\mathrm{BB}} * \operatorname{Var}(\mathrm{USL})_{\mathrm{BB}}+\mathrm{Cov}_{\mathrm{Tgt}}+\operatorname{Cov}_{\mathrm{BCM}}+\operatorname{Cov}\left(\delta_{\mathrm{beam}}^{\mathrm{MD}}, \delta_{\mathrm{beam}}^{\mathrm{USL}}\right)}{\operatorname{Var}(\mathrm{USL})}
$$




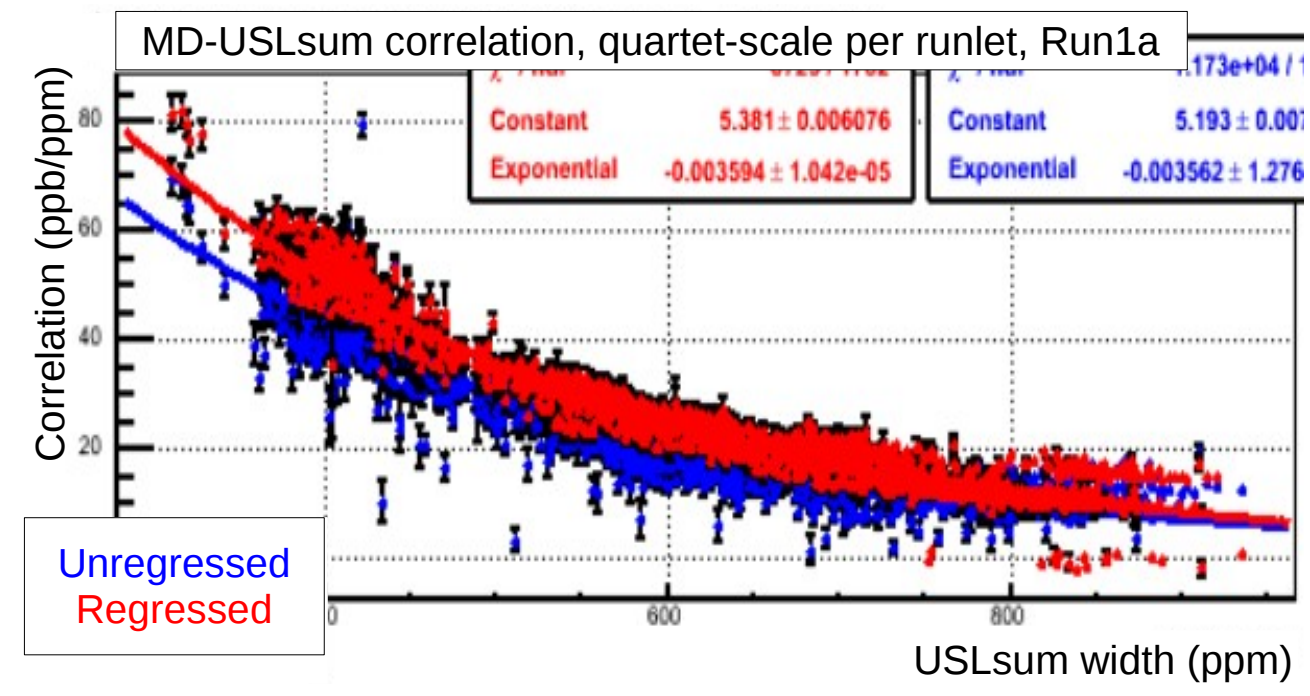

Figure 6.15: Dependence of the MD-USLsum correlation slope to the USLsum width, extracted at the quartet scale in every runlet in Run1a. The correlation falls like the inverse square of the USLsum width, asymptotically approaching the value of the correlation due to the BB component, exactly as we would expect from Eq. 6.9). Notice that the MD-USL correlation slope takes values about an order of magnitude higher than the $C_{\mathrm{MD}-\mathrm{BSL} u m}^{\mathrm{BB}}$ factor that we want to extract.

As the value of the $C_{\mathrm{MD} \text {-USL }}^{\mathrm{BB}}$ factor is suppressed relative to common noise contributions at the quartet time scale, we were unable to use this study for a precise estimation of its value. We attempted varying the unknown parameters with random number techniques to test the fit of the model-predicted correlations to the data, a technique inspired by the AMIAS method [161] which was the subject of this author's undergraduate thesis. Unfortunately at the quartet scale other unknown parameters dominate the uncertainty, such as the parametrization of the boiling noise in the USLsum.

However through this study we were able to demonstrate sufficient understanding of the different common-noise contributions to the correlation. These contributions should be suppressed like $1 / \sqrt{N_{\text {quartets }}}$ at longer time scales of averaging, where $N_{\text {quartets }}$ is the number of quartets included in each average. Therefore at longer time scales the relative contribution of the common noise is suppressed. This author has shown that the contribution of common noise should drop below $1 \%$ when asymmetries are averaged at the slug time scale in Run2. For this reason the asymmetries must be averaged at least at the slug scale in the extraction of the correlation, where common noise contributions are negligible. All considerations from Section 5.4 .2 about averaging at longer yet time scales apply here as well. We will mostly limit the correlation analysis to averaging at the slug scale.

The residual beam effects $\delta_{\text {beam }}$ however are not necessarily similarly suppressed by averaging, and will be discussed in the following subsection. 


\subsubsection{Residual beam effects}

The sinusoidal pattern of the octant dependence was seen in the previous chapter as clear evidence of residual dependence to $\mathrm{HC}$ beam parameters (Figure 5.23). In Figure 6.16 the same octant dependence can be seen in the correlation of individual MD bars to the USLsum asymmetry, at both the quartet and the slug time scale.

Since the USLumi monitors are effected by HC beam parameters, to some extent they can be considered as beam monitors. Therefore it is not surprising that for uncorrected asymmetries the correlation of MD bars to USLsum exhibits a dipole octant dependence. However the amplitude of that pattern is not completely suppressed even after beam corrections are applied through regression. The beam corrections are imperfect, both in the MD and the USLsum, and the residual beam effects bias the MD-USLsum correlation slope and give it a characteristic dipole pattern versus octant. This is the effect of the $\operatorname{Cov}\left(\delta_{\text {beam }}^{\mathrm{MD}}, \delta_{\text {beam }}^{\mathrm{USL}}\right)$ term in Eq. 6.10.
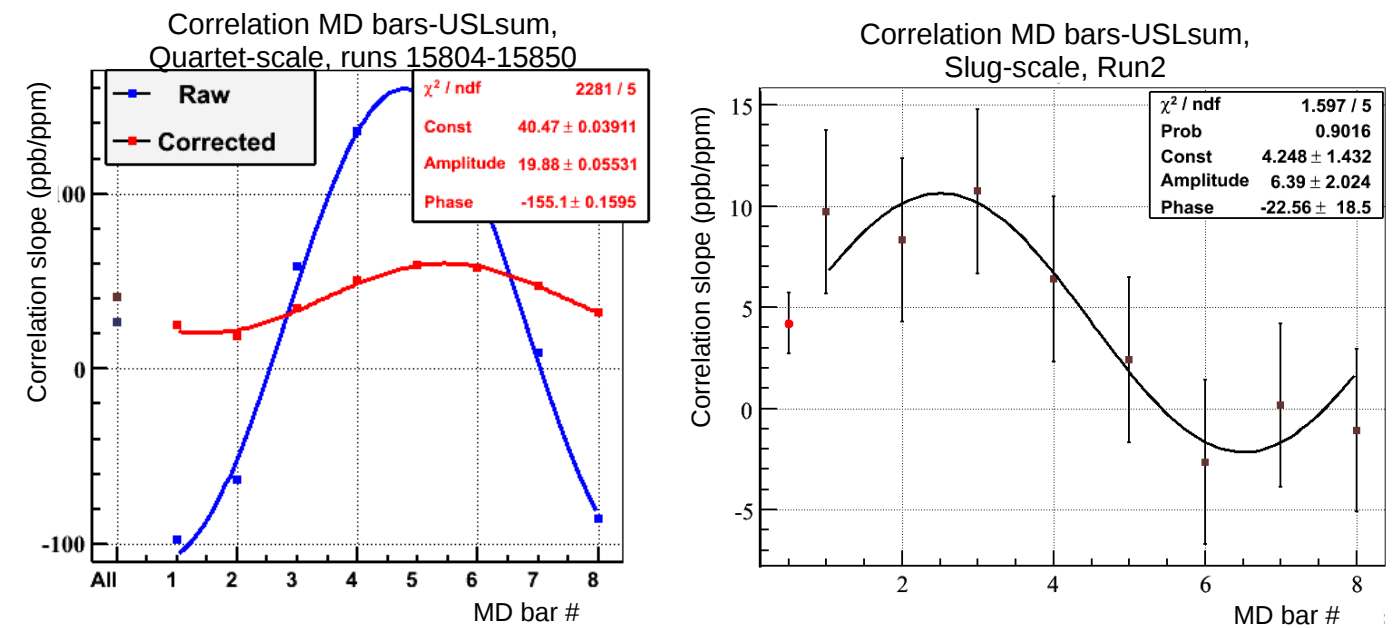

Figure 6.16: Correlation slope between MD bars and USLsum asymmetries plotted versus octant. Left: Correlation extracted at the quartet-scale over a subset of Wien8, plotted for uncorrected (blue) and regressed (red) asymmetries. Sinusoidal fit results correspond to the regressed asymmetries. Right: Correlations extracted at the Slug-scale over Run2, for asymmetries corrected through dithering. The characteristic dipole octant dependence can be seen at both the quartet and the slug time scale.

Notice that for regressed asymmetries at the quartet scale all correlations to the regression parameters (the HC beam differences) are suppressed by construction, and yet the MD-USLsum correlation still exhibits this pattern. Potentially this correlation accesses higher-order beam effects that are missed by the $A_{\text {beam }}$ correction. We had suspected the same for the residual correlation of the corrected MD to charge asymmetry, in the absence of bias from BCM monitor noise (Section 99).

The background detectors also have residual correlations to charge asymmetry after beam corrections, and they reveal a deeper connection between the background asymmetries and HC beam parameters. The regression sensitivities $S_{Q}^{\mathrm{Bkgd}}$ of background detectors to charge 


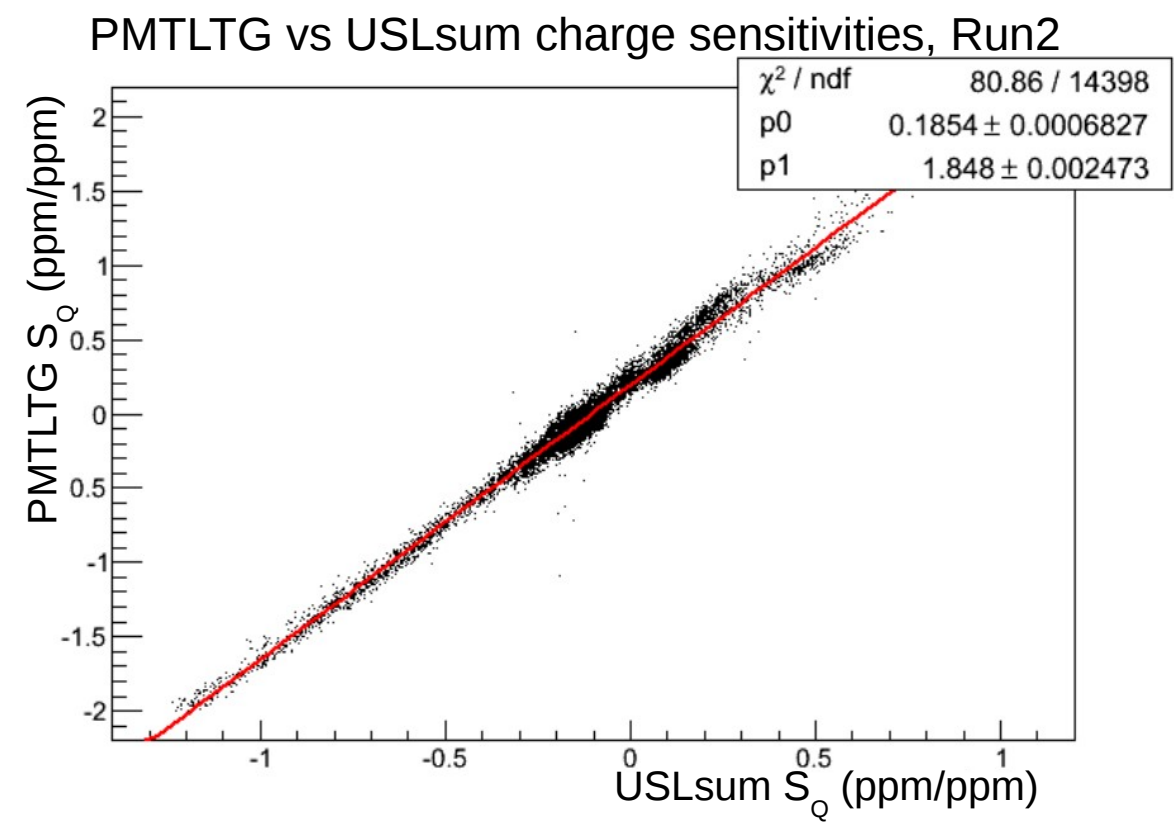

Figure 6.17: The charge sensitivities between the PMTLTG and USLsum asymmetries are very highly correlated in Run2, with a correlation slope that is identical to that of their measured asymmetries.

Table 6.7: Correlation slope between background detectors and the USLsum, in their measured asymmetries and charge sensitivities in Run2.

\begin{tabular}{l|cc} 
& \multicolumn{2}{|c}{ Correlation slope to USLsum, through: } \\
& Background asymmetries $A_{\mathrm{BB}}$ & Charge sensitivities $S_{Q}$ \\
\hline PMTLTG & $1.82 \pm 0.02$ & 1.85 \\
PMTONL & $1.44 \pm 0.03$ & 1.46 \\
MD9 & $0.13 \pm 0.01$ & 0.12
\end{tabular}

asymmetry exhibit the same scaling factors as the background asymmetries. In Figure 6.17 the regression sensitivities $S_{Q}$ of the PMTLTG and USLsum asymmetries to charge in the regression $5+1$ scheme are very highly correlated through Run2. Even though the sensitivities are binned at the runlet scale, the most basic time scale where regression sensitivities are extracted, there is surprisingly little noise in this correlation. Remarkably, the correlation slope between the charge sensitivities of background detectors is identical to that of their measured asymmetries, as listed in Table 6.1 $C_{\mathrm{LTG}-\mathrm{USL}}=1.82 \pm 0.02$. This statement is true for the other background detectors as well, with the results compared in Table 6.7. The same very high correlation can also be seen in regression sensitivities to other beam parameters [162, as well as from blocked-octant data including the $S_{Q}$ correlations with the blocked MD bars [163].

We had hypothesized that beam halo, which is associated with the generation of the beamline background component through scattering at the collimators, may constitute a "hidden variable" in the regression analysis. Furthermore scattering from the collimators is very likely associated with dithering residuals, highly correlated among background detectors (Section 5.2.3). Then 


\section{USLsum asymmetry vs Slug pairs, Run2}

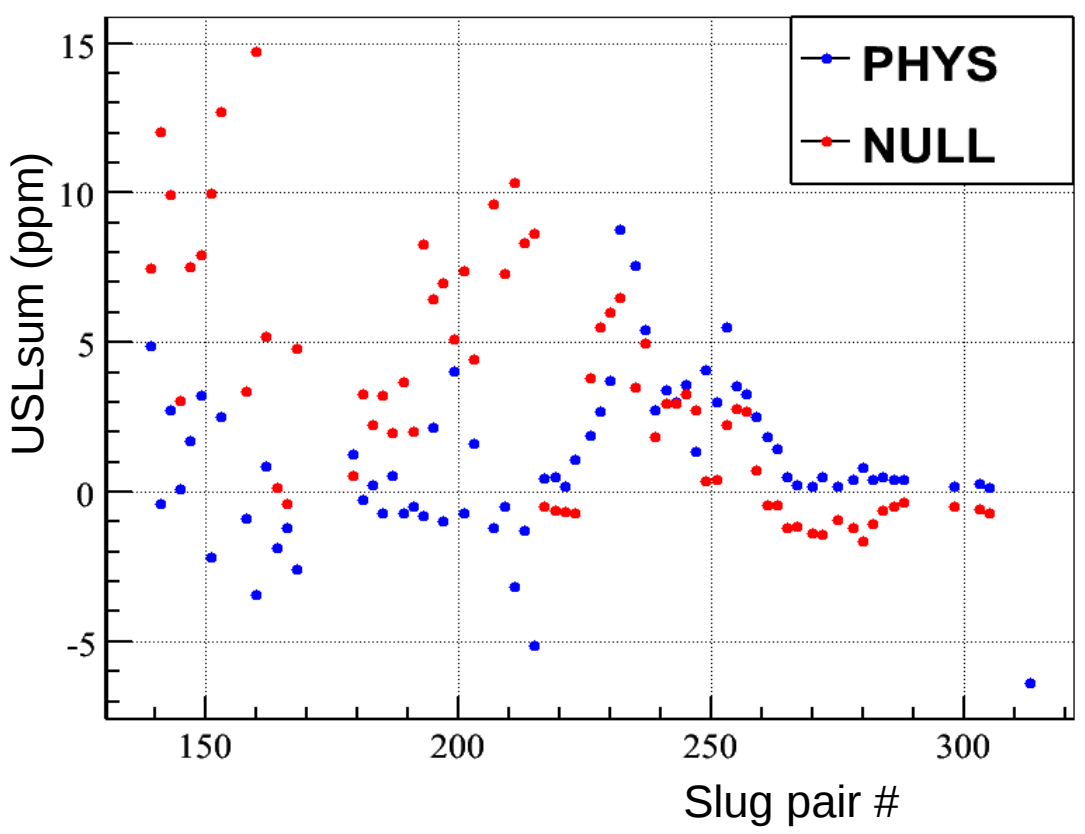

Figure 6.18: USLsum asymmetries in Run2, averaged over slug pairs (of opposite IHWP state, in the Sign-corrected formulation). The asymmetries are averaged in the Physics (blue) and Null (red) contributions in each slug pair.

the consistency of the results in Table 6.7 is very suggestive of the deeper connection between beamline background asymmetries and $\mathrm{HC}$ beam differences, where the former may be considered as a higher-order effect to the latter.

\subsubsection{Extraction of BB correction factor}

The beamline background correction factor $C_{\mathrm{MD}-\mathrm{USL}}^{\mathrm{BB}}$ is extracted here from the correlation slope between the slug-averaged MDall and USLsum asymmetries over Run2. This correlation slope is understood to potentially include residual effects from beam corrections, and our choice in details of the extraction are aimed to minimize these contributions. We will work in the Dithering correction scheme which was shown in the previous chapter to have better properties in removing long-scale residual correlations to beam parameters.

The asymmetries must be averaged over the slug scale or longer to ensure that common-noise contributions from target boiling and BCM resolution are rendered negligible, as discussed in Section 6.5.1. The correlation slopes between MDall and USLsum are extracted for asymmetries averaged over slugs and slug pairs in Table 6.8, in both the Residual and Sign-corrected formulations to remove the sign-changing Physics $A_{e p}$ asymmetry (Section 5.4.3). All results are extracted over the Modulation dataset of Run2.

While all results for the correction factor are consistent within their statistical precision, the discrepancies may be considered systematic and ascribed to residual effects from beam corrections 
Table 6.8: Correlation slope between MDall and USLsum over Run2, in various binning time scales and formulations.

\begin{tabular}{c|ll} 
& Modulation & Regression \\
\hline & \multicolumn{2}{|c}{ Slug binning } \\
Sign-corrected & $4.21 \pm 1.49$ & $4.23 \pm 1.49$ \\
Residual & $3.60 \pm 1.51$ & $3.98 \pm 1.53$ \\
\hline & \multicolumn{2}{|c}{} \\
& Slug-pair binning \\
Sign-corrected & $4.25 \pm 3.53$ & $4.78 \pm 3.54$ \\
Residual & $3.71 \pm 1.65$ & $4.10 \pm 1.67$
\end{tabular}

that are absorbed by the correction factor. That is obviously the case for the discrepancies between dithering and regression, but it can be assumed for the other choices as well. As discussed in Section 5.4 .2 different constructions of slug pairs cancel complementary parts of $\mathrm{HC}$ beam differences, and that is also the case for the background asymmetries. Specifically the Sign-corrected slug pairs of opposite IHWP states only allow access to the effects that survive in the Physics asymmetry combination. Due to good IHWP cancellation these effects are better suppressed compared to the Null combination (Figure 6.18), as is the case for the effect of HC beam parameters. The suppressed range is also the reason for the higher uncertainty in the correlation slopes extracted in that specific formulation.

For the correction factor in Run2 we will accept the value extracted over slugs in the Signcorrected formulation. There are potentially more residual beam effects in the slug scale compared to the cancellation achieved over slug pairs, and we accept that these may be absorbed in the correction factor. We accept the value from the Modulation correction scheme which was shown to achieve better cancellation of long-scale residual beam effects, although the value from regression is practically identical. The value we get for the Run2 correction factor, extracted from the fit shown in Figure 6.19:

$$
C_{\mathrm{MD}-\mathrm{USL}}^{\mathrm{BB}}=4.21 \pm 1.49 \text { (fit) } \mathrm{ppb} / \mathrm{ppm}
$$

The assigned uncertainty only corresponds to the statistical precision of the correlation slope extracted from the fit. The systematic discrepancy between the different choices should certainly not be neglected and will be accounted for in Section 6.7. In general the discrepancies between the different choices are well contained at the $10-15 \%$ of the correction factor 6.11. That is true for the different choices of beam corrections scheme, time scale of averaging and asymmetry formulation. It makes sense that these discrepancies are of the same size as they are all expected to be manifestations of residual beam effects. 


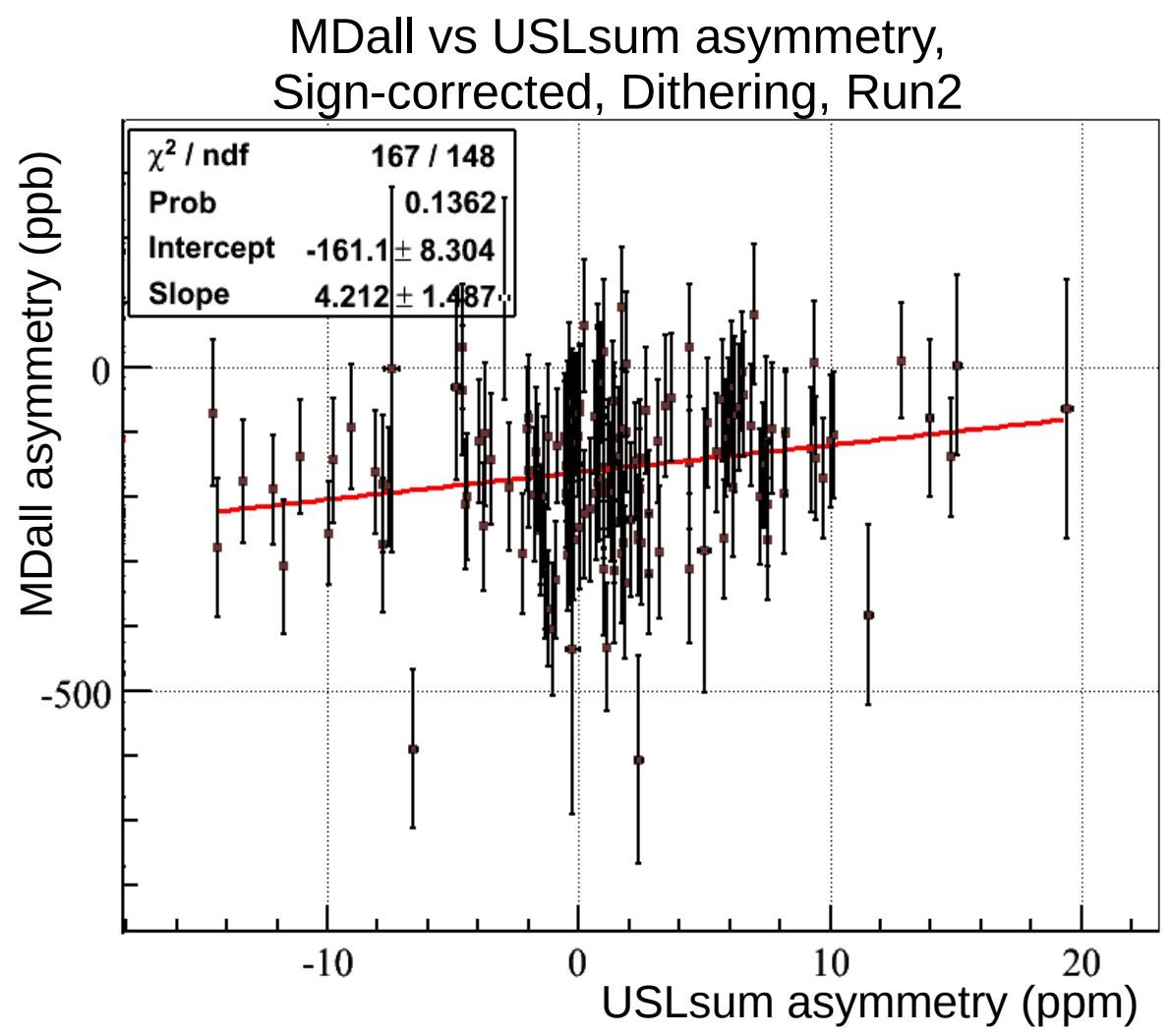

Figure 6.19: The correlation between MDall and USLsum asymmetries in the Sign-corrected formulation, averaged at the slug scale over the Run2 Modulation dataset. The extracted correlation slope is the correction factor $C_{\mathrm{MD}-\mathrm{USL}}^{\mathrm{BB}}$ for the $\mathrm{BB}$ correction 6.6 .

Finally, note that the value for the correction factor is in excellent agreement with the value expected from the simplistic model of beamline background signal fractions, formulated in Eq. 6.5). Using the values and errors from Table 6.6, the model prediction for the correction factor is:

$$
C_{\mathrm{MD}-\mathrm{USL}}^{\mathrm{BB}}[\text { model }] \approx f_{\mathrm{BB}}^{\mathrm{MD}} / f_{\mathrm{BB}}^{\mathrm{USL}} \approx 4.35 \pm 1.43 \mathrm{ppb} / \mathrm{ppm}
$$

in excellent consistency with 6.11), and also with the value of $4.5 \mathrm{ppb} / \mathrm{ppm}$ that was used in the $2013 Q_{\text {weak }}$ publication.

\subsubsection{Comparison with other background detectors}

For a comparison between different background detectors, the correlation slopes of the slugaveraged MD to the background asymmetries are extracted over the period in Run2 where dithering data exist and all background detectors were available. The latter requirement removes the period between Slugs 170-203 (including the entire Wien 8a) due to PMTLTG and PMTONL being unavailable. This may be significant as there are suggestions that residual beam effects are large in that period (Figure 5.22 .

Results for correction factors $C_{\mathrm{MD}-\mathrm{Bkgd}}^{\mathrm{BB}}$ corresponding to each background detector are listed 
Table 6.9: Correlation slopes of the MDall asymmetry to background asymmetries, averaged at the slug scale, in the subset of Run2 where all background detectors are available. Using the correlation slope to USLsum the expected result for other background detectors has been predicted (in parentheses) based on the very precise correlation slopes between background asymmetries in Run2.

\begin{tabular}{l|cccc} 
& USLsum & PMTLTG & PMTONL & MD9 \\
\hline Dithering & $4.78 \pm 1.61$ & $2.76 \pm 0.90$ & $3.46 \pm 1.11$ & $21.4 \pm 8.5$ \\
(expected) & - & $(2.63)$ & $(3.32)$ & $(36.8)$ \\
Regression & $5.34 \pm 1.62$ & $3.10 \pm 0.90$ & $3.77 \pm 1.11$ & $23.7 \pm 8.6$ \\
(expected) & - & $(2.93)$ & $(3.71)$ & $(41.1)$
\end{tabular}

in Table 6.9. Using the correlation slope to the USLsum asymmetry in this dataset (first column), we predicted the correlation slope on other background detectors based on the very precise correlation slopes between background asymmetries from Table 6.1

The correlation slopes of the MD asymmetry to the PMTLTG and PMTONL are in good agreement to the expected value as scaled from the correlation to the USLsum, although perhaps that expectation seems to be systematically a little lower. This means that PMTLTG and PMTONL would likely give the same correction on the MD as the USLsum, a statement that we will directly test in Section 6.6. If so, this redundancy gives us high confidence on this correction. On the other hand the prediction and consistency is not good on the MD9 detector. We explore the reason for this inconsistency in the following subsection.

\section{Effect of assigned uncertainty}

An important issue in the extraction of the correlation slope is the effect of the assigned uncertainty to the background asymmetries in each datum. This uncertainty if improperly assigned can bias the correlation slope (see discussion in Appendix C) and therefore our sensitivity to this choice should be investigated. We have generally experimented with three different choices in assigning the uncertainty to a background asymmetry average over a period of $N_{\text {quartets }}$ :

- The uncertainty of the background asymmetry can be assigned equal to the MDall uncertainty, including polarization weighting, thereby ensuring that the statistical contribution (weighting) will be the same as the contribution of that average to the full result. This is the main method to assign uncertainties of background asymmetries. It has been used in the extraction of the Run2 correction factor 6.11) from Figure 6.19.

- Alternatively the uncertainty can be assigned as $\sigma_{A}^{i} / \sqrt{N_{\text {quartets }}}$, where $\sigma_{A}^{i}$ is the width of the asymmetry distribution of background detector $i$. There may be bias introduced through this choice as higher asymmetry widths are usually associated with higher background asymmetries. 
Table 6.10: Assigned resolutions $\sigma_{\text {res }}^{i}$ and average distribution widths $\sigma_{A}^{i}$ (see text) for each background detector $i$, used in the study depicted in Figure 6.20. All values in units of ppm.

\begin{tabular}{l|cccc} 
& USLsum & PMTLTG & PMTONL & MD9 \\
\hline $\begin{array}{l}\text { Assigned } \\
\text { resolution }\end{array}$ & 150 & 1700 & 2600 & 3500 \\
\hline $\begin{array}{l}\text { Distribution } \\
\text { width }\end{array}$ & 517 & 1757 & 2944 & 3867
\end{tabular}

- Finally another choice is to assign the uncertainty as $\sigma_{\text {res }}^{i} / \sqrt{N_{\text {quartets }}}$, where $\sigma_{\text {res }}^{i}$ is the asymmetry "resolution" of background detector $i$. The resolution is a good measure of precision and this is the method we use for correlations to beam monitor differences, however for the background detectors these resolutions are not well defined.

To study the dependence of our result to the assigned uncertainty of background asymmetries, we extract the correlations by assigning "resolutions" $\sigma_{\text {res }}^{i}$ to each background detector $i$, similarly to the resolutions of beam monitors. The resolutions $\sigma_{\text {res }}^{i}$ were defined for this study as slightly smaller than the minimum observed asymmetry width for each detector $i$ over Run2 runlets, and are listed in Table 6.10. Also listed in the table is an estimation for the average asymmetry distribution width $\sigma_{A}^{i}$ in each detector over Run2. Both of these values correspond to the quartet time scale, i.e. they would be divided by $\sqrt{N_{\text {quartets }}}$ to get the error of the background asymmetry over a longer time period.

For this study the assigned resolution $\sigma_{\text {res }}^{i}$ of each background detector $i$ was scaled by a factor between 0 and 1.4, and the correlation with the MDall asymmetry was extracted for each value of the scaling factor. The results are plotted in Figure 6.20 versus the value of the scaling factor, where the correlation slope to all background detectors have been appropriately "normalized" to the correlation for the USLsum, based on the very precise correlation slopes between background detectors from Table 6.1, so that all background detectors can be drawn on the same axis and compared. The correlation slopes were also extracted using the quartet-scale asymmetry distribution widths $\sigma_{A}^{i}$ to assign the uncertainty of background detectors. These results are shown as off-colored inverted triangles, placed on an appropriate location on the horizontal axis according to the average quartet-scale widths over Run2, listed in Table 6.10 and expressed through the appropriate scaling to the corresponding resolution.

The figure reveals that the result for the correlation slope doesn't depend significantly on the assigned uncertainty of the background asymmetry for the USLsum, PMTLTG and PMTONL. The error of these correlation slopes similarly doesn't depend strongly on the assigned monitor uncertainty as it is dominated by the "noise" (non-BB component) in the MD asymmetry. It also shows that there is excellent agreement in the result from these background detectors 


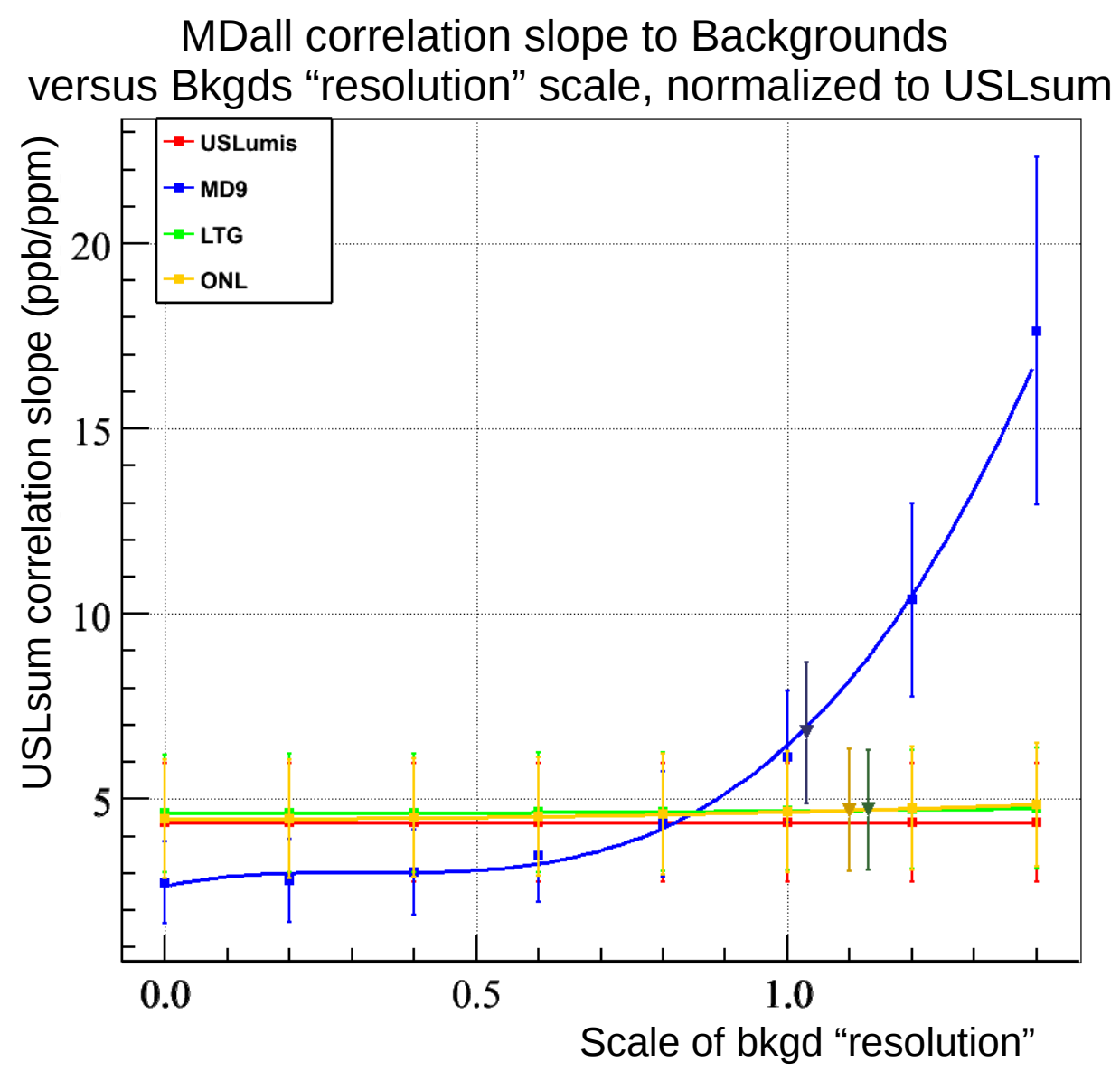

Figure 6.20: The correlation slope between regressed MDall and background asymmetries, extracted over Run2 at the slug scale, plotted versus the scaling of the assigned background asymmetry resolution $\sigma_{\text {res }}^{i}$. Each color corresponds to a different background detector. The correlation slopes to all background detectors have been normalized to the value of the correlation to the USLsum, based on the very precise correlation slopes between background detectors. Results plotted as off-colored inverted triangles correspond to background uncertainties assigned from the distribution widths $\sigma_{A}^{i}$ rather than the detector resolution. See text for more details.

when properly normalized to comparable values. Evidently there is good consistency between the background detectors in predicting the $\mathrm{BB}$ component in the $\mathrm{MD}$, which gives us great confidence in the procedure to extract the BB correction factor. Note that there is still a small (relative to the statistical uncertainty of the fit result) dependence of the result to the assigned uncertainty. This can be considered as a systematic effect and will be adequately covered by the assigned systematic uncertainty in our correction.

An exception is the MD9 detector which is shown to have a strong dependence on the assigned uncertainty. This is because the MD9 detector does not have a high "reliability" value to represent the $\mathrm{BB}$ component; therefore details of the assigned uncertainty are significant to predict that reliability and extract the appropriate value for the correlation slope. Understanding the large signal fraction that carries the elastic asymmetry and treating it properly is also a concern. For these reasons we will not consider the MD9 detector in our BB correction procedure. 
Notice that the extracted values for the correlation slope to USLsum in Table 6.10, while still statistically consistent with the value (6.11), appear to be systematically higher in the dataset examined here where Wien $8 \mathrm{a}$ is excluded. Since the correction factor is expected to absorb potential residual systematic effects which also vary in different periods, it is not surprising that excluding a period of unusually large residual effects shifts its value. As noted before, the appropriate effective correction factor is expected to vary over periods with different residual effects, and the value extracted over Run2 is the average over all these periods. The systematic uncertainty that will be assigned in Section 6.7 will allow for the variation of the BB correction factor over different periods.

\subsection{Beamline Backgrounds Correction in Run2}

Using the algorithm developed in the previous section we apply a correction for the false asymmetry in the MD due to asymmetric beamline backgrounds in Run2. The correction is applied in every runlet with appropriate polarization weighting, through Eq.6.6 and using the value of Eq.6.11 for the correction factor. Some statistical properties of the corrected asymmetry will be examined here, but a more detailed analysis will be reserved for Chapter 7.

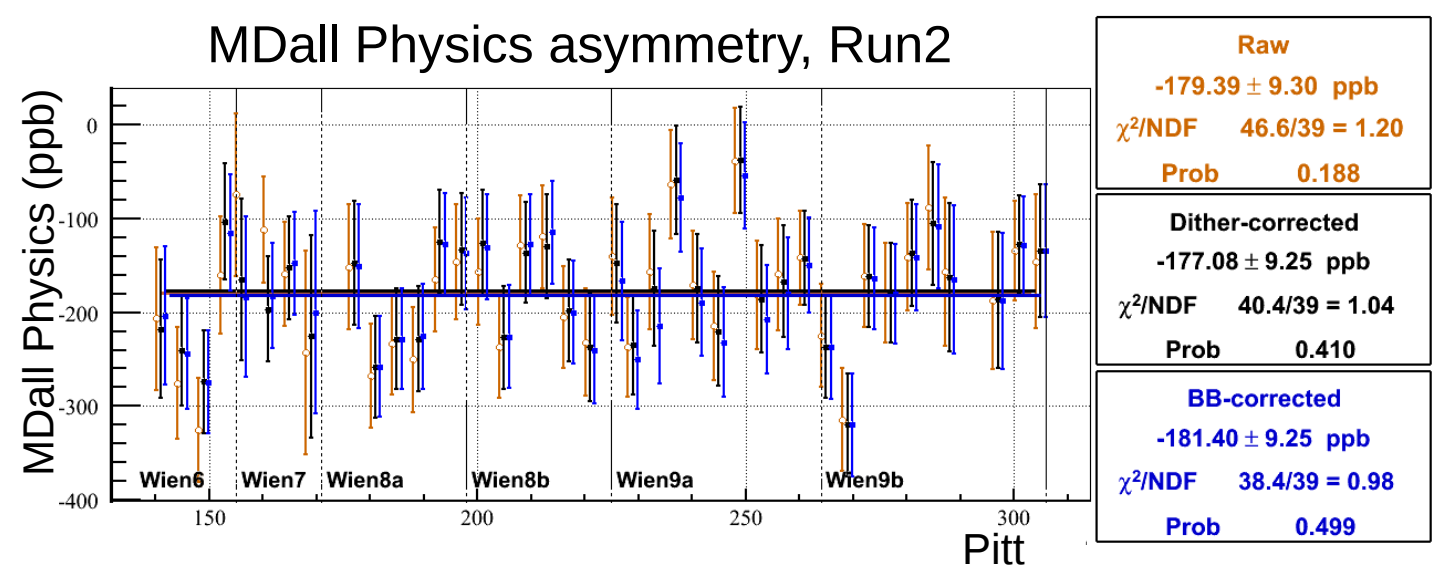

Figure 6.21: The MDall Physics asymmetry combination in Run2 averaged at the pitt scale. Data correspond to uncorrected (orange), then corrected for beam effects through dithering (black), and finally corrected for beamline backgrounds (blue), where the latter includes beam corrections.

The fit results over Run2 at the pitt scale are plotted in Figure 6.21, for data corrected for false beam asymmetries through dithering and then for the false BB asymmetry. The blinded result for the Physics asymmetry combination in Run2, corrected for the $A_{\text {beam }}$ and $A_{\mathrm{BB}}$ false asymmetries and normalized for polarization (but not including other systematic corrections), is $A^{\text {Phys }}=-181.40 \pm 9.25 \mathrm{ppb}$.

The $A_{\mathrm{BB}}$ correction is seen to further improve the fit probability to 0.499 , a value consistent with absence of residual systematic effects in Run2. The total polarization-weighted BB 
correction $A_{\mathrm{BB}}^{P}$ is the difference between the "Dither-corrected" and "BB-corrected" result from Figure 6.21. With a statistical uncertainty from the $35.4 \%$ precision of the BB correction factor from Eq.(6.11), the polarization-weighted BB correction in Run2 is:

$$
A_{\mathrm{BB}}^{P}(\text { Run2) }=4.32 \pm 1.53(\text { fit }) \mathrm{ppb}
$$

\section{Agreement from different background detectors}

Different background detectors can be used for the $A_{\mathrm{BB}}$ correction, with the corresponding correction factors taken from Table 6.9. For correcting through the PMTLTG, PMTONL, and MD9 detectors we have to make an estimation for the effect of the Physics $A_{e p}$ asymmetry in their signal through leakage, weighted by the appropriate signal fraction $f_{e p}^{i}$ on each detector $i$. For PMTLTG and PMTONL this is the complement to the $f_{\mathrm{BB}}^{i}$ factors in Table 6.6. There is some uncertainty introduced as these factors are not precisely known. This is not an issue for correcting through the USLsum which is considered to have $f_{\mathrm{ep}}^{U S L} \approx 0$.

The results for the Physics asymmetry are quoted in Table 6.11, for the subset of Run2 where dithering data and all background detectors are available. Again, this cut unfortunately removes a significant portion of data (more than 30 Slugs, including all of Wien8a), which in addition is suspected for residual beam effects. The result for the Physics asymmetry corrected for $A_{\mathrm{BB}}$ through the USLsum differs by $0.8 \mathrm{ppb}$ in the two dataset: 7 ]

The important comparison here is between results from different background detectors. The result from MD9 is the most discrepant but can be excluded based on the analysis of Section 6.5.4 The range of results from the other background detectors is $0.5 \mathrm{ppb}$ for the Physics and $0.8 \mathrm{ppb}$ for the Null combination. This is very good consistency between USLsum, PMTLTG and PMTONL, each giving a relatively independent estimation of the $A_{\mathrm{BB}}$ false asymmetry in the MD. Still we don't really make any use of this excellent agreement to reduce the uncertainty of the correction. We only argue that this is a strong confirmation for the BB correction procedure, especially considering that some added uncertainty is introduced by the estimation for the Physics asymmetry component in PMTLTG and PMTONL.

\subsection{Model Uncertainty of BB Correction}

Possible systematic effects that may bias the $A_{\mathrm{BB}}$ correction mainly concern residual effects from beam corrections. These account for discrepancies in the extracted correction factor between beam correction schemes and averaging formulations, and also residual correlations to beam

\footnotetext{
${ }^{7}$ Even if we ascribe this to residual false asymmetries, the assigned systematic uncertainty will be large enough to account for the $0.8 \mathrm{ppb}$ discrepancy.
} 
Table 6.11: Physics and Null MDall asymmetry combinations, with BB correction applied through different background detectors. These results are quoted over the subset of Run2 where dithering data exists and all of the background detectors are available.

\begin{tabular}{l|cc} 
& Physics & Null \\
\hline USLsum & $-180.3 \pm 10.1$ & $-5.7 \pm 10.1$ \\
PMTLTG & $-180.6 \pm 10.1$ & $-6.5 \pm 10.1$ \\
PMTONL & $-180.1 \pm 10.1$ & $-6.2 \pm 10.1$ \\
MD9 & $-179.0 \pm 10.1$ & $-16.5 \pm 10.1$
\end{tabular}

parameters for the main and background detectors. These discrepancies were generally of order 10-15\%. We argued that we can also ascribe to residual beam effects any possible time-variation of the appropriate correction factor.

Here we attempt to estimate the systematic uncertainty to the correction factor from all these effects by allowing it to vary over time, with the use of random numbers. Rather than considered constant, the correction factor is pulled randomly from a distribution over different periods. The mean value of the distribution is the accepted value of the correction factor from Eq. 6.11. The width of the distribution should be characteristic of the stability of the correction factor and therefore the systematic effects that we parametrize through its variation over time. As an estimate of the (in)stability of the correction factor we look at the correlations of the USLsum asymmetry to PMTLTG and PMTONL over every Wien period in Run2, plotted in Figure 6.22 The whole range of values for the PMTLTG-USLsum correlation slope extracted in every Wien, is $\sim 18 \%$ of the Run2 average value. For the PMTONL-USLsum correlation, if we neglect the Wien9b value which has a very large uncertainty (background asymmetries are small in Wien9b and the fit lever-arm is reduced; at these small asymmetries the uncertainty of the effect of the sign-changing Physics asymmetry also comes into consideration), then the whole range is $\sim 23 \%$ of its average value over Run2. We will conservatively estimate $23 \%$ for the appropriate randomization range of the correction factor in Run2. This is comfortably larger than the 10-15\% discrepancies observed between different correction schemes and averaging formulations.

The value of the correction factor will randomly be pulled from that distribution in every period, which we can be chosen to be in every pitt or Wien. The BB correction is then applied with a different (random) correction factor in every period and results are extracted for the average Physics and Null asymmetries. This procedure is repeated 100,000 times and ensembles of solutions are created.

Note that the choice of time scale for this segmentation, where a new random factor is used for each period/segment, is important for the result. A randomization width of $23 \%$ at the pitt scale will translate to a randomization of $23 \% / \sqrt{N_{\text {pitts }}}$ at the Wien scale through statistical averaging, where $N_{\text {pitts }}$ is the number of pitts in a Wien period. Therefore by randomizing at longer 

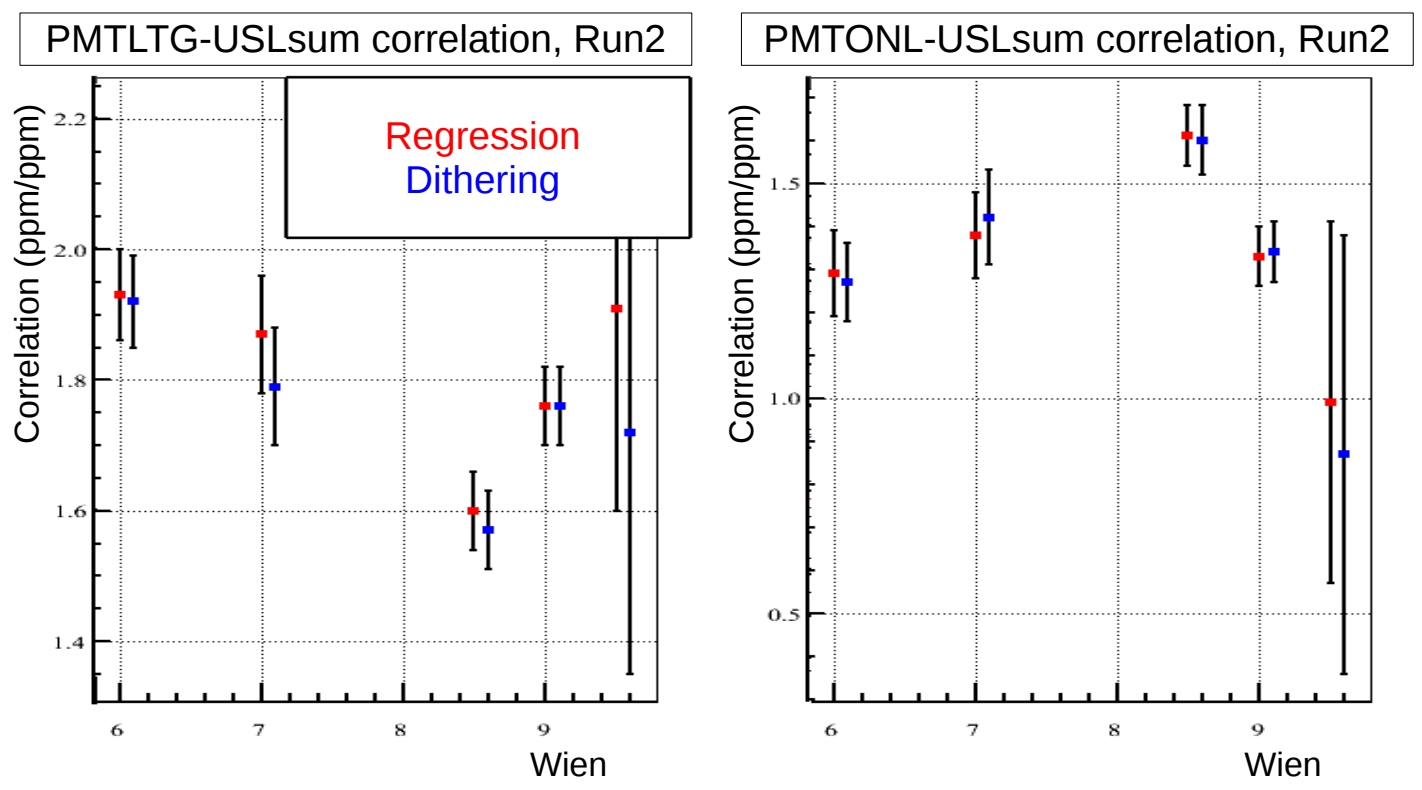

Figure 6.22: Correlation slopes between background asymmetries, extracted in every Wien period in Run2 for data binned at the slug scale.

time scales we allow for more randomization and uncertainty. We will conservatively choose to randomize at the Wien scale, which is also perhaps a better estimate for the characteristic time scale of the residual effects that are being parametrized.

The resulting distributions for the result of the Physics and Null asymmetries are plotted in Figure 6.23 The width of the Null asymmetry ensemble is higher, consistent with our expectation that residual systematic effects are more significant on the Null, due to better suppression on the Physics from the IHWP reversal. The width of the Physics distribution due to the $C_{\mathrm{MD}-\mathrm{BSL}}^{\mathrm{BB}}$ randomization, which parametrizes its potential time-variation and any residual systematic effects, is our estimation for the "model" uncertainty of the $A_{\mathrm{BB}}$ correction at 0.92 ppb.

With this model uncertainty, our estimation for the total polarization-weighted beamline background correction in Run2 becomes:

$$
A_{\mathrm{BB}}^{P}(\text { Run2 })=4.32 \pm 1.53(\text { fit }) \pm 0.92(\text { model }) \mathrm{ppb}
$$

From our treatment, this extra model uncertainty should account for residual effects from both $A_{\text {beam }}$ and $A_{\mathrm{BB}}$ corrections. Arguably any residual systematic effects from beam corrections should have already been accounted by the model uncertainty assigned to the $A_{\text {beam }}$ correction from the analysis of Section 5.6.1 So again we are being conservative and potentially doublecount the uncertainty due to these effects.

The appropriate "source" term for the beamline background asymmetry, that should be 

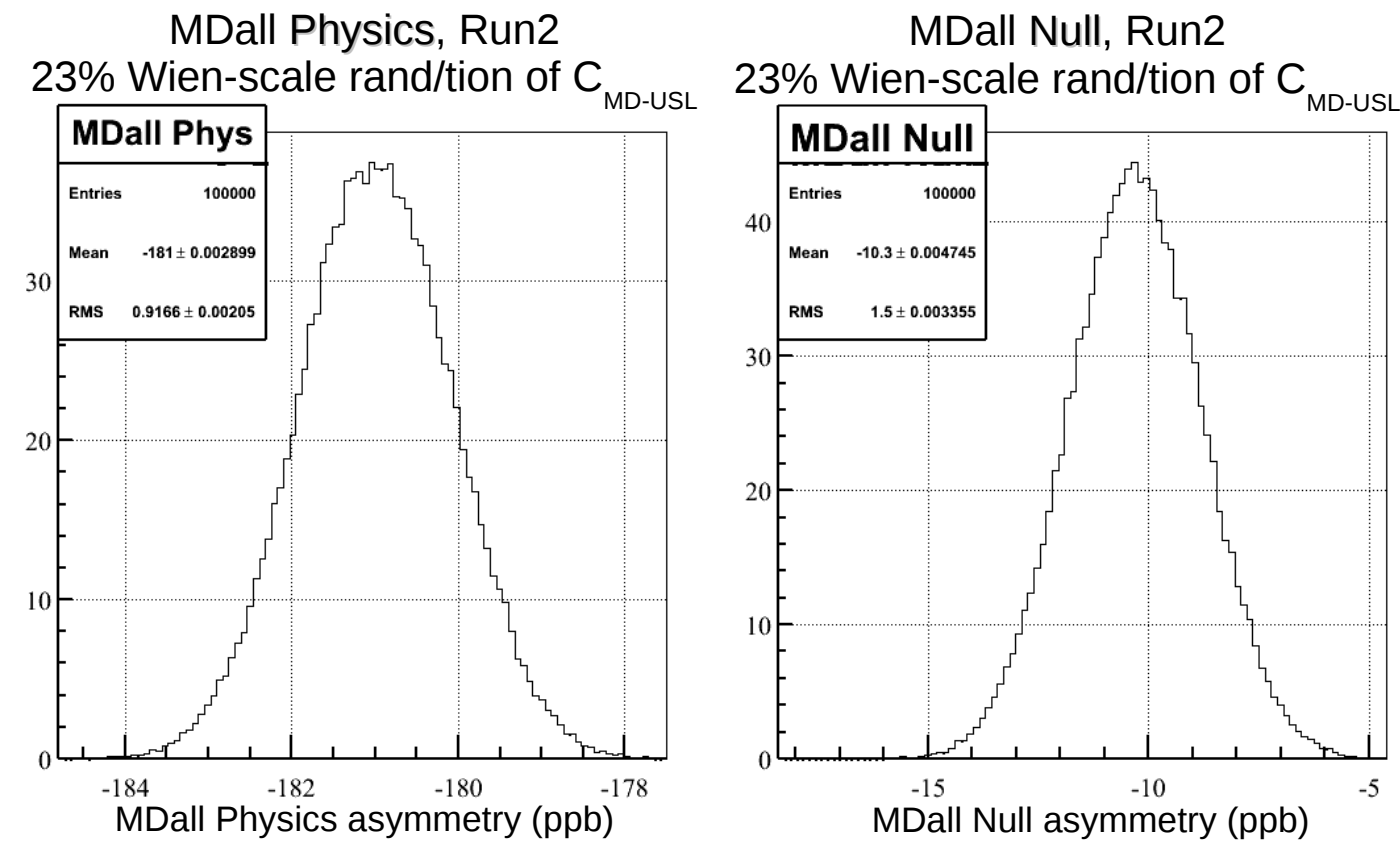

Figure 6.23: Ensemble distributions for Physics and Null asymmetries in Run2 after 100,000 iterations of the extraction, where the $\mathrm{BB}$ correction factor $C_{\mathrm{MD} \text {-USL }}^{\mathrm{BB}}$ has been randomly regenerated in every Wien from a distribution with a $23 \%$ width.

combined with the value 6.3 for $f_{2}$ to give the total $A_{\mathrm{BB}}^{P}(\mathrm{Run} 2)$ correction 6.14 , is:

$$
A_{2}^{P}(\mathrm{Run} 2)=2.238 \pm 0.552 \mathrm{ppm},
$$

where this value is also polarization-weighted.

\subsection{BB Correction in Run1}

We take a brief look at the beamline background analysis in Run1, which is not the main dataset examined in this thesis. For a clear comparison between Run1 and Run2, the USLsum asymmetries are plotted over the full $Q_{\text {weak }}$ dataset in Figure 6.24 The range of USLsum asymmetries in Run1 is significantly suppressed compared to Run2, and the same is true for the other background detector: 8 , Similar suppression is observed in the background yields and asymmetry widths in Run1 compared to Run2. It is not clear why are the beamline background asymmetries suppressed in Run1. It is possible that this is associated with the larger laser beam spot size in Run2.

This suppression complicates the $A \mathrm{BB}$ analysis for two reasons. First, the reduced range increases the fit uncertainty in the extraction of the correction factor $C_{\mathrm{MD}-\mathrm{USL}}^{\mathrm{BB}}$, as in Figure 6.19 Secondly, the suppression of the USLsum asymmetry variance Var(USL) which appears in the de-

\footnotetext{
${ }^{8}$ With the added complication that the background detectors (other than the USLumi bars) were in a different location in Run1.
} 
nominator of Eq. 6.10, amplifies the bias from noise and residual beam effects $\operatorname{Cov}\left(\delta_{\text {beam }}^{\mathrm{MD}}, \delta_{\text {beam }}^{\mathrm{USL}}\right)$ in the extracted correlation slope $C_{\mathrm{MD}-\mathrm{USL}}$, by as much as a factor of $\sim 20$ in the second part of Run1 where the range is most suppressed 9 . Note that the residual beam effects were also seen to be more significant in Run1, as suggested by the larger residual correlations to beam parameters and larger discrepancy between correction schemes in Section 5.6.2.

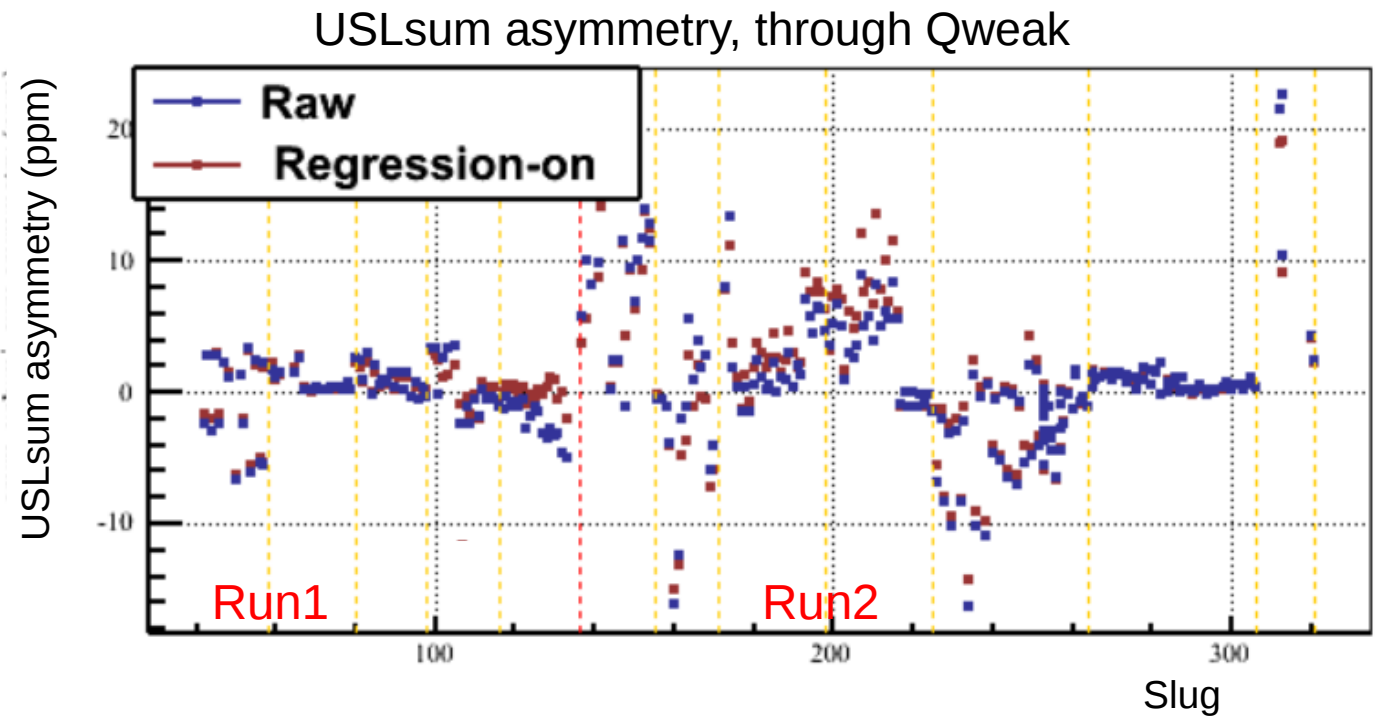

Figure 6.24: Slug-averaged USLsum asymmetry through $Q_{\text {weak }}$, uncorrected (blue) and regressed (red). Yellow dashed vertical lines mark Wien transitions, while the red dashed vertical line separates Run1 and Run2.

The Run1 beamline backgrounds correction factor is extracted from the slug-scale correlation between MDall and USLsum over Run1, from Figure 6.25.

$$
C_{\mathrm{MD}-\mathrm{USL}}^{\mathrm{BB}}(\mathrm{Run} 1)=9.84 \pm 7.26 \text { (fit) } \mathrm{ppb} / \mathrm{ppm}
$$

The uncertainty is much larger owing to the suppressed range of background asymmetries. Note that the background asymmetries in the first part of Run1 (before the long halt in production for bellows replacement on Slug 63) are larger and have the largest pull on the result. The extracted correction factor is consistent with the value 6.11 from Run2, which had a much smaller statistical uncertainty. We are currently exploring the possibility of using the Run2 value for the correction in Run1, given that the signal fraction $f_{\mathrm{BB}}^{\mathrm{MD}}$ was consistent in the blocked-octant studies from Run1 and Run2.

This analysis is still in progress, but here we will quote the beamline background correction in Run1 using the value from Eq. (6.16) for the correction factor. Following the Run2 procedure of the previous sections, including a $23 \%$ randomization of the correction factor for the model

\footnotetext{
${ }^{9}$ Derived from the reduction of the USLumi asymmetry variance.
} 


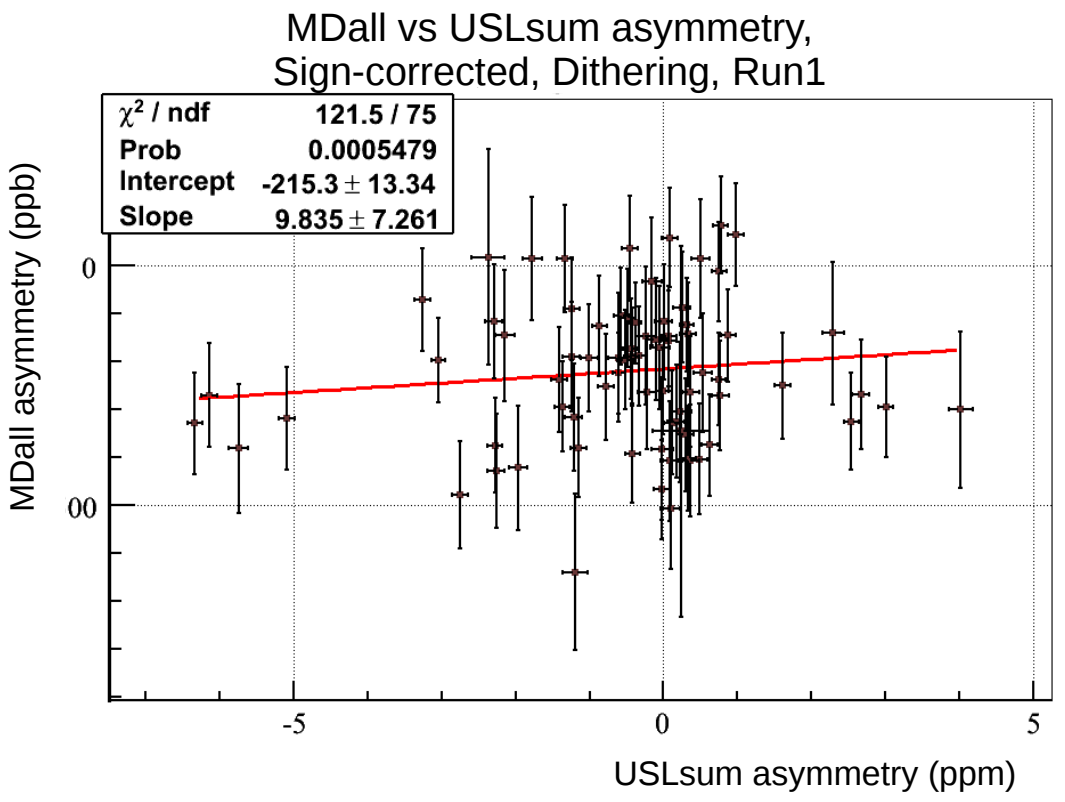

Figure 6.25: Fit between MD and USLsum asymmetries in Run1, averaged at the slug time scale. The correlation slope is the BB correction factor $C_{\mathrm{MD}-\mathrm{USL}}^{\mathrm{BB}}$ for Run1.

uncertainty, the polarization-weighted BB correction in Run1 is:

$$
A_{\mathrm{BB}}^{P}(\text { Run } 1)=-6.13 \pm 4.54(\text { fit }) \pm 1.24(\text { model }) \mathrm{ppb}
$$




\section{Chapter 7}

\section{Statistical Properties of the}

\section{Corrected Asymmetry}

In this chapter we examine statistical properties of the Physics and Null asymmetry combinations in the Run2 Modulation dataset, for the MD asymmetries that have been corrected for false beam asymmetries and beamline backgrounds. Other than weighting by appropriate polarization, no other systematic corrections have been applied. The asymmetries are only assigned the statistical uncertainty of the measurement without taking into account the error of the systematic corrections for $A_{\text {beam }}$ and $A_{\mathrm{BB}}$ from Eqs. (5.11) and 6.14 respectively. We have already examined the Physics asymmetry combination in our dataset segmented at the pitt scale in Figure 6.21. Studies of the statistical properties of the corrected asymmetries will allow us to discern the efficiency of the systematic corrections in removing false asymmetries from our measurement.

\subsection{Null Asymmetries}

Null asymmetry combinations $A_{\text {Null }}$ are arranged so that the "physics" $A_{e p}$ is cancelled and the expectation value of the combination is 0 (null), in the absence of residual systematic effects. Therefore any deviation of the Null asymmetry combination from zero may be an indication of residual systematic effects.

There is more than one way to construct the Null asymmetry combination and arrange for cancellation of $A_{e p}$ through the applied slow reversals. The reversals are separated into two classes: the "IHWP" reversal from the insertion of the IHWP in the source laser table, and the "Spin" reversal of the electron beam spin through the injector spin manipulation system 1

\footnotetext{
${ }^{1}$ Reversal of the electron beam spin through precession is also included in this category.
} 
This author defined three separate $A_{\text {Null }}$ combinations, each one accessing different classes of residual systematic effects. The naming scheme for the Null definitions is derived from the class of reversals used to cancel the $A_{e p}$ asymmetry, by not applying a sign-correction for the effect of that reversal. Or equivalently, the name of each Null combination is the reversal which should achieve cancellation of the residual systematic effects accessed by that combination.

Thus the defined Null combinations are:

- The "IHWP+Spin" Null definition, constructed by applying no sign-correction:

$$
A_{\mathrm{Null}}^{\mathrm{IHWP}+\mathrm{Spin}}=\frac{A_{\mathrm{IN}}+A_{\mathrm{OUT}}}{2},
$$

where $A_{\mathrm{IN} \text { (OUT) }}$ is the measured asymmetry under the IN (OUT) IHWP state, without any sign-corrections applied for either IHWP or Spin slow reversals. The sum of Eq.(7.1) is unweighted to ensure cancellation of the physics asymmetry. The IHWP+Spin Null combination accesses false asymmetries due to mechanical or direct electrical pick-up effects. These would depend only on the HV state of the Pockels cell and not change sign with IHWP or Spin reversals, i.e. the non-polarization class of effects on the laser table like HC steering from the PC, described in Section 4.6.3. As implied by the name of this Null combination, these effects should cancel through either the IHWP or the Spin reversal. This is the Null definition that was quoted in Section 5.6 .

- The "IHWP-only" Null definition is effectively the same as the previous one, but with sign-corrections applied for Spin reversals. It is constructed as

$$
A_{\text {Null }}^{\mathrm{IHW}-\text { only }}=\frac{[\mathrm{sgn}] A_{\mathrm{IN}}-[\mathrm{sgn}] A_{\mathrm{OUT}}}{2},
$$

where [sgn] is the appropriate sign to access the physics asymmetry, but the subtraction in the numerator effectively removes the sign-correction for the IHWP reversal. Therefore this Null combination gets the same benefit of cancellations through the Spin reversals as the "Physics" combination $A_{\text {Phys }}$, but not the same benefit of the IHWP reversal. Note that the only difference with the IHWP+Spin definition is a sign-correction applied for every Spin reversal. Therefore the fit $\chi^{2}$ and probability, quoted with respect to zero, will be identical for the two Null combinations.

- The "Spin-only" Null definition accesses false asymmetries due to "polarization" effects on the laser table (Section 4.6.1) that would change sign with IHWP and therefore survive in the $A_{\text {Phys }}$ combination, but should cancel under Spin reversal on the electron beam. The 
average Spin-only Null asymmetry over a long period is calculated as

$$
A_{\text {Null }}^{\text {Spin-only }}=\frac{[\operatorname{sgn}] A_{\mathrm{L}}-[\operatorname{sgn}] A_{\mathrm{R}}}{2}
$$

where $[\operatorname{sgn}] A_{\mathrm{L}, \mathrm{R}}$ is the average Physics asymmetry combination in each of the two opposite Spin reversal states (the product of the Wien and precession reversals), referred as "SpinLeft" and "Spin-Right" states. Note that these averages can only be extracted over the

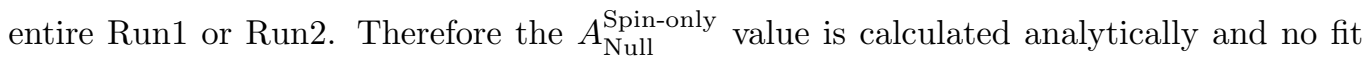
probability is defined for it.
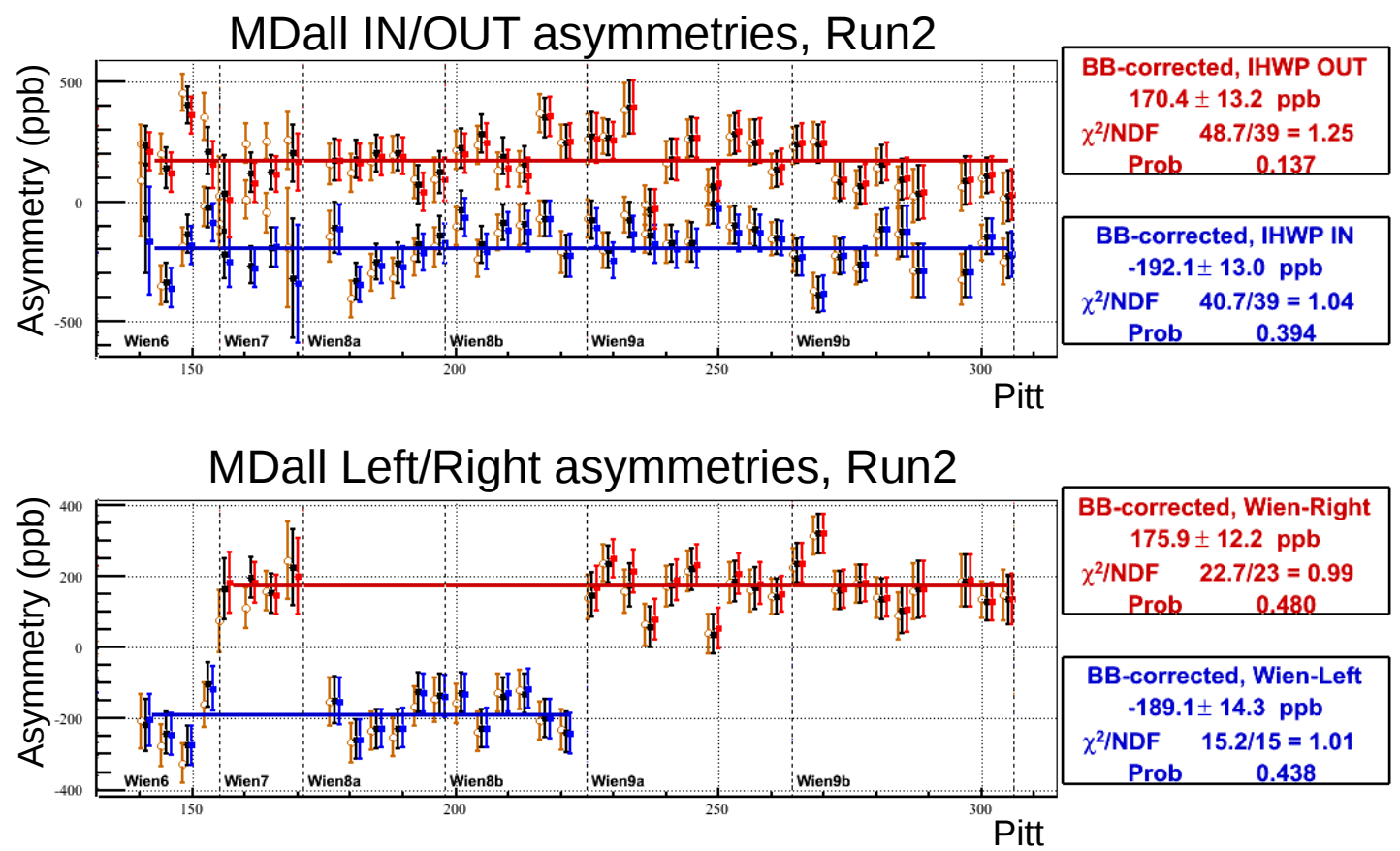

Figure 7.1: MDall asymmetries averaged at the pitt scale in Run2 and separated by IHWP (top) or Spin state (bottom). Data correspond to uncorrected (orange), corrected for beam asymmetries $A_{\text {beam }}$ through dithering (black) and finally corrected for beamline backgrounds (blue/red, depending on IHWP/Spin separation). Fit results are quoted for the BB-corrected asymmetries.

The separate contributions from the different IHWP and Spin states are plotted in Figure 7.1. These are the $A_{\mathrm{IN}(\mathrm{OUT})}$ and $A_{\mathrm{L}(\mathrm{R})}$ terms that correspond to the average IHWP IN (OUT) and Spin-Left (-Right) asymmetries, used in the construction of the Null combinations. Comparing to the BB-corrected $A_{\text {Phys }}$ from Figure 6.21 with a fit probability of 0.499 , the fit probability is always smaller for the separate contributions. The increased fit probability on the Physics asymmetry combination suggests better suppression of systematic effects achieved by the combination of the opposite reversal states. The increase is higher compared to the separate IHWP states, suggesting that the IHWP reversal is most important.

Through the separate terms we construct the three Null combinations, which are plotted in Figure 7.2 over Run2. Again we note that the fit probability is sequentially increased by the 
beam corrections followed by the BB correction, just as in the Physics asymmetry combination of Figure 6.21. However the fit probability for the Null combinations remains significantly smaller than for the Physics, owing to cancellations of residual systematic effects from the reversals. The average value over Run2 for all three Null definitions is consistent with zero at the $1 \sigma$ level, suggesting that all different classes of residual systematic effects cancel well over Run2.
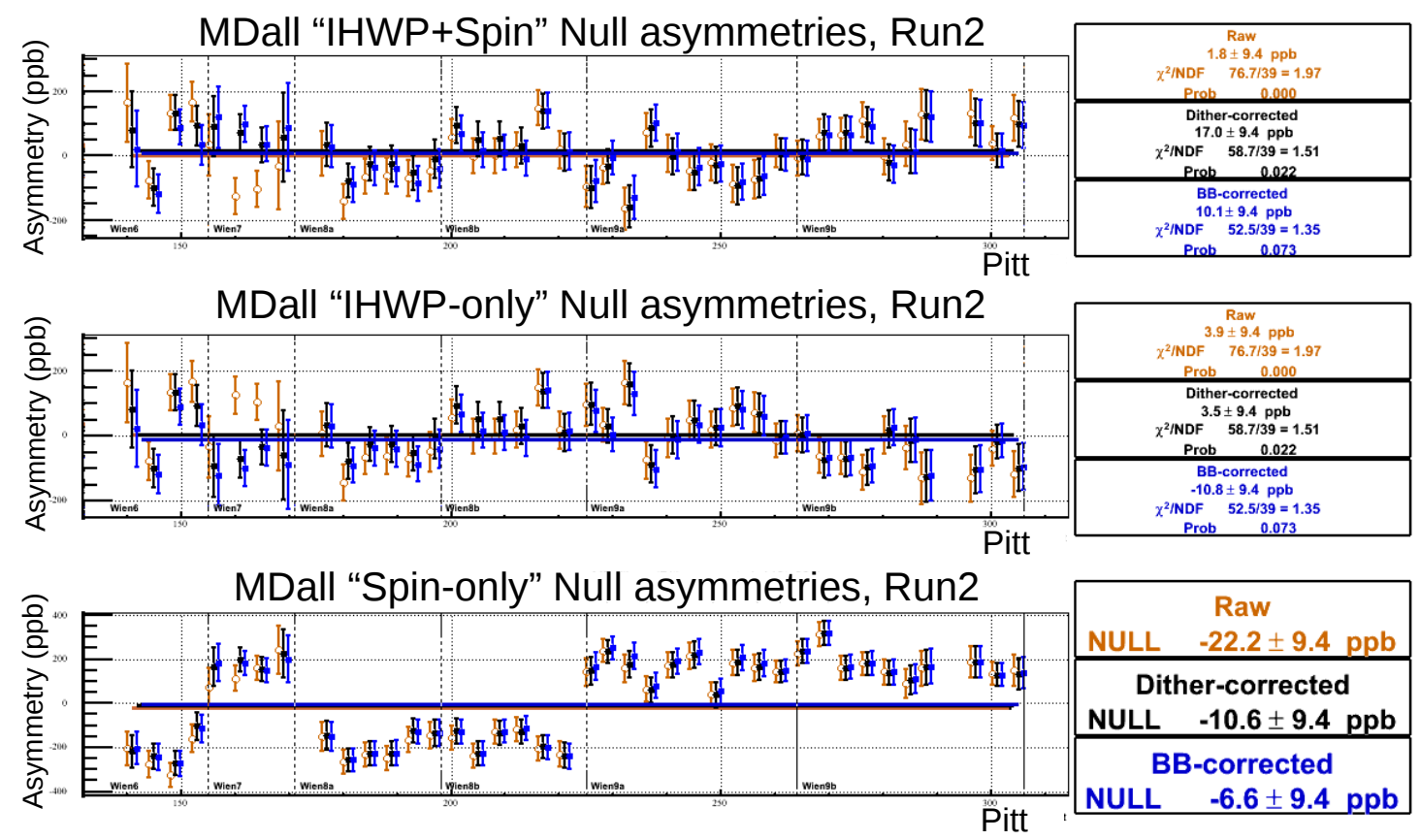

Figure 7.2: Three different definitions of the MDall Null asymmetry combination in Run2 averaged at the pitt scale. Fit probabilities and $\chi^{2} / \mathrm{NDF}$ are quoted with respect to zero.

For simplicity in the remainder of this chapter we only examine one of these Null definitions, the "IHWP+Spin" combination which should be most sensitive to residual false asymmetries.

\subsection{Beam and BB Corrections}

The total corrections from dithering and beamline backgrounds in every pitt are plotted in Figure 7.3 for the Physics and Null MDall asymmetry combinations. Beam corrections are generally larger in magnitude than BB corrections. Corrections on the Null asymmetry are also larger than the Physics asymmetry combination, again due to cancellations that only benefit the Physics asymmetry. The size of corrections on individual pitts can be of order of 100-200 ppb, much larger than the average $A_{\text {beam }}$ and $A_{\mathrm{BB}}$ corrections over Run2 (Eq. 5.11 and 6.14). The size of corrections is clearly larger before position feedback is applied through the helicity magnets around Slug 213. 


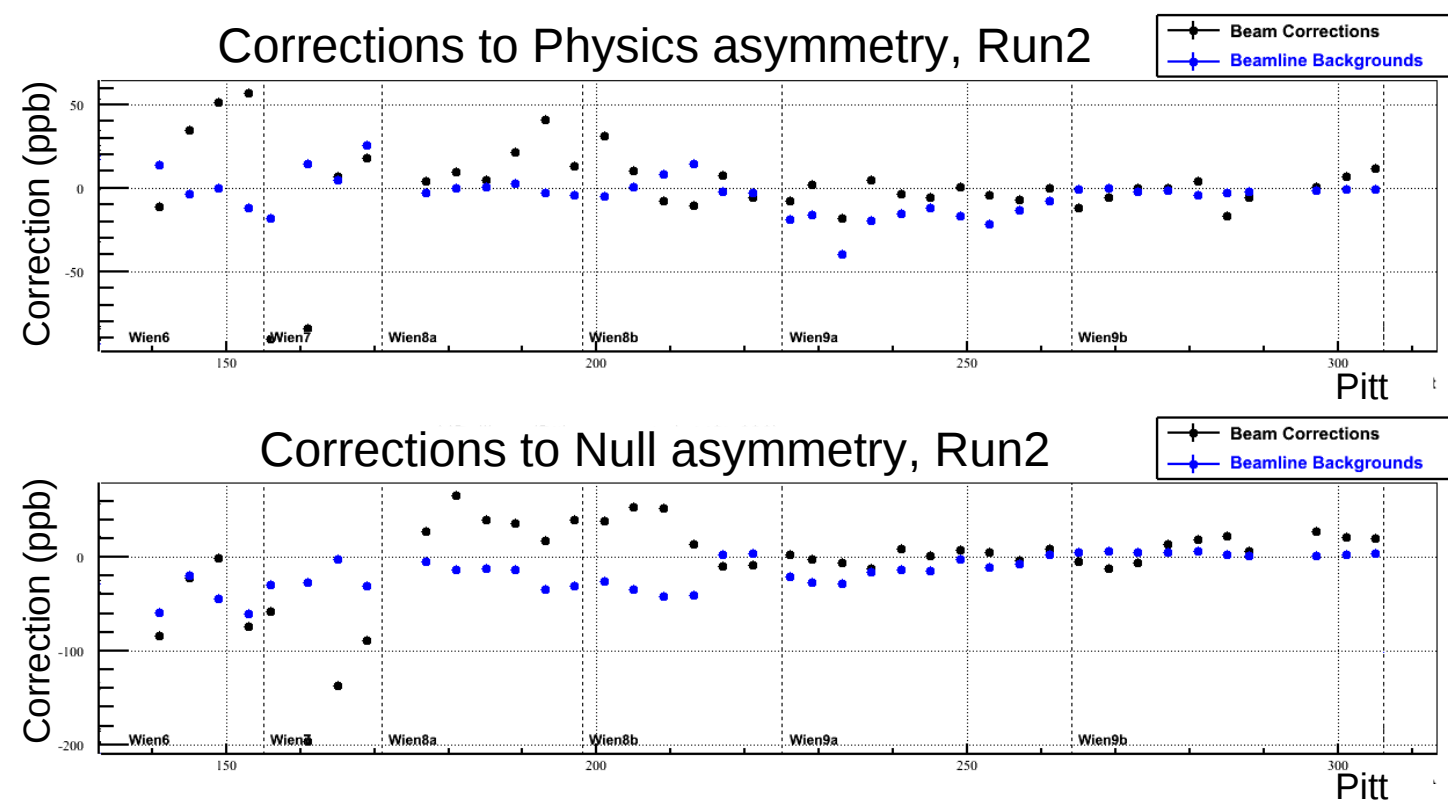

Figure 7.3: Applied corrections for false beam asymmetries $A_{\text {beam }}$ (black data) and beamline backgrounds $A_{\mathrm{BB}}$ (blue data) through Run2, averaged at the pitt scale and normalized for polarization. Corrections correspond to the Physics (top) and Null (bottom) MDall asymmetry combinations.

\subsection{Azimuthal Dependence of Asymmetries}

The average Physics and Null asymmetries over Run2 are plotted in Figure 7.4 for each MD octant. The figure reveals a significant sinusoidal (dipole) pattern in the azimuthal dependence of the results in Run2. For the Null asymmetry combination $A_{\text {Null }}$, the uncorrected results have a clear dipole amplitude of order $200 \mathrm{ppb}$, characteristic of false beam asymmetries, however the beam correction removes that amplitude convincingly. For the Physics asymmetry combination $A_{\text {Phys }}$ on the other hand, the dipole amplitude is smaller (of order $50 \mathrm{ppb}$ ) due to good reversal cancellations; but it is not adequately removed from the $A_{\text {beam }}$ correction. It is considered then that the remaining azimuthal dependence arises from residual transverse asymmetry $A_{T}$ in the scattered electrons, which is not expected to be removed through beam corrections. It is also consistent with the $A_{T}$ hypothesis that cancellation is achieved on individual bars for the Null but not for the Physics combination. The $\sim 50 \mathrm{ppb}$ dipole amplitude, with a transverse asymmetry of $\sim 4.8 \mathrm{ppm}$ as measured in dedicated studies (see Section 8.1 .3 , suggests a residual transverse polarization of $\sim 0.64^{\circ}$, averaged over Run2. The $\mathrm{BB}$ correction $A_{\mathrm{BB}}$ is much smaller by comparison to account for the observed azimuthal dependence.

The fit probabilities for the Run2 Physics and Null asymmetry combinations are plotted versus MD octant in Figure 7.5. For $A_{\text {Phys }}$, the beam corrections generally increase the fit probability by about an order of magnitude compared to raw data. The probability is seen to be higher for the average MDall compared to individual bars, suggesting that residual system- 


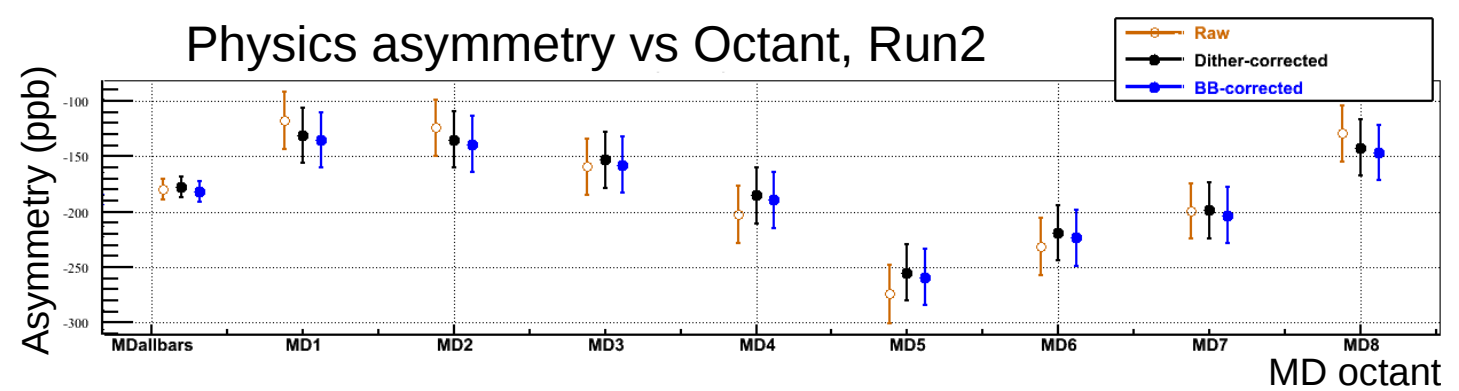

Null asymmetry vs Octant, Run2

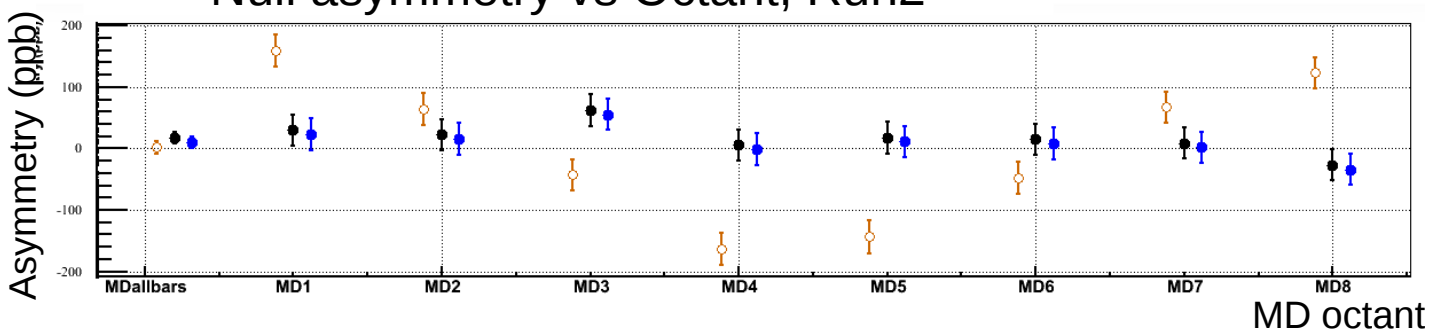

Figure 7.4: Physics (top) and Null (bottom) asymmetry combinations, extracted over Run2 and plotted versus MD octant. The values have been extracted and plotted for every MD octant. The average asymmetries are quoted in every octant for uncorrected (orange), corrected for $A_{\text {beam }}$ through dithering (black) and then corrected for $A_{\mathrm{BB}}$ (blue) data in Run2. The MDall combination is the left-most point.

atic effects cancel when opposite bars are combined. Very importantly, the residual transverse asymmetry that gives rise to the azimuthal dependence is expected to cancel on the MDall average.

On the other hand, the $A_{\text {Null }}$ asymmetry fit probability is improved through beam corrections by many orders of magnitude in most MD bars. We expect the effect of false beam asymmetries to be larger on the "IHWP+Spin" Null combination where we don't get the benefit of cancellations, but we see that fit probabilities of individual bars are very good after beam corrections. Therefore the $A_{\text {beam }}$ correction is successful in removing false beam effects, at least within the precision offered by individual bars. On individual bars the fit probability is larger on the Null combination, probably due to cancellation of the residual transverse asymmetry which changes sign under the IHWP reversal and only affects the Physics asymmetry. However the MDall average for the Null asymmetry has lower fit probability compared to individual MD bars, or the Physics combination. This can be interpreted to arise from the improved precision of the MDall average compared to individual bars (by a factor of $\sqrt{8}$ ) which allows increased sensitivity to residual false beam asymmetries. 


\section{Physics asymmetry probability vs Octant, Run2}
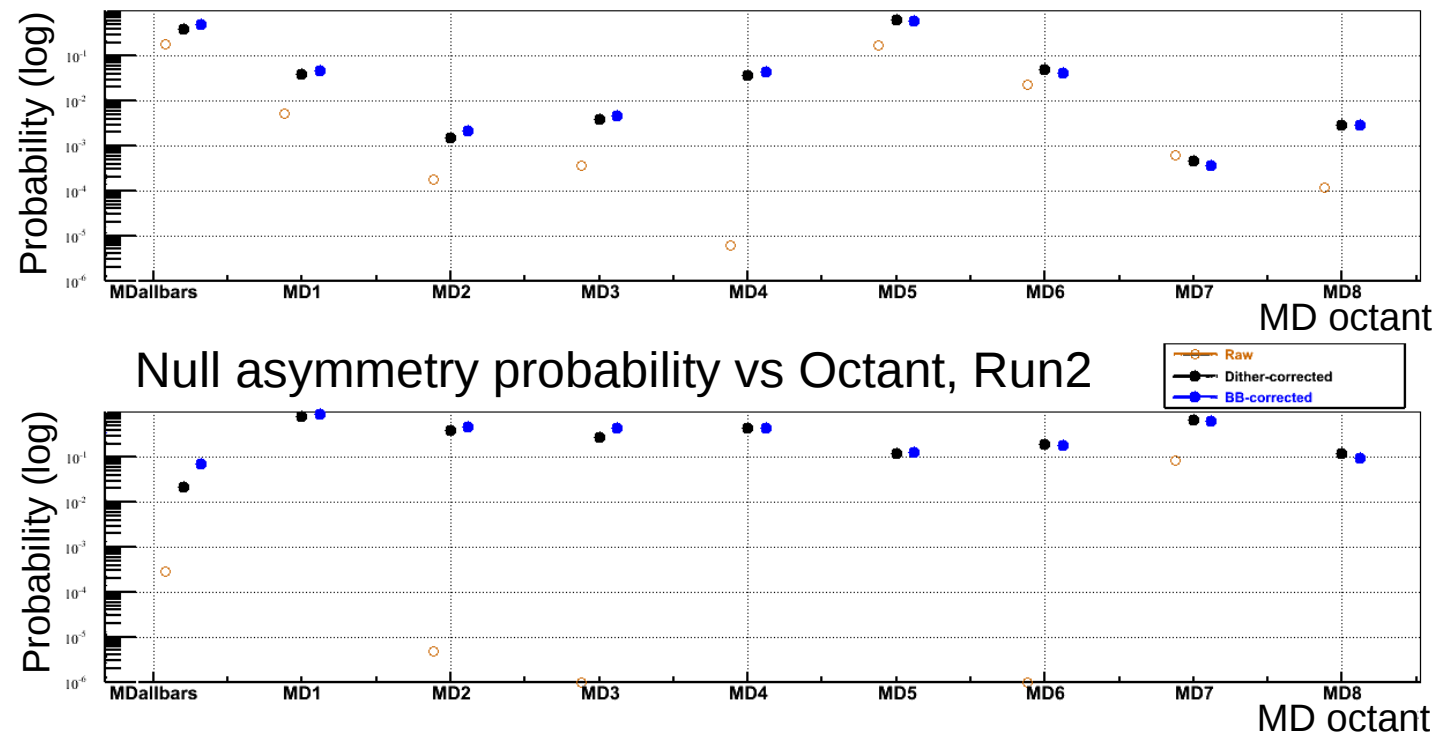

Figure 7.5: The fit probability for the Physics (top) and Null (bottom) asymmetry combinations, extracted over Run2 and plotted on a log scale versus MD octant. The probability is quoted in every octant for uncorrected (orange), corrected for $A_{\text {beam }}$ through dithering (black) and then corrected for $A_{\mathrm{BB}}$ (blue) asymmetries. The MDall combination is the left-most point.

\subsection{Auto-Correlation}

The auto-correlation function (ACF, also referred to as time correlation) of the Physics and Null MDall asymmetry combinations, segmented at the pitt scale over Run2, is plotted in Figure 7.6. $\mathrm{ACF}$ is the auto-correlation coefficient, a measure of the degree of correlation between measurements of the asymmetry separated by a number of pitts $N_{\text {pitts }}$, referred as the pitt "lag". For example, the ACF for a lag of $1\left(N_{\text {pitts }}=1\right)$ is the degree of correlation between all neighbouring pitts in Run2.

Of course we expect measurements of the asymmetry separated by many pitts (large pitt lag) to be uncorrelated. The Physics asymmetry measurements appear to have an ACF that is consistent with zero even at small values of pitt lag/separation, suggesting good suppression of residual systematic effects within the precision of individual pitts. The Null asymmetry on the other hand appears to have a significantly positive ACF value for small pitt lags. In other words the Null asymmetry measurement in any pitt is a good estimator of the Null asymmetry in the following few pitts, suggesting residual false effects with a characteristic time scale of a few pitts, or approximately a Wien. The Null ACF arguably swings negative for larger values of separation, which may be an indication of the effect of Wien reversals. 


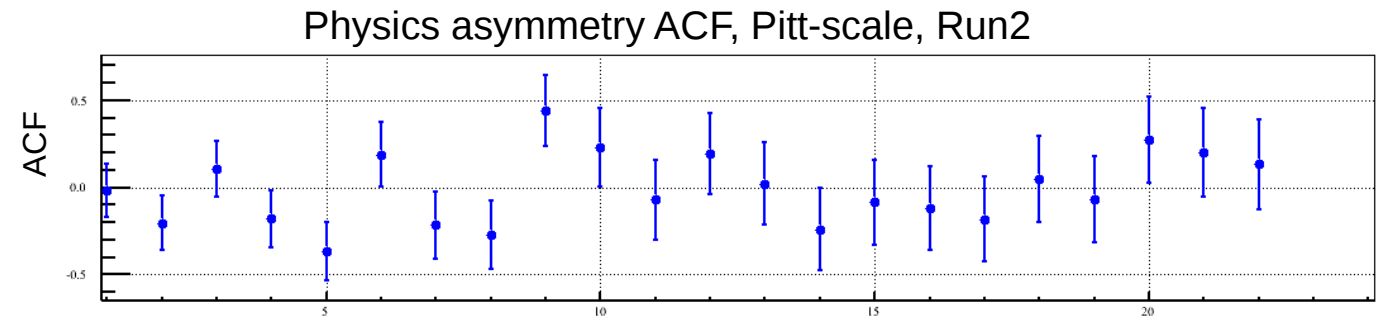

Pitt lag

Null asymmetry ACF, Pitt-scale, Run2

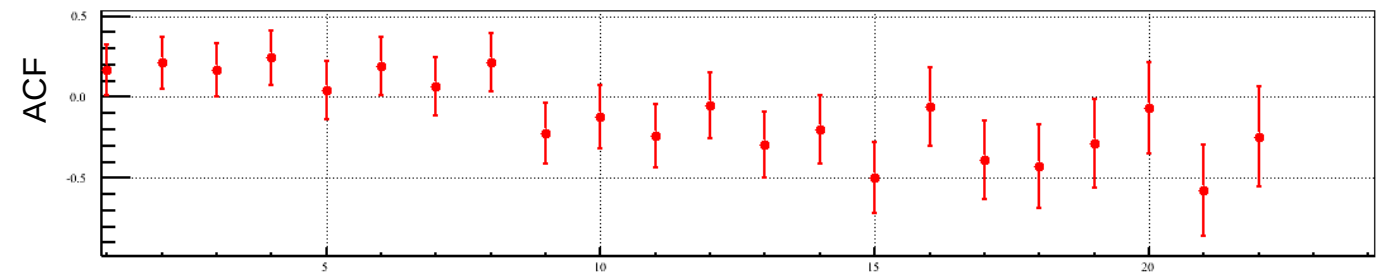

Pitt lag

Figure 7.6: The Auto-Correlation Function of the Physics (top) and Null (bottom) asymmetry combinations, for pitt-scale segmentation over Run2, is plotted versus the pitt "lag". All asymmetries are corrected for $A_{\text {beam }}$ and $A_{\mathrm{BB}}$. See text for details.

\subsection{Dependence to Time Scale of Averaging}

Up to now we have examined the statistical properties of the asymmetries segmented (averaged) at the pitt time scale. In this section we study the dependence of the fit probabilities to the averaging time scale. The fit probabilities were extracted for asymmetries averaged at the slug, pitt and Wien time scales, and are quoted in Table 7.1. Current results from Run1 are included for comparison, even though all analysis in this chapter has focused on the Run2 Modulation dataset.

At the slug scale the Physics fit probabilities are lower, even after the $A_{\text {beam }}$ and $A_{\mathrm{BB}}$ systematic corrections ${ }^{2}$. It is impressive that the fit probability for the uncorrected Physics asymmetry at the pitt scale is about as good as the fully corrected asymmetry averaged at the slug scale. By combining slugs of opposite IHWP states we achieve some cancellation of residual false asymmetries, as discussed in Section 5.4 .2 .

The Run2 Physics and Null asymmetry distributions are extracted in Figure 7.7, averaged at the Wien time scale. This is the same data plotted previously in Figures 6.21 and 7.2 , but averaged at a longer time scale. The small decrease in fit probability for the Null asymmetry at the Wien scale, compared to the pitt-scale probability, could be an indication of residual systematic effects becoming more prominent. This is consistent with the discussion on the ACF of the pitt-scale Null asymmetry from Figure 7.6 , suggesting that the Wien is a more characteristic time scale for these effects. Still the average over different periods in Run2 is consistent with

\footnotetext{
${ }^{2}$ There can be no Null asymmetry combination defined from individual slugs; we need at least two slugs of opposite IHWP state to define a Null combination.
} 
Table 7.1: Fit probabilities of Physics and Null asymmetry combinations, segmented over different time scales in Run1 and Run2.

\begin{tabular}{l|ccc|ccc|}
\multirow{2}{*}{} & \multicolumn{3}{|c|}{ Physics } & \multicolumn{3}{c|}{ Null } \\
\cline { 2 - 7 } & Uncorrected & $\begin{array}{c}\text { Dithering } \\
\text { correction }\end{array}$ & $\begin{array}{c}\text { BB } \\
\text { correction }\end{array}$ & Uncorrected & $\begin{array}{c}\text { Dithering } \\
\text { correction }\end{array}$ & $\begin{array}{c}\text { BB } \\
\text { correction }\end{array}$ \\
\cline { 2 - 7 } & \multicolumn{7}{|c|}{ Run1 } & & \\
\hline Slug & 0.000 & 0.000 & 0.002 & - & - & \\
\hline Pitt & 0.000 & 0.071 & 0.154 & 0.001 & 0.001 & 0.000 \\
\hline Wien & 0.019 & 0.101 & 0.481 & 0.002 & 0.000 & 0.000 \\
\hline & \multicolumn{7}{|c|}{ Run2 } & & \\
\hline Slug & 0.054 & 0.164 & 0.263 & - & - & 0.073 \\
\hline Pitt & 0.188 & 0.410 & 0.499 & 0.000 & 0.022 & 0.003 \\
\hline Wien & 0.054 & 0.607 & 0.829 & 0.000 & 0.000 & 0.003 \\
\hline
\end{tabular}

zero. On the other hand the fit probability for the Physics asymmetry combination increases at the Wien scale, suggesting that there are no signs of systematic effects even at the higher precision of the Wien averages, but only after correcting for $A_{\text {beam }}$. We conclude that there is no evidence of residual false asymmetries on our measurement, after we get the full benefit of systematic corrections $A_{\text {beam }}$ and $A_{\mathrm{BB}}$, azimuthal symmetry of the MD array, and cancellations from applied reversals.
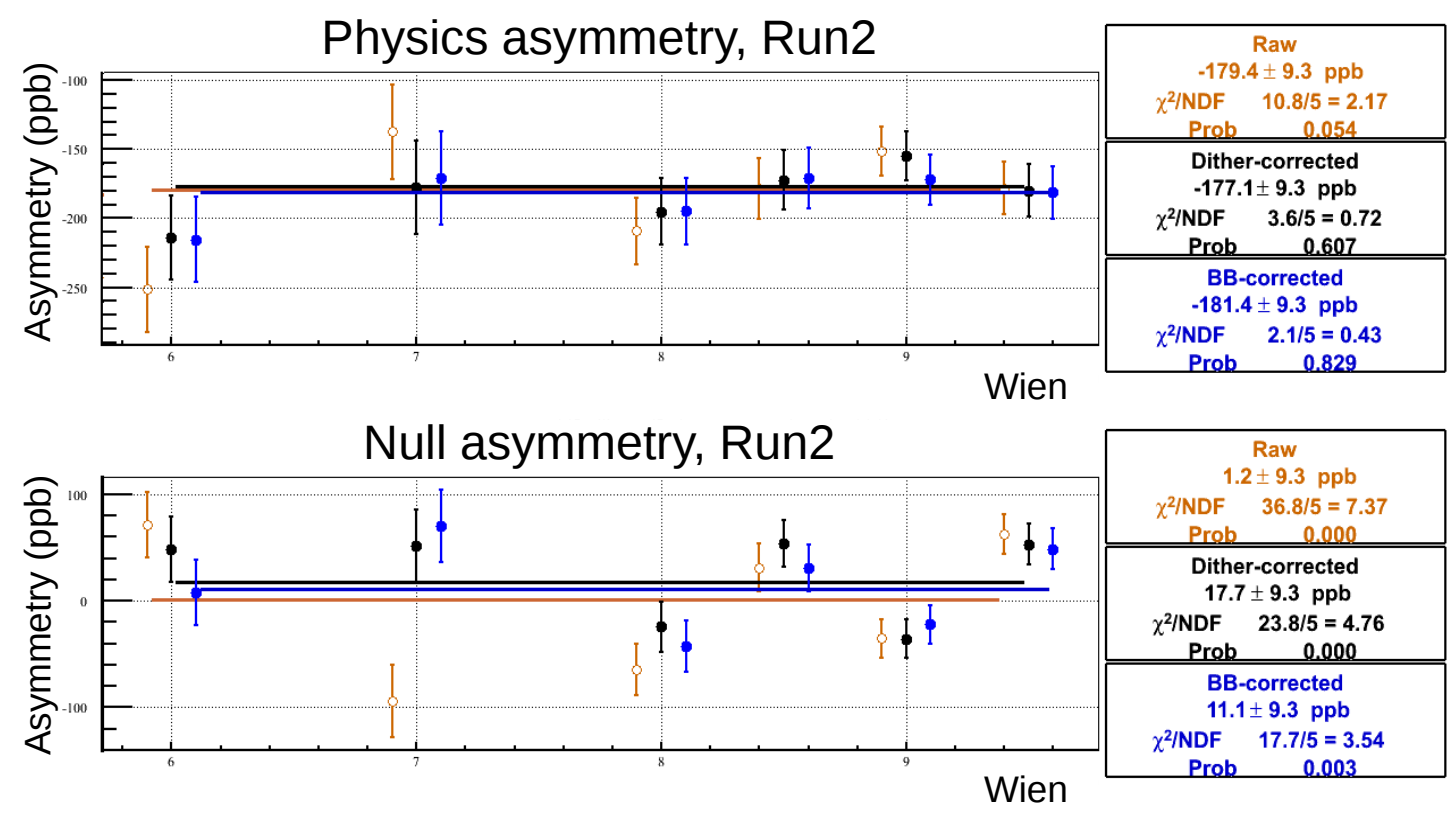

Figure 7.7: MDall Physics (top) and Null (bottom) asymmetry combinations in Run2, segmented at the Wien scale. Data shown for uncorrected 


\section{Chapter 8}

\section{Results and Conclusion}

In this chapter we extract the $\mathrm{PV}$ asymmetry $A_{\mathrm{PV}}$ for $\overrightarrow{e p}$ elastic scattering at $Q_{\text {weak }}$ kinematics. We will use the values calculated for the $A_{\text {beam }}^{P}$ and $A_{\mathrm{BB}}^{P}$ corrections in the previous chapters. We will adopt the best currently available values for all other systematic corrections, which vary in level of maturity. Then we go on to calculate the weak charge of the proton $Q_{w}^{p}$, the $C_{1 u}, C_{1 d}$ coupling parameters, and the weak mixing angle.

As discussed in Section 3.9 we will extract our result from the Modulation dataset of Run2. This is the same dataset whence we extracted our estimations for the total $A_{\text {beam }}^{P}$ and $A_{\mathrm{BB}}^{P}$ corrections. Furthermore, our result will include a blinding term, a randomized constant that has been added to prevent potential analysis bias based on comparison with the Standard Model prediction. The blinding term will only be revealed and subtracted after the procedure for all corrections is finalized. As we discuss next, we slightly modify the treatment of the $A_{\mathrm{BB}}$ correction in the formula to extract the PV asymmetry $A_{\mathrm{PV}}^{e p}$, compared to the treatment in the 2013 result.

\subsection{Extracting the Parity-Violating Asymmetry}

The parity-violating asymmetry can be extracted from the raw experimentally measured asymmetry after accounting for systematic effects. We use the formula

$$
\begin{gathered}
A_{\mathrm{PV}}^{e p}=\frac{R_{\mathrm{tot}}}{1-\sum_{b=1}^{4} f_{b}}\left(\frac{1}{P} A_{\mathrm{msr}}-\sum_{b=1,3,4} f_{b} A_{b}\right), \\
A_{\mathrm{msr}}=A_{\text {raw }}-A_{\text {beam }}-A_{\mathrm{BB}}-A_{T}-A_{L}-A_{\mathrm{PMT}-\mathrm{DD}}
\end{gathered}
$$

Note that the treatment of the $b=2$ beamline background correction $A_{\mathrm{BB}}$ is modified relative to Eq.3.3 which was used for the $2013 Q_{\text {weak }}$ result. Given that the beamline background 
correction was treated in Chapter 6 as a "beam" correction, it is absorbed here in the $A_{\mathrm{msr}}$ asymmetry in the same footing as the $A_{\text {beam }}$ correction of Chapter 5 . The signal fraction $f_{2}$ from beamline backgrounds should still be considered as separate from the elastic ep signal, and is included in the sum of background signal fractions in the denominator of Eq. 8.1. We have included a new correction $A_{\mathrm{PMT}-\mathrm{DD}}$ in the $A_{\mathrm{msr}}$ term, which will be discussed in Section 8.1.3

\subsubsection{Radiative corrections and experimental bias}

The $R_{\text {total }}$ factor accounts for radiative corrections and several effects that can create bias in our asymmetry measurement. It is defined as

$$
R_{\mathrm{total}}=R_{\mathrm{RC}} R_{\mathrm{Det}} R_{\mathrm{Bin}} R_{Q^{2}}
$$

- To facilitate interpretability and comparison, PV asymmetries are typically quoted at the tree-level. $R_{\mathrm{RC}}$ is a factor accounting for radiative corrections from multiple scattering and Brehmsstrahlung processes, including higher-order loop diagrams, that are included in our measurement (Figure 8.1). The effect of radiative corrections was deduced from GEANT3 simulations with and without Brehmsstrahlung, using methods described in [36, 164] via the Mo-Tsai prescription [165]. The $R_{\mathrm{RC}}=A_{\mathrm{elastic}}^{\mathrm{sim}} / A_{\mathrm{Mo} \text {-Tsai }}^{\mathrm{sim}}=1.0101 \pm 0.0007$ factor normalizes the measured asymmetry, which includes radiative corrections, so that the corrected value corresponds to the tree-level elastic asymmetry. $A_{\text {elastic(Mo-Tsai) }}^{\text {sim }}$ is the simulation result with the elastic Mo-Tsai generator.

- The $R_{\text {Det }}$ factor accounts for detector bias due to the measured light variation and nonuniform $Q^{2}$ distribution across the bars. The factor was deduced from GEANT4 simulations for the $Q^{2}$ distribution with and without light-weighting, as the ratio $R_{\text {Det }}=$ $A_{\text {unbiased }} / A_{\text {biased }}=0.9921 \pm 0.0044$.

- $R_{\text {Bin }}$ is an effective kinematic acceptance correction. This factor normalizes the measured asymmetry, which is an average $\left\langle A\left(Q^{2}\right)\right\rangle$ over the $Q^{2}$ distribution, to a value $A\left(\left\langle Q^{2}\right\rangle\right)$ that corresponds to the average calculated $\left\langle Q^{2}\right\rangle$ :

$$
R_{\text {Bin }}=\frac{A_{\text {tree }}\left(\left\langle Q^{2}\right\rangle\right)}{\left\langle A_{\text {tree }}\left(Q^{2}\right)\right\rangle}=0.980 \pm 0.005
$$

- Finally $R_{Q^{2}}$ represents the limited precision in calibrating the central $Q^{2}$. The current estimation for the error is $d Q^{2} / Q^{2}=1.28 \%$ [166], which for the average $\left\langle Q^{2}\right\rangle=0.02455 \mathrm{GeV}^{2}$ gives $R_{Q^{2}}=1.0000 \pm 0.016$. 

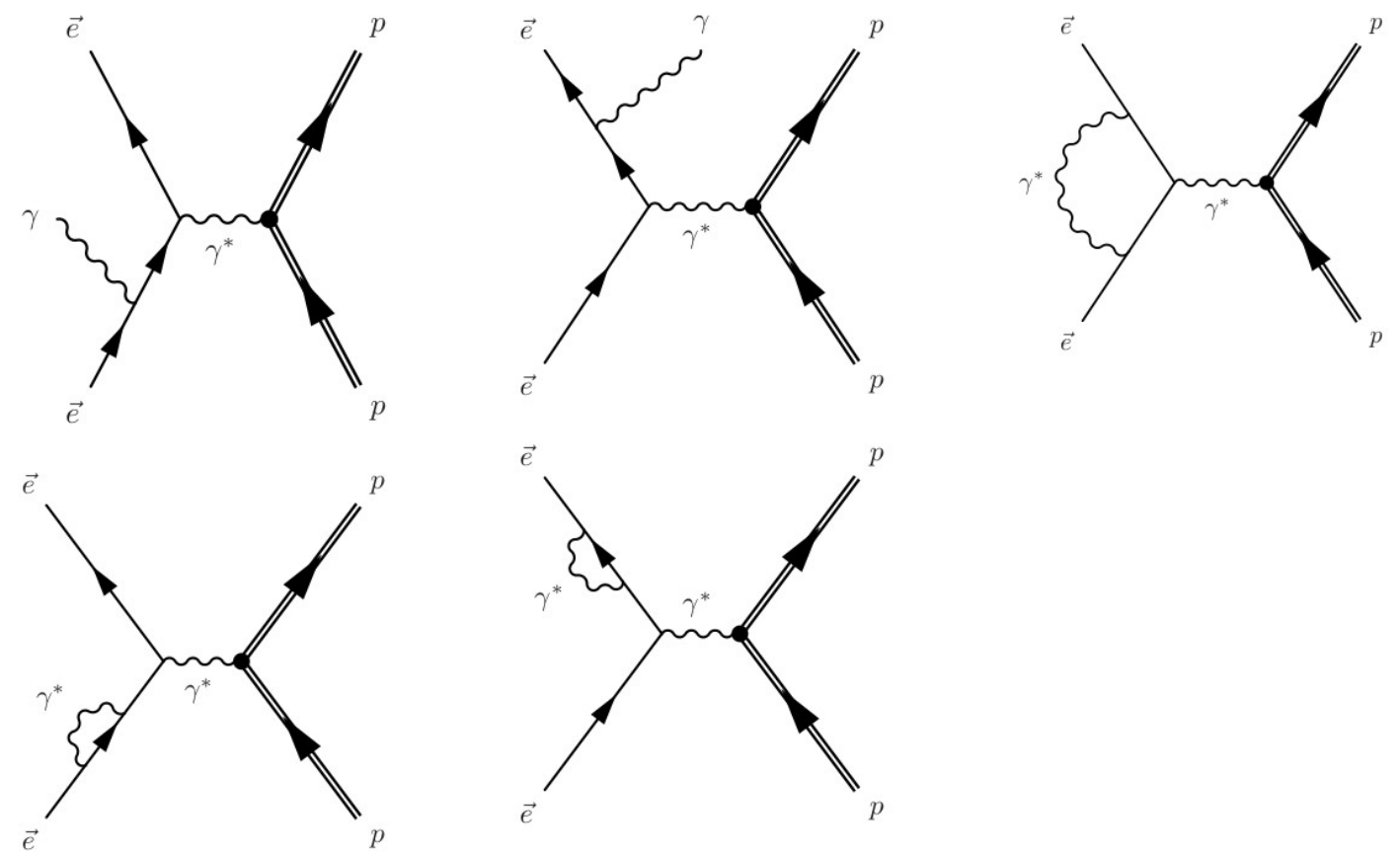

Figure 8.1: Initial (left) and final (middle) state QED radiative corrections in the $\overrightarrow{e p}$ interaction, and vertex loop correction (right).

The full bias correction then is:

$$
R_{\text {total }}=R_{\mathrm{RC}} R_{\text {Det }} R_{\text {Bin }} R_{Q^{2}}=0.982 \pm 0.017
$$

\subsubsection{Polarization}

In Run2 the polarization was extracted from a combination between the Møller and Compton polarimeters. The measurements from the two polarimeters are shown in Figure 8.2 over Run2. The measurements from each polarimeter include statistical plus point-to-point systematic errors, the latter drawn as the outer error bars. The stability of the beam polarization measured by the Compton polarimeter justifies the time interval chosen for the more infrequent Møller measurements. However, Møller measurements were always made immediately before and after changes in the polarized source that were known to affect the beam polarization: notably laser spot changes or reactivation of the injector photocathode.

The central value for the polarization is calculated as a combination of the two polarimeters over eight periods in Run2, separated by the vertical dashed lines. In each of the separate periods the polarization is assumed constant, except in the period beginning in run number 17316; that period is immediately after the Run2 cathode heat and reactivation (Section 4.4.1), when the following gradual increase in polarization is expected.

The systematic uncertainty for the polarization quoted from the Møller and Compton po- 
larimeters is $0.83 \%$ and $0.58 \%$ respectively, where all contributions to these uncertainties were listed in Section 3.8 . The largest contribution to the Møller systematic uncertainty is a conservative $0.5 \%$ uncertainty for the extrapolation of the Møller measurement at low current $(\lesssim 2 \mu \mathrm{A})$ to the high current of $Q_{\text {weak }}$. The largest contributions to the Compton uncertainty are associated with DAQ inefficiencies.

The overall agreement between the two polarimeters is good in Run2. The average ratio $P_{\text {Compton }} / P_{\text {Møller }}=1.0073 \pm 0.0016\left(\chi^{2} / \mathrm{NDF}=1.96\right)^{17}$ through Run2 suggests an average $0.73 \%$ systematic discrepancy, which is consistent within the systematic uncertainties. Extra normalization uncertainties are assigned to both results to account for this discrepancy, and the total polarization is extracted as their weighted average with a total uncertainty of $0.62 \%$. The result is shown in Figure 8.2 as the dashed horizontal line in each period with the yellow band denoting the uncertainty. The polarization "map" of Figure 8.2 was used in the analyses of previous chapters to weigh the asymmetries and corrections we developed. The average polarization value in the Modulation dataset examined in this dissertation, is extracted from the polarization map with appropriate weights from the MD asymmetry:

$$
\langle P\rangle=0.889 \pm 0.006
$$

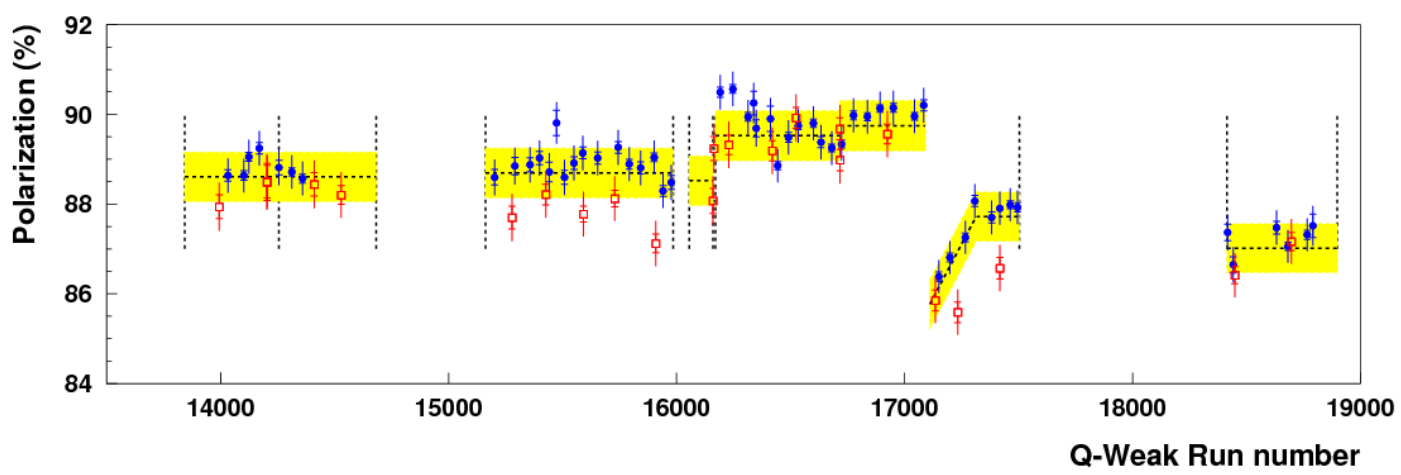

Figure 8.2: Polarization measurements versus run number through Run2, from the Møller (red) and Compton (blue) polarimeters. Each data point for the Compton corresponds to an average over about 30 hours. The uncertainties shown represent statistical and estimated systematic errors added in quadrature. The dotted line with the yellow band is the central value and total uncertainty for the polarization in Run2, including a scaling uncertainty.

\subsubsection{Measured asymmetry}

The measured asymmetry includes all "beam" corrections to the raw asymmetry. In a modification to the treatment of the $2013 Q_{\text {weak }}$ publication [55], we also include here the beamline

\footnotetext{
${ }^{1}$ The uncertainty and $\chi^{2}$ vary over different choices in forming the ratio, but the mean value is very stable.
} 
background correction $A_{\mathrm{BB}}$. A blinding term $A_{\text {blind }}$ has been added to the raw asymmetry, and would need to be subtracted to extract the physics asymmetry:

$$
A_{\mathrm{msr}}=A_{\text {raw }}-A_{\text {beam }}-A_{\mathrm{BB}}-A_{T}-A_{L}-A_{\mathrm{PMT}-\mathrm{DD}}-A_{\mathrm{blind}}
$$

Note that the values for $A_{\mathrm{raw}}^{P}, A_{\mathrm{beam}}^{P}$ and $A_{\mathrm{BB}}^{P}$ that we extracted in Chapters 5 and 6 were already corrected for polarization, whereas the polarization correction is applied separately in Eq.8.1). Therefore our extracted values must be scaled appropriately by the average polarization in the examined dataset.

- $A_{\text {raw }}$ is the raw MDall asymmetry before any applied corrections. The value $A_{\text {raw }}^{P}$ for the raw asymmetry in the Run2 Modulation dataset, properly weighted and corrected for polarization, was extracted in Eq. (5.9). We multiply that value by the average polarization (Eq. 8.3) to get the appropriate value for use in Eq. 8.1):

$$
A_{\text {raw }}=\langle P\rangle \cdot A_{\text {raw }}^{P}=-159.48 \pm 8.27 \text { (stat) } \mathrm{ppb},
$$

where the uncertainty of $A_{\text {raw }}$ corresponds to the statistical error of the $Q_{\text {weak }}$ measurement.

- $A_{\text {beam }}$ is the correction extracted in Chapter 5 for the false asymmetries arising from helicity-correlated beam properties. The appropriately weighted and corrected for polarization value given in Eq. (5.11) is multiplied by the average polarization in the Run2 Modulation dataset:

$$
A_{\text {beam }}=\langle P\rangle \cdot A_{\text {beam }}^{P}=-2.05 \pm 0.73 \mathrm{ppb}
$$

- $A_{\mathrm{BB}}$ is the correction for beamline backgrounds extracted in Chapter 6. The "source" asymmetry $A_{2}$ was extracted in Eq.66.15. However this asymmetry does not have a welldefined source and value that can be measured in a dedicated measurement. Rather it arises through mechanisms that are not well understood and changes with beam conditions, so that its value depends on the dataset examined. Hence this false asymmetry is treated as a "beam" asymmetry, although the necessary numbers to treat it as a background correction have also been given. However, the signal fraction $f_{2}$ in the main detector associated with this component is well-defined and separate from the elastic $f_{e p}$ fraction, therefore this dilution must be included in the denominator of Eq.8.1.

The appropriately weighted and corrected for polarization value given in Eq.(5.11) is mul- 
tiplied by the average polarization in our dataset:

$$
A_{\mathrm{BB}}=\langle P\rangle \cdot A_{\mathrm{BB}}^{P}=3.84 \pm 1.59 \mathrm{ppb}
$$

- $A_{T}$ is the false asymmetry arising from residual transverse polarization on the nominally longitudinal polarized electron beam. This parity-conserving asymmetry (often referred as "beam-normal single-spin asymmetry" $B_{n}$ ) was measured to be $B_{n}=-4.8 \pm 0.6 \mathrm{ppm}$ in dedicated studies where the beam polarization was set fully transverse, horizontal or vertical. As shown in Figure 8.3 the transverse asymmetry exhibits a sinusoidal azimuthal dependence for both vertical $(\mathrm{V})$ and horizontal $(\mathrm{H})$ transverse electron polarization:

$$
A_{T}= \begin{cases}A^{\mathrm{V}} \cos \left(\phi^{\mathrm{V}}+\phi_{0}^{\mathrm{V}}\right)+C^{\mathrm{V}} & \text { Vertical } \\ A^{\mathrm{H}} \sin \left(\phi^{\mathrm{H}}+\phi_{0}^{\mathrm{H}}\right)+C^{\mathrm{H}} & \text { Horizontal }\end{cases}
$$

where $A^{\mathrm{V}(\mathrm{H})}$ is the amplitude of the azimuthal modulation generated by $B_{n}, \phi^{\mathrm{V}(\mathrm{H})}$ is the azimuthal angle in the detector plane with $\phi=0$ being at beam-left (octant 1 ), and $\phi_{0}^{\mathrm{V}(\mathrm{H})}$ is an azimuthal phase offset, for vertical (horizontal) transverse polarization. The important quantity for $Q_{\text {weak }}$ is the constant $C^{\mathrm{V}(\mathrm{H})}$ which represents the "leakage" transverse asymmetry that survives the cancellation over the azimuthally symmetric detector. The leakage factors are characteristic of the breaking of the azimuthal symmetry in the detector array, therefore we can combine data from Run1 and Run2 to extract them. With appropriate sign corrections to estimate the effect for the Physics asymmetry combination, the vertical and horizontal transverse leakage is

$$
\begin{aligned}
& C^{\mathrm{V}}=12.4 \pm 55.1 \mathrm{ppb} \\
& C^{\mathrm{H}}=11.0 \pm 61.0 \mathrm{ppb}
\end{aligned}
$$

The leakage factors obtained from simulations based on survey results for the main detector misalignment are in agreement with the results from dedicated studies. The residual transverse polarization is extracted from fits to the corrected asymmetry versus octant with the functional form

$$
A(\phi)=-4.8 \mathrm{ppm} \times\left[P^{\mathrm{V}} \cos \phi-P^{\mathrm{H}} \sin \phi\right]+C,
$$

where $P^{\mathrm{V}(\mathrm{H})}$ is the residual component of vertical (transverse) beam polarization. The fit is repeated in every Wien period.

The current analysis for the estimation of this correction suggests that further cancellation 


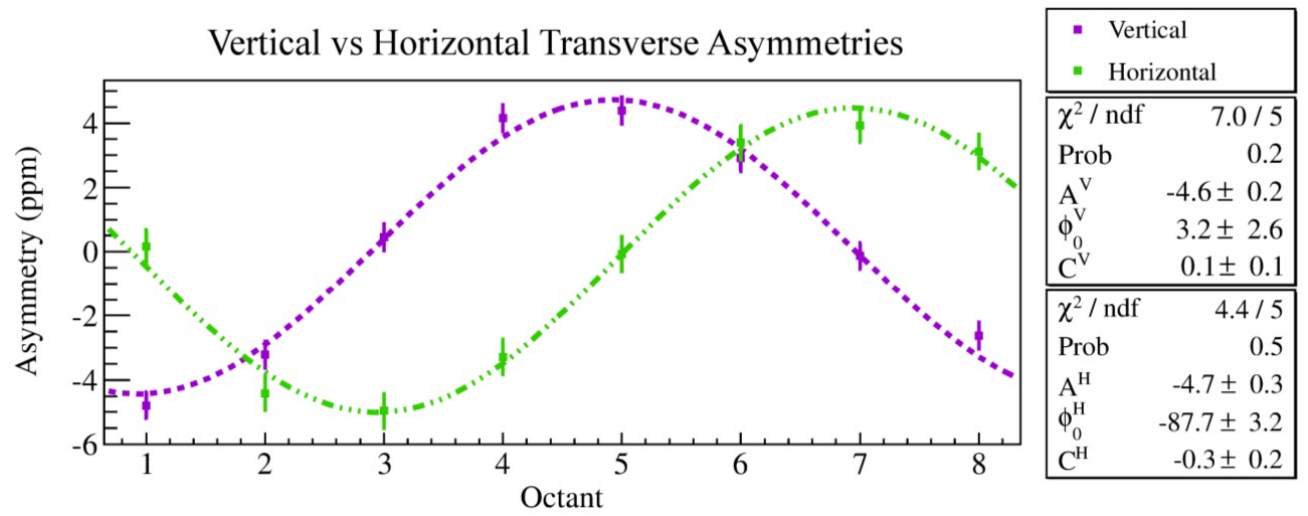

Figure 8.3: Comparison of the azimuthal dependence of the asymmetry measured with fully vertical (purple) and horizontal (green) transverse polarization, from a dedicated 2-hour long measurement in each case. The phase offset between the two orientations is $90^{\circ}$ as expected.

is achieved by combining the different Wien periods [167. However, the suppression over different Wien periods (at least in the dataset that we examine in this dissertation) is shown to be limited by the significant azimuthal dependence of corrected asymmetries on individual MD bars from Figure 7.4. From the discussion of Section 7.3 , the residual dipole amplitude of $\sim 50 \mathrm{ppb}$ suggests a significant $0.64^{\circ}$ transverse polarization in our dataset, which is probably mostly vertical given that the maximum deviations occur on the horizontal bars 1 and 5 (compare Figures 7.4 and 8.3 ). The measured leakage factors from Eq.8.8, while consistent with zero due to the azimuthal asymmetry, are only controlled within $\sim 1 \%$ of the size of the transverse asymmetry. We will then assign a $1 \%$ uncertainty on the size of the observed $50 \mathrm{ppb}$ amplitude in our dataset:

$$
A_{T}=0.0 \pm 0.5 \mathrm{ppb}
$$

- The linearity correction accounts for potential non-linearity in the PMT response. A zero correction is bounded within $0.5 \%$ by bench tests of the PMT and readout electronics, with a dependence on the gain applied to the PMT base [168. We conservatively assign a 2 ppb uncertainty:

$$
A_{L}=0 \pm 2 \mathrm{ppb}
$$

- A random blinding term is assigned from a uniform $\pm 60 \mathrm{ppb}$ distribution:

$$
A_{\text {blind }} \in[-60 \mathrm{ppb}, 60 \mathrm{ppb}]
$$

The purpose of the blinding term is to prevent analysis bias, and it will only be revealed and subtracted after all $Q_{\text {weak }}$ analyses are completed. Therefore the result quoted in this 
dissertation will be "blinded", i.e. it will include this currently unknown term.

There is one more correction to the measured asymmetry that has been recently discovered, described by the $A_{\mathrm{PMT}-\mathrm{DD}}$ term in Eq.8.4. We discuss this correction in the following subsection.

\section{Discrepancy between asymmetries of individual PMTs}

An interesting systematic effect has been discovered in the discrepancy of the asymmetry measured from the opposite individual PMTs on every MD bar, referred as positive and negative PMTs. Both PMTs collect Čerenkov light from the same quartz bar, but on opposite ends. The placement of these PMTs on every MD bar is shown in Figure 8.4. The difference between the raw asymmetry measured on positive and negative PMTs, referred as the positive-negative PMT "double-difference" (DD), is shown to be very large:

$$
A_{\mathrm{PMT}-\mathrm{DD}}=A_{\text {raw }}^{+}-A_{\text {raw }}^{-} \approx 300 \mathrm{ppb},
$$

which is as large as the PV asymmetry $A_{\mathrm{PV}}^{e p}$ we seek to extract. This quantity is shown to be remarkably stable throughout the experiment and consistent on all MD bar: ${ }^{2}$ (Figure 8.5).

Many models were proposed to account for the observed PMT DD. As an example, a SternGerlach effect on the electron beam was proposed, suggesting that the electron states of opposite helicity are separated spatially due to gradients on the field of the toroidal spectrometer. There is an interesting literature on the subject, beginning with Bohr and Pauli's assertion that such an effect cannot be observed 3 Recent reviews and possible applications on the creation of fully polarized electron beams can be found in 170, 171. This model is ruled out as a possible explanation for the observed $Q_{\text {weak }}$ PMT DD, as the spa-

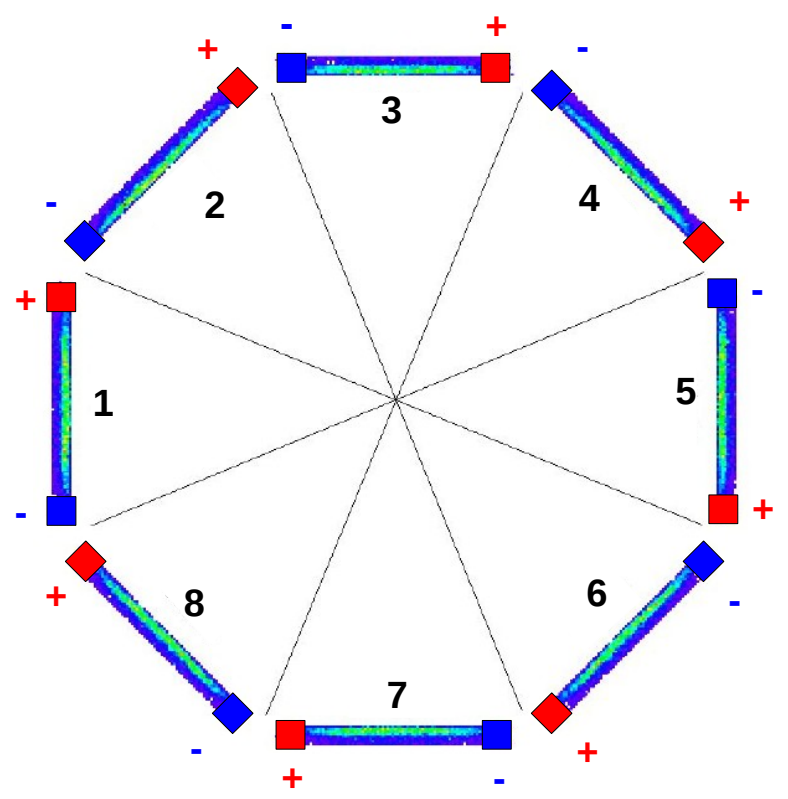

Figure 8.4: Placement of positive (red) and negative (blue) PMTs on every MD bar.

tial separation for our geometry and spectrometer is calculated to $\Delta x \lesssim 3 \cdot 10^{-15} \mathrm{~m}$, which is

\footnotetext{
${ }^{2}$ The PMT double difference acquires a small sinusoidal azimuthal dependence from imperfect cancellation of residual transverse asymmetry $A_{T}$ due to the small azimuthal separation of $\sim 5^{\circ}$ between the PMTs of each bar.

${ }^{3}$ Pauli's formulation is often quoted: "it is impossible to observe the spin of the electron, separated fully from its orbital momentum, by means of experiments based on the concept of classical particle trajectories" [169].
} 

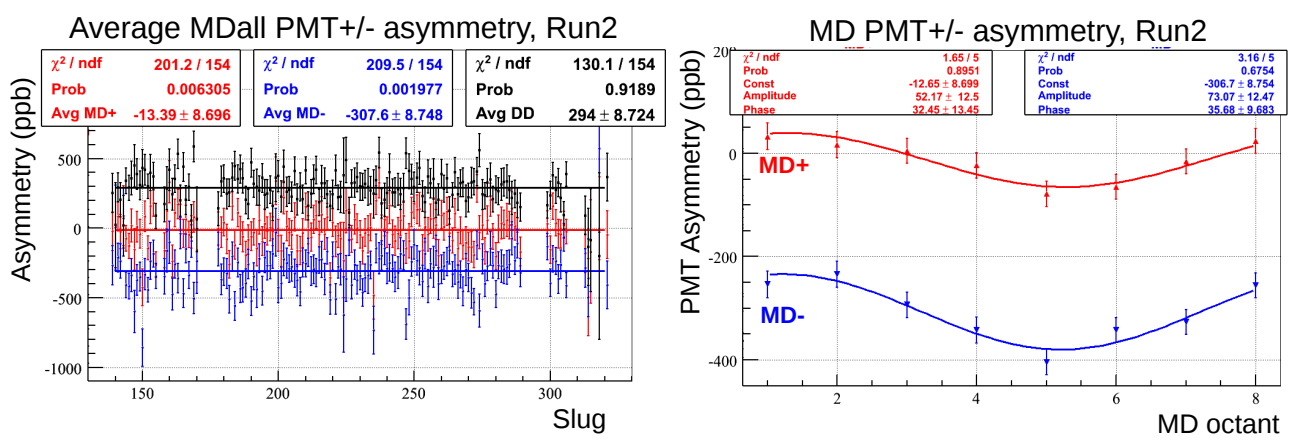

Figure 8.5: Left: The PV asymmetry $A_{\text {raw }}$ measured from the average of the positive (red) and negative (blue) PMTs on the main detector bars, plotted versus slug number in Run2. The difference between the two, referred as PMT double-difference $A_{\text {PMT-DD }}$ is also plotted in black. All three have been separately fitted to a constant over Run2. Right: The average asymmetries of positive (negative) PMTs over Run2, $A_{\text {raw }}^{+(-)}$, have been plotted in red (blue) versus MD octant and fitted to a sinusoid.

approximately 9 orders of magnitude smaller than what would be needed to account for the observed $A_{\mathrm{PMT}-\mathrm{DD}}$.

The observed PMT DD is shown to be caused by the $2 \mathrm{~cm}$ thick lead pre-radiators placed in front of the MD bars. In a dedicated dataset during commissioning where only half of the MD bars had pre-radiators installed, the double-difference was observed only on the preradiated bars. $A_{\text {PMT-DD }}$ is now believed to arise from left-right asymmetry of transversely polarized electrons scattering in the pre-radiators. Scattered electrons acquire a radial transverse polarization component through g-2 precession of their spin in the field of the QTor spectrometer, making this effect consistent on every MD octant. The radial transverse polarization scattering in the lead pre-radiator shifts the centroid of the light distribution deposited in the main detector bars in a manner that correlates to beam helicity. Due to light attenuation in the quartz Čerenkov bar the light collection on each PMT acquires a helicity dependence, giving rise to a "false" asymmetry of opposite sign in the two PMTs. The angle dependence of the scattered asymmetry and of light collection is also potentially an important contribution. These effects are shown to have the right sign to explain the observed $A_{\text {PMT-DD }}$, and could give roughly the right magnitude.

To first order this false asymmetry should cancel in the average of the MD PMTs. Simulations are currently performed to reproduce the size of the observed asymmetry, by modeling the convolution of the asymmetric position and angle distributions in the shower with the asymmetry of light collection and appropriate weighting. This should allow us to investigate the cancellation of the false asymmetry and tune parameters of light collection that can break the symmetry between PMTs. These studies are still in progress and are the primary outstanding issue in the $Q_{\text {weak }}$ analysis. It is estimated that any leakage false asymmetry can be constrained within $1 \%$ of the size of the false asymmetry, which we will use here as the systematic uncertainty from 
this correction:

$$
A_{\mathrm{PMT}-\mathrm{DD}}=0 \pm 3 \mathrm{ppb}
$$

This is the largest systematic uncertainty uncertainty contribution to $A_{\mathrm{msr}}$. It is hoped that when this analysis matures we will be able to constrain this effect to better than $3 \mathrm{ppb}$.

Collecting terms, our result for the measured asymmetry $A_{\mathrm{msr}}$ is:

$$
\begin{aligned}
A_{\text {msr }} & =A_{\text {raw }}-A_{\text {beam }}-A_{\mathrm{BB}}-A_{T}-A_{L}-A_{\mathrm{PMT}-\mathrm{DD}} \\
& =-161.26 \pm 8.27 \text { (stat) } \pm 4.04 \text { (syst) } \mathrm{ppb} \quad \text { (blinded) }
\end{aligned}
$$

We remind again that the unknown blinding term $A_{\text {blind }}$ is included in our determination of $A_{\mathrm{msr}}$, and therefore our result will be blinded.

\subsubsection{Background corrections}

There are four identified background sources that generate some signal in the main detector and give rise to false asymmetries. One of them, the $b=2$ beamline background asymmetry component, was treated as a beam correction and was included in $A_{\mathrm{msr}}$. The total false asymmetry in the MD from the remaining background sources is included in Eq.8.1p as $\sum_{b=1,3,4} f_{b} A_{b}$. The signal fraction $f_{b}$ and asymmetry $A_{b}$ from each background source must be estimated.

- The $b=1$ background component refers to electrons scattering from the target aluminum windows. The asymmetry of this component was measured in dedicated runs where the $\mathrm{LH}_{2}$ target was removed and electrons were scattering from a thick 4\% (0.04 radiation lengths) solid target, made from the same alloy as the windows and located near the downstream target window. Using simulations to estimate the effect of the different zlocation, energy loss in the $\mathrm{LH}_{2}$ target, and radiative effects associated with the thicker aluminum alloy, the asymmetry from the upstream and downstream windows is calculated from the thick target measurement:

$$
A_{\mathrm{US}(\mathrm{DS})}=A_{4 \%} \frac{A_{\mathrm{US}(\mathrm{DS})}^{\mathrm{sim}}}{A_{4 \%}^{\mathrm{sim}}}
$$

where $A_{\mathrm{US}(\mathrm{DS})}$ is the asymmetry of backgrounds scattering from the upstream (downstream) aluminum target window, and $A_{4 \%}$ is the dedicated measurement from the thick $4 \%$ aluminum target. Analysis by this author reduced by an order of magnitude the systematic uncertainty associated with the effect of the beamline background component during the dedicated $A_{4 \%}$ measurement. The asymmetry $A_{1}$, including the weighted contribution 
of each window, is

$$
A_{1}=\frac{R_{\mathrm{US}} A_{\mathrm{US}}+R_{\mathrm{DS}} A_{\mathrm{DS}}}{R_{\mathrm{US}}+R_{\mathrm{DS}}},
$$

where $R_{\mathrm{US}(\mathrm{DS})}$ is the MD rate from the upstream (downstream) aluminum target window. From these measurements and appropriately scaling for polarization we get:

$$
A_{1}=1.506 \pm 0.072 \mathrm{ppm}
$$

The total signal fraction in the MD from the target windows is calculated based on dedicated measurements with an evacuated target cell as $f_{1}=Y_{\text {empty }} / Y_{\mathrm{LH} 2}$, where $Y_{\text {empty (LH2) }}$ is the MD yield when the target cell is empty (full with the $\mathrm{LH}_{2}$ target). The $Y_{\text {empty }}$ measurement is verified through measurements on cold hydrogen gas of varying density. The $Y_{\text {empty }}$ yield must be corrected for radiative losses and ionization associated with the presence of the liquid hydrogen target, calculated through simulations and becnhmarked through the different solid targets. A light-weighting correction must also be applied to translate the measured rates to light yields in the MD bars. The current estimation for the signal fraction from the aluminum windows background in Run2, including radiative corrections [172]:

$$
f_{1}=0.02872 \pm 0.00057
$$

- The second background source is the beamline background component that was discussed in Chapter 6. The correction for this false asymmetry was included in the $A_{\mathrm{msr}}$ term in the previous section. The signal fraction from this component however is still separate from the elastic signal in the MD, and should be included in the denominator of Eq.8.1). The Run2 signal fraction has been extracted from the dedicated blocked-octant studies and given in Eq. 6.3):

$$
f_{2}=0.00193 \pm 0.00064
$$

- A potential source of soft neutral backgrounds may arise from elastic electrons scattering at the edges of the collimator openings, the concrete shielding wall, the spectrometer and its support structure. This is referred as the "QTor transport channel background", or just $b_{3}$. Some potential sources for this background are tracks 1 and 3 in Figure 3.7, arising from electrons scraping at the collimator edges, although shielding should prevent these tracks from reaching the MD. This source of background would not be included in the background signal measured during the dedicated blocked-octant studies of Section 6.3 where the collimator openings were blocked. Instead it is determined via the scintillation detectors during tracking measurements at low currents. Since they are insensitive to 
neutral particles, neutral backgrounds were identified as events that were seen by the main detector but not the trigger scintillator. The total neutral signal fraction thus measured includes the $f_{2}$ signal fraction that was measured in the blocked octant studies. Subtracting $f_{2}$ we get an estimation for the signal fraction of the QTor transport channel background component that is consistent with zero:

$$
f_{3}=0.00127 \pm 0.00138
$$

The asymmetry associated with this background component is estimated from simulations. The current best estimate is:

$$
A_{3}=-0.283 \pm 0.057 \mathrm{ppm}
$$

- The final background source comes from inelastic scattering events associated with $N \rightarrow$ $\Delta(1232)$ production. A simulation for the contribution of this component in the main detector is shown versus the spectrometer current as the solid blue line in Figure 8.6. amplified by an order of magnitude relative to the elastic signal plotted as the dashed red line. The experiment operated on the elastic "peak" at $8921 \mathrm{~A}$, where the elastic rate is maximal relative to backgrounds. At that point the signal fraction from this background source is estimated from GEANT3 simulations as:

$$
f_{4}=0.0002 \pm 0.0002
$$

A separate simulation analysis from GEANT4.9 is in excellent agreement with this result. The asymmetry associated from this component was measured directly with the spectrometer at the setting where the inelastic signal is maximal, at $6700 \mathrm{~A}$. The contribution from the elastic radiative tail is still dominant at that setting ( $\sim 75 \%$ of the signal) and must be calculated from simulations. The corrected result for the asymmetry of this background component is:

$$
A_{4}=-3.02 \pm 0.97 \mathrm{ppm}
$$

Then the total background correction is

$$
A_{\mathrm{Bkgd}}=\sum_{b=1,3,4} f_{b} A_{b}=42.287 \pm 2.361 \mathrm{ppb}
$$




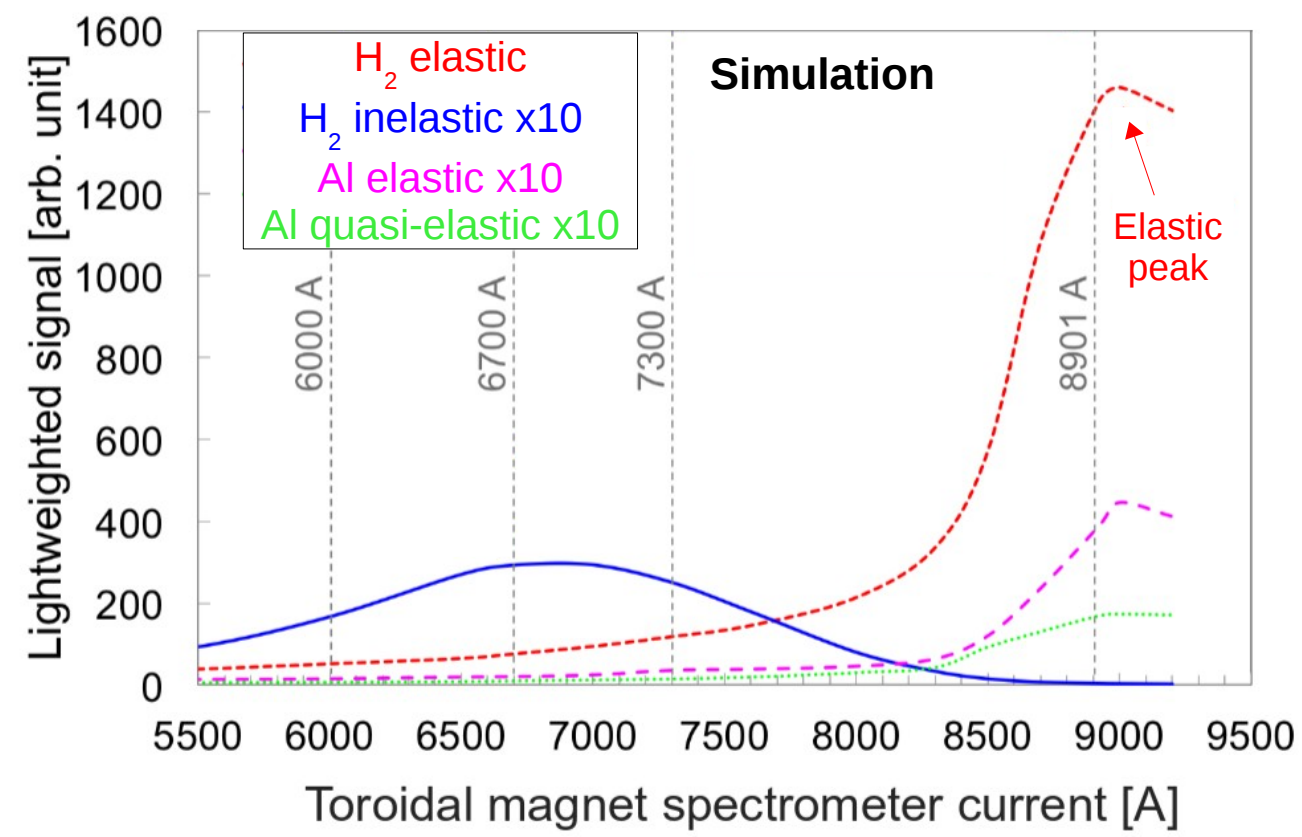

Figure 8.6: Simulated rates on the MD versus current on the toroidal spectrometer. Rates from four different sources are plotted in different colours and blown-up by an order of magnitude relative to the elastic $\overrightarrow{e p}$ signal. Nominal value for the magnet current during production was $8921 \mathrm{~A}$, at the peak of the elastic contribution, plotted in red. The maximum inelastic rate occurs at a spectrometer current of $6700 \mathrm{~A}$, where dedicated studies for the measurement of $A_{4}$ were performed.

The denominator in Eq. 8.1) accounts for the dilution of the elastic $\overrightarrow{e p}$ signal due to all background sources:

$$
f_{e p}=1-\sum_{b=1}^{4} f_{b}=0.9679 \pm 0.0016
$$

For simplicity we treat the $A_{\mathrm{Bkgd}}$ and $f_{e p}$ terms separately, and neglect the small effect from a correlated error component due to the common signal fractions $f_{b}$.

\subsubsection{The parity-violating asymmetry}

Collecting all terms, we extract the parity-violating asymmetry in the Run2 Modulation dataset:

$$
\begin{aligned}
A_{\mathrm{PV}}^{e p} & =\frac{R_{\mathrm{total}}}{f_{e p}}\left(\frac{A_{\mathrm{msr}}}{P}-A_{\mathrm{Bkgd}}\right) \\
& =-226.95 \pm 9.44 \text { (stat) } \pm 6.64(\text { syst }) \mathrm{ppb} \quad \text { (blinded) }
\end{aligned}
$$

We remind again that this result is blinded, as an unknown blinding term is included in $A_{\text {msr }}$. It is also preliminary as most of the quoted values are not final. The value and uncertainty we used for each term in Eq. (8.26) is given in Table 8.1 . 
Table 8.1: Assigned value and uncertainty for each term entering the extraction of $A_{\mathrm{PV}}$.

\begin{tabular}{l|c} 
Term & Value \\
\hline$R_{\text {total }}$ & $0.982 \pm 0.017$ \\
$\langle P\rangle$ & $0.889 \pm 0.006$ \\
$A_{\mathrm{msr}}$ & $42.287 \pm 2.361$ \\
$A_{\mathrm{Bkgd}}=\sum_{b=1,3,4} f_{b} A_{b}$ & $-161.264 \pm 8.268$ (stat) \pm 4.037 (syst) ppb \\
$f_{e p}=1-\sum_{b=1}^{4} f_{b}$ & $0.9679 \pm 0.0016$
\end{tabular}

\subsection{Extraction of the Weak Charge of the Proton}

As described in Section 2.4 the weak charge of the proton $Q_{w}^{p}$ can be extracted from the parityviolating asymmetry

$$
A_{\mathrm{PV}}^{e p}=\frac{g_{A}^{e}\left(\epsilon G_{E}^{\gamma p} G_{E}^{Z p}+\tau G_{M}^{\gamma p} G_{M}^{Z p}\right)+g_{V}^{e} \epsilon^{\prime} G_{M}^{\gamma p} G_{A}^{Z p}}{\epsilon\left(G_{E}^{\gamma p}\right)^{2}+\tau\left(G_{M}^{\gamma p}\right)^{2}}
$$

Uncertainties of hadronic structure encapsulated in the proton form factors of Eq. 8.27) are constrained through a global fit over the world dataset of parity-violating electron scattering results on nuclear targets. Rather than the Kelly parametrization of Eq. 2.19), we update the EM form factors through the Arrington-Sick parametrization [173, which uses a continued fraction $(\mathrm{CF})$ expansion:

$$
G_{\mathrm{CF}}\left(Q^{2}\right)=\frac{1}{1+\frac{b_{1} Q^{2}}{1+\frac{b_{2} Q^{2}}{1+\ldots}}}
$$

This parametrization was designed to be used as input for low- $Q^{2}$ analyses and including the effects of two-photon exchange corrections and correlations between different form factors. All other fit choices remain unchanged as in the early result. The $2013 Q_{\text {weak }}$ result is included as a separate point in the fit. As described in Section 2.4 the energy-dependent correction for the $\gamma-Z$ box diagram has been applied directly to each point, with the associated uncertainty folded into the systematic error of each measurement.

The data from proton targets in Figure 8.7 have been rotated to forward angles based on the results of the fit, as in Eq. 2.24). Our result from Eq. (8.26) is translated to $\bar{A}_{\mathrm{PV}}$ at $Q^{2}=$ $0.02455(\mathrm{GeV} / \mathrm{c})^{2}$, which is the average $\left\langle Q^{2}\right\rangle$ of the Run2 $Q_{\text {weak }}$ dataset. The blinded result for the weak charge of the proton is the intercept of the plot at $Q^{2}=0$ :

$$
Q_{w}^{p}(\mathrm{PVES})=0.0716 \pm 0.0053 \quad(\text { blinded })
$$

in excellent agreement with the Standard Model prediction, $Q_{w}^{p}(\mathrm{SM})=0.0710 \pm 0.0007$. However 


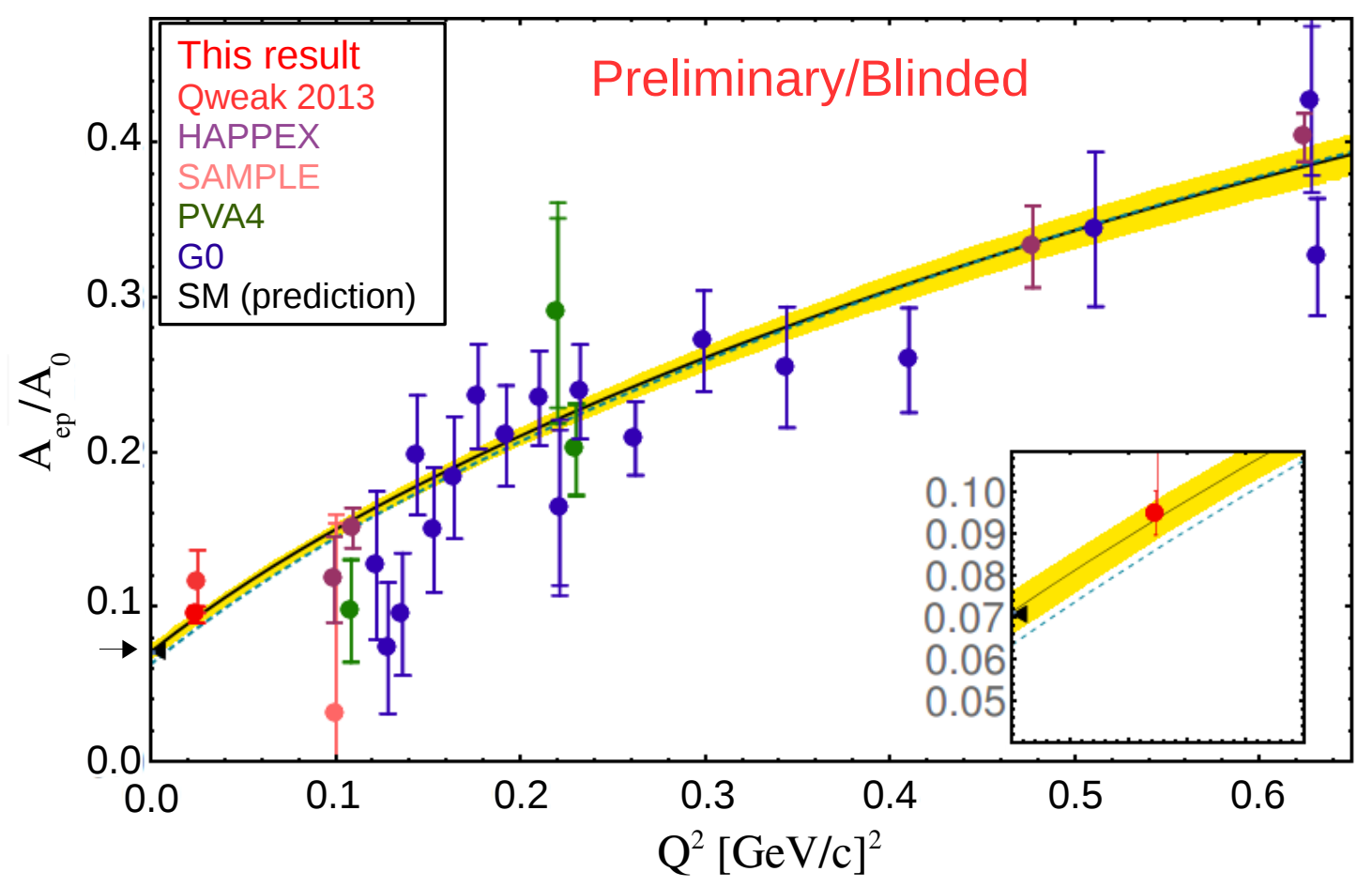

Figure 8.7: Fit over PVES data on nuclear targets, with our Run2 Modulation preliminary/blinded result 8.26 plotted at $Q^{2}=0.02455(\mathrm{GeV} / \mathrm{c})^{2}$. The fit is shown as the black line with the uncertainty given by the yellow band. The fit intercept is our result for the weak charge of the proton, in good agreement with the Standard Model prediction (black arrow). The dotted line is the 2013 result from Figure 2.5 before our preliminary/blinded Run2 measurement. The insert on the right is zoomed in at the low- $Q^{2}$ region.

the larger values of the blinding term still allow up to $4 \sigma$ deviations. Even with the limited dataset that we examined, the precision of our preliminary result is considerably higher than the 2013 extraction in Eq.2.25). Note that while our result in Eq.8.26) for the parity-violating asymmetry is smaller in magnitude than the 2013 Run0 measurement $[55]^{4}$, the result for the weak charge in Eq. (8.29) is larger. That is due to the large increase in the relative significance of the $Q_{\text {weak }}$ result compared to the rest of the dataset, the latter favouring a small value for $Q_{w}^{p}$.

Combining the PVES result with the APV constraints, the $C_{1 u}, C_{1 d}$ coupling parameters can be extracted separately from the combined fit:

$$
\begin{array}{ll}
C_{1 u}=-0.1872 \pm 0.0026 & \text { (blinded) } \\
C_{1 d}=0.3387 \pm 0.0028 & \text { (blinded) }
\end{array}
$$

with a correlation coefficient -0.941 and improved precision relative to the 2013 result. From these the weak charge of the neutron can also be extracted:

$$
\left.Q_{w}^{n}(\mathrm{PVES}+\mathrm{APV})=-0.9805 \pm 0.0066 \quad \text { (blinded }\right)
$$

\footnotetext{
${ }^{4}$ At a slightly smaller $Q^{2}$, but that is not a big effect.
} 


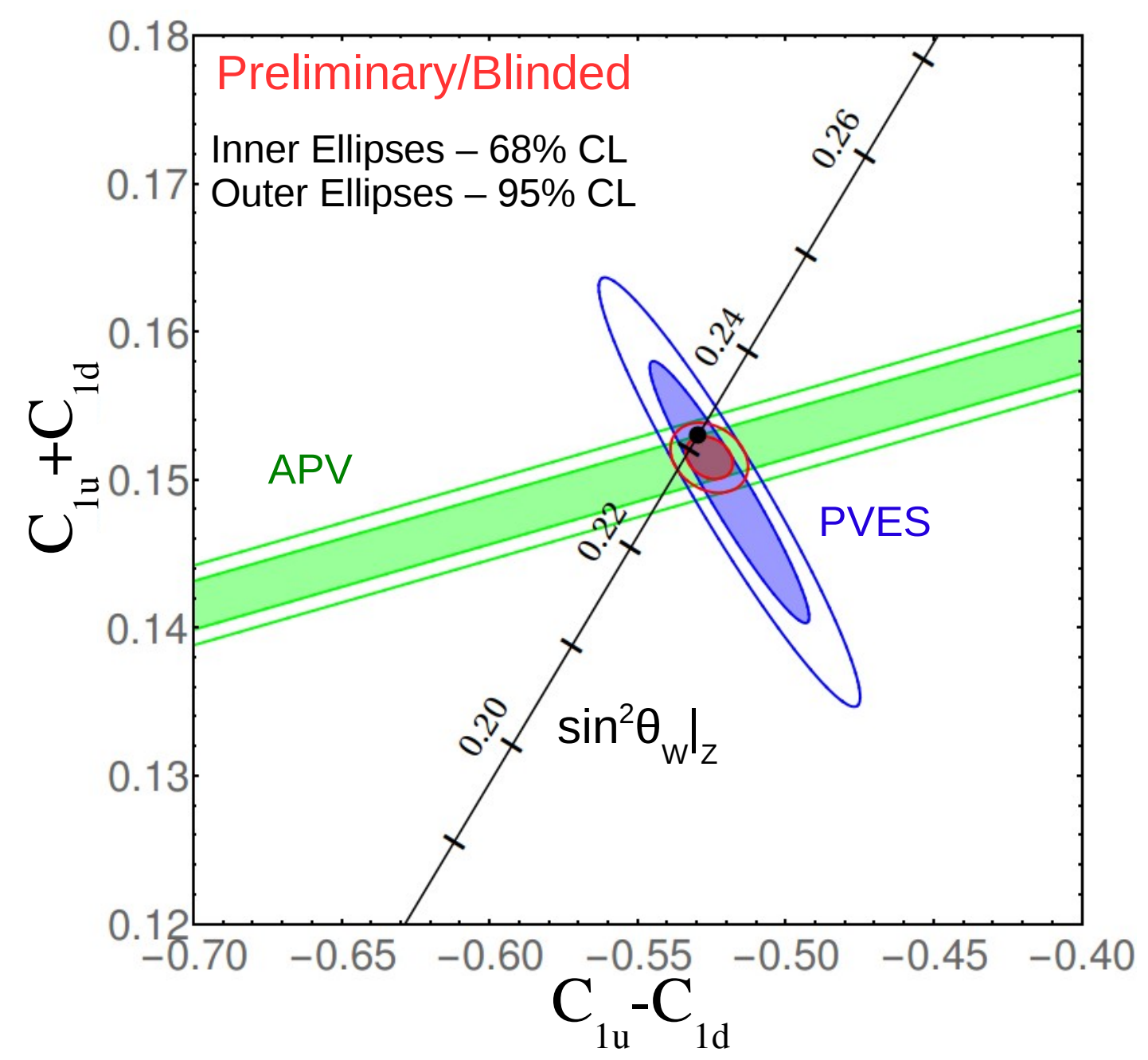

Figure 8.8: Combined constraints on the isovector and isoscalar combinations of the $C_{1 u}, C_{1 d}$ couplings, from the PVES result shown in Figure 8.7 (blue ellipses) and from the APV measurement on cesium (green band). The global constraints are shown as red ellipses. The SM prediction is the black dot, shown as a function of the weak mixing value at the $Z$ resonance.

Using the prescription of Eq.2.31) for quoting limits for new interactions, the precision of this measurement would translate to model-independent limits for the mass-coupling ratio of new interactions (95\% confidence level)

$$
\frac{\Lambda_{\text {new }}}{g_{\text {new }}} \gtrsim 7.2 \mathrm{TeV}
$$

This corresponds to compositeness scales $\left(g_{\text {new }}^{2}=4 \pi\right)$ of up to $\Lambda_{\text {new }}>25.6 \mathrm{TeV}$.

\subsection{Preliminary studies on model dependence}

The stability of the result for various choices in the global PVES fit is examined in Figure 8.9 . for a "trial" $Q_{\text {weak }}$ result $A_{\mathrm{PV}}^{\text {trial }}=-230 \pm 10 \mathrm{ppb}$. Under the "canonical" fit choices which are 
used in this dissertation, the trial value gives $Q_{w}^{p}[$ trial $]=0.0702 \pm 0.0048$, shown as the black point with the dashed and solid horizontal lines representing its mean value and $1 \sigma$ bound. The model dependence of the result is examined by repeating the extraction under different choices in the fit parameters and dataset:

- The red data points in Figure 8.9 correspond to different choices for the value of the dipole "mass" parameter $\lambda$ between 0.84 and $10 \mathrm{GeV}$ in the dipole parametrization of Eq.2.21, around the canonical choice $\lambda=1 \mathrm{GeV}$. Variation of the value assigned to this parameter is shown to have a small effect.

- The blue data examine different constraints on the isoscalar and isovector parts $(T=0$ and 1 respectively) of the proton axial form factor

$$
G_{A}^{(T=0,1)}(0)=-g_{A}\left(1+R_{A}^{(T=0,1)}\right)
$$

where $g_{A}$ is the nucleons axial charge extracted from neutron $\beta$-decay, and $R_{A}$ denotes process-dependent electroweak radiative corrections to the $\mathcal{V}(e) \times \mathcal{A}(p)$ scattering amplitude. The canonical choice in the global PVES fit uses the Zhu calculation [58] to constrain the isoscalar radiative correction $R_{A}^{(T=0)}$, although there is considerable uncertainty associated with the many-quark anapole contribution to that factor. Constraints on the isovector part have a negligible effect, however floating the isoscalar part in the fit is shown to shift the result by almost $1 \sigma$.

- Rather than using the dipole form of Eq.2.22, we examine the effect of employing $Q^{2}$ polynomials in the parametrization of the strange form factors (magenta points). The second-order polynomials have the form:

$$
\begin{aligned}
& G_{\mathrm{E}}^{s}=\rho^{s} Q^{2}+\rho_{2}^{s} Q^{4} \\
& G_{\mathrm{M}}^{s}=\mu^{s}+\mu_{2}^{s} Q^{2}
\end{aligned}
$$

- The orange points examine different combinations of experimental results to be included in the global PVES fit. The canonical choice is to include all results up to $Q^{2}=0.63 \mathrm{GeV}^{2}$. The largest shift comes from excluding PVES results from scattering on the deuteron (from G0 and SAMPLE) and on helium-4 (from HAPPEX), which are important in constraining the $G_{E}^{s}$ and $G_{A}$ form factors. It should be noted that removing the "heavy target" data and just using results on the proton, allows us to fit the neutral weak form factors $G_{E}^{Z p}$ and $G_{M}^{Z p}$ directly without using the flavour separation and charge symmetry assumption of Eq. (2.8). 
- The green points examine the effect of using the very precise strange form factors extracted from a direct lattice QCD calculation by Green et al. [4], referenced in Section 2.3 . This choice reduces the uncertainty of the extraction, and also the dependence of the result on including data from the deuteron and helium-4. Input from the lattice calculation of Shanahan et al. 174 is also examined, which finds unexpectedly small contribution from charge symmetry violation (CSV) on the electromagnetic form factors of the nucleon, smaller by an order of magnitude compared to the precision of current PVES studies.

- The striped points of Figure 8.9 refer to a stand-alone calculation of $Q_{w}^{p}$ from the trial $Q_{\text {weak }}$ asymmetry, not including any other PVES data. This result is not extracted from a global fit, rather the hadronic contribution in the $Q_{\text {weak }}$ result is simply calculated and subtracted. Based on the flavour decomposition of Eq. 2.7) and under the charge symmetry assumption, the neutral weak proton form factors can be expressed as

$$
G_{\mathrm{E}, \mathrm{M}}^{p, Z}=\left(1-4 \sin ^{2} \theta_{W}\right) G_{\mathrm{E}, \mathrm{M}}^{p, \gamma}-G_{\mathrm{E}, \mathrm{M}}^{n, \gamma}-G_{\mathrm{E}, \mathrm{M}}^{s}+G_{\mathrm{CSV}}
$$

where the last term accounts for potential charge symmetry violation effects. The standalone calculation uses the Arrington-Sick parametrization of the EM form factors (Eq. 8.28) and the Particle Data Group values for the weak mixing angle and radiative corrections to calculate the hadronic contribution. The effect of either using experimental constraints on $\rho_{s}, \mu_{s}$ and neglecting CSV contributions, or constraining these through the lattice calculations of [47] and [174] is seen to be negligible, and the calculation is in good agreement with the canonical result from the global PVES fit.

- Finally the results without the trial $Q_{\text {weak }}$ datum are shown on the greyed area on the right of Figure 8.9 for comparison, with or without the $2013 Q_{\text {weak }}$ result. The uncertainty is significantly higher without the trial $Q_{\text {weak }}$ result. We see again that the global PVES dataset pre- $Q_{\text {weak }}$ favours a small value for $Q_{w}^{p}$.

There is no uncertainty assigned to our result from Eq. 8.29 to account for the model dependence in the extraction of $Q_{w}^{p}$. The Collaboration is in the process of estimating the uncertainty contribution due to model dependence for the full $Q_{\text {weak }}$ result.

\subsection{Extraction of the Weak Mixing Angle}

Finally from our result we extract the value for the weak mixing angle $\sin ^{2} \hat{\theta}_{W}$. From Eq. 2.27 we get 73 :

$$
\sin ^{2} \hat{\theta}_{W}(0)=\frac{1}{4}\left[1+\Delta_{e}{ }^{\prime}-\frac{Q_{w}^{p}-\square_{W W}-\square_{Z Z}-\square_{\gamma Z}(0)}{\rho_{\mathrm{NC}}+\Delta_{e}}\right],
$$




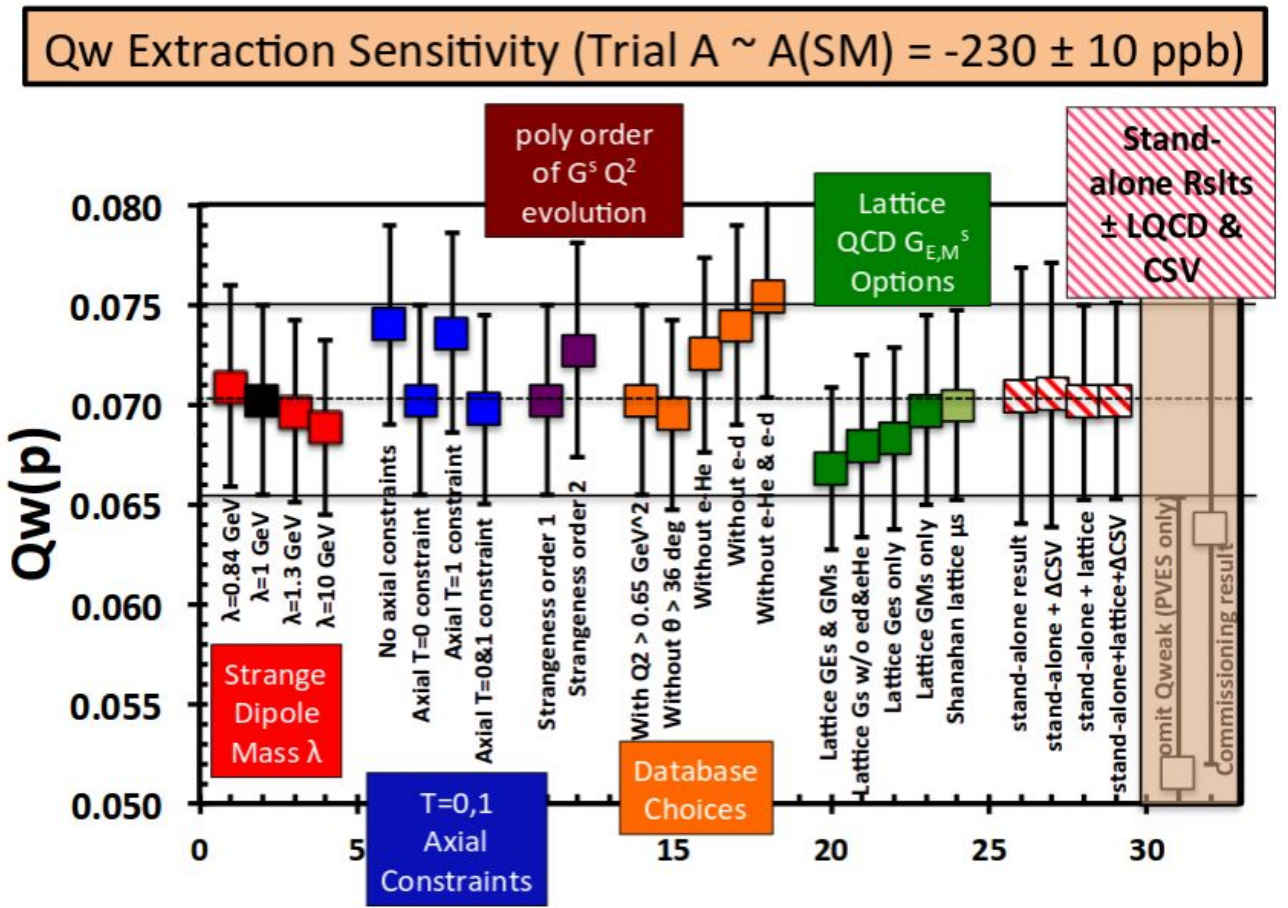

Figure 8.9: The effect of different fit choices on the $Q_{w}^{p}$ result, for a trial value of the final $Q_{\text {weak }}$ parity-violating asymmetry $A_{\mathrm{PV}}^{\text {trial }}=-230 \pm 10 \mathrm{ppb}$, near its expected value from the SM. The black point and the dotted line correspond to the canonical fit choices used in this dissertation, which give $Q_{w}^{p}[$ trial $]=0.0702 \pm 0.0048$, and the solid horizontal lines are the $1 \sigma$ bound. The $Q_{w}^{p}$ [trial] result is quoted under many different fit choices - see text for details. For reference the $Q_{w}^{p}$ without any $Q_{\text {weak }}$ data and from the 2013 result are shown on the far right, with much higher uncertainty. Reproduced from 175 .

where the various terms were introduced in Section 2.5. Note that the $\square_{\gamma Z}(0)$ correction only refers to the axial part of that term, since the vector term which was plotted in Figure 2.6 vanishes at threshold 5. Using expressions from [73] and inputs from the 2014 Particle Data Group Review [26], we calculate

$$
\begin{aligned}
& \Delta_{e}=-\frac{\alpha}{2 \pi}=-0.0011614097 \\
& \Delta_{e}{ }^{\prime}=-\frac{\alpha}{3 \pi}\left(1-4 \hat{s}^{2}\right)\left[\ln \left(\frac{M_{Z}^{2}}{m_{e}^{2}}\right)+\frac{1}{6}\right]=-0.0014208498 \\
& \square_{W W}=\frac{7 \hat{\alpha}}{4 \pi \hat{s}^{2}}=0.0183167299 \\
& \square_{Z Z}=\frac{\hat{\alpha}}{4 \pi \hat{s}^{2} \hat{c}^{2}}\left(\frac{9}{4}-5 \hat{s}^{2}\right)\left(1-4 \hat{s}^{2}+8 \hat{s}^{4}\right)=0.0019255186
\end{aligned}
$$

Also from [176] and [62] we get

$$
\rho_{\mathrm{NC}}=1+\Delta \rho=1.00833 \quad \square_{\gamma Z}(0)=0.0044(4)
$$

Using these inputs and for our blinded value of $Q_{w}^{p}$, which is calculated at $Q^{2}=0$ from our

\footnotetext{
${ }^{5}$ The small energy dependence of the axial part $\square_{\gamma Z}^{\mathrm{A}}[62]$ is currently not accounted for in the extraction of $Q_{w}^{p}$. This will be corrected in the extraction of the final $Q_{\text {weak }}$ result.
} 


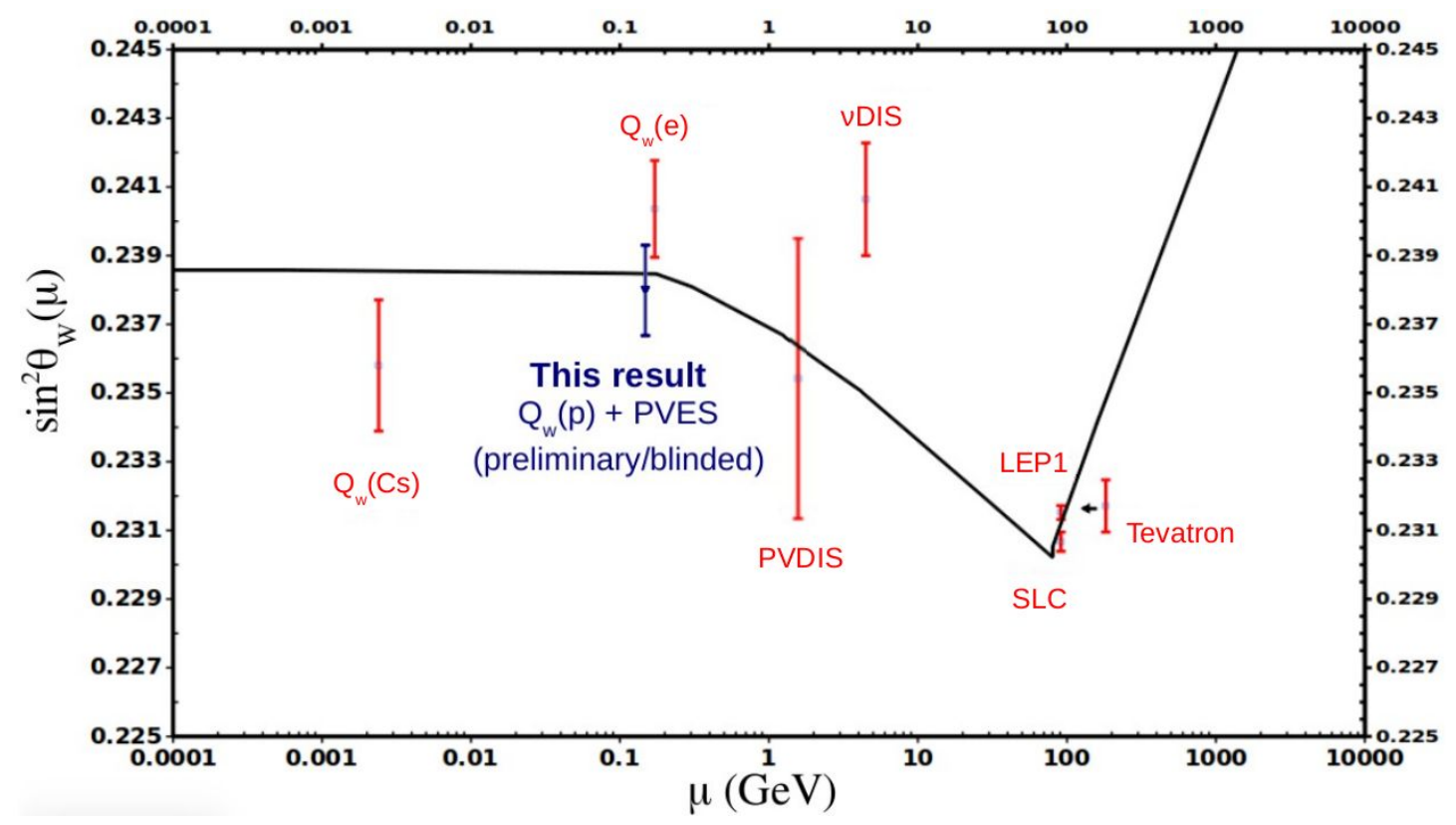

Figure 8.10: Our result for the weak mixing angle from Eq. 8.38 is the blue datum at $\mu=0.157 \mathrm{GeV}$. This result is extracted from the global PVES fit, including our preliminary analysis on the blinded Run2 Modulation dataset of $Q_{\text {weak }}$. Red data points are existing experimental determinations, while the black line is the theoretical prediction for the running of the weak mixing angle in the $\overline{\mathrm{MS}}$ scheme.

blinded Run2 value in the global PVES fit, we get

$$
\sin ^{2} \hat{\theta}_{W}(0)=0.23799 \pm 0.00132 \quad \text { (blinded) }
$$

Our result should be expressed at $Q^{2}=0.02455 \mathrm{GeV}^{2}$ where the $Q_{\text {weak }}$ measurement was performed. For that we also account for the very small running of the weak mixing angle. While this running is barely visible by eye on the theoretical curve, the $\sin ^{2} \hat{\theta}_{W}(0)$ value is only stable up to $Q=m_{e}=0.511 \mathrm{MeV}$ and is modified for higher $Q$. The appropriate modification for $Q_{\text {weak }}$ is calculated as $\sin ^{2} \hat{\theta}_{W}\left(Q^{2}=0\right)_{\mathrm{SM}}-\sin ^{2} \hat{\theta}_{W}\left(Q^{2}=0.02455 \mathrm{GeV}^{2}\right)_{\mathrm{SM}}=0.00018$. Then the weak mixing angle extracted from our blinded result in the global PVES fit and expressed at $Q_{\text {weak }}$ kinematics is

$$
\sin ^{2} \hat{\theta}_{W}(Q=0.157)=0.2378 \pm 0.0013 \quad \text { (blinded) }
$$

Our preliminary and blinded result is a $0.55 \%$ determination of the weak mixing angle and found to be in excellent agreement with the SM prediction. This preliminary analysis has similar absolute precision as E158 [177] for the most precise determination (in absolute terms) 
of $\sin ^{2} \hat{\theta}_{W}$ off the $Z$ pole.

\subsection{Concluding Discussion}

We have presented a measurement of the parity-violating asymmetry in elastic electron-proton scattering, extracted from the Run2 Modulation dataset of $Q_{\text {weak }}$, constituting approximately $56 \%$ of the full dataset. Our blinded result for the asymmetry is

$$
A_{\mathrm{PV}}=-226.95 \pm 9.44 \text { (stat) } \pm 6.64 \text { (syst) ppb (preliminary/blinded) }
$$

at $Q^{2}=0.02455(\mathrm{GeV} / \mathrm{c})^{2}$, where an unknown blinding term $A_{\text {blind }} \in[-60 \mathrm{ppb}, 60 \mathrm{ppb}]$ is included in the measured asymmetry. The weak charge of the proton extracted from a global fit of PVES data is

$$
Q_{w}^{p}(\mathrm{PVES})=0.0716 \pm 0.0053 \quad(\text { preliminary } / \text { blinded })
$$

which, in absence of the blinding term $A_{\text {blind }}$, would be in excellent agreement with the Standard Model prediction and place significant constraints on new physics models. Large deviations of $4 \sigma$ on $Q_{w}^{p}$ are still allowed for the largest values of $A_{\text {blind }}$. The real constraints on new physics in the electroweak sector will be provided by the full $Q_{\text {weak }}$ measurement, which is expected within 2016. Analysis is significantly matured since the early publication, but challenging systematic corrections remain to be controlled.

With the Large Hadron Collider in its second phase of operation, enhanced searches at the Cosmic Frontier and powerful precision programs at the Intensity Frontier performed or preparing around the world, including measurements of parity-violating electron scattering at Mainz and Jefferson Lab, the Standard Model is put to higher levels of scrutiny than ever before. A watershed moment for fundamental Physics may be very close, or alternatively a desert where no new interactions appear may extend to the GUT scale. In this context, many years since its inception and benefiting from the world PVES program, the full $Q_{\text {weak }}$ result is anticipated to measure up to the Standard Model soon, while paving the way with important experience and lessons for future ultra-precise measurements. 


\section{Appendix A}

\section{Definition of Pitts}

A "pitt" is a combination of nominally four consecutive slug periods, each characterized by a definite state of the insertable half-wave plate (IHWP) in the polarized source. Constructing the asymmetry over slugs of opposite IHWP state allows the creation of complimentary combinations, defined in Section 5.4 .2

Appropriate pitt definitions for the Modulation dataset take into account that certain slugs do not have good Modulation correction slopes and are excluded. In that case more than four consecutive slugs are included in a given pitt, considering that all pitts should have similar statistical power and roughly equal statistics from each IHWP state. Another requirement is that all slugs in a given pitt are from the same Wien period, ensuring stability of the underlying examined systematic effects.

The slug range included in each pitt, as defined by this author for the Modulation datasets of Run1 and Run2 for use in this dissertation, are given in Table A.1. One small exemption is the sole pitt in Wien10, which has very small statistical power and different systematic characteristics and is excluded from our analysis. 
Table A.1: The range of slugs included in each pitt, separated by Run and Wien periods. The identifying number for each pitt is simply the first slug number in its range.

\begin{tabular}{cc} 
Wun1 \\
Wien & Slug range in pitt \\
\hline Wien1 & $42-53$ \\
& $54-58$ \\
\hline & $59-61$ \\
& $62-65$ \\
Wien2 & $66-69$ \\
& $70-73$ \\
& $74-77$ \\
& $78-80$ \\
\hline \multirow{4}{*}{ Wien3 } & $81-85$ \\
& $86-89$ \\
& $90-93$ \\
& $94-98$ \\
\hline \multirow{4}{*}{ Wien4 } & $99-101$ \\
& $102-105$ \\
& $106-109$ \\
& $110-113$ \\
& $114-116$ \\
\hline & $117-121$ \\
Wien5 & $122-125$ \\
& $126-129$ \\
& $130-136$
\end{tabular}

\begin{tabular}{|c|c|}
\hline Wien & $\begin{array}{l}\text { Run2 } \\
\text { Slug range in pitt }\end{array}$ \\
\hline \multirow{5}{*}{ Wien6 } & $137-140$ \\
\hline & $141-144$ \\
\hline & $145-148$ \\
\hline & $149-152$ \\
\hline & $153-155$ \\
\hline \multirow{4}{*}{ Wien7 } & $156-160$ \\
\hline & $161-164$ \\
\hline & $165-168$ \\
\hline & 169-171 \\
\hline \multirow{7}{*}{ Wien8a } & $172-176$ \\
\hline & $177-180$ \\
\hline & $181-184$ \\
\hline & $185-188$ \\
\hline & 189-192 \\
\hline & $193-196$ \\
\hline & $197-200$ \\
\hline \multirow{6}{*}{ Wien8b } & $201-204$ \\
\hline & 205-208 \\
\hline & 209-212 \\
\hline & $213-216$ \\
\hline & $217-220$ \\
\hline & $221-225$ \\
\hline \multirow{10}{*}{ Wien9a } & $226-228$ \\
\hline & $229-232$ \\
\hline & $233-236$ \\
\hline & $237-240$ \\
\hline & $241-244$ \\
\hline & $245-248$ \\
\hline & 249-252 \\
\hline & $253-256$ \\
\hline & $257-260$ \\
\hline & $261-264$ \\
\hline \multirow{11}{*}{ Wien9b } & $265-268$ \\
\hline & 269-272 \\
\hline & $273-276$ \\
\hline & $277-280$ \\
\hline & $281-284$ \\
\hline & $285-287$ \\
\hline & 288-292 \\
\hline & 293-296 \\
\hline & $297-300$ \\
\hline & 301-304 \\
\hline & 305-306 \\
\hline Wien10 & $307-321$ \\
\hline
\end{tabular}




\section{Appendix B}

\section{Development of a Kerr Cell for Future PVES Measurements}

In Chapter 5 we described our efforts to correct for false "beam" asymmetries $A_{\text {beam }}$ arising from helicity-correlated beam properties. This correction is quite challenging in periods of $Q_{\text {weak }}$ where the $\mathrm{HC}$ beam differences are large and poorly controlled. In Chapter 4 we presented the procedures to optimize the Jefferson Lab polarized source and suppress HC beam parameters. Even small higher moments of the beam charge asymmetry distribution, such as the beam spot size and halo, can couple to non-linear mechanisms in the accelerator to produce complex beam asymmetries, potentially including the large neutral "beamline" backgrounds that were presented in Chapter 6 .

Future PVES experiments, such as MOLLER [96], SOLID [178] and PREX-II [179] aim to measure smaller asymmetries with higher precision, and will therefore need to achieve better control of $A_{\text {beam }}$ asymmetries. Realizing kinematic damping of the beam phase space through well-matched acceleration will be crucial, but still better control is required for potential sources of false asymmetries in the polarized source, and especially during the fast helicity reversal. Future experiments also require even faster helicity reversal to minimize the noise contribution from target boiling that limits statistical precision. For MOLLER the design specification is a reversal rate of $1920 \mathrm{~Hz}$, twice as fast as $Q_{\text {weak }}$. Significant progress is required to achieve faster transition of the beam polarization to minimize measurement dead-time, with minimal "ringing" effects. Considerable progress was realized for the fast helicity reversal of $Q_{\text {weak }}$ through piezoelectric Pockels crystals (Section 4.3.3), and further improvements may be possible through shaping of the initial high voltage pulse and feedback to actively damp ringing oscillations. There are however inherent limitations in Pockels crystals that may interfere with the design specifications of 
future experiments for faster transitions with better control of $\mathrm{HC}$ beam properties.

A potential solution for future ultra-precise PVES measurements is the development of the technology to use Kerr materials to replace Pockels crystals [180. These materials make use of the quadratic electro-optic Kerr effect to control the polarization of light. In Kerr media the change of their refractive index in the direction of the applied electric field is proportional to the square of the field strength:

$$
\Delta n=\lambda K E^{2} \quad,
$$

where $\lambda$ is the wavelength of light and $K$ is the Kerr constant of the material. The Kerr effect is generally much weaker than the linear Pockels effect, therefore only centrosymmetric materials (gases, liquids, and some crystals) where the linear electro-optic effect is absent are appropriate. A liquid Kerr cell holds the promise of overcoming many inherent limitations of Pockels crystals. In principle it should complete the change in its birefringence state much faster without any ringing effect that is associated with crystal vibrations. It should also be free of birefringence gradients arising from crystal defects and strain, as well as the non-polarization lensing effect due to crystal piezo-electric deformation.

There are also however many practical challenges associated with the use of Kerr materials for our purposes. The weakness of the effect requires very high voltages (of order $30 \mathrm{kV}$, presenting safety concerns) and small electrode spacing to achieve the required quarter-wave retardation. The exotic centrosymmetric materials come with disadvantages, for example nitrobenzene $\left(\mathrm{C}_{6} \mathrm{H}_{5} \mathrm{NO}_{2}\right)$ which exhibits the highest Kerr constant $\left(\sim 2 \cdot 10^{-10} \mathrm{~cm} / \mathrm{V}^{2}\right)$ is poisonous. Since the DC Kerr effect requires the electric field to be applied transversely to the propagation direction, the transverse electric field of the light itself can create a variation of the index of refraction of the material via the optical or AC Kerr effect. This can give rise to non-linear optical effects such as self-focusing, turning a charge asymmetry in the incident light into a spot size asymmetry.

In a Kerr cell the symmetry is provided by the electric field rather than the material, but non-uniformities in the electric field may arise due to charge screening effects (Figure B.1). Such birefringence gradients give rise to position differences in the context of a PVES measurement. Finally, since the Kerr effect is proportional to the square of the electric field a change in the voltage polarity will not reverse the helicity of the resulting beam. Thus two successive Kerr cells are needed to generate states of opposite helicity, as passive plates on a single Kerr cell create field non-uniformities. But having a separate cell generate each state is also a natural source of helicity-correlated asymmetries.

While the challenges are significant and the feasibility of using a Kerr cell to control polarization in a future PVES measurement remains to be proven, the potential benefits of this technology may prove essential on the future PVES program. 


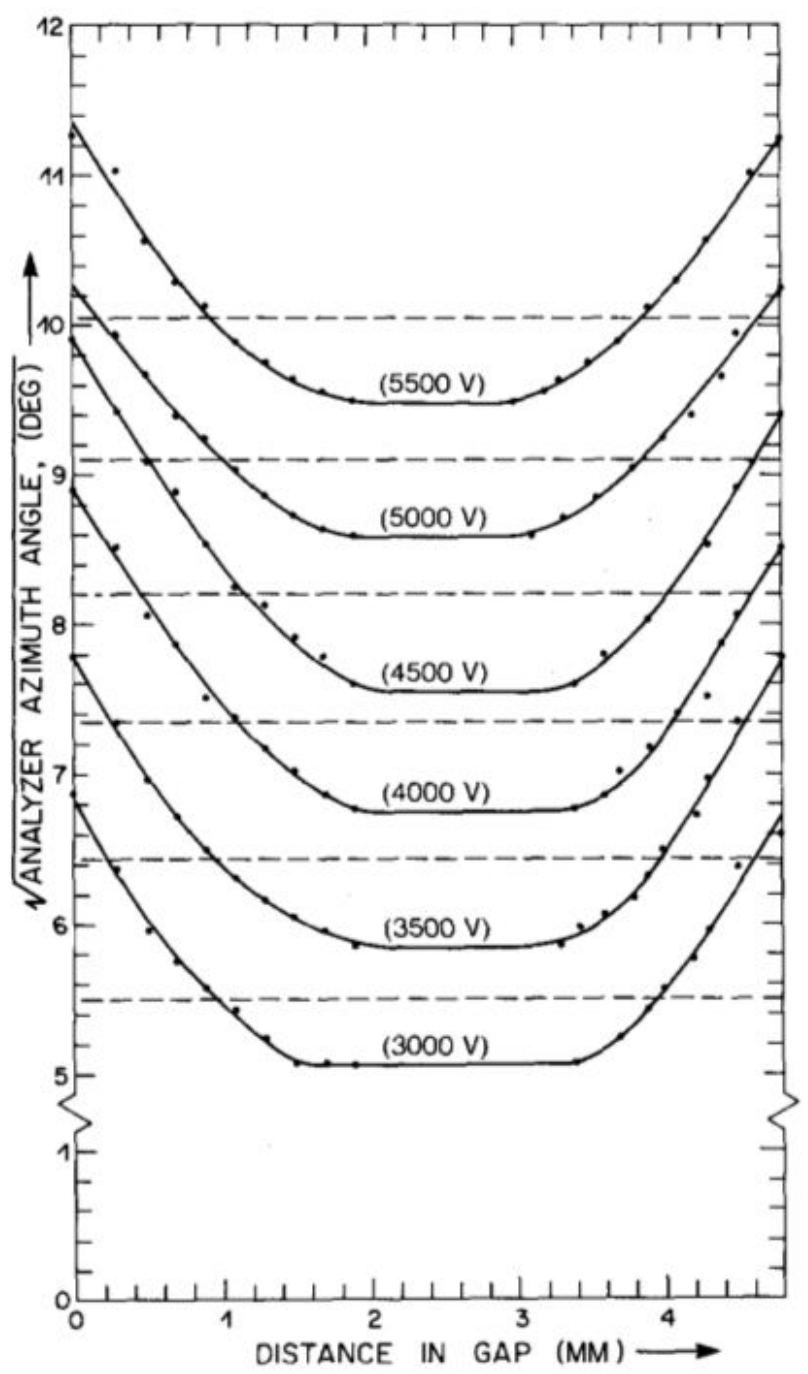

Figure B.1: Experimental data on a nitrobenzene Kerr cell from 181. The vertical axis is proportional to the induced birefringence and the horizontal axis is distance along the entrance face of the Kerr cell. The different lines correspond to different applied voltages. The birefringence gradients arise from accumulation of space charge in the bulk of the dielectric near the electrodes, resulting in voltagedependent optical non-uniformities. 


\section{Appendix C}

\section{Robust Algorithm to Extract}

\section{Regression Parameters}

Regression here does not refer to the specific algorithm to extract sensitivities of the raw detector to beam parameters, which was presented and characterized in Chapter 5 . Here we are concerned with the general regression problem of modeling the relationship between a variable of interest, typically the (corrected) MD asymmetry, and a set of predictive variables/monitors. In this Appendix we study different regression approaches to understand potential sources of bias in the extracted correlation. Ideas from these studies were essential in guiding the development of a correction for the beamline background asymmetry, as well as identifying the beam correction schemes that fail to remove false asymmetries from the main detector.

\section{C.1 Ordinary least squares}

Ordinary least squares (OLS) is a very simple algorithm to fit a function to data, and this discussion will be limited to the simplest case of a straight-line fit. Each data point is defined by pairs of values for the "independent" variable $x$ and the "dependent" variable $y,\left(X_{i}, Y_{i}\right)$, and potentially an uncertainty on the value of the dependent variable, $\sigma_{y}$. The goal of OLS fitting then is to extract the optimal regression parameters $a, b$ of the model $y=a+b x$.

Define $x_{i}, y_{i}$ as the values of the variables predicted by the model at the $i^{\text {th }}$ point, i.e. $y_{i}=a+b x_{i}$ for every point $i$, where $a, b$ are the best available regression parameters. The $x_{i}, y_{i}$ values are also called the expectation values of $X_{i}, Y_{i}$, or in the context of some models that we will see later, the least- squares-adjusted points. Then the goal of the OLS algorithm is to minimize the vertical distances of the data from the best regression line. The optimal regression 


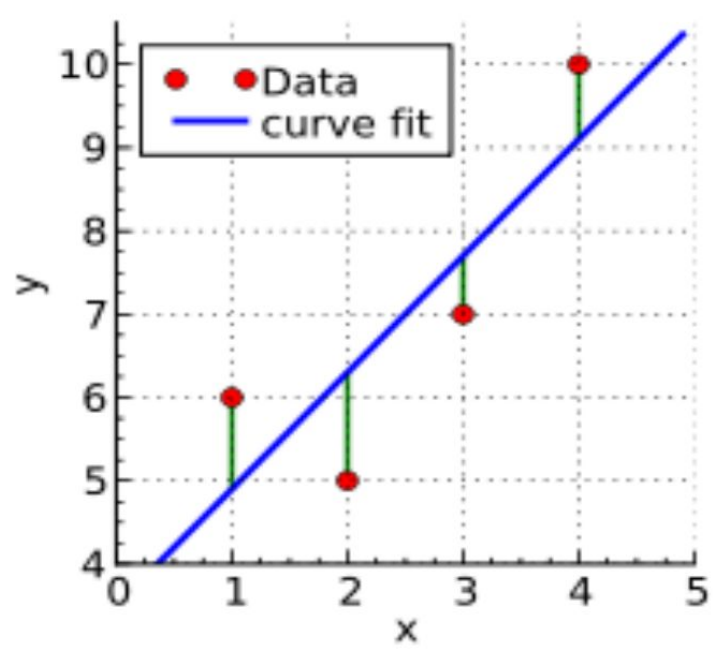

Figure C.1: Ordinary least squares fit to data.

parameters $a, b$ minimize the function:

$$
\chi^{2}=\sum_{i=1}^{N} w_{y_{i}}\left(y_{i}-Y_{i}\right)^{2}
$$

A common choice for the weight $w_{y}$ of each datum is $w_{y_{i}}=1 / \sigma_{y_{i}}^{2}$.

Note that very often in OLS potential errors in the independent variable $x$ are neglected. The great advantage of this algorithm is its simplicity which allows an analytic solution for the regression parameters that minimize the $\chi^{2}$ sum in Eq. C.1. But the simplicity of the OLS algorithm comes at a price. When both variables contain errors any distinction between dependent and independent variables is ambiguous. This can be seen clearly by exchanging the role of the dependent and independent variables and fitting the regression line $x=a^{\prime}+b^{\prime} y$. When the errors are significant one finds $b^{\prime} \neq 1 / b$. The OLS algorithm is not symmetric between the dependent and independent variables.

\section{C.2 Symmetric algorithms}

There have been many algorithms that attempt to incorporate errors of both variables into the extraction of the regression parameters, with a widely varying terminology for these procedures. Authors have used terms such as major axis regression, errors in variables models, rigorous least squares, orthogonal regression, total least squares, maximum likelihood estimation [182, 183]. One can attempt to include the uncertainty of the independent variable in the weight of each datum by projecting the $x$-uncertainty onto the $y$-direction, e.g.:

$$
w_{i}=1 /\left(\sigma_{y_{i}}^{2}+b^{2} \sigma_{x_{i}}^{2}\right),
$$




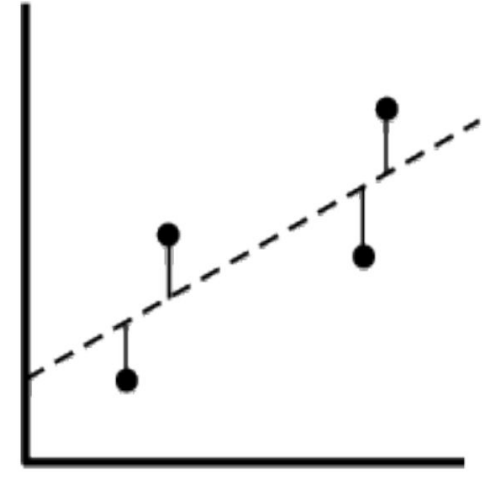

vertical offsets

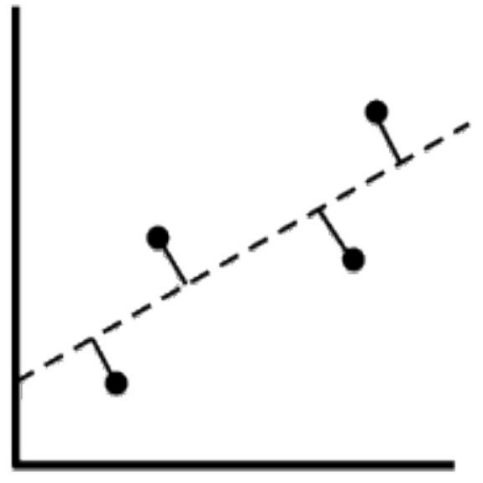

perpendicular offsets

Figure C.2: Minimization of vertical versus perpendicular offsets.

where an intermediate estimation of the slope $b$ may be used. This however is just a projection of the horizontal uncertainty onto the vertical, where the problem then reduces to simple OLS. This is still asymmetric between the two variables. When the $y$-errors are underestimated the result is that the uncertainty of the regression parameters is underestimated; but when the $x$ errors are underestimated then regression bias occurs, i.e. the regression parameters are shifted from their true values.

An algorithm that is symmetric between the two variables will attempt to minimize the weighted sum of the perpendicular distances between each datum and the best regression line, rather than just the vertical distances. The optimal regression parameters will then minimize the function:

$$
\chi^{2}=\sum_{i=1}^{N}\left[w_{y_{i}}\left(y_{i}-Y_{i}\right)^{2}+w_{x_{i}}\left(x_{i}-X_{i}\right)^{2}\right]
$$

Again a simple choice for the weights is $w_{y_{i}}=1 / \sigma_{y_{i}}^{2}, w_{x_{i}}=1 / \sigma_{x_{i}}^{2}$.

Finally we must account for correlation between the errors of the two variables, i.e. the case when the covariance of the $x$ and $y$ errors is not zero. If $r_{i}$ is the correlation coefficient between $x$ and $y$ errors of the $i^{\text {th }}$ point, then the function to minimize is [184]:

$$
\chi^{2}=\sum_{i=1}^{N}\left[w_{y_{i}}\left(y_{i}-Y_{i}\right)^{2}+w_{x_{i}}\left(x_{i}-X_{i}\right)^{2}-2 r_{i} \sqrt{w_{x_{i}} w_{y_{i}}}\left(x_{i}-X_{i}\right)\left(y_{i}-Y_{i}\right)\right] \frac{1}{1-r_{i}^{2}}
$$

The correlation of errors means that the $x$ and $y$ uncertainties of a datum are not orthogonal and should be "rotated". In the case of $Q_{\text {weak }}$ averaging and fitting over a 5-minute runlet, where each datum is an average of asymmetries over that runlet, the correlation of errors may be understood as the correlation coefficient of the quartet-level bivariate distribution between the 

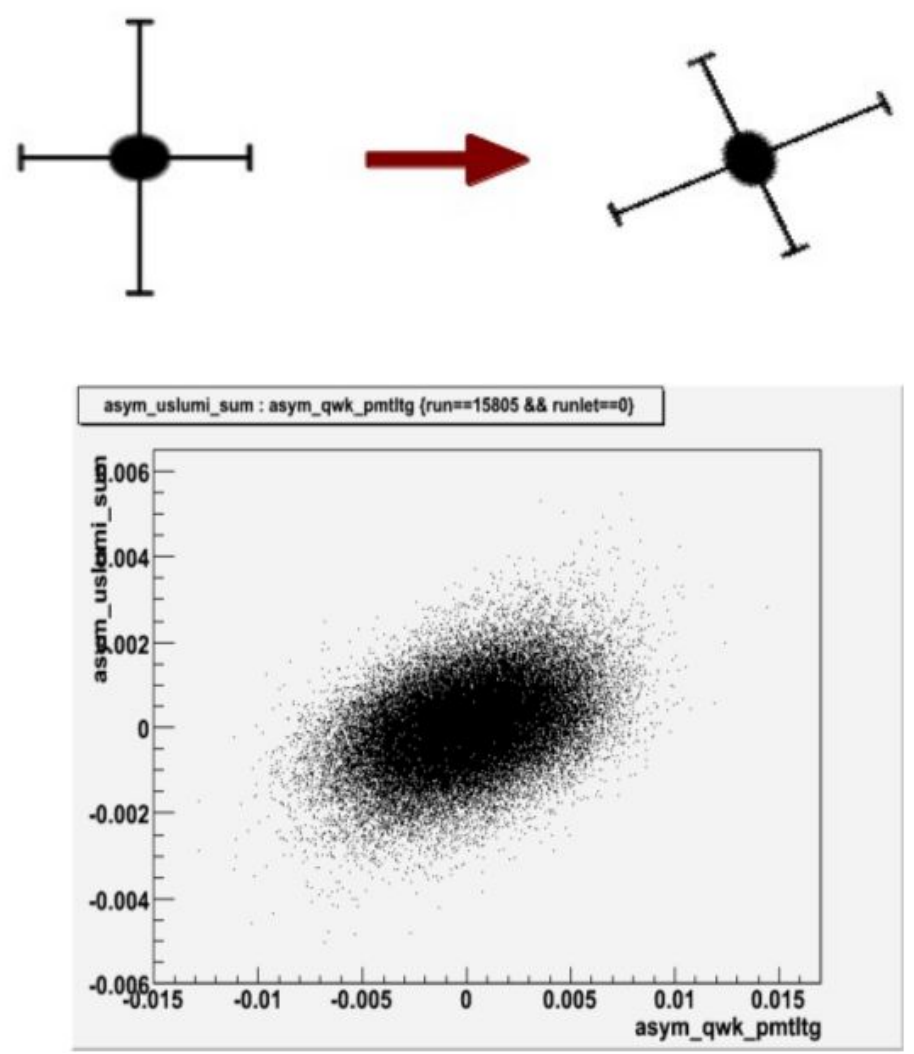

Figure C.3: Correlation in the USLsum and PMTLTG asymmetries over a 5-minute runlet, 15805.0.

variables in that runlet. In Figure C.3 the bivariate distribution of the USLsum and PMTLTG asymmetries is shown in a runlet. This is also shown in the correlation among beam parameters in Figure 5.6. A proper runlet-level fit should account for this correlation of errors, although the significance of this effect averages away at longer time scales. In a quartet-level fit the expected common noise between the detectors should be estimated, but it is not straightforward to understand the common sources of noise for any two detectors. We were quite successful at understanding most of the common noise between the MDall and USLsum asymmetry combinations in Figure 6.14.

\section{C.3 York's algorithm}

Based on Deming's work [185], York [6] and others in the late 60's gave a general solution to minimize the $\chi^{2}$ function C.3 and identified the regression slope as the root of a "least-squares 
cubic" equation. The 2004 York et al. paper [186] describes best the computational algorithm to extract the regression parameters and shows that it is equivalent to other methods. A brief functional description of the algorithm follows. After an approximate initial value of the slope is chosen, for example from OLS, the regression slope is then extracted iteratively. In each iteration the previous estimate of the slope is used to calculate a new and improved estimate, until convergence is reached. In each iteration the slope estimate is given by the very compact form:

$$
b=\frac{\sum W_{i} \beta_{i} V_{i}}{\sum W_{i} \beta_{i} U_{i}},
$$

where $U_{i}=X_{i}-\bar{X}, V_{i}=Y_{i}-\bar{Y}, \bar{X}=\sum W_{i} X_{i} / \sum W_{i}, \bar{Y}=\sum W_{i} Y_{i} / \sum W_{i}$,

$$
W_{i}=\frac{w_{x_{i}} w_{y_{i}}}{w_{x_{i}}+b^{2} w_{y_{i}}-2 b r_{i} \alpha_{i}} \quad, \quad \alpha_{i}=\sqrt{w_{x_{i}} w_{y_{i}}}
$$

and

$$
\beta_{i}=W_{i}\left[\frac{U_{i}}{w_{y_{i}}}+\frac{b V_{i}}{w_{x_{i}}}-\left(b U_{i}+V_{i}\right) \frac{r_{i}}{\alpha_{i}}\right]
$$

After convergence is reached for the slope, the intercept may then be estimated simply as

$$
\alpha=\bar{Y}-b \bar{X}
$$

\section{C.4 Example with $Q_{\text {weak }}$ data}

Using the same data as in Figure C.3 from runlet 15805.0, the regression parameters of the correlation between USLsum and PMTLTG asymmetries are extracted in two cases, where the two variables exchange roles of dependent/independent variables (Figure C.4). The data has been profiled for clarity. Profiling the data is taking the average $y$-value in each $x$-bin and is therefore asymmetric and neglects variations in $x$.

The black lines in Figure C.4 are the results of the standard TH1::Fit command and their regression parameters are given in the statistics boxes, p1 being the regression slope. The parameters of the red lines have been extracted using the iterative algorithm described in Section C.3 with the result for slopes given in red font. The discrepancy is much larger in the left plot of the figure where the PMTLTG is treated as the independent variable. The PMTLTG errors are much larger and more significant than the USLsum errors, therefore neglecting them creates much larger regression bias: the extracted slopes differ by a factor of 7 between the two methods. Neglecting the USLsum erorrs, as in the right plot of Figure C.4 the bias is smaller. In both cases neglecting the errors of the independent variable leads to an underestimation of the regression slope, as is predicted by errors-in-variables models. 

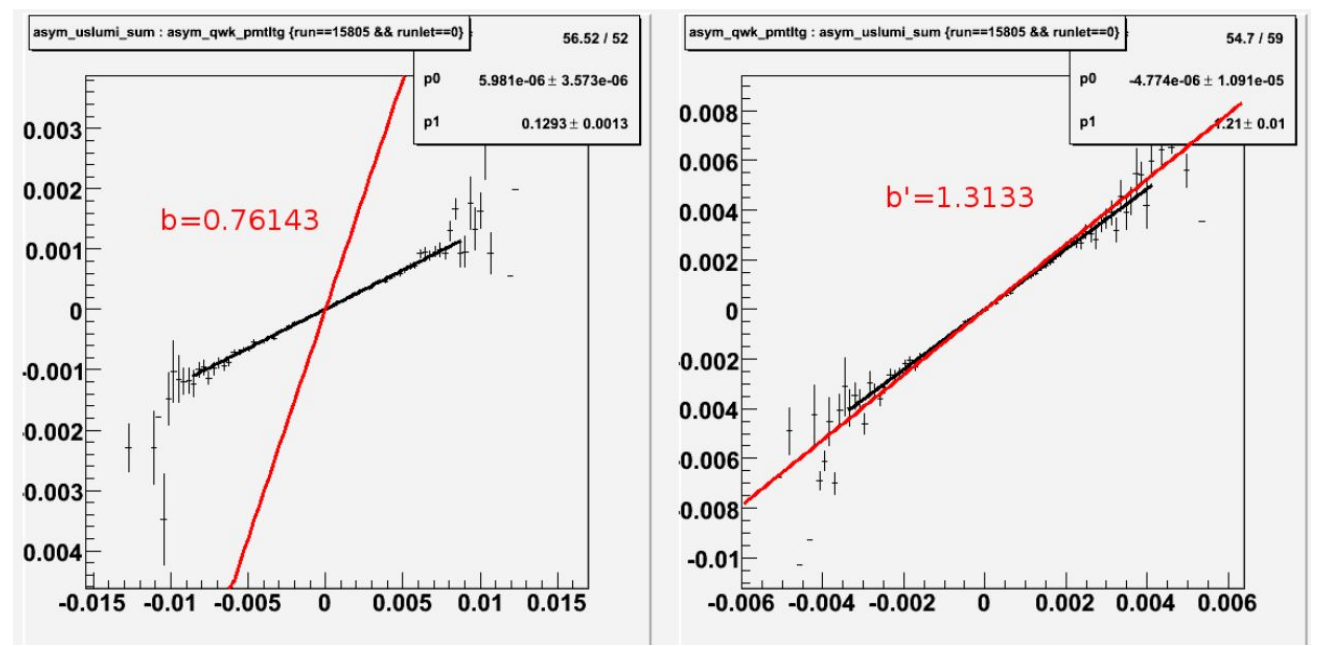

Figure C.4: Fit between the USLsum and PMTLTG asymmetries, exchanging roles as independent and dependent variables (left and right plots) over the same data set. The black lines and fit statistics are extracted from the standard TH1::Fit ROOT command. The red lines correspond to the regression parameters extracted with the symmetric method.

It is almost hard to believe that the red line in the left plot of Figure C.4 is the correct regression line, but the proof is in the pudding: the slopes of the red regression lines, extracted with the algorithm of Section C.3 are almost exact reciprocals of each other:

$$
b_{Y \text { ork }}=0.76143 \approx \frac{1}{1.3133}=\frac{1}{b_{\text {York }}^{\prime}}
$$

The asymmetric calculation of the standard ROOT fit on the other hand fails to produce unambiguous fit results, as is evidenced by the non-reciprocity of the extracted slopes:

$$
b_{\mathrm{ROOT}}=0.1293 \approx \frac{1}{7.73} \neq \frac{1}{b_{\mathrm{ROOT}}^{\prime}}
$$




\section{Bibliography}

[1] C. N. Yang and R. L. Mills. Conservation of isotopic spin and isotopic gauge invariance. Phys. Rev., 96:191, 1954.

[2] W. Heisenberg. Über den Bau der Atomkerne. Z. Physik, 77:1, 1932.

[3] J. Schwinger. Ann. Phys., 2:407, 1957.

[4] M. Gell-Mann. Phys. Rev., 92:833, 1953.

[5] J. Bardeen, L. N. Cooper, and J. R. Schrieffer. Phys. Rev., 108:1175, 1957.

[6] Y. Nambu and G. Jona-Lasinio. Phys. Rev., 122:345, 1961.

[7] J. Goldstone. Field theories with "superconductor" solutions. Il Nuovo Cimento, 19(1):154-164, 1961.

[8] J. Goldstone, A. Salam, and S. Weinberg. Phys. Rev., 127:965, 1962.

[9] P. W. Anderson. Plasmons, gauge invariance, and mass. Physical Review, 130, 1962.

[10] P. W. Higgs. Phys. Lett., 12:132, 1964.

[11] F. Englert and R. Brout. Phys. Rev. Lett., 13:321, 1964.

[12] G. S. Guralnik, C. Hagen, , and T. W. B. Kibble. Phys. Rev. Lett., 13:585, 1964.

[13] Steven Weinberg. A model of leptons. Phys. Rev. Lett., 19:1264-1266, Nov 1967.

[14] Abdus Salam. Weak and electromagnetic interactions. In Elementary Particle Physics, pages 367-377. Almqvist \& Wiskell, Nobel Symposium No. 8 edition, 1968.

[15] F. J. Hasert et al. Observation of neutrino-like interactions without muon or electron in the Gargamelle neutrino experiment. Phys. Lett. B, 46:121, 1973.

[16] L. L. Lewis et al. Phys. Rev. Lett., 39:795, 1977.

[17] C. Y. Prescott et al. Phys. Lett. B, 77:347, 1978. 
[18] C. Rubbia. Experimental observation of the intermediate vector bosons $W^{+}, W$, and $Z^{0}$. Reviews of Modern Physics, 57(3):699, 1985.

[19] Arnaudon et al. Measurement of the mass of the $\mathrm{Z}$ boson and the energy calibration of LEP. Physics Letters B, 307:187193, 1993.

[20] Georges Aad et al. Observation of a new particle in the search for the Standard Model Higgs boson with the ATLAS detector at the LHC. Phys.Lett.B, 2012. 1207.7214.

[21] S. Chatrchyan et al. (CMS Collaboration). Phys. Lett. B, 716:30, 2012.

[22] Nima Arkani-Hamed et al. Phys.Lett. B, 429:263-272, 1998. hep-ph/9803315.

[23] J.M. Campbell et al. Report of the Snowmass 2013 energy frontier QCD working group. October 2013. hep-ph/1310.5189.

[24] G. W. Bennett et al. Phys. Rev. Lett., 89:101804, 2002.

[25] M. Davier et al. Eur. Phys. J. C, 71:1515, 2011.

[26] J. Beringer et al. (Particle Data Group Collaboration). Phys. Rev. D, 86:010001, 2014.

[27] T. Lee and C. Yang. Question of parity conservation in weak interactions. Physical Review, 104(1):254, 1956.

[28] C. Wu, E. Ambler, R. Hayward, D. Hoppes, , and R. Hudson. Experimental test of parity conservation in beta decay. Physical review, 105, 1957.

[29] R. G. Sachs. Nucleon electromagnetic form factors at high momentum transfer. Phys. Rev. Lett., 1964.

[30] J.C. Bernauer et al. (A1 Collaboration). Phys.Rev. C, 90:015206, 2014.

[31] M.J. Musolf, T.W. Donnelly, J. Dubach, S.J. Pollock, S. Kowalski, and E.J. Beise. Intermediate-energy semileptonic probes of the hadronic neutral current. Physics Reports, 239(1-2):1178, 1994.

[32] D.B. Kaplan and A. Manohar. Strange matrix elements in the proton from neutral- current experiments. Nuclear Physics B, 310(3-4):527547, 1988.

[33] Qweak Collaboration. The Qweak experiment: A search for new physics at the TeV scale via a measurement of the protons weak charge. 2007. arXiv:1202.1255v2 [physics.ins-det].

[34] D.M. Webber et al. (MuLan Collaboration). Phys. Rev. Lett., 106:041803, 2011. 
[35] R. Carlini et al. Measurements of parity violation in electron scattering. CEBAF Letter of Intent, 1990.

[36] K. A. Aniol et al. (HAPPEX Collaboration). Phys. Rev. Lett., 82:1096, 1999.

[37] K. A. Aniol et al. (HAPPEX Collaboration). Constraints on the nucleon strange form factors at $Q^{2} \sim 0.1 \mathrm{GeV}^{2}$. Phys. Lett. B, 635:275, 2006.

[38] K. A. Aniol et al. (HAPPEX Collaboration). Parity-violating electron scattering from He-4 and the strange electric form factor of the nucleon. Phys. Rev. Lett., 96:022003, 2006.

[39] A. Acha et al. (HAPPEX collaboration). Precision measurements of the nucleon strange form factors at $Q^{2} \sim 0.1(\mathrm{GeV})^{2}$. Phys. Rev. Lett., 98:032301, 2007.

[40] D. S. Armstrong et al. (G0 Collaboration). Strange quark contributions to parity-violating asymmetries in the forward G0 electron proton scattering experiment. Phys. Rev. Lett., 95:092001, 2005.

[41] D. Androic et al. (G0 Collaboration). Phys. Rev. Lett., 104:012001, 2010.

[42] F. E. Maas et al. (A4 Collaboration). Measurement of strange quark contributions to the nucleons form factors at $Q^{2}=0.230(\mathrm{GeV} / \mathrm{c})^{2}$. Phys. Rev. Lett., 93:022002, 2004 .

[43] F. E. Maas et al. Evidence for strange quark contributions to the nucleons form factors at $Q^{2}=0.108(\mathrm{GeV} / \mathrm{c})^{2}$. Phys. Rev. Lett., 94:152001, 2005.

[44] S. Baunack et al. (PVA4 Collaboration). Phys. Rev. Lett., 102:151803, 2009.

[45] D. T. Spayde et al. (SAMPLE Collaboration). Phys. Lett. B, 583:79, 2004.

[46] T. M. Ito et al. (SAMPLE Collaboration). Phys. Rev. Lett., 92:102003, 2004.

[47] J. Green et al. High-precision calculation of the strange nucleon electromagnetic form factors. Phys. Rev. D, 92:031501, 2015.

[48] P. Wang, D. B. Leinweber, and A. W. Thomas. Phys. Rev. C, 79:065202, 2009.

[49] R. D. Young, J. Roche, R. D. Carlini, and A. W. Thomas. Phys. Rev. Lett., 97:102002, 2006.

[50] Kent Paschke, Anthony Thomas, Robert Michaels, and David Armstrong. Strange vector form factors from parity-violating electron scattering. J.Phys.Conf.Ser., 299:012003, 2011.

[51] P. L. Anthony et al. (SLAC E158 Collaboration). Observation of parity nonconservation in Møller scattering. Physical review letters, 92(18):181602, 2004. 
[52] X. Zheng et al. (PVDIS Collaboration). Measurement of parity violation in electron-quark scattering. Nature, 506, 2014.

[53] Liu J, McKeown RD, and Ramsey-Musolf MJ. Phys.Rev. C, 76:025202, 2007.

[54] Gonzalez-Jimenez R, Caballero J, and Donnelly T. 1111.6918, 2011.

[55] D. Androic et al. (Qweak Collaboration). First determination of the weak charge of the proton. Phys.Rev.Lett. 111, 14, 141803, 2013. arXiv:1307.5275 [nucl-ex].

[56] R. D. Young, R. D. Carlini, A. W. Thomas, and J. Roche. Phys. Rev. Lett., 99:122003, 2007.

[57] J. J. Kelly. Phys. Rev. C, 70:068202, 2004.

[58] S. Zhu, S. J. Puglia, B. R. Holstein, and M. J. Ramsey-Musolf. Nucleon anapole moment and parity-violating ep scattering. Phys. Rev. D, 62(3):033008, 2000.

[59] H. Budd, A. Bodek, and J. Arrington. Modeling quasi-elastic form factors for electron and neutrino scattering. 2003. arXiv:hep-ex/0308005 [hep-ph].

[60] M. Gorchtein and C. J. Horowitz. Phys. Rev. Lett., 102:091806, 2009.

[61] A. Sibirtsev et al. Phys. Rev. D, 82:013011, 2010.

[62] P. G. Blunden et al. Phys. Rev. Lett., 107:081801, 2011.

[63] B. C. Rislow and C.E. Carlson. Phys. Rev. D, 83:113007, 2011.

[64] M. Gorchtein et al. Phys. Rev. C, 84:015502, 2011.

[65] B. C. Rislow and C.E. Carlson. Phys. Rev. D, 88:013018, 2013.

[66] N. L. Hall et al. Phys. Rev. D, 88:013011, 2013.

[67] M. Gorchtein et al. 2013. arXiv:1311.4586 [hep-ph].

[68] N.L. Hall, P.G. Blunden, W. Melnitchouk, A.W. Thomas, and R.D. Young. Quark-hadron duality constraints on $\gamma Z$ box corrections to parity-violating elastic scattering. 2015.

[69] X. Zheng et al. (PVDIS Collaboration). Measurements of parity-violating asymmetries in electron-deuteron scattering in the nucleon resonance region. Physical Review Letters, 111:082501, 2013.

[70] C. S. Wood, S. C. Bennett, D. Cho, B. P. Masterson, J. L. Roberts, C. E. Tanner, and C. E. Wieman. Science, 275:1759, 1997. 
[71] L3 Collaboration. Phys.Lett.B, 623:26-36, 2005.

[72] S. Bethke. arXiv:0908.1135 [hep-ph].

[73] J. Erler et al. The weak charge of the proton and new physics. Phys.Rev. D, 68, 2003.

[74] M. Veltman. Nucl. Phys. B, 123:89, 1977.

[75] W. A. Bardeen, A. J. Buras, D. W. Duke, and T. Muta. Phys. Rev. D, 18:3998, 1978.

[76] S. Weinberg. Phys. Lett. B, 91:51, 1980.

[77] W. J. Marciano and A. Sirlin. Phys. Rev. Lett., 46:163, 1981.

[78] J. Erler et al. The weak mixing angle at low energies. Phys.Rev. D, 72, 2005.

[79] K.S. Kumar, Sonny Mantry, W.J. Marciano, and P.A. Souder. Low energy measurements of the weak mixing angle. Ann.Rev.Nucl.Part.Sci., 63, 2013.

[80] Peskin ME and Takeuchi T. Phys.Rev.Lett., 65, 1990.

[81] Marciano WJ and Rosner JL. Phys.Rev.Lett., 65:2963, 1990.

[82] M. J. Ramsey-Musolf. Low-energy parity-violation and new physics. Phys. Rev. C, 60(1):015501, 1999.

[83] S.G. Porsev, K. Beloy, and A. Derevianko. Precision determination of electroweak coupling from atomic parity violation and implications for particle physics. Physical Review Letters, 102(18):181601, 2009.

[84] V.A. et al. Dzuba. Revisiting parity non-conservation in cesium. Phys.Rev.Lett., 109:203003, 2012. arXiv:1207.5864 [hep-ph].

[85] G.P. Zeller et al. (NuTeV Collaboration). A departure from prediction; Electroweak results from NuTeV. 2002.

[86] J.T. Londergan and A.W. Thomas. Charge symmetry violation corrections to determination of the Weinberg angle in neutrino reactions. Physical Review D, 67(11):111901, 2003.

[87] A.D. Martin et al. Parton distributions incorporating QED contributions. The European Physical Journal C-Particles and Fields, 39(2), 2005.

[88] I.C. Cloet, W. Bentz, and A.W. Thomas. Isovector EMC effect and the NuTeV anomaly. Physical Review Letters, 102(25):252301, 2009. 
[89] P. L. Anthony et al. (SLAC E158 Collaboration). Precision measurement of the weak mixing angle in møller scattering. Phys. Rev. Lett., 95(8):081601, 2005.

[90] J. Erler et al. Weak polarized electron scattering. Annual Review of Nuclear and Particle Science, 64:269-298, 2014.

[91] Davoudiasl H. Low $Q^{2}$ weak mixing angle measurements and rare Higgs decays. 2015.

[92] Davoudiasl H, Lee HS, and Marciano WJ. Phys.Rev. D, 85:115019, 2012.

[93] Phys.Rev.Lett., 109:031802, 2012.

[94] Czarnecki A and Marciano WJ. Phys.Rev. D, 53:1066, 1996. hep-ph/9507420.

[95] G. Aad et al. (ATLAS Collaboration). 2015. arXiv:1505.07645 [hep-ex].

[96] J. Benesch et al. (MOLLER Collaboration). The MOLLER experiment: An ultra-precise measurement of the weak mixing angle using Møller scattering. 2014. arXiv:1411.4088 [nucl-ex].

[97] T.Allison et al. (Qweak Collaboration). Nucl. Instrum. Methods Phys. Res., Sect. A, $781: 105,2015$.

[98] Christoph W. Leemann, David R. Douglas, and Geoffrey A. Krafft. The continuous electron beam accelerator facility: CEBAF at the Jefferson Laboratory. Annu. Rev. Nucl. Part. Sci., 2001.

[99] C.K. Sinclair et al. Development of a high average current polarized electron source with long cathode operational lifetime. Phys.Rev.ST Accel.Beams, 10:023501, 2007.

[100] Donald Geesaman et al. Reaching for the horizon: The 2015 long range plan for nuclear science. 2015.

[101] G.R. Smith, S. Covrig, and J. Dunne. 2009. JLab Technical Design Note JLAB-PHY-091313.

[102] G.R. Smith. Il Nuovo Cimento C, 159:035N04, 2012.

[103] Katherine E. Myers. The First Determination of the Protons Weak Charge Through ParityViolating Asymmetry Measurements in Elastic $e+p$ and $e+A l$ Scattering. PhD thesis, The George Washington University, 2012.

[104] Peiqing Wang. Magnetic field simulation and mapping for the Qweak experiment. Master's thesis, The University of Manitoba, 2007. http://hdl.handle.net/1993/2454. 
[105] Spectrosil 2000 fused quartz material properties. http://www.matweb.com/search/ datasheettext. aspx?matguid=9d934f588f3f44df9396996e94b61c7a

[106] Custom readout electronics, designed and built by TRIUMF, Vancouver, Canada.

[107] John Leacock. Measuring the Weak Charge of the Proton and the Hadronic Parity Violation of the $N-\Delta$ Transition. $\mathrm{PhD}$ thesis, Virginia Polytechnic Institute and State University, 2012. http://www.osti.gov/scitech/servlets/purl/1057572.

[108] J. Pan. Towards a Precision Measurement of Parity-Violating e-p Elastic Scattering at Low Momentum Transfer. PhD thesis, University of Manitoba, Canada, 2012. http: //mspace.lib.umanitoba.ca/jspui/handle/1993/8124.

[109] K.B. Unser. Design and preliminary tests of a beam intensity monitor for LEP. In Proceedings of the 1989 IEEE Particle Accelerator Conference, 1989.

[110] Mark Pitt, 2014. https://www.jlab.org/conferences/moller/fri-pm-talks/Pitt. pdf.

[111] Tom Powers et al. Design, commissioning and operational results of wide dynamic range bpm switched electrode electronics. volume 390, page 257, 1997.

[112] A.S. Hofler et al. Performance of the CEBAF arc beam position monitors. Conf.Proc. C, 930517, 1993.

[113] Buddhini P. Waidyawansa. A 3\% Measurement of the Beam Normal Single Spin Asymmetry in Forward Angle Elastic Electron-Proton Scattering using the Qweak Setup. PhD thesis, Ohio University, 2014.

[114] C. Yan, R. Carlini, and D. Neuffer. Beam energy measurement using the Hall C beam line. 1993.

[115] Valery Lebedev. OptiM - Computer code for linear and non-linear optics calculations. http://pbar.fnal.gov/organizationalchart/lebedev/OptiM/optim.htm

[116] M. Hauger et al. A high-precision polarimeter. Nuclear Instruments and Methods in Physics Research Section A, 462, 2001.

[117] M. Loppacher. Møller polarimetry for CEBAF Hall C. Master's thesis, University of Basel, Switzerland, 1996.

[118] K. Halbach and R.F. Holsinger. Particle Accelerators, 7:213, 1976. 
[119] Joshua Magee. The Qweak Run 2 Moller polarimetry analysis. Technical report, 2014. Qweak Document 1955 [Collaboration access].

[120] C.Y. Prescott. Technical report. SLAC-TN-73-001.

[121] Amrendra Narayan. Determination of electron beam polarization using electron detector in Compton polarimeter with less than $1 \%$ statistical and systematic uncertainty. PhD thesis, Mississippi State University, 2015.

[122] T.B. Humensky et al. SLAC's polarized electron source laser system and minimization of electron beam helicity correlations for the E-158 parity violation experiment. Nucl.Instrum.Meth. A, 521, 2004.

[123] K.D. Paschke. AIP Conference Proceedings, 853:1149, 2008.

[124] Dave Mack. Excess noise factors from the helicity gate scan at 60 and $145 \mu \mathrm{A}$. Qweak Analysis \& Simulation elog 101 [Collaboration access].

[125] P. King. Swing shift summary 28/04/2011.

[126] Richard Jones. http://zeus.phys.uconn.edu/qweak/filters/Random.cgi.

[127] J. Hansknecht and M. Poelker. Phys. Rev. ST Accel. Beams, 9:063501, 2006.

[128] E. S. Fulkerson et al. Driving Pockels cells using avalanche transistor pulsers. 11th IEEE International Pulse Power Conference, 1997.

[129] PVX-4110 pulse generator. https://www.jlab.org/accel/inj_group/laser2001/ pockels_files/sppvx-4110.pdf.

[130] OC-100-HG opto-coupler Voltage Multipliers Inc. http://www.voltagemultipliers.com.

[131] Pockels cell driver response tests. https://www.jlab.org/accel/inj_group/laser2001/ pockels.htm.

[132] J. M. Grames et al. Proceedings of the 2007 Particle Accelerator Conference, 2007.

[133] T. Maruyama et al. Appl. Phys. Lett., 85:2640, 2004.

[134] T.B. Humensky et al. Probing The Standard Model And Nucleon Structure Via Parityviolating Electron Scattering. PhD thesis, Princeton University, 2003.

[135] K. Aulenbacher. SLAC 432-Rev., 1994. SLAC, Stanford, CA.

[136] A. Adderley et al. Physical Review Special Topics: Accelerator Beams, 13, 2010. 
[137] M. Breidenbach et al. Nucl. Instrum. Methods Phys. Res., Sect. A, 350:1, 1994.

[138] R. A. Mair et al. Anisotropies in strain and quantum efficiency of strained GaAs grown on GaAsP. Phys. Lett. A, 212:231, 1996.

[139] C. E. Carlson and M. Vanderhaeghen. Annu. Rev. Nucl. Part. Sci., 57, 2007.

[140] R. Silwal. Probing the Strangeness Content of the Proton and the Neutron Radius of $208 \mathrm{~Pb}$ using Parity-Violating Electron Scattering. PhD thesis, University of Virginia, Charlottesville, 2012.

[141] G.D. Cates et al. Nucl. Instr. and Meth. A, 278, 1989.

[142] M. Kargiantoulakis. Spot size asymmetry coupling to QE hole effect, 2012. Qweak Beam elog 225 [Collaboration access].

[143] R. A. Mair et al. Phys. Lett. A, 212:231, 1996.

[144] Pavel Evtushenko. Large dynamic range beam diagnostics for high current LINACs. http: //web.mit.edu/lns/PEB_Workshop/talks/\%20P.Evtushenko.pdf.

[145] Douglas H. Beck and Mark L. Pitt. Beam optics for electron scattering parity-violation experiments. Eur Phys J A, 2005.

[146] E.D. Courant and H.S. Snyder. Theory of the alternating gradient synchrotron. Annals Phys., 3, 1958.

[147] Y. Chao. Measuring and matching transport optics at Jefferson Lab. Procceedings of the 2003 Particle Accelerator Conference, 2003.

[148] Rakitha Beminiwattha. A Measurement of the Weak Charge of the Proton through Parity Violating Electron Scattering using the Qweak Apparatus. PhD thesis, Ohio University, 2013.

[149] Compact nested steering magnets, parts no. HRC 334 and no. HRC 335, from Haimson Research Corporation, Santa Clara, CA.

[150] Kent Paschke. Double peaking. Qweak Analysis \& Simulation elog 729 [Collaboration access].

[151] M.Tiefenbach. Double peaking in 3c12 x-position. https://logbooks.jlab.org/entry/ 1566487

[152] Peng Zang. Monitors correlations. Qweak Analysis \& Simulation elog 1389 [Collaboration access]. 
[153] Dave Mack. The apparent nonlinearity in Run I is dominated by BCM noise: A simulation. Qweak Analysis \& Simulation elog 1216 [Collaboration access].

[154] Manolis Kargiantoulakis. Comparison between "residual" and "sign-corrected" methods. Qweak Analysis \& Simulation elog 1447 [Collaboration access].

[155] Peng Zang. Dithering correction error - systematic. Qweak Analysis \& Simulation elog 1514 [Collaboration access].

[156] Peng Zang. Dithering correction error - statistic. Qweak Analysis \& Simulation elog 1511 [Collaboration access].

[157] Adesh Subedi. Determination of the weak charge of the proton through parity violating asymmetry measurements in the elastic e+p scattering. $\mathrm{PhD}$ thesis, Mississippi State University, 2014.

[158] Mark Dalton. C laser phase changes interception. https://hallcweb.jlab.org/hclog/ 1202_archive/120228085527.html.

[159] Wade Duvall. [W-shutter] Run 2 correlations. Qweak Analysis \& Simulation elog 836 [Collaboration access].

[160] Greg Smith. Target boiling/target noise. Technical report, 2011. Qweak Document 1353 [Collaboration access].

[161] C. N. Papanicolas and E. Stiliaris. A novel method of data analysis for hadronic physics. 2012. arXiv:1205.6505 [hep-ph].

[162] Peng Zang. Correlation between uslumisum, pmtonl and pmtltg. Qweak Analysis \& Simulation elog 1102 [Collaboration access].

[163] Wade Duvall. Charge slope correlations during the W-shutter running. Qweak Analysis \& Simulation elog 1482 [Collaboration access].

[164] K. A. Aniol et al. (HAPPEX Collaboration). Phys. Rev. C, 69:065501, 2004.

[165] L. W. Mo and Y. S. Tsai. Rev. Mod. Phys., 41, 1969.

[166] David Armstrong. $Q^{2}$ Determination. Qweak Document 2178 [Collaboration access].

[167] Buddhini Waidyawansa. Transverse leakage error for Qweak. Qweak Document 2245 [Collaboration access].

[168] Rob Mahurin. Description of Qweak PMT linearity tests using the EEL126 test stand. Technical report, 2013. Qweak Document 1838 [Collaboration access]. 
[169] W. Pauli. Handbuch der Physik, 1, 1958. edited by S. Fl"gge.

[170] H. Batelaan, T.J. Gay, and J.J. Schwendiman. Stern-Gerlach effect for electron beams. Phys.Rev.Lett., 79:4517-4521, 1997.

[171] H. Majlesi. Observing the spin of free electrons in action. 2014. arXiv:1504.07963 [quant$\mathrm{ph}]$.

[172] Josh Magee. Aluminum dilution status report. Technical report, 2015. Qweak Document 2266 [Collaboration access].

[173] J. Arrington and I. Sick. Precise determination of low-Q nucleon electromagnetic form factors and their impact on parity-violating e-p elastic scattering. Physical Review C, 76:035201, 2007.

[174] P.E. Shanahan et al. Charge symmetry violation in the electromagnetic form factors of the nucleon. Physical Review D, 91:113006, 2015.

[175] G. Smith. Model sensitivities in the extraction of Qw(p) from PVES data. 2015. Presented at the 2015 Fall Meeting of the APS Division of Nuclear Physics.

[176] K.G. Chetyrkin, J.H. Kuehn, and M. Steinhauser. Corrections of order $\mathcal{O}\left(G_{F} M_{t}^{2} \alpha_{s}^{2}\right)$ to the $\rho$ parameter. Phys.Lett. B, 351:331-338, 1995.

[177] Jens Erler and Shufang Su. The weak neutral current. Progress in Particle and Nuclear Physics, 71:119 - 149, 2013. Fundamental Symmetries in the Era of the LHC.

[178] P. Souder et al. Precision measurement of parity-violation in deep inelastic scattering over a broad kinematic range. 2009. http://www.jlab.org/exp_prog/proposals/10/ PR12-10-007.pdf.

[179] K. Paschke et al. (Hall A Collaboration). PREX-II: Precision parity-violating measurement of the neutron skin of lead. 2011. http://www.jlab.org/exp_prog/proposals/11/ PR12-11-101.pdf.

[180] M. M. Dalton. Demands on polarized electron sources by future experiments in parity violating electron scattering. PoS, PSTP2013:032, 2013.

[181] Artur W. Knudsen. The Kerr effect in nitrobenzene. American Journal of Physics, 43, 1975.

[182] J. R. Macdonald and W. J. Thompson. Least-squares fitting when both variables contain errors: Pitfalls and possibilities. Am. J. Phys., 60(1), 1992. 
[183] C. A. Cantrell. Technical note: Review of methods for linear least-squares fitting of data and application to atmospheric chemistry problems. Atmos. Chem. Phys., 8, 2008.

[184] D. York. Least squares fitting of a straight line with correlated errors. Earth and Planet. Sci. Lett., 5, 1969.

[185] W.E.Deming. Statistical adjustmant of data. 1943.

[186] D. York, N. M. Evensen, M. L pez Martinez, and J. De Basabe Delgado. Unified equations for the slope, intercept, and standard errors of the best straight line. 72(3), 2004. 